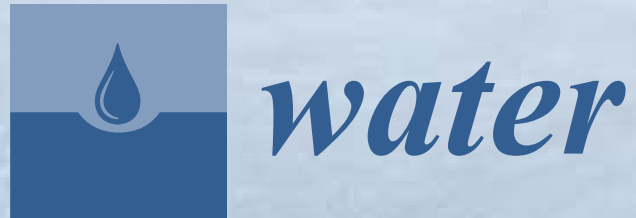

\title{
Turbulence in River and Maritime
} Hydraulics

\section{Michele Mossa, Donatella Termini and Peter A. Davies \\ Printed Edition of the Special Issue Published in Water}


Turbulence in River and Maritime Hydraulics 



\section{Turbulence in River and Maritime Hydraulics}

Special Issue Editors

Michele Mossa

Donatella Termini

Peter A. Davies 
Michele Mossa

Polytechnic University of Bari

Italy
Donatella Termini

University of Palermo

Italy
Peter A. Davies

University of Dundee

UK

\section{Editorial Office}

MDPI

St. Alban-Anlage 66

4052 Basel, Switzerland

This is a reprint of articles from the Special Issue published online in the open access journal Water (ISSN 2073-4441) in 2018 (available at: https://www.mdpi.com/journal/water/special_issues/ turbulence)

For citation purposes, cite each article independently as indicated on the article page online and as indicated below:

LastName, A.A.; LastName, B.B.; LastName, C.C. Article Title. Journal Name Year, Article Number, Page Range.

ISBN 978-3-03897-594-6 (Pbk)

ISBN 978-3-03897-595-3 (PDF)

(C) 2019 by the authors. Articles in this book are Open Access and distributed under the Creative Commons Attribution (CC BY) license, which allows users to download, copy and build upon published articles, as long as the author and publisher are properly credited, which ensures maximum dissemination and a wider impact of our publications.

The book as a whole is distributed by MDPI under the terms and conditions of the Creative Commons license CC BY-NC-ND. 


\section{Contents}

About the Special Issue Editors $\ldots \ldots \ldots \ldots \ldots \ldots \ldots$ vii

Preface to "Turbulence in River and Maritime Hydraulics" $\ldots \ldots \ldots \ldots \ldots \ldots$. . . . . .

Michele Mossa, Donatella Termini and Peter Davies

Turbulence in River and Maritime Hydraulics

Reprinted from: Water 2018, 10,963, doi:10.3390/w10070963 . . . . . . . . . . . . . .

Simone Ferrari, Maria Grazia Badas and Giorgio Querzoli

On the Effect of Regular Waves on Inclined Negatively Buoyant Jets

Reprinted from: Water 2018, 10, 726, doi:10.3390/w10060726 . . . . . . . . . . . . . 4

Laura Maria Stancanelli, Rosaria Ester Musumeci and Enrico Foti

Computational Fluid Dynamics for Modeling Gravity Currents in the Presence of Oscillatory

Ambient Flow

Reprinted from: Water 2018, 10, 635, doi:10.3390/w10050635 . . . . . . . . . . . . 22

Christy Ushanth Navaratnam, Jochen Aberle, Jie Qin and Pierre-Yves Henry

Influence of Gravel-Bed Porosity and Grain Orientation on Bulk Flow Resistance

Reprinted from: Water 2018, 10, 561, doi:10.3390/w10050561 . . . . . . . . . . . . . . 40

Lorenzo Melito, Matteo Postacchini, Giovanna Darvini and Maurizio Brocchini

Waves and Currents at a River Mouth: The Role of Macrovortices, Sub-Grid Turbulence and

Seabed Friction

Reprinted from: Water 2018, 10, 550, doi:10.3390/w10050550 . . . . . . . . . . . . . 56

Michele Mossa and Peter A. Davies

Some Aspects of Turbulent Mixing of Jets in the Marine Environment

Reprinted from: Water 2018, 10, 522, doi:10.3390/w10040522 . . . . . . . . . . . . . 85

Nadia Penna, Mauro De Marchis, Olga B. Canelas, Enrico Napoli, António H. Cardoso and

Roberto Gaudio

Effect of the Junction Angle on Turbulent Flow at a Hydraulic Confluence

Reprinted from: Water 2018, 10, 469, doi:10.3390/w10040469 . . . . . . . . . . . . . 105

Didem Yılmazer, Ayşe Yüksel Ozan and Kubilay Cihan

Flow Characteristics in the Wake Region of a Finite-Length Vegetation Patch in a Partly Vegetated Channel

Reprinted from: Water 2018, 10, 459, doi:10.3390/w10040459 . . . . . . . . . . . . . 128

Paolo Peruzzo, Francesca De Serio, Andrea Defina and Michele Mossa

Wave Height Attenuation and Flow Resistance Due to Emergent or Near-Emergent Vegetation

Reprinted from: Water 2018, 10, 402, doi:10.3390/w10040402 . . . . . . . . . . . . . . . 144

Sara Pascolo, Marco Petti and Silvia Bosa

Wave-Current Interaction: A 2DH Model for Turbulent Jet and Bottom-Friction Dissipation

Reprinted from: Water 2018, 10, 392, doi:10.3390/w10040392 . . . . . . . . . . . . . . 157 
Diana De Padova, Maurizio Brocchini, Federica Buriani, Sara Corvaro, Francesca De Serio, Michele Mossa and Stefano Sibilla

Experimental and Numerical Investigation of Pre-Breaking and Breaking Vorticity within a Plunging Breaker

Reprinted from: Water 2018, 10, 387, doi:10.3390/w10040387 . . . . . . . . . . . . . . . . 174

Gustavo Tomas, Tobias Bleninger, Colin D. Rennie and Henrique Guarneri

Advanced 3D Mapping of Hydrodynamic Parameters for the Analysis of Complex Flow Motions in a Submerged Bedrock Canyon of the Tocantins River, Brazil

Reprinted from: Water 2018, 10, 367, doi:10.3390/w10040367

Donatella Termini and Tommaso Moramarco

Dip Phenomenon in High-Curved Turbulent Flows and Application of Entropy Theory

Reprinted from: Water 2018, 10, 306, doi:10.3390/w10030306 . . . . . . . . . . . . . 210

Mouldi Ben Meftah and Michele Mossa

Turbulence Measurement of Vertical Dense Jets in Crossflow

Reprinted from: Water 2018, 10, 286, doi:10.3390/w10030286

Nikolaos Th. Fourniotis, Georgios M. Horsch and Georgios A. Leftheriotis

On the Hydrodynamic Geometry of Flow-Through versus Restricted Lagoons

Reprinted from: Water 2018, 10, 237, doi:10.3390/w10030237 . . . . . . . . . . . . . . . . 244

Sandro Longo, Maria Clavero, Luca Chiapponi and Miguel A. Losada

Invariants of Turbulence Reynolds Stress and of Dissipation Tensors in Regular Breaking Waves

Reprinted from: Water 2017, 9, 893, doi:10.3390/w9110893 . . . . . . . . . . . . . . 260 


\section{About the Special Issue Editors}

Michele Mossa is Professor of Hydraulics at the Polytechnic University of Bari (Italy). He is chief scientist of the Coastal Engineering Laboratory-LIC and a member of the board of directors of the National Consortium of Universities for Marine Sciences (CoNISMa) for the Polytechnic University of Bari. The main topics of his research are related to environmental and maritime hydraulics.

Donatella Termini is Professor of Hydraulics at the University of Palermo (Italy). Her research activity mainly regards fluvial processes and water distribution network models. Her present research efforts include the prediction of river morphological evolution, both through experimental investigations and by the development of numerical simulation codes.

Peter A. Davies is Professor of Fluid Dynamics at the University of Dundee (UK), where he works in environmental and geophysical fluid mechanics, including buoyancy-driven flows, rotating flows, turbulent mixing, internal waves, and wastewater disposal. 



\section{Preface to "Description of the Cover Image"}

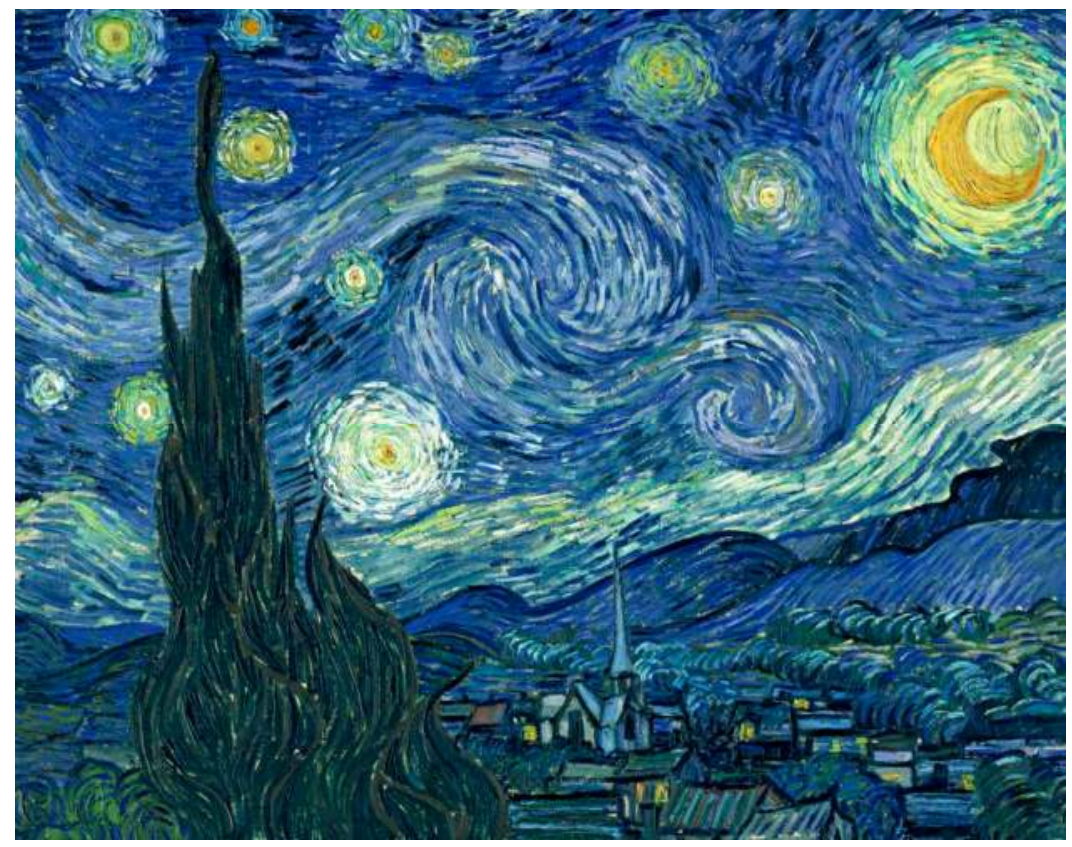

Starry Night, Vincent van Gogh, 1889 Oil on canvas, $73 \times 92 \mathrm{~cm}, 28^{3} / 4 \times 36^{1 / 4}$ in Museum of Modern Art, New York City.

Art experts have long marvelled at the emotional chaos apparent in the later paintings of the Dutch artist Vincent Van Gogh. Perhaps that is because the images reflect light in a way that mimics the physics of turbulence. At least that is the view of a team of physicists led by Jose Luis Aragon of the National Autonomous University of Mexico, who analysed several of VanGogh's later paintings, including Starry Night, Road with Cypress and Star and Wheat Field with Crows. Mathematically, they studied how the luminosity, which is a measure of the total amount of visible light reflected off the paintings, varied across the canvas. Specifically, they analysed the likelihood that two points a distance D apart would have the same, or similar, brightness. In each of the paintings, they found that points further apart were statistically less likely to have similar luminosities. This in itself is not surprising. But this probability decreased in a very simple way, in proportion to the distance between the points, D, raised to some power. This pattern is significant. The very same pattern characterizes variations in fluid velocity at different points in a churning, turbulent liquid, a property called Kolmogorov scaling, after the Russian physicist Andrei Kolmogorov. "Some art critics, have said that Van Gogh's paintings give the impression of looking through a turbulent atmosphere," says team member Gerardo Naumis. "But we were very, very surprised by the close link to Kolmogorov's theory."

Also surprising is the difference between Van Gogh's later and earlier work .The pattern of luminosity in the paintings Van Gogh created during periods of emotional calm bears little resemblance to real-world turbulence. A strong visual sense of turbulence, apparent also in the mathematical analysis, appeared only in paintings created during times when he was psychologically disturbed. Van Gogh painted his famous Starry Night, for example, during a year spent in an asylum. In contrast, he completed his Self-Portrait with Pipe and Bandaged Ear 
in a state of self-described "absolute calm". Mathematically, this work lacks the signature of turbulence. Naumis speculates that there could be some link between fluid turbulence and the dynamics of neural processes in disturbed individuals, and that mathematics might provide a means for detecting psychic disturbance through the analysis of drawings. "The work so far is only a first step," he says. "We need to apply it to patients and see if it works".

(By Mark Buchanan, New Scientist, 15 July 2006) 


\section{Preface to "Turbulence in River and Maritime Hydraulics"}

Understanding of the role of turbulence in controlling transport processes is of paramount importance for the preservation and protection of aquatic ecosystems, the minimization of the deleterious consequences of anthropogenic activity, and the successful sustainable development of river and maritime areas. In this context, the present Special Issue collects 15 papers which provide a representation of the present understanding of turbulent processes and their effects in river and maritime environments. The presented collection of papers is not exhaustive, but it highlights the key priority areas and knowledge gaps in this field of research.

Michele Mossa, Donatella Termini, Peter A. Davies Special Issue Editors 



\title{
Editorial
}

\section{Turbulence in River and Maritime Hydraulics}

\author{
Michele Mossa ${ }^{1}$, Donatella Termini ${ }^{2}$ and Peter Davies ${ }^{3, *}$ \\ 1 Department of Civil, Environmental, Land, Building Engineering and Chemistry, Polytechnic University of \\ Bari, 70125 Bari, Italy; michele.mossa@poliba.it \\ 2 Dipartimento di Ingegneria Civile, Ambientale, Aerospaziale, dei Materiali, Polytechnique School, \\ University of Palermo, 90128 Palermo, Italy; donatella.termini@unipa.it \\ 3 Department of Civil Engineering, University of Dundee, Dundee DD1 4HN, UK \\ * Correspondence: p.a.davies@dundee.ac.uk; Tel.: +44-138-238-4346
}

Received: 11 July 2018; Accepted: 20 July 2018; Published: 23 July 2018

\begin{abstract}
Understanding of the role of turbulence in controlling transport processes is of paramount importance for the preservation and protection of aquatic ecosystems, the minimisation of deleterious consequences of anthropogenic activity, and the successful sustainable development of river and maritime areas. In this context, the present Special Issue collects 15 papers which provide a representation of the present understanding of turbulent processes and their effects in river and maritime environments. The presented collection of papers is not exhaustive but it allows for highlighting key priority areas and knowledge gaps in this field of research.
\end{abstract}

Keywords: rivers; maritime areas; turbulent processes

\section{Introduction to the Special Issue}

As the contents of this Special Issue of Water illustrate, there remains an enduring fascination with the classical topic of turbulence and the properties of turbulent flows in an environmental context. Despite many years of investigation and many major advances, the subject continues to offer interesting and difficult research challenges. Nowhere are these challenges more prominent than in the fields of environmental fluid mechanics and environmental hydraulics, where an understanding of the role(s) of turbulence in controlling transport processes (mass, heat, solute, momentum) is crucial for the sensitive management of natural resources, the preservation and protection of aquatic ecosystems, the minimisation of deleterious consequences of anthropogenic activity, and the successful sustainable development of river and maritime areas. In order to achieve these goals, there is an urgent need for research on the fundamental properties and action(s) of turbulence to enable and guide appropriate implementation of the above objectives. Modelling investigations (analytical, laboratory, numerical) have key roles to play in this process (in conjunction with carefully-designed field measurement and monitoring campaigns), if the demands and standards of regulatory agencies are not only formulated correctly but also applied effectively. The papers contained within this issue provide an illustration of the current activity and the strength of these approaches.

The importance of recognising and understanding turbulent processes in the aquatic environment has increased with: (1) the nonlinear global economic development that has occurred in the past 50 years; (2) the recognition that such growth has the potential for (and in many cases has caused) significant negative impacts on such environments; and (3) the unrelenting social and political opposition to such consequences. These factors have taken place during a period in which energy sources have shifted and diversified and demands on water for domestic and industrial consumption have grown. All of these developments have resulted in the emergence of new and important problems in environmental fluid mechanics that rely on an understanding of turbulent processes for their amelioration or solution. In some cases, the existing research tools required to prepare responses to new external environmental threats are poorly developed or inadequate. 
In the river and maritime context, recent examples are: (1) Desalination and solution mining activities to meet, respectively, water and gas storage needs, with resultant discharge of extremely high concentration brine into sensitive marine waters populated by vulnerable flora and fauna; (2) increased flood protection measures that affect sediment distributions in rivers and coastal zones and affect the state of bordering wetlands; and (3) traffic management (tunnels, bridges, harbours, etc.) that disrupt and modify established natural flow and wave conditions. A common and continued theme in this context for many years has been the pollution of the coastal marine environment as a result of anthropogenic activities associated with, for example: (1) The discharge of domestic and industrial waste water; (2) the unregulated and accidental release of petroleum and hydrocarbon products from marine traffic and offshore exploration/production installations; (3) the run-off of animal waste and fertiliser-derived nutrients from agricultural land; and (4) the construction of marine structures and waste disposal and treatment systems. All of these processes affect population health, marine ecology, economic prosperity, commercial operations, and environmental sustainability. Likewise, all of the scientific and engineering challenges associated with tackling the negative consequences require detailed knowledge of the hydrodynamic state of the affected zones. This is particularly important when it is clear that the tools available to satisfy regulatory authority guidelines may no longer be adequate for some environmental scenarios.

From a demand point of view, burgeoning interest in understanding the nature of turbulence in river and marine regions has been stimulated in recent years by: (1) The identification of numerous, previously-overlooked or previously-intractable turbulence processes controlling key environmental phenomena; (2) the recognition of the inter-connected roles of turbulent flow, sediment, vegetation, waves, boundary conditions, and the spatial and temporal distributions of contaminant sources in determining the state of the aquatic environment; and (3) the realisation of the importance of multiand inter-disciplinary approaches to meet, target, and solve ecosystem challenges. Concurrent with the above developments, there has been significant "supply" growth in computational tools to develop new and powerful numerical modelling approaches to such problems, together with technological improvements in measurement devices for laboratory and field conditions to enable increased spatial and temporal resolution to be achieved for the distribution of key environmental parameters (e.g., velocity, vorticity, solute, contaminants, dissolved oxygen etc.) in turbulent flows relevant to the problems outlined above.

The wide-ranging survey above surely confirms that no justification is required to assess recent progress in understanding turbulence and turbulent processes through the medium of this thematic Special Issue. The constituent papers have been assembled from the work of individuals and groups of researchers working in a wide range of research areas in river and maritime environments, in order to provide a timely indication of the breadth and depth of current research effort in these areas. Not least, the contents of the papers serve to highlight key priority areas, knowledge gaps, and growth points in research activity in river, coastal, estuarine, and near-shore environments. The 15 papers contained within the Special Issue reflect the range of problems where new high quality data from laboratory and CFD (Computational Fluid Dynamics) modelling studies, and field measurements aid the interpretation of classical flow features in complex flows in: (1) Open channels, river mouths and river canyons [1-8]; (2) breaking waves and wave-current interactions in coastal zones [9-14]; and (3) gravity currents in natural channels [15]. Most significantly, the papers provide examples of where emerging problems associated with the disposal of dense brine into the aquatic environment are being tackled by detailed measurements of the turbulence properties of the flows.

The choice of papers for inclusion was dictated primarily by the timeliness of the work and the focus of the studies on turbulence and turbulent processes applicable to the chosen environmental zones. Overviews of a particular topic of research in individual areas of expertise and exposure of the current and future challenges associated with these areas were particularly encouraged. Rigorous reviewing protocols were applied in selecting the papers for inclusion.

Conflicts of Interest: The authors declare no conflict of interest. 


\section{References}

1. Navaratnam, C.U.; Aberle, J.; Qin, J.; Henry, P. Influence of Gravel-Bed Porosity and Grain Orientation on Bulk Flow Resistance. Water 2018, 10, 561. [CrossRef]

2. Penna, N.; De Marchis, M.; Canelas, O.B.; Napoli, E.; Cardoso, A.H.; Gaudio, R. Effect of the Junction Angle on Turbulent Flow at a Hydraulic Confluence. Water 2018, 10, 469. [CrossRef]

3. Yllmazer, D.; Ozan, A.Y.; Cihan, K. Flow Characteristics in the Wake Region of a Finite-Length Vegetation Patch in a Partly Vegetated Channel. Water 2018, 10, 459. [CrossRef]

4. Peruzzo, P.; De Serio, F.; Defina, A.; Mossa, M. Wave Height Attenuation and Flow Resistance Due to Emergent or Near-Emergent Vegetation. Water 2018, 10, 402. [CrossRef]

5. Tomas, G.; Bleninger, T.; Rennie, C.D.; Guarneri, H. Advanced 3D Mapping of Hydrodynamic Parameters for the Analysis of Complex Flow Motions in a Submerged Bedrock Canyon of the Tocantins River, Brazil. Water 2018, 10, 367. [CrossRef]

6. Termini, D.; Moramarco, T. Dip Phenomenon in High-Curved Turbulent Flows and Application of Entropy Theory. Water 2018, 10, 306. [CrossRef]

7. Ben Meftah, M.; Mossa, M. Turbulence Measurement of Vertical Dense Jets in Crossflow. Water 2018, 10, 286. [CrossRef]

8. Fourniotis, N.T.; Horsch, G.M.; Leftheriotis, G.A. On the Hydrodynamic Geometry of Flow-Through versus Restricted Lagoons. Water 2018, 10, 237. [CrossRef]

9. Ferrari, S.; Badas, M.G.; Querzoli, G. On the Effect of Regular Waves on Inclined Negatively Buoyant Jets. Water 2018, 10, 726. [CrossRef]

10. Melito, L.; Postacchini, M.; Darvini, G.; Brocchini, M. Waves and Currents at a River Mouth: The Role of Macrovortices, Sub-Grid Turbulence and Seabed Friction. Water 2018, 10, 550. [CrossRef]

11. Mossa, M.; Davies, P.A. Some Aspects of Turbulent Mixing of Jets in the Marine Environment. Water 2018, 10, 522. [CrossRef]

12. Pascolo, S.; Petti, M.; Bosa, S. Wave-Current Interaction: A 2DH Model for Turbulent Jet and Bottom-Friction Dissipation. Water 2018, 10, 392. [CrossRef]

13. De Padova, D.; Brocchini, M.; Buriani, F.; Corvaro, S.; De Serio, F.; Mossa, M.; Sibilla, S. Experimental and Numerical Investigation of Pre-Breaking and Breaking Vorticity within a Plunging Breaker. Water 2018, 10, 387. [CrossRef]

14. Longo, S.; Clavero, M.; Chiapponi, L.; Losada, M.A. Invariants of Turbulence Reynolds Stress and of Dissipation Tensors in Regular Breaking Waves. Water 2017, 9, 893. [CrossRef]

15. Stancanelli, L.M.; Musumeci, R.E.; Foti, E. Computational Fluid Dynamics for Modeling Gravity Currents in the Presence of Oscillatory Ambient Flow. Water 2018, 10, 635. [CrossRef]

(C) 2018 by the authors. Licensee MDPI, Basel, Switzerland. This article is an open access article distributed under the terms and conditions of the Creative Commons Attribution (CC BY) license (http:/ / creativecommons.org/licenses/by/4.0/). 


\title{
Article \\ On the Effect of Regular Waves on Inclined Negatively Buoyant Jets
}

\author{
Simone Ferrari, Maria Grazia Badas and Giorgio Querzoli * \\ Dipartimento di Ingegneria Civile, Ambientale e Architettura (DICAAR), University of Cagliari, \\ via Marengo 2, 09123 Cagliari, Italy; ferraris@unica.it (S.F.); mgbadas@unica.it (M.G.B.) \\ * Correspondence: querzoli@unica.it; Tel.: +39-070-675-5308
}

Received: 28 February 2018; Accepted: 31 May 2018; Published: 3 June 2018

\begin{abstract}
The target of this paper is to measure the modifications that regular waves induce on the geometrical features and dilution of inclined negatively buoyant jets. In order to achieve this aim, we have carried out a set of experiments in a wavemaker-equipped flume, by measuring the concentration fields via light-induced fluorescence, a non-intrusive and full-field image analysis technique. The wave and jet parameters were selected in order to simulate the case of a typical discharge of brine, from a desalination plant, into the Mediterranean Sea, and compare it to a reference case, i.e., the same jet discharging into a stagnant water body. The mean concentration fields were obtained, as well as the geometrical features and dilution of the jets. The three main effects of waves on inclined negatively buoyant jets are the bifurcation (i.e., the separation in two branches), the rotation of the point of maximum height and the oscillation of the impact point around a fixed position different from the stationary one, and the reduction in size of the sea region interested by the discharge; this last effect increases with the wave period. As a consequence, under waves with high period and amplitude, the dilution of inclined negatively buoyant jets tends to decrease.
\end{abstract}

Keywords: inclined negatively buoyant jets; regular waves; dilution; sea discharges

\section{Introduction}

A negatively buoyant jet (NBJ) is the phenomenon that develops when a fluid is discharged, with a non-negligible momentum, in a less dense fluid. As the jet tends to fall downwards, it is typically released upwards, with a certain angle to the horizontal, in order to increase the path length and, consequently, the final dilution. Due to the several practical applications of inclined NBJs (see Ferrari and Querzoli, 2010 [1], for a list), many researches during the last few years focused on this topic.

In particular, many experimental investigations on NBJs released into a stationary receiving body can be found in recent years. Qiao et al. (2017, [2]) carried out an experimental and theoretical study on the internal hydraulics of NBJs from diffusers for sewage disinfection. Bashitialshaaer et al. (2012, [3] and 2015, [4]), measured in a laboratory model the geometrical features and the dilution of inclined NBJs. Seo and Song $(2015,[5])$ tested the influence of different diffuser types on the dispersion of NBJs for the particular application of the discharge of cooled water. Christodoulou et al. (2015, [6]) focused on the achieved dilution at the impact point and on its dependence on the NBJ inclination and on the densimentric Froude number. Oliver et al. (2013, [7]) removed the bottom boundary to find dilutions not affected by the stratification of dense effluent at the bottom. Geyer et al. (2012, [8]) experimentally obtained a chart of the dependence of flow types on Richardson, Reynolds and Weber numbers. Papakonstantis et al. (2011-a [9] and 2011-b [10]) experimentally studied the geometrical characteristics (via image analysis techniques) and the concentration (via probes) of inclined NBJs. In other experiments, velocity measurements were performed (e.g., Crowe et al., 2012 [11] and 2016 [12], Besalduch et al., 2013 [13] and 2014 [14]), highlighting, among the other results, the different turbulent quantities in the upper and lower boundaries of NBJs. 
The number of experimental investigations on the discharge of NBJs into a non-stationary receiving body are much less. Malcangio et al., 2016 [15], studied in the laboratory the turbulent features of vertical buoyant jets discharged into a transversal current. Hajikandi and Barjastehmaleki $(2015,[16])$ and Lai and Lee (2014, [17]) experimentally studied the mixing of inclined NBJs discharged into, respectively, a co-flow and a perpendicular cross-flow. Yang et al. (2005, [18]) experimentally investigated NBjs with a different angle discharged in a cross flow. Davies et al. (2001, [19]) and Davies and Ahmed (1996, [20]) studied, with both laboratory and numerical simulations, the peculiar case of an NBJ discharged horizontally into a rotating homogeneous fluid. Moreover, Stancanelli et al. (2018, [21]), experimentally investigated a buoyancy current in the presence of regular waves.

Regarding the jets released into a wave environment, some experimental researches on horizontal NBJs, on positively buoyant jets and on simple jets can be found in the literature. Bas et al. (2012, [22]) focused their experiments on the effect of the wave direction (discharge in the wave direction or in the opposite direction) on the dilution of horizontal NBJ. They found a higher dilution for the discharge in the opposite direction to the wave propagation, compared to both the dilution obtained in a stagnant receiving environment and the dilution of the co-flow discharge. Lin et al. (2011, [23]), investigated experimentally the turbulent properties of horizontal NBJs and non-buoyant jets under regular progressive waves via Particle Image Velocimetry (PIV). They found that dilution is increased by the wave motion. The same authors (Lin et al. 2013, [24]) measured, via PIV, the mean velocity and turbulence properties in a horizontal (positively, neutrally and negatively) buoyant jet discharged in the opposite direction to the wave propagation. They found that the width and the dilution of the positively and negatively buoyant jets increased in the wave receiving environment. Also Chin (1987, [25]) and Sharp (1986, [26]) observed experimentally that the dilution of a buoyant jet in a wave environment is significantly higher than in a stagnant one.

Many other Authors found similar results about the increased dilution due to the larger spreading caused by waves also on simple jets (e.g., Xu et al., 2016-a [27] and 2016-b [28], Hsiao et al., 2011 [29], Chang et al., 2009 [30], Ryu et al., 2005 [31], Mossa, 2004 [32], Chyan and Hwung, 1993 [33], Chyan et al., 1991 [34]). In this context, the classification formulated by Chyan and Hwung (1993, [33]) for the regions of a jet interacting with waves is particularly relevant: They identified three regions, namely the jet deflection region, the transition region and the developed jet region. In the jet deflection region, the jet still has most of its initial momentum and so it tends to preserve its shape; the influence of the wave on the jet deflection region is consequently an oscillation of the jet around its stationary position. This behavior has been confirmed by the experiments of Mossa (2004, [32]), Chyan et al. (1991, [34]) and Sharp (1986, [24]): They have shown that this oscillation has a considerable effect on the jet dilution. In the developed jet region, a periodic deflection, observed by Chyan and Hwung (1993, [31]) and Chin (1987, [26]), was found to be one of the main reasons for the higher dilution reached by a jet released in the presence of waves.

Although the increase of the dilution of a jet has been measured in many experimental investigations, to the best of the authors' knowledge the effects of regular waves on inclined (not horizontal or vertical) NBJs were never investigated before. For this reason, Ferrari and Querzoli, 2015 [35], performed a preliminary series of experiments that highlighted significant differences among the behavior of simple or positively buoyant jets and the case of the inclined negatively buoyant jets. As a consequence, a new set of carefully designed experiments were performed, with the main aim of better characterizing the peculiar behavior of negatively buoyant jets in a wavy environment and, in particular, of measuring the changes in the most important parameter for the design of environmentally friendly sea outfalls, i.e., the achieved dilution. In the present paper we present the results arising from this new set of experiments.

This paper is structured as follows: In Section 2, the experimental set-up, the run parameters and the data elaboration are briefly described; in Section 3, the results are shown and discussed; in Section 4, the conclusions are drawn. 


\section{Materials and Methods}

The experimental set-up simulates a typical configuration of a submarine outfall, i.e., the discharge from an orifice in the lateral wall of a pipe laid down on the sea bottom. A solution of water, sodium-sulphate and fluorescein was released, through a sharp-edged orifice outlet with a diameter $D=4.0 \mathrm{~mm}$ and an angle to the horizontal $\theta=67^{\circ}$, into a flume with glass walls. The solution came from a constant head tank to a cylindrical pipe with a $25.0 \mathrm{~mm}$ diameter. The sodium-sulphate was employed to increase the density of the solution, the fluorescein to allow concentration measurements via a Light Induced Fluorescence (LIF) technique (a non-intrusive and full-field image analysis technique). The flume is $21.00 \mathrm{~m}$ long and $0.30 \mathrm{~m}$ wide and it is equipped with a piston-type wavemaker, on one side, and an absorbing beach, on the opposite side, to minimize the reflections. The wavemaker can produce monochromatic or random waves. The water depth $d$ was kept constant at $0.40 \mathrm{~m}$.

The key parameter controlling the behavior of negatively buoyant jets is the densimetric Froude number Fr:

$$
F r=\frac{U}{\sqrt{\frac{g D\left(\rho_{D}-\rho_{R}\right)}{\rho_{R}}}}
$$

where $U$ is the outlet mean velocity, $g$ the gravitational acceleration, $\rho_{D}$ the discharged fluid density and $\rho_{R}$ the receiving fluid density. The Reynolds number is defined here as $R e=U D / v$, where $v$ is the kinematic viscosity of the discharged fluid. Two sets of experiments were performed, with Fr equal to 18.0 and 28.0 , and a $R e$ of $10^{3}$ (higher than the critical value of around 500 for the present set-up, as shown by Ferrari and Querzoli, 2010 [1]) and five monochromatic waves, with wave periods $T$ of $0.50 \mathrm{~s}, 1.00 \mathrm{~s}$ and $1.50 \mathrm{~s}$ (corresponding to wave lengths $L$ of $0.39 \mathrm{~m}, 1.56 \mathrm{~m}$ and $3.51 \mathrm{~m}$ ) and wave amplitudes $A$ of $5.00 \mathrm{~mm}$ and $12.50 \mathrm{~mm}$ (see Table 1). A reference run with a NBJ released in a stagnant environment was performed for each Fr. The ratio of the water depth $d$ to the wave length $L$ was in the deep-water regime $(d / L=1.02)$ or in the intermediate-depth water regime $(d / L=0.26$ and 0.11$)$; the ratio between the wave height $H=2 A$ and $L$ was between 0.003 and 0.026 , typical values of a long wave. The experimental set-up dimensions and wave parameters were chosen in order to simulate a typical submerged discharge in the Mediterranean Sea, with respect to the geometrical similarity (scale model $K_{L}=L_{M} / L_{P}=1 / 40$; the subscript $M$ stands for Model and the subscript $P$ for Prototype), to the kinematic similarity and to the dynamic (Froude) similarity, achieved through the respect of the following equations (see e.g., Von Ellenrieder and Dhanak, 2016 [36]):

$$
\begin{gathered}
A_{M}=A_{P} K_{L}, \\
T_{M}=T_{P} \sqrt{K_{L}} .
\end{gathered}
$$

In Figure 1, the data of the wave period, $T$, versus the wave height $H$ recorded by the Alghero wave buoy (in the north-western sea of Sardinia) of the RON-Rete Ondametrica Nazionale (Italian national wavemetric system [37]) from 2002 to 2014 are shown. These data were employed to define the wave parameters of the present experiments. As a matter of fact, by using Equations (2) and (3), the simulated prototype waves have $H=2 A=0.40-1.00 \mathrm{~m}$ and $T=3.16-6.32-9.49 \mathrm{~s}$, hence their values are within the range of those registered by the Alghero wave buoy.

The jet is discharged rightwards, in the opposite direction to the wave propagation and the flume is long enough to avoid an accumulation of dense fluid on the bottom during the duration of the experiment.

The axial vertical jet section was illuminated by a light sheet and a 3-CCD video-camera placed orthogonal to the light sheet. The experiments were recorded with a frame rate of $25 \mathrm{fps}$, a resolution of $720 \times 576$ pixels and a bit depth of 8 bit per color. As a consequence, the recorded images showed a bright jet on a dark background and, as the fluorescein concentration was very low, a linear relationship between the light intensity and the salt concentration holds true (Troy and Kosseff, 2005 [38], Sutton et al., 2008 [39]), so we used light intensity as a proxy for the salt concentration. 
The free surface levels were measured via the image analysis technique developed by Ferrari et al., 2016 [40]; the framed zone for the wave height measurement had a spatial resolution of around $11.62 \mathrm{px} / \mathrm{mm}$, so the $10 \mathrm{~mm}$ height wave was measured with a resolution of around $\pm 0.86 \%$ and the $25 \mathrm{~mm}$ height wave with a resolution of around $\pm 0.34 \%$. The resolution in the concentration measurements is around $0.4 \%$. The resolution on the measured distances $(X / D$ and $Y / D)$ depends on the spatial resolution of the investigation area, which is not the same for every experiment and varies from a minimum of $14.26 \mathrm{px} / \mathrm{cm}$ (run 7) to $20.89 \mathrm{px} / \mathrm{cm}$ (run 6). In addition, following Bendat and Piersol, 2010 [41], the uncertainty of the statistics presented in this paper was estimated from their standard deviation and was then computed over all the recorded images for each experiment (5000 for $T=0.5 \mathrm{~s}, 10000$ for $T=1.0 \mathrm{~s}, 15,000$ for $T=1.5 \mathrm{~s}$ ); the maximum uncertainty varies from a minimum of $3.35 \%$ (run 3 ) to a maximum of $4.52 \%$ (run 8 ).

Table 1. Main parameters for the experiments; $A$ is the wave amplitude, $H$ the wave height, $T$ the wave period, $L$ the wave length, $d$ the water depth $(0.40 \mathrm{~m}), F r$ the densimetric Froude number; experiments 1 and 7 were performed without waves as reference cases.

\begin{tabular}{cccccccc}
\hline Exp. & $A[\mathrm{~mm}]$ & $H[\mathrm{~mm}]$ & $T[\mathrm{~s}]$ & $L[\mathrm{~m}]$ & $d / L$ & $H / L$ & $F r$ \\
\hline 1 & 0 & 0 & - & - & - & - & 18.0 \\
2 & 5.00 & 10.00 & 0.50 & 0.39 & 1.02 & 0.026 & 18.0 \\
3 & 5.00 & 10.00 & 1.00 & 1.56 & 0.26 & 0.006 & 18.0 \\
4 & 12.50 & 25.00 & 1.00 & 1.56 & 0.26 & 0.015 & 18.0 \\
5 & 5.00 & 10.00 & 1.50 & 3.51 & 0.11 & 0.003 & 18.0 \\
6 & 12.50 & 25.00 & 1.50 & 3.51 & 0.11 & 0.007 & 18.0 \\
7 & 0 & 0 & - & - & - & - & 28.0 \\
8 & 5.00 & 10.00 & 0.50 & 0.39 & 1.02 & 0.026 & 28.0 \\
9 & 5.00 & 10.00 & 1.00 & 1.56 & 0.26 & 0.006 & 28.0 \\
10 & 12.50 & 25.00 & 1.00 & 1.56 & 0.26 & 0.015 & 28.0 \\
11 & 5.00 & 10.00 & 1.50 & 3.51 & 0.11 & 0.003 & 28.0 \\
12 & 12.50 & 25.00 & 1.50 & 3.51 & 0.11 & 0.007 & 28.0 \\
\hline
\end{tabular}

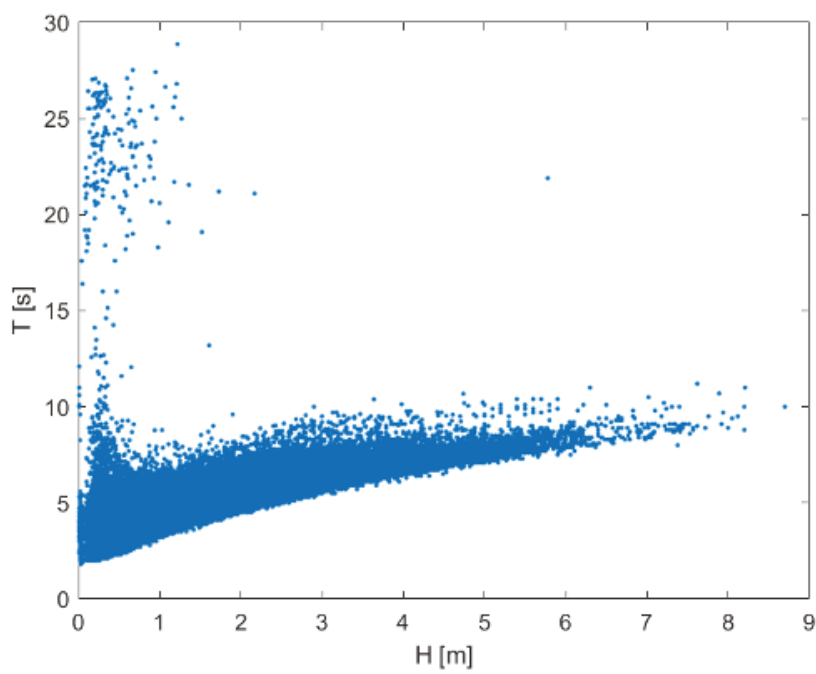

Figure 1. Wave period $T$ versus wave height $H$ recorded by the Alghero wave buoy in the period 2002-2014; data from the Rete Ondametrica Nazionale (Italian National wavemetric System) [37].

In Figures 2 and 3, two instantaneous visualizations of an inclined negatively buoyant jet with $F r=28.0$, released respectively in a stagnant and in a wavy environment with $T=1.00 \mathrm{~s}$ and $A=12.5 \mathrm{~mm}$, are shown. High concentrations are indicated by pale grey, low concentrations by dark grey. Figure 2 shows that an inclined NBJ released in a stagnant environment has an ascent region (where the initial 
momentum prevails on the negative buoyancy) followed by a descent region (where the negative buoyancy prevails). Moreover, the jet luminosity close to the outlet is high (as indicated by the pale grey color) and it tends to reduce along the jet path, pointing out the dilution caused by the entrainment of external fluid into the jet. As a matter of fact, the external fluid has null concentration, so its color is black. Few seconds of this experiment are shown in the movie "Run7_Ferrari_et_al.mp4", available as Supplementary Material. Figure 3 shows the modifications induced on the NBJ (the same of Figure 2) by the waves: Even if these modifications will be discussed in depth in Section 3, it is apparent that this jet, even though it still has an ascent and a descent region, has a different shape and is lower and shorter than the one released in the stagnant environment. Few seconds of this experiment are shown in the movie "Run10_Ferrari_et_al.mp4", available as Supplementary Material.

An image of the background was obtained by recording, before each run, 100 images without the NBJ and computing their point-by-point average. This image of the background was then subtracted from each image with the NBJ, and the resulting images (field of light intensity) were normalized by the grey level measured at the outlet, corresponding to the initial concentration $C_{0}$. The subtraction of the background from the images with the jet allows us to remove sources of light not linked to the discharged fluid concentration. The subtraction of the background explains why the cylindrical pipe with the outlet on its wall is visible in Figures 2 and 3 (before the background removal) and not visible in Figures 4-6, Figures 10 and 11 (after the background removal). Under the assumption of ergodicity, the non-dimensional fields of the mean concentration $C / C_{0}$ were obtained by time averaging the measured values of $C / C_{0}$ on each pixel. The value of $C / C_{0}$ is reported in false colors in Figures 4-6, Figures 10 and 11, according to the color bar shown on the left of each Figure (dark red is linked to the highest concentrations, dark blue to the lowest ones). As a consequence, $C / C_{0}$ is a measure of the reduction of the mean concentration compared to the outlet concentration $C_{0}$ : For instance, $C / C_{0}=1$ (dark red) means that the concentration $C$ in that point is the same as $C_{0}$ (no dilution), $C / C_{0}=0.5$ (green) means that the concentration $C$ in that point is one half of $C_{0}, C / C_{0}=0$ (dark blue) means that the concentration $C$ in that point is zero (external fluid not reached by the jet). The $x$-axis and $y$-axis are non-dimensionalised by the outlet diameter $D$, with the origin on the outlet.

The jet axis was assumed as the locus of the concentration maxima on the jet cross-sections (on the non-dimensional mean concentration fields) and it was computed via the iterative procedure described in Ferrari and Querzoli [1]. The stagnant case jet axis was determined on the non-dimensional mean concentration fields shown in Figure 10a for $F r=18.0$ and in Figure 4 and Figure 11a for $F r=28.0$. This stagnant case jet axis is drawn as a white line in Figures 4-6, Figures 10 and 11 for comparison with the features of the NBJ with the same $F r$ released into a receiving body affected by regular waves. Moving along the jet axis, the color change from dark red to blue (i.e., from high concentrations to low concentration) highlights the dilution due to the entrainment. As shown in Figures 2 and 4, an inclined NBJ has an ascent and a descent region, so its axis will have a non-symmetrical parabolic-like shape, with the origin coinciding with the outlet (green star in Figure 4, with $X / D=0, Y / D=0$ ), a point of maximum height (defined as the highest point reached by the jet axis, with coordinates $X / D=X_{h} / D$, $Y / D=Y_{h} / D$ ) and an impact point (defined as the point where the jet axis reaches again the outlet height, with coordinates $X / D=X_{d} / D, Y / D=0$ ). The point of maximum height and the impact point for the stagnant case are drawn, respectively, as a white circle and a white asterisk in Figures 4-6, Figures 10 and 11. The vertical distance between the jet origin and the point of maximum height is defined as the maximum height (vertical orange line in Figure 4) and the horizontal distance between the jet origin and the impact point is defined as the impact distance (horizontal orange line in Figure 4).

The runs in a wavy environment were also analyzed by dividing the wave period in 8 phases equally spaced in time by a $\Delta T=T / 8$ : phase 1 is taken when the wave trough is above the outlet, phase 5 corresponds approximately to the moment when the wave crest is above the outlet. In Figure 5 the phase averaged $C / C_{0}$ field for phase $1 / 8$ is shown. For the NBjs released into a wave environment, the impact point was measured as the point of maximum height of $C / C_{0}$ on the horizontal line $Y / D$ $=0$ for $X / D>X_{h} / D$ (i.e., beyond the horizontal coordinate of the point of maximum height; yellow 
asterisk in Figures 5 and 6), while the point of maximum height for the jet undergoing a bifurcation (see Section 3.1.1 for the phenomenological discussion) was measured in the upper branch of the two branches caused by the bifurcation. In Figures 5 and 6, the point of maximum height is drawn as a magenta circle. In Figure 5, the jet axis of the upper branch as a magenta line, the jet axis of the lower branch as a black line and the jet axis of the descent branch as a yellow line.

See Ferrari and Querzoli, 2010 [1], for more details on the experimental configuration simulating the discharge of inclined NBJs and data elaboration; see Ferrari and Querzoli, 2015 [35] and Ferrari et al., 2016 [40] for more details on the wavemaker system.

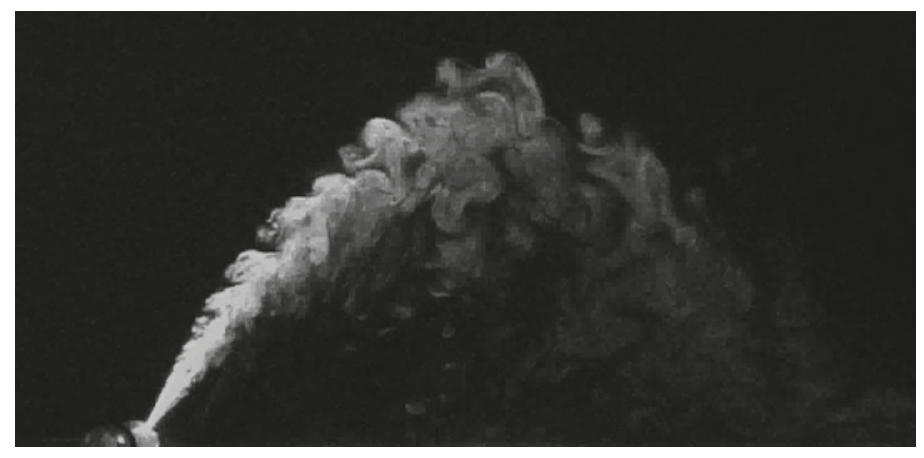

Figure 2. An instantaneous visualization of the concentration field for an inclined negatively buoyant jet with $F r=28.0$ and $\theta=67^{\circ}$ released into a stagnant receiving body.

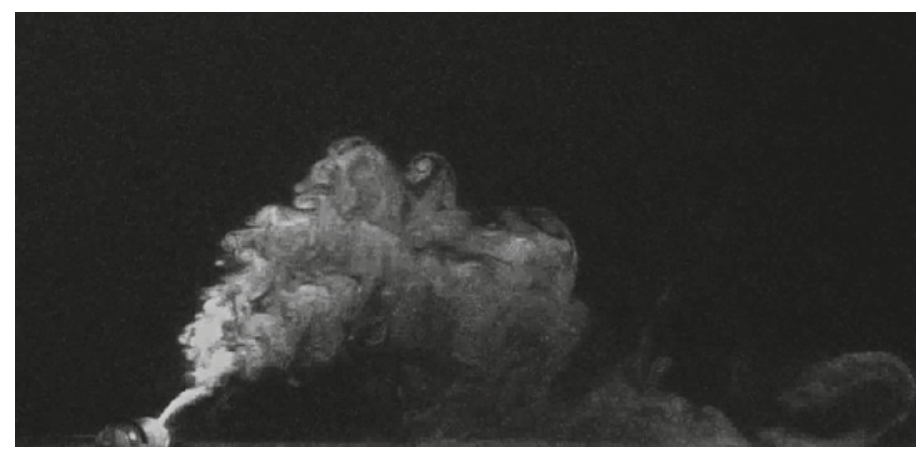

Figure 3. An instantaneous visualization of the concentration field for the same inclined NBJ of Figure 2 released into a receiving body affected by regular waves with $A=12.5 \mathrm{~mm}$ and $T=1.00 \mathrm{~s}$. 


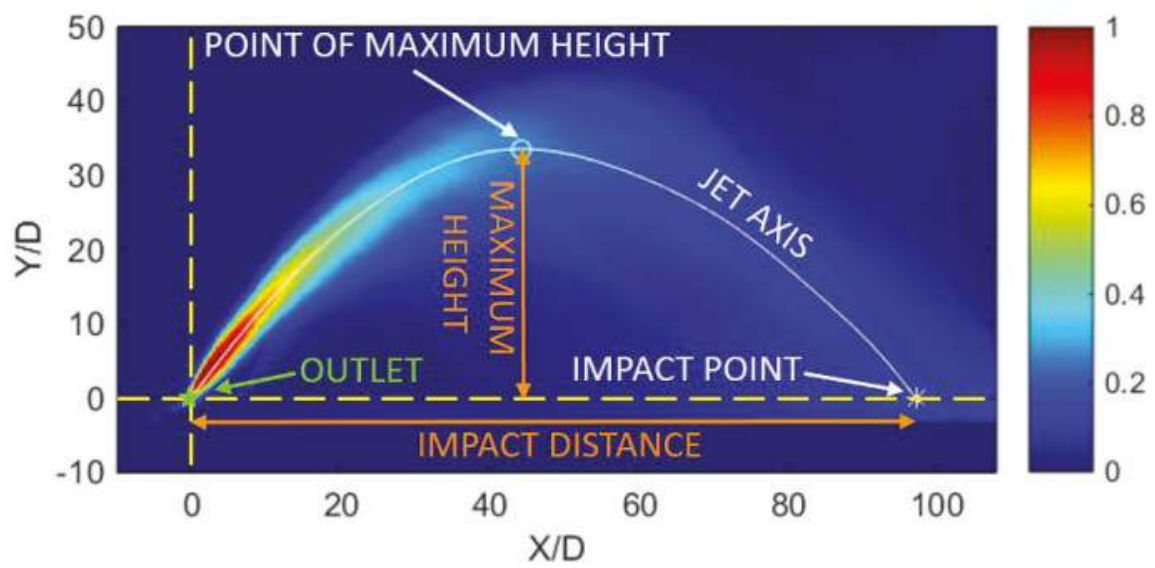

Figure 4. Non-dimensional mean concentration $C / C_{0}$ fields for the same inclined NBJ of Figure 2 released into a stagnant receiving body, with the jet geometrical features; the maximum uncertainty in the mean concentration measurement is $\pm 4.41 \%$.

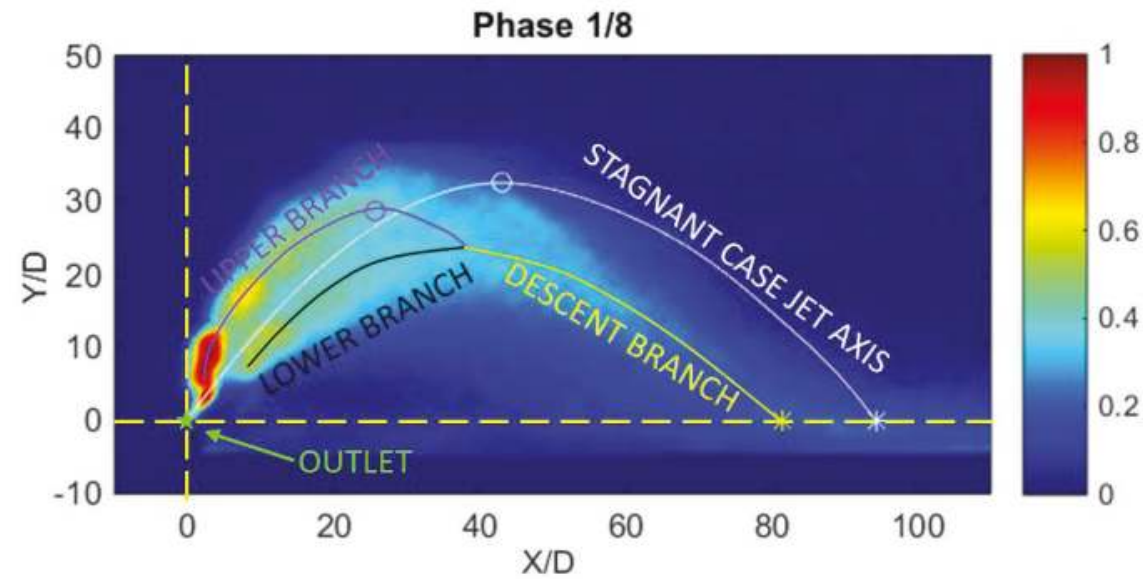

Figure 5. Non-dimensional mean concentration fields for the same NBJ of Figure 3, in phase 1/8, with the jet geometrical features; the maximum uncertainty in the mean concentration measurement is $\pm 4.38 \%$.

\section{Results}

This section is organized as follows: In Section 3.1, the focus is on the temporal evolution of the phenomenon, so, for each of the eight phases, the mean concentration fields (3.1.1), the maximum height and impact point (3.1.2), and the concentration profiles (3.1.3) are shown; in Section 3.2 the overall mean concentration fields, computed performing the time average on all the images for each run, are presented; eventually, the measured dilutions are reported in Section 3.3. 


\subsection{Temporal Evolution}

\subsubsection{Mean Concentration Fields}

To better highlight the temporal behaviour of a negatively buoyant jet released upwards with a certain angle to the horizontal when a wave is passing through, in Figure 6 the non-dimensional mean concentration field of an NBJ with $F r=28.0$ subjected to regular waves with $T=1.00 \mathrm{~s}$ and $A=12.5 \mathrm{~mm}$ has been divided into 8 phases; in addition, to ease the comparison with the stationary case (shown in Figure 4), on each image the stagnant case jet axis (white line) is superimposed. The images of Figure 6 are shown as a movie in "Run10_mean_phase_Ferrari_et_al.mp4", available as Supplementary Material. To highlight the differences between the stationary and the wave case, a white circle and a white asterisk indicate, respectively, the point of maximum height and the impact point for the stationary case, whilst a magenta circle and a yellow asterisk indicate, for each phase, the wave case maximum height point and impact point.

Close to the outlet $(X / D=0, Y / D=0)$, the NBJ is deflected upwards or downwards, so it oscillates around the stationary position (pointed out by the white line, i.e., the stationary case jet axis): So in this region the NBJ tends to resist the wave because it tends to preserve its initial direction, as it still retains most of its initial momentum. This behavior is similar to the simple jet one and this region can be identified as the jet deflection region, in agreement with the findings of Chyan and Hwung (1993, [33]) and Mossa (2004 [32]). This oscillation is linked to the position of the wave above the jet which, in turn, is linked to the phase: In phase 1 the wave trough is above the outlet, so there is the largest leftward deflection, while when the wave crest is above the outlet (phase 5), the jet experiences the largest rightward deflection. It is interesting to note that this oscillation is larger upwards than downwards; this is presumably due to the velocity field generated by the interaction between the wave motion (mostly horizontal in that region) and the cylindrical pipe simulating the pipe (visible in Figures 2 and 3). Velocities tend to be higher and directed upwards close to the outlet, whilst they tend to be lower and horizontal in the lower zone. Consequently, the deflection which pushes the jet upwards tends to carry it towards regions with higher velocity and this causes this larger displacement upward.
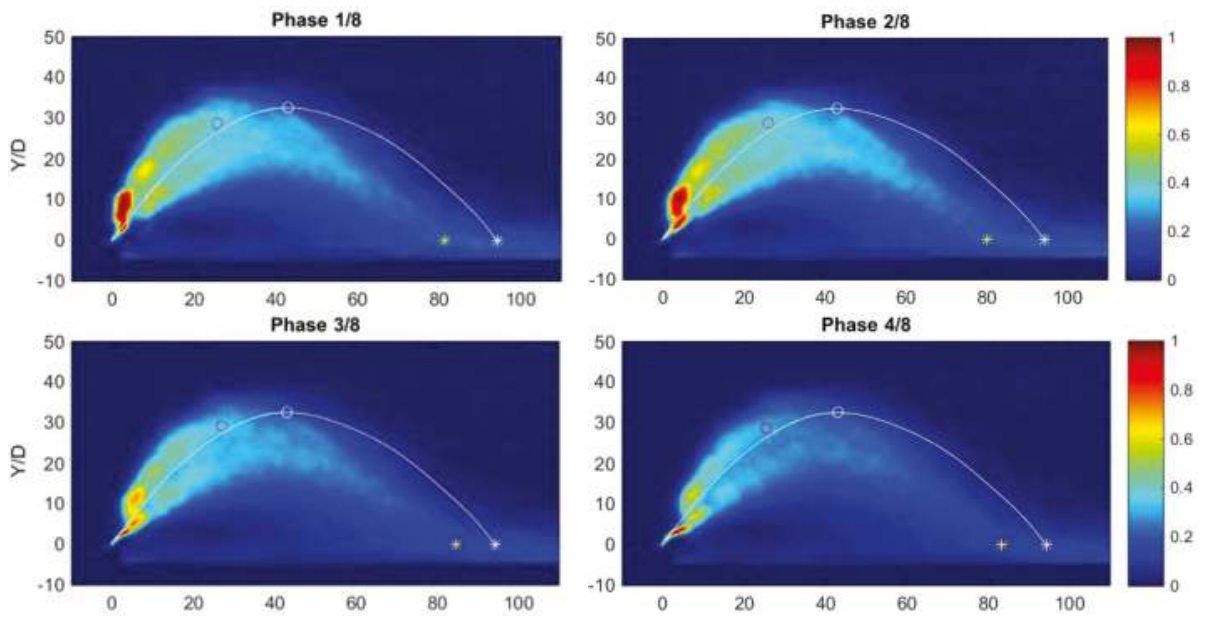

Figure 6. Cont. 

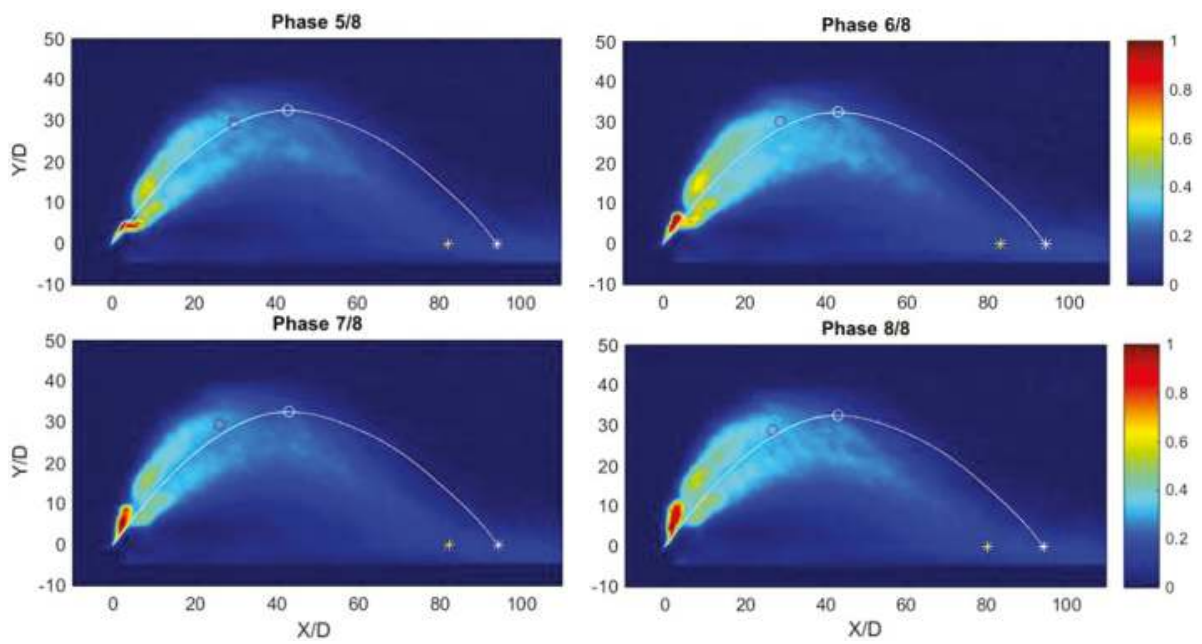

Figure 6. Non-dimensional mean concentration fields for the same NBJ of Figure 3, divided into 8 phases; the white line is the stagnant case jet axis; the maximum uncertainty in the mean concentration measurement is $\pm 4.38 \%$.

Coming back to the observation of Figures 5 and 6, and moving along the jet axis, it is noticeable how, after the jet deflection region, if the wave is intense enough (i.e., with $T$ and $A$ high enough), the NBJ bifurcates; this is the transition region. The jet, slowed down by the enhanced entrainment of low-momentum fluid, is divided into two branches, one mainly higher (magenta line in Figure 5) and one lower (black line in Figure 5) than the stationary jet axis (white line in Figure 5). Eventually, the two branches merge to build up again a single jet flow in the descent branch (yellow line in Figure 5); this is the developed jet region. This bifurcation is sharpened by the fact that the change in the direction is not gradual but, instead, the NBJ in the jet deflection region tends to abruptly move from one extreme to the other.

\subsubsection{Geometrical Features}

The point of maximum height of the NBJ released into a wavy environment is in the upper branch of the bifurcated jet (magenta asterisk in Figures 5 and 6 ) and tends to be lower than the same point of the stagnant case jet (white circle in Figures 5 and 6); a similar consideration stands for the impact point (compare the yellow asterisk, i.e., the wave case impact point, with the white asterisk, i.e., the stagnant case impact point, in Figures 5 and 6). For this reason, in Figure 7 the trajectories followed by the points of maximum height for the wave cases (colored lines: blue circles for $T=0.5 \mathrm{~s}$ and $A=5.0 \mathrm{~mm}$, green asterisks for $T=1.0 \mathrm{~s}$ and $A=5.0 \mathrm{~mm}$, magenta stars for $T=1.0 \mathrm{~s}$ and $A=12.5 \mathrm{~mm}$, cyan xs for $T=1.5 \mathrm{~s}$ and $A=5.0 \mathrm{~mm}$ and red $+\mathrm{s}$ for $T=1.5 \mathrm{~s}$ and $A=12.5 \mathrm{~mm}$ ) are compared with the coordinates of the maximum height point for the stagnant case (a single black square, as it does not move in the stagnant case). Similarly, in Figure 8 the oscillation of the impact distances for the wave cases in the various wave phases (colored lines, the same as in Figure 7) are compared with the constant impact distance for the stationary case (black line). In both Figures 7 and 8 , the left subfigure refers to the NBJ with $F r=28$ and the right subfigure to the NBJ with $F r=18$.

By looking at Figures 7 and 8, it is possible to note the peculiar behavior of an NBJ released into a wavy environment: The maximum height and the impact distance of the NBJs released in wavy environment are shorter than those of the stationary case for all the different waves tested here. In particular, the ratio between the maximum height for the wave cases and the stagnant case varies from a maximum value of about $0.86(T=0.5 \mathrm{~s}, A=5.0 \mathrm{~mm})$ to a minimum of about $0.36(T=1.5 \mathrm{~s}$, 
$A=12.5 \mathrm{~mm})$ for $F r=28.0$, and from a maximum of about $0.83(T=0.5 \mathrm{~s}, A=5.0 \mathrm{~mm})$ to a minimum of about $0.32(T=1.5 \mathrm{~s}, A=12.5 \mathrm{~mm})$ for $F r=18.0$.

Moreover, by looking at Figure 7 it is noticeable that the trajectories of the point of maximum height tend to be rotations around a fixed point, even if, in some cases, the closed trajectories are quite flattened. The point of maximum height tends to be lower and closer to the outlet when the wave parameters change: When the wave period $T$ and amplitude $A$ increase, the distance from the stationary position increases. If, on one hand, it is clear that the strongest waves (red,$+ T=1.5 \mathrm{~s}$ and $A=12.5 \mathrm{~mm}$ ) have the strongest impact on this phenomenon, on the other the wave period $T$ seems to have the biggest role on the decrease of the maximum height; as a matter of fact, the curves in Figure 7 are sorted by increasing wave period $T$.
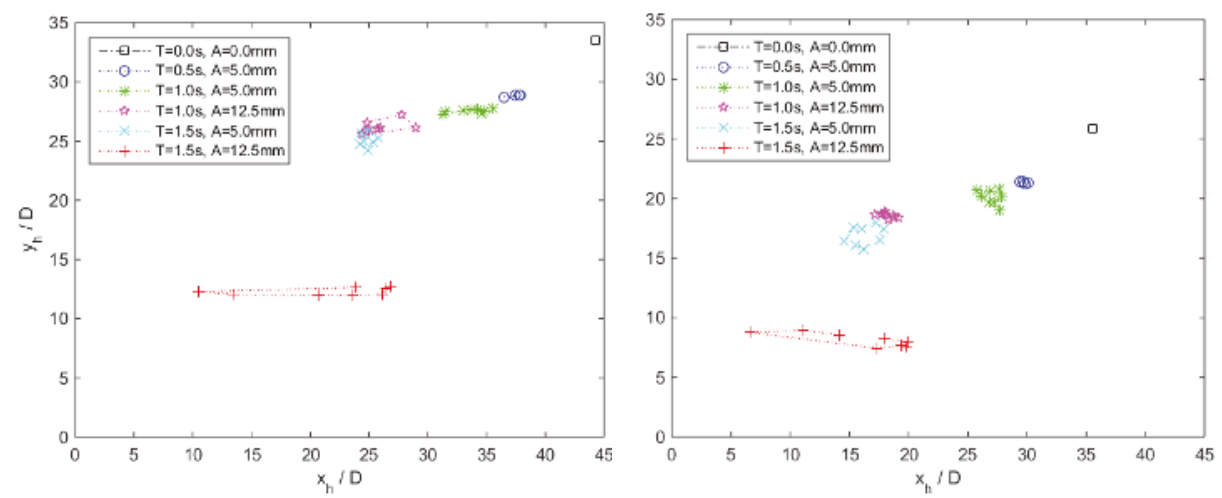

Figure 7. Trajectories of the maximum height points for the NBJs with $F r=28.0$ (left) and $F r=18.0$ (right) released into a receiving body affected by regular waves (wave parameters in the legend).

The impact point (Figure 8) oscillates around a fixed point which is different from the stationary impact point. Different to the point of maximum height, the impact point seems more influenced by the wave amplitude $A$; as a matter of fact, in Figure 8, the curves relative to $A=5.0 \mathrm{~mm}$ are generally closer to the stationary position than the ones with $A=12.5 \mathrm{~mm}$. The only exception to this rule is the case of $T=0.5 \mathrm{~s}$ and $A=5.0 \mathrm{~mm}$ for $F r=28.0$ (blue circles on the left of Figure 8); an explanation for this will be given in the Section 3.2. Similar to the maximum height, also the impact distance of the wave cases shows a reduction in size with respect to the stagnant one. In particular, the ratio between the impact distance for the wave cases and the stagnant case varies from a maximum value of about $0.94(T=1.5 \mathrm{~s}, A=5.0 \mathrm{~mm})$ to a minimum of about $0.51(T=1.5 \mathrm{~s}, A=12.5 \mathrm{~mm})$ for $F r=28.0$, and from a maximum of about $0.97(T=0.5 \mathrm{~s}, A=5.0 \mathrm{~mm})$ to a minimum of about $0.54(T=1.5 \mathrm{~s}, A=12.5 \mathrm{~mm})$ for $F r=18.0$.

In summary, close to the outlet, the NBJ oscillates around the stationary position, as the simple jets; conversely, the NBJ maximum height point rotates around a different position from the stationary maximum height point and the NBJ impact point oscillates around a different position from the stationary impact point.

The reduction of the maximum height and impact distance in the wave cases showed in Figures 7 and 8 confirms the contraction of NBJs when discharged into a wavy environment. This contraction is probably due to the periodic oscillation of the initial direction of the NBJ that causes its bifurcation and a consequent increase of the entrainment; the larger amount of low-momentum external fluid trapped into the NBJ causes a premature slowdown (when compared to the stagnant case). 

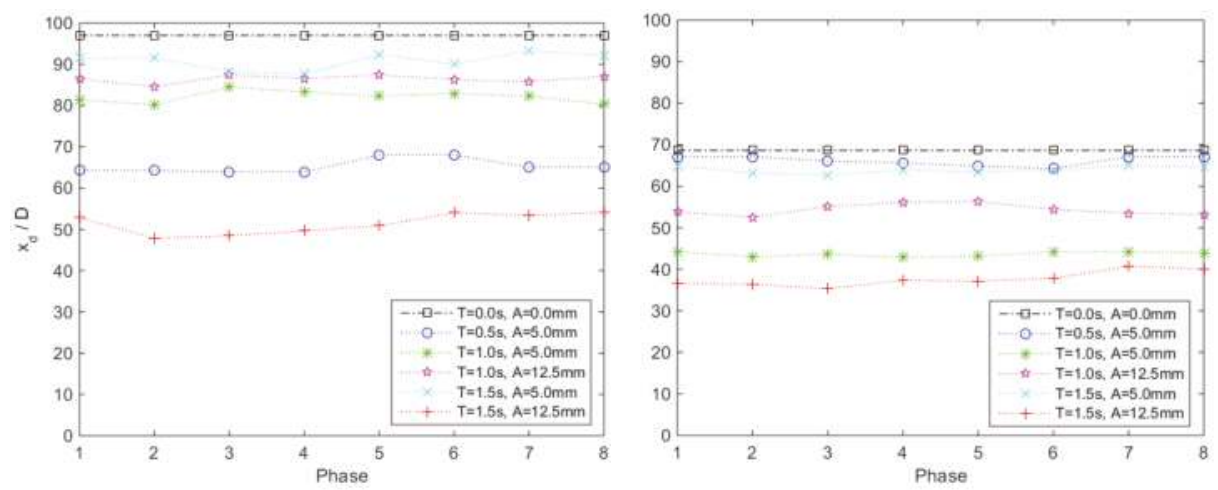

Figure 8. Non-dimensional impact distance $x_{d} / D$ for the NBJs with $F r=28.0$ (left) and $F r=18.0$ (right) released into a receiving body affected by regular waves (wave parameters in the legend).

\subsubsection{Concentration Profiles}

In Figure 9 , the cross-sectional profiles of the non-dimensional mean concentration $C / C_{0}$ measured on the point of maximum height ( $\mathrm{a}$ and c, vertical profiles) and on the impact point ( $\mathrm{b}$ and $\mathrm{d}$, horizontal profiles), in eight different phases of the wave cycle, are shown, for two NBJs with $F r=28.0$ (a and b) and $F r=18.0$ ( $\mathrm{c}$ and $\mathrm{d}$ ) released into a receiving body affected by a wave motion with $T=1.00 \mathrm{~s}$ and $A=12.5 \mathrm{~mm}$. The different colors highlight the different phases the profiles are measured in. The bimodal distribution of the concentration maxima in Figures 7c and 9a confirms what was previously stated about the bifurcation of NBJs released in a wave environment.

In the impact point, the various profiles have a single peak because here the NBJ is in the developed jet region, where it tends to merge again in a single entity (the yellow line in Figure 5).

The differences among the concentration values in the various phases highlight the different concentration dilutions achieved by the NBJ in the different phases.

\subsection{Overall Time Averaged Concentration Fields}

In Figures 10 and 11, the mean concentration fields, computed performing the time average over all the images recorded on each experiment and not divided by phases, are shown: Figure 10 concerns the inclined negatively buoyant jets with $F r=18.0$, while Figure 11 concerns the ones with $F r=28.0$. In particular, the Figures 10a and 11a show the inclined NBJs released into a stagnant receiving body, whilst the others (Figures 10b-f and 11b-f) show the inclined NBJs released into a wavy environment.

By comparing the concentration field of the NBJ released into a stagnant environment with those of the NBJs released into a wavy environment, it is apparent how the interaction with the waves causes a contraction of the jet, which tends to be less high and shorter (with a decrease of the maximum height and of the impact distance compared to the stagnant cases, as stated before). This contraction tends to increase as the wave period $T$ and amplitude $A$ increase.

Moreover, for the highest $T$ and $A$ (Figures $10 \mathrm{~d}-\mathrm{f}$ and $11 \mathrm{~d}-\mathrm{f}$ ), the distribution of the concentration is bimodal, confirming a bifurcation of the NBJ into two branches, one higher and one lower than the stationary position. These two branches tend to behave as two NBJs released with different angles to the horizontal, to eventually merge when the higher branch falls down on the lower one, causing an increase in the concentration in the lower branch, which is clearly visible in Figures $10 \mathrm{f}$ and $11 \mathrm{f}$ at $X / D \approx 15$ and $Y / D \approx 10$. This bifurcation, caused by the oscillation of the initial region of the NBJ around the stationary position, becomes more pronounced when the wave amplitude $A$ increases, as it is possible to see from a comparison of the Figure $10 \mathrm{~d}, \mathrm{f}$ (or Figure $11 \mathrm{~d}$,f, with $A=12.5 \mathrm{~mm}$ ) with Figure $10 \mathrm{~b}, \mathrm{c}, \mathrm{e}$ (or Figure $11 \mathrm{~b}, \mathrm{c}, \mathrm{e}$, with $A=5.0 \mathrm{~mm}$ ). If in the first two Figures the bifurcation is more 
evident, in the other three it is almost absent or very weak. As a matter of fact, in Figures $10 \mathrm{~b}$ and $11 \mathrm{~b}$ the effect of the wave begins to be noticeable only very close to the point of maximum height, where the initial momentum of the NBJ has been almost completely spent. In Figures 10c and 11c, concerning a wave with $T=1.00 \mathrm{~s}$ and $A=5.0 \mathrm{~mm}$, the oscillation of the initial region of the NBJ caused by the wave is still present, because the jet widens more than in the stationary case, but it is not sufficient to create the bifurcation. This could suggest that the bifurcation is more linked to high-amplitude waves.

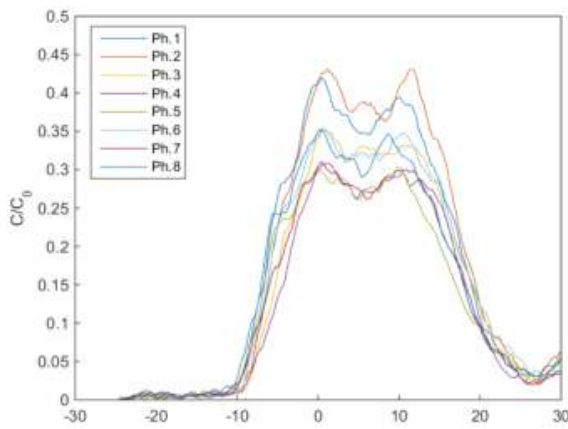

(a)

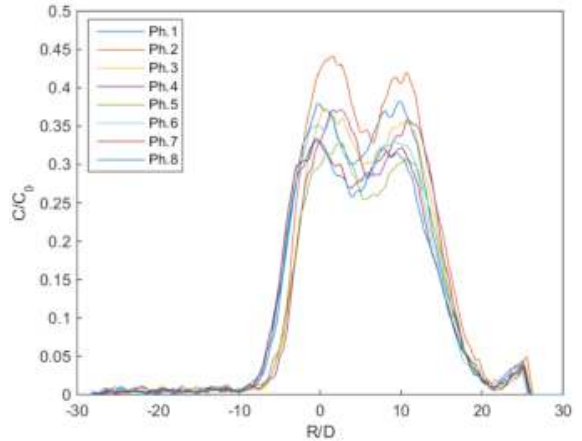

(c)

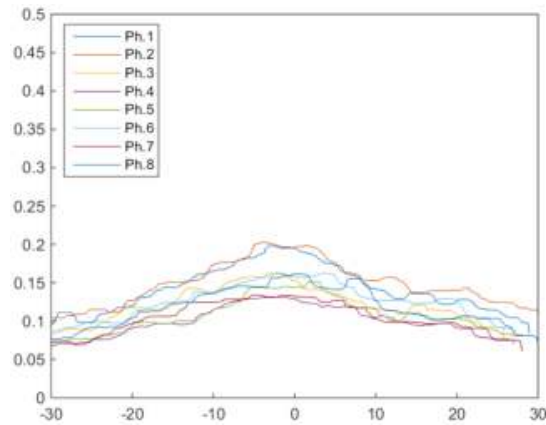

(b)

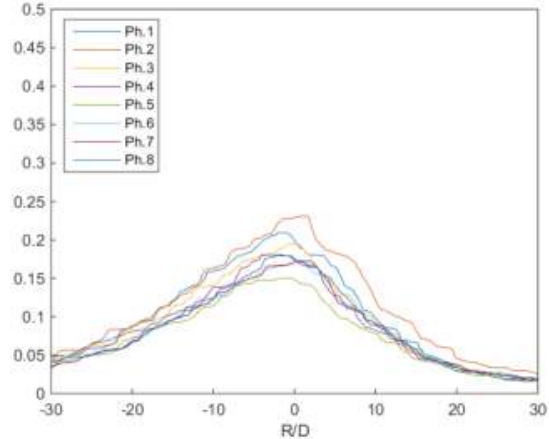

(d)

Figure 9. Cross-sectional non-dimensional mean concentration profiles for a NBJ with $F r=28.0(\mathbf{a}, \mathbf{b})$ and for a NBJ with $F r=18.0$ (c,d) released in a receiving body affected by regular waves with $T=1.00 \mathrm{~s}$ and $A=12.5 \mathrm{~mm} ;(\mathrm{a}, \mathrm{c})$ vertical profiles on the point of maximum height; $(\mathrm{b}, \mathrm{d})$ horizontal profiles on the impact point; $R / D$ is the non-dimensional span-wise abscissa.

The reduction of the maximum height of the NBJ is mainly caused by the bifurcation. As a matter of fact, because of the strong deflection upwards and downwards imposed by the bifurcation (see Figures $10 \mathrm{f}$ and 11f), the jet uses a portion of its initial momentum to return to the initial direction and, consequently, it cannot reach the same height of the stagnant case. When the wave deflects the jet downwards, the trajectory is similar to the one of a NBJ released with a lower angle to the horizontal, i.e., the jet tends to reach a lower maximum height (see Ferrari and Querzoli, 2010, [1]).

The reduction of the impact distance is mainly caused by a combination of the bifurcation and of the rotation of the maximum height point.; the fluid in the lower branch finds the opposition of the oscillating fluid trapped between the bottom and the jet itself, while the fluid in the upper branch, as stated before, falls down on the lower branch, causing an additional mixing and a slowing down of the NBJ.

In Figure $11 \mathrm{~b}$, the effect of the wave with $T=0.5 \mathrm{~s}$ and $A=5.0 \mathrm{~mm}$ on the impact point (compare with Figure 8) can be explained. The wave is too weak to slow down the NBJ enough, so, when the 
NBJ comes close to the point of maximum height, it has covered a distance long enough to consume most of its initial momentum and, consequently, it undergoes the rotatory movement imposed by the waves, with an additional stirring that lets the jet fall down almost vertically, with a consequent strong reduction of the impact distance.

In summary, the main effect of the wave amplitude $A$ is the bifurcation of the NBJ, while the one of the wave period $T$ is more linked to the additional stirring, with a rotatory motion, imposed to the transition region of the NBJ. Both these phenomena contribute to consume the initial momentum of the NBJ and to decrease the size of the sea region interested by the NBJ itself. An alternative explanation of these effects of the waves on NBJs could be linked to the Stokes drift. For this reason, we have planned to perform Lagrangian velocity measurements in the future.

(a)

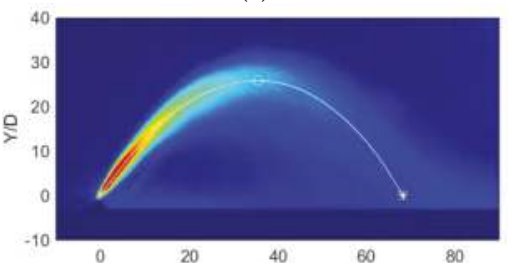

(c)

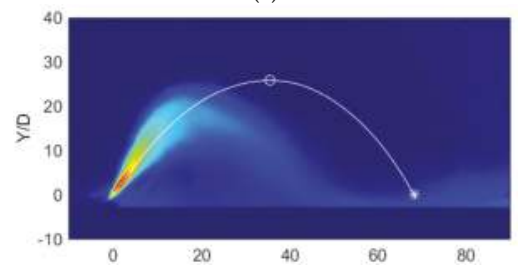

(e)

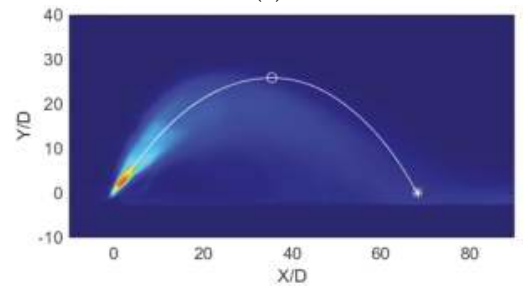

(b)

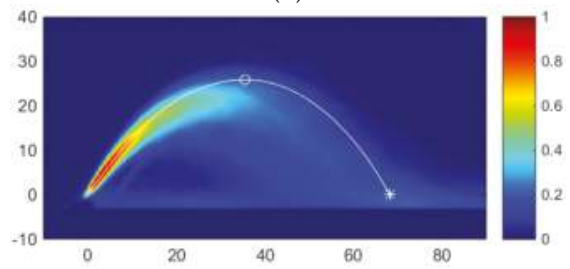

(d)

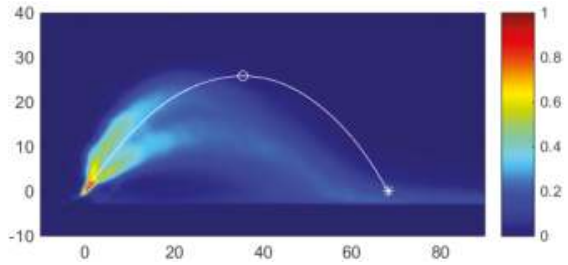

(f)

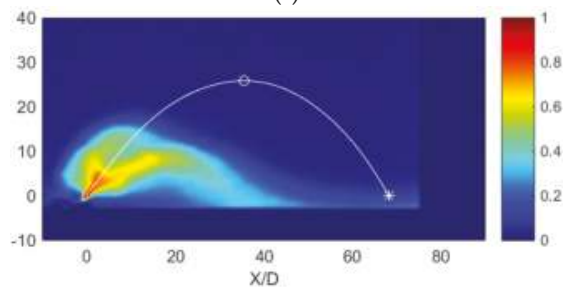

Figure 10. Non-dimensional mean concentration fields for an NBJ with $\mathrm{Fr}=18.0$ released into a receiving body affected by regular waves with $T=0.0 \mathrm{~s}$ and $A=0.0 \mathrm{~mm}$ (stagnant case, a), $T=0.5 \mathrm{~s}$ and $A=5.0 \mathrm{~mm}(\mathbf{b}), T=1.0 \mathrm{~s}$ and $A=5.0 \mathrm{~mm}(\mathbf{c}), T=1.0 \mathrm{~s}$ and $A=12.5 \mathrm{~mm}(\mathrm{~d}), T=1.5 \mathrm{~s}$ and $A=5.0 \mathrm{~mm}$ (e), and $T=1.5 \mathrm{~s}$ and $A=12.5 \mathrm{~mm}$ (f), with the stagnant case jet axis; the maximum uncertainty in the mean concentration measurement is $\pm 3.44 \%$ in (a), $\pm 3.93 \%$ in (b), $\pm 3.35 \%$ in (c), $\pm 3.72 \%$ in (d), $\pm 3.45 \%$ in $(\mathbf{e}), \pm 3.99 \%$ in (f).

\subsection{Dilution}

In order to study the effect of waves on the dilution of inclined negatively buoyant jets, we can define the ratio of the dilution of jets under waves to the dilution of jets in a stagnant environment, if $R_{D I L}$ : if $R_{D I L}$ is higher than one, the waves increase the dilution and vice versa. In Figure $12, R_{D I L}$ has been plotted versus $F r$ for all the experiments. The horizontal dotted black line at $R_{D I L}=1$ highlights the stagnant case, while, as in the previous Figures, colored lines highlight the wave cases (blue circles for $T=0.5 \mathrm{~s}$ and $A=5.0 \mathrm{~mm}$, green asterisks for $T=1.0 \mathrm{~s}$ and $A=5.0 \mathrm{~mm}$, magenta stars for $T=1.0 \mathrm{~s}$ and $A=12.5 \mathrm{~mm}$, cyan $x \mathrm{~s}$ for $T=1.5 \mathrm{~s}$ and $A=5.0 \mathrm{~mm}$ and $\mathrm{red}+\mathrm{s}$ for $T=1.5 \mathrm{~s}$ and $A=12.5 \mathrm{~mm}$ ). The dilution 
is measured, in all the cases, as the inverse of the maximum non-dimensional concentration at the impact point on the overall time-averaged fields, in order to avoid phase variations (see Figure 9b,d).

Figure 12 shows that, differently from what was found by investigations on simple jets or positively buoyant jets, the dilution not always increases for NBJs released in a wavy environment. In particular, the waves with $A=5.0 \mathrm{~mm}$ and $T=0.5 \mathrm{~s}, T=1.0 \mathrm{~s}$ enhance the dilution $\left(R_{D I L}=1.81\right.$ for $A=5.0 \mathrm{~mm}, T=0.5 \mathrm{~s}, F r=18.0 ; R_{D I L}=1.08$ for $A=5.0 \mathrm{~mm}, T=1.0 \mathrm{~s}, F r=18.0 ; R_{D I L}=1.26$ for $A=5.0 \mathrm{~mm}, T=0.5 \mathrm{~s}, F r=28.0 ; R_{D I L}=1.08$ for $A=5.0 \mathrm{~mm}, T=1.0 \mathrm{~s}, F r=28.0$ ), whilst the remaining waves (with higher values of $A$ and $T$ ) reduce the dilution. The lowest values for $\mathrm{R}_{\mathrm{DIL}}$ have been measured for the strongest wave $(A=12.5 \mathrm{~mm}, T=1.0 \mathrm{~s})$ for both the $F r\left(R_{D I L}=0.36\right.$ for $F r=28.0$; $R_{\text {DIL }}=0.45$ for $F r=18.0$ ).

This result can be explained by two of the effects of the waves on the NBJ: The reduction in size of the sea region interested by the discharge and the bifurcation.

(a)

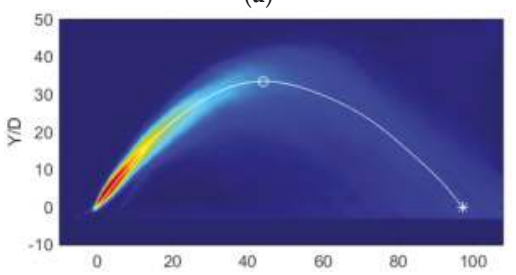

(c)

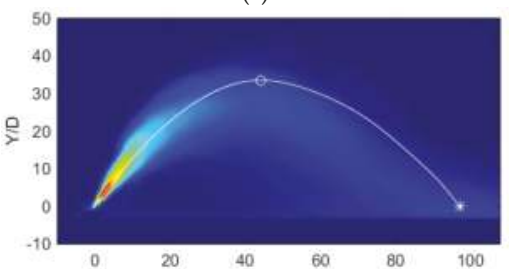

(e)

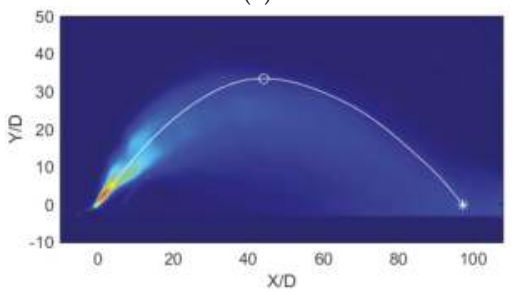

(b)

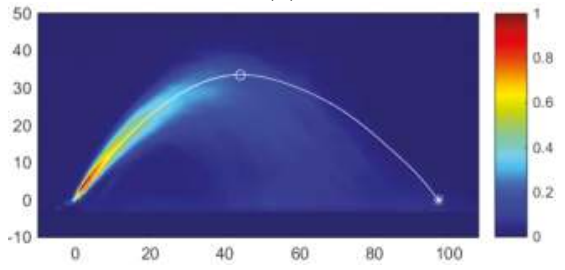

(d)

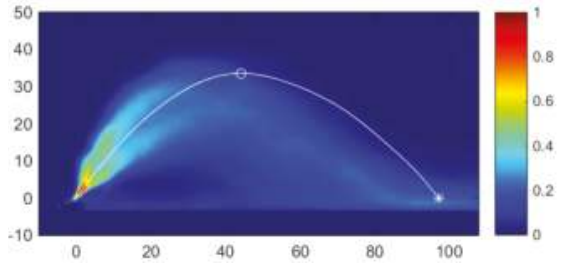

(f)

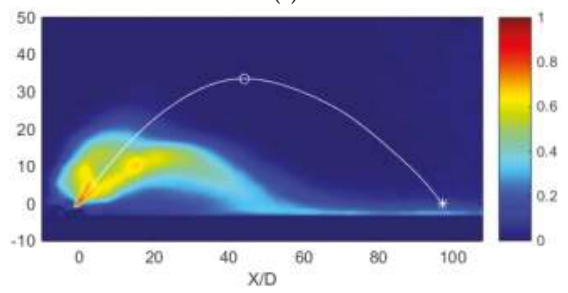

Figure 11. Non-dimensional mean concentration fields for an NBJ with $F r=28.0$ released into a receiving body affected by regular waves with $T=0.0 \mathrm{~s}$ and $A=0.0 \mathrm{~mm}$ (stagnant case, a), $T=0.5 \mathrm{~s}$ and $A=5.0 \mathrm{~mm}(\mathbf{b}), T=1.0 \mathrm{~s}$ and $A=5.0 \mathrm{~mm}(\mathbf{c}), T=1.0 \mathrm{~s}$ and $A=12.5 \mathrm{~mm}(\mathbf{d}), T=1.5 \mathrm{~s}$ and $A=5.0 \mathrm{~mm}$ (e) and $T=1.5 \mathrm{~s}$ and $A=12.5 \mathrm{~mm}(\mathrm{f})$, with the stagnant case jet axis; the maximum uncertainty in the mean concentration measurement is $\pm 4.41 \%$ in (a), $\pm 4.52 \%$ in (b), $\pm 4.16 \%$ in (c), $\pm 4.38 \%$ in (d), $\pm 4.28 \%$ in (e), $\pm 4.31 \%$ in (f).

Because of the contraction, the NBJ is forced by the waves to remain in a smaller region compared to the stagnant case, so the path available for mixing is decreased and this can reduce (or even cancel) the additional stirring caused by the rotation. Moreover, if the NBJ is forced into a smaller region, it will tend to re-entrain some jet fluid instead of entraining external fluid.

The bifurcation has a conflicting role: On one hand, it increases the surface available for entrainment, but, on the other hand, the lower boundary of the upper branch and the upper boundary 
of the lower branch tend to exchange jet fluid between themselves and not to entrain external fluid, reducing the dilution. Moreover, as shown in the previous subsections, the upper branch tends to fall down over the lower one, causing an increase in concentration along the path of the wave case NBJ.

The authors cited in the introduction found that discharging a horizontal NBJ or a simple jet in the opposite direction to the wave propagation increases in size the region occupied by the jet and, as a consequence, its dilution. In the present case, an inclined NBJ (in particular with the strongest waves) experiences, as previously seen, a reduction in size of the region occupied by the jet in comparison with the stagnant case which, consequently, leads to a lower dilution.

For these reasons, the waves that cause the worst dilutions are the ones with $A=12.5 \mathrm{~mm}$. On the contrary, the two waves with $A=5.0 \mathrm{~mm}$ and $T=0.5 \mathrm{~s}, T=1.0 \mathrm{~s}$ enhance the dilution, because the rotation imposed by the waves on the NBJs is intense enough to compensate their contraction (which, anyway, is smaller than in the other cases).

In summary, the peculiar nature of inclined NBJs, which tend to behave in a limited region of space and cannot reach an asymptotic state like some simple or positively buoyant jets, leads to the conclusion that the wave motion not always enhances their dilution. In particular, the strongest waves tested in the present work tend to reduce the dilution, whilst the weakest ones tend to enhance it.

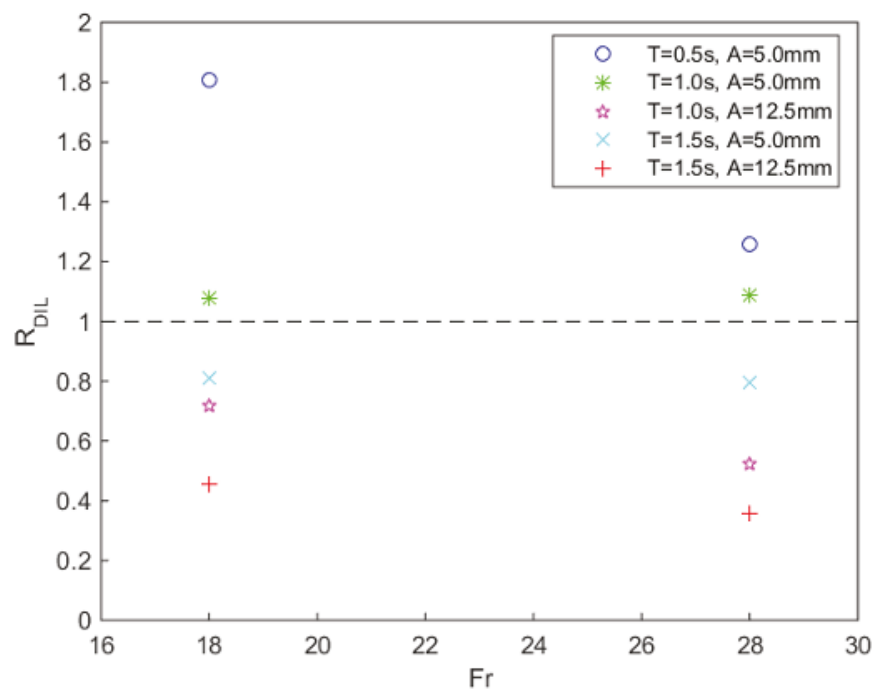

Figure 12. $R_{D I L}$ versus $F r ; R_{D I L}$ is the ratio of the dilution of NBJs under waves to the dilution of jets in stagnant environment; (wave parameters in the legend).

\section{Conclusions}

With the target to measure the modifications that regular waves induce on the geometrical features and on the dilution of inclined negatively buoyant jets, we have carried out an experimental campaign on a laboratory model of a submerged outfall discharging brine under some typical conditions of the Mediterranean Sea. In particular, the concentration fields were measured via a non-intrusive and full-field light-induced fluorescence technique.

Differently from simple jets under regular waves, in inclined negatively buoyant jets the point of maximum height rotates and the impact point oscillates around a fixed position, which is different from that of the stationary case. Both the maximum height and the impact distance are smaller than the corresponding ones without waves and are more influenced by the wave amplitude. The oscillation of the initial region of the negatively buoyant jet causes a bifurcation, more pronounced when the 
wave amplitude $A$ increases. As a consequence of the previous two effects, the region interested by the discharge is reduced in size by the wave action.

Differently from simple or positively buoyant jets, the dilution of inclined negatively buoyant jets is not always enhanced by waves. Specifically, the strongest waves tested in the present work tend to reduce the dilution, whilst the weakest ones tend to enhance it. As usually the stagnant receiving body case is considered the worst one for dilution, designers should pay particular attention in the case of dense discharges, because in regions often affected by strong waves the wavy receiving body conditions might be more critical for dilution and, consequently, for environmental preservation.

Supplementary Materials: The following are available online at http://www.mdpi.com/2073-4441/10/6/726/s1, The movie "Run7_Ferrari_et_al.mp4" depicts few seconds of Run 7 of Table 1, i.e., a NBJ with Fr $=28$ released in a stagnant environment. The movie "Run10_Ferrari_et_al.mp4" depicts few seconds of Run 10 of Table 1, i.e., the same NBJ of the previous movie but released into a receiving body affected by regular waves with $A=12.5 \mathrm{~mm}$ and $T=1.00 \mathrm{~s}$. The movie "Run10_mean_phase_Ferrari_et_al.mp4" depicts the non-dimensional mean concentration fields for the same NBJ of the previous movie, divided into 8 phases; the white line is the stagnant case jet axis, the white circle is the stagnant case point of maximum height and the white asterisk is the stagnant case impact point.

Author Contributions: S.F. and G.Q. conceived, designed and performed the experiments; S.F., M.G.B. and G.Q. analyzed the data and wrote the paper.

Acknowledgments: The present research was funded by the University of Cagliari. The authors very much appreciate all the help provided by Mr. Antonio Mascia.

Conflicts of Interest: The authors declare no conflict of interest.

\section{References}

1. Ferrari, S.; Querzoli, G. Mixing and re-entrainment in a negatively buoyant jet. J. Hydraul. Res. 2010, 48, 632-640. [CrossRef]

2. Qiao, Q.S.; Choi, K.W.; Chan, S.N.; Lee, J.H.W. Internal hydraulics of a chlorine jet diffuser. J. Hydraul. Eng. 2017, 143, 06017022. [CrossRef]

3. Bashitialshaaer, R.; Larson, M.; Persson, K.M. An experimental investigation on inclined negatively buoyant jets. Water 2012, 4, 720-738. [CrossRef]

4. Bashitialshaaer, R.; Persson, K.M.; Larson, M. New criteria for brine discharge outfalls from desalination plants. Environ. Sci. Eng. 2015, 149, 451-467. [CrossRef]

5. Seo, I.W.; Song, C.G. Dispersion of negatively buoyant effluent jetted from ORV. KSCE J. Civ. Eng. 2015, 19, 1164-1173. [CrossRef]

6. Christodoulou, G.C.; Papakonstantis, I.G.; Nikiforakis, I.K. Desalination brine disposal by means of negatively buoyant jets. Desalin. Water Treat. 2015, 53, 3208-3213. [CrossRef]

7. Oliver, C.J.; Davidson, M.J.; Nokes, R.I. Removing the boundary influence on negatively buoyant jets. Environ. Fluid Mech. 2013, 13, 625-648. [CrossRef]

8. Geyer, A.; Phillips, J.C.; Mier-Torrecilla, M.; Idelsohn, S.R.; Oñate, E. Flow behaviour of negatively buoyant jets in immiscible ambient fluid. Exp. Fluids 2012, 52, 261-271. [CrossRef]

9. Papakonstantis, I.G.; Christodoulou, G.C.; Papanicolaou, P.N. Inclined negatively buoyant jets 1: Geometrical characteristics. J. Hydraul. Res. 2011, 49, 3-12. [CrossRef]

10. Papakonstantis, I.G.; Christodoulou, G.C.; Papanicolaou, P.N. Inclined negatively buoyant jets 2: Concentration measurements. J. Hydraul. Res. 2011, 49, 13-22. [CrossRef]

11. Crowe, A.T.; Davidson, M.J.; Nokes, R.I. Maximum height and return point velocities of desalination brine discharges. In Proceedings of the 18th Australasian Fluid Mechanics Conference, Launceston, Australia, 3-7 December 2012.

12. Crowe, A.T.; Davidson, M.J.; Nokes, R.I. Velocity measurements in inclined negatively buoyant jets. Environ. Fluid Mech. 2016, 16, 503-520. [CrossRef]

13. Besalduch, L.A.; Badas, M.G.; Ferrari, S.; Querzoli, G. Experimental studies for the characterization of the mixing processes in negative buoyant jets. Eur. Phys. J. WoC 2013, 45, 01012. [CrossRef]

14. Besalduch, L.A.; Badas, M.G.; Ferrari, S.; Querzoli, G. On the near field behavior of inclined negatively buoyant jets. Eur. Phys. J. WoC 2014, 67, 02007. [CrossRef] 
15. Malcangio, D.; Ben Meftah, M.; Mossa, M. Physical modelling of buoyant effluents discharged into a cross flow. In Proceedings of the 2016 IEEE Workshop on Environmental, Energy, and Structural Monitoring Systems, Bari, Italy, 13-14 June 2016. [CrossRef]

16. Hajikandi, H.; Barjastehmaleki, S. Negatively buoyant jets at low froude numbers in a co-flow. Proc. Inst. Civ. Eng. Water Manag. 2015, 168, 129-138. [CrossRef]

17. Lai, C.C.K.; Lee, J.H.W. Initial mixing of inclined dense jet in perpendicular crossflow. Environ. Fluid Mech. 2014, 14, 25-49. [CrossRef]

18. Yang, Z.-H.; Huai, W.-X.; Dai, H.-C. Experimental investigation into hot water slot jets with negatively buoyancy in cross flow. J. Hydrodyn. 2005, 17, 412-417.

19. Davies, P.A.; Käse, R.H.; Ahmed, I. Laboratory and numerical model studies of a negatively-buoyant jet discharged horizontally into a homogeneous rotating fluid. Geophys. Astro Fluid 2001, 95, 127-183. [CrossRef]

20. Davies, P.A.; Ahmed, I. Laboratory studies of a round, negatively buoyant jet discharged horizontally into a rotating homogeneous fluid. Fluid Dyn. Res. 1996, 17, 237-274. [CrossRef]

21. Stancanelli, M.; Musumeci, R.E.; Foti, E. Dynamics of gravity currents in the presence of surfacewaves. J. Geophys. Res. Ocean. 2018. [CrossRef]

22. Bas, B.; Sedat Kabdasli, M.; Nur Erturk Bozkurtoglu, S.; Zafer Seker, D. Effect of wave direction on discharged brine dilution. Fresenius Environ. Bull. 2012, 21, 3093-3100.

23. Lin, J.F.; Hsiao, S.C.; Hsu, T.W.; Chang, K.A. Experimental study of a negatively buoyant horizontal jet in wave environment. Proc. Int. Conf. Offshore Mech. Arct. Eng. 2011, 6, 191-198. [CrossRef]

24. Lin, J.F.; Hsiao, S.C.; Hsu, T.W.; Chang, K.A. Buoyancy effect on turbulent round jet under regular waves. J. Waterw. Port. Coast. Ocean. Eng. 2013, 139, 190-208. [CrossRef]

25. Chin, D.A. Influence of surface waves on outfall dilution. J. Hydraul. Eng. 1987, 113, 1006-1018. [CrossRef]

26. Sharp, J.J. The effects of waves on buoyant jets. Proc. Inst. Civ. Eng. 1986, 81, 471-475.

27. Xu, Z.; Chen, Y.; Tao, J.; Pan, Y.; Sowa, D.M.A.; Li, C.-W. Three-dimensional flow structure of a non-buoyant jet in a wave-current coexisting environment. Ocean. Eng. 2016, 116, 42-54. [CrossRef]

28. Xu, Z.S.; Chen, Y.P.; Tao, J.F.; Pan, Y.; Zhang, C.K.; Li, C.W. Modelling of a non-buoyant vertical jet in waves and currents. J. Hydrodyn. 2016, 28, 778-793. [CrossRef]

29. Hsiao, S.C.; Hsu, T.W.; Lin, J.-F.; Chang, K.A. Mean and turbulence properties of a neutrally buoyant round jet in a wave environment. J. Waterw. Port. Coast. Ocean. Eng. 2011, 137, 109-122. [CrossRef]

30. Chang, K.A.; Ryu, Y.; Mori, N. Parameterization of neutrally buoyant horizontal round jet in wave environment. J. Waterw. Port. Coast. Ocean. Eng. 2009, 135, 100-107. [CrossRef]

31. Ryu, Y.; Chang, K.A.; Mori, N. Dispersion of neutrally buoyant horizontal round jet in wave environment. J. Hydraul. Eng. 2005, 131, 1088-1097. [CrossRef]

32. Mossa, M. Experimental study on the interaction of non buoyant jets and waves. J. Hydraul. Res. 2004, 42, 13-28. [CrossRef]

33. Chyan, J.M.; Hwung, H.H. On the interaction of a turbulent jet with waves. J. Hydraul. Res. 1993, 31, 791-810. [CrossRef]

34. Chyan, J.; Hwung, H.; Chang, Y. Wave effects on the mean flow characteristics of turbulent round jets. In Environmental Hydraulics; Lee, J., Cheung, Y., Eds.; Balkema: Rotterdam, The Netherlands, 1991.

35. Ferrari, S.; Querzoli, G. Laboratory experiments on the interaction between inclined negatively buoyant jets and regular waves. Eur. Phys. J. WoC 2015, 92, 02018. [CrossRef]

36. Von Ellenrieder, K.D.; Dhanak, M.R. Hydromechanics. In Springer Handbook of Ocean Engineering; Dhanak, M.R., Xiros, N.I., Eds.; Springer: New York, NY, USA, 2016; ISBN 978-3-319-16648-3.

37. RON-Rete Ondametrica Nazionale (Italian National wavemetric System). Available online: http://dati. isprambiente.it/dataset/ron-rete-ondametrica-nazionale/ (accessed on 14 March 2018).

38. Troy, C.D.; Koseff, J.R. The generation and quantitative visualization of internal breaking waves. Exp. Fluids 2005, 38, 549-562. [CrossRef]

39. Sutton, J.A.; Fisher, B.T.; Fleming, J.W. A laser-induced fluorescence measurement for aqueous fluid flows with improved temperature sensitivity. Exp. Fluids 2008, 45, 869-881. [CrossRef] 
40. Ferrari, S.; Badas, M.G.; Querzoli, G. A non-intrusive and continuous-in-space technique to investigate the wave transformation and breaking over a breakwater. Eur. Phys. J. WoC 2016, 114, 02022. [CrossRef]

41. Bendat, J.S.; Piersol, A.G. Random Data: Analysis and Measurement Procedures, 4th ed.; Wiley and Sons: Hoboken, NJ, USA, 2010; pp. 1-640. ISBN 978-0-470-24877-5.

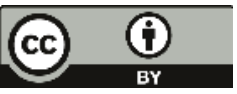

(C) 2018 by the authors. Licensee MDPI, Basel, Switzerland. This article is an open access article distributed under the terms and conditions of the Creative Commons Attribution (CC BY) license (http:/ / creativecommons.org/licenses/by/4.0/). 


\title{
Computational Fluid Dynamics for Modeling Gravity Currents in the Presence of Oscillatory Ambient Flow
}

\author{
Laura Maria Stancanelli ${ }^{*}{ }^{\dagger}$, Rosaria Ester Musumeci ${ }^{\dagger}$ and Enrico Foti \\ Department of Civil Engineering and Architecture, Via Santa Sofia 64, 95125 Catania, Italy; \\ rmusume@unict.it (R.E.M.); efoti@unict.it (E.F.) \\ * Correspondence: lmstanca@unict.it; Tel.: +39-095-375-2729 \\ + These authors contributed equally to this work.
}

Received: 15 February 2018; Accepted: 8 May 2018; Published: 14 May 2018

\begin{abstract}
Gravity currents generated by lock release are studied in the case of initially quiescent ambient fluid and oscillating ambient fluid (regular surface waves). In particular, the dynamics of the density currents are investigated by means of CFD numerical simulations. The aim is to evaluate the influence of the ambient fluid velocity field on the observed mixing and turbulent processes. Results of two different turbulence closure models, namely the standard $k-\varepsilon$ turbulence model and the LES model, are analyzed. Model predictions are validated through comparison with laboratory measurements. Results show that the $k-\varepsilon$ model is able to catch the main current propagation parameters (e.g., front velocity at the different phases of the evolution of the current, gravity current depth, etc.), but that a LES model provides more realistic insights into the turbulent processes (e.g., formation of interfacial Kelvin-Helmholtz billows, vortex stretching and eventual break up into 3D turbulence). The ambient fluid velocity field strongly influences the dynamics of the gravity currents. In particular, the presence of an oscillatory motion induces a relative increase of mixing at the front (up to $25 \%$ ) in proximity of the bottom layer, and further upstream, an increase of the mixing process (up to $60 \%$ ) is observed due to the mass transport generated by waves. The observed mixing phenomena observed are also affected by the ratio between the gravity current velocity $v_{f}$ and the horizontal orbital velocity induced by waves $u_{w}$, which has a stronger impact in the wave dominated regime $\left(v_{f} / u_{w}<1\right)$.
\end{abstract}

Keywords: CFD; Kelvin-Helmholtz; billow; lobe; cleft; gravity current; surface waves

\section{Introduction}

Gravity currents are mainly horizontal flows moving under the influence of gravity and generated by buoyancy differences. Gravity currents are phenomena of great interest in the field of engineering and geophysics with numerous important environmental and industrial applications [1,2]. These include: the outflow of brackish waters [3] referred to as viscous gravity currents, pyroclastic flows [4] referred to as particle-laden gravity currents and mud and debris flows $[5,6]$ referred to as concentrated flows.

The propagation of gravity currents under oscillatory wave regimes is quite relevant in coastal regions, especially to understand the processes acting during the continuous natural or artificial discharges of fluids having a different density than the ambient fluid, e.g., river plumes, desalination plant, industrial discharges, etc. [3,7]. Notwithstanding the fact that the discharge of fresh or brackish water in the sea is frequent, the effect of the wave motion on the propagation in coastal regions of the salt-brackish wedge has not been systematically investigated yet [8].

In the absence of waves, extensive laboratory investigations have been carried out in the field of viscous gravity currents. Several geometries of the flow domain have been investigated, for example: smooth bottom [9], rough beds [10], sloping bottoms [11-14], the presence of obstacles [15] and 
stratified ambient fluid [16]. Experimental studies usually involve hydraulic flumes filled with a lower density fluid, and the current is generated by means of lock or point release of a higher density fluid. In the former approach, the heavier fluid is contained in a lock, whose gate is suddenly removed, while in the latter approach, the heavier fluid is released from a point source. The analysis of the advancing front and of the instabilities has often been carried out through image analysis. In this case, images are acquired from the side glass wall of the tank. Such an experimental setting allows for width-averaged density measurements in a 2D configuration, which could result in a limited analysis in terms of turbulence structures. Indeed, studies adopting 2D numerical simulation were successful in the description of turbulence structures; for example, Dai [17] describes the gravity current propagation in the acceleration phase, during which three-dimensional interactions are not important. However, Cantero et al. [18] points out the importance of three-dimensional processes governing the interface between heavy and light fluids, which first roll up by baroclinic generation of Kelvin-Helmholtz vortices and then undergo sudden breakup and decay to small-scale turbulence. In such a case, numerical three-dimensional analyses, as the one carried out by Ottolenghi et al. [19], should be applied. In fact, unless one adopts a very complex and expensive high-speed camcorder and 3D particle image velocimetry [20], it is extremely difficult to have information on the 3D turbulent processes that influence mixing by just considering 2D lab data. Measurements of the instantaneous bed shear stress distribution are nearly impossible to achieve experimentally [21]. Indeed, detailed measurements of the velocity and density fields within the gravity current are seldom available from experimental studies [21]. High-resolution numerical simulations can overcome the lack of information previously mentioned, providing also information on the global energy balance at different stages of the density current evolution [22-26]. In the past, numerical simulations provided important information of the entrainment mechanisms characteristic of the gravity current, and important progress is summarized as follows. The application of direct numerical simulations provided important results in order to clarify the instability mechanism that governs the formation of the complex lobe-and-cleft pattern commonly observed at the leading edge of gravity currents [22,23]. Ooi et al. [21] investigated, using large eddy simulations (LES), the compositional gravity current flows produced by the instantaneous release of a finite volume and heavier lock fluid in a rectangular horizontal plane channel. The LES numerical simulations provided insightful results, describing the development of turbulent structures during the slumping phase and the buoyancy-inertia phase. High-resolution two-dimensional Navier-Stokes simulations provided interesting results on the entrainment mechanisms governing the gravity current propagating downslope. In particular, the interface roll-up and vortex overturns were studied varying parameters as the depth ratio and the slope angle [17]. The entrainment and mixing in unsteady gravity currents were studied by Ottolenghi et al. [19] performing LES simulations, focusing on the influence of the aspect ratio and density difference. The results showed that irreversible mixing is detected during the entire development of the flow, not only during self-similar phases, but also during the slumping phase.

The interaction between gravity currents and oscillatory motion has been investigated only in a few works [8,27-29]. $\mathrm{Ng}$ and Fu [27] studied numerically the spreading of viscous gravity currents propagating in intermediate and deep water depth conditions, observing that wave-induced streaming flow acting at the bottom is responsible for changes in the gravity current velocity speed. Robinson et al. [8] were the first to analyze in laboratory the influence of the orbital motion induced by the presence of regular progressive free-surface water waves on the gravity current propagation. They adopted a point release technique and observed the self-similar phase of the two fronts respectively propagating under regular surface waves in deep water condition. Musumeci et al. [28] investigated the propagation of gravity currents under regular surface waves, modeling the phenomenon both experimentally and numerically. The gravity currents were modeled assuming low density difference and intermediate water depth conditions and adopted the lock-exchange problem for the generation of the gravity current. They focused on the front spreading evolution, comparing the experimental evidence with numerical results, and on the capability of the numerical model to 
reproduce the 2D turbulence at the interface. Viviano et al. [30] investigated the turbulence observed during the interaction between waves and gravity currents. They adopted a simple 2D numerical model that couples a Boussinesq-type of model for surface waves and a gravity current model for stratified flows. The velocity is decoupled into a wave-related component and a density gradient-related component. Turbulence is described by two alternative approaches: a simple subgrid Smagorinsky formulation, and the Smagorinsky formulation with a depth uniform eddy viscosity. Such a model designed for engineering applications needs a previous careful calibration process to choose the calibration parameters of the Smagorinsky formulation. The recent work of Stancanelli et al. [29] has explored a larger dataset compared to the one presented by Musumeci et al. [28] evaluating the change of front velocity and mixing at the front of the current for a large number of wave types and different density fluid ratio conditions. They show that the front velocity is related to the Lagrangian mass transport induced by the surface waves, while the mixing observed at the front is related to the orbital motion.

The aim of the present work is to numerically investigate the influence of the ambient fluid velocity field on the mixing processes and the formation of three-dimensional turbulent structures generated by the density current propagation. The objective of the present study is also to explore the dynamics of density currents adopting different turbulence models and to discuss the possibility to adopt them for engineering applications. The numerical simulation of high Reynolds number flows is hampered by model accuracy if the Reynolds-averaged Navier-Stokes (RANS) equations are used, and by computational cost if a more sophisticated model, such as direct or large-eddy simulations (LES), is adopted [31]. Here, we highlight the performance of numerical models in a very complex flow, such as the superimposition of gravity currents and surface waves. 3D flow structures are discussed not only at the front, but also along the entire gravity current and at the bottom boundary. To the authors' knowledge, this has never been attempted before. Indeed, previous works [28-30] were able only to comment on the 2D features of the turbulence structures, not taking into account small-scale structures. Numerical modeling is carried out by means of a computational fluid dynamics model (CFD). Two different turbulence closure schemes are used, namely the standard $k-\varepsilon$ turbulence model [32] and the LES model [33]. A volume of fluid (VOF) model is used to account for free surface effects [34]. The capability of the two turbulence closures to predict various important dynamics of density current propagation in the presence of waves (i.e., propagation speed, gravity current height and density profile) is discussed by comparing the numerical results with the laboratory experiments of Stancanelli et al. [29]. Results highlight how not only the current propagation, but also the turbulent structures and consequently the density field are significantly affected by the nonlinear interaction between the gravity current and the regular surface waves.

\section{Materials and Methods}

\subsection{Model Description}

The CFD computational model used in the present work is the FLOW-3D model distributed by Flow Science Inc., which is considered a powerful tool thanks to its capabilities of accurately predicting free-surface flows. In particular, in FLOW-3D, the free surface is modeled by the volume of fluid (VOF) technique. The VOF method consists of three ingredients: a scheme to locate the surface, an algorithm to track the surface as a sharp interface moving through a computational grid and a means of applying boundary conditions at the surface. Such a model is described in Hirt and Nichols [34]. Since its commercial release, FLOW-3D has been used in research, providing to engineers valuable insight into many physical flow processes [35-38].

A variety of turbulence models for simulating turbulent flows, including the Prandtl mixing length model, the one-equation model and the standard two-equation $k-\varepsilon$ model, the re-normalization group (RNG) scheme and the large eddy simulation (LES) model, are available within FLOW-3D. These turbulence models have been well tested and documented in the relevant technical literature [1]. 
The standard $k-\varepsilon$ model and the LES model are considered here, since the first one is able to catch the main characteristics of the flow at a relatively low computational cost, while the LES scheme is more sophisticated and is able to account in a physical way for the effect of the smallest unresolved scales on the larger ones in a flow. FLOW-3D employs the finite difference/control volume method to discretize the computational domain. In particular, the physical domain to be simulated is decomposed by using Cartesian grids composed of variable size hexahedral cells. Applications are presented later in Section 3.

The following continuity equation and momentum equations are solved along with the turbulent closure $k-\varepsilon$ equations:

$$
\begin{gathered}
\frac{\partial u_{i} A_{i}}{\partial x_{i}}=0 \\
\frac{\partial u_{i}}{\partial t}+\frac{1}{V_{f}} u_{j} A_{j} \frac{\partial u_{i}}{\partial x_{j}}=-\frac{1}{\rho} \frac{\partial p}{\partial x_{i}}+g_{i}+f_{i}
\end{gathered}
$$

where:

$$
\begin{gathered}
\rho V_{f} f_{i}=\tau_{b, i}-\frac{\partial A j S_{i, j}}{\partial x_{j}} \\
S_{i i}=-2 \mu_{t o t}\left[\frac{\partial u_{i}}{\partial x_{i}}\right], S_{i j}=-2 \mu_{t o t}\left[\frac{\partial u_{i}}{\partial x_{j}}+\frac{\partial u_{j}}{\partial x_{i}}\right]
\end{gathered}
$$

where $u_{i}$ is the mean velocity, $p$ is the pressure, $A_{i}$ is the fractional open area open to flow in the $i$ direction, $V_{f}$ is the fractional volume open to flow, $g$ represents the gravity acceleration, $f_{i}$ represents the viscous acceleration, $S_{i j}$ is the strain rate tensor, $\tau_{b, i}$ is the wall shear stress, $\rho$ is the density of water, $\mu_{t o t}$ is the total dynamic viscosity including the effect of turbulence $\mu_{\text {tot }}=\mu+\mu_{T}$, with $\mu$ being the dynamic viscosity and $\mu_{T}$ the eddy viscosity. For the $k-\varepsilon$ model, the eddy viscosity is approximated as:

$$
\mu_{T}=\frac{\rho C_{\mu} k^{2}}{\varepsilon}
$$

where the following closure equations for the turbulent kinetic energy $k$ and the dissipation rate $\varepsilon$ are:

$$
\begin{aligned}
\frac{\partial k}{\partial t}+u_{j} \frac{\partial k}{\partial x_{j}}=\tau_{i j} \frac{\partial u_{i}}{\partial x_{j}} & -\varepsilon+\frac{\partial}{\partial x_{j}}\left[\frac{1}{\rho}\left(\frac{\mu_{t}}{\sigma_{k}}+\mu\right) \frac{\partial k}{\partial x_{j}}\right] \\
\frac{\partial \varepsilon}{\partial t}+u_{j} \frac{\partial \varepsilon}{\partial x_{j}} & =\frac{\partial}{\partial x_{j}}\left[\left(\frac{\mu_{t}}{\sigma_{\varepsilon}}+\mu\right) \frac{\partial \varepsilon}{\partial x_{j}}\right] \\
& +C_{1 \varepsilon} \frac{\varepsilon}{k} \tau_{i j} \frac{\partial u_{i}}{\partial x_{j}}-C_{2 \varepsilon} \frac{\varepsilon^{2}}{k}
\end{aligned}
$$

The constant coefficients are chosen based on the classical model of Launder and Spalding [39]: $C_{\mu}=0.09\left(C_{1 \varepsilon}=1.44, C_{2 \varepsilon}=1.92, \sigma_{k}=1.00, \sigma_{\varepsilon}=1.30\right)$.

Regarding the LES model [40,41], a Smagorinsky approach [42] is used to approximate the eddy viscosity as:

$$
\mu_{T}=\rho(c L)^{2}\left(e_{i j} e_{i j}\right)^{0.5} \frac{\rho C_{\mu} k^{2}}{\varepsilon}
$$

where the constant $c=0.2, C_{\mu}=0.09$ as in the $k-\varepsilon$ model, the strain rate tensor is given by:

$$
e_{i j}=\frac{1}{2}\left(\frac{\partial u_{i}}{\partial x_{j}}+\frac{\partial u_{j}}{\partial x_{i}}\right)
$$

and the characteristic length scale is defined as:

$$
L=(\delta x \delta y \delta z)^{1 / 3}
$$


It is worth pointing out that the two different turbulence models have different computational costs. Piomelli [31] argued that the cost of a calculation scales like the Reynolds number to the power 2.4 for LES. The computational cost of LES model is about 4-100-times higher than that required by the RANS model [43,44].

Additionally, FLOW-3D is able to simulate the free surface wave motion, considering both regular linear [45] or nonlinear waves and irregular waves. In particular, three nonlinear wave theories are used for nonlinear wave generation: the fifth-order Stokes wave theory [46], the Fourier series method for Stokes and cnoidal waves [47] and McCowan's theory for solitary waves [48,49].

\subsection{Flume Tests}

The experiments presented here, used for validating the numerical results, are those carried out at the small-scale wave flume of the Hydraulic Laboratory of the University of Catania. The experimental apparatus is the one adopted and presented by Musumeci et al. [28] and Stancanelli et al. [29]. In the following, we present a brief description of the experimental apparatus and of the experimental procedure, as well as the controlling parameters of the tests used for validation. More detailed information can be found in the cited literature.

The flume is $9 \mathrm{~m}$ long, $0.5 \mathrm{~m}$ wide and $0.7 \mathrm{~m}$ high. A piston-type wave maker is located at the initial section $(x=0 \mathrm{~m})$ of the flume, while at the opposite side, a porous beach minimizes wave reflection. In order to carry out classical lock exchange tests, the flume is partitioned by a Perspex sluice gate (at $x=5.10 \mathrm{~m}$ ). Salt water, having density $\rho_{1}$, is present at the wave maker side of the gate and fresh water, having density $\rho_{0}<\rho_{1}$, at the onshore side.

Full-depth two-dimensional lock-exchange experiments have been carried out with and without regular waves (see Figure 1). At the beginning of each test, samples of the two fluids are collected and then analyzed to measure the density difference. The generation of the gravity currents is performed by manually opening the sluice gate. During the tests performed in the presence of regular waves, the wave maker is activated and the sluice gate is removed only when the first wave is approaching the lock position. The laboratory experimental observations, video-recorded from the side wall, provide information about the geometric and kinematic characteristics of the front propagation. The parameters investigated are the shape, depth and velocity of the current, as well as width-averaged maps of the density field.

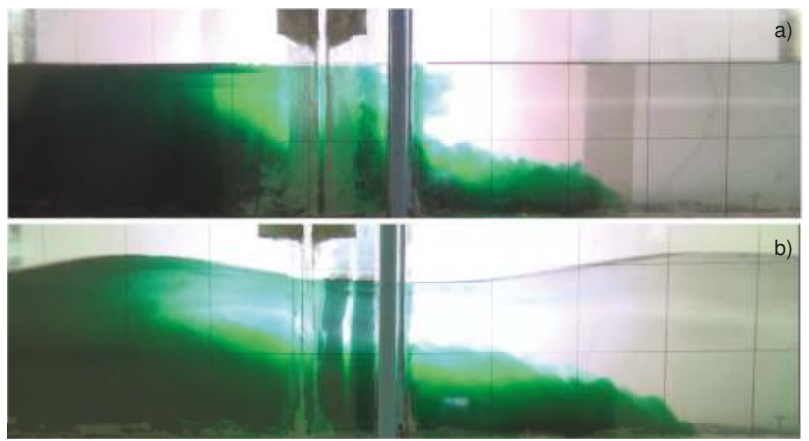

Figure 1. Gravity current propagation during full-depth two-dimensional lock-exchange experiments: (a) in the presence of initially quiescent ambient fluid; (b) when regular surface waves are superimposed on the current.

The controlling parameters of the experiments are the initial still water level within the flume $H$, the salt water density $\rho_{1}$, the fresh water density $\rho_{0}$, the reduced gravity $g^{\prime}=g\left(\rho_{1}-\rho_{0}\right) / \rho_{0}$ where $g$ is the gravitational acceleration, the wave height $H_{w}$ and the wave period $T_{w}$. All experimental results 
refer to gravity current propagation during the slumping phase, characterized by a constant velocity advancement of the front. The density difference is always such that the Boussinesq approximation $\left(\rho_{1} / \rho_{0} \sim 1\right)$ is satisfied. Table 1 reports the values of the controlling parameters of the tests used for model validation. The selected tests include gravity currents characterized by different reduced gravity and different wave conditions. From the dataset of Stancanelli et al. [29], the particular case of the current-dominated regime $\left(v_{f} / u_{w}>1\right)$ has been investigated here (Case No. 6 with $v_{f} / u_{w}=1.3$ ), as well as different wave-dominated regimes (Case Nos. 2, 4, 5 with $v_{f} / u_{w}=0.7-0.8$ ). The wave conditions correspond to: shorter regular surface waves (i.e., Case Nos. 2, 4, 5) and longer surface waves (i.e., Case No. 6). Test cases in the absence of waves are also presented as a benchmark (i.e., Case 1 and Case 3).

Table 1. Controlling parameters of the experimental tests selected to validate the numerical simulations.

\begin{tabular}{ccccccc}
\hline \multirow{2}{*}{ Run } & $\boldsymbol{H}$ & $\rho_{\mathbf{1}}$ & $\rho_{\mathbf{0}}$ & $g^{\prime}$ & $H_{w}$ & $T_{w}$ \\
\cline { 2 - 7 } & $\mathbf{( \mathbf { c m } )}$ & $\mathbf{( \mathbf { k g } / \mathbf { m } ^ { \mathbf { 3 } } )}$ & $\mathbf{( \mathbf { k g } / \mathbf { m } ^ { 3 } )}$ & $\mathbf{( \mathbf { m } / \mathbf { s } ^ { \mathbf { 2 } } )}$ & $\mathbf{( \mathbf { c m } )}$ & $\mathbf{( s )}$ \\
\hline Case 1 & 20.3 & 1010 & 998 & 0.13 & - & - \\
Case 2 & 20.3 & 1010 & 998 & 0.13 & 4.22 & 0.71 \\
Case 3 & 20.3 & 1006 & 998 & 0.08 & - & - \\
Case 4 & 20.3 & 1006 & 998 & 0.08 & 4.22 & 0.71 \\
Case 5 & 20.3 & 1010 & 998 & 0.13 & 2.86 & 0.84 \\
Case 6 & 20.3 & 1010 & 998 & 0.13 & 1.50 & 1.32 \\
\hline
\end{tabular}

\section{Numerical Simulations}

Simulations are performed for flow conditions that correspond to the laboratory experimental setup described in Section 2.2. The computational flume is $9 \mathrm{~m}$ long, $0.5 \mathrm{~m}$ wide and $0.7 \mathrm{~m}$ high. The dimensions are the same as the experimental flume.

The computational grid system is composed of different nested meshes (see Figure 2): two coarser ones (Mesh 1 and Mesh 2, with cubic cells having size $0.01 \mathrm{~m}$ ) for defining the two different fluid regions, namely the saltier water and the fresh water; a finer grid to more accurately solve the interface region between the two fluids (Mesh 3, whose cell size is $0.005 \mathrm{~m}$ ); and a finer grid at the bottom (Mesh 4, with cell size $0.003 \mathrm{~m}$ ). The latter grid permits one to better investigate turbulent structures that develop at the bottom, as lobe and cleft instabilities. The choice of the grid size is the result of a preliminary analysis carried out following the suggestions of Ooi et al. [21], Boris et al. [50], Kyrousi et al. [51] (grid spacing is equal to 0.01-0.05 H).

All boundaries of the flow domain are defined as no-slip smooth walls, except the free surface where a constant pressure is selected as the boundary condition. A zero-gradient boundary condition is used at the initial interface of the two fluid mesh blocks. The dynamics of the gravity currents is considered independent of the gate opening operation, since the time scale of current propagation and wave-current interaction is $10^{2}$ larger than the time scale of gate opening. Moreover, the analysis is carried out about a water depth of 10 from the lock position. Therefore, in the measuring area, the effects of operations at the gate can be assumed to be negligible.

For the case of gravity currents in the presence of waves, at the offshore end of the saltier side, a regular wave field is generated and enters the domain. The wave is assumed to come from a flat bottom reservoir, which is located outside the computational domain. For the description of the wave motion, the Fourier series method for Stokes and cnoidal waves, which possesses higher order of accuracy than other wave theories [52], is selected. Such a method is selected since in intermediate waters, as the present ones, cnoidal waves are a better representation than linear waves of the experimentally-generated constant-shape waves [53]. Moreover, in such a case, in order to avoid wave reflection from the onshore boundary, an absorbing layer at the end of the tank is adopted. Such an absorbing layer mimics the effect of the porous beach in the experiments. 


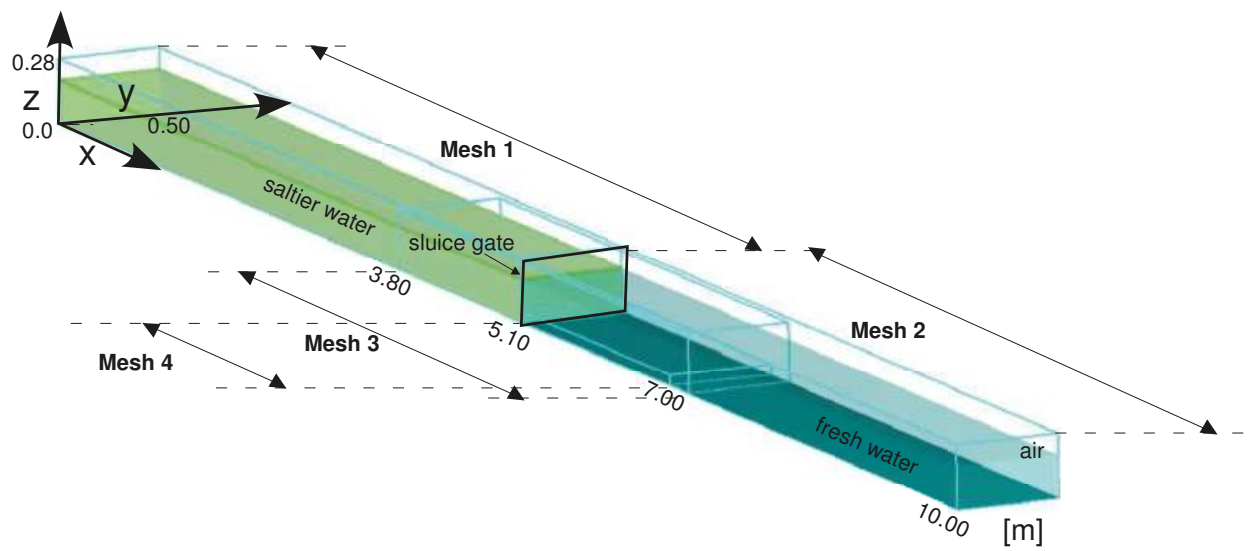

Figure 2. Computational domain and boundary conditions used for the CFD simulations of gravity currents.

\section{Results}

The CFD model was used to simulate different experimental tests characterized by different density ratios and different ambient fluid conditions (presence and absence of waves). The model is applied both to a classical lock exchange problem with initially quiescent ambient fluid (see Test No. 1 and No. 3) and to reproduce lock exchange experiences in the presence of short regular surface waves (i.e., Case Nos. 2, 4, 5) and long surface waves (i.e., Case No. 6). The simulated flow conditions (i.e., water depth, salinity difference, wave characteristics, etc.) are the same as the experimental ones reported in Table 1. Simulations adopting the $k-\varepsilon$ turbulence model and the LESturbulence model are compared. An Intel(R) Core(TM) i7-4790 CPU 3.60-GHz processor has been used to run all the numerical simulations. On this processor, simulations adopting the $k-\varepsilon$ turbulence model required about $4.12 \times 10^{5}$ of CPU time, whereas the LES turbulence model required about $9.1 \times 10^{5}$ of CPU time.

The validation of the numerical model is performed by comparing the experimental results of Stancanelli et al. [29] of the front position with the numerical results. Figure 3 reports the experimental results, the numerical results and the predictions of the model of Huppert and Simpson [54] for Test No. 1 and No. 3, which are characterized by different reduced gravity values. The well-known model of Huppert and Simpson [54] has been proposed to predict the front evolution in the slumping phase, and it has been calibrated on a set of experimental data. A linear behavior is recognizable, indicating that the observed gravity currents are in the constant-velocity phase (slumping phase). The $k-\varepsilon$ and the LES simulations agree fairly well with each other in terms of the front positions. Indeed, both numerical results show a linear trend characterized by the same angular coefficient. The slope of the linear trend of the front advancement indicates that for lower reduced gravity (Test No. 3), the averaged front velocity is of about $5.4 \mathrm{~cm} / \mathrm{s}$, which is equal to the value measured in the lab $\left(v_{f-\text { meas }}=5.4 \mathrm{~cm} / \mathrm{s}\right)$; while for higher reduced gravity (Test No. 1), it indicates an averaged velocity of $6.2 \mathrm{~cm} / \mathrm{s}$, which is slightly smaller than the measured value reported in Stancanelli et al. [29] $\left(v_{f-\text { meas }}=6.4 \mathrm{~cm} / \mathrm{s}\right)$. In general, as should be expected, an increase of the reduced gravity is responsible for an increase of the front velocity.

The influence of the adopted closure schemes is appreciable when comparing the front shape. Figure 4 shows the gravity current front after $t=12.8 \mathrm{~s}$ from the sluice gate opening respectively for (a) the experimental results of Stancanelli et al. [29], the numerical results applying (b) the $k-\varepsilon$ model and (c) the LES model. The gravity current shape of the experimental evidence, defined applying a density concentration threshold equal to 0.96 , is reported as a dash-dotted green line over the numerical results. As expected, the $k-\varepsilon$ model tends to smooth out the shape of the interface showing also 
a front with a round shape (Figure $4 \mathrm{~b}$ ). The LES turbulence model, instead, reproduces a sharper front (Figure 4c), which agrees better with the experimental observations (Figure 4a). Furthermore, the dynamics at the interface between the two fluids is better reproduced by the LES model, since mixing processes induced by the shear between the two fluids and at the bottom induced by the wall effect are better represented.
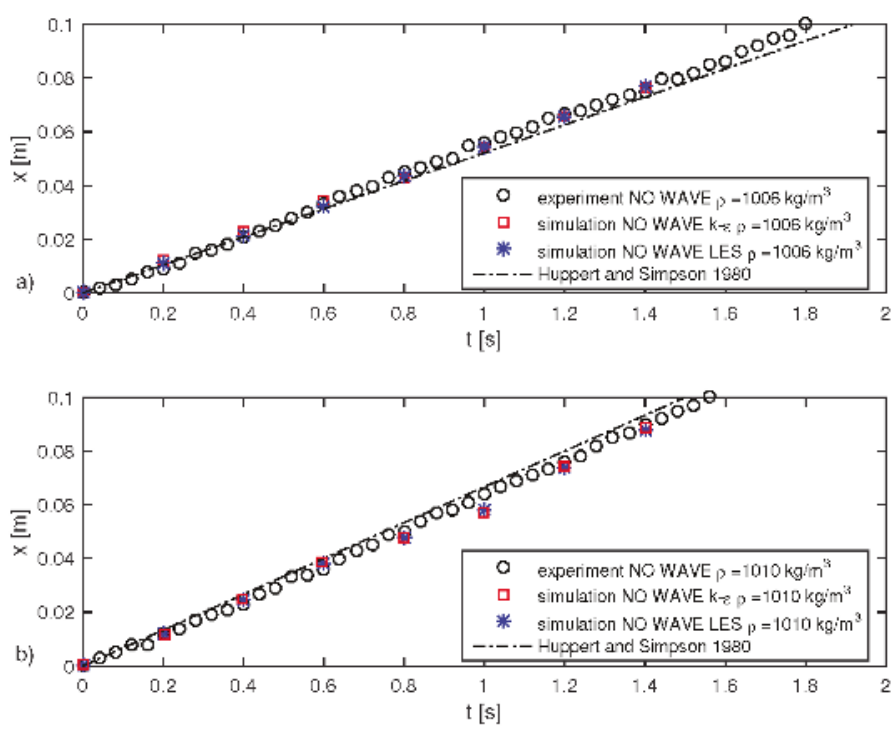

Figure 3. Front position of the gravity current in quiescent ambient fluid. The numerical results are compared with the results of the experimental campaign of Stancanelli et al. [29] and with the model of Huppert and Simpson [54]: (a) Test No. 3, reduced gravity equal to $0.08 \mathrm{~m} / \mathrm{s}^{2}$; (b) Test No. 1, reduced gravity equal to $0.10 \mathrm{~m} / \mathrm{s}^{2}$.

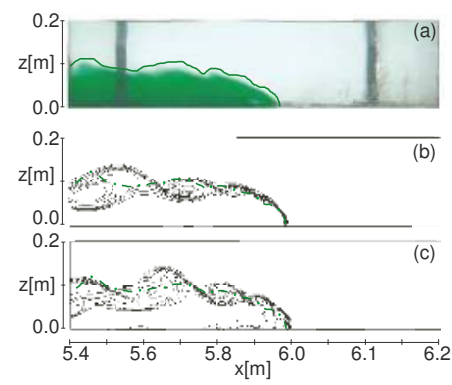

Figure 4. Density contour map observed and calculated for Case 3 after $t=12.8 \mathrm{~s}$ from the gate opening: (a) using the light intensity to infer the dye (salt) concentration during the experiments; (b) $k-\varepsilon$ model simulation; (c) LES model simulation. In both density maps, 10 contour layers are used, with values in the range $998-1006 \mathrm{~kg} / \mathrm{m}^{3}$. The shape of the experimental gravity current (density concentration threshold 0.96) is indicated with a dash-dotted green line on the numerical results.

Both turbulence models are able to reproduce the development of K-H billows. Such turbulent structures are mainly 2D. This allows one to investigate them also using width-average measurements acquired at the side wall. However, the experimental observations fail to describe small-scale instabilities. For investigating such instabilities, we analyze the results of the LES model, which is 
more reliable, and it enables us also to observe the presence of 3D structures, such as lobe and cleft instabilities interacting with K-H billows. In the numerical simulations, the lobe and cleft development is observed $5 \mathrm{~s}$ after the sluice gate opening. The development of these instabilities caused the loss of the coherent structure of the K-H billows. In Test 1, when the density current propagates in initially quiescent ambient fluid, the small instabilities at the interface appear first in the region just behind the front and then propagate further downstream (see Figure $5 a, b$ ). This result is in agreement with the results of Cantero et al. [18], which observed firstly that fluids roll up by baroclinic generation of Kelvin-Helmholtz vortices and then the breakup and decay of these vortices into small-scale turbulence structures, which propagate upstream with time. In the presence of waves (Test 2), a similar generation of $\mathrm{K}-\mathrm{H}$ billows at the interface and of lobe and clefts at the bottom occurs only during an initial phase. After such a short transitory, the effects of the wave-induced motion can be clearly recognized. In particular, 3D instabilities are formed all over the interface of the gravity current as the response of the non-linear interaction with the superimposed wave field (see Figure $5 \mathrm{c}, \mathrm{d}$ ). It appears that for an experimental duration less than $4 \mathrm{~s}$, the presence of waves does not play a role; while for longer durations, a significant contribution of the orbital motion is observed. In particular, the intensity of the turbulence is reduced at the interface near the saltier front.

LES- classical lock exchange

$t=8 s$

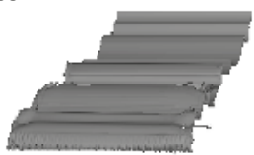

a)

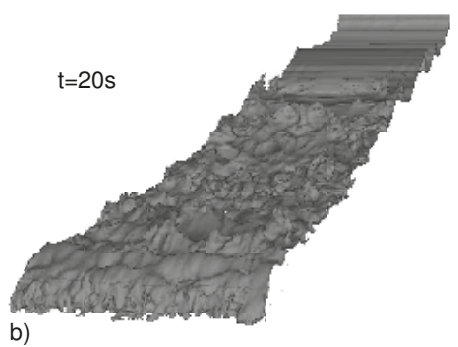

LES- lock exchange with waves

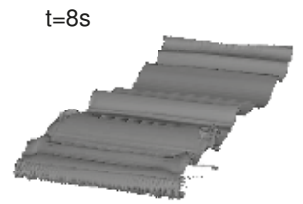

c)

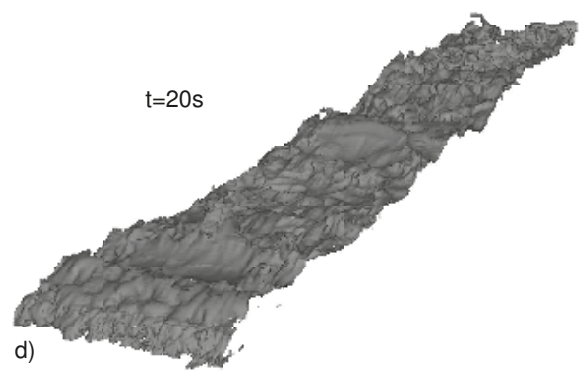

Figure 5. Iso-surface representation of density current $\left(\rho=1004 \mathrm{~kg} / \mathrm{m}^{3}\right)$ adopting the LES turbulence model, respectively: for the Test 1 simulation modeled at the following time steps: (a) $t=8 \mathrm{~s}$ and (b) $t=20 \mathrm{~s}$; for the Test 2 simulation modeled at the following time steps: (c) $t=8 \mathrm{~s}$ and (d) $t=20 \mathrm{~s}$.

In order to better clarify the influence of the wave motion, the turbulent structures observed at the middle longitudinal section are represented adopting the Q-criterion (see Figure 6). Also in this case, we can compare the condition of quiescent ambient fluid (Case 1) and of superimposed surface waves (Cases 5 and 6). It can be noticed for all cases presented that the region behind the front (linear extension of about $0.6 \mathrm{~m}$ ) is strongly turbulent. In particular, a turbulent layer close to the bottom boundary of the channel is identified along the $x$-axis from the front $(x=6.36 \mathrm{~m})$ until behind the section $x=5.50 \mathrm{~m}$. Such a turbulent layer, responsible for quasi-streamwise vortices and hairpin vortices, was also observed in the experimental and numerical analysis of Cantero et al. [18] and of Kyrousi et al. [51], and it will be further discussed in the following. Turbulence structures that govern the region upstream $(4.50 \mathrm{~m}<x<5.50 \mathrm{~m})$ show a regular pattern with cores of the vortices alined along an ideal horizontal in the presence of quiescent ambient fluid. Instead, for both analyzed wave conditions, the cores of 
the vortices are losing the coherent characteristic of the K-H billows. Relevant differences between the absence and the presence of waves can be noticed in the upstream region $(3.80 \mathrm{~m}<x<4.50 \mathrm{~m})$ where the appearance of numerous small turbulence structures is identified in the presence of the oscillatory motion. Such phenomena are responsible for an increased mixing in the presence of waves (see Figure $7 \mathrm{~b}, \mathrm{c})$, which is predominantly for the test case characterized by a wave-dominated $\left(u_{w}>v_{f}\right)$ regime (Figure $7 \mathrm{c})$. As could be reasonably expected, the current-dominated $\left(v_{f}>u_{w}\right)$ regime shows turbulence features more similar to the quiescent ambient fluid case. The spatial distribution of these wave-induced small-scale vortices differs for the two type of waves. In particular, for the longer wave case, they are distributed vertically along the water column, while for shorter waves, an increment of the presence of small structures close to the bottom boundary layer is observed. We believe that the generation of the small-scale vortices, in Test No. 5 and No. 6, is dependent on the mass transport induced by the wave propagation. This assumption is also supported by different studies [55-58] on the wave-induced turbulence, which argued that the turbulent kinetic energy dissipation rate is mainly influenced by a component of the mass transport, the Stokes drift.

In the upper layer, the waves induce a mass transport in the opposite direction of the lighter front propagation (offshore direction), which results in a rearrangement of the density gradient (see Figure 7). In the lower region, in the case of shorter waves, the mass transport has a stronger offshore-directed negative component, while in the presence of longer waves, it could have a positive component close to the bottom [29]. The presented results (Figures 6 and 7) show that the different wave regime is responsible for a change of the density gradient that changes the density field with a variation up to $60 \%$ compared to the case in the absence of waves.

Q-criterion for vortex structures
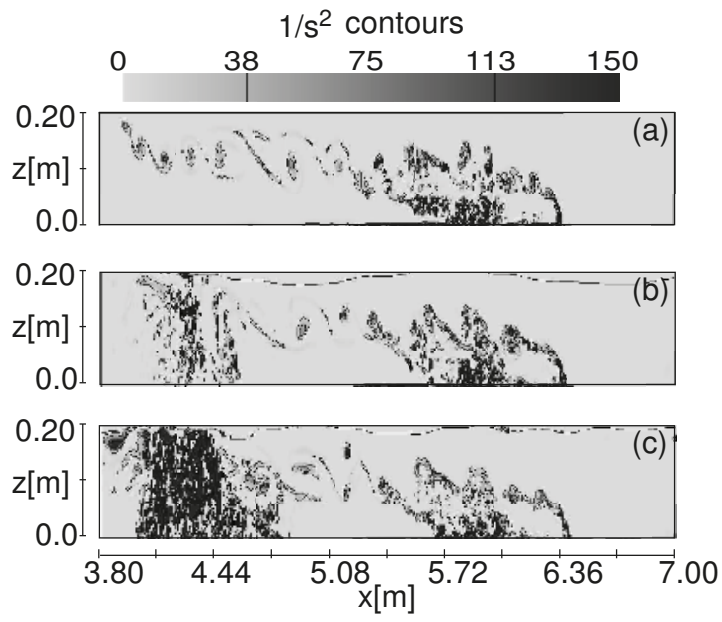

Figure 6. Q-criterion representation of vortex structures at the time step $t=17.4 \mathrm{~s}$ (LES model) for gravity current characterized by the same reduced gravity value $\left(0.13 \mathrm{~m} / \mathrm{s}^{2}\right)$, but different ambient fluid regimes, respectively for: (a) quiescent ambient fluid; (b) presence of shorter regular waves (Case 5); (c) presence of longer regular waves (Case 6). 


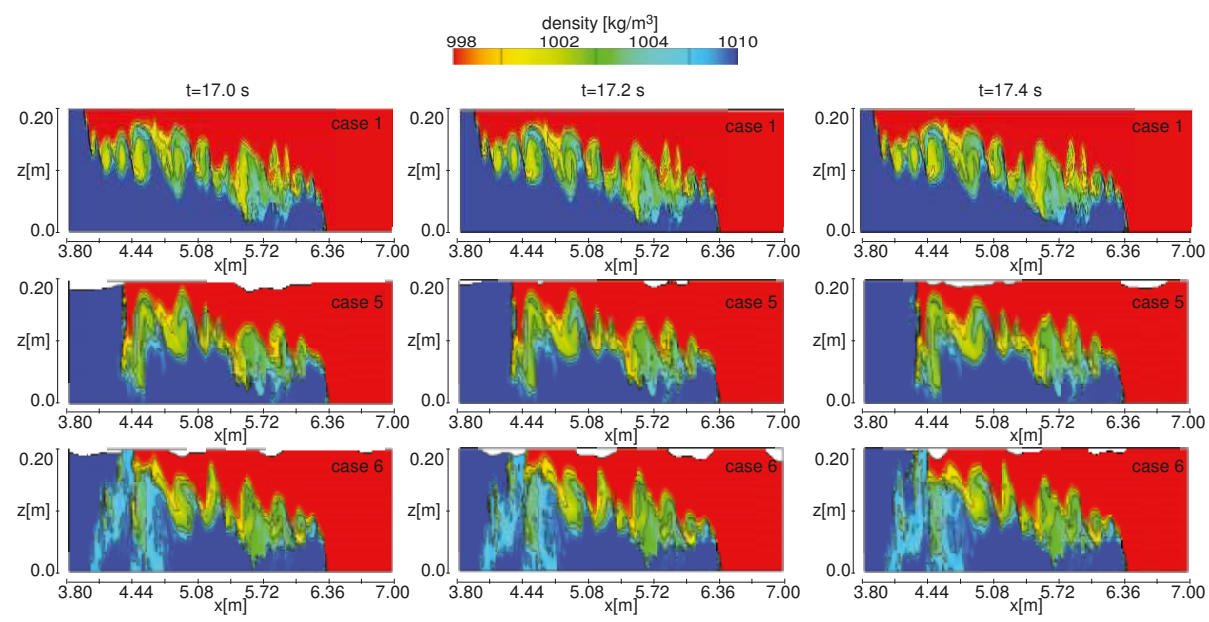

Figure 7. Density maps at different time steps (LES model) for gravity current characterized by the same reduced gravity value $\left(0.13 \mathrm{~m} / \mathrm{s}^{2}\right)$, but different ambient fluid regimes, respectively for: (first row) quiescent ambient fluid (Case 1); (second row) presence of shorter regular waves (Case 5); (third row) presence of longer regular waves (Case 6).

The processes at the bottom are herein described through $\mathrm{x}$-velocity and density maps, presented in Figure 8. Velocity maps show characteristic features of gravity current propagation [18] in the absence of waves, with a mean component similar to the front velocity. For the wave combined flow, spatial velocity oscillations are in accordance with the wave phase. The amplitude of velocity oscillations is of the same order of the maximum horizontal orbital velocity at the bottom $\left(u_{w}=8 \mathrm{~cm} / \mathrm{s}\right)$. For the gravity current propagation in the absence of waves and in the presence of waves in all cases, the shape of quasi-streamwise vortices is recognizable from the density maps acquired during a wave period (Figure 8). For the case in the presence of waves, the mixing process is slightly enhanced, and such a phenomenon could be due to shear velocity induced by the presence of waves in the bottom boundary layer.

Results in terms of vertical profiles of density and horizontal velocity are presented for the front region, always comparing simulations in the absence and in the presence of waves (see Figure 9). Figure $9 \mathrm{a}, \mathrm{b}$ shows the 2D density maps indicating with a dash line the section where the profiles presented in Figure 9e,f are gathered during a time period of $1.2 \mathrm{~s}$ (about $2 T_{w}$ ) $16 \mathrm{~s}<t<17.2 \mathrm{~s}$ after gate opening. Simulation results show that oscillatory motion induces a thicker mixing layer. Indeed, in the absence of waves, the mixing layer thickness is about $20 \%$ of the water depth $H$ (Figure 9c), while in the presence of waves it increases up to $50 \%$ of the water depth $H$ (Figure 9e). Symmetric velocity profiles are recovered for quiescent ambient fluid, while asymmetric ones are obtained in the presence of waves. In initially quiescent ambient fluid, the velocity profiles collapse onto each other, confirming that the density current is in the constant velocity phase (slumping) (Figure 9d). The asymmetry of the velocity profiles in the presence of waves is caused by the propagation of waves under intermediate water depth that induces a velocity field within a wave cycle, varying in relation to the wave period (i.e., crest, though, etc) and influencing the flow along the entire water depth. Moreover, focusing on the lower part of Figure 9f, which describes the velocity of the heavier fluid, we observe an oscillation of the velocity values reaching peak values $(13 \mathrm{~cm} / \mathrm{s})$, which are two-times greater than the mean velocity observed in the absence of waves $(6.5 \mathrm{~cm} / \mathrm{s})$. Comparison between experimental results and simulation of gravity currents characterized by the same reduced gravity $\left(0.13 \mathrm{~m} / \mathrm{s}^{2}\right)$ and the same ambient flow conditions is presented in Figure 10. Both the initially quiescent fluid and the presence of waves, $H_{w} / L_{w}=0.06$, are considered here. Results are in dimensionless form at a section located $1.5 \mathrm{H}$ upstream of the front, with the elevation scaled by the local current depth $h_{f}$. Both numerical 
and experimental profiles confirm that the presence of waves is responsible for an increased mixing compared to the no wave case. The model tends to overestimate such an effect. However, it should be also considered that numerical results are obtained at the centerline of the flume, while the measured concentration profiles are width-averaged.

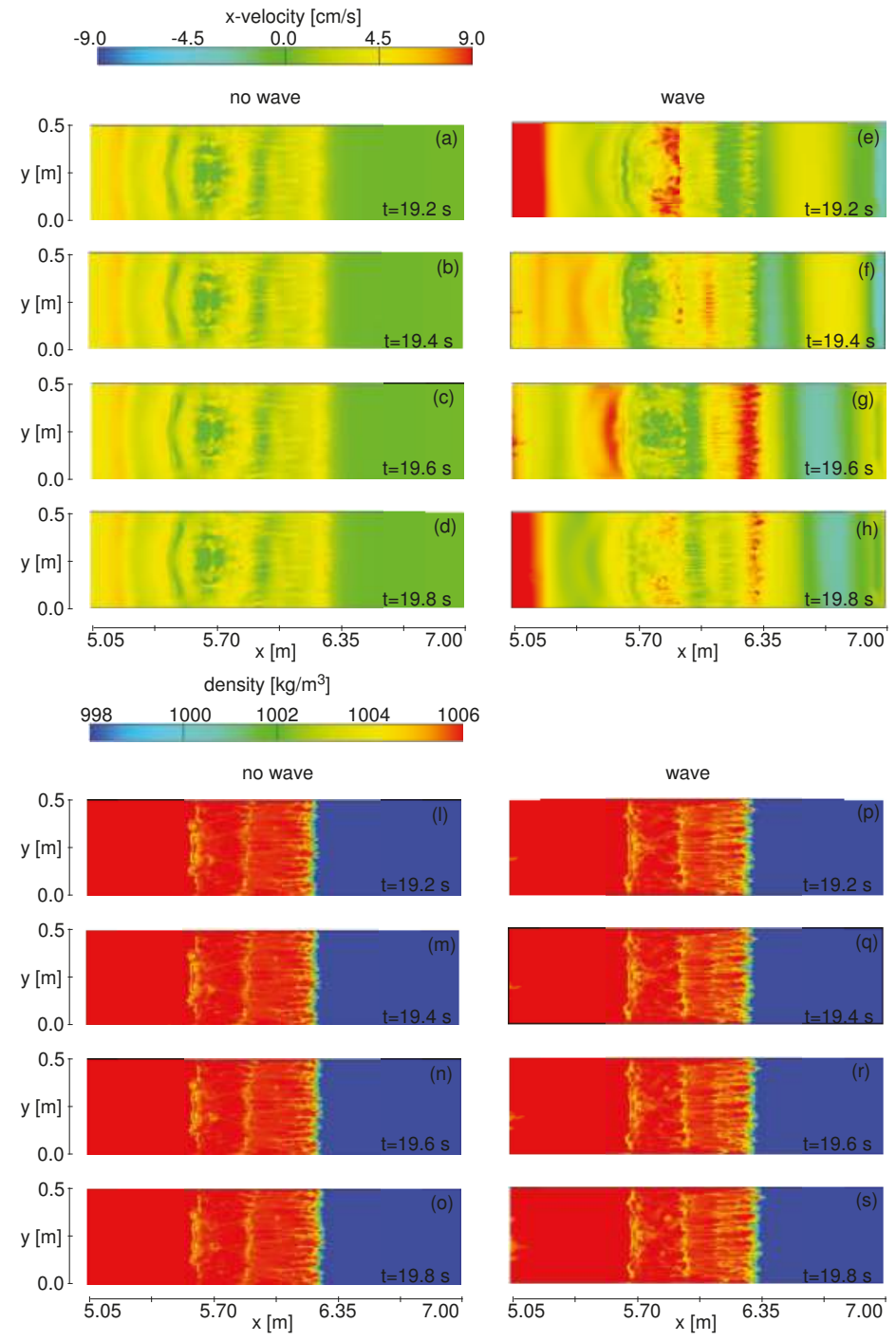

Figure 8. Maps of the x-velocity component and density at the bottom, respectively for Test No. 3 (absence of wave) and Test No. 4 (presence of waves). The maps are presented with a time interval of $0.2 \mathrm{~s}$ covering the time period of $0.8 \mathrm{~s}$, a duration equal to the wave period of Test No. 4 .

Analysis of the gravity current within a wave cycle shows the time and spatial oscillation of the depth of the gravity current $\Delta_{h_{f}}$. Stancanelli et al. [29] have observed this phenomenon and have also evaluated it in relation to the orbital particle trajectory under the water waves. Figure 11 shows data from both the experimental campaign of Stancanelli et al. [29] and the simulations, which are 
compared with the maximum vertical displacement of the wave-induced particle trajectory $2 B[29,59]$. The comparison between the gravity current depth oscillations predicted by the $k-\varepsilon$ model and the LES model with the experimental results (Case 2) shows a small overprediction for the LES model (5\%) and a quite substantial under-prediction (30\%) for the $k-\varepsilon$ model. The numerical results of the LES simulations agree fairly well with the experimental observations (errors less than $10 \%$ ). For shorter waves $\left(H_{w} / L_{w}<0.02\right)$, both numerical and experimental results show a stronger relationship $\Delta_{h_{f}}-2 B$, indicating a greater dependency on the wave regimes.
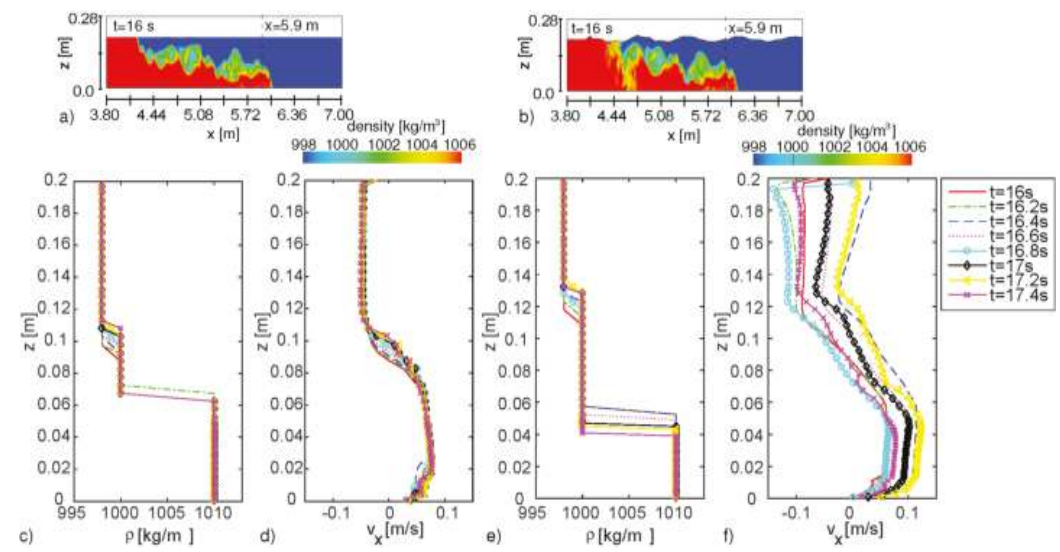

Figure 9. LES simulation results of gravity currents. Case 3: (a) density map at $t=16 \mathrm{~s}$ reporting the section $x=5.9 \mathrm{~m}$ of the profiles; (c) density profiles and (d) velocity profiles acquired from $t=16 \mathrm{~s}$ to $t=17.2 \mathrm{~s}$ with a time step of $0.2 \mathrm{~s}$. Case 4 : (b) density map at $t=16 \mathrm{~s}$ reporting the section $x=5.9 \mathrm{~m}$ of the profiles; (e) density profiles and (f) velocity profiles acquired from $t=16 \mathrm{~s}$ to $t=17.2 \mathrm{~s}$ with a time step of $0.2 \mathrm{~s}$.
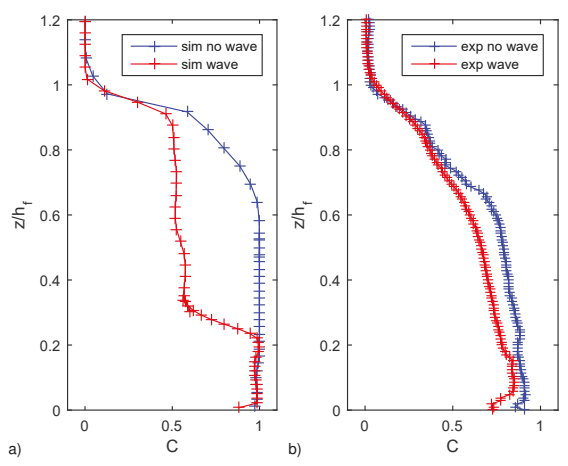

Figure 10. Comparison between concentration profiles acquired at $t=11.6 \mathrm{~s}$ and at a section located $1.5 \mathrm{H}$ upstream of the front respectively for LES simulations (a) and for the experimental results of Stancanelli et al. [29] (b). The profiles refer to the gravity currents with reduced gravity of $0.13 \mathrm{~m} / \mathrm{s}^{2}$ propagating in quiescent ambient fluid (no wave) and in the presence of regular surface waves $\left(H_{w} / L_{w}=0.06\right)$. For the numerical simulations, they are relative to the centerline of the flume, while for the experimental results, they are width average measurements. 


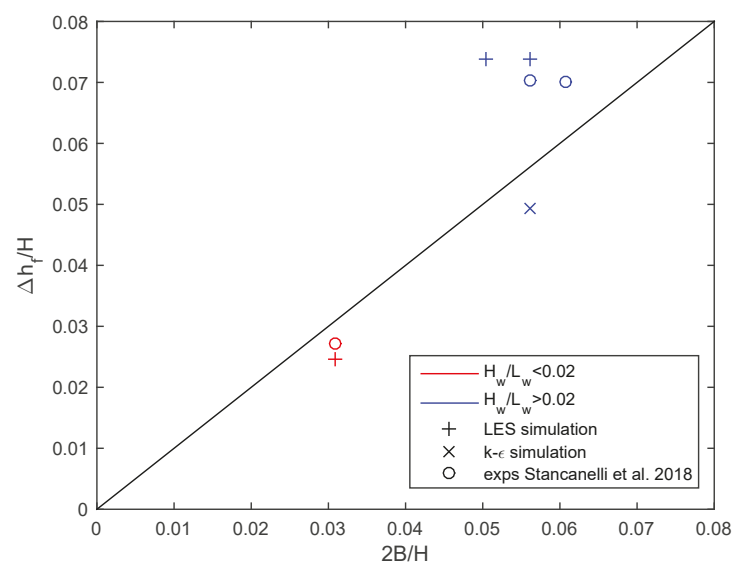

Figure 11. Comparison between the observed maximum oscillation of the gravity current depth $\Delta_{h_{f}}$ (at $x=5.10 \mathrm{~m}$ ) and the maximum vertical displacement of the wave-induced particle trajectory $2 B$. Data from the experimental campaign of Stancanelli et al. [29] and the simulation results.

\section{Conclusions}

Gravity currents in the absence and in the presence of regular surface waves are investigated by means of a CFD model, focusing on understanding the physics that governs the gravity current dynamics in relation to the ambient fluid field velocity.

Comparisons with experimental data are carried out in order to validate the capability of the adopted CFD model. The influence of the adopted turbulence closure method on the description of the dynamics of the propagation of the gravity current is evaluated. The computational cost required for the LES model is more then twice that needed for running the $k-\varepsilon$ model. The gravity current development in terms of the average velocity of the front is well reproduced by both the $k-\varepsilon$ model and the LES model. The prediction of average front velocity is equal for both models, although it is slightly underestimated, $5 \%$, in the case of higher reduced gravity by both models. Differences are noticed in terms of shear-induced instabilities causing a different mixing pattern at the interface. Comparison with the experimental shape of the gravity current shows that the location of the K-H billows is better reproduced by the LES model. The influence of the ambient fluid on the dynamics of the gravity currents is then analyzed with the more reliable LES scheme. The presence of lobe and clefts at the bottom of the front is visible after a few seconds from the generation of the gravity currents ( $5 \mathrm{~s}$ ) for both conditions of ambient flow (Case 1 No. waves, Case 2 with waves). The density iso-surfaces show the formation of both $\mathrm{K}-\mathrm{H}$ billows and 3D turbulent instabilities at the interface of the gravity current. The first ones appear suddenly after the front generation, while the latter ones are observed later. In the case of density currents in quiescent ambient fluid, the 3D instabilities develop at the front and propagate further upstream. In the presence of waves, such instabilities are present along all the density current, showing a lower intensity if compared with the case in the absence of waves. Moreover, the lighter front propagating in the opposite direction of the waves generates small-scale turbulent structures. Such turbulent structures are presented in both cases of shorter and longer waves, and their distribution is influenced by the mass transport induced by the wave propagation. Such evidence is also supported by the studies of Huang and Qiao [60], who observed that the turbulence induced by the surface wave decays in relation to the Stokes drift. The latter together with the Eulerian velocity describes the mass transport. The number of such small vortices seems to be related to the ratio between the front velocity and the horizontal orbital velocity $\left(v_{f} / u_{w}\right)$. For the wave-dominated regime $\left(v_{f} / u_{w}<1\right)$, a much larger number of vortices appear, also close to 
the bottom. It follows that in the presence of waves, the dynamics of the propagating density current appears strongly modified. The orbital motion is responsible for the increase of the mixing processes at the front. This is clearly observed at the bottom, where the relative density difference between the no wave and the wave case is about $20 \%$. In the region of the negative front, the difference between the no wave and the wave case is even larger, reaching up to $60 \%$. Further analyses on the mixing process acting at the front are carried out in terms of the vertical profiles of density and horizontal velocity. The density profiles show that the thickness of the observed mixing layer is equal to $20 \%$ of the water depth $H$ in initially quiescent ambient fluid and to $50 \%$ of the water depth $H$ in the presence of waves. The velocity profiles indicate that the gravity currents in the presence of waves advance following a periodic development, which is repeated for each wave cycle. The velocity values of the heavier fluid oscillate reaching peak values two-times greater than the velocity values in the absence of waves. Finally, wave-induced oscillation of the gravity current depth has been analyzed in comparison with the experimental results, and the LES model is more accurate (error less than 10\%), showing results in accordance with the experimental evidence. For shorter waves, the gravity current depth oscillation is mainly influenced by the orbital motion, while for longer waves, phenomena such as instantaneous mixing or diffusion can influence the measure of the gravity current depth.

The present research allowed also the analysis of the small-scale vortices induced by waves, which are responsible for the development of different mixing processes. This phenomenon was never observed in previous works dealing with gravity current in the presence of waves. Indeed, due to the instrumentation constraints, the experimental campaign [28,29] could not investigate such phenomena. Previous numerical investigations [27] were focused on the propagation velocity, or just on larger structures captured by a simplistic turbulence closure [30] where calibration procedure are needed prior to application. Certainly, further investigations are needed for a more comprehensive understanding of the interaction between the wave-induced turbulence with the gravity current turbulence.

Future works will investigate the dynamics of density current propagation combined with oscillatory wave motion during the buoyancy-inertia phase. In initially quiescent ambient fluid, the density current during the buoyancy-inertia phase is composed by a heavier fluid head and a diluted tail, and the front velocity is no longer constant, as it was during the slumping phase. In such a highly variable system, it would be interesting to evaluate the effects on the gravity current dynamics of the periodic disturbances induced by the superimposed surface wave field. Moreover, the process of energy exchanges will be investigated, considering the balance between the kinetic and the potential energy.

Author Contributions: R.E. Musumeci has conceived of and designed the experiments. L.M. Stancanelli has performed the experimental tests and the numerical simulations. R.E. Musumeci and L.M. Stancanelli have analyzed the data. All three authors have equally contributed to writing the paper.

Acknowledgments: This work has been partly funded by the EU-funded project HYDRALAB PLUS (Proposal Number 64110) and by the INTERREG Italia-Malta project NEWS (C1 - 3.2 - 60).

Conflicts of Interest: The authors declare no conflict of interest. The founding sponsors had no role in the design of the study; in the collection, analyses or interpretation of data; in the writing of the manuscript; nor in the decision to publish the results.

\section{References}

1. Ooi, S.K.; Constantinescu, G.; Weber, L. A numerical study of intrusive compositional gravity currents. Phys. Fluids (1994-Present) 2007, 19, 076602. [CrossRef]

2. Huppert, H.E. Gravity currents: A personal perspective. J. Fluid Mech. 2006, 554, 299-322. [CrossRef]

3. Stancanelli, L.M.; Musumeci, R.E.; Cavallaro, L.; Foti, E. A small scale Pressure Retarded Osmosis power plant: Dynamics of the brackish effluent discharge along the coast. Ocean Eng. 2017, 130, 417-428. [CrossRef]

4. Cagnoli, B.; Piersanti, A. Stresses at the base of dry and dense flows of angular rock fragments in 3-D discrete element modeling: Scaling of basal stress fluctuations versus grain size, flow volume and channel width. J. Volcanol. Geotherm. Res. 2018, 349, 230-239. [CrossRef] 
5. Stancanelli, L.M.; Lanzoni, S.; Foti, E. Mutual interference of two debris flow deposits delivered in a downstream river reach. J. Mt. Sci. 2014, 11, 1385-1395. [CrossRef]

6. Lanzoni, S.; Gregoretti, C.; Stancanelli, L.M. Coarse-grained debris flow dynamics on erodible beds. J. Geophys. Res. Earth Surf. 2017, 122, 592-614. [CrossRef]

7. Palomar, P.; Losada, I. Desalination in Spain: Recent developments and recommendations. Desalination 2010, 255, 97-106. [CrossRef]

8. Robinson, T.; Eames, I.; Simons, R. Dense gravity currents moving beneath progressive free-surface water waves. J. Fluid Mech. 2013, 725, 588-610. [CrossRef]

9. Rottman, J.W.; Simpson, J.E. Gravity currents produced by instantaneous releases of a heavy fluid in a rectangular channel. J. Fluid Mech. 1983, 135, 95-110. [CrossRef]

10. Nogueira, H.I.; Adduce, C.; Alves, E.; Franca, M.J. Image analysis technique applied to lock-exchange gravity currents. Meas. Sci. Technol. 2013, 24, 047001. [CrossRef]

11. Britter, R.; Linden, P. The motion of the front of a gravity current travelling down an incline. J. Fluid Mech. 1980, 99, 531-543. [CrossRef]

12. Choi, S.U.; Garcia, M. Spreading of gravity plumes on an incline. Coast. Eng. J. 2001, 43, 221-237. [CrossRef]

13. Lombardi, V.; Adduce, C.; Sciortino, G.; La Rocca, M. Gravity currents flowing upslope: Laboratory experiments and shallow-water simulations. Phys. Fluids 2015, 27, 016602. [CrossRef]

14. He, Z.; Zhao, L.; Lin, T.; Hu, P.; Lv, Y.; Ho, H.C.; Lin, Y.T. Hydrodynamics of Gravity Currents Down a Ramp in Linearly Stratified Environments. J. Hydraul. Eng. 2017, 143, 04016085. [CrossRef]

15. Lane-Serff, G.; Beai, L.; Hadfield, T. Gravity Current Flow Over Obstacles. J. Fluid Mech. 1995, 292, 39-53. [CrossRef]

16. Maxworthy, T.; Leilich, J.; Simpson, J.E.; Meiburg, E.H. The propagation of a gravity current into a linearly stratified fluid. J. Fluid Mech. 2002, 453, 371-394. [CrossRef]

17. Dai, A. High-resolution simulations of downslope gravity currents in the acceleration phase. Phys. Fluids 2015, 27, 076602. [CrossRef]

18. Cantero, M.I.; Balachandar, S.; García, M.H.; Bock, D. Turbulent structures in planar gravity currents and their influence on the flow dynamics. J. Geophys. Res. Oceans 2008, 113. [CrossRef]

19. Ottolenghi, L.; Adduce, C.; Inghilesi, R.; Armenio, V.; Roman, F. Entrainment and mixing in unsteady gravity currents. J. Hydraul. Res. 2016, 54, 541-557. [CrossRef]

20. Akutina, Y.; Mydlarski, L.; Gaskin, S.; Eiff, O. Error analysis of 3D-PTV through unsteady interfaces. Exp. Fluids 2018, 59, 53. [CrossRef]

21. Ooi, S.K.; Constantinescu, G.; Weber, L. Numerical simulations of lock-exchange compositional gravity current. J. Fluid Mech. 2009, 635, 361-388. [CrossRef]

22. Härtel, C.; Meiburg, E.; Necker, F. Analysis and direct numerical simulation of the flow at a gravity-current head. Part 1. Flow topology and front speed for slip and no-slip boundaries. J. Fluid Mech. 2000, 418, 189-212. [CrossRef]

23. Härtel, C.; Carlsson, F.; Thunblom, M. Analysis and direct numerical simulation of the flow at a gravity-current head. Part 2. The lobe-and-cleft instability. J. Fluid Mech. 2000, 418, 213-229. [CrossRef]

24. Blanchette, F.; Strauss, M.; Meiburg, E.; Kneller, B.; Glinsky, M.E. High-resolution numerical simulations of resuspending gravity currents: Conditions for self-sustainment. J. Geophys. Res. Oceans 2005, 110. [CrossRef]

25. Cantero, M.I.; Balachandar, S.; García, M.H.; Ferry, J.P. Direct numerical simulations of planar and cylindrical density currents. J. Appl. Mech. 2006, 73, 923-930. [CrossRef]

26. Ottolenghi, L.; Adduce, C.; Inghilesi, R.; Roman, F.; Armenio, V. Mixing in lock-release gravity currents propagating up a slope. Phys. Fluids 2016, 28, 056604. [CrossRef]

27. Ng, C.O.; Fu, S.C. On the propagation of a two-dimensional viscous density current under surface waves. Phys. Fluids (1994-Present) 2002, 14, 970-984. [CrossRef]

28. Musumeci, R.E.; Viviano, A.; Foti, E. Influence of regular surface waves on the propagation of gravity currents: experimental and numerical modeling. J. Hydraul. Eng. 2017, 143, 04017022. [CrossRef]

29. Stancanelli, L.M.; Musumeci, R.E.; Foti, E. Dynamics of gravity currents in the presence of surfface waves. J. Geophys. Res. Oceans 2018. [CrossRef]

30. Viviano, A.; Musumeci, R.E.; Foti, E. Interaction between waves and gravity currents: description of turbulence in a simple numerical model. Environ. Fluid Mech. 2018, 18, 117-148. [CrossRef]

31. Piomelli, U. Wall-layer models for large-eddy simulations. Prog. Aerosp. Sci. 2008, 44, 437-446. [CrossRef] 
32. Harlow, F.H.; Nakayama, P.I. Turbulence Transport Equations. Phys. Fluids 1967, 10, 2323-2332. [CrossRef]

33. Spalart, P. Strategies for turbulence modeling and simulations. Int. J. Heat Fluid Flow 2000, 21, 252-263. [CrossRef]

34. Hirt, C.; Nichols, B.D. Volume of fluid (VOF) method for the dynamics of free boundaries. J. Comput. Phys. 1981, 39, 201-225. [CrossRef]

35. Parsaie, A.; Haghiabi, A.H.; Moradinejad, A. CFD modeling of flow pattern in spillway's approach channel. Sustain. Water Resour. Manag. 2015, 1, 245-251. [CrossRef]

36. Tsai, C.P.; Chen, Y.C.; Sihombing, T.O.; Lin, C. Simulations of moving effect of coastal vegetation on tsunami damping. Nat. Hazards Earth Syst. Sci. 2017, 17, 693-702. [CrossRef]

37. Bayon, A.; Valero, D.; García-Bartual, R.; Vallés-Morán, F.J.; López-Jiménez, P.A. Performance assessment of OpenFOAM and FLOW-3D in the numerical modeling of a low Reynolds number hydraulic jump. Environ. Model. Softw. 2016, 80, 322-335. [CrossRef]

38. Bayon, A.; Toro, J.P.; Bombardelli, F.A.; Matos, J.; López-Jiménez, P.A. Influence of VOF technique, turbulence model and discretization scheme on the numerical simulation of the non-aerated, skimming flow in stepped spillways. J. Hydro-Environ. Res. 2018, 19, 137-149. [CrossRef]

39. Launder, B.E.; Spalding, D. The numerical computation of turbulent flows. Comput. Methods Appl. Mech. Eng. 1974, 3, 269-289. [CrossRef]

40. Pope, S.B. Turbulent Flows; Cambridge University Press: Cambridge, UK, 2000.

41. Sagaut, P. Large Eddy Simulation for Incompressible Flows: An Introduction; Springer Scientific Computation: Berlin/Heidelberg, Germany, 2006.

42. Smagorinsky, J. General circulation experiments with the primitive equations: I. The basic experiment. Mon. Weather Rev. 1963, 91, 99-164. [CrossRef]

43. Cheng, Y.; Lien, F.; Yee, E.; Sinclair, R. A comparison of large eddy simulations with a standard k- $-\varepsilon$ Reynolds-averaged Navier-Stokes model for the prediction of a fully developed turbulent flow over a matrix of cubes. J. Wind Eng. Ind. Aerodyn. 2003, 91, 1301-1328. [CrossRef]

44. Meiburg, E.; Radhakrishnan, S.; Nasr-Azadani, M. Modeling gravity and turbidity currents: Computational approaches and challenges. Appl. Mech. Rev. 2015, 67, 040802. [CrossRef]

45. Airy, G. Tides and Waves. Encycl. Metrop. 1845, 102, 241-396.

46. Fenton, J.D. A Fifth-Order Stokes Theory for Steady Waves. J. Waterw. Port Coast. Ocean Eng. 1985, 111, 361-388. [CrossRef]

47. Fenton, J.D. Numerical methods for nonlinear waves. In Advances in Coastal and Ocean Engineering; World Scientific: Singapore, 2011; pp. 241-324.

48. McCowan, J. On the solitary wave. Lond. Edinb. Dublin Philos. Mag. J. Sci. 1891, 32, 45-58. [CrossRef]

49. Munk, W. The solitary wave theory and its application to surf problems. Ann. N. Y. Acad. Sci. 1949, 51,376-424. [CrossRef]

50. Boris, J.P.; Grinstein, F.F.; Oran, E.S.; Kolbe, R.L. New insights into large eddy simulation. Fluid Dyn. Res. 1992, 10, 199-228. [CrossRef]

51. Kyrousi, F.; Leonardi, A.; Roman, F.; Armenio, V.; Zanello, F.; Zordan, J.; Juez, C.; Falcomer, L. Large Eddy Simulations of sediment entrainment induced by a lock-exchange gravity current. Adv. Water Resour. 2018, 114, 102-118. [CrossRef]

52. USACE. Coastal Engineering Manual, EM 1110-2-1100; U.S. Army Corps of Engineers: Washington, DC, USA, 2006.

53. Svendsen, I.A. Introduction to Nearshore Hydrodynamics; World Scientific: Singapore; Hackensack, NJ, USA, 2006; Volume 24.

54. Huppert, H.E.; Simpson, J.E. The slumping of gravity currents. J. Fluid Mech. 1980, 99, 785-799. [CrossRef]

55. Mcwilliams, J.C.; Sullivan, P.P.; Moeng, C.H. Langmuir turbulence in the ocean. J. Fluid Mech. 1997, 334, 1-30. [CrossRef]

56. Teixeira, M.; Belcher, S. On the distortion of turbulence by a progressive surface wave. J. Fluid Mech. 2002, 458, 229-267. [CrossRef]

57. Kantha, L.H.; Clayson, C.A. On the effect of surface gravity waves on mixing in the oceanic mixed layer. Ocean Model. 2004, 6, 101-124. [CrossRef]

58. Ardhuin, F.; Jenkins, A.D. On the interaction of surface waves and upper ocean turbulence. J. Phys. Oceanogr. 2006, 36, 551-557. [CrossRef] 
59. Dalrymple, R.A.; Dean, R.G. Water Wave Mechanics for Engineers and Scientists; Prentice-Hall: Englewood Cliffs, NJ, USA, 1991.

60. Huang, C.J.; Qiao, F. Wave-turbulence interaction and its induced mixing in the upper ocean. J. Geophys. Res. Oceans 2010, 115. [CrossRef]

(C) 2018 by the authors. Licensee MDPI, Basel, Switzerland. This article is an open access article distributed under the terms and conditions of the Creative Commons Attribution (CC BY) license (http:/ / creativecommons.org/licenses/by/4.0/). 
Article

\title{
Influence of Gravel-Bed Porosity and Grain Orientation on Bulk Flow Resistance
}

\author{
Christy Ushanth Navaratnam ${ }^{1, *}$, Jochen Aberle ${ }^{1,2}$, Jie Qin ${ }^{3}$ and Pierre-Yves Henry ${ }^{1}$ \\ 1 Department of Civil and Environmental Engineering, Norwegian University of Science and \\ Technology (NTNU), S.P.Andersens veg 5, 7491 Trondheim, Norway; \\ jochen.aberle@tu-braunschweig.de (J.A.); pierre-yves.henry@ntnu.no (P.-Y.H.) \\ 2 Leichtweiß-Institut für Wasserbau, Technische Universität Braunschweig, 38106 Braunschweig, Germany \\ 3 College of Harbour, Coastal and Offshore Engineering, Hohai University, Xikang road \#1, Nanjing 210098, \\ China; jqin@hhu.edu.cn \\ * Correspondence: christy.ushanth.navaratnam@ntnu.no; Tel.: +47-73-595-158
}

Received: 19 March 2018; Accepted: 20 April 2018; Published: 26 April 2018

\begin{abstract}
This paper presents results from experiments that were carried out to study the effect of porosity and grain orientation on flow resistance. Experiments were performed over three rough surfaces; a water-worked gravel-bed, its non-porous facsimile (cast-bed) and the rotated cast-bed (cast tiles rotated through $180^{\circ}$ ). The first two beds were used to isolate the influence of gravel-bed porosity on the bulk flow resistance and the rotated cast was used to study effect of the grain orientation on the flow resistance. The results showed that the rotated cast-bed exerted the highest flow resistance whereas the porous water-worked gravel-bed was, for comparable hydraulic boundary conditions, characterized by slightly higher flow resistance than its non-porous counterpart. The results from the bulk flow analysis were substantiated by a preliminary analysis of flow velocity data.
\end{abstract}

Keywords: flow resistance; roughness; gravel-bed rivers; casting technique

\section{Introduction}

Gravel bed rivers represent an important stream-type in the fluvial environment. Gravel beds are, in general, characterized by a large roughness influencing hydraulic and fluvial processes, which in turn govern the turbulent flow structure, flow resistance, sediment transport, and morphological development. Although these processes have been in the focus of research for a long time, there is still a lack of knowledge with regard to near bed flow structure, flow resistance (e.g., [1-3]) and exchange processes between the main stream and groundwater flow (e.g., $[4,5])$. This is partly associated with the fact that many studies have focused mainly on the determination of roughness coefficients as a function of characteristic grain-sizes thereby neglecting the structure of gravel beds.

Traditional methods quantify flow resistance through Manning's roughness coefficient $n$, Chézy's flow resistance factor $C$ or Darcy-Weisbach's friction factor $f$ [6]. These are interrelated and can be written as a function of the bulk velocity $U$, water depth $h$ and energy slope $S_{f}$. As an example, the Darcy-Weisbach friction factor $f$ is defined as

$$
f=8 \frac{u_{*}^{2}}{U^{2}}
$$

where $u_{*}$ denotes the shear velocity which, for uniform flow conditions, can be determined according to $u_{*}=\left(g h S_{f}\right)^{0.5}$ with $g=$ gravitational acceleration. Gravel-bed roughness is often described by a characteristic grain size of the bed material (e.g., $d_{50}, d_{84}$ or $d_{90}$ ) and is linked to the friction factor via empirical or semi-empirical relationships (e.g., [6-10]). However, gravel-beds are characterized by a 
high degree of irregularities (grain shape, orientation, packing pattern, etc.), multiple roughness scales (e.g., small and meso-scale bedforms such as pebble clusters, steps, pools, etc. [11]) and therefore roughness properties may vary independently of grain size [12]. Some studies suggested that the standard deviation of bed-elevations may be used as characteristic vertical roughness scale (e.g., [3,13-15]) and further studies focused on the determination of characteristic horizontal roughness scales through the analysis of longitudinal profiles or digital elevation models, respectively, using spectral analysis, correlation functions and structure functions (e.g., $[12,13,16,17])$. Despite the application of surface structure analyses, there is still no consensus regarding the interpretation of the results or the most appropriate measure of bed roughness [18,19].

Moreover, it has often been tacitly assumed in flow resistance studies that fluvial beds may be considered as non-porous structures despite the fact that natural gravel-beds are typically composed of a coarse surface layer and a porous subsurface layer. This assumption implies that a porous and non-porous bed with an identical surface structure would be characterized by exactly the same flow resistance. However, the flow over porous beds is characterized by mass and momentum exchange across the sediment water interface due to the pressure gradients driving the flow in and out of the bed [20-22]. The exchange processes, also known as hyporheic exchange, are assumed to have a distinct effect on the near bed flow field and hence flow resistance. In fact, compared to flow resistance studies, only few studies have focused on the influence of bed porosity on flow resistance (e.g., [23-26]). These studies have shown that porous beds impose higher flow resistance than similar non-porous beds.

The present paper investigates this topic further by analyzing experimental data which was acquired over a porous gravel-bed armor layer and its impermeable facsimile. In an additional experimental series, the facsimile was rotated through $180^{\circ}$ so that the bulk flow analysis could be extended towards the investigation of the effect of grain-orientation on flow resistance. Within this paper, we focus on the effect of bed porosity on bulk flow characteristics such as the friction factor $f$, while more detailed considerations regarding the near bed turbulent flow pattern will be presented in a follow up study. The manuscript is organized as follows: the next Section presents a brief review on the significance of bed porosity for flow resistance; Section 3 describes the experimental setup and methodology, and the results are presented and discussed in Section 4. Conclusions are drawn in Section 5 .

\section{Background}

The effect of bed porosity on flow patterns has been investigated in several studies based on data acquired in laboratory and numerical experiments over beds composed of different artificial roughness elements. Most of these studies have revealed that the flow resistance over permeable beds is larger than the flow resistance exerted by their impermeable counterparts (e.g., [23-28]). Moreover, various studies have indicated a dependency of the friction factor with the relative thickness of the permeable layer and the Reynolds-number $R e=U h / v$, where $v$ is the kinematic viscosity of the fluid (e.g., $[24,26,29])$. The increase of flow resistance with $R e$ has been associated with shear penetration into the porous bed [26-28] and the associated exchange of momentum between the surface flow and the flow in the porous medium which increases the Reynolds shear-stress in the near bed region and hence the flow resistance $[25,27,29]$. In this context, Breugem et al. [25] found that flow resistance increases with bed permeability or $R e_{K}=\sqrt{K} u_{*} / v$, where $K$ is the permeability, and Manes et al. [27] showed that the characteristic length scale of the turbulent flow over a permeable wall can be defined by the depth of shear penetration. This penetration depth is related to the zero-plane position which may be determined from the velocity profiles above the bed [27].

Comparing flow patterns over a single layer of gravel grains and multiple grain layers, Manes et al. [28] highlighted the dependency flow resistance considerations from the definition of bulk parameters. Assuming that the effective hydrodynamic roughness is related to the thickness of the interface, i.e., the region where the surface and sub-surface flow interacts, and not to the size of the grains composing the bed, the friction factors of the non-porous bed were determined using 
the fixed channel bottom as datum for bulk flow parameters such as flow depth and velocity. On the other hand, for the quantification of the friction factors over the porous beds, Manes et al. [28] used the roughness crest as datum to account for the unknown spatial extent of the interface which depends on flow characteristics. This means that the flow in the interfacial sublayer (the region between the roughness crests and troughs) was intentionally neglected in the bulk analysis presented in the study of Manes et al. [28]. Defining the roughness crest as datum for the determination of water depth will result in larger values for the bulk velocities and smaller water depths compared to the case where the roughness trough is defined as datum. Thus, the use of different datums will be associated with different estimates of the friction factor. Moreover, the subsurface flow-rate is typically difficult to measure in experimental studies and is often not considered separately, i.e., it is often assumed that the discharge used in the experiments represents the surface flow rate, although a small portion of the flow is conveyed through the subsurface layer. For lower discharges, this may hamper the analysis of flow resistance data from bulk analyses.

Most of the aforementioned studies have focused on the analysis of data obtained over beds composed of rather regular roughness elements of similar size, and studies with real gravel-beds remain rare. However, such investigations are needed to account for the non-uniform porosity variation from the crest of the gravel-bed layer to the undisturbed subsurface layer (see [30,31]). The recent study of Cooper et al. [32] used a casting technique to reproduce a non-porous section of $0.4 \mathrm{~m}$ length and width of a water-worked gravel surface that was created in a $8.2 \mathrm{~m}$ long and $0.6 \mathrm{~m}$ wide flume. The flow patterns over the permeable and non-permeable test section were compared based on velocity measurements obtained with Particle Image Velocimetry (PIV). Focusing on the near-bed region, Cooper et al. [32] concluded that the flow resistance imposed by the non-porous surface was higher than that by the porous water-worked gravel-bed, which is contrary to the previous findings. Cooper et al. [32] explained their findings from the analysis of the flow velocity data acquired at the roughness crest. They observed higher double-averaged velocities (velocities averaged in the time and space domain) over the gravel-bed than over the reproduced section and hypothesized that the higher efficiency in the momentum transfer and lower kinetic energy over the porous gravel-bed is a strong indicator that less energy was extracted from the mean flow.

To summarize, all studies related to the effect of bed-porosity revealed that flow resistance is altered by the porosity. More specifically, most studies found that a porous bed offers higher flow resistance than a comparable impermeable bed. This has been associated with the shear penetration and momentum exchange over the porous medium caused by large-scale vortical structures. On the other hand, the study carried out by Cooper et al. [32] over a gravel-bed concluded that flow resistance over a non-porous water-worked gravel-bed is larger than over its permeable counterpart. Some possible explanations for these contradicting results are discussed in Section 4 of this paper, as the experimental methodology of our study is similar to the one used by Cooper et al. [32]. It is worth mentioning that experiments presented in the following section were already ongoing when the study of Cooper et al. [32] was published. Thus, the results of the experiments can be used to shed more light on the influence of bed gravel-bed porosity on flow resistance.

\section{Experimental Setup and Procedure}

\subsection{Experimental Facility}

Experiments were conducted in a $12.5 \mathrm{~m}$ long $1 \mathrm{~m}$ wide and $1 \mathrm{~m}$ deep closed-circuit tilting flume in the hydraulics laboratory at the Norwegian University of Science and Technology (NTNU), Trondheim, Norway. The flume, schematically shown in Figure 1, has a $12.5 \mathrm{~m}$ long glass-sided working section and a $2 \mathrm{~m}$ long inlet section consisting of a head tank and flow-conditioning section. In the experiments, the flow was recirculated by two centrifugal pumps and the flow rate was regulated by electronic motor speed controllers and the valves at each pump. The flow rate was measured by Euromag MUT1000 EL inductive discharge meters (IDM) with an accuracy of $0.1 \%$, installed at the pipes leading 
from the pumps to the inlet tank. The maximum flow rate of each pump was $0.225 \mathrm{~m}^{3} / \mathrm{s}$ resulting in a maximum discharge capacity of $0.45 \mathrm{~m}^{3} / \mathrm{s}$. Polyvinyl chloride (PVC) rods with a diameter of $20 \mathrm{~mm}$ were installed in the inlet tank to condition the flow, and the inlet tank was separated from the flume channel by a honeycomb panel which served as flow straightener. The water depth could be adjusted by both a weir installed at the downstream end of the flume and by adding or draining water from the flume using drain valves. Dependent on the experiment, water levels in the flume were determined from pressure measurements using 4 pressure taps located at the flume bottom or from water surface elevation measurements using 8 Microsonic ultrasonic sensors (accuracy of $1 \%$ ). The distance between the static pressure taps was $1.25 \mathrm{~m}$ with the first tap being located $6.875 \mathrm{~m}$ from the beginning of the working section. The ultrasonic sensors were installed in the same flume reach where the static pressure tubes were located. The spacing between the acoustic sensors varied from $0.4 \mathrm{~m}$ to $0.7 \mathrm{~m}$ to avoid acoustic interferences of the signals emitted from adjacent sensors.

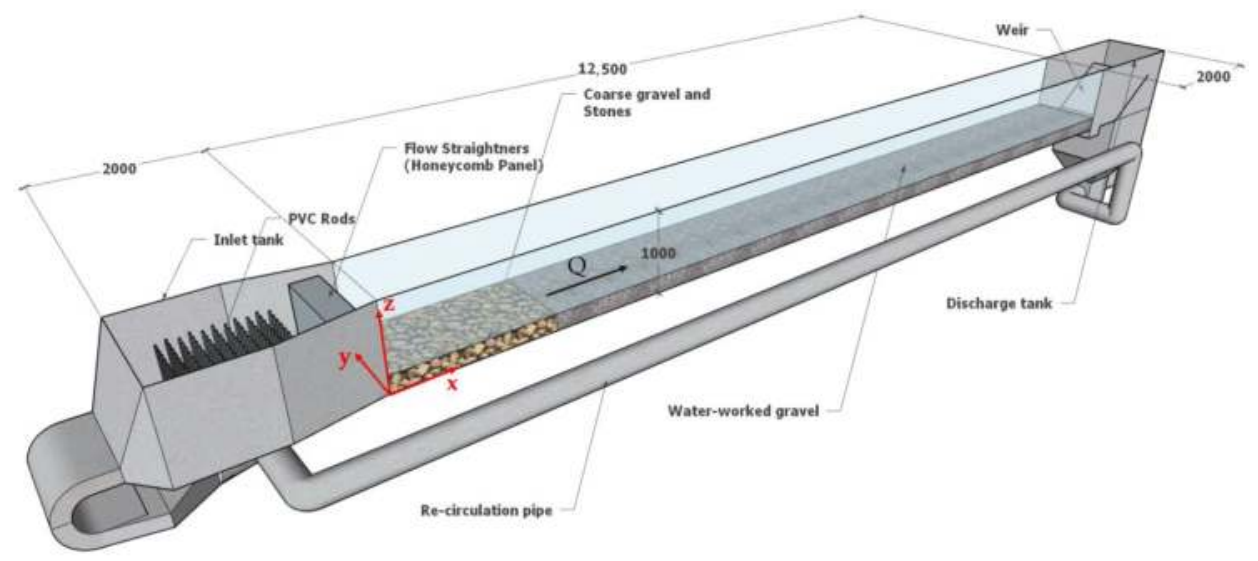

Figure 1. Isometric view of the 3D drawing of the recirculating flume (the pumps, which are located below the inlet tank, are not shown). All units are in [mm].

\subsection{Bed Types}

The first series of measurements was carried out over a water worked gravel-bed armor layer with a porous subsurface. For the generation of the armor layer, a $0.20 \mathrm{~m}$ high and $10.31 \mathrm{~m}$ long gravel layer consisting of a well-mixed sediment mixture $(0.64 \mathrm{~mm}<d<64 \mathrm{~mm}$; see Figure 2 for the grain-size distribution) was placed in the flume. The sediment mixture was similar to the one used in the study by Aberle and Nikora [16]. The bed was screeded flat and surface compacted to ensure that the bed slope was parallel to the flume slope. The gravel layer was retained by a L-shape perforated sill at its upstream and downstream end to allow for subsurface flow during the experiments. The $2.19 \mathrm{~m}$ long flume section between the gravel-bed and the upstream flow straightener was formed by coarse gravel to prevent scouring (Figure 1).

The gravel layer was water-worked with quasi-uniform flow conditions for a discharge of $Q=0.2 \mathrm{~m}^{3} / \mathrm{s}$, a bed and water surface slope of $S_{b}=S_{w}=0.0027$, respectively, and a water depth of $h=0.24 \mathrm{~m}$. The eroded sediment was collected in a basket placed downstream of the gravel layer and the surface was considered to be armored when the sediment transport rate became less than $2.0 \mathrm{~kg} / \mathrm{h} / \mathrm{m}$, i.e., the same criterion was used as in Aberle et al. [33]. After the armoring, the bed topography was scanned over a total length of $7 \mathrm{~m}$ using an Acuity AR200-100 laser displacement meter attached to a traversing system spanning the flume length. The diameter of the footprint of the laser beam ranged between 55 and $250 \mu \mathrm{m}$ dependent on the distance of the laser to the bed surface, and the accuracy of the bed elevation measurements was $30.5 \mu \mathrm{m}$. The coordinate system had its origin 
$x, y, z=(0,0,0)$ at the beginning of working section, the span wise direction $y=0$ was at the right glass wall of the flume and the vertical coordinate $z=0$ at the plastic channel bottom (see Figure 1). In total, 660 longitudinal profiles were recorded at a span wise step distance of $\Delta y=1 \mathrm{~mm}$. The scanned section did not include the topography near the glass walls (approx. $170 \mathrm{~mm}$ on either side) due to the setup of the traversing system. Each longitudinal profile was recorded with a sampling frequency of $100 \mathrm{~Hz}$ and a traverse speed of $2000 \mathrm{~mm} / \mathrm{min}$ resulting in a longitudinal resolution of $\Delta x=0.33 \mathrm{~mm}$. Spikes in the scanned data were removed manually during the post processing and the final data were used to produce digital elevation models (DEM) of the gravel and the cast surfaces, respectively. The scans will be analyzed in Section 4.1 .

Porosity measurements were carried out before and after armoring using the water displacement method (WDM) to obtain the vertical distribution of the porosity of both the initial and water-worked gravel-bed (see [31] for details). In addition to the WDM, the porosity distributions were also derived from the laser scans. The corresponding results will be presented in Section 4.1.

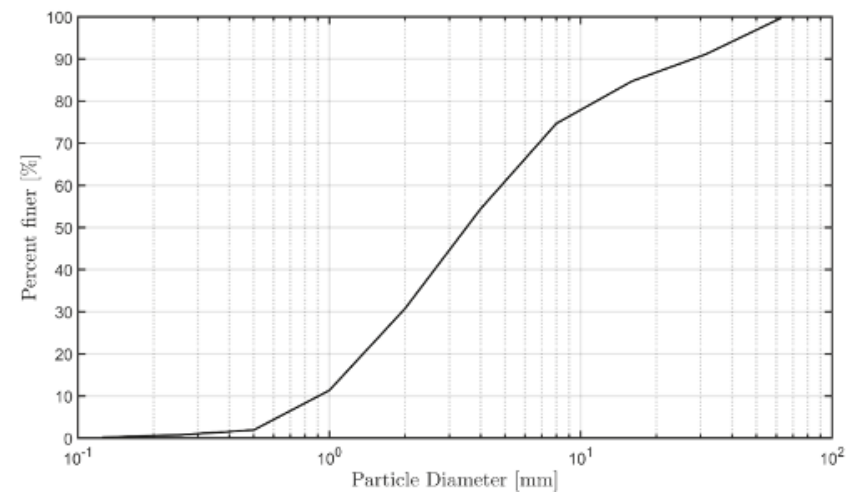

Figure 2. Grain size distribution curve of the gravel mixture.

In order to study the effect of the bed subsurface porosity on the flow, a $7 \mathrm{~m}$ long section of the armored gravel-bed was reproduced using the casting technique reported in Spiller and Rüther [34], Navaratnam et al. [35] after completion of the hydraulic measurements over the gravel-bed (the latter are described below in Section 3.3). For the preparation of the molds, a thin steel plate was vertically inserted $1 \mathrm{~cm}$ into the gravel layer at both ends of the $7 \mathrm{~m}$ long section (at $x=3250 \mathrm{~mm}$ and 10,250 $\mathrm{mm}$, respectively; see Figure 3) and a bi-component silicon mixture was poured layer-by-layer onto the section. The initial layer was very thin so that the silicon did not alter the grain orientation and did not penetrate the sub-surface. This facilitated the removal of gravel grains from the silicon-mold. It was also ensured that the silicon seeped enough into the interstices in the roughness layer, i.e., into the pore space between the roughness crest and trough. All silicon layers were poured before the initial layer solidified completely to guarantee bonding between the layers. The last silicon layer submerged the highest grain elevation by $2 \mathrm{~cm}$ enabling the proper handling of the mold. The final silicon-body was screeded horizontally and allowed to cure for one day. After solidification of the silicon, the two steel plates were removed, and the silicon mat was cut in half and removed from the flume with the help of a crane. The two resulting silicon mats were manually cleaned from sediment particles.

For the preparation of the cast surface, the two silicon mats were further subdivided into two mats of $2.3 \mathrm{~m}$ and $1.2 \mathrm{~m}$ length, respectively, resulting in a total of 4 molds which originated from Sections 2-5 in Figure 3. Each mold was placed inside a wooden frame and levelled horizontally to avoid the introduction of artificial slopes in the cast. The cast consisted of polyurethane resin mixed with a filling powder (ATH-Aluminum trihydrate) in a volume ratio of 1:2.5 (resin:filler). The filler powder reduced the heat emission from the exothermal reaction during curing so that the shrinkage of 
the cast was limited to $1 \%$. The edges of the cast pieces were polished to remove irregularities due to leakage and small irregularities in the wooden frame.

The final cast pieces were placed in the flume so that tiles $2-5$ were accurately reproducing the original gravel surface. Copies of the $1.2 \mathrm{~m}$ long tiles (tiles 1, 6 and 7 in Figure 3; marked in orange) were cut to the required length and placed at the upstream and downstream end of the bed so that all parts of the bed were completely immobile. Small gaps between the tiles and between the tiles and the glass wall, respectively, were filled with silicon and clay to make it water tight. Both, the upstream and downstream end were sealed to avoid flow development under the cast. After its installation, the cast-bed was scanned using the laser displacement meter.

The third bed configuration was installed following the completion of the hydraulic measurements over the cast-bed to investigate the effect of grain orientation on hydraulic roughness. For this purpose, the cast pieces (except pieces 1,6 and 7) were rotated through 180 degrees and, after sealing the gaps, the bed was scanned again.

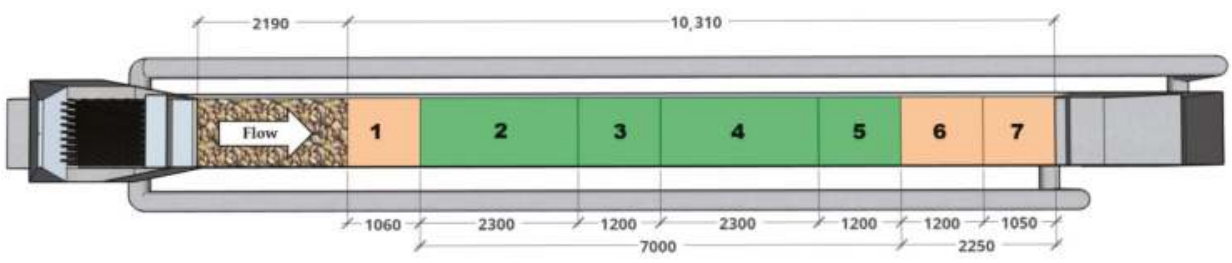

Figure 3. Plan view of the flume consisting the working section; colored section and numbers denote the position of the cast tiles placement. All units are in [mm].

\subsection{Hydraulic Measurements}

Hydraulic measurements were carried out over each of the three surfaces for a total of seven different hydraulic boundary conditions. Three different shear velocities were achieved and the range of relative submergence $h / k$ in the gravel-bed tests varied between 3.6 and 9.3 (see Table 1). The roughness height $k$ was derived from the laser scan data and corresponded to the difference $Z_{99}-Z_{01}$, where, $Z_{99}$ and $Z_{01}$ denote the 99 th and 1st percentile of the distribution of bed elevations, respectively; the datum for the water depth $h$ was at the mean bed elevation.

For the gravel-bed tests, the water surface slope was determined using the data from the four pressure taps at the flume bottom. For the cast-bed, the solid bottom prevented the measurements from pressure taps and the water surface slopes were determined using the data from the 8 ultrasonic sensors. Additional tests in which multiple measurements were taken for a particular water surface slope indicated that the results from both measurement systems gave the same mean slope with the same order of magnitude of the deviation from the mean. Although uniform flow conditions were targeted, it was difficult to match the water surface slope exactly with the bed slope due to the mild slopes and the discrete nature of the water surface slope measurements. Both slopes were similar, but still showed some differences so that the St. Venant equation was used to determine the friction velocity [36]:

$$
u_{*}=\left[g h S_{b}-g h\left(S_{b}-S_{w}\right)\left(1-F r^{2}\right)\right]^{1 / 2}
$$

where $h$ denotes the water depth and Fr the Froude number which is defined as $F r=U /(g h)^{0.5}$. Equation (2) represents a simplified version of the St. Venant equation which is obtained by assuming that the slopes are very small. In the tests over the impermeable facsimiles (cast), the discharge was adjusted to ensure the same water surface slope and water depth as in the testes over the permeable bed. 
Table 1. Hydraulic boundary conditions applied for the measurements over the water-worked gravel-bed. The bulk flow velocity was determined from the equation of continuity $U=Q / A$ where $A=h b$ denotes the cross-sectional area with $b=$ flume width $(1 \mathrm{~m})$.

\begin{tabular}{cccccccccc}
\hline Test & $S_{b}$ & $S_{w}$ & $\begin{array}{c}h \\
{[\mathrm{~m}]}\end{array}$ & $\begin{array}{c}h / k \\
{[-]}\end{array}$ & $\begin{array}{c}Q \\
{\left[\mathrm{~m}^{3} / \mathrm{s}\right]}\end{array}$ & $\begin{array}{c}\boldsymbol{U} \\
{[\mathrm{m} / \mathrm{s}]}\end{array}$ & $\boldsymbol{F r}$ & $\boldsymbol{R e}$ & $\begin{array}{c}\boldsymbol{u}_{*} \\
{[\mathrm{~m} / \mathrm{s}]}\end{array}$ \\
\hline BC1 & 0.0018 & 0.00134 & 0.137 & 3.6 & 0.056 & 0.41 & 0.35 & 55890 & 0.043 \\
BC2 & 0.0015 & 0.00103 & 0.178 & 4.7 & 0.076 & 0.43 & 0.32 & 76270 & 0.043 \\
BC3 & 0.0015 & 0.00103 & 0.236 & 6.2 & 0.121 & 0.51 & 0.34 & 120787 & 0.050 \\
BC4 & 0.0020 & 0.00159 & 0.215 & 5.7 & 0.124 & 0.58 & 0.40 & 124107 & 0.059 \\
BC5 & 0.0013 & 0.00083 & 0.293 & 7.7 & 0.156 & 0.53 & 0.31 & 156412 & 0.050 \\
BC6 & 0.0010 & 0.00051 & 0.353 & 9.3 & 0.168 & 0.48 & 0.25 & 167987 & 0.043 \\
BC7 & 0.0015 & 0.00098 & 0.319 & 8.4 & 0.200 & 0.63 & 0.35 & 199883 & 0.057 \\
\hline
\end{tabular}

Velocity measurements were taken using the TSI stereoscopic particle image velocity system (SPIV; 2-dimensional 3-component velocimetry). The PIV measurements were carried out at $x \approx 8000 \mathrm{~mm}$ and $y \approx 500 \mathrm{~mm}$, i.e., in the centerline of tile number 4 (see Figure 3). The laser sheet was formed by a Nd:YAG (neodymium-doped yttrium aluminum garnet) double-pulsed laser and was aligned normal to the bed surfaces and parallel to the flume walls. The flow was seeded with polyamide particles of $55 \mu \mathrm{m}$ diameter. Two high speed 4 Mega Pixel CCD (Charge-coupled device) cameras captured the particle images at a frequency of $20 \mathrm{~Hz}$ for a period of $150 \mathrm{~s}$, producing 3000 image pairs per camera i.e., 12,000 images in total. The images were post-processed and analyzed using the TSI Insight $4 \mathrm{G}$ software. An interrogation area of $32 \times 32$ pixels with $50 \%$ overlapping was set to increase the probability that the seeding particles close to the edges of the interrogation area correlated well. The resulting grid size of the vector field was $16 \times 16$ pixels which corresponded to a spatial resolution of $1.56 \mathrm{~mm} \times 1.56 \mathrm{~mm}$ in streamwise and vertical direction respectively.

\section{Results and Discussions}

\subsection{Comparability of the Surfaces}

The vertical distributions of the porosity for initial gravel (surface-compacted), water-worked gravel, cast and the rotated cast are presented in Figure 4. For the water-worked gravel-bed, the porosity obtained from the WDM measurements decreased from $\phi=1$ at the roughness top to an absolute minimum just above the roughness trough before reaching an approximately constant value in the sub-surface. The increase in porosity close to the plastic bottom of the flume was associated with the combined effect of capillary action and poor sorting of gravel at the bottom [31]. The bulk porosity of the water-worked gravel-bed was $\phi=0.31$ whereas the bulk porosity of the surface compacted gravel was $\phi=0.26$; this difference was due to the larger height of the interfacial sublayer of the water-worked bed compared to the surface compacted bed. Note that the porosity values $\phi>1$ for the WDM measurements in Figure 4 are associated with the large spatial scale of the measurements (spanning the whole gravel-bed) and the accuracy of the measurements (see also [30]). The porosity distribution of the water-worked gravel-bed obtained by the WDM measurement matched the porosity distribution derived from the laser scans gravel-bed from the roughness crest $(z / H=1)$ to $z / H \approx 0.85$, where $H$ denotes the height of the bed measured from the flume bottom. Below $z / H \approx 0.85$, the results from the two methods deviate since the laser scan could not capture the pore space in the subsurface layer, i.e., the measurement range of the laser scan was restricted to the distance from the roughness crest to the roughness trough so that $\phi=0$ at the roughness trough for these measurements.

For the cast-beds, the porosity varied from $\phi=1$ at the roughness top to $\phi=0$ at the roughness trough. These distributions were solely obtained from the analysis of the laser scan data as the non-porous structure of the bed prevented the use of the WDM. The comparison of the porosity distributions derived from the laser scan measurements nearly collapse on a single line which is a first indicator of the accurate reproduction of the bed surface. However, a difference can be observed for 
the rotated cast just above the roughness trough and $\phi<0.2$. This is associated with the placement of the tile and is described below in some more detail.

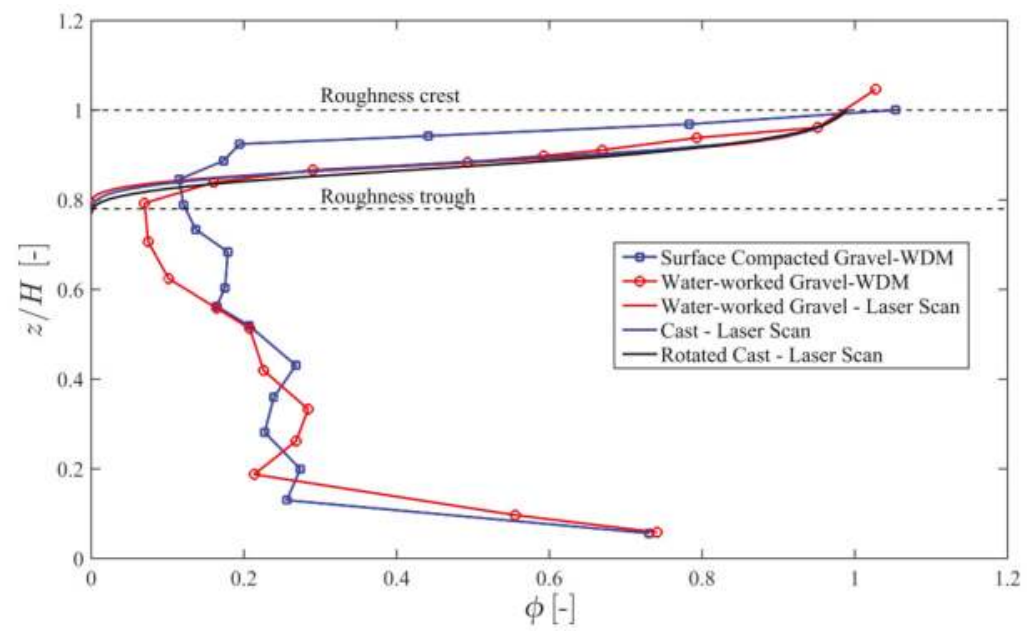

Figure 4. The vertical distribution of the porosity for all surfaces, $z$ is the distance from the flume bottom and $H$, the total height of the bed.

The digital elevation models of the surfaces obtained from the laser scan, shown in Figure 5, were used to compare the geometrical properties of the three bed configurations and to ensure accurate placement of the cast pieces. The following analysis focuses on the $4.65 \mathrm{~m}$ long section over which the water surface slope was measured (tiles 3 to 5 in Figure 3) and which contained the PIV-measurement area. For the analysis, the DEMs were detrended and the origin of the vertical coordinate corresponds to the mean bed level.

The visual comparison of the permeable gravel-bed (Figure 5a) with its impermeable counterpart (cast; Figure 5b) indicates a good agreement between the two surfaces. The only visible difference is at the transitions between the tiles in Figure $5 \mathrm{~b}$ at $x=6750 \mathrm{~mm}$ and $9050 \mathrm{~mm}$. The match of the cast-bed with the water worked gravel-bed can be analyzed by comparing the distributions of the bed elevations (Figure 6a) as well as the histogram of the observed differences in vertical elevations at each DEM grid point between the gravel and cast-bed (Figure 6b). The histograms shown in Figure 6a reveal a good agreement between the gravel and cast-bed, and the histogram of bed elevation differences (Figure 6b) indicates that most of the values characterizing the deviation of the two surfaces are within the range of $\pm 5 \mathrm{~mm}$. It is worth noting that the histogram in Figure $6 \mathrm{~b}$ includes, besides the effects of shrinkage and the joints between the tiles, the tile-alignment as it was based on the subtraction of the two DEMs. The good match of the gravel-bed with the cast-bed can further be substantiated by the statistical moments of the surfaces which are presented in Table 2. The standard deviation of bed elevations $\sigma$, skewness and kurtosis are directly comparable and fall into the typical range for armored gravel-bed surfaces as defined by Coleman et al. [3].

The distribution of bed elevations for the rotated cast differs slightly from the distribution of the cast. Strictly speaking, both surfaces should be characterized by identical histograms, however, the observed differences in Figure 6 a can be associated with very small transverse slopes induced when replacing the cast sections and due to discontinuities between the tiles. The latter were more pronounced for the rotated cast than for the cast and led to an increased number of measurement errors with the laser displacement meter due to its measurement principle. Despite these differences, Table 2 indicates still a good agreement of the statistical moments of the rotated bed with the ones for the original and cast-bed. 

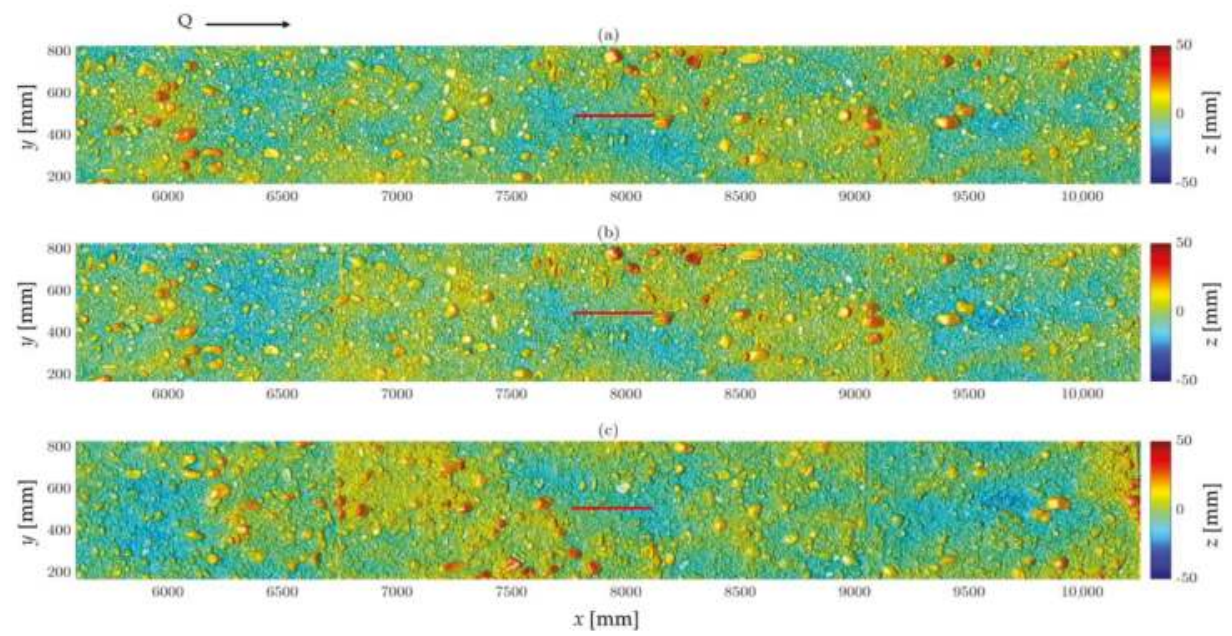

Figure 5. Digital elevation models (DEMs) of (a) gravel, (b) cast and (c) rotated cast surface respectively, thick horizontal red line indicates the position of the laser sheet (flow direction is from left to right).
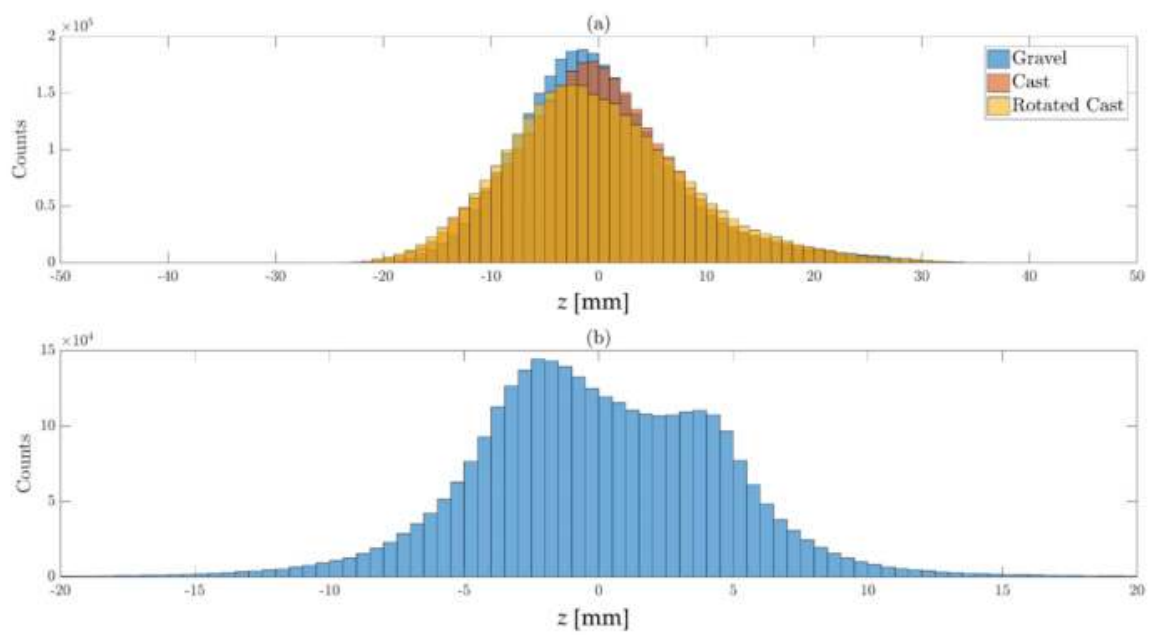

Figure 6. (a) Histograms of the vertical elevation of all three surfaces; (b) histograms of the difference in the vertical elevations between gravel and cast surface.

Table 2. Statistical moments for the three surfaces.

\begin{tabular}{ccccc}
\hline Bed Surface & $\begin{array}{c}\text { Roughness Height, } k \\
{[\mathrm{~mm}]}\end{array}$ & $\begin{array}{c}\text { Standard Deviation } \sigma \\
{[\mathrm{mm}]}\end{array}$ & $\begin{array}{c}\text { Skewness } \\
{[-]}\end{array}$ & $\begin{array}{c}\text { Kurtosis } \\
{[-]}\end{array}$ \\
\hline Gravel & 38.1 & 7.57 & 0.76 & 1.03 \\
Cast & 38.6 & 7.96 & 0.50 & 0.66 \\
Rotated Cast & 40.6 & 8.52 & 0.56 & 0.49 \\
\hline
\end{tabular}

The beds were also compared on the basis of normalized 2D-second order structure functions (2DSSF). For this purpose, the filtered normalized 2DSSF were determined according to the 
method described in detail in Qin et al. [17]. In brief, filtered 2DSSFs show only values in areas which are characterized by statistical significant correlations. The latter are determined by subdividing the surface in small tiles of a certain length scale and reshuffling them to create new surfaces. For these new surfaces, 2DSSF are calculated and used for statistical significance testing. The method is based on Monte Carlo simulations (see [17] for details) and for the present analysis, the reshuffled quadratic sub-areas were characterized by a side length of $d_{84}$ of the original gravel-bed. The statistical significance testing carried out here was based on a significance level $5 \%$ and 100 Monte-Carlo simulations.

Figure 7 shows the filtered 2DSSFs for the three surfaces and the sub-plots reveal areas of high spatial correlation at small spatial lags (the minimum value of 0 indicates perfect spatial correlation and a value of 1 a lack of spatial correlation). The area of high correlation for small spatial lags are characterized by a central ellipse, and the spatial extent of the main axes of the central ellipses can be interpreted as characteristic length scales in the horizontal plane [19]. The benefit of the filtered 2DSSF is that these length scales can be clearly defined and the resulting values of $100 \mathrm{~mm}(96 \mathrm{~mm}$ for the rotated bed) for the long and $40 \mathrm{~mm}$ for the short axis (for all three beds) substantiates the high accuracy that was achieved when reproducing the bed.

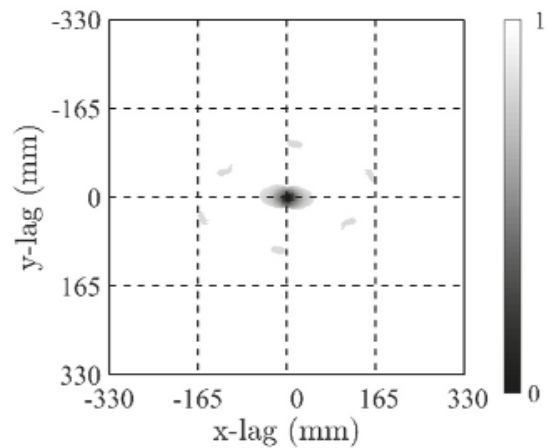

(a)

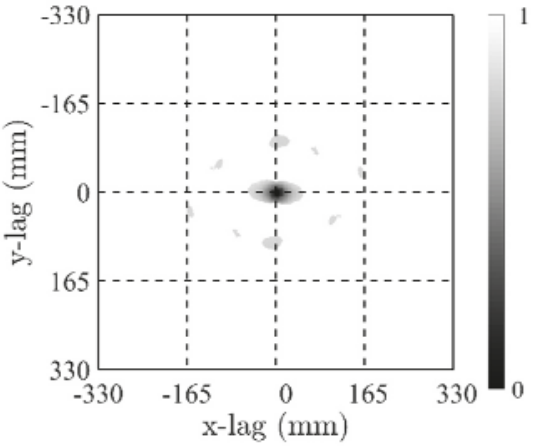

(b)

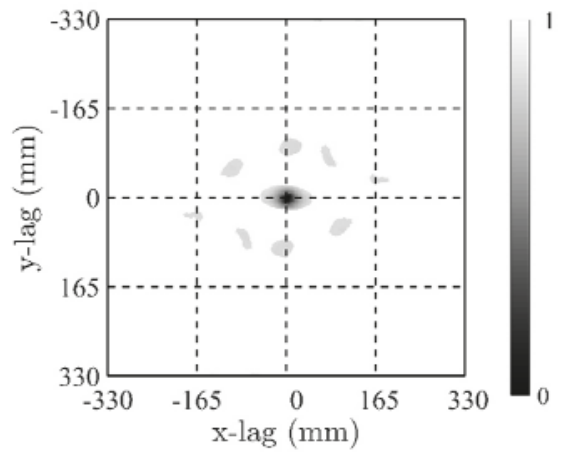

(c)

Figure 7. The $2 \mathrm{D}$ second-order structure function of the surfaces filtered by the upper $5 \%$ confidence limit, values lower than the upper $5 \%$ confidence limit are preserved. The greyscale corresponds to the values of the normalized structure function which ranges from 0 to 1 , where the value of 0 defines perfect spatial correlation. Sub-figures $(\mathbf{a}-\mathbf{c})$ correspond to gravel, cast and rotated cast surfaces respectively. 
The 2DSSFs can also be used to investigate the mean alignment of larger particles on the bed. For the present case, the orientation of the long axis of the ellipses deviates 5 degrees from the flow direction indicating that most of the larger particles are nearly aligned with their long axis in flow direction. This is in agreement with previous studies investigating the surface structure of stable armour layers [16]. Note that the patterns at larger spatial lags in Figure 7 reflect grain structures larger than individual grains. However, despite the fact that they are statistically significant, their value indicates only a low spatial correlation so that these patterns should not be interpreted [17].

\subsection{Flow Resistance}

The good agreement between the geometry of the original bed with its counterparts is a prerequisite for the detailed analysis of differences in hydraulic resistance. Figure 8 shows $(8 / f)^{0.5}$ as a function of relative submergence $(h / k)$ for the three surfaces. The lowest values of $(8 / f)^{0.5}$, i.e., a higher Darcy-Weisbach friction factor $f$, was observed for the tests over the rotated bed for relative submergences $h / k<6$. For relative submergences $h / k>6$, the difference in $(8 / f)^{0.5}$ between the rotated bed and the cast became smaller which may be due to the increasing submergence. Moreover, the uncertainty associated with the water surface slope measurements increased for the highest submergence for which $S_{w}$ was rather small $(\sim 0.05 \%)$. On the other hand, in all tests with the rotated cast-bed it was necessary to decrease the discharge to obtain the same water levels as in the tests with the cast-bed (discharge reductions of up to $6 \%$ were required; the average was $4 \%$ ). The higher flow resistance exerted by the rotated cast-bed shows that the orientation of the grains on the surface has a significant influence on flow resistance, i.e., the water working results in a more hydraulically efficient bed configuration (e.g., [37]). For example, rotating the fixed cast-bed means that the lee-areas of grains, where small grains typically settle during armoring, become exposed to the flow. Rotating the cast-bed, these particles are directly exposed to the flow but cannot be eroded as they are part of the cast. As a consequence these areas are characterized by a less hydrodynamic shape than comparable frontal areas of real water-worked beds as the associated drag coefficient changes with the shape and orientation [38]. Consequently, the rotated cast-bed imposes a higher resistance on the flow than the cast-bed. This result thus implies that bed roughness cannot solely be described by a characteristic grain size and that both surface structure and grain orientation play a vital role for the determination of flow resistance $[14,18,19]$.

Figure 8 further reveals small differences in flow resistance between the porous gravel-bed and its impermeable counterpart. For the two lowest submergences $(h / k=3.6$ and 4.7$),(8 / f)^{0.5}$ is larger for the porous gravel-bed than for the impermeable cast-bed while for the larger relative submergences this trend is reversed. This means that, for the two lowest submergences, larger friction factors were obtained for the cast-bed than for the porous gravel-bed. These two experiments were carried out with discharges of $Q=0.056 \mathrm{~m}^{3} / \mathrm{s}$ and $0.076 \mathrm{~m}^{3} / \mathrm{s}$, respectively, for the gravel-bed and $Q=0.056 \mathrm{~m}^{3} / \mathrm{s}$ and $0.074 \mathrm{~m}^{3} / \mathrm{s}$ respectively, for the cast-bed to obtain identical water depths $h$. Noting that a certain amount of the flow is conveyed through the subsurface in the porous-bed tests, the discharge used for the calculations of the bulk values in Table 1 should, strictly speaking, be reduced to account for subsurface flow. The experimental setup did not allow for the measurement of the subsurface flow rate but assuming a flow rate of approx. $0.001-0.002 \mathrm{~m}^{3} / \mathrm{s}$, computations indicated that for these two cases the flow resistance of the porous gravel-bed would be slightly larger than for the cast-bed $(h / k=3.7)$ or approximately equal $(h / k=4.7)$. The significance of subsurface flow rate gradually decreases with increasing discharge (and hence increasing relative submergence) so that the results for the experiments carried out with $h / k \geq 5.7$ (discharges $Q \geq 0.121 \mathrm{~m}^{3} / \mathrm{s}$; Table 1 ) are less affected, i.e., in these tests the flow resistance of the porous bed was larger than for the cast-bed.

The literature review revealed that flow resistance over porous beds depends on $\operatorname{Re}$ (e.g., [24]), and therefore $f$ is plotted as a function of Re in Figure 9. Regarding the comparison of $f$ obtained for the experiments over the cast-bed and the rotated cast-bed, Figure 9 yields the same conclusions as before; the rotated bed is characterized by higher flow resistance for all boundary conditions except 
for $R e \approx 170,000$ corresponding to the test which was carried out with the lowest water surface slope $(\sim 0.05 \%)$. For $\operatorname{Re}>100,000(h / k \geq 5.7)$, Figure 9 reveals again that the porous gravel bed showed a higher resistance to the flow than its non-porous counterpart. In fact, for the experiments over the cast-bed the discharge had to be increased for $h / k \geq 5.7$ to obtain the same water depth and shear velocity as in the porous-bed experiments, which confirms the observed trend. The deviation of the friction factors for the tests carried out for the lowest $R e$ values would be mitigated if subsurface flow would be accounted for (see above).

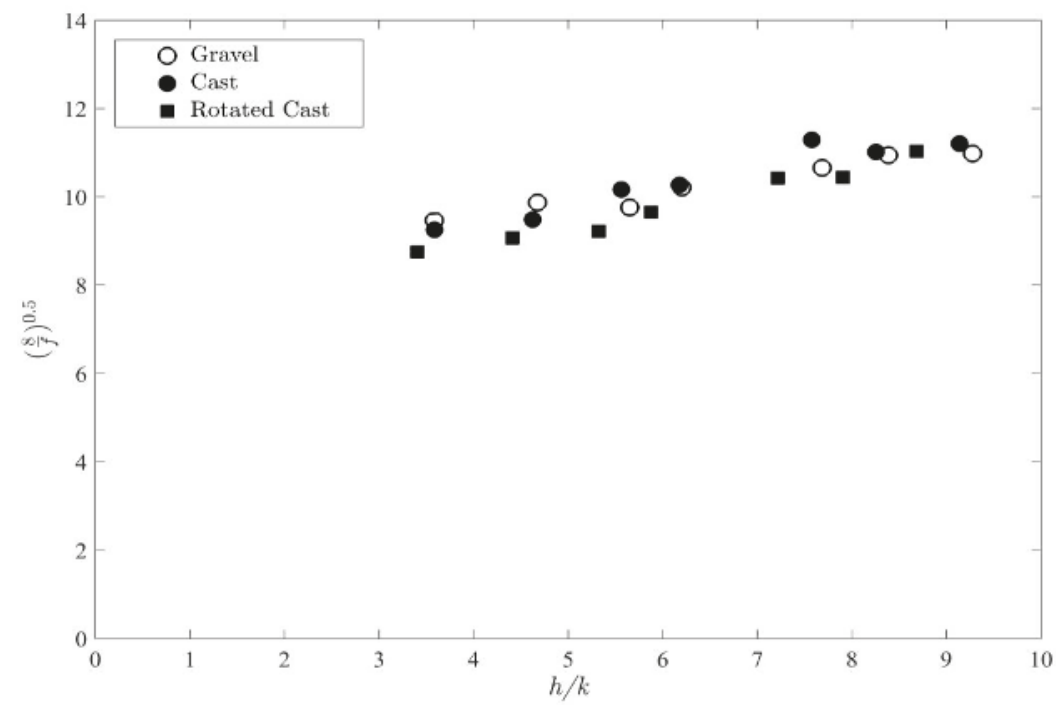

Figure 8. $(8 / f)^{0.5}$ as function of relative submergence $(h / k)$.

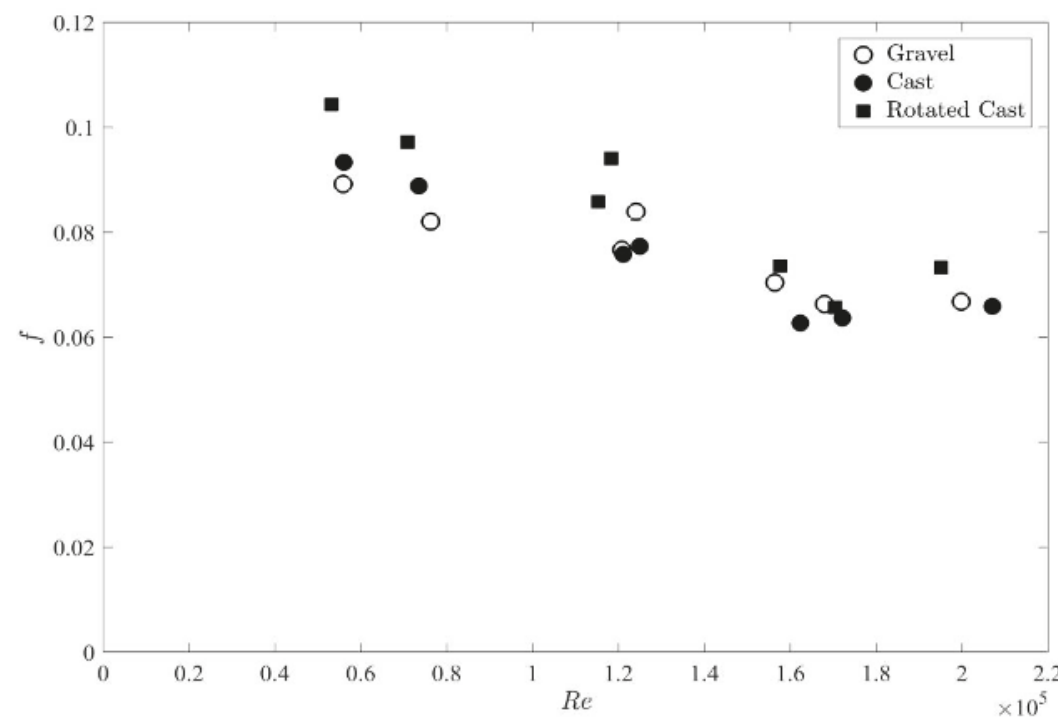

Figure 9. The variation of bulk friction factors with Re for the tested bed surfaces. 
The above discussion regarding the differences in friction factors needs to be extended regarding the surface properties. For example, the cast-bed was characterized by slightly larger $k$-values and standard deviations $\sigma$ than the porous bed (Table 2). These values indicate that the cast-bed may be slightly 'rougher' in geometrical terms than the gravel-bed, and this may contribute to the observed trend at lower submergences (and hence low $R e$ ). Similarly, the rotated cast-bed was characterized by larger $k$ - and $\sigma$-values than the cast-bed, which in turn means that the rotated cast-bed was already rougher due to its placement. However, the differences in $k$ and $\sigma$ are rather small (less than $2 \mathrm{~mm}$ for $k$ and $0.56 \mathrm{~mm}$ for $\sigma$ ) so that it can be hypothesized that this effect is negligible.

The comparison of the porous and non-porous bed can be further investigated by a preliminary and qualitative analysis of the double averaged longitudinal velocity distributions (i.e., time-averaged PIV-velocities, which were then spatially averaged in planes parallel to the mean bed elevation). Figure 10 shows exemplarily the velocity distributions, normalized with the shear velocities obtained by Equation (2), for three different boundary conditions (BC2, BC3 and BC4) corresponding to the three different shear velocities (Table 1$)$. The origin of the vertical axis $(z=0)$ in Figure 10 corresponds to the mean bed elevation. The global roughness crest (i.e., the roughness crest of the scanned section) was $23 \mathrm{~mm}$ above the mean bed elevation whereas the local roughness crest (i.e., the roughness crest of the shorter PIV section) was only $8 \mathrm{~mm}$ above the mean bed level.

The three normalized velocity profiles over the gravel-bed tests (open symbols in Figure 10) nearly collapse on a single line and show the expected logarithmic shape above the roughness crest. The three profiles over the cast-beds (solid symbols) deviate slightly, especially the profile for BC2. Note that the dip in the velocity profile for BC2 at $z \approx 160 \mathrm{~mm}$ is due to a plastic glass which was placed at the water surface to avoid the refraction of laser sheet caused by surface waves. In order to cross-check the data, the velocity profiles were integrated to estimate the discharge and for all presented profiles, the calculated discharge was similar to the discharge used in the experiments.

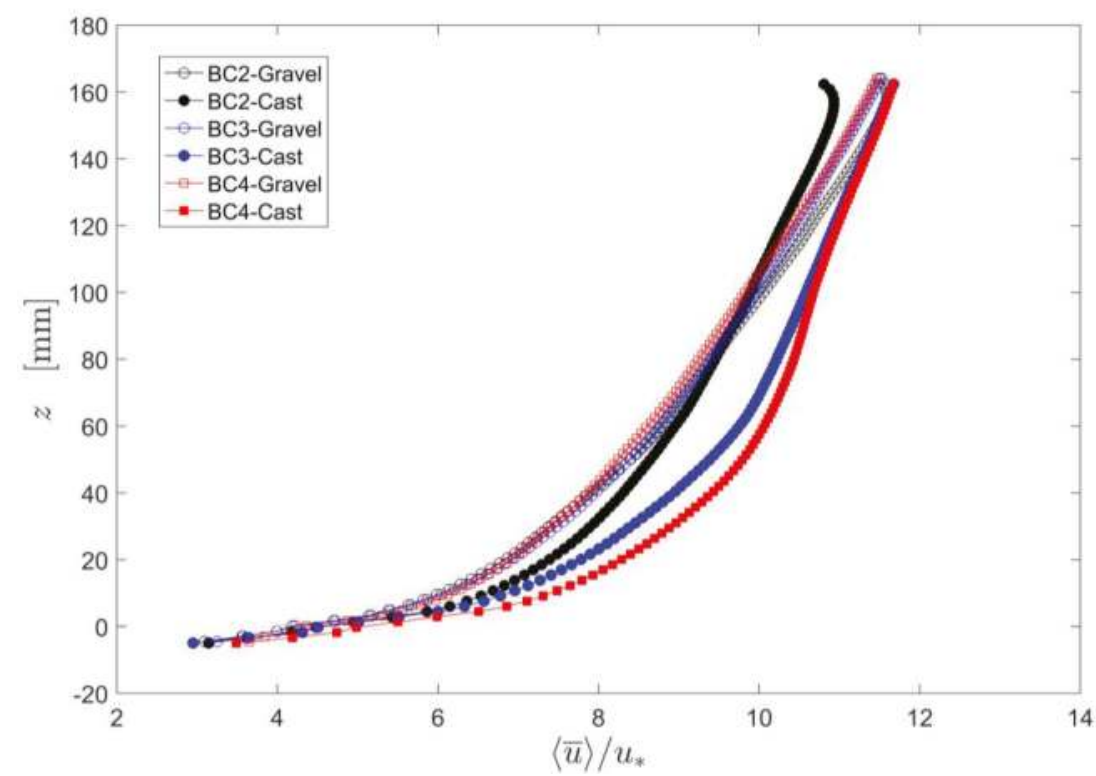

Figure 10. The double-averaged longitudinal velocities normalized with the shear velocities. Open and filled symbols denote water worked gravel-bed and cast-bed respectively while the colors represent different boundary conditions. 
The comparison of the profiles over the gravel-bed with the ones over the cast-bed shows that, for the same boundary condition, the velocities above the crest in the near-bed region $(8 \mathrm{~mm}<z<80 \mathrm{~mm}$ ) are larger over the cast-bed than over the porous bed. While the aforementioned cast-bed profile for $\mathrm{BC} 2$ shows larger velocities than the gravel-bed profile up to $z \approx 80 \mathrm{~mm}$, the velocity profiles for BC3 and $\mathrm{BC} 4$ show higher values for the cast-bed up to $z \approx 140 \mathrm{~mm}$ before nearly matching the velocities over the gravel-bed.

The smaller velocities over the crest of the water-worked gravel-bed in the near-bed region are additional evidence that a porous water-worked gravel-bed imposes higher flow resistance. It is interesting to note that in the interfacial sublayer (i.e., $z<8 \mathrm{~mm}$ ), the flow velocities are partly larger over the water-worked gravel-bed than over the cast, especially below the mean bed elevation $(z<0)$. This can be explained by the 'no slip' condition for the non-porous cast-bed while, due to the porosity of the subsurface layer, such a condition does only exist at the gravel-particle surfaces over the gravel-bed. Note that due to the limitations of the PIV-setup, the velocity profiles could not be measured to the roughness trough. Nonetheless, these preliminary results of the PIV data further confirm the results from the bulk-flow analysis that the flow resistance over the porous gravel-bed is larger than over the cast-bed.

The presented results together with the results of the qualitative analysis of the double-averaged velocity profiles can be used to discuss the different results regarding the influence of porosity reported by Cooper et al. [32]. The present study is based on experiments carried out over a casted surface which covered nearly the entire flume area. On the other hand, the length and width of the cast-bed in [32] was limited, corresponding roughly to about $5 \%$ of the total water-worked area, and the control of the sub-surface flow was not clearly stated by Cooper et al. [32]. Moreover, the cast tile was placed in the middle of the water-worked gravel and hence the transition from the gravel-bed to the cast-bed could affect the flow patterns; however, here we can only speculate about this effect. On the other hand, it is interesting to note that the range of relative submergence $(h / k)$ in Cooper et al. [32] varied between 3.1 and 4.6 with Re ranging between 64,000 and 84,000. The results presented in Figures 8 and 9 indicate that for comparable relative submergences and Re-values the cast-bed is 'rougher' which coincides with the findings of Manes et al. [28], Cooper et al. [32] although the behavior of the velocity profiles deviates from the one reported by Cooper et al. [32].

\section{Summary and Conclusions}

The present study presents results from an experimental program aiming at the investigation of the effect of gravel-bed porosity and grain orientation on bulk flow resistance. Experiments were carried out over three different surfaces; a water-worked gravel-bed, its non-porous counterpart (cast-bed), and the rotated cast-bed. The quality of the reproduced beds was shown and discussed based on laser-scan data and statistical parameters. Focusing on the analysis of bulk-flow parameters, the results showed that the rotated cast exerted the highest flow resistance which yielded to the conclusion that not only the surface structure but also its alignment regarding the flow direction (i.e., grain orientation) has a major influence on flow resistance. The results also confirmed the findings from studies carried out over artificial beds that a porous gravel-bed imposes higher flow resistance than its non-porous counterpart for comparable relative submergences. In the analysis of the data, the importance of subsurface flow-rates was briefly highlighted, especially for low relative submergences and hence slightly reduced surface flow rates. The subsurface flow rate has often been neglected in flume studies dealing with the determination of flow resistance, and this can hamper the comparability of results from experiments which were carried out over impermeable beds. The results of the present study have practical implications. For example, colmation processes, i.e., the settling of fine particles in the hyporheic zone, can change the porosity of the sub-surface, and hence the flow resistance (or vice versa). Having analyzed bulk flow characteristics in this paper, we will use the PIV-data for the detailed analysis of flow patterns over the three beds to investigate the effect of porosity on the near bed turbulent flow field and turbulence characteristics in our upcoming analyses. 
Author Contributions: Christy Ushanth Navaratnam and Jochen Aberle conceived and designed the experiments. Christy Ushanth Navaratnam performed the experiments and analyzed the data with support from Jie Qin, Pierre-Yves Henry and Jochen Aberle. All authors contributed to write the manuscript.

Funding: This research received no external funding.

Acknowledgments: Authors are thankful to C. Manes for providing the data from his study which helped to compare results between these studies.

Conflicts of Interest: The authors declare no conflict of interest.

\section{References}

1. Nikora, V.; Koll, K.; McEwan, I.; McLean, S.; Dittrich, A. Velocity Distribution in the Roughness Layer of Rough-Bed Flows. J. Hydraul. Eng. 2004, 130, 1036-1042. [CrossRef]

2. Aberle, J.; Koll, K.; Dittrich, A. Form induced stresses over rough gravel-beds. Acta Geophys. 2008, 56, 584-600. [CrossRef]

3. Coleman, S.E.; Nikora, V.I.; Aberle, J. Interpretation of alluvial beds through bed-elevation distribution moments. Water Resour. Res. 2011, 47, W11505. [CrossRef]

4. Vollmer, S. Einfluß der Oberflächenströmung auf die Permeable Gewässersohle; Universität Karlsruhe (TH): Karlsruhe, Germany, 2005.

5. Detert, M. Hydrodynamic Processes at the Water-Sediment Interface of Sreambeds. Doctoral Thesis, Universität Karlsruhe (TH), Karlsruhe, Germany, 2008.

6. Chow, V.T. Open-Channel Hydraulics; McGraw-Hill, Inc.: Singapore, 1959.

7. Keulegan, G.H. Laws of Turbulent Flow in Open Channels. J. Res. Natl. Bureau Standards 1938, 21, 707-741. [CrossRef]

8. Hey, R.D. Flow Resistance in Gravel-Bed Rivers. J. Hydraul. Div. 1979, 105, 365-379.

9. Bathurst, J.C. Flow Resistance Estimation in Mountain Rivers. J. Hydraul. Eng. 1985, 111, 625-643. [CrossRef]

10. Ferguson, R. Flow resistance equations for gravel- and boulder-bed streams. Water Resour. Res. 2007, 43, W05427. [CrossRef]

11. Hendrick, R.R.; Ely, L.L.; Papanicolaou, A.N. The role of hydrologic processes and geomorphology on the morphology and evolution of sediment clusters in gravel-bed rivers. Geomorphology 2010, 114, 483-496. [CrossRef]

12. Nikora, V.I.; Goring, D.G.; Biggs, B.J.F. On gravel-bed roughness characterization. Water Resour. Res. 1998, 34, 517-527. [CrossRef]

13. Smart, G.M.; Duncan, M.J.; Walsh, J.M. Relatively Rough Flow Resistance Equations. J. Hydraul. Eng. 2002, 128, 568-578. [CrossRef]

14. Aberle, J.; Smart, G.M. The influence of roughness structure on flow resistance on steep slopes. J. Hydraul. Res. 2003, 41, 259-269. [CrossRef]

15. Yochum, S.E.; Bledsoe, B.P.; David, G.C.L.; Wohl, E. Velocity prediction in high-gradient channels. J. Hydrol. 2012, 424-425, 84-98. [CrossRef]

16. Aberle, J.; Nikora, V. Statistical properties of armored gravel bed surfaces. Water Resour. Res. 2006, 42, W11414. [CrossRef]

17. Qin, J.; Aberle, J.; Henry, P.-Y.; Wu, T.; Zhong, D. Statistical significance of spatial correlation patterns in armoured gravel beds. J. Hyraul. Res. 2018, in press.

18. Powell, D.M. Flow resistance in gravel-bed rivers: Progress in research. Earth-Sci. Rev. 2014, 136, 301-338. [CrossRef]

19. Flack, K.A.; Schultz, M.P. Review of Hydraulic Roughness Scales in the Fully Rough Regime. J. Fluids Eng. 2010, 132, 041203. [CrossRef]

20. Tonina, D.; Buffington, J.M. Hyporheic exchange in gravel bed rivers with pool-riffle morphology: Laboratory experiments and three-dimensional modeling. Water Resour. Res. 2007, 43, W01421. [CrossRef]

21. Boano, F.; Harvey, J.W.; Marion, A.; Packman, A.I.; Revelli, R.; Ridolfi, L.; Wörman, A. Hyporheic flow and transport processes: Mechanisms, models, and biogeochemical implications. Rev. Geophys. 2014, 52, 603-679. [CrossRef] 
22. Marion, A.; Nikora, V.; Puijalon, S.; Bouma, T.; Koll, K.; Ballio, F.; Tait, S.; Zaramella, M.; Sukhodolov, A.; O'Hare, M.; et al. Aquatic interfaces: A hydrodynamic and ecological perspective. J. Hydraul. Res. 2014, 52, 744-758. [CrossRef]

23. Zagni, A.F.E.; Smith, K.V.H. Channel flow over permeable beds of graded spheres. J. Hydraul. Div. 1979, 102, 207.

24. Zippe, H.J.; Graf, W.H. Turbulent boundary layer flow over permeable and non-permeable rough surfaces. J. Hydraul. Res. 1983, 21, 51-65. [CrossRef]

25. Breugem, W.P.; Boersma, B.J.; Uittenbogaard, R.E. The influence of wall permeability on turbulent channel flow. J. Fluid Mech. 2006, 562, 35-72. [CrossRef]

26. Manes, C.; Pokrajac, D.; McEwan, I.; Nikora, V. Turbulence structure of open channel flows over permeable and impermeable beds: A comparative study. Phys. Fluids 2009, 21, 125109. [CrossRef]

27. Manes, C.; Poggi, D.; Ridolfi, L. Turbulent boundary layers over permeable walls: Scaling and near-wall structure. J. Fluid Mech. 2011, 687, 141-170. [CrossRef]

28. Manes, C.; Pokrajac, D.; Nikora, V.I.; Ridolfi, L.; Poggi, D. Turbulent friction in flows over permeable walls. Geophys. Res. Lett. 2011, 38, L03402. [CrossRef]

29. Kuwata, Y.; Suga, K. Direct numerical simulation of turbulence over anisotropic porous media. J. Fluid Mech. 2017, 831, 41-71. [CrossRef]

30. Aberle, J. Measurements of armour layer roughness geometry function and porosity. Acta Geophys. 2007, 55, 23-32. [CrossRef]

31. Navaratnam, C.U.; Aberle, J.; Daxnerová, J. An Experimental Investigation on Porosity in Gravel Beds. In Free Surface Flows and Transport Processes; Springer International Publishing: Cham, Germany, 2018; pp. 323-334.

32. Cooper, J.R.; Ockleford, A.; Rice, S.P.; Powell, D.M. Does the permeability of gravel river beds affect near-bed hydrodynamics? Earth Surf. Process. Landf. 2018, 43, 943-955. [CrossRef]

33. Aberle, J.; Dittrich, A.; Koll, K.; Schoneboom, T. Sohlnahes turbulentes Strömungsfeld. In BAW-Workshop: Boden- und Sohl-Stabilität-Betrachtungen an der Schnittstelle zwischen Geotechnik und Wasserbau; Bundesanstalt für Wasserbau: Karlsruhe, Germany, 2004.

34. Spiller, S.; Rüther, N. Artificial reproduction of the surface structure in a gravel bed. In 2nd IAHR Europe Conference; TU Munich: Munich, Germany, 2012.

35. Navaratnam, C.U.; Aberle, J.; Spiller, S.M. Evaluation of the accuracy of a bed casting technique. In River Flow 2016; CRC Press: St. Louis, MO, USA, 2016; pp. 398-403.

36. Graf, W.H.; Song, T. Bed-shear stress in non-uniform and unsteady open-channel flows. J. Hydraul. Res. 1995, 33, 699-704. [CrossRef]

37. Leopold, L.B.; Langbein, W.B. The Concept of Entropy in Landscape Evolution. In Theoretical Papers in the Hydrologic and Geomorphic Sciences; United States Government Printing Office: Washington, DC, USA, 1962; pp. A1-A20.

38. Cassan, L.; Roux, H.; Garambois, P.-A. A Semi-Analytical Model for the Hydraulic Resistance Due to Macro-Roughnesses of Varying Shapes and Densities. Water 2017, 9, 637. [CrossRef]

(c) 2018 by the authors. Licensee MDPI, Basel, Switzerland. This article is an open access article distributed under the terms and conditions of the Creative Commons Attribution (CC BY) license (http:/ / creativecommons.org/licenses/by/4.0/). 
Article

\title{
Waves and Currents at a River Mouth: The Role of Macrovortices, Sub-Grid Turbulence and Seabed Friction
}

\author{
Lorenzo Melito *, Matteo Postacchini, Giovanna Darvini and Maurizio Brocchini \\ Department of DICEA, Università Politecnica delle Marche; Via Brecce Bianche 12, 60131 Ancona, Italy; \\ m.postacchini@univpm.it (M.P.); g.darvini@univpm.it (G.D.); m.brocchini@univpm.it (M.B.) \\ * Correspondence: 1.melito@pm.univpm.it; Tel.: +39-071-220-4912
}

Received: 7 February 2018; Accepted: 20 April 2018; Published: 25 April 2018

\begin{abstract}
Numerical experiments of wave-current interaction have been performed to investigate the evolution and dissipation of horizontal large-scale vortical structures generated by differential wave breaking and current shearing at river mouths. Specific focus is on the role played by turbulence of scales smaller than the water depth and by seabed friction on the dissipation of the mentioned macrovortices. The analysis reveals two regions of turbulence generation: at the river mouth, and along the curved seaward boundary of the shoal. In the latter zone, macrovortices are formed due to differential wave breaking induced by the sudden variation in water depth and enhanced by opposing currents which favour wave steepening. Such vortices are then advected towards the shore. Among the dissipative mechanisms, dissipation induced by seabed friction is deemed dominant, in particular in the most shallow waters of the shoal. Sub-grid turbulence dissipation, conversely, is more efficient offshore, although exerting some effect also over the shoal when supported by the continuous action of waves.
\end{abstract}

Keywords: river mouth; flow mixing; nonlinear shallow water equations; macrovortices; sub-grid turbulence; seabed friction

\section{Introduction}

At estuaries, river mouths and inlets in a broader sense, waves and outflowing currents give rise to a very wide spectrum of interactions, which, in turn, has effects on the hydrodynamics and morphology of coastal and estuarine environments. Notwithstanding its relevance, though, this strongly nonlinear interplay is still far to be fully understood, and increasing research efforts have been spent over the last decades to study the phenomena connected with wave-current interactions.

A common and easily detectable feature of interacting waves and currents is wave blocking, an occurrence in which the incoming waves are gradually blocked (with part of their energy reflected back to the open sea) by a current flowing in the opposite direction [1,2]. Wave blocking can considerably reduce the energy of sea waves penetrating the river mouth or estuary, since it leads to local wave steepening and breaking and global energy dissipation. The wave frequency spectrum also experiences a downshift, with the high-frequency energy of wind waves being both dissipated by breaking and partially transferred to low-frequency components such as infragravity waves [3]. Currents themselves are also modified by the interaction with waves; experimental investigations have shown that, because of opposing waves, the current velocity profile loses its typical logarithmic shape, with the current strength decreasing near the bottom and slightly increasing near the mean water level [4]. The effects of waves on the current profiles is also highly dependent on the propagation angle of the waves with respect to the current [5]. In the light of the above, it is clear that the coastal areas located at the convergence of marine and riverine forcings are regions of extreme complexity and 
their study via experimental and observational means is very difficult. The analysis of the dynamics of wave-current interaction at river mouths has seen a notable increase in recent years thanks to the increasing use of numerical models (e.g., [6-9]).

Coastal environments where waves and currents coexist are also characterized by a high level of turbulence, which promotes water mixing in both vertical and horizontal directions. Turbulence at the mouth of rivers and in the nearby shallow areas is often supported by large-scale, coherent vortical structures with rotation axis perpendicular to the seabed, commonly known as macrovortices $[10,11]$. Within a shallow-water framework, large eddies are seen to gradually form after the rearrangement of the vorticity field [12], generated because of wave breaking spatial unevenness due to varying topography in both cross-shore and alongshore directions and also because of the mentioned wave-current interaction. Differential wave breaking leads to breakers of finite length and injection of vorticity at the lateral edges of the breaker $[10,13]$. Finite-length breakers leading to vorticity generation are also likely to occur because of variabilities in the incoming wave field, or in the presence of cross-sea, i.e., several wave trains approaching the shore from different directions [14]. Finite-length breakers are a source of considerable vorticity $\omega$ that is injected into the fluid [15]; assuming, for the sake of simplicity, a bore (shock discontinuity) with a straight and finite front propagating in the $x$-direction, so that the $y$-direction is the cross-flow direction, the amount of potential vorticity generated across the jump or bore, $\Delta \Omega$, may be evaluated as

$$
\Delta \Omega=-\left[\frac{2 d_{2}}{g d_{1}\left(d_{1}+d_{2}\right)}\right]^{\frac{1}{2}} \frac{\mathrm{d} E_{d}}{\mathrm{~d} y},
$$

where $\Omega=\omega / d$ is the potential vorticity, $d_{1}$ and $d_{2}$ are the water depths upstream and downstream of the shock, respectively, and $E_{d}$ is the specific rate of energy dissipation, which is strictly related to the jump in water height across the shock:

$$
E_{d}=\frac{\left[d_{2}-d_{1}\right]^{3}}{4 d_{1} d_{2}} .
$$

According to relation (1), potential vorticity is generated wherever there is a large cross-flow gradient in $E_{d}$ and, hence, an abrupt cross-flow change in $\left(d_{2}-d_{1}\right)$ [16,17]. This situation often happens at the edges of finite-length breakers.

Once macrovortices are well-formed thanks to the reorganization of injected vorticity, they are often advected towards the shore by the action of waves and self-advection, this forcing the vortex to move along the isobaths [10].

A number of studies ascertained that macrovortices are a strong agent in several phenomena and processes occurring in the nearshore, such as flow mixing [18-20], entrainment and distribution of sediment [21,22] and advection of passive tracers [23]. As a natural consequence, macrovortices are crucial in defining the geomorphological response of many environments, such as barred beaches [15], riverine systems [24-26] and compound channels [27,28]. Some recent studies [29-31] also highlighted the role both of river jet instability and of macrovortices for the morphological evolution of estuarine and deltaic structures.

Although a complete representation of turbulent motions at all time and space scales requires important computational efforts, a simplified yet suitable description of the underlying structure of macrovortices can be achieved by means of relatively simple numerical models, such as depth-averaged solvers based on the nonlinear shallow water equations (NSWEs). These models neglect the vertical structure of the described flows by integrating the flow quantities over the water depth, but are still efficient tools to catch the relevant features of wave-current interactions and 2-dimensional large-scale vorticity.

Such simplified models can be further complemented by coupling with Large Eddy Simulation (LES) models to improve their capability of describing vortical flows [26,32-34]. In a LES approach, 
only the "large" turbulent motions, i.e., those occurring at a length scale greater than a predefined filter size (usually taken as the computational grid size), is resolved exactly. The dissipative effect of 3D turbulence occurring at length scales smaller than the flow depth, the so-called "sub-grid scale", is accounted for by suitable Sub-Grid Scale (SGS) closure models that connect the physical properties of small-scale turbulence with those of the mean flow field [35]. LES schemes for the modeling of vorticity and turbulence have received considerable research attention over the last decades (see, as examples, $[26,32,34]$ ) and have been successfully applied to simulate vortical and turbulent patterns in fluvial-like flows $[24,28,36]$. LES has been proven particularly appropriate to model vortical structures that are generated by shear instabilities within the flow [37]. A LES approach has been adopted to describe frictional effects and jet instability in the work of Canestrelli et al. [30], although their study neglected the effects of wind waves.

The purpose of the present work is to investigate the main mechanisms of flow mixing, with focus on macrovortices, in a simplified river mouth environment, and to better understand the role of seabed friction and sub-grid-scale turbulence in draining energy from such macrovortices. This is done through a parametric, numerical analysis of wave-current interactions performed with a depth-averaged shallow water solver. The effect of frictional dissipation is introduced through a classical Chezy-like formulation for bed stresses; dissipation by sub-grid turbulence is accounted for through a simple horizontal LES (HLES) model. The main vortical and turbulent patterns are therefore observed and discussed.

The paper is organized as follows. In Section 2 a brief description of the numerical framework in use is presented. Section 3 is devoted to a description of the numerical simulations, the main findings of which are illustrated and discussed in Section 4. Concluding remarks are finally given in Section 5.

\section{Materials and Methods}

\subsection{The Nonlinear Shallow Water Equations: The Analytics}

The nonlinear shallow water equations are a set of depth-averaged, hyperbolic equations which describes the conservation of mass and momentum in shallow waters. For a more complete treatment of the problem, the standard NSWEs are complemented with additional terms accounting for flow dissipation due to bottom friction and small-scale turbulent motions.

The system of the NSWEs in non-conservative form is:

$$
\begin{aligned}
d_{, t}+(u d)_{, x}+(v d)_{, y} & =0, \\
u_{, t}+u u_{, x}+v u_{, y}+g d_{, x} & =-g z_{b, x}-B_{x}+F_{x}, \\
v_{, t}+u v_{, x}+v v_{, y}+g d_{, y} & =-g z_{b, y}-B_{y}+F_{y}
\end{aligned}
$$

where $(x, y, z)$ is an orthogonal Cartesian system of spatial coordinates and $t$ represents time. The cross-shore $x$-coordinate points toward the shore and has its origin at the seaward boundary of the domain of interest (see Figure 1). The datum for the vertical coordinate $z$ is located at the still water level $z=0 . \mathbf{v}=(u, v)$ is the vector of the depth-averaged velocities; $d=\eta-z_{b}$ is the water depth, where $\eta$ is the elevation of the water surface and $z_{b}$ is the seabed location, both measured from the still water level; $g$ is the acceleration of gravity. Subscripts after commas represent partial differentiation with respect to space or time.

The terms $B_{x}$ and $B_{y}$ appearing at the right-hand side of Equations (3b) and (3c) represent the contributions due to seabed friction. When a Chezy-type formulation is used for modelling bed shear stresses, $B_{x}$ and $B_{y}$ are defined as follows:

$$
B_{x}=c_{\tau} \frac{|\mathbf{v}| u}{d} \quad \text { and } \quad B_{y}=c_{\tau} \frac{|\mathbf{v}| v}{d}
$$

where $c_{\tau}$ is the dimensionless Chezy friction coefficient, usually of order $10^{-2}-10^{-3}$ [38]. 


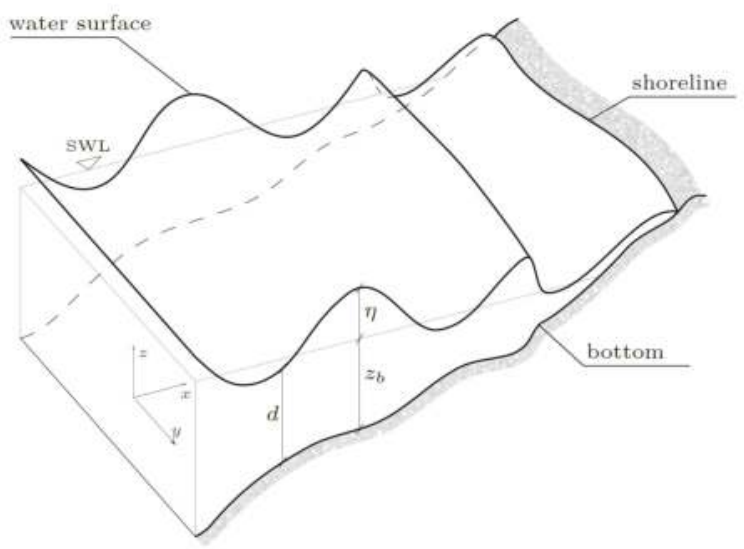

Figure 1. Schematics of the nearshore and representation of the involved variables.

For the purposes of our simulations, $c_{\tau}$ is computed as a function of the initial water depth, using the formulation proposed by van Rijn [39] in case of a hydraulically rough flow:

$$
c_{\tau}=\frac{g}{\left(18 \log \frac{12 d}{k_{s}}\right)^{2}},
$$

where $k_{s}$ is the bed roughness.

The additional terms $F_{x}$ and $F_{y}$ here account for the viscous dissipation due to sub-grid turbulence, i.e., the turbulence that develops at length scales smaller than the water depth and thus not directly resolved. The friction terms $B_{x}$ and $B_{y}$ and the viscous dissipation terms $F_{x}$ and $F_{y}$ are included among the source terms as they are part of a specific "source" system, as described in the following.

The description of turbulent structures with scales smaller than the water depth is performed by means of a Horizontal Large Eddy Simulation (HLES) model to the likes of those adopted for numerical applications in riverine environments (e.g., [24,28,36,40]). Therefore, only the "large" turbulent motions, i.e., those occurring at a length scale greater than a predefined filter size (in this case the water depth), are directly resolved. The dissipative effect of turbulence at sub-grid scales (SGS) is usually accounted for with appropriate models that link the properties of "small" turbulence to those of the resolved flow. Approaches like this are, in fact, suitable for implementation in depth-averaged solvers, since they lump all the complex mechanisms related to sub-grid turbulence in a simple model in which only mean properties are explicitly used. Of course, a proper physical description of 3D turbulence and all related phenomena is hindered in depth-averaged models, which neglect the essential contibution of vertical velocities and gradients to the development of turbulence. In spite of this, simplified approaches, such as HLES, can still be used satisfactorily to reach a suitable broad description of such complex mechanisms.

An SGS model based on the variable eddy viscosity formulation proposed by Grosso et al. [41] is used in our solver. To implement it, the momentum balance Equations (3b) and (3c) are complemented with terms $F_{x}$ and $F_{y}$, representing the dissipative body forces due to the effects of sub-grid turbulence. They are introduced with a typical Laplacian-type formulation as in the following:

$$
\begin{aligned}
& F_{x}=\frac{\left(d T_{x x}\right)_{, x}+\left(d T_{x y}\right)_{, y}}{d}, \\
& F_{y}=\frac{\left(d T_{x y}\right)_{, x}+\left(d T_{y y}\right)_{, y}}{d} .
\end{aligned}
$$


$T_{x x}, T_{y y}$ and $T_{x y}$ are the components of the Reynolds stress tensor for turbulent flows, for which a simple closure is considered [24]:

$$
T_{x x}=2 v_{T} u_{, x}, \quad T_{y y}=2 v_{T} v, y, \quad T_{x y}=v_{T}\left(u_{, y}+v, x\right),
$$

where $v_{T}$ is a depth-averaged effective eddy viscosity, defined as a function of the water depth:

$$
v_{T}=\lambda g^{1 / 2} d^{3 / 2} .
$$

Such eddy viscosity formulation is clearly compatible with the shallow-water approach of the model, as the velocity scale is taken to be the phase speed of waves in shallow waters, i.e., $\sqrt{g d}=g^{1 / 2} d^{1 / 2}$, and the length scale is the water depth $d$.

From an operative point of view, Expressions (6) and (7) are included in the source terms along with the bed friction terms $B_{x}$ and $B_{y}$, and used to solve the "source" problem (20). Implementing a second-order centered difference scheme to discretize problem (20) over a time step $\Delta t$, the following expressions for the depth-averaged velocities $u$ and $v$ at mesh node $(i, j)$ are obtained:

$$
\begin{aligned}
& u_{i, j}^{n+1}=u_{i, j}^{n}+v_{T} \Delta t\left[2 u_{, x x}+u_{, y y}+v_{, x y}\right]+\frac{v_{T}}{d} \Delta t\left[5 d_{, x} u_{, x}+\frac{5}{2} d_{, y} u_{, y}+\frac{5}{2} d_{, y} v, x\right], \\
& v_{i, j}^{n+1}=v_{i, j}^{n}+v_{T} \Delta t\left[2 v_{, y y}+v_{, x x}+u_{, x y}\right]+\frac{v_{T}}{d} \Delta t\left[5 d_{, y} v_{, y}+\frac{5}{2} d_{, x} u_{, y}+\frac{5}{2} d_{, x} v_{, x}\right] .
\end{aligned}
$$

Through Expressions (10) and (11), the values of the depth-averaged velocities $u_{i, j}$ and $v_{i, j}$ are "corrected" by the viscous contributions, to give account of the effect of sub-grid turbulence. The presence and size of such "correction" is determined by the value of the calibration parameter $\lambda$ appearing in the definition of the variable eddy viscosity (9). If $\lambda=0$, the turbulent sub-grid scales are not modeled and the HLES technique is deactivated: the dissipative terms (6) and (7) are not included in the numerical model and the corrective contribution due to viscosity in (10) and (11) is not present, the only corrections being those due to friction. A value of $\lambda \neq 0$ activates the HLES terms and includes their dissipative effects into the numerical solution. When solving Equations (10) and (11), it is assumed that the water depth $d$ is constant, hence the only flow variables that effectively change are the velocities $u_{i, j}$ and $v_{i, j}$. A conservative form of Equations (10) and (11), in line with the use of a conservative formulation for the NSWE system detailed in the following, is therefore not required. The HLES formulation explained above has been successfully tested and validated against experimental evidence in earlier works (e.g., [41-43]).

\subsubsection{Dimensional Analysis}

We now perform a dimensional analysis of the terms of system (3), useful for an introductory assessment of the role of frictional and viscous dissipation forces. By defining a length scale for horizontal distances $L_{0}$ and a length scale for vertical distances $d_{0}$, a reference velocity $\sqrt{g d_{0}}$ and a time scale $t_{0}=L_{0} /\left(g d_{0}\right)$ can be derived. The dimensionless forms (labeled with a tilde) of the variables appearing in system (3) are then defined as follows:

$$
\begin{gathered}
\tilde{x}=\frac{x}{L_{0}}, \quad \tilde{y}=\frac{y}{L_{0}}, \quad \tilde{t}=t \frac{\sqrt{g d_{0}}}{L_{0}}, \\
\tilde{u}=\frac{u}{\sqrt{g d_{0}}}, \quad \tilde{v}=\frac{v}{\sqrt{g d_{0}}}, \quad \tilde{d}=\frac{d}{d_{0}}, \quad \tilde{z}_{b}=\frac{z_{b}}{d_{0}}, \quad \tilde{v}_{T}=\frac{v_{T}}{\sqrt{g d_{0}} d_{0}}, \\
\tilde{T}_{x x}=T_{x x} \frac{L_{0}}{g d_{0}^{2}}, \quad \tilde{T}_{y y}=T_{y y} \frac{L_{0}}{g d_{0}^{2}}, \quad \tilde{T}_{x y}=T_{x y} \frac{L_{0}}{g d_{0}^{2}} .
\end{gathered}
$$


The NSWE system (3), expanded by means of Equations (4), (6) and (7), is thus made dimensionless with scales (12):

$$
\begin{aligned}
& \tilde{d}_{, \tilde{t}}+(\tilde{u} \tilde{d})_{, \tilde{x}}+(\tilde{v} \tilde{d})_{, \tilde{y}}=0, \\
& \tilde{u}_{, \tilde{t}}+\tilde{u} \tilde{u}_{, \tilde{x}}+\tilde{v} \tilde{u}_{, \tilde{y}}+\tilde{d}_{, \tilde{x}}=-g \tilde{z}_{b, \tilde{x}}-\frac{c_{\tau}}{\epsilon} \frac{|\tilde{\mathbf{v}}| \tilde{u}}{\tilde{d}}+\epsilon \frac{\left(\tilde{d} \tilde{T}_{x x}\right)_{, \tilde{x}}+\left(\tilde{d} \tilde{T}_{x y}\right)_{, \tilde{y}}}{\tilde{d}}, \\
& \tilde{v}_{, \tilde{t}}+\tilde{u} \tilde{v}_{, \tilde{x}}+\tilde{v} \tilde{v}, \tilde{y}+\tilde{d}_{, \tilde{y}}=-g \tilde{z}_{b, \tilde{y}}-\frac{c_{\tau}}{\epsilon} \frac{|\tilde{\mathbf{v}}| \tilde{v}}{\tilde{d}}+\epsilon \frac{\left(\tilde{d} \tilde{T}_{x y}\right)_{, \tilde{x}}+\left(\tilde{d} \tilde{T}_{y y}\right)_{, \tilde{y}}}{\tilde{d}},
\end{aligned}
$$

where $\epsilon=d_{0} / L_{0}$ is a dimensionless parameter defined by the ratio between vertical and horizontal length scales. By inspection of Equations (3b) and (3c), it can be readily seen that $B_{x}, B_{y} \propto P_{\tau}=c_{\tau} / \epsilon$; the dissipation of flow momentum due to frictional effects is, therefore, more relevant in very shallow waters $(\epsilon \ll 1)$. On the other hand, the viscous dissipation terms are proportional to $P_{\lambda}=\lambda \epsilon$ and are thus dominant over intermediate-to-deep waters, where $\epsilon \approx 0.1-1$.

\subsection{The Nonlinear Shallow Water Equations: The Numerics}

For the NSWE system (3) to be solvable by numerical techniques, it must be cast in a conservative form, which is the most suitable for our purposes since it allows to properly treat flow discontinuities or shocks. Within this numerical framework, in fact, bores and breaking waves can be treated through a synthetic shock-capturing approach by substituting them with jump discontinuities in flow properties. For further details about conservative and non-conservative formulations see [44]. An apt conservative vectorial form of the NSWEs is

$$
\mathbf{U}_{, t}+\mathbf{F}(\mathbf{U})_{, x}+\mathbf{G}(\mathbf{U})_{, y}=\mathbf{S}(\mathbf{U}),
$$

where $\mathbf{U}$ is the vector of conserved variables, which for the chosen conservative form are defined as $d$, $u d$, and $v d . \mathbf{F}(\mathbf{U})$ and $\mathbf{G}(\mathbf{U})$ are the vectors of fluxes and $\mathbf{S}(\mathbf{U})$ is the vector of source terms:

$$
\begin{gathered}
\mathbf{U}=\left[\begin{array}{c}
d \\
u d \\
v d
\end{array}\right], \\
\mathbf{F}(\mathbf{U})=\left[\begin{array}{c}
u d \\
u^{2} d+\frac{1}{2} g d^{2} \\
u v d
\end{array}\right], \quad \mathbf{G}(\mathbf{U})=\left[\begin{array}{c}
v d \\
u v d \\
v^{2} d+\frac{1}{2} g d^{2}
\end{array}\right], \\
\mathbf{S}(\mathbf{U})=\left[\begin{array}{c}
0 \\
-g d z_{b, x}-B_{x} d+F_{x} d \\
-g d z_{b, y}-B_{y} d+F_{y} d
\end{array}\right] .
\end{gathered}
$$

The hyperbolic system of NSWEs (3) is then numerically solved by means of a finite-volume scheme based on the Weighted Averaged Flux (WAF) method developed by Toro [44,45]. The hydrodynamic numerical solver employed in the present work, which is described in detail and validated in [38] as well as through previous experiences with various configurations (e.g., $[27,42,43,46,47])$, is used to solve the conservative form (14) of the NSWEs and hence update the flow variables $u, v$ and $d$ in a time-marching procedure. The NSWE system (14) is preliminarily split up into two homogeneous, 1-dimensional systems and one "source" system, which are solved separately:

$$
\begin{gathered}
\mathbf{U}_{, t}+\mathbf{F}(\mathbf{U})_{, x}=0, \\
\mathbf{U}_{, t}+\mathbf{G}(\mathbf{U})_{, y}=0,
\end{gathered}
$$




$$
\mathbf{U}, t=\mathbf{S}(\mathbf{U}) \text {. }
$$

The equivalence between the "split" systems and the original one is proven in [44].

Labeling with $H_{x}$ and $H_{y}$ the solving schemes for the homogeneous systems (18) and (19), respectively, and with $S$ the scheme for the "source" system (20), the numerical procedure that advances the flow variables from $\mathbf{U}^{n}=\left(u^{n}, v^{n}, d^{n}\right)$ to $\mathbf{U}^{n+1}=\left(u^{n+1}, v^{n+1}, d^{n+1}\right)$, with the bed depth $z_{b}^{n}$ remaining constant, is summarised as follows:

$$
\mathbf{U}^{n+1}=\frac{1}{2}\left\{H_{x y}\left[S\left(\mathbf{U}^{n}\right)\right]+S\left[H_{x y}\left(\mathbf{U}^{n}\right)\right]\right\}
$$

where $n$ is the present integration time and $H_{x y}$ is a numerical operator defined as

$$
H_{x y}(\mathbf{U})=\frac{1}{2}\left\{H_{x}\left[H_{y}(\mathbf{U})\right]+H_{y}\left[H_{x}(\mathbf{U})\right]\right\} .
$$

The arithmetic means figuring in both Equations (21) and (22) are performed to rule out the unavoidable numerical inaccuracies originating from considering 1-dimensional operators separately instead of using a full 2-dimensional approach for the resolution of system (14).

The 1-dimensional schemes $H_{x}$ and $H_{y}$ are numerically implemented through a finite-volume discretization. As an example, applying a finite-volume approach to scheme $H_{x}$, which solves (18), gives the following approximate formula for the unknown averaged vector $\mathbf{U}^{n+1}$ :

$$
\mathbf{U}_{i}^{n+1}=\mathbf{U}_{i}^{n}+\frac{\Delta t}{\Delta x}\left(\mathbf{F}_{i-\frac{1}{2}}^{n+\frac{1}{2}}-\mathbf{F}_{i+\frac{1}{2}}^{n+\frac{1}{2}}\right)
$$

where $i$ represents the grid point of interest, $\Delta x$ is the grid size in the $x$ direction, $\Delta t$ is the time step (automatically determined and adapted according to the mesh size through the CFL condition), and $\mathbf{F}_{i \pm \frac{1}{2}}^{n+\frac{1}{2}}$ are unknown numerical flux vectors, to be evaluated via the WAF method.

The "source" system (20) can be easily solved using standard methods for sets of ordinary differential equations. In scheme $S$ the frictional terms $B_{x}$ and $B_{y}$ are discretized via a second-order midpoint method. The viscous terms $F_{x}$ and $F_{y}$, on the other hand, are second-order discretized and implemented through the HLES model, described in Section 2.1.

\subsection{The River Mouth Model}

The basic version of the NSWE solver described in the previous section, as presented in [38], accepts a wave input in the form of a time series of both elevation of water surface and water depth-averaged orbital velocity. The input is applied at the grid nodes of the seaward domain boundary. This approach is consistent with the numerical framework, since the forcing of the mentioned quantities is equivalent to imposing the boundary values of the Riemann invariants propagating along the characteristics curves of the NSWEs.

In order to make the solver able to reproduce complex flow configurations typical of estuarine environments, where the incoming waves interact with an outflowing current, the solver is complemented with a simple estuarine model.

A window of "river mouth nodes" or "estuary nodes" can be defined within the landward edge of the computational domain, through which the input river current can enter the domain. The estuary window is defined by the longshore $y$-coordinate of its center, $y_{m, e}$ (the estuary center $x$-coordinate, $x_{m, e}$, being automatically assumed as the $x$-coordinate of the landward domain boundary), and its width $L_{e}$. The spatial limits of the estuary window are, therefore

$$
y_{\min , e}=y_{m, e}-\frac{L_{e}}{2}, \quad y_{\max , e}=y_{m, e}+\frac{L_{e}}{2} .
$$


The mesh nodes at the landward domain boundary falling between $y_{\text {min,e }}$ and $y_{\text {max }, e}$ are labelled as "estuary nodes". At these nodes, similarly to what happens at the seaward boundary, a time history of current velocity and/or water level elevation is prescribed to simulate the entrance of river discharges into the basin.

The estuarine input is given as a time history for the depth-averaged water velocity $\mathbf{U}_{b}=\left(u_{b}, v_{b}\right)$ and water elevation $\eta_{b}$. The values of the time history are equally spaced in time by a constant interval $\Delta t_{b}$, which does not need to be equal to the time step determined by the solver, as the computation is automatically adapted to satisfy the CFL stability condition, which is varying at each step. To encompass different real-world situations, the estuarine input is allowed to enter at different angles with respect to the basin. To allow for the propagation of the estuary input at a variable angle of incidence, a simple numerical procedure for the evaluation of the boundary condition values to be imposed at estuary nodes has been developed, which is briefly explained in the following.

Two distinct cases may occur: the input may enter the domain perpendicularly to the boundary edge (at an angle $\theta=0$ with respect to the outer-pointing normal to the edge), or obliquely (at an angle $\theta \neq 0$ ). Although the numerical treatment for the first scenario can be seen as a special case of the procedure used for the second scenario, we treat the two cases separately for clarity.

- Case I: estuary input perpendicular to the boundary $(\theta=0)$. All the estuary nodes "read" the same boundary conditions from the time history simultaneously since they are "touched" by the input signal at the same time. Moreover, since the estuary input is perpendicular to the normal of the domain edge, the estuary input velocity $\mathbf{U}_{b}$ is already oriented in the $x$-direction and has no $y$-component, so that $\mathbf{U}_{b}=\left(u_{b}, 0\right)$.

As said previously, the estuarine boundary conditions (in terms of imposed fluid velocity $u_{b}$ and water elevation $\eta_{b}$ ) are sampled at a constant interval $\Delta t_{b}$ that a priori is not the same as the solver-defined, variable time interval $\Delta t$; therefore, it is likely that the current solution time level $t$ falls between two consecutive time instants $t_{m}$ and $t_{m+1}$ in the estuary input time series, so that $t_{m} \leq t \leq t_{m+1}$. Then the exact values of fluid elevation $\hat{\eta}_{b}$ and velocity $\hat{u}_{b}$ to impose at all estuary nodes at the solution level $t$ are then obtained through linear interpolation between the boundary condition values at times $t_{m}$ and $t_{m+1}$ :

$$
\hat{\eta}_{b}=\eta_{b, m}+\left(\eta_{b, m+1}-\eta_{b, m}\right) \frac{t-t_{m}}{t_{m+1}-t_{m}}
$$

and

$$
\hat{u}_{b}=u_{b, m}+\left(u_{b, m+1}-u_{b, m}\right) \frac{t-t_{m}}{t_{m+1}-t_{m}},
$$

where $\eta_{b, m}$ and $u_{b, m}$ are the prescribed boundary values for fluid elevation and velocity at time $t_{m}$ of the input time series, and $\eta_{b, m+1}$ and $u_{b, m+1}$ are the same boundary values at time $t_{m+1}$ of the input time series. The extrapolated values $\hat{\eta}_{b}$ and $\hat{u}_{b}$, finally, are imposed at all estuary nodes by adding them to the existing values of water depth $d_{n}$ and velocity $u_{n}$. The updated values are then carried on to the next solution step, $t_{n+1}=t_{n}+\Delta t$. Hence, for the generic estuary node,

$$
d_{n+1}=d_{n}+\hat{\eta}_{b} \quad \text { and } \quad u_{n+1}=u_{n}+\hat{u}_{b}
$$

- $\quad$ Case II: estuary input oblique to the boundary $(\theta \neq 0)$. Since the fronts of the estuarine input are propagating obliquely, the estuary nodes are "touched" by a single boundary condition value at different times according to the signal propagation velocity (in other terms, at a fixed solution time $t_{n}$, different estuary nodes "read" different boundary values from the input time history). Moreover, the estuary input fluid velocity $\mathbf{U}_{b}$ is now oriented at an angle with respect to the $x$-direction, and thus can be decomposed into an $x$-component, $u_{b}$, and a $y$-component, $y_{b}$. Each component alters the corresponding nodal velocity as effect of the estuarine boundary condition. 
Since each estuary node "reads" different boundary values of water elevation $\eta_{b}$ and velocity $\mathbf{U}_{b}$ at the same solution time, a key problem is to understand which boundary values each node "read", and how to compute them from the input time history. This is done by assuming that the estuarine input propagates according to the characteristic propagation speed or celerity $\sqrt{g d}$.

Refer to the left panel in Figure 2. Let $\left(x_{0}, y_{0}\right)$ be the coordinates of the first estuary node interested by the estuary input (the "first contact" node hereinafter), and $(\hat{x}, \hat{y})$ the coordinates of another estuary node different from the first. At the start of the numerical simulation $\left(t_{n}=0\right)$, the estuary input interests only the "first contact" node. At the following times (solution time levels $t_{n+1}$, $\left.t_{n+2} \ldots\right)$, the input value that only interested the "first contact" node has travelled into the domain (along the direction defined by the angle $\theta$ ) and perhaps has interested other nodes $(\hat{x}, \hat{y})$ of the estuary window. The time $\partial \hat{t}$ that a boundary condition value would take to travel from the "first contact" node $\left(x_{0}, y_{0}\right)$ to any other estuary node $(\hat{x}, \hat{y})$ along the direction defined by $\theta$ is evaluated with the following formula:

$$
\partial \hat{t}(\hat{x}, \hat{y})=\frac{(\hat{x} \cos \theta+\hat{y} \sin \theta)-\left(x_{0} \cos \theta+y_{0} \sin \theta\right)}{\sqrt{g d_{0}}} .
$$

The quantity appearing at the numerator in (28) represents the linear distance between the generic estuary node $(\hat{x}, \hat{y})$ and the "first contact" node $\left(x_{0}, y_{0}\right)$, evaluated along the direction defined by $\theta$, i.e., the direction of propagation of the estuary input; this is the "space" covered by the traveling boundary condition. The denominator in (28) is the celerity of the estuary input signal, approximately evaluated using $d_{0}$, the water depth at the "first contact" node.

We can now find, for a specific solution time level, which boundary conditions $\left(\hat{\eta}_{b}, \hat{\mathbf{U}}_{b}\right)$ each estuary node "reads" from the time history. Reference to the right panel in Figure 2 is made in the following. Suppose that at a generic solution time $t_{n}>0$, node $A$ reads the boundary conditions for the water elevation $\left(\hat{\eta}_{b, A}, \hat{\mathbf{U}}_{b, A}\right)$. At the same time, $t_{n}$, node $B$, which is temporally ahead of $A$ by a time $\partial t_{A B}$ which is evaluated by means of Equation (28) with $(\hat{x}, \hat{y})=\left(x_{B}, y_{B}\right)$, reads boundary conditions $\left(\hat{\eta}_{b, B}, \hat{\mathbf{U}}_{b, B}\right)$. If $t_{A}$ is the "reference" time instant at which the boundary conditions for node $A$ are evaluated from the input time history, the evaluation time $t_{B}$ for node $B$ is:

$$
t_{B}=t_{A}-\partial t_{A B}=t_{A}-\frac{\left(x_{B} \cos \theta+y_{B} \sin \theta\right)-\left(x_{A} \cos \theta+y_{A} \sin \theta\right)}{\sqrt{g d_{0}}},
$$

As for Case I, since the evaluation times $t_{A}$ and $t_{B}$ most likely fall between two consecutive user-defined time levels in the time history (identified by the little gray dots in the input time history in the right panel of Figure 2), the exact values to be imposed at each node can be obtained by linear interpolation between the two adjacent boundary condition values, by use of relations like (25) and (26). Once each estuary node has read the proper (interpolated) boundary conditions for both water level $\hat{\eta}_{b}$ and fluid velocity $\hat{\mathbf{U}}_{b}=\left(\hat{u}_{b}, \hat{v}_{b}\right)$, these values alter the water depth, $x$-velocity and $y$-velocity for the respective estuary nodes, and the altered values are carried on to the next solution step:

$$
d_{n+1}=d_{n}+\hat{\eta}_{b}, \quad u_{n+1}=u_{n}+\hat{u}_{b}, \quad \text { and } \quad v_{n+1}=v_{n}+\hat{v}_{b},
$$

where $d_{n}, u_{n}$ and $v_{n}$ are the water depth, the $x$-directed and the $y$-directed components of the depth-averaged fluid velocity of the specific estuary node at solution time level $n$.

Through simple mathematical arguments it can be seen that Case II is a generalization of Case I. The numerical procedure above explained for a slanted estuary input, therefore, can be also applied to a perpendicular input without loss of generality. 

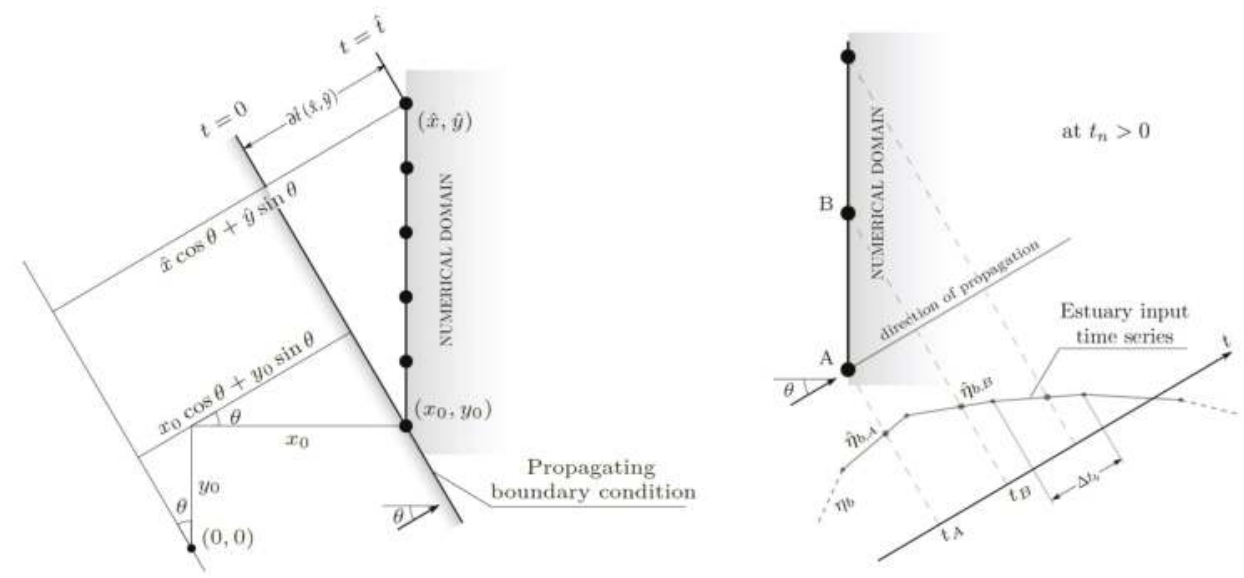

Figure 2. (Left) geometric scheme for the case of an estuary boundary condition entering the domain at an angle $\theta$. A single propagating boundary condition is read by the "first contact" node $\left(x_{0}, y_{0}\right)$ at time $t=0$, and reaches node $(\hat{x}, \hat{y})$ after a time $\partial \hat{t}$. (Right) scheme for the evaluation of the boundary conditions for water level $\hat{\eta}_{b}$ (and fluid velocity $\hat{\mathbf{U}}_{b}$ ) for each estuary node, at a generic solution time $t_{n}>0$.

\section{Numerical Tests}

The inlet configuration chosen for the simulations presented in our study has been reproduced in strict resemblance with the one conceived by Olabarrieta et al. [9] (left panel of Figure 3) for the analysis of wave-current interactions at a tidal inlet. The study by Olabarrieta et al. (OL14 hereinafter) aimed at assessing the physical processes involved in the interaction between currents, gravity waves, and bathymetry at inlets. We here use the same configuration for three main scopes: (i) to assess the validity of the river mouth modelling described in the previous section; (ii) to illustrate how macrovortices are generated by differential breaking at the mouth and shoal edges; (iii) to perform a parametric analysis of the role of sub-grid turbulence and seabed friction on the evolution and dissipation of the macrovortices. The results of these analyses are reported, in the same sequence, in the next section.

The inlet area is characterized by a main channel about $300 \mathrm{~m}$ wide and $5 \mathrm{~m}$ deep at the central axis, which opens to a semicircular, symmetric ebb shoal zone of minimum depth $2 \mathrm{~m}$, extending $1 \mathrm{~km}$ offshore of the inlet mouth. The main channel also crosses the shoal and connects the inlet to the offshore area. The beach exhibits a Dean equilibrium profile given by the expression $d=A x^{\frac{2}{3}}$, where $d$ is the water depth, $x$ is the cross-shore distance, measured offshore from the mouth, and the profile scale factor $A$ is set equal to 0.05 in agreement with the numerical setup by OL14. The computational domain generated for our simulations is $3 \mathrm{~km}$ long in the cross-shore direction and $4 \mathrm{~km}$ long in the alongshore direction, and has been discretized into a computational mesh with node spacings $\Delta x=10 \mathrm{~m}$ and $\Delta y=25 \mathrm{~m}$ in the $x$ and $y$ direction respectively. The resulting mesh can be seen in the right panel of Figure 3 and allows to describe the chosen waves (see below) with at least 15 nodes per wavelength.

The bed roughness $k_{s}$ for the evaluation of the Chezy seabed friction coefficient $c_{\tau}$ (see (5)) is set equal to $2 \times 10^{-4} \mathrm{~m}$, in agreement with OL14. For all tests, the increase in apparent bed roughness and friction coefficient due to waves has not been considered. 

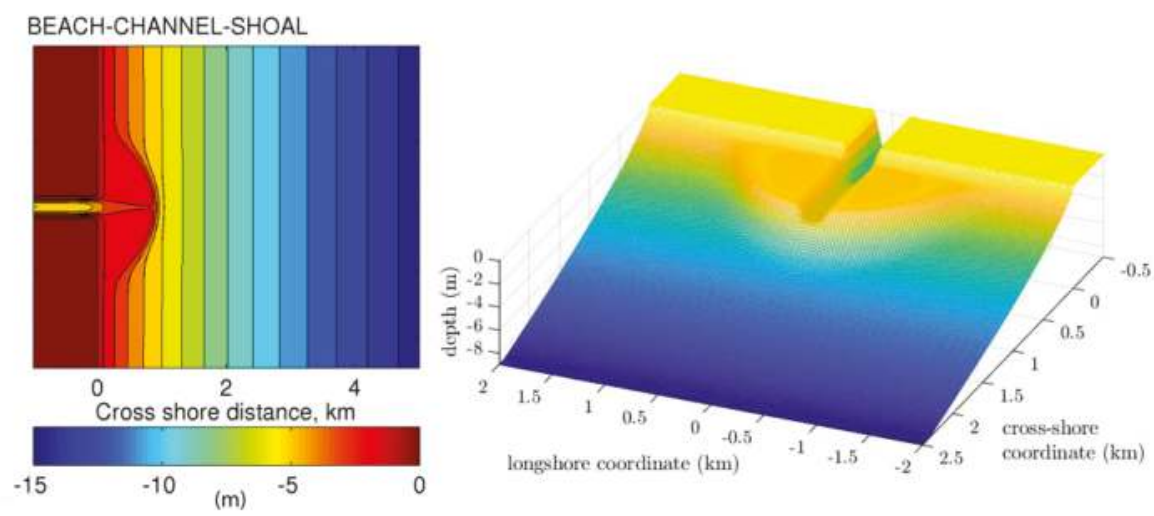

Figure 3. Sketches of a shoal bathymetry. (Left) plan view of the idealized configuration of an inlet mouth with shoal used in the tests by OL14 (image reprinted with permission). (Right) 3-dimensional view of the central portion of the shoal bathymetry used in our simulations.

To evaluate the capability of the solver to reproduce the hydrodynamics typical of a river mouth aim (i) above, benchmarking tests have been performed beforehand and a comparison with the results of the study by OL14 has been made. Our NSWE solver is depth-averaged and wave-resolving; OL14, conversely, applied the wave-averaged COAWST solver [48] for their numerical simulations. The data set used for the benchmarking coincides with that used by OL14 for their wave-current interaction tests in condition of strong outflow, and differs from that used for the analyses aimed at scopes (ii) and (iii) of above. In particular, for the benchmark tests an outflowing current of $1.1 \mathrm{~m} / \mathrm{s}$ has been opposed to three distinct wave conditions: no waves, a spectral wave of significant height $H_{s}=0.5 \mathrm{~m}$, and a spectral wave of significant height $H_{s}=1.5 \mathrm{~m}$. These two wave regimes have been generated by means of a JONSWAP spectrum with a peak period $T_{p}$ set to $10 \mathrm{~s}$. In all our benchmarking tests a value of the eddy viscosity parameter $\lambda=0$ is used. The results of the benchmarking analysis are presented in Section 4.1.

Moreover, a simple sensitivity test has been executed to inspect the influence of the mesh size over the definition and evolution of the numerical solution, with specific focus on the vorticity which, being defined by velocity derivatives, is the variable most sensitive to mesh refinement. To this end, test TW1, with waves interacting with the river current and no dissipation mechanism activated, has been performed with two different mesh resolutions. In addition to the "reference" mesh size $(10 \mathrm{~m} \times 25 \mathrm{~m})$, a mesh with tighter node spacings $\Delta x=7.5 \mathrm{~m}$ and $\Delta y=15 \mathrm{~m}$ in the $x$ and $y$ direction have been chosen for the new test TW1.M2. The results of such comparison are given in Section 4.2.1.

To assess the role of waves, frictional and viscous dissipative forces on the global hydrodynamics and vorticity patterns, a parametric analysis with different wave conditions, bed friction coefficient $\mathcal{c}_{\tau}$, and eddy viscosity parameter $\lambda$ has been performed. Two wave conditions have been used: no waves, and a representative monochromatic sinusoidal wave of height $1.5 \mathrm{~m}$ and period $15 \mathrm{~s}$. As for frictional effects, some tests have been undertaken disregarding the seabed friction, whereas others include a friction coefficient $c_{\tau}$ evaluated according to Equation (5). Finally, the tests used to assess the role of sub-grid turbulence have been run using a calibration parameter $\lambda=0.1$. This value has been chosen in agreement with the eddy viscosity model (9), to match the average real-case field conditions and obtain an average horizontal eddy viscosity in the order of $1 \mathrm{~m}^{2} / \mathrm{s}$ over the shoal zone, where the water depth is (2-3) $\mathrm{m}$. A value of $1 \mathrm{~m}^{2} / \mathrm{s}$ has been also used for the tests in OL14, based on [49]. 
In all tests, a current of speed $1.1 \mathrm{~m} / \mathrm{s}$ flowing out of the river mouth is used. This value corresponds to the current velocity for the tests with strong outflow conditions proposed in the work by OL14. Table 1 shows a summary of the tests made for the parametric analysis and the dissipative contributions (friction, viscosity, both, or none) activated in each of them; the results are presented in Sections 4.2.2 and 4.2.3. All simulations have been run for a time long enough to achieve some sort of steady state in terms of rotational flow energy.

Table 1. Summary of the performed numerical tests.

\begin{tabular}{|c|c|c|c|c|c|c|}
\hline Test & Current & Wave & Wave Direction & Friction & Viscosity & Mesh \\
\hline TC1 & $1.1 \mathrm{~m} / \mathrm{s}$ & - & - & - & - & $10 \mathrm{~m} \times 25 \mathrm{~m}$ \\
\hline TC2 & $1.1 \mathrm{~m} / \mathrm{s}$ & - & - & $k_{s}=2 \times 10^{-4}$ & - & $10 \mathrm{~m} \times 25 \mathrm{~m}$ \\
\hline TC3 & $1.1 \mathrm{~m} / \mathrm{s}$ & - & - & - & $\lambda=0.1$ & $10 \mathrm{~m} \times 25 \mathrm{~m}$ \\
\hline TC4 & $1.1 \mathrm{~m} / \mathrm{s}$ & 一 & - & $k_{s}=2 \times 10^{-4}$ & $\lambda=0.1$ & $10 \mathrm{~m} \times 25 \mathrm{~m}$ \\
\hline TW1 & $1.1 \mathrm{~m} / \mathrm{s}$ & $H=1.5 \mathrm{~m} ; T=15 \mathrm{~s}$ & $0^{\circ}$ & - & - & $10 \mathrm{~m} \times 25 \mathrm{~m}$ \\
\hline TW2 & $1.1 \mathrm{~m} / \mathrm{s}$ & $H=1.5 \mathrm{~m} ; T=15 \mathrm{~s}$ & $0^{\circ}$ & $k_{s}=2 \times 10^{-4}$ & - & $10 \mathrm{~m} \times 25 \mathrm{~m}$ \\
\hline TW3 & $1.1 \mathrm{~m} / \mathrm{s}$ & $H=1.5 \mathrm{~m} ; T=15 \mathrm{~s}$ & $0^{\circ}$ & - & $\lambda=0.1$ & $10 \mathrm{~m} \times 25 \mathrm{~m}$ \\
\hline TW4 & $1.1 \mathrm{~m} / \mathrm{s}$ & $H=1.5 \mathrm{~m} ; T=15 \mathrm{~s}$ & $0^{\circ}$ & $k_{s}=2 \times 10^{-4}$ & $\lambda=0.1$ & $10 \mathrm{~m} \times 25 \mathrm{~m}$ \\
\hline TW1.1 & $1.1 \mathrm{~m} / \mathrm{s}$ & $H=1.5 \mathrm{~m} ; T=15 \mathrm{~s}$ & $0^{\circ}$ & - & - & $10 \mathrm{~m} \times 25 \mathrm{~m}$ \\
\hline TW1.2 & $1.1 \mathrm{~m} / \mathrm{s}$ & $H=1.5 \mathrm{~m} ; T=15 \mathrm{~s}$ & $10^{\circ}$ & - & - & $10 \mathrm{~m} \times 25 \mathrm{~m}$ \\
\hline TW1.3 & $1.1 \mathrm{~m} / \mathrm{s}$ & $H=1.5 \mathrm{~m} ; T=15 \mathrm{~s}$ & $20^{\circ}$ & - & - & $10 \mathrm{~m} \times 25 \mathrm{~m}$ \\
\hline TW1.4 & $1.1 \mathrm{~m} / \mathrm{s}$ & $H=1.5 \mathrm{~m} ; T=15 \mathrm{~s}$ & $30^{\circ}$ & - & - & $10 \mathrm{~m} \times 25 \mathrm{~m}$ \\
\hline TW4.1 & $1.1 \mathrm{~m} / \mathrm{s}$ & $H=1.5 \mathrm{~m} ; T=15 \mathrm{~s}$ & $0^{\circ}$ & $k_{s}=2 \times 10^{-4}$ & $\lambda=0.1$ & $10 \mathrm{~m} \times 25 \mathrm{~m}$ \\
\hline TW4.2 & $1.1 \mathrm{~m} / \mathrm{s}$ & $H=1.5 \mathrm{~m} ; T=15 \mathrm{~s}$ & $0^{\circ}$ & $k_{s}=10^{-3}$ & $\lambda=0.1$ & $10 \mathrm{~m} \times 25 \mathrm{~m}$ \\
\hline TW4.3 & $1.1 \mathrm{~m} / \mathrm{s}$ & $H=1.5 \mathrm{~m} ; T=15 \mathrm{~s}$ & $0^{\circ}$ & $k_{s}=2 \times 10^{-4}$ & $\lambda=0.5$ & $10 \mathrm{~m} \times 25 \mathrm{~m}$ \\
\hline TW4.4 & $1.1 \mathrm{~m} / \mathrm{s}$ & $H=1.5 \mathrm{~m} ; T=15 \mathrm{~s}$ & $0^{\circ}$ & $k_{s}=10^{-3}$ & $\lambda=0.5$ & $10 \mathrm{~m} \times 25 \mathrm{~m}$ \\
\hline TW1.M1 & $1.1 \mathrm{~m} / \mathrm{s}$ & $H=1.5 \mathrm{~m} ; T=15 \mathrm{~s}$ & $0^{\circ}$ & - & - & $10 \mathrm{~m} \times 25 \mathrm{~m}$ \\
\hline TW1.M2 & $1.1 \mathrm{~m} / \mathrm{s}$ & $H=1.5 \mathrm{~m} ; T=15 \mathrm{~s}$ & $0^{\circ}$ & - & - & $7.5 \mathrm{~m} \times 15 \mathrm{~m}$ \\
\hline
\end{tabular}

To better inspect the possible changes of vorticity patterns with wave direction, test TW1 (featuring interacting current and waves, with neither friction nor viscosity dissipation; see Table 1) has been repeated four times, with four different angles of wave approach: $0^{\circ}, 10^{\circ}, 20^{\circ}$, and $30^{\circ}$, respectively. The resulting vorticity maps are presented and discussed in Section 4.2.4.

To evaluate the effect of the magnitude of friction and sub-grid turbulence dissipation mechanisms into the development of vortical patterns, test TW4 (waves interacting with current, both friction and viscosity dissipation activated) has been repeated four times. In each test the intensity of frictional effects and viscous dissipation have been altered, respectively by changing the bed roughness $k_{s}$ and the viscosity calibration parameter $\lambda$, giving rise to four different combinations of friction and viscosity. The results of this analysis is given in Section 4.2.5.

Finally, we propose some simple considerations on the global amount of large-scale vorticity present in the basins. To do this, use is made of the Okubo-Weiss (OW) parameter for 2-dimensional vortical flows [50,51] which is defined as follows:

$$
\mathrm{OW}=s_{n}^{2}+s_{s}^{2}-\omega^{2},
$$

where $s_{n}$ and $s_{s}$ are the normal and shear components of strain, respectively, and $\omega$ is the vorticity for 2-dimensional flows:

$$
s_{n}=\frac{\partial u}{\partial x}-\frac{\partial v}{\partial y}, \quad s_{s}=\frac{\partial v}{\partial x}+\frac{\partial u}{\partial y} \quad \text { and } \quad \omega=\frac{\partial v}{\partial x}-\frac{\partial u}{\partial y} .
$$

According to the $O W$ criterion the vortical flow can be roughly divided into two types of regions. Where $O W>0, s_{n}^{2}+s_{s}^{2}>\omega^{2}$ and the flow is dominated by fluid straining more than vorticity. 
Conversely, where $O W<0, s_{n}^{2}+s_{s}^{2}<\omega^{2}$ and the rotational effects dominate over deformation, giving birth to vortices [23].

An integral Okubo-Weiss parameter $\Psi_{x y}$, furthermore, is defined as follows:

$$
\Psi_{x y}=\int_{y} \int_{x} O W^{\prime} \mathrm{d} x \mathrm{~d} y
$$

where

$$
O W^{\prime}=\left\{\begin{array}{l}
O W \text { when } O W \leq 0 \\
0 \text { when } O W>0
\end{array}\right.
$$

For each of the tests discussed in Sections 4.2.1, 4.2.2, and 4.2.3, a numerical integration of only the negative $O W$ over the computational domain is performed via application of the trapezoidal method. The obtained "integral OW parameter" (33) is, thus, used as a proxy to evaluate the overall amount of large-scale vortical energy in the flow, as a function of time. Small oscillations in the time series may indicate the organization and rapid dissipation of moderate-sized vortices, which account for only a modest increase of vortical energy over the whole domain. A steady and consistent increase in the time series over a time interval, conversely, is caused by the development of one or more macrovortices increasing their intensity and size.

\section{Results and Discussion}

From here onwards and whenever reasonable, the involved variables and figures are made dimensionless by setting the vertical length scale $d_{0}$ of Equations (12) equal to the still water depth at the offshore boundary of the domain, the horizontal length scale $L_{0}$ equal to the cross-shore extension of the shoal region from the channel mouth, and using the derived time scale $t_{0}=L_{0} / \sqrt{g d_{0}}$. In addition to the already defined dimensionless quantities (12), dimensionless forms for vorticity $\omega$ and the integral Okubo-Weiss parameter $\Psi_{x y}(33)$ are as follows:

$$
\tilde{\omega}=\omega t_{0}, \quad \tilde{\Psi}_{x y}=\Psi_{x y} \frac{t_{0}^{2}}{L_{0}^{2}}=\frac{\Psi_{x y}}{g d_{0}} .
$$

Some considerations on the magnitude of friction-based and viscosity-based dissipation are also proposed by means of the parameters $P_{\tau}$ and $P_{\lambda}$ (see Section 2.1.1), where the length scaling parameter $\epsilon \approx 10^{-3}$ over the shoal and $\epsilon \approx 10^{-2}$ offshore of the shoal, where depths are larger.

\subsection{Evaluation of the Model for the River Mouth}

For the sake of evaluating the performance of our estuarine model, a comparison of velocity contour fields is presented in Figure 4, where the results of the tests of OL14 are shown in the left column, while the results from the corresponding benchmarking simulations, averaged over $100 \mathrm{~s}$ (10 wave periods), are shown in the right column.

Our depth-averaged, wave-resolving model manages to represent the main hydrodynamical features of the wave-current interaction displayed in the solution by OL14, such as the current speed increase at the mouth due to the gradual reduction of water depth, the jet current spreading over the shoal, and the seaward jet extension and thinning. The numerical tests by OL14 highlight a significant spreading of the river jet with medium-to-strong alongshore currents at the edge of the shoal (see middle and bottom left panels). On the other hand, our simulations show that the current jet undergoes a weaker longshore spreading and a significant generation of large-scale eddies (see middle and bottom right panels). Both differences suggest that our NSWE, wave-resolving solver produces a stronger breaking-induced dissipation of the incoming waves, which: (a) are less effective in pushing the river jet back to the coast and spread it in the alongshore, and (b) transfer, via differential breaking, a significant amount of energy to macrovortices. 

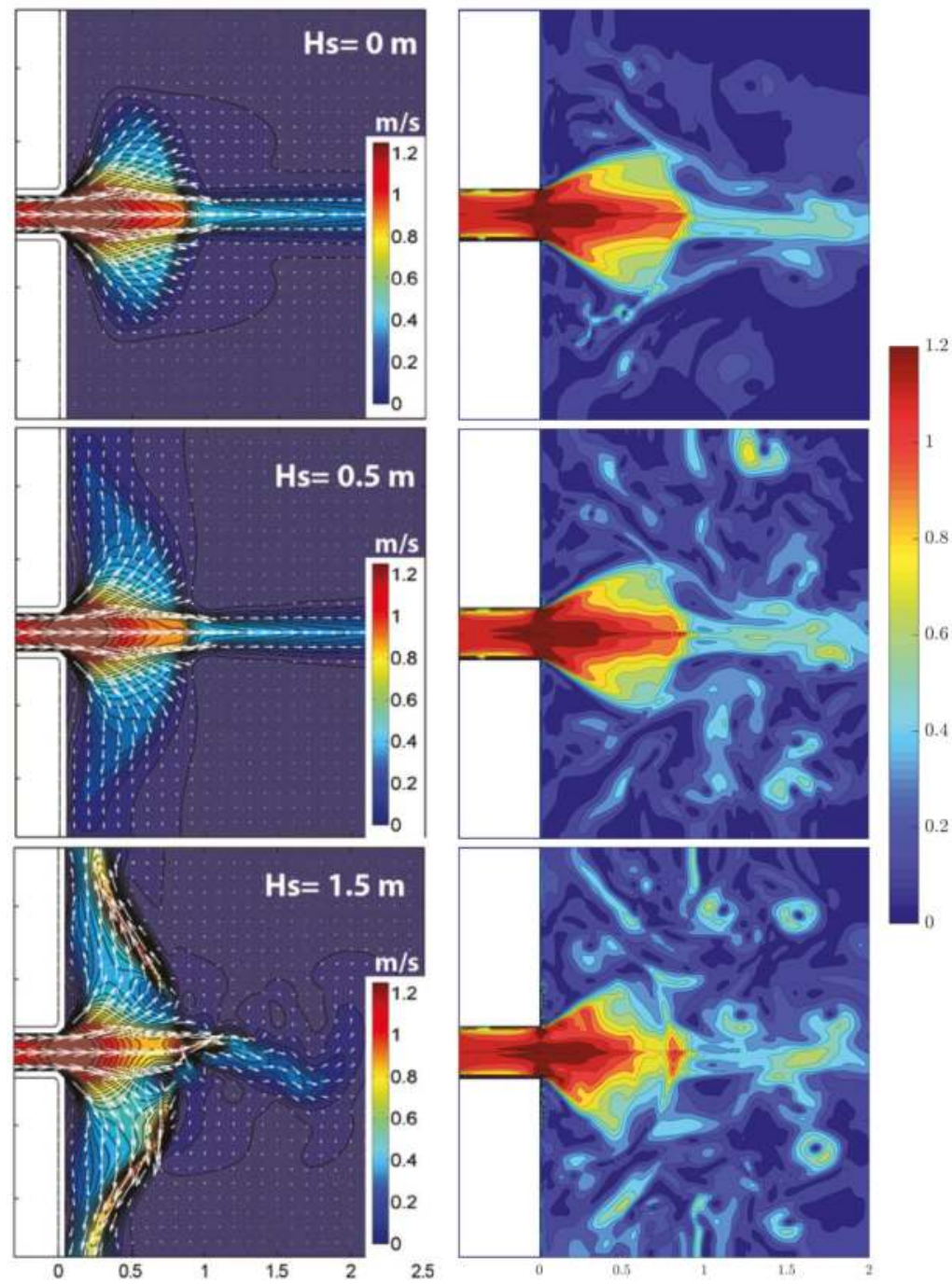

Figure 4. Comparison of velocity contour fields for a $1.1 \mathrm{~m} / \mathrm{s}$ current (corresponding to strong outflow conditions in OL14) opposed to three different wave regimes (from top to bottom: no waves; waves with $H_{s}=0.5 \mathrm{~m}$; waves with $H_{s}=1.5 \mathrm{~m}$ ). (Left) velocity fields for the tests performed by OL14 (image reprinted with permission). (Right) velocity fields obtained using our NSWE solver and averaged over 10 wave periods.

Figure 5 shows a comparison of the velocity computed along the main central axis. Results from the numerical simulations by OL14 are shown in the left panel, while the corresponding results from our benchmarking NSWE simulations are shown in the right panel. Both test campaigns show a similar global behavior, with the current gaining speed outside the mouth due to a gradual reduction of water depth and strong wave-breaking-induced undertow ( $x \approx 0-0.3 \mathrm{~km})$, then decreasing over the shoal mainly because of momentum loss and the action of bottom friction $(x \approx 0.2-0.9 \mathrm{~km})$, and finally experiencing a decay at the transition between the ebb shoal and the beach profile. The latter 
decay, which is gentle except for the case with $H_{S}=1.5 \mathrm{~m}$ in the test by OL14, is rather sharp in all NSWE simulations.

Notwithstanding some differences, mainly to be credited to the different nature of the two numerical solvers, our flow circulation model well represents the hydrodynamics of a simplified river mouth environment. Moreover, it also suggests a significant role of macrovortices in the energy redistribution of the wave-current interaction.
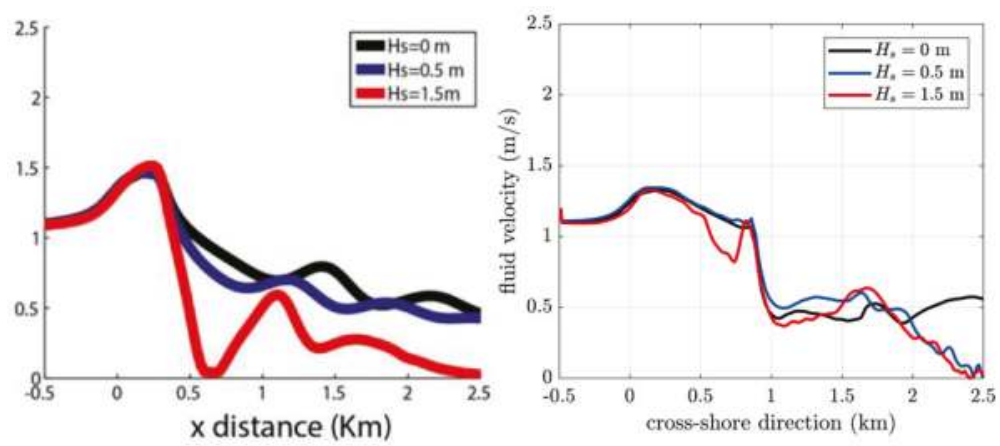

Figure 5. Comparison of velocity along the mouth and shoal central axis as a function of the significant wave height of the opposing waves. (Left) velocity along the middle axis of the domain for the tests performed by OL14 (image reprinted with permission). (Right) velocity along the middle axis of the domain obtained using our NSWE solver and averaged over 10 wave periods.

\subsection{Vorticity Patterns and Global Vorticity}

\subsubsection{Sensitivity to Mesh Size}

Figure 6 shows a preliminary comparison of vorticity maps for assessing the role of the mesh size on the simulated vorticity patterns that are thoroughly discussed in the next sections. Results from test TW1.M1, featuring a coarse mesh of $10 \mathrm{~m} \times 25 \mathrm{~m}(0.01 \times 0.025$ in dimensionless units $)$, are shown in the top panels. The bottom panels are relative to the test TW1.M2, performed with a finer mesh $(7.5 \mathrm{~m} \times 15 \mathrm{~m}$, or $0.0075 \times 0.015$ in dimensionless units). Both tests feature a setup that corresponds to test TW1 (interacting waves and currents with no friction nor viscosity). The vorticity maps for the present tests, as well as for those in the next sections, have been extrapolated at four different time instants to show in a compact way the evolution of large-scale vortical structures.

The comparison reveals that vorticity generation patterns are qualitatively quite similar, with the bulk of vorticity injection focused along the border of the shoal in a symmetrical way. Well-formed macrovortices arise at the shoal tip, as a product of the interactions between breaking waves and outflowing currents, and gradually migrate outwards following the shoal curvature. In both tests mouth vortices, i.e., vortical structures developing at the corners of the channel mouth due to lateral spreading of the outflowing current, are absent. The increased spatial resolution in test TW1.M2, however, allows for more macrovortices to arise along the shoal edge; comparatively, test TW1.M1, which features a coarser mesh, presents wider zones of non-organized vorticity that has not yet rearranged into vortical structures. This is particularly visible in the inner parts of the shoal (top panels in Figure 6).

Figure 7 shows the time series of the (dimensionless) integral $O W$ parameter $\tilde{\Psi}_{x y}$ for preliminary tests TW1.M1 and TW1.M2.

Different mesh sizes inevitably have an impact on the numerical evaluation of vorticity, since the values of the space derivatives of velocities are different when different discretization lengths are assumed (see (32)). This presumably reflects into the different amplitudes and periods of the oscillations 
in $\tilde{\Psi}_{x y}$. A discrepancy between the two time series can also be seen at the earlier times of the simulations, approximately at $\tilde{t}=4-11$. This is mainly due to the fine mesh test TW1.M2 giving less vorticity in the inner parts of the shoal than the coarse mesh test TW1.M1. Notwithstanding this, the similar values and long-term overall growth of $\tilde{\Psi}_{x y}$ suggest that the amount of vortical energy injected into the basin by flow processes is roughly the same for both tests, regardless of the chosen mesh size.

$\mathrm{i}=4.7$
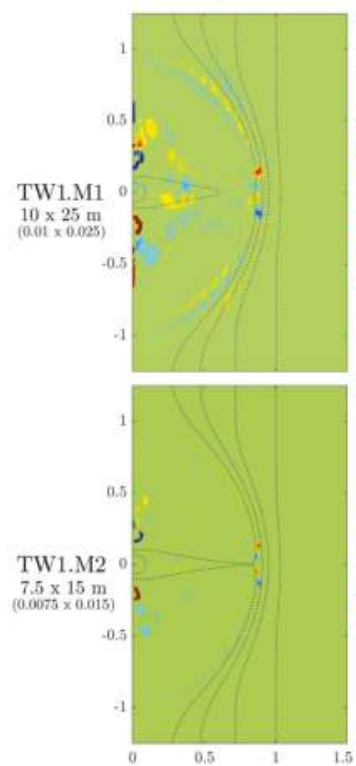

$\mathrm{t}=9.4$
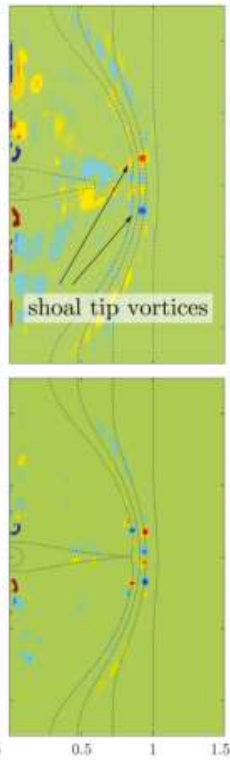

$\mathrm{t}=18.8$
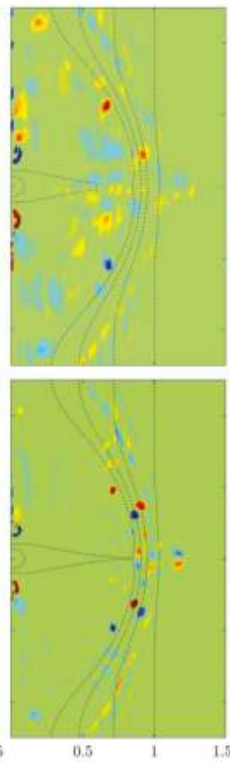

$\mathrm{i}=28.1$

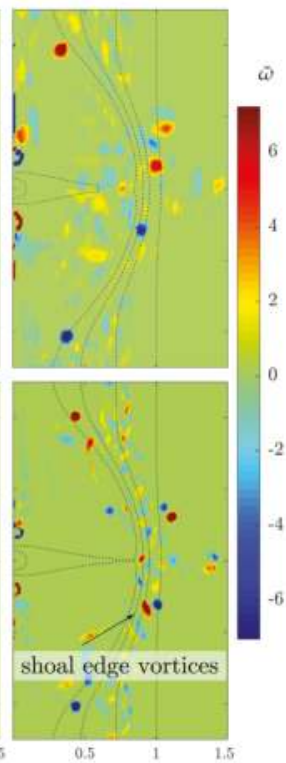

Figure 6. Time evolution of vorticity for test TW1 as a function of the mesh size. Test TW1.M1 has been run with a coarser mesh of size $10 \mathrm{~m} \times 25 \mathrm{~m}(0.01 \times 0.025$ in dimensionless units). Test TW1.M2 has been run with a finer mesh of size $7.5 \mathrm{~m} \times 15 \mathrm{~m}(0.0075 \times 0.015$ in dimensionless units). Each column represents a single time level (from left to right: $\tilde{t}=4.7,9.4,18.8$, and 28.1). Red tones represent clockwise vorticity; blue tones represent anticlockwise vorticity. The dotted lines show the bathymetry contours of the shoal edge and the main channel.

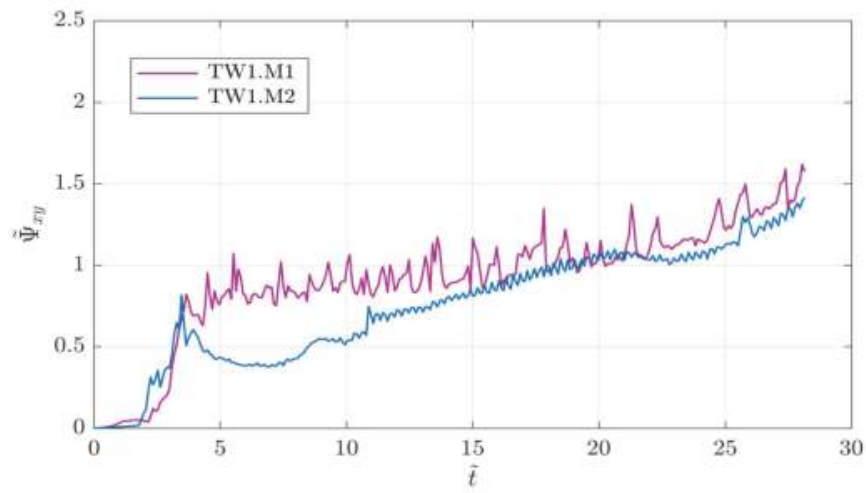

Figure 7. Time series of the dimensionless domain-integral $O W$ parameter $\tilde{\Psi}_{x y}$ for tests TW1.M1 and TW1.M2. 


\subsubsection{Vorticity Patterns with No Waves}

Figure 8 gives vorticity maps for the four numerical simulations carried out with only an outflowing current spreading over the shoal and no opposing waves. In each of these tests, labeled TC1 to TC4 (see Table 1), a unique combination of dissipative mechanisms has been activated, to better inspect the influence of both frictional and sub-grid turbulence effects.
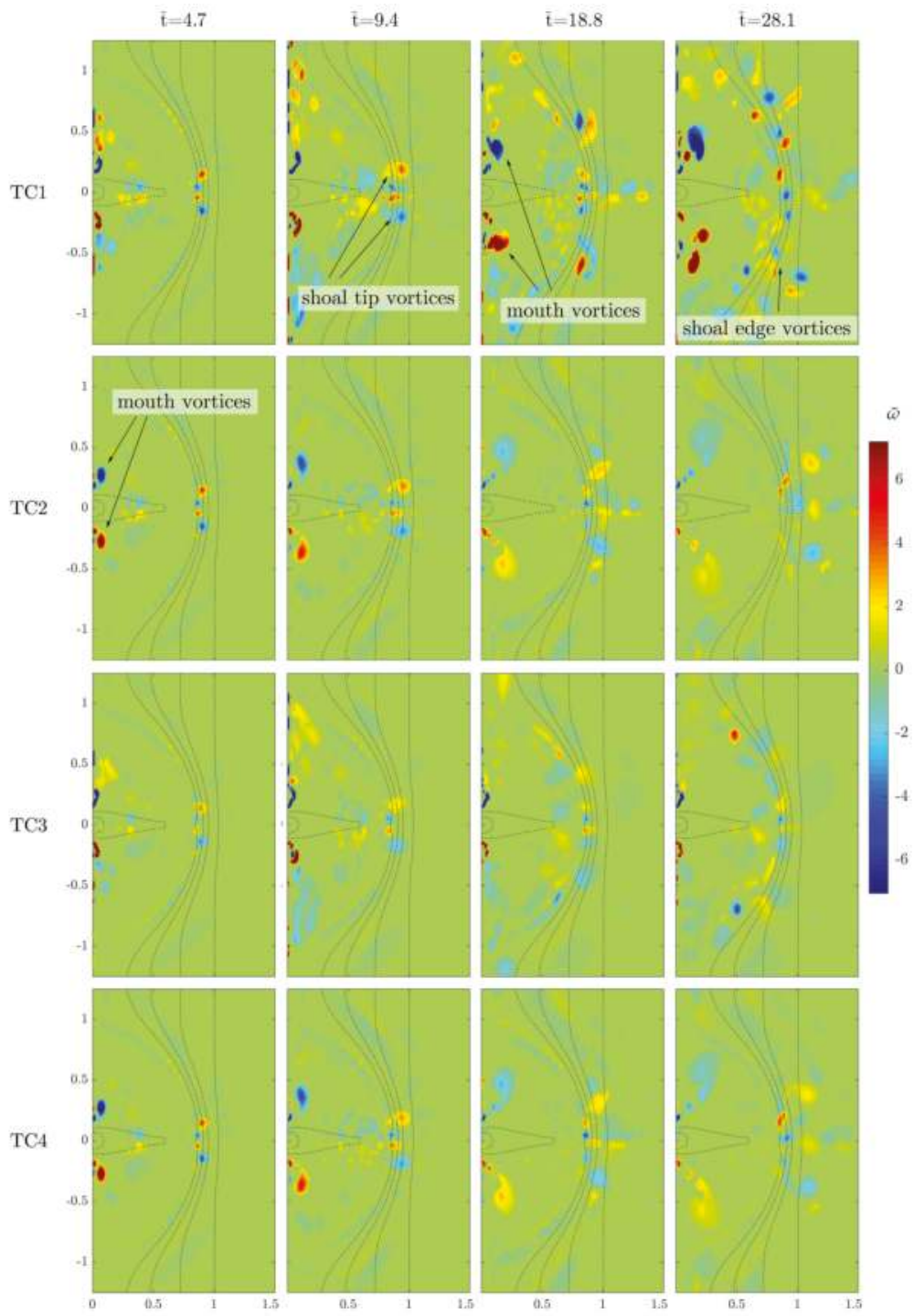

Figure 8. Time evolution of vorticity for the four tests with river current only. Each row represents a single test (from top to bottom: TC1 to TC4). Each column represents a single time level (from left to right: $\tilde{t}=4.7,9.4,18.8$, and 28.1). Red tones represent clockwise vorticity; blue tones represent anticlockwise vorticity. The dotted lines show the bathymetry contours of the shoal edge and the main channel. 
All tests show a relevant generation of vorticity in two regions of the domain of interest: at the river mouth edges, where the river current exits the channel $(\tilde{x} \approx 0-0.1)$, and at the shoal edge, where the shoal connects to the beach through a steep bottom gradient $(\tilde{x} \approx 1-1.2)$. Although the global structure of the vortical flows originating at these regions is rather similar throughout the tests, the intensity and temporal evolution of vorticity, as well as its alongshore distribution to some extent, are highly dependent on the dissipation mechanisms at work.

In test TC1, the reference case (first row in Figure 8), no dissipation mechanism is active and a great amount of vorticity is seen near the river mouth since the earlier times of the simulation, due to the lateral spreading of the river current in the longshore direction. At later times $(\tilde{t}>10)$ two intense macrovortices, approximately $100 \mathrm{~m}$ in diameter, develop in correspondence of the mouth corners and are gradually advected towards the inner parts of the shoal, as the current spreads (see the arrows in the third panel of the first row). Further, a pair of oppositely-signed macrovortices initially develops at the shoal tip ( $\tilde{x} \approx 0.8-1$; see the arrows in the second panel of the first row), mainly due to lateral velocity gradients occurring at the boundaries between the main channel and the shoal plains, and are gradually advected laterally. Time passing, further turbulence develops along the boundary of the shoal (see the arrow in the fourth panel of the first row), organizes into vortices and grows unimpeded to values of about $\tilde{\omega}=4.5-5$ in absolute value, since the dissipative effects of both sub-grid turbulence and bed friction are neglected.

Since the bed friction coefficient is inversely proportional to the water depth (see Equations (4) and (5)), the contribution of seabed friction to the global dissipation of vorticity is expected to be more important in shallow waters, i.e., over the shoal and along the shoreline. This is confirmed in test TC2, where only the frictional dissipative effects are accounted for (second row in Figure 8). In fact, the mouth corner macrovortices generated at the start of the simulation are soon dissipated as their size increases and the wavenumber of the related turbulent motion decreases. The shoal tip macrovortices are also dissipated, albeit to a lesser extent, due to the effect of friction being less intense seaward of the shoal $(\tilde{x}>1)$, where the water depth is larger. On the other hand, the vorticity at the shoal boundary, which is rather intense in test TC1, is absent here, indicating that the dissipative effect of seabed friction is noticeable also at the transition between the shoal and the beach, characterized by a steep bottom gradient.

For test TC3 (third row in Figure 8) the HLES routine described in Section 2.1 is activated, modeling the dissipation due to the sub-grid turbulence, while the seabed friction is neglected. The impact of the sub-grid turbulence dissipation on the vorticity patterns is evident at the most seaward parts of the shoal, where it is effective in limiting (although not suppressing altogether) the evolution and reorganization of macrovortices with respect to the reference case (test TC1). Sub-grid turbulence dissipation is effective also within the shoal, reducing size and intensity of the macrovortices at the river mouth corners. This is likely due to the considerable transversal velocity gradients occurring right outside of the mouth, where the rapidly spreading current interacts with the quiescent water on the shoal. This leads to large values of the velocity gradients in the inner shoal, which, in turn, induce an appreciable dissipation of flow energy and momentum (see Equations (10) and (11)).

Finally, for test TC4 (fourth row in Figure 8) both frictional effects and sub-grid turbulence contributions concur to dissipate the large-scale turbulence of the macrovortices. The vorticity patterns are similar to those of test TC2, where only friction-based dissipation is present: this suggests that, when both dissipation sources are present, large-scale dissipation by friction tends to dominate over the small-scale dissipation by sub-grid turbulence. Moreover, with respect to test TC2, the additional presence of sub-grid turbulence is effective in further restraining the formation of the shoal edge macrovortices. 


\subsubsection{Vorticity Patterns with Waves}

Figure 9 shows vorticity maps for the same test cases illustrated above, but for each simulation the river current is now opposed by a monochromatic sinusoidal wave. These tests are labelled TW1 to TW4 (see Table 1).

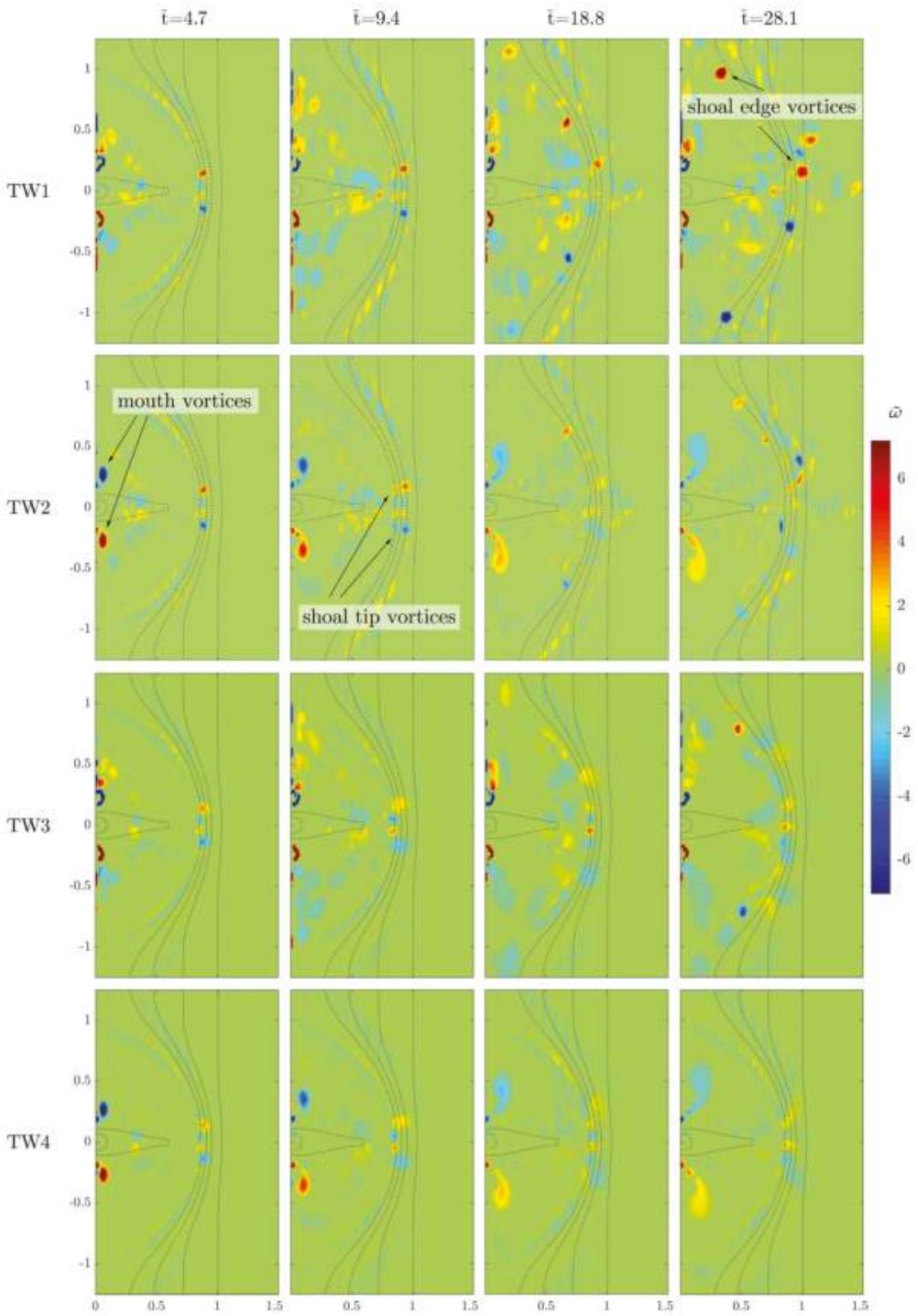

Figure 9. Time evolution of vorticity for the four tests with a monochromatic wave opposing the river current. Each row represents a single test (from top to bottom: TW1 to TW4). Each column represents a single time level (from left to right: $\tilde{t}=4.7,9.4,18.8$, and 28.1). Red tones represent clockwise vorticity; blue tones represent anticlockwise vorticity. The dotted lines show the bathymetry contours of the shoal edge and the main channel. 
The dissipation-free test TW1 (first row in Figure 9) shows the presence of organized turbulence developing at the tip of the shoal, similarly to what occurs for test TC1. This may imply that in this zone the process of vortex generation is mainly dominated by current-related dynamics, with the effect of incoming waves being toned down in comparison. The maximum values for dimensionless vorticity in this test are around $\tilde{\omega}=5.5-6$ in absolute value. The vorticity structure of the wave-current interaction in this test is also characterized by a distinctive pattern which is roughly symmetrical with respect to the domain central axis. When waves are present, symmetrical vortices are formed, because of differential wave breaking, over the shoal edge and are advected diagonally towards the shore by the action of waves and interaction with the bathymetry, following the curvature of the shoal boundary itself. Such vortices are clearly visible in the last two panels of the first row in Figure 9. This behavior is in contrast to the essentially lateral advection experimented by the large eddies of test TC1 (see the last two panels in the first row in Figure 8). Vortex generation is mainly due to the previously cited depth-induced differential breaking experimented by the waves when they reach the shoal boundary. The portions of the wave fronts entering the shoal abruptly steepen and break because of the reduction of water depth, the steepening process being further enhanced by the effect of the opposing current. At the same time, the most lateral parts of the wave fronts are not yet broken since they are traveling over milder slopes. At the breaker lateral edges vorticity is injected into the flow and reorganizes into coherent vortices over time due to continuous and localized wave breaking. Once generated, these macrovortices migrate towards the shore in response to their interaction with the bathymetry, as illustrated by Brocchini and co-workers [10,11]. Furthermore, broken waves are also effective within the shoal, as they prevent the formation of the river mouth eddies.

For test TW2 (second row in Figure 9), only dissipation through bed friction is accounted for. The simulation notably shows the development of mouth vortices that are gradually dissipated, still being rather visible by the end of the test (last panel of the second row in Figure 9). The shoal boundary eddies are also similarly generated, albeit they are rapidly dampened as they travel inside the shoal due to friction.

Shoal boundary vortices are the main feature also in test TW3, in which only the sub-grid turbulence dissipation is active (third row in Figure 9). The small-scale turbulence dissipation of test TW3, however, is effective in reducing the size of the generated macrovortices when compared to the reference case TW1. Sub-grid viscosity-based turbulence has a greater effect when waves are present with respect to the no-wave condition (see test TC3), since waves generate strong, sign-changing velocity gradient in the alongshore direction that are continuous in time. This activates considerable and repeated dissipation by viscosity since such dissipation is dependent on the velocity gradients (see (10) and (11)).

Injection and reorganization of turbulence along the shoal boundary, as an effect of wave breaking, is also noticeable in test TW4 (fourth row in Figure 9), albeit the presence of both frictional and small-scale viscous dissipation strongly hinders the size and shoreward advection of generated macrovortices. Mouth vortices are very weak.

\subsubsection{Vorticity Patterns as a Function of Wave Direction}

Test TW1 (waves interacting with current, no friction nor viscosity dissipation; see top row in Figure 9) has been repeated four times with different directions of wave attack, in order to inspect the effect of wave direction on the vorticity patterns. Figure 10 shows vorticity maps for the four runs. From the top to the bottom row, the four angles of wave attack are $0^{\circ}, 10^{\circ}, 20^{\circ}$, and $30^{\circ}$ with respect to the $x$-direction. In all panels the waves are approaching the shoreline from the bottom right angle. Test TW1.1, with a wave attack perpendicular to the seaward domain boundary, corresponds to test TW1 (see Section 4.2.3 and Figure 9); a slightly different notation for test names has been chosen for coherence in the nomenclature. 

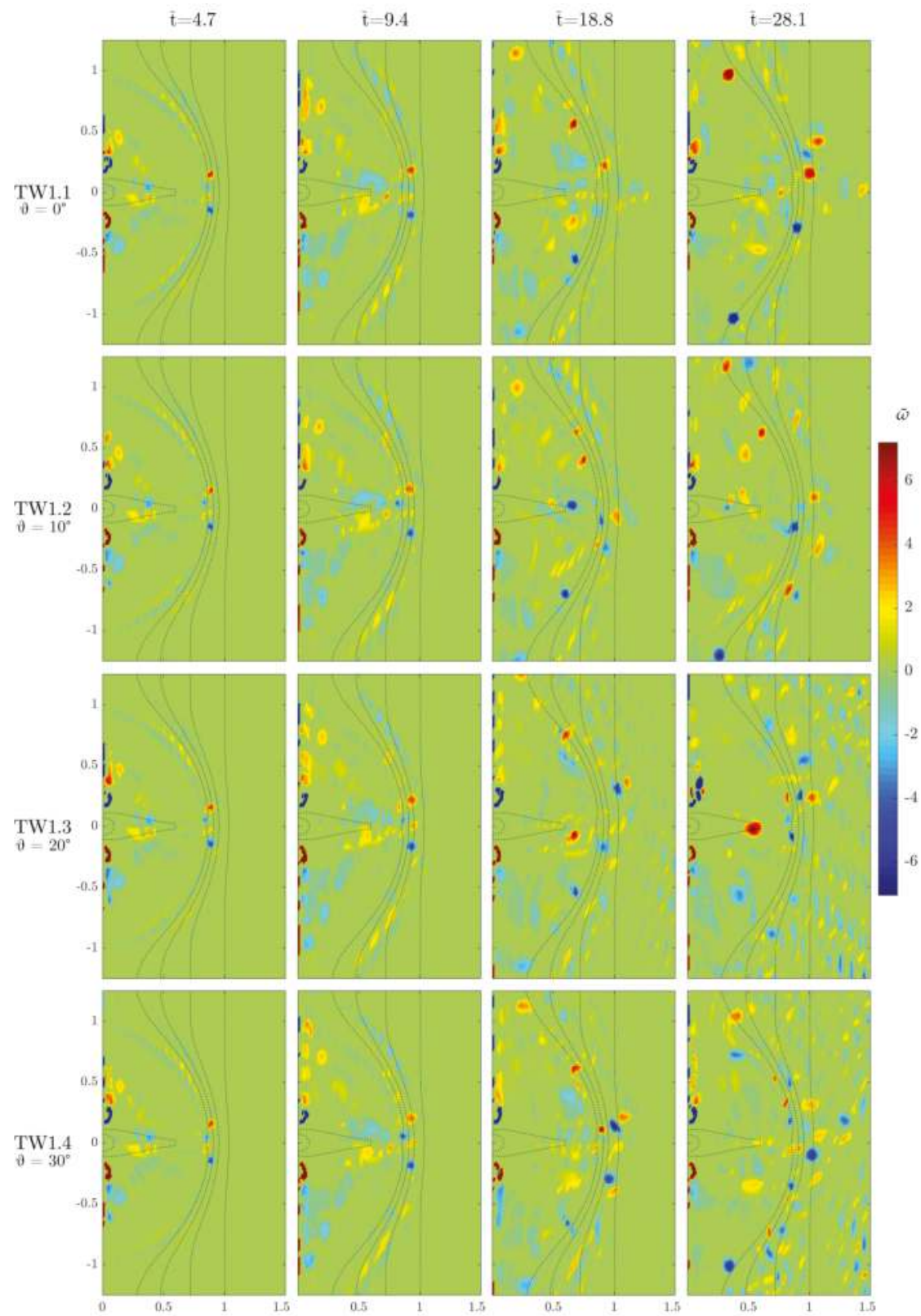

Figure 10. Time evolution of vorticity for test TW1 as a function of wave direction. Each row represents a different wave direction (from top to bottom: $\theta=0^{\circ}, 10^{\circ}, 20^{\circ}$, and $30^{\circ}$ ). Each column represents a single time level (from left to right: $\tilde{t}=4.7,9.4,18.8$, and 28.1). Red tones represent clockwise vorticity; blue tones represent anticlockwise vorticity. The dotted lines show the bathymetry contours of the shoal edge and the main channel. 
The performed tests reveal that different angles of wave approach do not break in a significant way the predominantly symmetrical pattern of vorticity already evidenced in the previous tests. This can be explained with the fact that the incoming waves surf through a considerable distance (more than $2 \mathrm{~km}$ ) into the domain before hitting the shoal. As a side effect, wave fronts become more and more parallel to the shore thanks to well-known effects of refraction, and thus impact the shoal border at roughly the same angle, regardless of their direction upon entering the domain.

At earlier times in all tests $(\tilde{t}<10)$, shoal tip vortices are formed at the same location than in tests TW (see Figure 9), suggesting that the wave direction is not a determinant variable in the birth and evolution of such vortices, which are, conversely, mainly dominated by the interaction between the outflowing current and the waves at the shoal border.

\subsubsection{Sensitivity to Friction and Eddy Viscosity}

To evaluate the effect of the magnitude of friction and sub-grid turbulence dissipation mechanisms into the development of turbulence, test TW4 has been repeated four times. In each test the intensity of frictional effects and viscous dissipation have been altered, respectively by changing the bed roughness $k_{s}$ and the eddy viscosity calibration parameter $\lambda$. Figure 11 shows the vorticity maps for the four iterations, labeled TW4.1 to TW4.4 (see also Table 1). Test TW4.1 corresponds to the reference test TW4 discussed in Section 4.2.3 and shown in the bottom row of Figure 9.

In test TW4.2 (second row in Figure 11) the action of dissipation due to friction is larger than in the reference case TW4.1, because of an increase in bed roughness $k_{s}$, while the sub-grid turbulence dissipation is unaltered. This leads to Chezy friction coefficients $c_{\tau} \approx 10^{-3}$ and $P_{\tau} \approx 1$ over the shoal and near the numerical shoreline, while $P_{\tau} \approx 10^{-1}$ off the shoal. The viscosity-related parameter is $P_{\lambda} \approx 10^{-4}$ over the shoal, and $P_{\lambda} \approx 10^{-3}$ outside the shoal. Therefore, frictional effects dominate over viscous dissipation. The increased effect of friction is visible through a slight reduction in the size and intensity of the mouth vortices. The extension of the region interested by mouth vorticity is reduced by frictional effects also during the phase of vortex dissipation (last two panels in the second row of Figure 11). The shoal tip vorticity, conversely, remains largely unaltered when increasing the friction; this suggests that, although the dissipative effect of friction is greater in magnitude than those of viscosity $\left(P_{\tau}=10^{-1}>P_{\lambda}=10^{-3}\right.$ out of the shoal), the friction-based contribution only slightly affects the vortex morphology at the shoal tip and borders, similarly to the eddy viscosity contribution, mainly due to the increased water depths.

Test TW4.3 features an increase in the dissipative range of sub-grid turbulence with respect to the reference case. This is achieved by an increment of the eddy viscosity calibration parameter $\lambda$ from 0.1 to 0.5 (see Equation (9)). The size of frictional dissipation is unchanged. This leads to $P_{\lambda} \approx 10^{-3}$ on the shoal and $P_{\lambda} \approx 10^{-2}$ off the shoal; whereas $P_{\tau} \approx 10^{-1}-1$ over the shoal and $P_{\tau} \approx 10^{-2}-10^{-1}$ outside the shoal. In this test, friction still manages to exert a dominant role, especially in the shallow waters of the shoal, where the evolution of mouth vortices is dampened. However, the increase in viscosity dissipation has the notable consequence of reducing vorticity at the shoal tip and borders, while also causing a slight loss of coherence of the mouth vortices. All the above is confirmed by the vorticity maps shown in the third row in Figure 11. While exerting some effects also over the shoal, mainly through a reduction of the region of turbulence at the channel mouth, sub-grid turbulence has the greatest effects on the shoal tip vortices, which are strongly damped even during the earlier stages of the simulations $(\tilde{t} \leq 10$; compare the first and third row of Figure 11).

Finally, in test TW4.4 (fourth row in Figure 11) both friction and sub-grid-turbulence dissipation are increased. $P_{\tau} \approx 1$ over the shoal and $P_{\tau} \approx 10^{-1}$ outside the shoal; $P_{\lambda} \approx 10^{-3}$ over the shoal and $P_{\lambda} \approx 10^{-2}$ outside the shoal, with $P_{\lambda}$ increasing as the water depth grows. Therefore, friction is still dominant over viscosity at the shoal, while the balance becomes less skewed towards the shoal edge and offshore. The relative vorticity maps, thus, show a mix of the features described for tests TW4.2 and TW4.3: namely, a decrease of vortex intensity and size for mouth vortices (thanks to 
the enhanced friction-based dissipation), and a reduction of turbulence at the shoal tip (due to the enhanced viscosity-based dissipation).
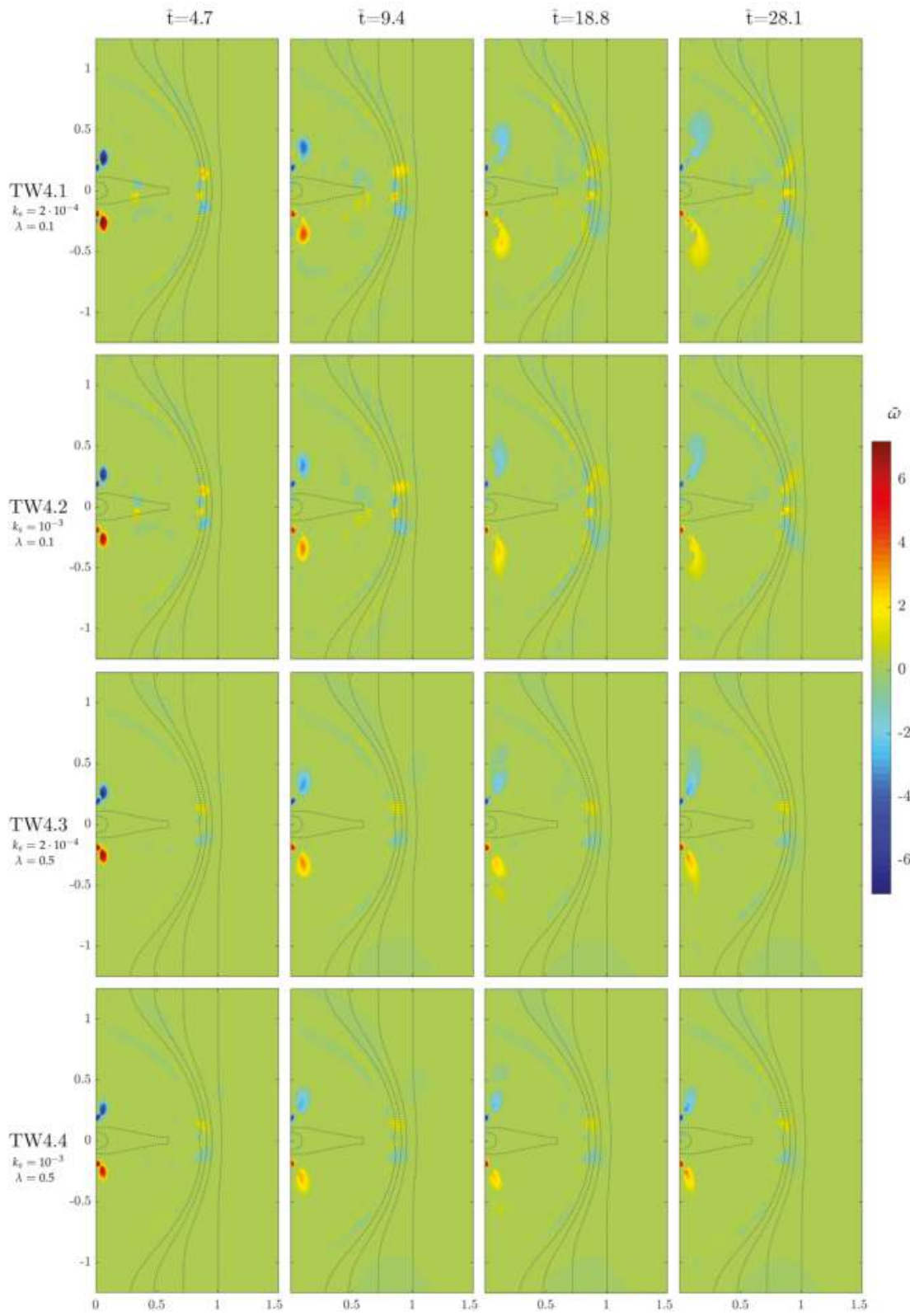

Figure 11. Time evolution of vorticity for test TW4 as a function of the intensity of friction and viscosity. The adopted values for the bed roughness $k_{s}$ and the eddy viscosity calibration parameter $\lambda$ are given along with each test name. Each column represents a single time level (from left to right: $\tilde{t}=4.7,9.4$, 18.8, and 28.1). Red tones represent clockwise vorticity; blue tones represent anticlockwise vorticity. The dotted lines show the bathymetry contours of the shoal edge and the main channel. 


\subsubsection{Global Vorticity for Currents and Waves Plus Currents}

The time series of the integral dimensionless OW parameter $\tilde{\Psi}_{x y}$ for the tests TC and TW, presented in Sections 4.2.2 and 4.2.3, are illustrated in Figure 12. Note that the time series for test TW1 (purple line in the bottom panel) is the same as the purple line in Figure 7 (test TW1.M1).
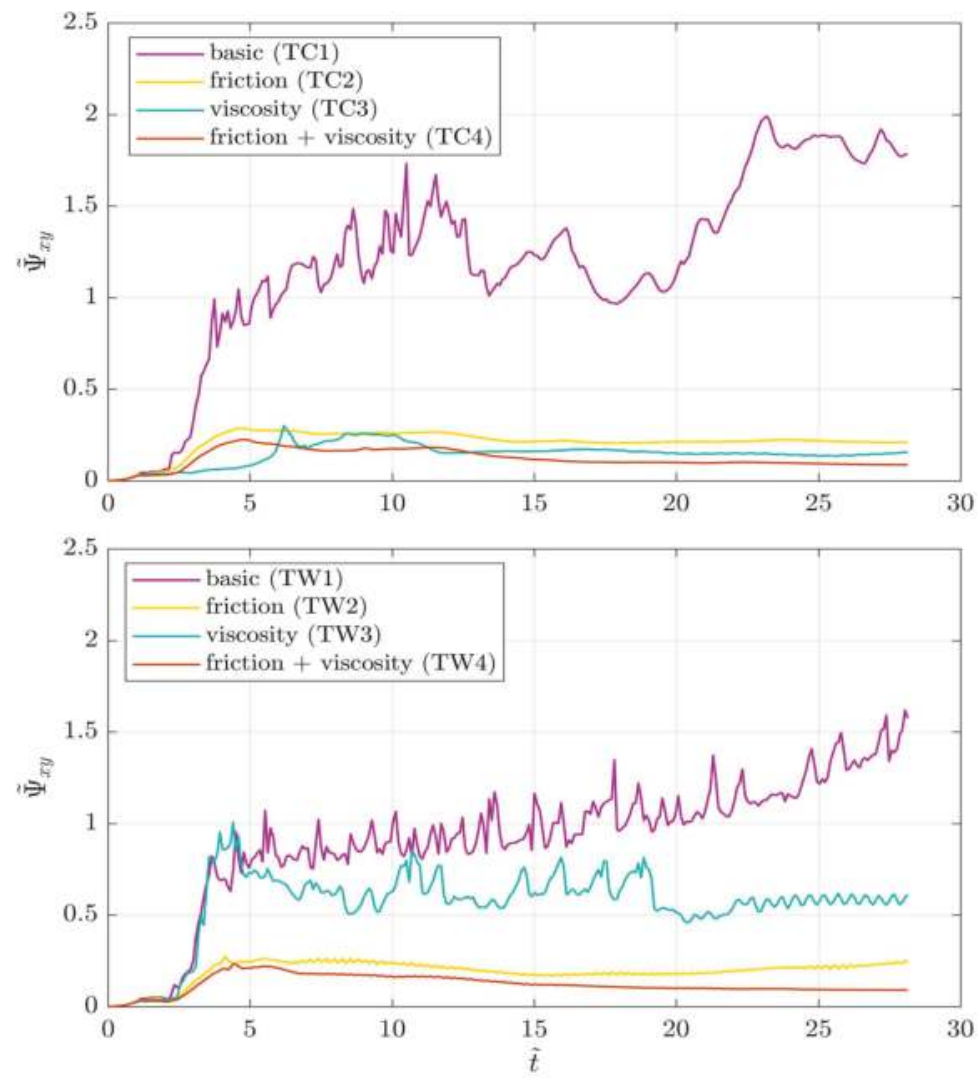

Figure 12. Time series of the dimensionless domain-integral $O W$ parameter $\tilde{\Psi}_{x y}$ as a function of the dissipation mechanisms. Top: tests with river current only (TC1 to TC4). Bottom: tests with a monochromatic wave opposing the river current (TW1 to TW4).

The irregular behavior of the time series of $\tilde{\Psi}_{x y}$ for the dissipation-free simulation with only river current (test TC1; purple line in the top panel) suggests that, when neither friction nor small-scale turbulence dissipation are accounted for, injection and dissipation of vorticity into the basin occur at a wide range of time scales. When waves are accounted for (test TW1; purple line in the bottom panel) the oscillations in $\tilde{\Psi}_{x y}$ are more regular, with a clearly detectable rising trend ascribable to the continuous injection of vorticity promoted by breaking waves on the shoal boundary. The presence of waves into the domain, therefore, seems to have a regularizing effect on the evolution of turbulence.

The introduction of turbulence dissipation by seabed friction (tests TC2 and TW2; yellow lines) results in a friction magnitude parameter $P_{\tau} \approx 10^{-1}-1$ (into the shoal) to $10^{-2}-10^{-1}$ (outside the shoal), whereas clearly $P_{\lambda}=0$ as viscosity-related dissipation is deactivated. Frictional dissipation results in a noticeable decrease of the overall amount of vortical energy for both test cases. The greater magnitude of $P_{\tau}$ on the shoal, moreover, implies that the dissipative action of seabed friction is stronger in that 
zone: this is also corroborated by the lower amount of vorticity in the shoal area given by tests TC2 and TW2 (second rows in Figures 8 and 9) in comparison with the respective "reference" tests TC1 and TW1 (first rows in the same Figures). The strong influence of dissipation by friction is also apparent by inspection of the dimensionless $O W$ parameter. $\tilde{\Psi}_{x y}$ for the test with river current only (test TC2, top panel) is reduced by $75-80 \%$ in the earlier stages $(\tilde{t}<20)$ mainly due to the consistent reduction in shoal vorticity, and by up to $90 \%$ in the final stages $(\tilde{t}>20)$ with respect to test TC1. For the test with interacting waves and currents (test TW2, bottom panel) the reduction in $\tilde{\Psi}_{x y}$ with respect to test TW1 grows steadily in time, from $65 \%$ at the beginning of the run, up to $85 \%$ in the ending stages of the simulation. Furthermore, friction favours the emergence of a steady state for the global vorticity for both tests. This implies that the action of friction is competitive with the stirring of vorticity promoted by currents and waves, and the order of magnitude of both processes (stirring by waves/currents and dissipation by friction) is the same over the test duration.

The mechanism of turbulence dissipation through small-scale turbulence (tests TC3 and TW3; cyan lines) acts in different ways, according to the test case. For both tests, the viscosity-related magnitude parameter $P_{\lambda} \approx 10^{-4}$ over the shoal and $P_{\lambda} \approx 10^{-3}$ outside the shoal; $P_{\tau}=0$ since friction-based dissipation is deactivated. For the test with river current only (test TC3, top panel) sub-grid turbulence acts to a considerable decrease of global vortical energy, comparable to the reduction caused by frictional mechanisms. Albeit being smaller in magnitude with respect to the standard friction-based dissipation $\left(P_{\tau} \approx 10^{-2}-1\right.$ for tests TC2 and TW2), sub-grid turbulence dissipation mechanisms seem to be competitive in dissolving the vorticity injected by a river current only. On the other hand, when the current is opposed by waves (test TW3, bottom panel), the reduction in $\tilde{\Psi}_{x y}$ is generally smaller (between $35 \%$ and $65 \%$ with respect to test TW1); this may suggest that the action of waves is effective in injecting a greater amount of vorticity, which is absent in the tests without waves. The action of viscosity, moreover, is greater outside of the shoal thanks to the greater depths, and leaves unorganized turbulence inside of the shoal. The regularization of the oscillations in $\tilde{\Psi}_{x y}$, though, means a reduction and regularization of the time scale and intensity of vorticity stirring, especially towards the end of the simulation (the oscillations become more regular at $\tilde{t}>20$ for test TW3). In summary, in both tests small-scale turbulence manages to dissipate a variable amount of the vorticity generated during the initial stages of the runs and to keep the remaining vorticity roughly constant within the basin.

Finally, the combination of dissipation by seabed friction and sub-grid turbulence (orange lines) is the most effective in dissipating the overall vorticity, although the relative importance of the two factors for the dissipation process differs between the test cases and between each other $\left(P_{\tau} \approx 10^{-2}-1\right.$ and $\left.P_{\lambda} \approx 10^{-4}-10^{-3}\right)$. Seabed friction and sub-grid dissipation are deemed to play a similar role when vorticity is stirred by the river current only (see the comparable reduction of $\tilde{\Psi}_{x y}$ by both friction and viscosity in the top panel of Figure 12). On the other hand, the action of friction is enhanced with respect to viscosity-based dissipation, if turbulence is injected by the concurrent action of current and waves (see the far greater reduction of $\tilde{\Psi}_{x y}$ induced by friction, with respect to the sub-grid-turbulence-induced, in the bottom panel of Figure 12).

\section{Conclusions}

Numerical simulations via a finite-volume numerical solver for shallow water flows have been exploited to achieve a better understanding of the dynamics of flow mixing and turbulence at an ideal river mouth configuration, as well as the role of bottom friction and sub-grid turbulence on the definition and temporal evolution of large coherent vortical structures. Flow dissipation by bed friction has been introduced through a simple Chezy-like approach. The effect of sub-grid vertical mixing has been accounted for through a suitable HLES formulation, in which the dissipative action of small-scale 3D turbulence is introduced through a global, depth-averaged eddy viscosity coefficient.

The performances of such wave-resolved model have been preliminarily tested against existing numerical simulations described in the work of Olabarrieta et al. [9], based on a wave-averaged solver. Both models are able to catch the main features of the wave-current interaction at a river mouth, albeit 
with some differences. The vertical-resolving model of OL14 seems to properly describe the alongshore currents observed in the field, but does not seem to give account of the potential vorticity generated at a river jet (e.g., see [52]). On the other hand, the wave-resolving NSWE model of Brocchini et al. [38] clearly reproduces macrovortices due to flow gradients caused by both the river jet and differential wave breaking, but predicts much weaker alongshore currents. This is in line with the strong wave breaking predicted by the NSWE model, which in turn leads to a considerable reduction of wave energy and generation of more intense macrovortices.

The analyses have identified two main regions of vorticity generation: at the inlet mouth due to current spreading, and along the shoal border as a consequence of the interaction between current, incoming waves, and bathymetry. In the definition and evolution of vorticity patterns, dissipation by means of friction seems to be generally dominant over sub-grid turbulence-based dissipation, especially over the shoal.

When only an outflowing river current is considered, dissipation through seabed friction is shown to be a strong agent in reducing the global amount of vorticity over the shoal and at the river mouth, where the mouth vortices are soon dissipated. At the shoal edge and further offshore seabed friction is not as effective, inducing only a mild reduction in vortex size and intensity. Conversely, dissipation through sub-grid turbulence has a greater effect at the shoal boundary, where the current slows down by feeling the increasing depth and develops negative velocity gradients, which are the fundamental contributions to viscosity-based dissipation.

When the interaction of current and waves is investigated, a significant amount of vorticity is generated, by differential wave braking, at the shoal edges and rolls up in the form of macrovortices. Wave breaking is also promoted by enhanced steepening thanks to the opposing current leaving the shoal. The shoal vortices are then advected towards the shore due to their interaction with the topography and the continuous action of waves. Again, seabed friction is shown to be the most important agent of regularization and dissipation of large-scale macrovortices within the shoal. Nonetheless, sub-grid turbulent motions also reduce the vorticity intensity at the shoal border, due to repeated velocity gradients. Waves are also effective in suppressing mouth vortices within the shoal.

Author Contributions: L.M. conceived, performed and discussed the numerical simulations and was responsible for the paper write-up. M.P. provided collaboration and suggestions to improve the quality of the tests. M.B. and G.D. supervised both analyses and writing.

Acknowledgments: The financial support from the Office of Naval Research Global (UK) NICOP EsCoSed Project (Research Grant N62909-13-1-N020) and MORSE Project (Research Grant N62909-17-1-2148) is acknowledged.

Conflicts of Interest: The authors declare no conflict of interest.

\section{Abbreviations}

The following abbreviations are used in this manuscript:

HLES Horizontal Large Eddy Simulation

LES Large Eddy Simulation

NSWE Nonlinear Shallow Water Equations

OL14 (the work of) Olabarrieta et al. (2014)

OW Okubo-Weiss (parameter)

SGS Sub-Grid Scale

WAF Weighted Averaged Flux

\section{References}

1. Shyu, J.H.; Phillips, O.M. The blockage of gravity and capillary waves by longer waves and currents. J. Fluid Mech. 1990, 217, 115-141. [CrossRef]

2. Ris, R.; Holthuijsen, L. Spectral modelling of current induced wave-blocking. In Proceedings of the 25th International Conference on Coastal Engineering, Orlando, FL, USA, 2-6 September 1996; American Society of Civil Engineers: Reston, VA, USA, 1997. 
3. Chawla, A.; Kirby, J.T. Monochromatic and random wave breaking at blocking points. J. Geophys. Res. 2002, 107, 4-1-4-19. [CrossRef]

4. Kemp, P.H.; Simons, R.R. The interaction of waves and a turbulent current: waves propagating against the current. J. Fluid Mech. 1983, 130, 73-89. [CrossRef]

5. Olabarrieta, M.; Medina, R.; Castanedo, S. Effects of wave-current interaction on the current profile. Coast. Eng. 2010, 57, 643-655. [CrossRef]

6. Davies, A.G.; Soulsby, R.L.; King, H.L. A numerical model of the combined wave and current bottom boundary layer. J. Geophys. Res. 1988, 93, 491-508. [CrossRef]

7. Kim, H.; O'Connor, B.A.; Park, I.; Lee, Y. Modeling effect of intersection angle on near-bed flows for waves and currents. J. Waterw. Port Coast. Ocean Eng. 2001, 127, 308-318. [CrossRef]

8. Olabarrieta, M.; Warner, J.C.; Kumar, N. Wave-current interaction in Willapa Bay. J. Geophys. Res. 2011, 116, doi:10.1029/2011JC007387. [CrossRef]

9. Olabarrieta, M.; Geyer, W.R.; Kumar, N. The role of morphology and wave-current interaction at tidal inlets: An idealized modeling analysis. J. Geophys. Res. Oceans 2014, 119, 8818-8837. [CrossRef]

10. Brocchini, M.; Kennedy, A.; Soldini, L.; Mancinelli, A. Topographically controlled, breaking-wave-induced macrovortices. Part 1. Widely separated breakwaters. J. Fluid Mech. 2004, 507, 289-307. [CrossRef]

11. Kennedy, A.B.; Brocchini, M.; Soldini, L.; Gutierrez, E. Topographically controlled, breaking-wave-induced macrovortices. Part 2. Changing geometries. J. Fluid Mech. 2006, 559, 57-80. [CrossRef]

12. McWilliams, J.C. The emergence of isolated coherent vortices in turbulent flow. J. Fluid Mech. 1984, 146, $21-43$. [CrossRef]

13. Bühler, O. On the vorticity transport due to dissipating or breaking waves in shallow-water flow. J. Fluid Mech. 2000, 407, 235-263. [CrossRef]

14. Postacchini, M.; Brocchini, M.; Soldini, L. Vorticity generation due to cross-sea. J. Fluid Mech. 2014, 744, $286-309$. [CrossRef]

15. Bühler, O.; Jacobson, T.E. Wave-driven currents and vortex dynamics on barred beaches. J. Fluid Mech. 2001, 449, 313-339. [CrossRef]

16. Peregrine, D. Surf Zone Currents. Theor. Comput. Fluid Dyn. 1998, 10, 295-309. [CrossRef]

17. Piattella, A.; Brocchini, M.; Mancinelli, A. Topographically controlled, breaking-wave-induced macrovortices. Part 3. The mixing features. J. Fluid Mech. 2006, 559, 81-106. [CrossRef]

18. Best, J.L.; Roy, A.G. Mixing-layer distortion at the confluence of channels of different depth. Nature 1991, 350, 411-413. [CrossRef]

19. Gaudet, J.M.; Roy, A.G. Effect of bed morphology on flow mixing length at river confluences. Nature 1995, 373, 138-139. [CrossRef]

20. Provenzale, A. Transport by coherent barotropic vortices. Annu. Rev. Fluid Mech. 1999, 31, 55-93. [CrossRef]

21. Mazumder, R. Turbulence-particle interactions and their implications for sediment transport and bedform mechanics under unidirectional current: Some recent developments. Earth Sci. Rev. 2000, 50, 113-124. [CrossRef]

22. Lesieur, M.; Begou, P.; Briand, E.; Danet, A.; Delcayre, F.; Aider, J.L. Coherent-vortex dynamics in large-eddy simulations of turbulence. J. Turbul. 2003, 4, 1-24. [CrossRef]

23. Elhmaïdi, D.; Provenzale, A.; Babiano, A. Elementary topology of two-dimensional turbulence from a Lagrangian viewpoint and single-particle dispersion. J. Fluid Mech. 1993, 257, 533-558. [CrossRef]

24. Yossef, M.F.; Klaassen, G.J. Reproduction of groynes-induced river bed morphology using LES in a 2-D morphological model. In Proceedings of the International Conference on Fluvial Hydraulics, Louvain-la-Neuve, Belgium, 4-6 September 2002; Volume 2, pp. 1099-1108, River Flow 2002.

25. Yossef, M.; de Vriend, H. Mobile-bed experiments on the exchange of sediment between main channel and groyne fields. In River Flow 2004; CRC Press: Boca Raton, FL, USA, 2004; pp. 127-133.

26. Keylock, C.; Hardy, R.; Parsons, D.; Ferguson, R.; Lane, S.; Richards, K. The theoretical foundations and potential for large-eddy simulation (LES) in fluvial geomorphic and sedimentological research. Earth Sci. Rev. 2005, 71, 271-304. [CrossRef]

27. Soldini, L.; Piattella, A.; Mancinelli, A.; Bernetti, R.; Brocchini, M. Macrovortices-induced horizontal mixing in compound channels. Ocean Dyn. 2004, 54, 333-339. [CrossRef] 
28. Van Prooijen, B.C.; Battjes, J.A.; Uijttewaal, W.S.J. Momentum exchange in straight uniform compound channel flow. J. Hydraul. Eng. 2005, 131, 175-183. [CrossRef]

29. Rowland, J.C.; Dietrich, W.E.; Stacey, M.T. Morphodynamics of subaqueous levee formation: Insights into river mouth morphologies arising from experiments. J. Geophys. Res. 2010, 115, doi:10.1029/2010JF001684. [CrossRef]

30. Canestrelli, A.; Nardin, W.; Edmonds, D.; Fagherazzi, S.; Slingerland, R. Importance of frictional effects and jet instability on the morphodynamics of river mouth bars and levees. J. Geophys. Res. Oceans 2014, 119, 509-522. [CrossRef]

31. Fagherazzi, S.; Edmonds, D.A.; Nardin, W.; Leonardi, N.; Canestrelli, A.; Falcini, F.; Jerolmack, D.J.; Mariotti, G.; Rowland, J.C.; Slingerland, R.L. Dynamics of river mouth deposits. Rev. Geophys. 2015, 53, 642-672. [CrossRef]

32. Piomelli, U. Large-eddy simulation: Achievements and challenges. Prog. Aerosp. Sci. 1999, 35, 335-362. [CrossRef]

33. Lesieur, M.; Metais, O.; Comte, P. Large-Eddy Simulations of Turbulence; Cambridge University Press: Cambridge, UK, 2005.

34. Moeng, C.H.; Sullivan, P. Large-Eddy Simulation. In Encyclopedia of Atmospheric Sciences; Elsevier: New York, NY, USA, 2015; pp. 232-240.

35. Clark, R.A.; Ferziger, J.H.; Reynolds, W.C. Evaluation of subgrid-scale models using an accurately simulated turbulent flow. J. Fluid Mech. 1979, 91, 1-16. [CrossRef]

36. Nadaoka, K.; Yagi, H. Shallow-water turbulence modeling and horizontal large-eddy computation of river flow. J. Hydraul. Eng. 1998, 124, 493-500. [CrossRef]

37. Hinterberger, C.; Fröhlich, J.; Rodi, W. Three-dimensional and depth-averaged large-eddy simulations of some shallow water flows. J. Hydraul. Eng. 2007, 133, 857-872. [CrossRef]

38. Brocchini, M.; Bernetti, R.; Mancinelli, A.; Albertini, G. An efficient solver for nearshore flows based on the WAF method. Coast. Eng. 2001, 43, 105-129. [CrossRef]

39. Van Rijn, L.C. Principles of Sediment Transport in Rivers, Estuaries and Coastal Seas; Aqua Publications: Blokzijl, The Netherlands, 1993.

40. van Vossen, B.; Uittenbogaard, R. Subgrid-scale model for quasi-2D turbulence in shallow water. In Shallow Flows; Jirka, G.H., Uijttewaal, W.S.J., Eds.; Taylor \& Francis: London, UK, 2004; pp. 575-582.

41. Grosso, G.; Brocchini, M.; Piattella, A. Modeling horizontal coastal flows: assessing the role of coastal contributions. In Applied and Industrial Mathematics in Italy II; World Scientific: Singapore, 2007; pp. 410-421.

42. Piattella, A. On Mixing in Natural Shallow Flows. Ph.D. Thesis, Università Politecnica delle Marche, Ancona, Italy, 2003.

43. Postacchini, M.; Othman, I.K.; Brocchini, M.; Baldock, T.E. Sediment transport and morphodynamics generated by a dam-break swash uprush: Coupled vs uncoupled modeling. Coast. Eng. 2014, 89, 99-105. [CrossRef]

44. Toro, E.F. Riemann Solvers and Numerical Methods for Fluid Dynamics; Springer: Berlin, Germany, 2009.

45. Toro, E.F. Riemann problems and the WAF method for solving the two-dimensional shallow water equations. Phil. Trans. R. Soc. A 1992, 338, 43-68. [CrossRef]

46. Brocchini, M. Bore-generated macrovortices on erodible beds. J. Fluid Mech. 2013, 734, 486-508. [CrossRef]

47. Briganti, R.; Torres-Freyermuth, A.; Baldock, T.E.; Brocchini, M.; Dodd, N.; Hsu, T.-J.; Jiang, Z.; Kim, Y.; Pintado-Patiño, J.C.; Postacchini, M. Advances in numerical modelling of swash zone dynamics. Coast. Eng. 2016, 115, 26-41. [CrossRef]

48. Warner, J.C.; Armstrong, B.; He, R.; Zambon, J.B. Development of a Coupled Ocean-Atmosphere-WaveSediment Transport (COAWST) modeling system. Ocean Model. 2010, 35, 230-244. [CrossRef]

49. Madsen, P.A.; Rugbjerg, M.; Warren, I.R. Subgrid Modelling in Depth Integrated Flows. In Coastal Engineering 1988; American Society of Civil Engineers: Reston, VA, USA, 1989.

50. Okubo, A. Horizontal dispersion of floatable particles in the vicinity of velocity singularities such as convergences. Deep Sea Res. Oceanogr. Abstr. 1970, 17, 445-454. [CrossRef] 
Water 2018, 10, 550

51. Weiss, J. The dynamics of enstrophy transfer in two-dimensional hydrodynamics. Phys. D 1991, 48, $273-294$. [CrossRef]

52. Falcini, F.; Jerolmack, D.J. A potential vorticity theory for the formation of elongate channels in river deltas and lakes. J. Geophys. Res. 2010, 115, doi:10.1029/2010JF001802. [CrossRef]

(c) (1)

(C) 2018 by the authors. Licensee MDPI, Basel, Switzerland. This article is an open access article distributed under the terms and conditions of the Creative Commons Attribution (CC BY) license (http:/ / creativecommons.org/licenses/by/4.0/). 
Article

\title{
Some Aspects of Turbulent Mixing of Jets in the Marine Environment
}

\author{
Michele Mossa ${ }^{1, *}$ and Peter A. Davies ${ }^{2}$ \\ 1 Department of Civil, Environmental, Land, Building Engineering \& Chemistry, Polytechnic University of Bari, \\ Via E. Orabona 4, 70125 Bari, Italy \\ 2 Department of Civil Engineering, University of Dundee, Dundee DD14HN, UK; p.a.davies@dundee.ac.uk \\ * Correspondence: michele.mossa@poliba.it; tel.: +39-080-596-3289
}

Received: 6 March 2018; Accepted: 13 April 2018; Published: 21 April 2018

\begin{abstract}
Prominent among environmental problems is the pollution of the coastal marine zone as a result of anthropogenic activities. On this point, while studies of jets in still water and in crossflows have been developed in many research centres, studies on jets interacting with waves are still rare. The present study analyses turbulent, non-buoyant water jets issued into a wave environment. A comparison of the time-averaged and phase-averaged velocity components has been carried out, in order to highlight the flow patterns in the two configurations. The experimental data have also been compared with others in the literature, such as the relationship between the dimensionless, longitudinal, time-averaged velocities of the jet mean axis and the distance from the source. Such comparisons reveal a good agreement. Furthermore, using the analogy between the equation of the turbulent transport of a solute concentration and the equation of the turbulent kinetic energy, the paper presents also estimates of the turbulence diffusion coefficients and advection terms of jets in a wave environment. The experimental results are compared with jets in still water. With the presence of waves, the turbulence length-scales in the streamwise direction vary, contributing to an increase in streamwise turbulent diffusion, relative to the condition of the same jet in still water. The analysis of the jet streamwise advection term reveals that it increases in the case of jets in a wave environment, as compared to no-wave conditions.
\end{abstract}

Keywords: jets; waves; turbulence; mixing; diffusion; advection

\section{Introduction}

The explosive global economic growth initiated in the 1960s has surely left its mark on hydraulic engineering and on a wide spectrum of environmental problems. Prominent among these problems is the pollution of the coastal marine environment as a result of anthropogenic activities associated with, for example, (i) the discharge of domestic and industrial wastewater; (ii) the unregulated and accidental release of petroleum and hydrocarbon products from marine traffic and offshore exploration/production installations; (iii) the run-off of animal waste and fertiliser-derived nutrients from agricultural land; and (iv) the construction of marine structures and waste disposal and treatment systems. All these consequences have an impact on population health, marine ecology, economic prosperity, commercial operations, and environmental sustainability. The impact is felt throughout the spheres of water management (drinking water, industrial water, bathing water, irrigation, hydropower, etc.), navigation (currents, ice formation, shoaling, erosion involving dredging, protection works, etc.), flood protection (forecasting, dike placement, regulation of discharges), traffic management (tunnels, bridges, harbours, etc.), near- and offshore activities (modifications to coastal currents and eddies, ice drifts, blow-outs, etc.), and commercial and leisure fishing (modifications to currents, salinity, temperature, oxygen content; formation of fronts, etc.). The prediction and management of 
pollution problems is surely becoming more and more pressing, with massive consequences for the environmental and ecological health of coastal waters and their surrounding communities [1-5].

In the present article, focus is placed upon the importance of turbulent mixing and entrainment processes for typical near-shore and estuarial conditions where the effective and safe disposal of wastewater is the principal objective. Attention is directed towards the role of turbulent jets [6-8] in accomplishing this mixing. In the marine environment, such jets are almost always associated with wastewater outfalls that have been constructed to convey treated wastewater from onshore treatment plants to zones appropriately far from shore (i) to prevent contamination of the waters in the near-shore region and (ii) to minimise the harmful effects of the discharge on humans and indigenous flora and fauna. The monographs by Wood et al. [9] and Roberts et al. [10] and the chapter by Tate et al. [11] provide comprehensive entry points to the extensive relevant literature on this topic. Finally, it is noted that, though the emphasis here is on the behaviour and properties of the turbulent buoyant jets, the dilution (and eventual fate) of contaminants discharged within the effluents is controlled also by the spatio-temporal structure of the ambient turbulence field in the receiving waters. In most cases, this structure is determined by the receiving water bathymetry and topography and the strength and variability of wind, tides, heat exchange, evaporation, ice formation, barometric pressure variation, gravity, etc. The spatio-temporal structure of the ambient turbulence field and the modifying role of (i) density stratification and (ii) vertical variations in mean flow is crucially important for the prediction of entrainment and mixing of turbulent jets discharged from wastewater outfalls. It is an area of research that requires further investigation.

The sea has always been the final destination for water-borne waste products coming from the land. In recent years, effects such as jet momentum, buoyancy, current, stratification, etc., on the processes of mixing and transport have received more and more attention [12-16], but wave action also plays a very important role in many cases [17-19]. While there are several studies in the literature on jets and their interaction with currents $[9,20,21]$, few deal with jet-wave interaction, with the majority emphasizing the importance of a wave flow field in diffusion processes [22-33] and the necessity of experimental tests to explain jet-wave interaction dynamics and possibly confirm the validity of mathematical models present in literature [34]. The topic is of great engineering interest for environmental problems [9,35-39].

Shuto and $\mathrm{Ti}$ [17] carried out experiments to investigate the dilution rate of plumes with waves at the free surface. The authors found that the dilution rate is inversely proportional to the square of the ratio of the water depth to the outlet diameter and is proportional to the ratio of the discharge velocity to a characteristic horizontal velocity of the ambient fluid. Sharp [19] pointed out that longitudinal velocity component profiles of jets with waves show twin peaks in some jet cross sections (so-called "dumb-bell effect", typical of sewage discharged into a tidal estuary). Chyan and Hwung [27] carried out experimental studies using a combined LDA (Laser Doppler Anemometry) and LIF (Laser-Induced Fluorescence) system to obtain a combined analysis of velocity components and concentration of nonbuoyant jets vertically discharged in a wave environment. Using flow visualisation, they identified three regions of a jet-wave interaction: (1) deflection region, (2) transition region, and (3) developed jet region. Chyan and Hwung [27] noted that the process of periodic jet deflection allows a large volume of water from the external environment to pour into this jet. The mechanism, which was called the "wave tractive mechanism", adds to that of classic entrainment, thereby improving the dilution process. Koole and Swan [28] analysed 2D non-buoyant jet dispersion in a flow field of regular waves. They presented velocity profiles, standard deviation of the turbulent velocity components, and Reynolds shear stresses.

The studies cited above illustrate that some investigations have been undertaken on the interaction between jets and waves in the past, but there is still a need for further detailed analysis of this problem, utilizing data collected with modern instrumentation. This is a main motivation for the present study and the main novel component. 
The principal purpose of this article is to highlight aspects of turbulent mixing in the marine environment (particularly for jet flows) in order (i) to raise awareness of the range of problems that still remain to be addressed and solved and (ii) to indicate future developments, directions, and perspectives in this field. The oft-cited dictum "Dilution is the Solution to Pollution" summarises the overall design objectives underlying many marine wastewater disposal systems, illustrating why environmental flows characterised by dilution and mixing processes are of great interest for researchers and practitioners. In the present article, recent theoretical analyses and experimental results are presented for some case studies of jets in a wave environment (see Figure 1).

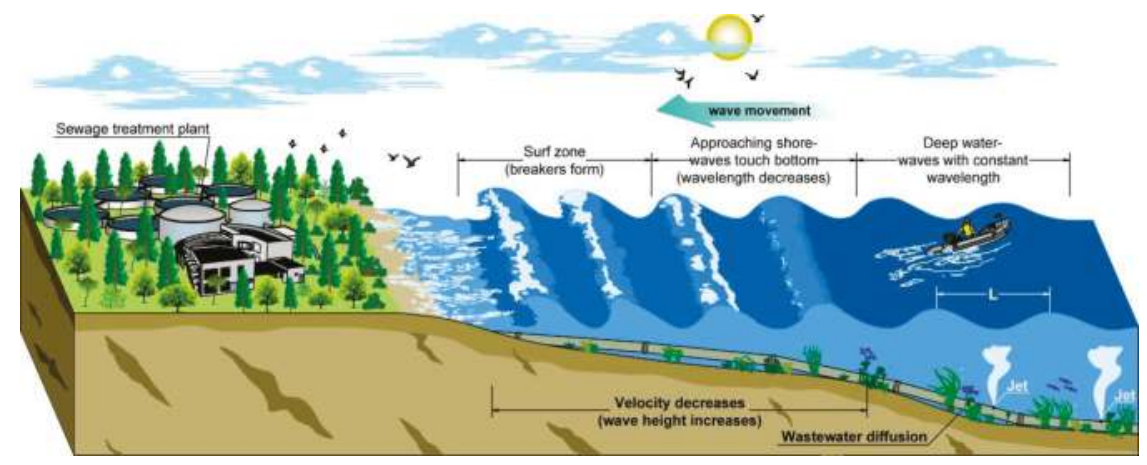

Figure 1. Typical release of jets in wave environment. The discharge region is located offshore of the surf zone.

The material to be presented here shows that deeper knowledge of these complex environmental flows should be pursued for research, technical, and engineering interests. Because of the increasing stress placed on water resources throughout the world, a resurgence and reinvention of hydraulic engineering should be considered, in the sense that hydraulic research will be more and more a cooperation with other experts and that researchers must respond to the need to manage and protect natural resources. Considering all these aspects, the old motto with which this summary starts could be changed to "Dilution is (not always) the Solution to Pollution".

\section{Materials and Methods}

\subsection{Momentum Equations}

The fundamental equations governing the problem can be derived from the Navier-Stokes equations. Any physical quantity is split into the steady mean flow component, the fluctuation component due to the statistical contribution of the wave, and the fluctuation component of the turbulence [40]. Therefore, the $u_{i}(i=1,2,3)$ velocity components can be expressed as follows:

$$
u_{i}\left(x_{i}, t\right)=\left\langle u_{i}\right\rangle\left(x_{i}, t\right)+u_{i}^{\prime}\left(x_{i}, t\right)=U_{i}\left(x_{i}\right)+\widetilde{u}_{i}\left(x_{i}, t\right)+u_{i}^{\prime}\left(x_{i}, t\right)
$$

where the angular brackets " $\langle>$ " denote an operator representing an ensemble average, the tilde symbol indicates the fluctuations due to the wave statistical contribution (or oscillating components), the prime symbol indicates the turbulent fluctuations, and the capital letters or the over-bar indicate the steady mean flow (time-averaged components). In addition, $t$ is time and the $x_{i}$ are the coordinates of a Cartesian frame.

Using the Cartesian tensor notation, the ensemble average of the motion equations for turbulent nonbuoyant jet flow under wave action is 


$$
\frac{\partial\left\langle u_{i}\right\rangle}{\partial t}+\frac{\partial\left\langle u_{i}\right\rangle\left\langle u_{j}\right\rangle}{\partial x_{j}}=\frac{1}{\rho} \frac{\partial}{\partial x_{j}}\left(-\langle p\rangle \delta_{i j}+\mu\left(\frac{\partial\left\langle u_{i}\right\rangle}{\partial x_{j}}+\frac{\partial\left\langle u_{j}\right\rangle}{\partial x_{i}}\right)-\rho\left\langle u_{i}^{\prime} u_{j}^{\prime}\right\rangle\right)
$$

where $\rho$ is the water density, $\delta_{i j}$ is the Kronecker delta, $p$ is the hydrodynamic pressure, and $\mu$ is the dynamic viscosity. The motion of the incompressible fluid is periodic, so the average over the period $T$ of Equation (2) becomes

$$
\frac{\partial}{\partial x_{j}}\left(U_{i} U_{j}+\overline{\widetilde{u}_{i} \widetilde{u}_{j}}+\overline{u_{i}^{\prime} u_{j}^{\prime}}\right)=\frac{1}{\rho}\left(-\frac{\partial P}{\partial x_{j}} \delta_{i j}+\mu \frac{\partial^{2} U_{i}}{\partial x_{j} \partial x_{j}}\right) .
$$

\subsection{Transport of Tracers and Turbulent Kinetic Energy}

The time-averaged, turbulent transport of a solute concentration is described by the following equation:

$$
\frac{\partial \bar{c}}{\partial t}+\frac{\partial \overline{u_{i} c}}{\partial x_{i}}=K_{i i} \frac{\partial^{2} \bar{c}}{\partial x_{i}^{2}}
$$

where $c\left(x_{1}, x_{2}, x_{3}\right)$ is the solute concentration and $K_{i i}$ are the coefficients for dispersion. For further details see Mossa et al. [32,33].

In the analysis of the flow-dispersion interaction, the turbulent kinetic energy is important in determining the turbulent dispersion coefficient and, thus, the mass transport. For high Reynolds numbers, assuming that the production term is of order of the dissipation term, the equation of the turbulent kinetic energy is

$$
\frac{\partial k}{\partial t}+\frac{\partial \overline{u_{i}} k}{\partial x_{i}}=D_{k} \frac{\partial k}{\partial x_{i}}
$$

where

$$
k=\frac{1}{2} \overline{u_{i}^{\prime} u_{i}^{\prime}}
$$

is the time-averaged turbulent kinetic energy, which, in the case of $2 \mathrm{D}$ velocity measurements, can be estimated as proposed by Svendsen [41] with the following equation

$$
k=\frac{1.33}{2}\left(\overline{u^{\prime} u^{\prime}}+\overline{v^{\prime} v^{\prime}}\right) .
$$

The parameter $D_{k}$ is the turbulent diffusion coefficient, which can be expressed as the product of a length scale and a velocity scale. A physical meaningful velocity scale is $\sqrt{k}$. Consequently, we consider

$$
D_{k}=l \sqrt{k}
$$

with $l$ the integral length scale associated with turbulent eddies. Equation (5) is formally analogous to Equation (4) and, therefore, assuming that the Prandtl number is $\mathrm{O}(1)$, the cross-correlation between the time-averaged turbulent kinetic energy and the $U, V$, and $W$ velocity components could be analysed and related to the time-averaged solute concentration $C$ transport by the mean flow $U C, V C$, and $W C$.

Furthermore, analogously to Equation (6), Tanino and Nepf [42] assumed that the net dispersion coefficients of Equation (1) could be set equal to

$$
K_{i i}=\alpha \sqrt{k} l_{i}
$$

where the scale factor $\alpha$ could be different for horizontal and vertical diffusion, even if generally it is of $\mathrm{O}(1)$. In the present study, the integral length scale $l_{i}$ is evaluated by multiplying the integral time scale $T_{u}$ by the local time-averaged velocity $u_{i}$, where $T_{u}$ is estimated by the autocorrelation function of the turbulent velocity fluctuations. 


\subsection{Experimental Procedure}

Experiments were carried out in a wave channel at the hydraulics laboratory of the Polytechnic University of Bari (Bari, Italy). The channel is about $45 \mathrm{~m}$ long and $1 \mathrm{~m}$ wide with walls made up of crystal plane sheets $1.2 \mathrm{~m}$ high, supported by iron frames with a centre-to-centre distance of about $0.44 \mathrm{~m}$, where resistance probes for wave profile measurements may be placed. Figures 2 and 3 show drawings of the laboratory system and the jet characteristics, respectively.

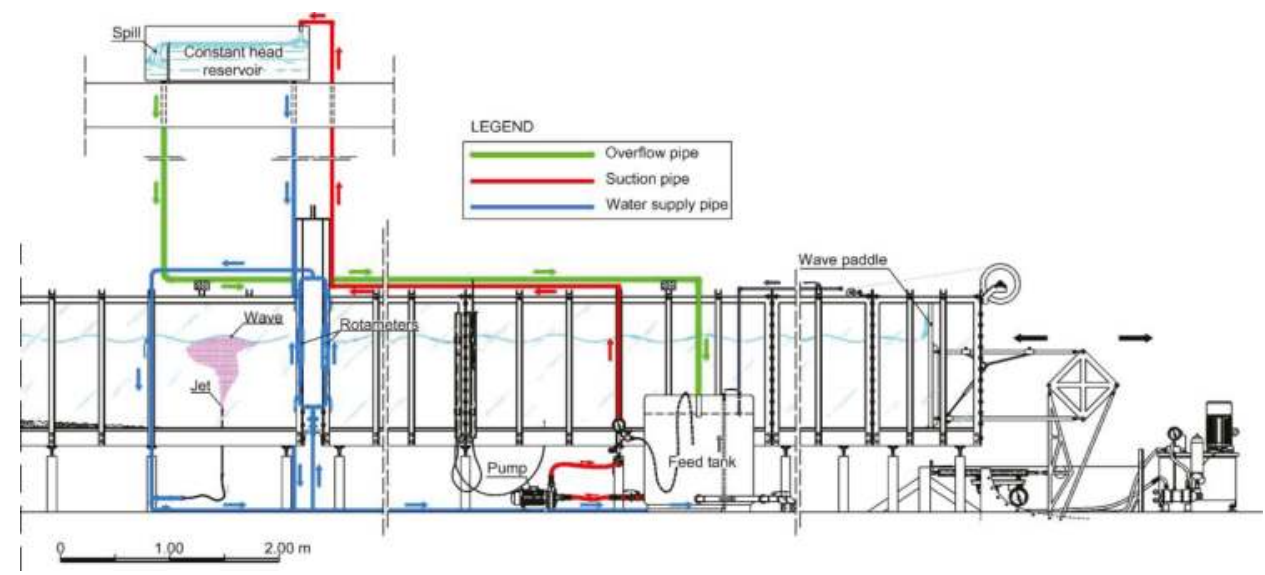

Figure 2. Drawing of the experimental setup.

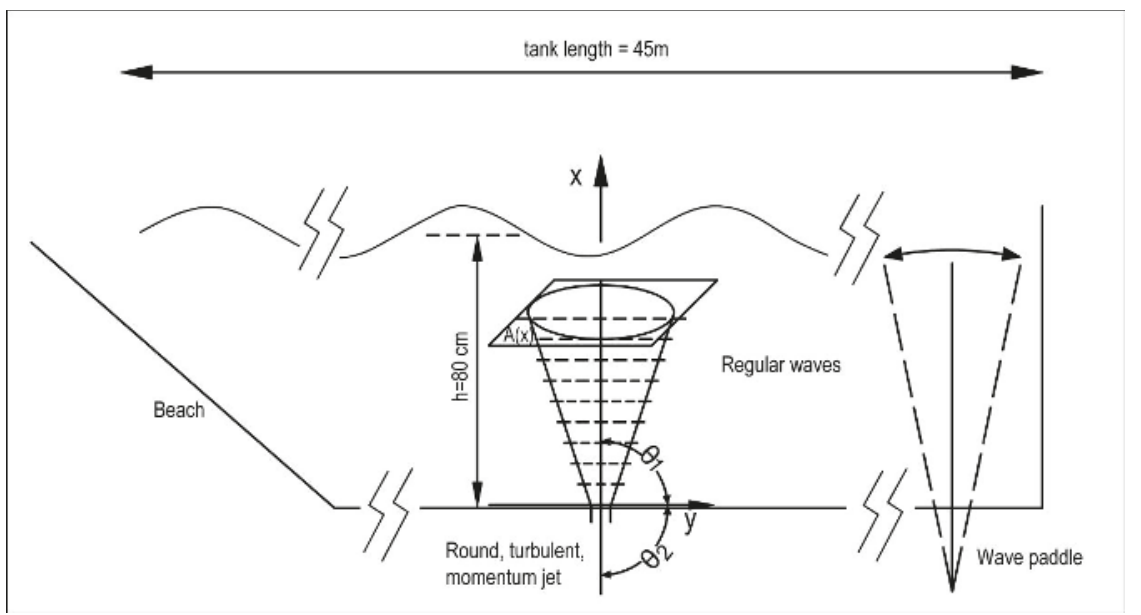

Figure 3. Jet defining drawing.

This study was carried out for a vertical turbulent nonbuoyant jet discharged in a stagnant environment and for the same jet interacting with progressive wave flow fields, in the intermediate range between deep and shallow water. During testing, the mean water depth near the paddle was $h=0.8 \mathrm{~m}$.

The velocity field was measured by using a backscatter, two-component four-beam fibre-optic LDA system (by Dantec Dynamics A/S, Skovlunde, Denmark). Particularly, the LDA system consists of a $5 \mathrm{~W}$ water-cooled argon-ion laser (version Innova 70, by Coherent Inc., Santa Clara, CA, USA), 
a 2D fibre flow transmitter (by Dantec Dynamics A/S, Skovlunde, Denmark, which comprises a transmitter providing colour separation and frequency shifting of the laser beam; fibre manipulators for optimum coupling of the laser light into optical fibres; a probe head with a fibre-optical connection to the transmitter; and back-scatter receiving optics including colour filters, colour separation, and photodetectors), a probe with a diameter of $85 \mathrm{~mm}$ (by Dantec Dynamics A/S, Skovlunde, Denmark, with a focal length of $310 \mathrm{~mm}$ and a beam spacing of $60 \mathrm{~mm}$ ), and a signal processor (version 58N40 FVA-Flow Velocity Analyzer enhanced, by Dantec Dynamics A/S, Skovlunde, Denmark). The accuracy of velocity measurements is $\pm 2 \%$. Through an AD/DA-Analog to Digital/Digital to Analog board (Keithley Metrabyte model DAS 50/4), the laser Doppler data can be correlated with up to four 12-bit channels of auxiliary inputs coming from four transducers. Therefore, for the configurations of jets with waves, the measurement system allows us to assess-at the same time as the velocity components-the wave elevation profile, by use of a resistance wave gauge placed in the transversal section of the channel crossing the laser measurement volume. The entire system is assisted by a process computer (Figure 4).

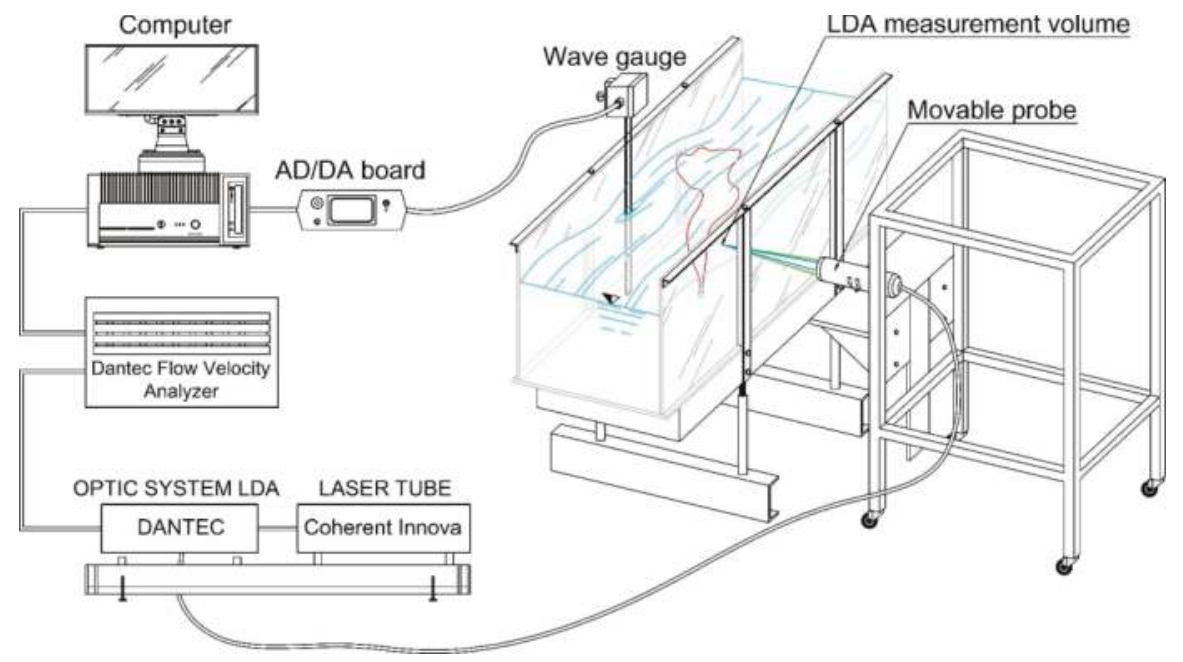

Figure 4. Sketch of the LDA system with the wave gauge, the AD/DA board and the process computer.

Figure 5a-f show the different parts of the complex experimental apparatus, which comprises the LDA system, the resistance wave gauge system, and the wavemaker system. 


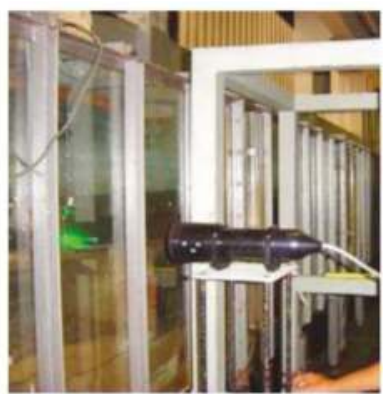

a)

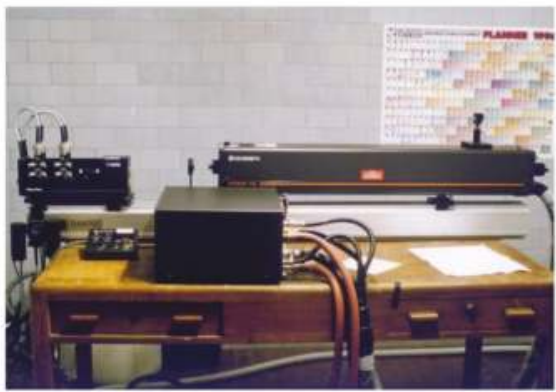

c)

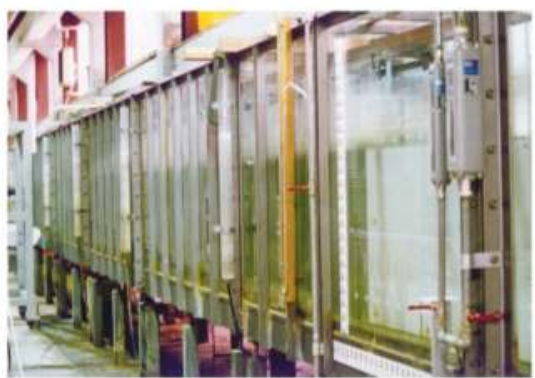

e)

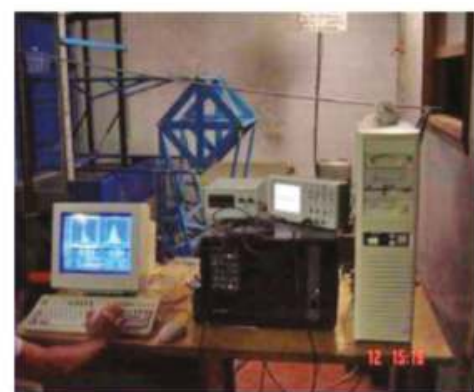

b)

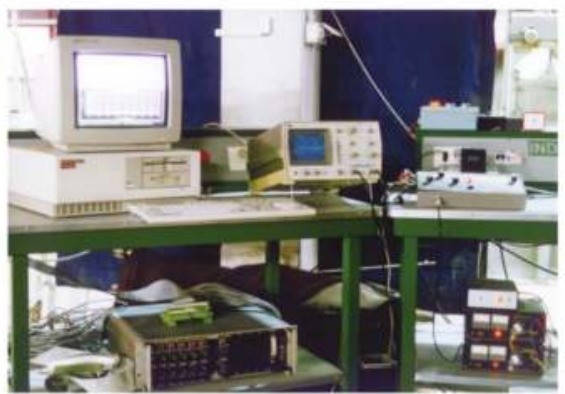

d)

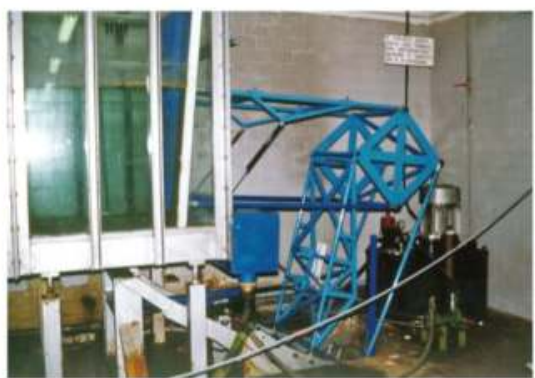

f)

Figure 5. Experimental apparatus: (a) LDA probe; (b) Dantec FVA signal processor and process computer; (c) Laser Coherent Innova and Dantec 2D Fibre Flow optics; (d) process computer with an AD/DA board for the wavemaker control; (e) a part of the wave channel where the jets were discharged; (f) the wavemaker.

The vertical nonbuoyant jet under consideration was introduced through a $2.01 \mathrm{~mm}$ insidediameter circular nozzle, with a volume flow $Q_{0}$ rate equal to $22.22 \mathrm{~cm}^{3} / \mathrm{s}$; a discharge velocity $U_{0}$ equal to $6.42 \mathrm{~m} / \mathrm{s}$; a momentum flux $M=Q_{0} U_{0}$ at the nozzle equal to $1.43 \times 10^{-4} \mathrm{~m}^{4} / \mathrm{s}^{2}$; and Reynolds number, based on the nozzle diameter, equal to 13,482 . The discharge scale length $L_{Q}=Q_{0} / M^{0.5}$, which measures the distance over which the port geometry influences the jet behaviour, is equal to $1.9 \mathrm{~mm}$ (comparable with the nozzle diameter). The round nozzle was located about $11 \mathrm{~m}$ from the wavemaker at $16.7 \mathrm{~cm}$ from the bottom of the channel. The velocity components measured in the present study are $u$ in the $x$ direction (see Figure 3; velocity component parallel to the jet axis $x$, i.e., 
vertical velocity component, conventionally established as positive if oriented upward) and $v$ in the horizontal direction (conventionally established as positive if oriented onshore).

Two categories of flow were considered; firstly, jet discharged into still water (i.e., configuration without waves) and, secondly, jet discharged into regular wave trains, generated in the channel, with a wave period for each configuration of $2.00 \mathrm{~s}$ (Test 1), $1.43 \mathrm{~s}$ (Test 2), and $1.00 \mathrm{~s}$ (Test 3), respectively. For the tests with waves, Table 1 (movies in supplementary) shows the wave height $(H)$, the wave length $(L)$, the wave period $(T)$, the wave steepness $H / L$, the relative depth $h / L$, and $L_{w}=M^{1 / 2} / \widetilde{v}_{w}$. The latter term is the length scale of the region from the nozzle dominated by the initial jet momentum compared to the wave-induced momentum, where $\widetilde{v}_{w}$, the values of which are also shown in the table, is a crossflow velocity scale of the wave motion, defined as [27]

$$
\widetilde{v}_{w}=\frac{H g}{h \pi \sigma} \tanh (k h)
$$

with $\sigma=2 \pi / T$ the wave angular frequency and $k=2 \pi / L$ the wave number. The values of the relative depths $h / L$ show that the jets of Table 1 are discharged in a wave transitional zone, i.e., between shallow and deep waters.

Table 1. Main characteristics of the jet configurations with waves.

\begin{tabular}{cccccccc}
\hline Test with Waves & $H[\mathrm{~cm}]$ & $\boldsymbol{L}[\mathrm{m}]$ & $\boldsymbol{T}[\mathrm{s}]$ & $\boldsymbol{H} / \boldsymbol{L}$ & $h / L$ & $\widetilde{v}_{w}[\mathrm{~m} / \mathrm{s}]$ & $\boldsymbol{L}_{w}[\mathrm{~m}]$ \\
\hline 1 & 4.20 & 5.10 & 2.00 & 0.0082 & 0.16 & 0.039 & 0.31 \\
2 & 4.40 & 3.05 & 1.43 & 0.014 & 0.26 & 0.036 & 0.33 \\
3 & 4.13 & 1.56 & 1.00 & 0.027 & 0.51 & 0.026 & 0.47 \\
\hline
\end{tabular}

The wave flow field in the channel can be described with the Stokes second-order theory according to the classic Le Méhauté abacus [43]. The reflection coefficient in the channel is not greater than $9 \%$.

In the cases of jets interacting with waves, ensemble-averaged velocities were obtained by phase-averaging the measured signals separated by the wave period. The results, which represented the phase-averaged velocities at different phases of a wave cycle, were averaged to yield the time-averaged velocities. The turbulent velocity fluctuations were obtained by subtracting the phase-averaged velocities from the original time series. For further details, see [32,33].

\section{Results and Discussion}

\subsection{Jet Flow Patterns, and Phase- and Time-Averaged Velocities}

The jet flow in the unsteady and periodic wave flow field has the characteristics shown in Figure 6, which reports a sequence of pictures of the jet of Run 1 of Table 1 taken at one-eighth of the wave period, in comparison with the flow field of the same jet issued in still water. 


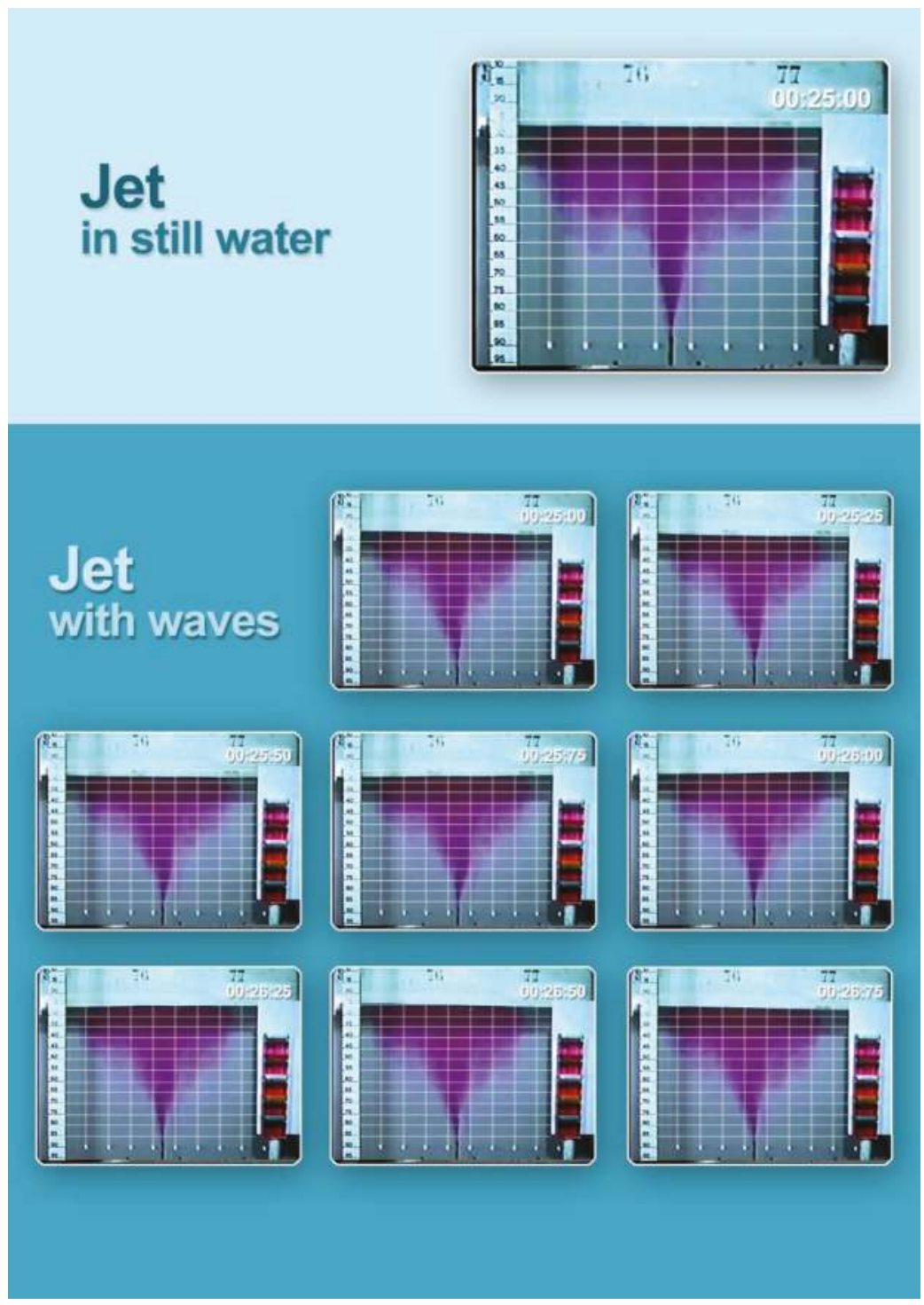

Figure 6. Flow patterns of the jet issued into still water and a time sequence of images of the same jet in a wave flow field of period $T$ at different wave phases separated by $T / 8$ (Test 1 of Table 1 ). The grid helps to show the jet oscillations.

The grid enables us to better show (i) the difference between the cases of jets with and without waves and (ii) the jet oscillations in the configurations where waves are present. Particularly, the jet issued into still water shows an enlargement that is described well in the literature [20,32,33]. Furthermore, in the case of the same jet with waves, the flow pattern resembles that of a jet discharged into a cross-current when the wave horizontal velocity dominates and that of a co-stream jet or jet in opposing flow when the vertical wave velocity dominates. As the wave motion changes periodically, different features will prevail by turns. The most impressive conclusion that we can obtain from these 
images is the enlargement of the jet area when waves are present compared with the turbulent jet in still water, which suggests an enhancement of the dilution and, therefore, a positive effect of the wave motion during the initial mixing processes.

The flow patterns are confirmed with the results of the phase-averaged velocity vectors shown in Figure 7 for Run 1 of Table 1.

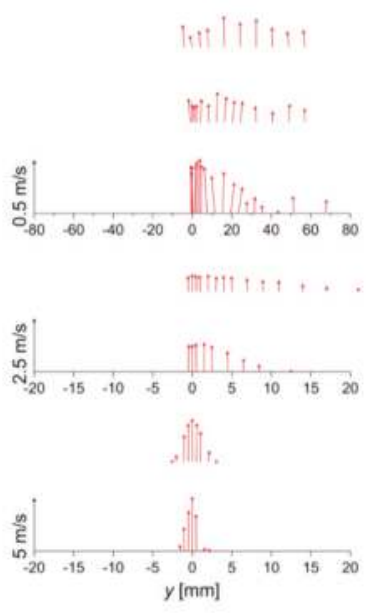

a
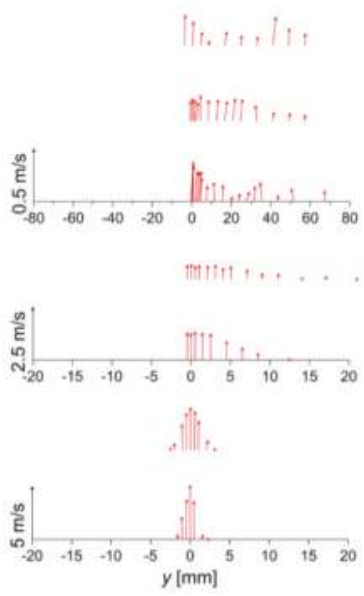

C

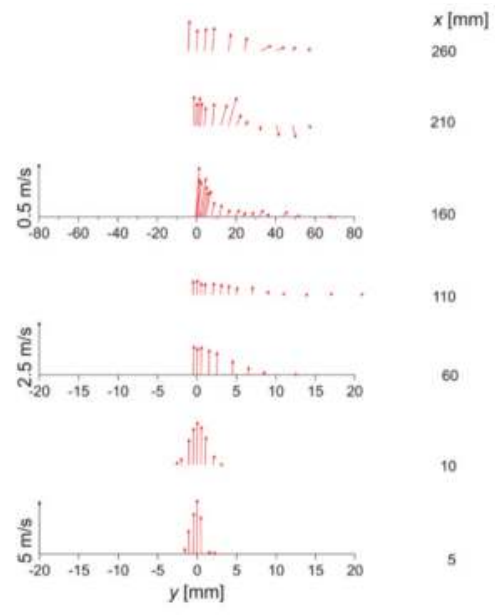

b

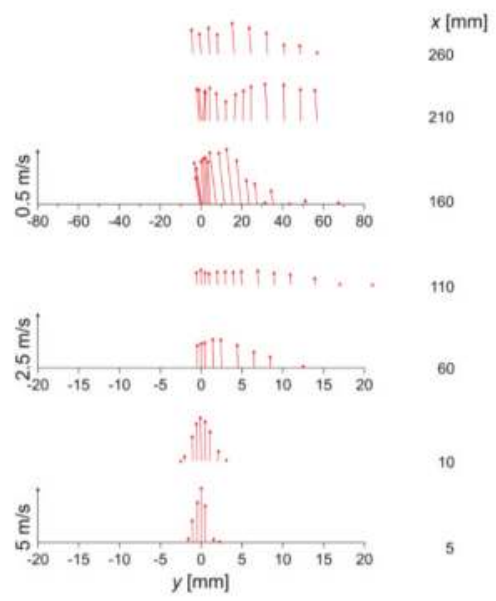

d

Figure 7. Phase-averaged flow patterns of velocity vectors of Run 1 of Table 1 acquired at a time rate of one-quarter of the wave period: (a) wave trough to crest passage; (b) under the wave crest; (c) wave crest to trough passage; (d) under the wave trough.

In this figure, it is possible to see the jet deflection back and forth by wave action, which agrees with the results of flow visualisation. The larger oscillations are present closer to the free surface, where the wave action is greater. 
Figure 8 shows the time-averaged velocity vectors of Run 1 of Table 1 and of the same jet in still water.

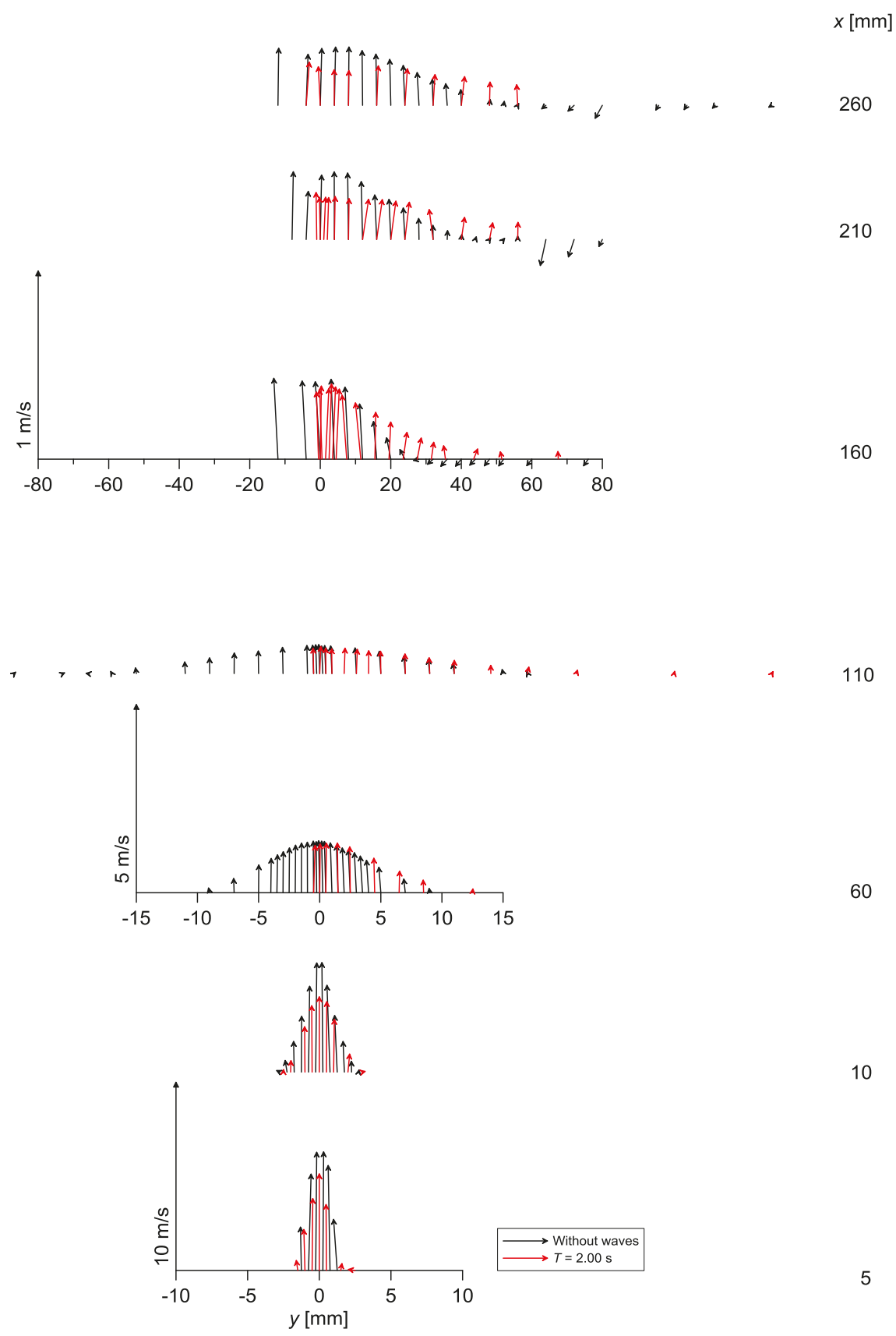

Figure 8. Time-averaged velocity vectors of Run 1 of Table 1 and of the same jet in still water. 
The comparison enables us to highlight the differences between the two configurations. Particularly, the figure shows that the velocity profiles of jets discharged in a wave environment are even flatter and wider than those of the same jet discharged in a stagnant environment. These profiles clearly indicate the existence of a relapse flow, as observed also by [13]. In the region farther from the nozzle, vertical velocity profiles point out, at times clearly, the presence of two peaks. In the case of the jet with waves, it is possible to see also the presence of twin peaks, as a typical result of the jet deflection due to periodic wave motion $[27,32,33]$.

Figure 9 shows the values of the square root of the turbulent kinetic energy $\sqrt{k}$ nondimensionalised by $U_{0}$ of Run 1 of Table 1 . Close to the nozzle, specifically at $x / D<60$ and $x<<L_{w}$, the values of $\sqrt{k}$ are similar with and without waves, indicating that the impact of the wave on the turbulent kinetic energy is small. This trend is also confirmed by the other runs. In this region it is possible to affirm that the turbulence is dominated by the jet and, therefore, this is the region with jet-generated shear. Farther from the nozzle, i.e., when $x$ is closer to or greater than $L_{w}$, the values of $\sqrt{k}$ are higher with the presence of waves. In this region the turbulence of the jet is also affected by the waves (region of jet-wave-generated shear).

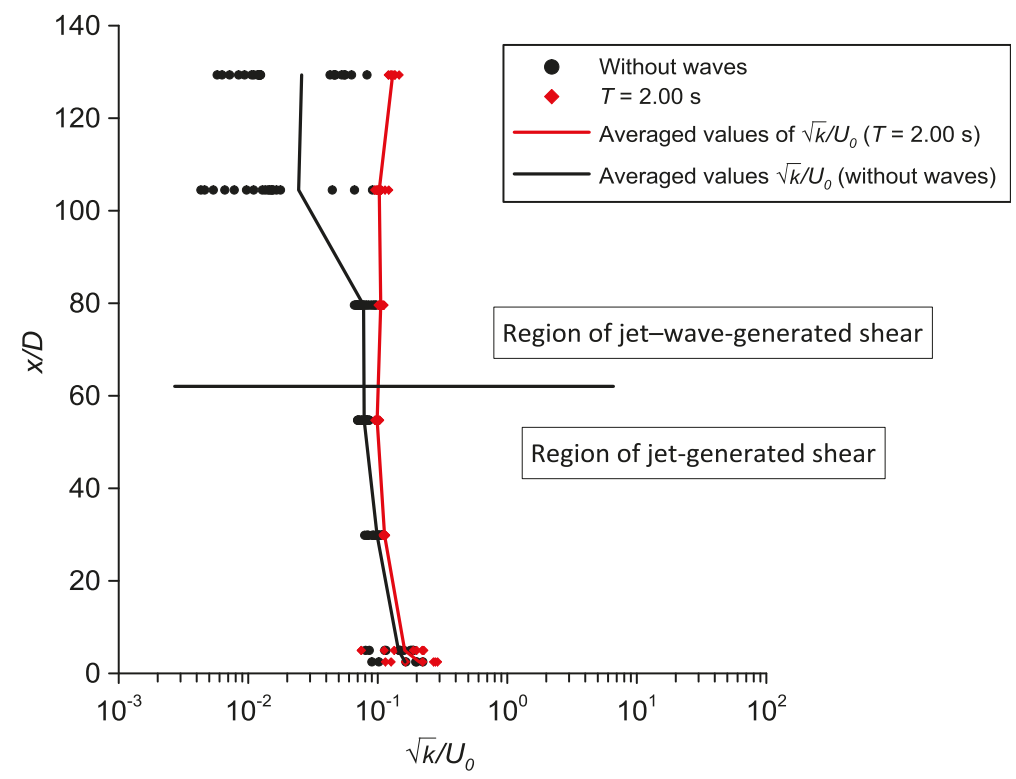

Figure 9. Values of $k^{0.5} / U_{0}$ with the definition of the region with jet-generated turbulence (at the bottom) and the region with jet-wave-generated turbulence (at the top).

Figure 9 shows also that, in the case of the absence of waves, the values of $k$ reduce at a higher distance from the nozzle. On the contrary, in the case of the jet with waves, $\mathrm{O}(k)$ is almost constant in the upper region, demonstrating that the waves feed the jet turbulence.

\subsection{Comparison with Previous Investigations}

Figure 10 shows the values of the mean axial velocity $U_{c}$ made dimensionless by the jet exit velocity $U_{0}$. The results of the present study are compared with those of Albertson et al. [44], Corrsin and Uberoi [45], Wygnanski and Fieldler [46], and Chyan and Hwung [27]. In order to highlight the differences of the experimental conditions, Table 2 shows the main flow conditions and instrumentation used by the authors of the previous investigations. 
It is important to consider that the classic relationships reported in Figure 10 can be applied in the fully developed flow region of a jet [20]. Furthermore, in the case of Run 1 of Table 1 of the present paper, where the measurements have also been assessed closer to the free surface where the wave action is more pronounced, Figure 10 shows that the last three points cannot be described by the classic laws, in agreement with Chyan and Hwung [27]. Therefore, using the experimental points of the jet's fully developed region where the wave action is not more pronounced (i.e., until the section at $110 \mathrm{~mm}$ from the nozzle in the case of the present study), the absolute values of the relative errors between the experimental and theoretical values of $U_{0} / U_{c}$ have a mean value of $7.06 \%$ using the relationship by Albertson et al. [44], 6.29\% using the relationship by Corrsin and Uberoi [45], and 6.72\% using the relationship by Wygnanski and Fiedler [46]. It is possible to conclude that in this region the experimental results of the present paper are well fitted by the classic literature relationships.

Table 2. Main flow conditions and instrumentation used by the authors of the previous investigations.

\begin{tabular}{|c|c|c|c|c|c|c|c|}
\hline Authors & Runs & $\begin{array}{c}\text { Jet Diameter/ } \\
\text { Jet Slot }\end{array}$ & Fluid & $\begin{array}{l}\text { Wave } \\
\text { Period }\end{array}$ & $h / L$ & $U_{0}$ & Velocity Measurements \\
\hline Albertson et al. [44] & All & $\begin{array}{l}\text { From } 0.08 \mathrm{~cm} \\
\text { to } 2.54 \mathrm{~cm}\end{array}$ & Air & - & - & $\begin{array}{l}\text { From } 12.19 \mathrm{~m} / \mathrm{s} \\
\text { to } 54.86 \mathrm{~m} / \mathrm{s}\end{array}$ & $\begin{array}{l}\text { Hypodermic needle with water } \\
\text { differential manometer or sensitive } \\
\text { two-liquid gauge, midget } \\
\text { spoke-vane anemometer }\end{array}$ \\
\hline $\begin{array}{l}\text { Corrsin and } \\
\text { Uberoi [45] }\end{array}$ & All & $2.54 \mathrm{~cm}$ & Air & - & - & $\begin{array}{l}\text { From } 19.81 \mathrm{~m} / \mathrm{s} \\
\text { to } 35.05 \mathrm{~m} / \mathrm{s}\end{array}$ & $\begin{array}{l}\text { Hypodermic needle total-head tube, } \\
\text { Chromel-Alumen thermocouple } \\
\text { (for mean velocity) and hot-wire } \\
\text { anemometer (turbulent } \\
\text { velocity components) }\end{array}$ \\
\hline $\begin{array}{l}\text { Wygnanski and } \\
\text { Fiedler [46] }\end{array}$ & All & $2.64 \mathrm{~cm}$ & Air & - & - & $\begin{array}{l}\text { From } 51 \mathrm{~m} / \mathrm{s} \text { to } \\
72 \mathrm{~m} / \mathrm{s}\end{array}$ & $\begin{array}{l}\text { Constant-temperature anemometers and } \\
\text { linearisers with standard hot wires }\end{array}$ \\
\hline \multirow{7}{*}{$\begin{array}{l}\text { Chyan and } \\
\text { Hwung [27] }\end{array}$} & A & $2.3 \mathrm{~mm}$ & \multirow{7}{*}{ Water } & $1.16 \mathrm{~s}$ & 0.1470 & $83.7 \mathrm{~cm} / \mathrm{s}$ & \multirow{7}{*}{ LDA } \\
\hline & B & $2.3 \mathrm{~mm}$ & & $0.85 \mathrm{~s}$ & 0.2251 & $84.7 \mathrm{~cm} / \mathrm{s}$ & \\
\hline & $\mathrm{C}$ & $2.3 \mathrm{~mm}$ & & $0.75 \mathrm{~s}$ & 0.2731 & $83.6 \mathrm{~cm} / \mathrm{s}$ & \\
\hline & E & $2.3 \mathrm{~mm}$ & & $0.64 \mathrm{~s}$ & 0.3598 & $83.2 \mathrm{~cm} / \mathrm{s}$ & \\
\hline & $\mathrm{F}$ & $2.3 \mathrm{~mm}$ & & $0.52 \mathrm{~s}$ & 0.5343 & $83.4 \mathrm{~cm} / \mathrm{s}$ & \\
\hline & G & $2.3 \mathrm{~mm}$ & & - & - & $82.5 \mathrm{~cm} / \mathrm{s}$ & \\
\hline & $\mathrm{H}$ & $5.0 \mathrm{~mm}$ & & - & - & $62.4 \mathrm{~cm} / \mathrm{s}$ & \\
\hline
\end{tabular}

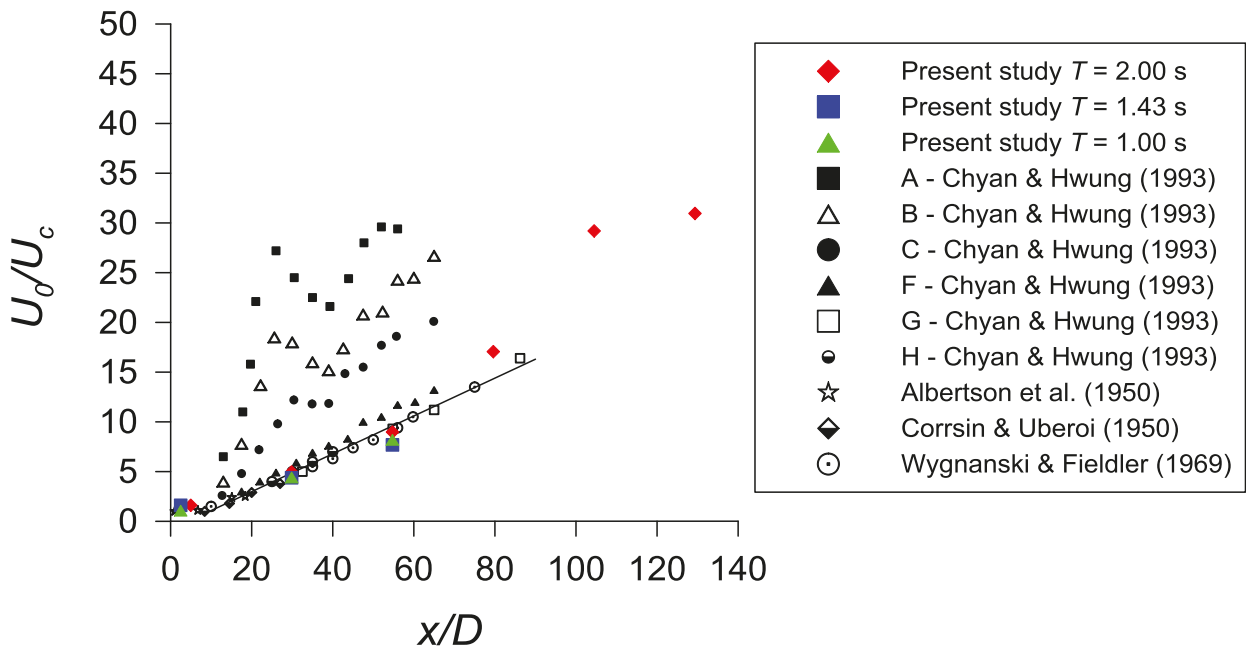

Figure 10. Values of $U_{0} / U_{c}$ along the jet mean axis. 
Using a dimensional analysis approach, Chyan and Hwung [27] proposed the following equation:

$$
\frac{U_{c}}{\widetilde{v}_{w}}=C_{w} \frac{x}{L_{w}}
$$

with $C_{w}$ depending on the relative depth $h / L$. Figure 11 shows that Equation (11) is confirmed by the present study.

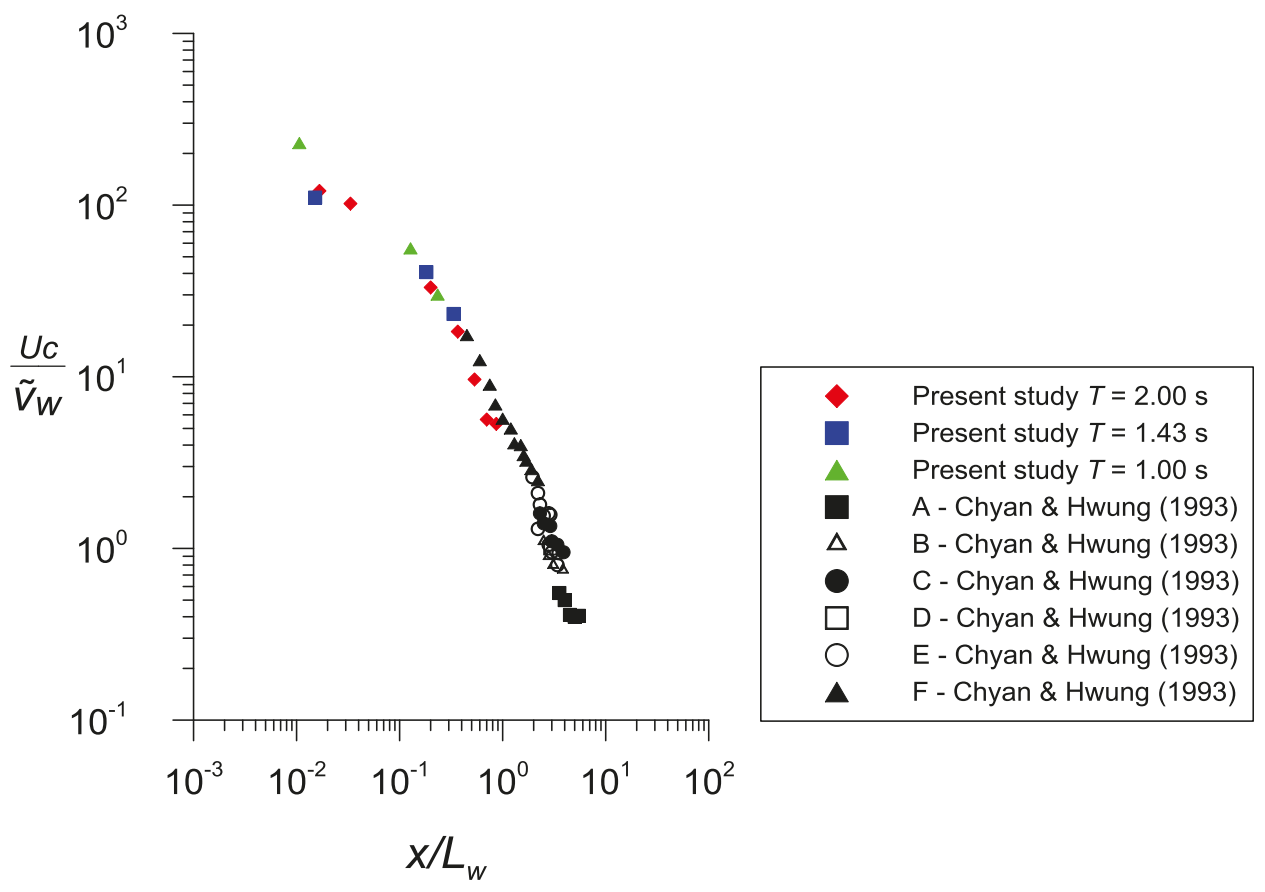

Figure 11. Distribution of the dimensionless values of $U_{c}$ along the dimensionless distance.

\subsection{Turbulent Diffusion Coefficients and Advection Terms}

Figure 12 show the cross profile of the streamwise turbulent diffusion coefficient, $K_{x x}$, for Run 1 of Table 1 and for the same jet issued in still water estimated from Equation (9). It is possible to see that $K_{x x}$ is greater in the case of the jet with waves.

Figure 13 shows the vertical profiles of the advection term $U k$ of Run 1 of Table 1 and of the same jet issued in still water. The other configurations of jets with waves, not reported for the sake of brevity, confirm these results. It is possible to conclude that the longitudinal advection term is greater in the case of jets with waves. 
$x[\mathrm{~mm}]$
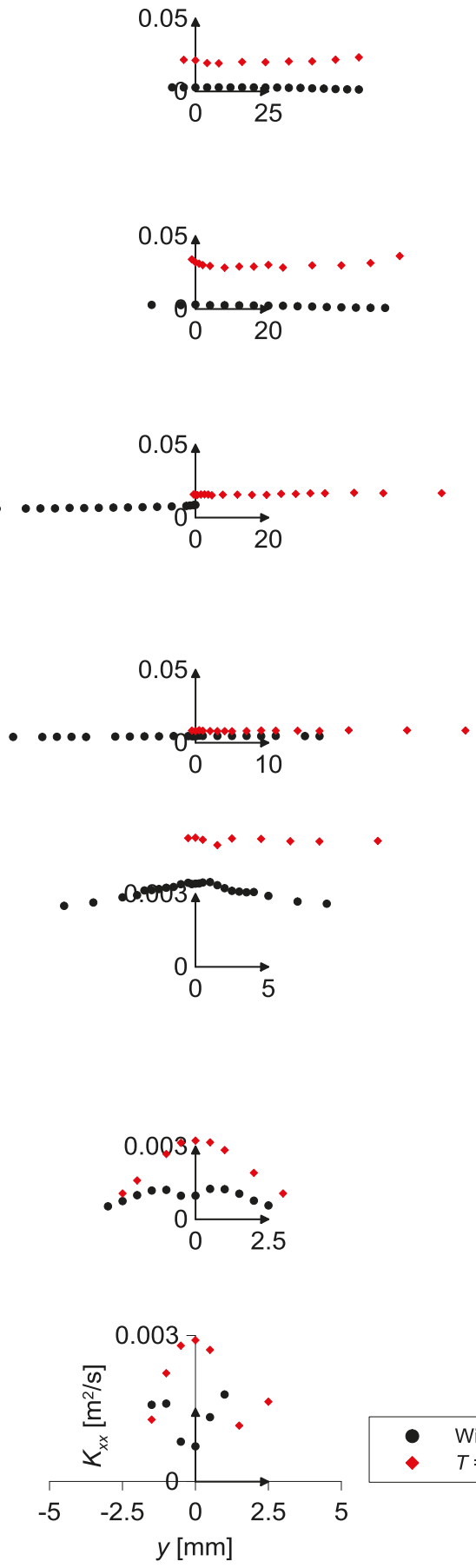

- Without waves

Figure 12. Values of $K_{x x}$ of Run 1 of Table 1 and of the same jet without waves. 

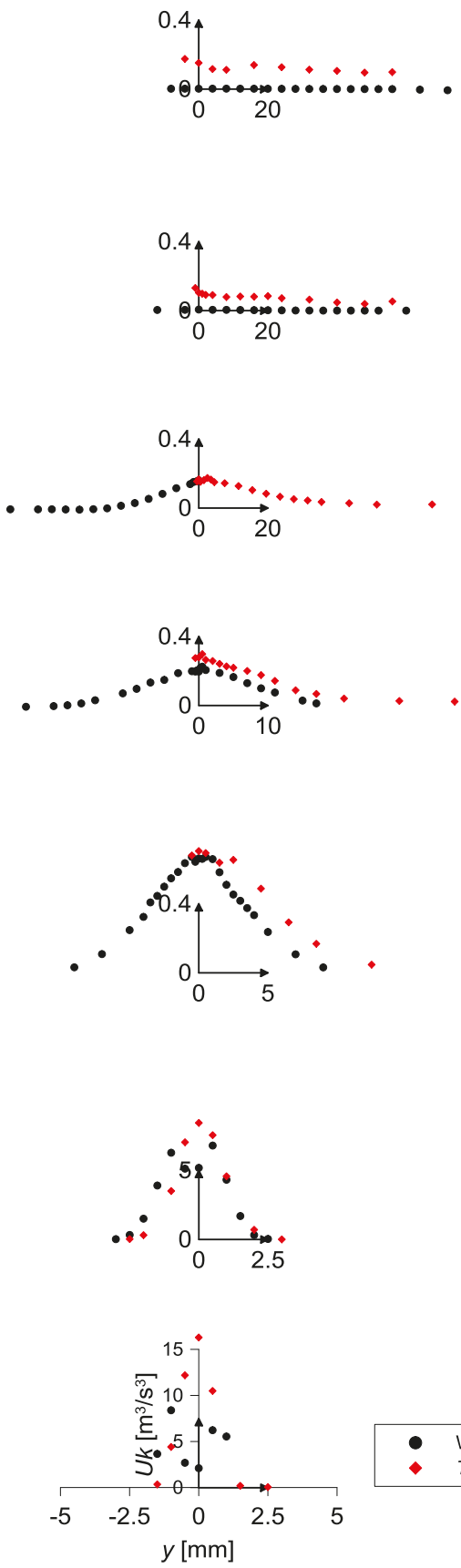

- Without waves

- $T=2.00 \mathrm{~s}$

Figure 13. Values of $U k$ of Run 1 of Table 1 and of the same jet without waves. 
Figure 14 shows the values of the streamwise turbulent diffusion coefficient $K_{x x}$ of each cross section of the jet as a function of the distance from the nozzle. The dots in Figure 14 represent individual estimates at all $y$ positions in the analysed cross sections and the lines represent their averages.

Figure 15 shows the values of $U k$ of each cross section of Run 1 of Table 1 and of the same jet without waves. The dots in Figure 13 represent individual estimates at all $y$ positions in the analysed cross sections with the lines representing the averages.

For the sake of brevity, Figures 14 and 15 show the data of Run 1 of Table 1 and of the same jet without waves, but the results are confirmed also by the other analysed configurations with waves. The averaged values of Figures 14 and 15 demonstrate that the streamwise diffusion coefficient $K_{x x}$ and the streamwise advection of the jet with waves are greater than those of the same jet issued into still water. Furthermore, the differences of values of $K_{x x}$ for the jets with and without waves increase with the distance from the nozzle.

In any case, the advection term is of the same order of magnitude at a distance from the nozzle of $\mathrm{O}\left(<L_{w}\right)$, i.e., in the jet region where the initial jet momentum is of the order of the wave-induced momentum. These results are better shown in Figures 14 and 15 with the lines of averaged values, which enable us to quantify the difference between the cases with and without waves. In the region closer to the nozzle, the longitudinal advection terms of jets issued into a wave environment or into still water are comparable, demonstrating that the wave action is less pronounced. From the experimental results presented above, it is possible to conclude that the presence of waves increases both the diffusion and advection processes in the longitudinal direction in the jet region where the wave-induced momentum is greater than the initial jet momentum.

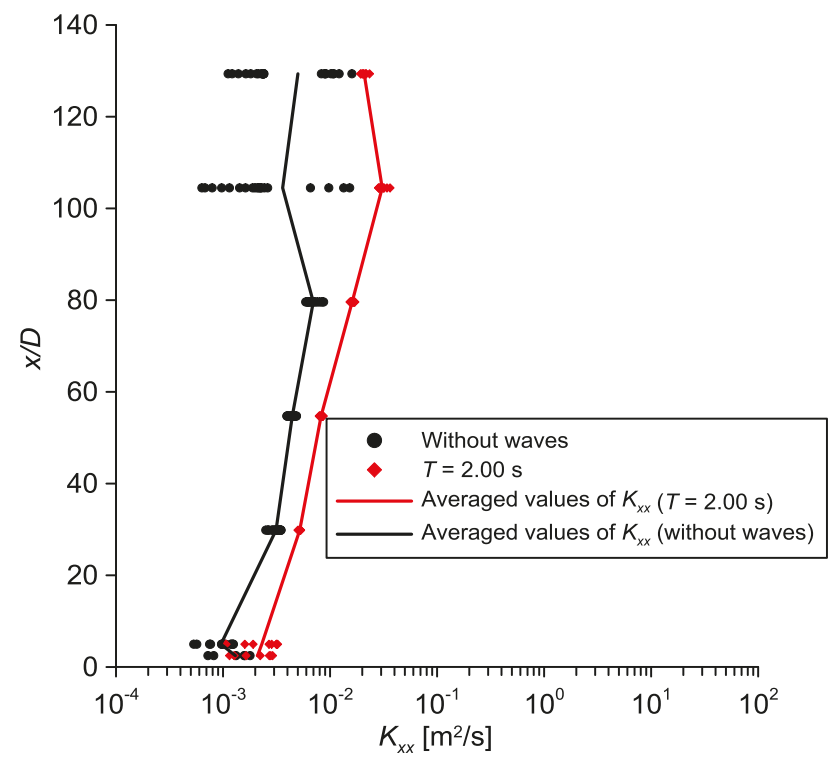

Figure 14. Values of $K_{x x}$ of Run 1 of Table 1 and of the same jet without waves with lines of the averaged values of each cross section. 


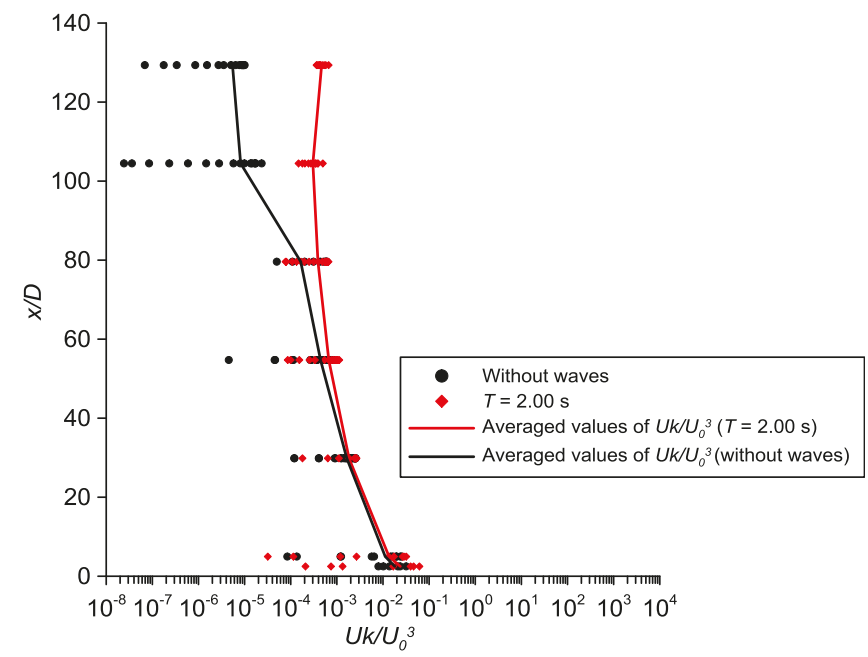

Figure 15. Values of $U k$ of Run 1 of Table 1 and of the same jet without waves with lines of the averaged values of each cross section.

\section{Conclusions}

Turbulent jets flowing in currents or still water have been widely examined because of their relevance to many environmental conditions. This study examines turbulent nonbuoyant jets issued into a wave environment. The main conclusions can be summarised as follows:

1. The analysis of the time-averaged and phase-averaged velocity vectors shows that the flow pattern resembles that of a jet discharged into a cross-current when the wave horizontal velocity dominates and that of a co-stream jet or jet in opposing flow when the vertical wave velocity dominates. Furthermore, the jet experiences an enlargement of its area when waves are present compared with the same jet in still water, which suggests an enhancement of the dilution and, therefore, a positive effect of the wave motion during the initial mixing processes. The velocity profiles of jets discharged in a wave environment are even flatter and wider than those of the same jet discharged in a stagnant environment.

2. The analysis of the turbulent kinetic energy $k$ nondimensionalised by $U_{0}$ shows that close to the nozzle, specifically when $x<<L_{w}$, the values are similar with and without waves, indicating that the impact of the waves on the turbulent kinetic energy is small. In this region it is possible to affirm that the turbulence is dominated by the jet and, therefore, this is a region with jet-generated shear. Farther from the nozzle, i.e., when $x$ is closer to or greater than $L_{w}$, the values of $k$ are higher with the presence of waves. In this region the turbulence of the jet is also affected by the waves. Furthermore, in the case of the jet with waves, $\mathrm{O}(k)$ is almost constant in the upper region, demonstrating that the waves feed the jet turbulence. On the contrary, in the case of the absence of waves, the values of $k$ reduce at higher distance from the nozzle.

3. The values of the mean axial velocity $U_{c}$ normalised by the jet exit velocity $U_{0}$ show that the configurations analysed in the present paper are well fitted by the classic literature laws for the measurement points in the jet's fully developed region where the wave effect is not pronounced.

4. The experimental data agree with Chyan and Hwung's [27] law of the dimensionless $U_{c}$ velocity as a function of $x / L_{w}$.

5. Using the analogy between the equation of the turbulent transport of a solute concentration and the equation of the turbulent kinetic energy, the jet net dispersion coefficients have been 
evaluated. In contrast to the case of jets in still water, in the runs with waves, the streamwise turbulent diffusion is increased.

6. The presence of waves increases both the diffusion and advection processes of the jet in the longitudinal direction, mainly in the region dominated by the wave-induced momentum, i.e., at distance from the nozzle greater than the length scale $L_{w}$.

Supplementary Materials: The following are available online at http://www.mdpi.com/2073-4441/10/4/522/s1. The two movies depict Run 1 of Table 1 of the paper (file jet_with_waves_run_1_Mossa.avi) and the same jet issued into still water (file jet_in_still_water.avi). A dye called Rhodamine B (C28H31CIN2O3) was used to visualize the jet flow pattern.

Author Contributions: M.M. conceived the experimental set up and carried out the experiments; both authors contributed to analysis of the data, writing of the paper, and discussions and review of the manuscript.

Funding: Peter Davies acknowledges the support of the UK Engineering and Physical Sciences Research Council (Grant EP/G066124/1).

Conflicts of Interest: The authors declare no conflict of interest.

\section{References}

1. Mossa, M. Field measurements and monitoring of wastewater discharge in sea water. Estuar. Coast. Shelf Sci. 2006, 68, 509-514. [CrossRef]

2. De Serio, F.; Mossa, M. A laboratory study of irregular shoaling waves. Exp. Fluids 2013, 54, 1536. [CrossRef]

3. Ben Meftah, M.; De Serio, F.; Malcangio, D.; Mossa, M.; Petrillo, A.F. Experimental study of a vertical jet in a vegetated crossflow. J. Environ. Manag. 2015, 164, 19-31. [CrossRef] [PubMed]

4. Armenio, E.; De Serio, F.; Mossa, M. Analysis of data characterizing tide and current fluxes in coastal basins. Hydrol. Earth Syst. Sci. 2017, 21, 3441. [CrossRef]

5. De Serio, F.; Mossa, M. Environmental monitoring in the Mar Grande basin (Ionian Sea, Southern Italy). J. Environ. Sci. Pollut. Res. 2016, 23, 12662-12674. [CrossRef] [PubMed]

6. List, E.J. Turbulent jets and plumes. Annu. Rev. Fluid Mech. 1982, 14, 189-212. [CrossRef]

7. Davies, P.A.; Valente Neves, M.J. Recent Research Advances in the Fluid Mechanics of Turbulent Jets and Plumes; NATO ASI Series, Series E: Applied Sciences; Springer Science \& Business Media: New York, USA, 2012; Volume 255, ISBN 978-94-010-4396-0. [CrossRef]

8. Lee, J.H.W.; Chu, V.H. Turbulent Jets and Plumes-A Lagrangian Approach; Kluwer Academic Publishers: Dordrecht, the Netherlands, 2003; ISBN 1402075200.

9. Wood, I.R.; Bell, R.G.; Wilkinson, D.L. Ocean Disposal of Wastewater; Advanced Series on Ocean Engineering; World Scientific: Singapore, 1993; Volume 8.

10. Roberts, P.J.W.; Salas, H.J.; Reiff, F.M.; Libhaber, M.; Labbe, A.; Thomson, J.C. Marine Wastewater Outfalls and Treatment Systems; IWA Publishing: Colchester, UK, 2010; ISBN 1843391899.

11. Tate, P.M.; Scaturro, S.; Cathers, B. Marine outfalls. In Springer Handbook of Ocean Engineering; Dhanak, M.R., Xiros, M.I., Eds.; Springer: Cham, Switzerland; Berlin, Germany, 2016; pp. 711-740, ISBN 978-3-319-16648-3.

12. Jirka, G.H.; Harleman, D.R.F. Stability and mixing of a vertical plane buoyant jet in confined depth. J. Fluid Mech. 1979, 94, 275-304. [CrossRef]

13. Papanicolaou, P.N.; List, E.J. Investigation of round vertical turbulent buoyant jets. J. Fluid Mech. 1988, 195, 341-391. [CrossRef]

14. Atkinson, J.F.; Wolcott, S.B. Interfacial mixing induced by mean shear and an oscillating grid. J. Hydraul. Eng. 1990, 116, 397-413. [CrossRef]

15. Smith, S.H.; Mungal, M.G. Mixing, structure and scaling of the jet in crossflow. J. Fluid Mech. 1998, 357, 83-122. [CrossRef]

16. Jirka, G.H. Large scale flow structures and mixing processes in shallow flows. J. Hydraul. Res. 2001, 39, 567-573. [CrossRef]

17. Shuto, N.; Ti, L.H. Wave effects on buoyant plumes. In Proceedings of the 14th Conference on Coastal Engineering, Copenhagen, Denmark, 24-28 June 1974; pp. 2199-2209.

18. Ger, A.M. Wave effects on submerged buoyant jets. In Proceedings of the 18th IAHR, Cagliari, Italy, 10-14 September 1979; pp. 295-300. 
19. Sharp, J.J. The effects of waves on buoyant jets. Proc. Inst. Civ. Eng. 1986, 81, 471-475.

20. Rajaratnam, N. Turbulent Jets; Elsevier Scientific Publishing Company: Amsterdam, The Netherlands; Oxford, UK; New York, NY, USA, 1976.

21. Fischer, H.B.; List, E.J.; Koh, R.C.Y.; Imberger, J.; Brooks, N.H. Mixing in Inland and Coastal Waters; Academic Press: New York, NY, USA, 1979.

22. Peregrine, D.H. Interaction of water waves and currents. Adv. Appl. Mech. 1976, 16, 9-117.

23. Roberts, P.J.W. Ocean outfall dilution: Effects of currents. J. Hydraul. Div. 1980, 106, 769-782.

24. Ismail, N.M.; Wiegel, R.L. Opposing wave effect on momentum jets spreading rate. J. Waterw. Port Coast. Ocean Eng. 1983, 109, 465-483. [CrossRef]

25. Chin, D.A. Influence of surface waves on outfall dilution. J. Hydraul. Eng. 1987, 113, 1006-1017. [CrossRef]

26. Yoon, S.B.; Liu, P.L.-F. Effects of opposing waves on momentum jets. J. Waterw. Port Coast. Ocean Eng. 1990, 116, 545-557. [CrossRef]

27. Chyan, J.-M.; Hwung, H.-H. On the interaction of a turbulent jet with waves. J. Hydraul. Res. 1993, 31, 791-810. [CrossRef]

28. Koole, R.; Swan, C. Measurements of a 2-D non-buoyant jet in a wave environment. Coast. Eng. 1994, 24, 151-169. [CrossRef]

29. Calabrese, M.; Di Natale, M. Diffusione di un getto liquido sommerso in presenza di un moto ondoso stazionario. In Proceedings of the XXIV Convegno di Idraulica e Costruzioni Idrauliche, Napoli, Italy, 20-22 September 1994; Volume I, pp. 241-254. (In Italian)

30. Nash, J.D.; Jirka, G.H. Buoyant surface discharges into unsteady ambient flows. Dyn. Atmos. Oceans 1996, 24, 75-84. [CrossRef]

31. Wu, S.; Rajaratnam, N.; Katopodis, C. Oscillating vertical plane turbulent jet in shallow water. J. Hydraul. Res. 1998, 36, 229-234. [CrossRef]

32. Mossa, M. Experimental study on the interaction of non-buoyant jets and waves. J. Hydraul. Res. 2004, 42, 13-28. [CrossRef]

33. Mossa, M. Behavior of non-buoyant jets in a wave environment. J. Hydraul. Eng. 2004, 130, 704-717. [CrossRef]

34. Chin, D.A. Model of buoyant-jet-surface-wave interaction. J. Waterw. Port Coast. Ocean Eng. 1988, 114, 331-345. [CrossRef]

35. Davies, P.A.; Mofor, L.A.; Valente Neves, M.J. Comparisons of remotely sensed observations with modeling predictions for the behaviour of wastewater plumes from coastal discharges. Int. J. Remote Sens. 1997, 18, 1987-2019.

36. Lee, J.H.W.; Wilkinson, D.L.; Wood, I.R. On the head-discharge relation of a "duckbill" elastomer check valve. J. Hydraul. Res. 2001, 39, 619-627. [CrossRef]

37. Cuthbertson, A.J.S.; Malcangio, D.; Davies, P.A.; Mossa, M. The influence of a localised region of turbulence on the structural development of a turbulent, round, buoyant jet. Fluid Dyn. Res. 2006, 38, 683-698. [CrossRef]

38. Mossa, M.; De Serio, F. Rethinking the process of detrainment: Jets in obstructed natural flows. Sci. Rep. 2016, 6, 39103. [CrossRef] [PubMed]

39. Mossa, M.; Ben Meftah, M.; De Serio, F.; Nepf, H.M. How vegetation in flows modifies the turbulent mixing and spreading of jets. Sci. Rep. 2017, 7, 6587. [CrossRef] [PubMed]

40. Hussain, A.K.M.F.; Reynolds, W.C. The mechanics of an organized wave in turbulent shear flow. J. Fluid Mech. 1970, 41, 241-258. [CrossRef]

41. Svendsen, I.A. Analysis of surf zone turbulence. J. Geophys. Res. 1987, 92, 5115-5124. [CrossRef]

42. Tanino, Y.; Nepf, H.M. Lateral dispersion in random cylinder arrays at high Reynolds number. J. Fluid Mech. 2008, 600, 339-371. [CrossRef]

43. Le Méhauté, B. An Introduction to Hydrodynamics and Water Waves; Water Wave Theories, II, TR ERL 118-POL-3-2; U.S. Department of Commerce, ESSA: Washington, DC, USA, 1969.

44. Albertson, M.L.; Dai, Y.B.; Jensen, R.A.; Rouse, H. Diffusion of submerged jets. Trans. ASCE 1950, 115, $639-664$.

45. Corrsin, S.; Uberoi, M.S. Further experiments on the flow and heat transfer in a heated turbulent air jet. NACA Rep. 1950, 998, 1-17.

46. Wygnanski, I.J.; Fiedler, H.E. Some measurements in the self-preserving jet. J. Fluid Mech. 1969, 38, 577-612.

(C) 2018 by the authors. Licensee MDPI, Basel, Switzerland. This article is an open access article distributed under the terms and conditions of the Creative Commons Attribution (CC BY) license (http:/ / creativecommons.org/licenses/by/4.0/). 


\title{
Effect of the Junction Angle on Turbulent Flow at a Hydraulic Confluence
}

\author{
Nadia Penna ${ }^{1, *}$, Mauro De Marchis ${ }^{2}$, Olga B. Canelas ${ }^{3}$, Enrico Napoli ${ }^{4}$, António H. Cardoso ${ }^{3}$ \\ and Roberto Gaudio ${ }^{1}$ \\ 1 Dipartimento di Ingegneria Civile, Università della Calabria, 87036 Rende, Italy; roberto.gaudio@unical.it \\ 2 Dipartimento di Ingegneria Civile e Ambientale, Università degli Studi di Enna 'Kore', 94100 Enna, Italy; \\ mauro.demarchis@unikore.it \\ 3 CERIS, Instituto Superior Tecnico, Universidade de Lisboa, 1649-004 Lisbon, Portugal; \\ olga.birjukova@ist.utl.pt (O.B.C.); antonio.cardoso@ist.utl.pt (A.H.C.) \\ 4 Dipartimento di Ingegneria Civile, Ambientale, Aerospaziale, dei Materiali, \\ Università degli Studi di Palermo, 90133 Palermo, Italy; enrico.napoli@unipa.it \\ * Correspondence: nadia.penna@unical.it; Tel.: +39-0984-496-553
}

Received: 17 February 2018; Accepted: 8 April 2018; Published: 12 April 2018

\begin{abstract}
Despite the existing knowledge concerning the hydrodynamic processes at river junctions, there is still a lack of information regarding the particular case of low width and discharge ratios, which are the typical conditions of mountain river confluences. Aiming at filling this gap, laboratory and numerical experiments were conducted, comparing the results with literature findings. Ten different confluences from $45^{\circ}$ to $90^{\circ}$ were simulated to study the effects of the junction angle on the flow structure, using a numerical code that solves the 3D Reynolds Averaged Navier-Stokes (RANS) equations with the $k-\epsilon$ turbulence closure model. The results showed that the higher the junction angle, the wider and longer the retardation zone at the upstream junction corner and the separation zone, and the greater the flow deflection at the entrance of the tributary into the post-confluence channel. Furthermore, it was shown that the maximum streamwise velocity does not necessarily increase with the junction angle and that it is not always located in the contraction section.
\end{abstract}

Keywords: channel confluences; junction angle; flow deflection zone; flow retardation zone; flow separation zone; numerical modelling; PANORMUS

\section{Introduction}

A confluence is a geomorphological node where typically two upstream channels merge into a single downstream channel, giving rise to complex 3D flow patterns. By definition, the main stream is the longest of the two intersecting channels, whereas the tributary is the shortest. Considering that $Q_{m}$ and $U_{m}$ are the discharge and the mean velocity of the upstream main channel, and $Q_{t}$ and $U_{t}$ are the discharge and the mean velocity of the tributary channel, respectively, the discharge ratio between the two rivers $\left(Q_{r}\right)$ was defined by Best [1] as $Q_{t} / Q_{m}$. Similarly, the ratio between the tributary width $\left(B_{t}\right)$ and the main channel width $\left(B_{m}\right)$ is known as width ratio $\left(B_{r}\right)$.

Although Taylor [2] was the first to study an asymmetrical channel confluence, proposing a $1 \mathrm{D}$ approach for the calculation of the water depth ratio between the upstream and downstream channels, the complete description of the flow field within a channel confluence is attributed to Best [1]. In fact, he distinguished six elements within a confluence (Figure 1): (1) a retardation zone at the upstream junction corner (commonly referred to as flow stagnation zone, even though it is characterized by reduced velocity that never decreases to zero); (2) a flow deflection zone, where each stream enters the confluence; (3) a flow separation zone, beyond the downstream junction corner; (4) an area of maximum velocity, located downstream of the confluence; (5) a flow recovery area, 
where the post-confluence flow tends to become unaffected by the confluence hydrodynamics; and (6) two main shear layers, caused by strong velocity gradients between the separation zone and the surrounding flow and between the flows coming from the tributary and main channels.

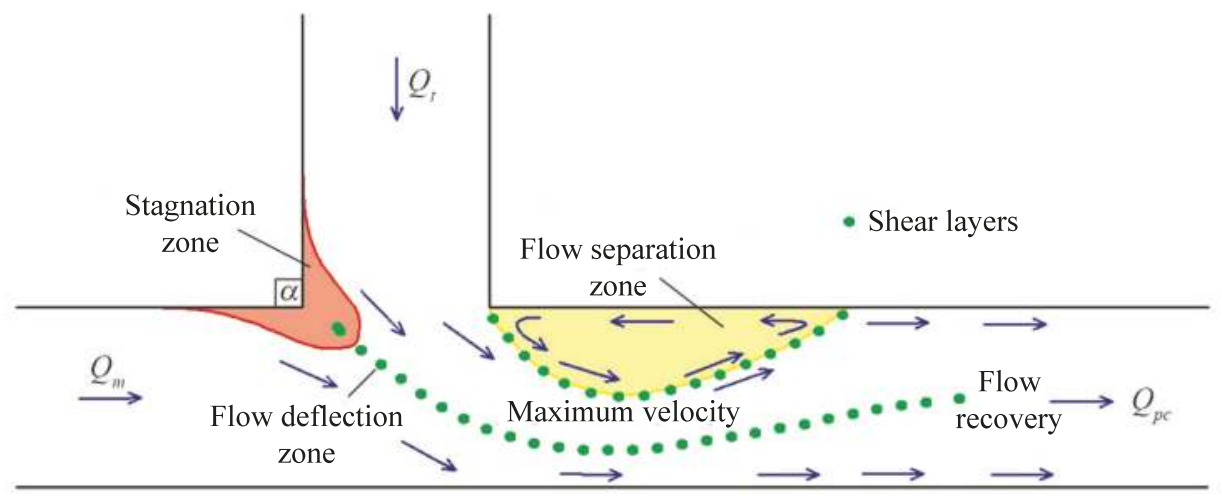

Figure 1. Conceptual model of flow dynamics at river confluences, modified from Best [1]. $Q_{p c}$ is the post-confluence discharge; $\alpha$ is the junction angle.

These zones depend, essentially, on the geometry (such as number of adjoining channels, cross-sectional area, junction angle $\alpha$, degree of concordance in elevation of the channel beds at the entrance to the confluence), flow and sediment parameters (such as $Q_{r}$ between the two streams, bed material, sediment transport). The effects of these factors have been examined with both experimentally derived junctions in laboratory (e.g., [1,3,4]), and field investigations (e.g., [5-9]), recently integrated with numerical modelling (e.g., [10-12]). In particular, with laboratory experiments, it was possible to isolate and accentuate the effect of certain parameters and processes under controlled conditions, which then were varied by using numerical simulations in order to overcome limitations of the physical models.

Initially, focusing on the basic case of asymmetrical confluences with a fixed concordant bed and subcritical flow, most attention in the experimental campaigns found in the literature has been paid to determine relationships for the calculation of the flow depth or the energy loss at the junction (e.g., $[13,14])$. Subsequently, the determination of the shape and the extent of the separation zone has become the main objective of the research in the field of open-channel confluences, owing to its importance in (1) confining the effective width of the post-confluence channel towards the opposite wall of the junction, giving rise to the adjacent zone of maximum velocity and bed shear stress, and (2) in the overall head losses at junctions, as well as sediment and solute balances [15]. For instance, through vertical photography of surface dye traces, Best and Reid [16] showed that the width and length of the flow separation zone increases with both $\alpha$ and the discharge ratio. Biron et al. [3] conducted a series of experiments in order to demonstrate the influence of the elevation of bed discordance on the characteristic zones of a confluence, with respect to the case of concordant beds. They showed that the difference in bed elevation destroys the flow deflection at the bed and creates a distortion of the mixing layer between the flows, resulting in fluid upwelling at the downstream junction corner. This phenomenon is responsible for the absence of a flow separation zone near the bed at the downstream junction corner and the lack of a zone of marked flow acceleration in the post-confluence channel. Gurram et al. [17], with their experimental work, yielded expressions for the pressure force on the lateral side walls, the ratio of flow depths in the junction point, the momentum correction coefficients, the extent of the separation zone determined with dye injections, and the lateral momentum contribution. They found that the separation zone increases with increasing values of Froude number $(F r), Q_{r}$ and $\alpha$. Shumate [18] studied the general junction flow features 
occurring in a $90^{\circ}$ confluence. In particular, he found that the separation zone is larger near the surface, both in length and width. Moreover, up to a threshold, the separation zone lengthens as $Q_{r}$ increases. Based on new confluence experiments, the detailed characteristics of the separation zone were analysed by Wang et al. [19] and Qing-Yuan et al. [20]. Specifically, Wang et al. [19] observed a zone of separation immediately downstream of the junction branch channel, the maximum and minimum velocity regions at the upstream and downstream in the confluent channel, and a shear plane developed between the two combining flows downstream of the confluent channel. Secondary circulations in different directions at the highest and lowest velocity zones were observed as well. Qing-Yuan et al. [20] used two different methods based on velocity streamlines and velocity isolines to investigate the geometry of the separation zone, which presented different forms with variations of distance to the flume bottom. Furthermore, they showed that the discharge ratio also influences the separation zone from bottom to surface. Also in the experiments of Biswa et al. [21], the width and the length of the flow separation zone increase with the contribution from the tributary channel to the discharge in the post-confluence channel $\left(Q_{p c}\right)$, while its shape remains practically unchanged. The most recent experimental campaigns were conducted separately by Schindfessel et al. [22] and by Birjukova et al. [23], investigating the time-averaged flow patterns in a $90^{\circ}$ confluence when $Q_{t}$ largely exceeds $Q_{m}$ [22] and in a $70^{\circ}$ confluence when $Q_{m}$ largely exceeds $Q_{t}$ [23]. In the first case, the separation zone was wider with respect to the literature reference cases, and a recirculating eddy appeared in the upstream main channel, confining the main channel incoming discharge and inducing a zone of reduced downstream momentum in its wake. Moreover, a new recirculation cell appeared with respect to the confluences studied by Gurram et al. [17] and Shumate [18], resulting in a change of sign of the lateral surface velocity near the bank opposite to the junction. Birjukova et al. [23] observed that, in their conditions, the separation zone limits the effective lateral flow cross-section, and hence results in the added acceleration of the mainstream flow near the downstream junction corner. Finally, Yuan et al. [24] analysed the distortion of the shear layer developed from the upstream junction corner at a $90^{\circ}$ open-channel confluence with a small width-to-depth ratio. They found that the distortion of the shear layer resulted in an increase in occurrence probabilities of ejection and sweep events within the shear layer, which were related to the turbulence presenting vortices induced by the wall.

In order to compare the experimental tests found in the literature, concerning laboratory asymmetrical confluences with fixed beds, Table 1 shows for each of them a brief overview of the geometrical and hydraulic conditions adopted. Moreover, it shows for each of the numerical simulations found in the literature: (1) the respective reference of the experimental tests used for the validation of the models and (2) the geometrical and hydraulic conditions. 
Table 1. Experimental tests and numerical studies on channel confluences in chronological order.

\begin{tabular}{|c|c|c|c|c|c|}
\hline Reference & Study ${ }^{a}$ & $\begin{array}{c}\text { Reference of the } \\
\text { Experimental } \\
\text { Test }\end{array}$ & $Q_{r}$ & $B_{r}$ & $\alpha\left({ }^{\circ}\right)$ \\
\hline Webber and Greated [13] & $\mathrm{E}$ & - & $0.25 \div 4.00$ & 1.00 & $30,60,90$ \\
\hline Lin and Soong [25] & $\mathrm{E}$ & - & $0.18 \div 1.40$ & 1.00 & 90 \\
\hline Best and Reid [16] & $\mathrm{E}$ & - & $0.11 \div 9.00$ & 1.00 & $15,45,70,90$ \\
\hline Ramamurthy et al. [14] & $\mathrm{E}$ & - & $0.30 \div 1.50$ & 0.99 & 90 \\
\hline Biron et al. [3] & $\mathrm{E}$ & - & 1.23 & 0.67 & 30 \\
\hline Gurram et al. [17] & $\mathrm{E}$ & - & $0.33 \div 3.00$ & $0.60 \div 1.00$ & $30,60,90$ \\
\hline Hsu et al. [26] & $\mathrm{E}$ & - & $0.11 \div 8.90$ & 1.00 & 90 \\
\hline Hsu et al. [27] & $\mathrm{E}$ & - & $0.09 \div 9.31$ & 1.00 & $30,45,60$ \\
\hline Shumate [18] & $\mathrm{E}$ & - & $0.09 \div 11.00$ & 1.00 & 90 \\
\hline Bradbrook et al. [28] & $\mathrm{N}$ & Biron et al. [3] & - & 1.00 & $30,45,60$ \\
\hline Biron et al. [10] & $\mathrm{N}$ & Biron et al. [3] & $0.67,1.74$ & 1.00 & $30,60,90$ \\
\hline Wang et al. [19] & $\mathrm{E}$ & - & 0.77 & 0.40 & 30 \\
\hline Qing-Yuan et al. [20] & $\mathrm{E}$ & - & 0.36 & 0.50 & 90 \\
\hline Biswa et al. [21] & $\mathrm{E}$ & - & $0.48 \div 2.09$ & 1.00 & 90 \\
\hline Shakibainia et al. [29] & $\mathrm{N}$ & Shumate [18] & $0.33,1.00,3.00$ & $0.66,1.00$ & $15,45,90,105$ \\
\hline Bonakdari et al. [30] & $\mathrm{N}$ & Wang et al. [19] & 0.77 & 0.40 & 30 \\
\hline Djordjevic [12] & $\mathrm{N}$ & Shumate [18] & 0.71 & 1.00 & 90 \\
\hline Yang et al. [31] & $\mathrm{N}$ & Shumate [18] & 3.00 & 1.00 & 90 \\
\hline Schindfessel et al. [22] & $\mathrm{E}$ & - & $3.00,19.00$ & 1.00 & 90 \\
\hline Birjukova et al. [23] & $\mathrm{E}$ & - & 0.11 & 0.15 & 70 \\
\hline Brito et al. [32] & $\mathrm{N}$ & Birjukova et al. [23] & 0.11 & 0.15 & 70 \\
\hline Schindfessel et al. [33] & $\mathrm{N}$ & Schindfessel et al. [22] & $0,3.00,19.00$ & 1.00 & 90 \\
\hline Yuan et al. [24] & $\mathrm{E}$ & - & 0.65 & 1.00 & 90 \\
\hline Schindfessel et al. [15] & $\mathrm{N}$ & Schindfessel et al. [22] & 3 & 1.00 & 90 \\
\hline
\end{tabular}

${ }^{a}$ E: experimental study; N: numerical study.

In fact, using as a reference some of the experimental tests of Table 1 for the calibration of their models, several authors have performed Computational Fluid Dynamics (CFD) simulations to investigate detailed flow structures at confluences, changing the geometry, the hydraulic conditions or both. In particular, most of the CFD simulations have been conducted using the Reynolds Averaged Navier-Stokes (RANS) equations or Large Eddy Simulation (LES) models. For instance, the data of Biron et al. [3] were taken into account for the simulations of Bradbrook et al. [28] and Biron et al. [10]. Shakibainia et al. [29] used SSIIM 2.0, a RANS-based turbulent model, to reproduce the experiments of Shumate [18]. The same model and experimental test were used by Djordjevic [12] to study the effects of upstream planform curvature and bed elevation discordance between the tributary and main channels on the confluence hydrodynamics. However, Yang et al. [31], by using the commercial software FLUENT (ANSYS Inc., Canonsburg, PA, USA) and, again, the data of Shumate [18], showed how the adoption of dynamic meshes could give much higher accuracy than that of Volume of Fluid (VoF) or rigid lid method. The experimental tests of Birjukova et al. [23] were reproduced by Brito et al. [32], comparing the results obtained with different turbulence closure models for solving the RANS equations. More recently, Schindfessel et al. [33] used LES of the OpenFOAM suite to investigate the flow patterns for three different $Q_{r}$, considering the experimental tests by Schindfessel et al. [22]. They demonstrated that the tributary flow impinges on the opposing bank when the tributary flow becomes sufficiently dominant, causing a recirculating eddy in the upstream 
channel of the confluence, which induces significant changes in the incoming velocity distribution. The changed flow patterns also influence the mixing layer and the flow recovery. In their successive work, Schindfessel et al. [15] analysed the influence of the cross-sectional shape on the flow patterns in a $90^{\circ}$ confluence. They showed that the shape of the cross-section, and more particularly the geometry of the downstream corner, can induce lateral currents directed into the separation zone.

Despite the extensive research in hydrodynamic processes at junctions, it is evident how there is still a lack of information regarding channel confluences characterized by low discharge and width ratios. On a prototype scale, these conditions are characteristic of highly channelized mountain-river confluences and not common for low-land confluences, which, on the contrary, have inspired most of the literature studies on that topic $[4,34]$. Moreover, only a few studies have analysed the changes in the flow dynamics at a confluence as a function of the junction angle and all of them are referred to $B_{r}=1$, except the cases investigated by Gurram et al. [17] and Shakibainia et al. [29], for which the minimum value of $Q_{r}$ was set equal to 0.33 . Therefore, the research works in this field are not comprehensive and the extension of the existing knowledge to not yet explored conditions must be pursued.

In light of the above considerations, the paper describes the effects of the junction angle on the flow structure of a confluence with fixed concordant beds and both low width and discharge ratios, on the basis of the experimental campaign carried out by Birjukova et al. [23].

To this end, numerical simulations were initially carried out through the open-source numerical model known as PANORMUS (PArallel Numerical Open-souRce Model for Unsteady flow Simulation; [35]), available at www.panormus3d.org, which was adapted on purpose to the cases studied hereinafter. Then, the results of the numerical simulations were compared to the experimental data of Birjukova et al. [23] to validate the consistency of the model. Besides the original layout of the physical model, other nine layouts with different $\alpha$ values from $45^{\circ}$ to $90^{\circ}$ were considered, in order to analyse their effects with particular attention paid to: (1) the extension of the retardation zone in the main and tributary channels; (2) the deflection of the tributary flow from the junction angle; (3) the length and width of the separation zone; and (4) the maximum streamwise velocity in the flow structure of the confluence.

Therefore, this study contributes to a better understanding of the flow processes occurring in a channel confluence, seeking to answer the following two main questions:

1. What happens at low width and discharge ratios when junction angle is modified? How does this modification influence the flow dynamics?

2. What do lower flow and width ratios imply with respect to the literature cases?

\section{Description of Experiments and Simulations}

\subsection{Laboratory Flume: Set-up and Measurements}

The experiment was conducted in the horizontal rectangular concrete flume of the Hydraulics Laboratory of the Instituto Superior Técnico (IST), Lisbon, Portugal (Figure 2a,b) as described in Birjukova et al. [23]. The length of the main and tributary channels were $L_{m}=12 \mathrm{~m}$ and $L_{t}=4.5 \mathrm{~m}$, respectively, and the junction was located $5 \mathrm{~m}$ downstream of the inlet of the main channel, forming a $70^{\circ}$ confluence angle. The width of the main and tributary channels were $B_{m}=1 \mathrm{~m}$ and $B_{t}=0.15 \mathrm{~m}$, respectively, resulting in a low $B_{r}$ equal to 0.15 . Regarding the hydraulic conditions, the main channel discharge was set equal to $Q_{m}=0.044 \mathrm{~m}^{3} \mathrm{~s}^{-1}$, whereas the tributary one to $Q_{t}=0.005 \mathrm{~m}^{3} \mathrm{~s}^{-1}$, with $h_{m}=h_{t}=0.11 \mathrm{~m}$, where $h_{m}$ is the main channel flow depth upstream the confluence and $h_{t}$ is the tributary flow depth. The discharge ratio, $Q_{r}$, assumes a low value equal to 0.114 . To guarantee the development of a fully turbulent flow at cross-section no. 1 (Figure 2b), a layer of gravel (with $d_{50}=6 \mathrm{~mm}$ ) was placed on the channel bed. 


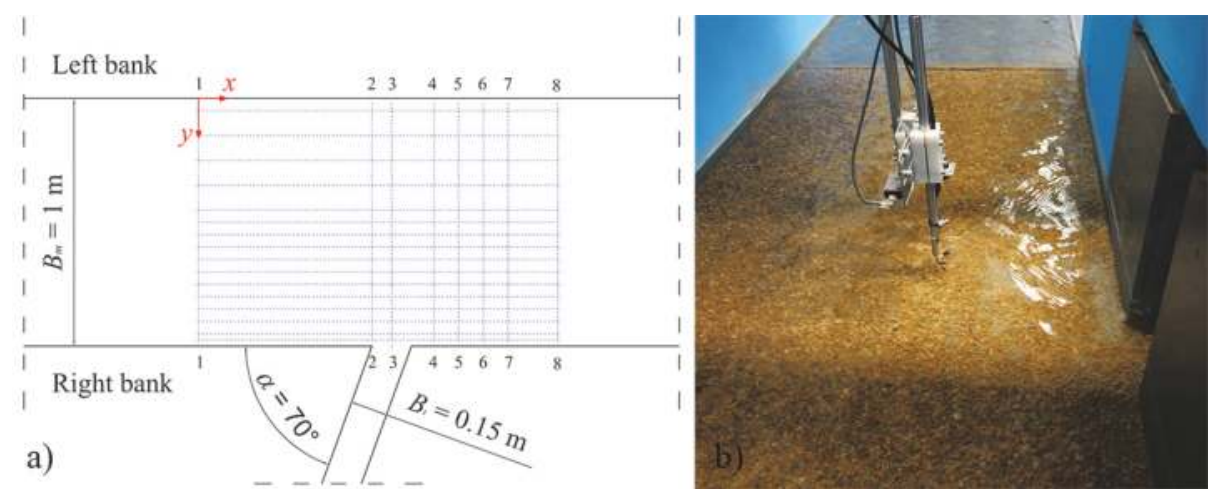

Figure 2. (a) plan view and (b) photograph of the experimental facility used by Birjukova et al. [23] with the measurement grid. Streamwise coordinates of the measured cross-sections: $x_{1}=0 \mathrm{~m}, x_{2}=0.7 \mathrm{~m}$, $x_{3}=0.78 \mathrm{~m}, x_{4}=0.95 \mathrm{~m}, x_{5}=1.05 \mathrm{~m}, x_{6}=1.15 \mathrm{~m}, x_{7}=1.25 \mathrm{~m}, x_{8}=1.45 \mathrm{~m}$.

An Acoustic Doppler Velocimeter (ADV), with a sampling frequency of $100 \mathrm{~Hz}$ and acquisition time per point equal to $90 \mathrm{~s}$, was used to measure the flow field at the confluence, considering the sampling grid depicted in Figure 2a. In particular, the origin of the coordinate system is fixed at cross-section no. $1,70 \mathrm{~cm}$ to the upstream junction corner at the left bank of the main channel. The horizontal axes $x$ and $y$ are directed in the streamwise and spanwise directions, respectively, whereas the vertical axis $z$, not shown in Figure 2a, starts at the bottom and is directed towards the water free surface. As many as 128 vertical profiles were considered in this study: eight cross-section and 16 lateral positions, starting at $2.5 \mathrm{~cm}$ from the right wall of the main channel, with a step of $5 \mathrm{~cm}$ from $y=0.05 \mathrm{~m}$ to $0.55 \mathrm{~m}$ and of $10 \mathrm{~cm}$ from $y=0.55 \mathrm{~m}$ to $0.95 \mathrm{~m}$. Each profile is constituted by 18 measuring points (i.e., 11 points at a vertical displacement of $2 \mathrm{~mm}$ in the lower $\approx 30 \%$ of the flow depth and seven points with a step of $7 \mathrm{~mm}$ in the upper flow region). Note that no velocity measurements were undertaken in the tributary channel.

\subsection{Analysis of the Experimental Data}

The analysis of the experimental data is a crucial aspect for the correct interpretation of the results obtained with numerical simulations. In fact, the consistency of the model is evaluated on the basis of this preliminary investigation, which reveals the actual flow pattern found in the laboratory confluence.

The time-averaged components of the velocity vector $(\bar{u}, \bar{v}, \bar{w}$, in the streamwise, spanwise and vertical directions, respectively) measured at cross-section no. 1 , normalized with the mean flow velocity upstream to the confluence $\left(U_{m 0}=Q_{m} /\left(B h_{m}\right)=0.38 \mathrm{~ms}^{-1}\right)$ are shown in Figure 3 , where $\hat{y}$ is defined as $y / B_{m}$ and $z$ as $z / h_{m}$. Specifically, $U_{m 0}$ was used as a reference, even though, from the analysis of the ADV data, a mean velocity equal to $0.41 \mathrm{~ms}^{-1}$ was obtained, with a relative error with respect to $U_{m 0}$ equal to $-8 \%$. This difference can be attributed to the fact that the ADV measurements do not cover the entire width and depth of the channel, along cross-section no. 1. 


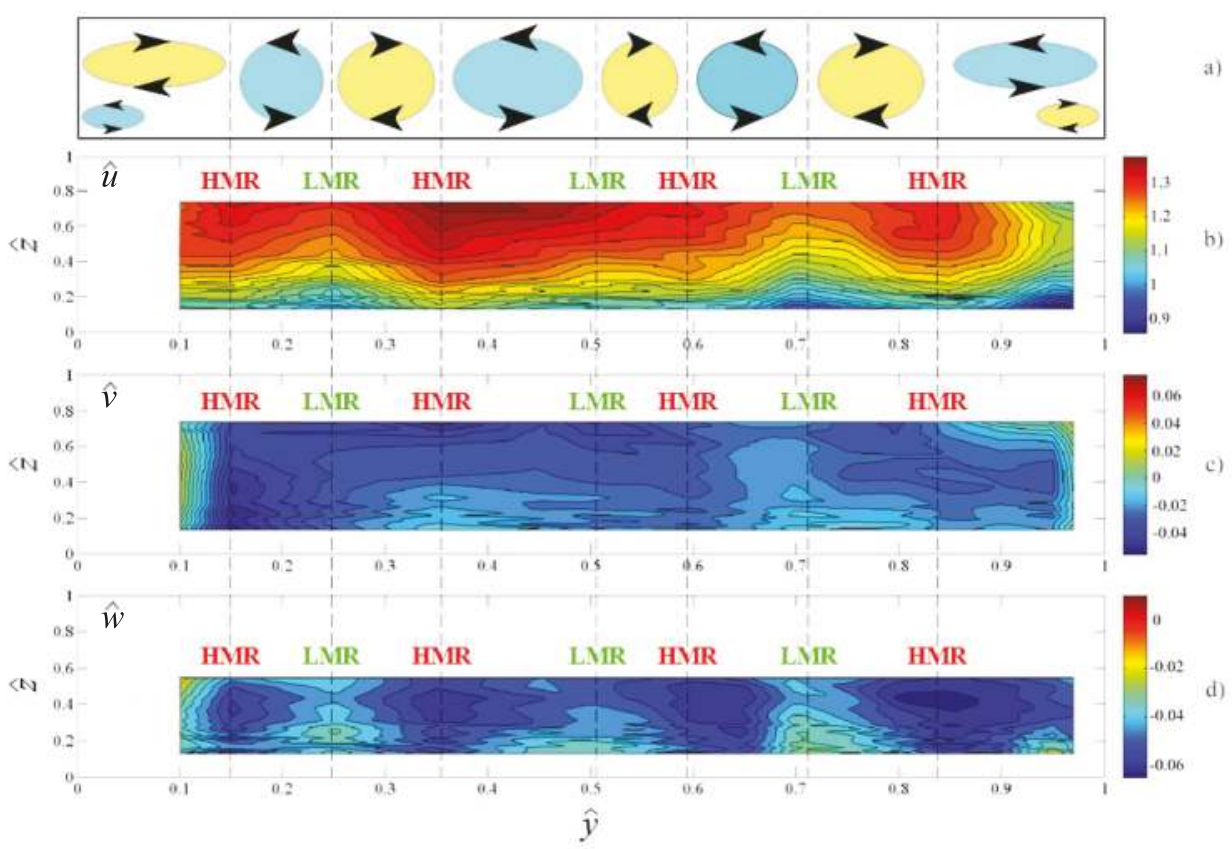

Figure 3. Patterns of some hydrodynamic variables at cross-section no. 1: (a) schematic of secondary currents; (b) normalized streamwise velocity component, $\hat{u}=\bar{u} / U_{m 0} ;(\mathbf{c})$ normalized spanwise velocity component, $\hat{v}=\bar{v} / U_{m 0} ;(\mathbf{d})$ normalized vertical velocity component, $\hat{w}=\bar{w} / U_{m 0}$.

The analysis of such patterns reveals the presence of a comparatively strong secondary currents over the entire width of the cross-section (Figure 3a), even if, as the experiment was designed with an aspect ratio $B_{m} / h_{m}=8.8$, either a weak or inexistent secondary circulation pattern would be expected in the central region of the channel. The reason behind the existence of such strong heterogeneity in Figure $3 \mathrm{~b}$ derives, essentially, from a combination of two processes. In fact, near the lateral walls, the presence of the secondary currents is due to the amplification of the surface-corner vortex caused by the difference in roughness between the bank and the bed [36,37], whereas, along the width of the main channel, the turbulent secondary flows are attributed to the spanwise heterogeneity in roughness height, which causes low-momentum regions (LMRs) spanwise-adjacent to high-momentum regions (HMRs) (their spanwise positions are labelled in Figure 3). In fact, the layer of gravel was not uniformly glued on the channel bed and, therefore, different topographical scales were arranged in a highly irregular manner. Several studies showed how the formation of these vortical structures are influenced by the roughness height (e.g., [38-40]). In general, the identified LMR and HMR patterns tend to occur at spanwise locations of recessed and elevated roughness (relative to the mean elevation), respectively, with the swirling motions residing at spanwise locations of intense spanwise gradients in topographical height [39]. The same trend could be recognized in the other cross-sections (see Figures 4 and 5). 


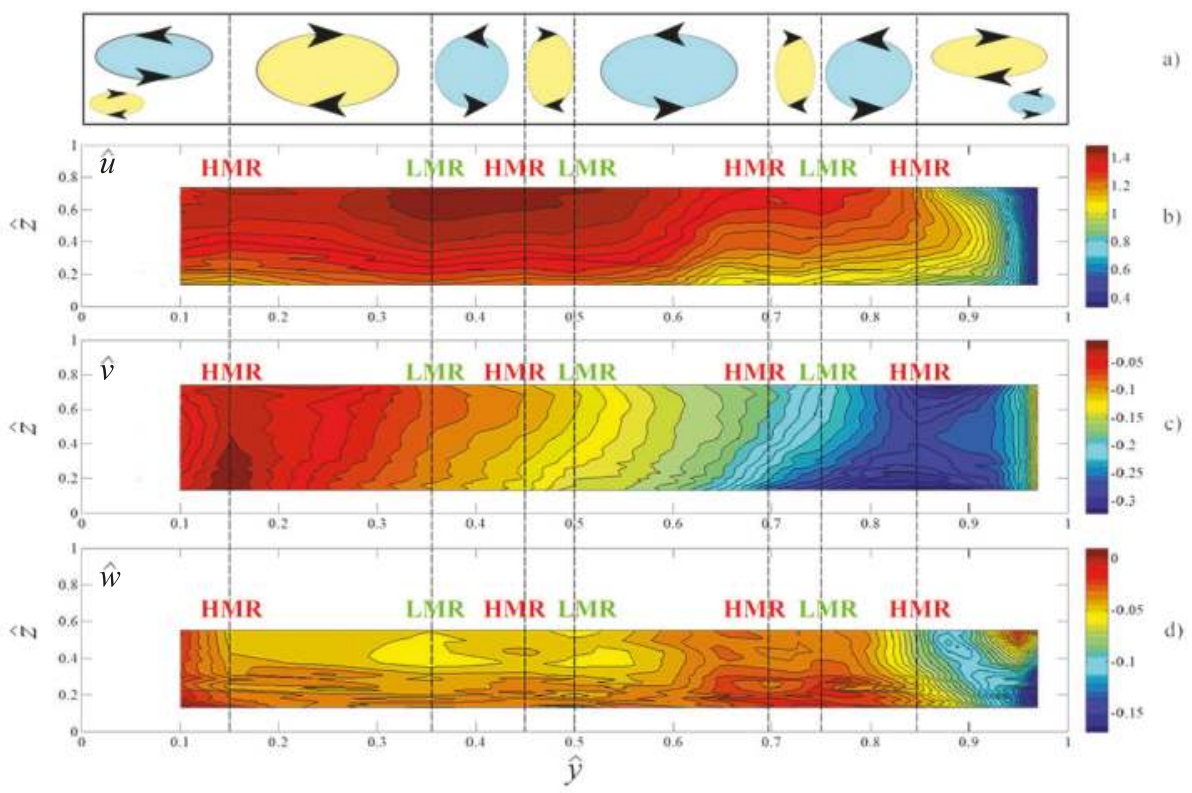

Figure 4. Patterns of some hydrodynamic variables at cross-section no. 2: (a) schematic of secondary currents; (b) normalized streamwise velocity component, $\hat{u}=\bar{u} / U_{m 0}$; (c) normalized spanwise velocity component, $\hat{v}=\bar{v} / U_{m 0} ;(\mathbf{d})$ normalized vertical velocity component, $\hat{w}=\bar{w} / U_{m 0}$.

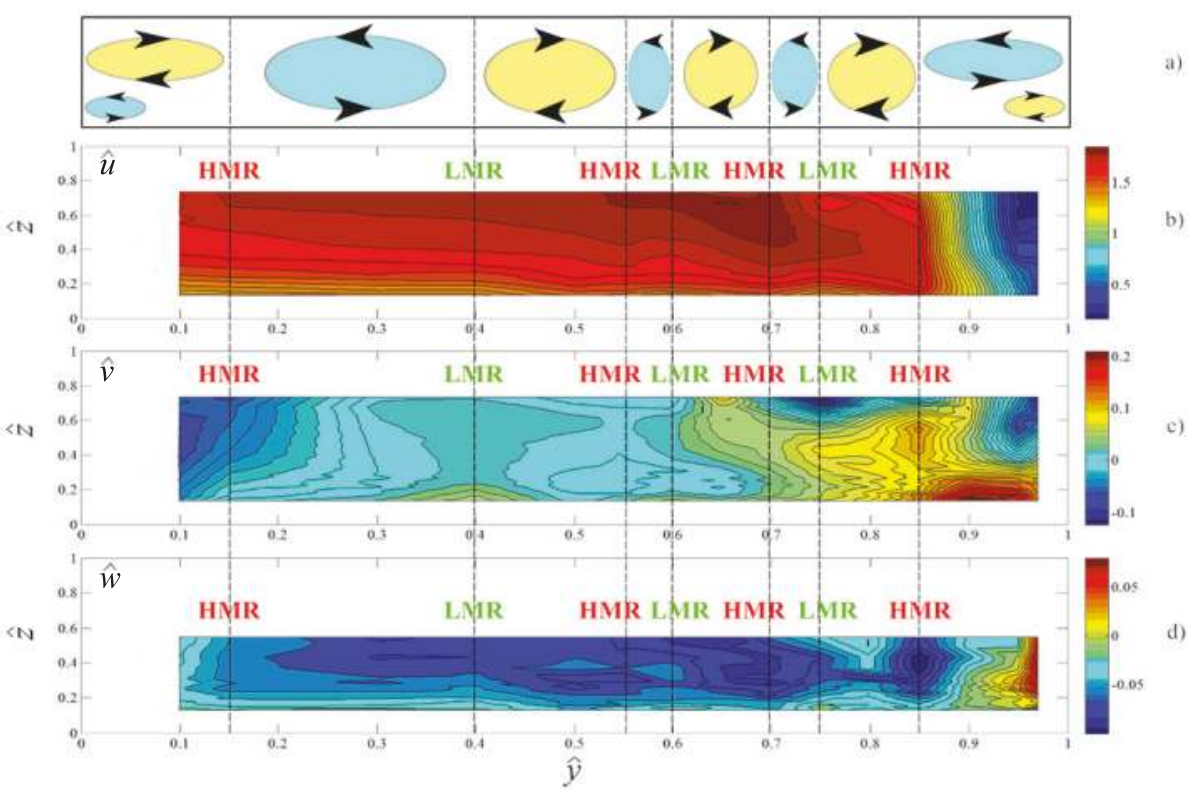

Figure 5. Patterns of some hydrodynamic variables at cross-section no. 7: (a) schematic of secondary currents; (b) normalized streamwise velocity component, $\hat{u}=\bar{u} / U_{m 0}$; (c) normalized spanwise velocity component, $\hat{v}=\bar{v} / U_{m 0} ;(\mathbf{d})$ normalized vertical velocity component, $\hat{w}=\bar{w} / U_{m 0}$. 


\subsection{Computational Domain and Validation of the Numerical Model}

The numerical simulations have been carried out by using the finite-volume numerical code PANORMUS (second-order accurate both in time and space). It solves the 3D RANS equations with the $k-\epsilon$ turbulence closure by using a finite-volume method on a three-dimensional, non-orthogonal, structured grid. For the time advancement of the solution, the numerical model uses the explicit Adams-Bashforth method, whereas to overcome the pressure-velocity decoupling, typical of incompressible flows, the fractional-step technique is employed. The predictor-corrector system is solved through the Line Successive OverRelaxation method (L-SOR) algorithm. For details on the numerical model and the mathematical formulation of the RANS equations, see [41]. The model is resolved in a computational domain reproducing the experimental laboratory facility. Specifically, the length of the tributary was determined arbitrarily because no velocity measurements were available from the experimental campaign, which would be used to validate the numerical results in the tributary. The confluence domain, after a grid sensitivity analysis, was discretized in $128 \times 96 \times 32$ cells in the streamwise, spanwise and vertical directions, respectively, by using a MATLAB (The MathWorks, Inc., Natick, MA, USA) code programmed on purpose. This resolution was selected so that the implementation of a finer grid did not show a considerable difference in the results. The mesh was refined near the walls and in the confluence zone (Figure 6), in order to obtain a finer discretisation close to the momentum transfer regions and the boundaries. A non-uniform grid was also used in the vertical direction, which was refined near the bottom and near the free-surface (imposed at $0.114 \mathrm{~m}$ above the bottom). The computational resolution is fine enough to reproduce the experiments and give a new insight into the dynamics of the channel confluence.

Both in the main and tributary channels, the flow is driven with assigned inflow conditions. Specifically, at the inlet of the two channels, a mean velocity profile is imposed to achieve the same values of flow rate $Q_{m}$ and $Q_{t}$ as in the experimental channel by means of a centrifugal pump. The logarithmic law of the wall was used near the solid boundaries (lateral walls and bottom) and null derivatives for all variables and hydrostatic pressure distribution were prescribed at the outflow boundary (Neumann-type boundary conditions). At the upper boundary, the free slip condition was adopted, which is justified as the tested Fr numbers (defined with the flow depth and mean velocity in the incoming and downstream channels) were smaller than 0.5 [11]. At the bottom wall, an equivalent roughness height of $3 \mathrm{~mm}$ (thus, lower than $d_{50}$ ) was considered for all simulations, in order to take into account the difference in roughness between the vertical walls and the channel bed. Such value was verified successively to be acceptable by comparing the experimental and numerical data.

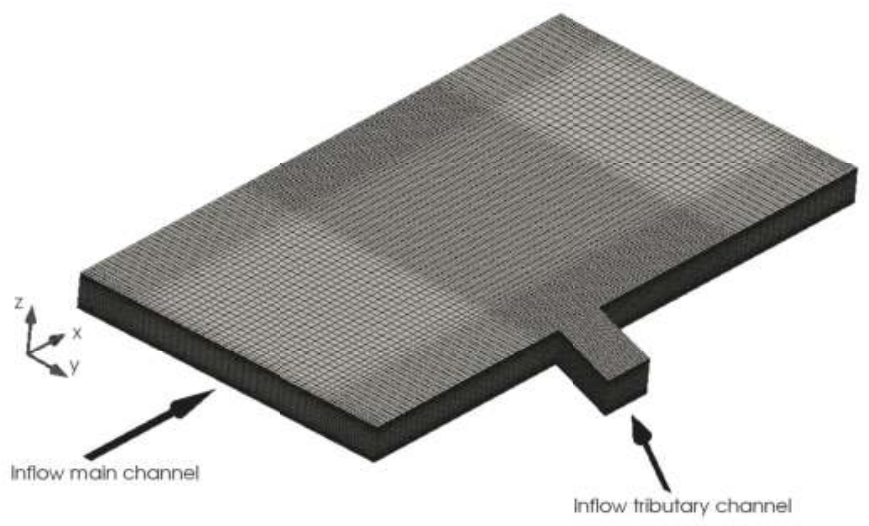

Figure 6. Computational domain of the confluence. 
The measured flow field at cross-section no. 1 and $Q_{m}$ were used to calibrate the inflow in the main channel, whereas, for the tributary, only $Q_{t}$ was taken into account.

Figure 7a shows the comparison between the measured and the simulated velocity profiles of the non-dimensional streamwise component at eight lateral positions along cross-section no. 1. Despite the presence of secondary currents, the results obtained with PANORMUS demonstrated a satisfactory agreement between the experimental and numerical data in the central part of the main channel (from $\hat{y}=0.35$ to $\hat{y}=0.75$ ). Here, the major discrepancies occurred in the lower part of the velocity profiles, near the bottom, where the velocity values were slightly underestimated owing to the use of the wall function.

To quantify such discrepancies, Figure $7 \mathrm{~b}$ shows the matching between measured $\left(\bar{u}_{m}\right)$ and simulated $\left(\bar{u}_{s}\right)$ streamwise velocity components, from $\hat{y}=0.35$ to $\hat{y}=0.75$, with the perfect agreement line and the $\pm 10 \%$ and $\pm 15 \%$ bands. It is evident that the maximum deviation is confined between $-10 \%$ and $+10 \%$. Moreover, Figure $7 \mathrm{c}$ shows the Root Mean Square Error (RMSE) obtained for each velocity profile and calculated as $\sqrt{\frac{1}{N} \sum_{i=1}^{N}\left(\bar{u}_{m_{i}}-\bar{u}_{s_{i}}\right)^{2}}$, where $N$ is the total number of measurement points along the vertical direction.

Figures 8 and 9 show the same comparisons along cross-section no. 3, in correspondence of the tributary channel, and along four different longitudinal profiles. It is clearly visible that the maximum deviation, in both cases, is generally confined between $-15 \%$ and $+10 \%$.

a)

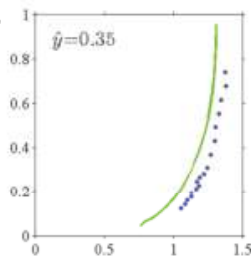

a

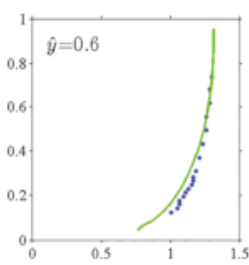

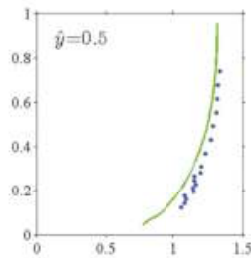
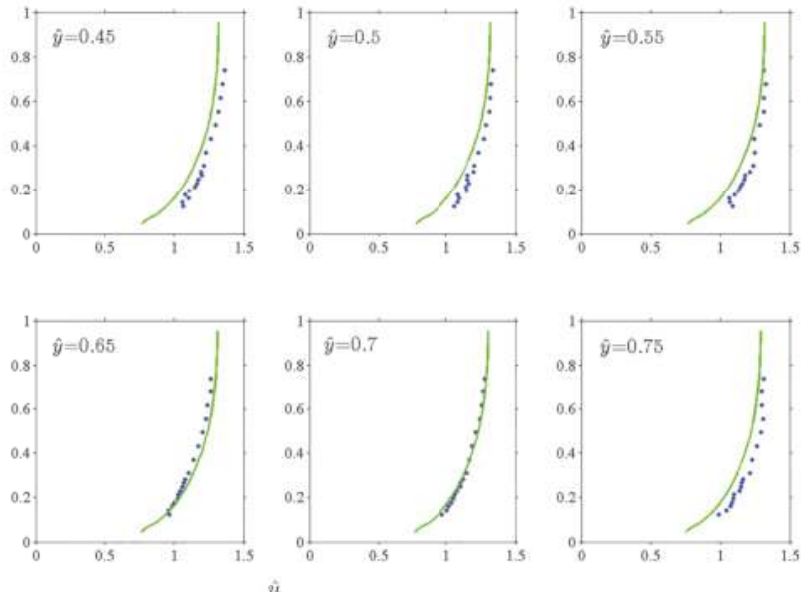

b)

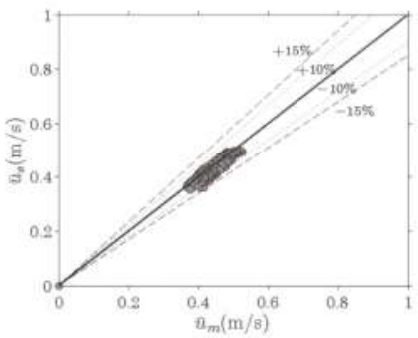

c)

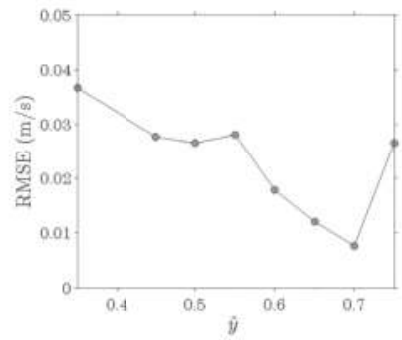

Figure 7. (a) comparison of measured (blue dots) and simulated (green lines) non-dimensional streamwise velocity component profiles in cross-section no. 1; (b) comparison of measured and simulated streamwise velocity components; (c) Root Mean Square Error (RMSE) of the streamwise velocity component profiles from $\hat{y}=0.35$ to $\hat{y}=0.75$ in cross-section no. 1 . 
a)
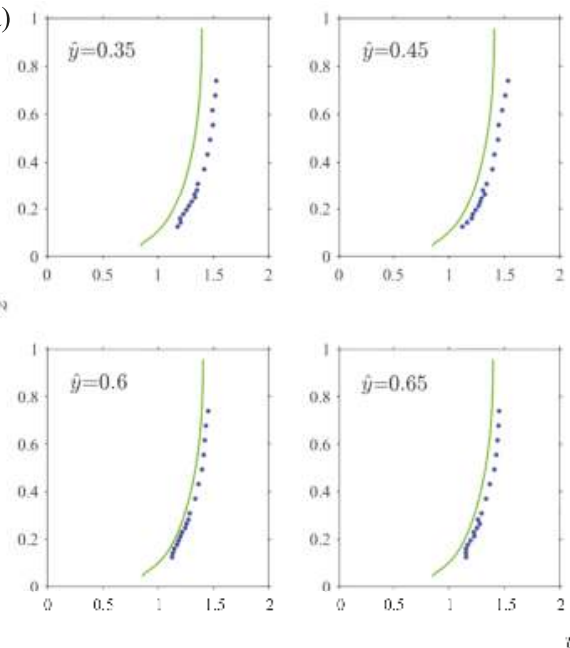

b)

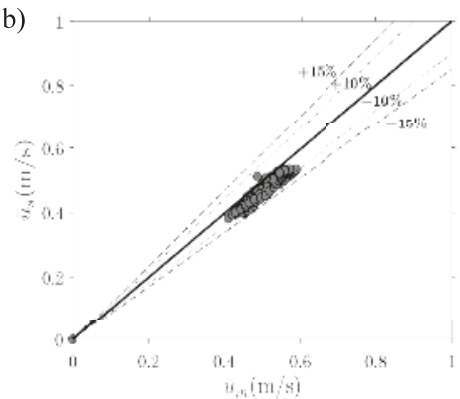

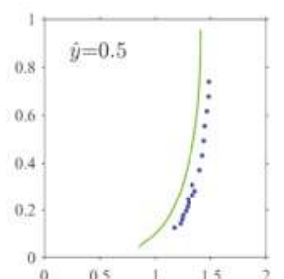
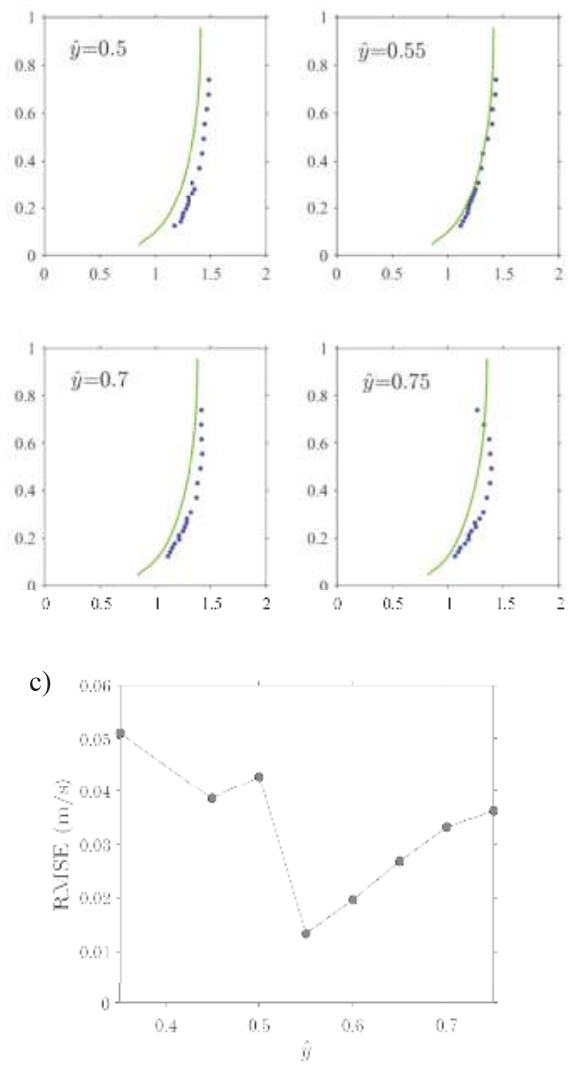

Figure 8. (a) comparison of measured (blue dots) and simulated (green lines) non-dimensional streamwise velocity component profiles in cross-section no. 3 ; (b) comparison of measured and simulated streamwise velocity components; (c) RMSE of the streamwise velocity component profiles from $\hat{y}=0.35$ to $\hat{y}=0.75$ in cross-section no. 3 .

However, it is important to highlight that, near the internal wall, where strong flow recirculation occurs, differences in measured and estimated values were detected (as an example, see Figure 10). This behaviour was not unexpected. In fact, similar deviation in the separation zone was shown in other literature studies (i.e., [29,42]), using different experimental data and numerical models.

For this reason, the model was also validated considering the shape of the separation zone in terms of its length $\left(L_{S}\right)$ and width $\left(B_{S}\right)$.

Different techniques were employed in the past for accurately determining the separation zone (e.g., $[15,20])$. However, since this is a region of decreased downstream momentum, the delineation of the separation zone extension is not an easy task. Generally, for a given $z$ coordinate, the length of the separation zone is defined as the distance from the downstream corner of the junction to the reattachment point. In addition, according to the boundary layer theory, the separation zone starts when the velocity near the wall is zero or negative. Therefore, the general hypothesis that considers the downstream junction corner as the starting point of the separation zone (known as the separation point) is misleading. Especially in the case of low $\alpha$ angles, it is expected that the flow arriving from the tributary channel tends to adhere to the wall downstream of the confluence and, only when the flow velocity can no longer overcome adverse pressure gradient, flow separation occurs. 
Therefore, the separation point is not coincident with the downstream junction corner, but it is located downstream of it.

a)
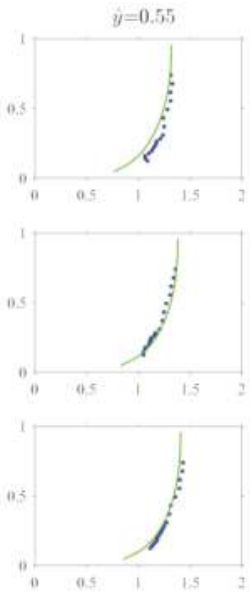
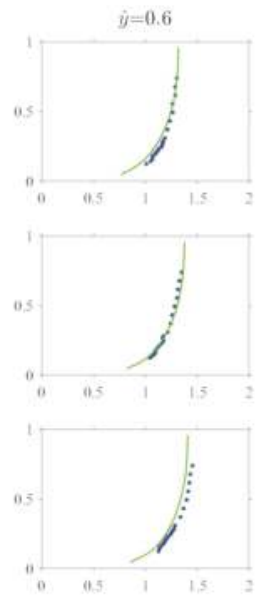

b)

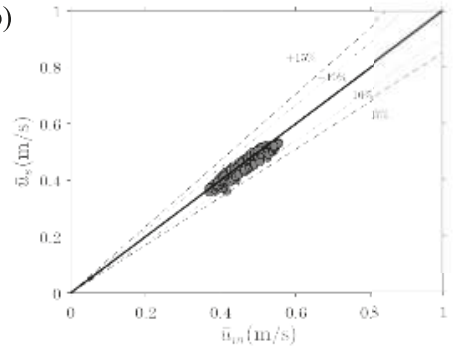

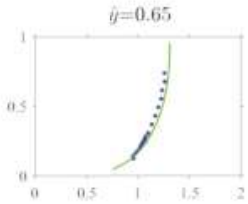
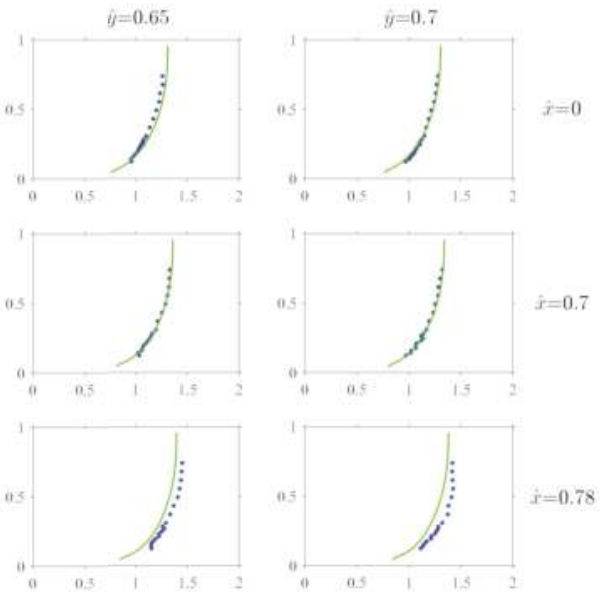

Figure 9. (a) comparison of measured (blue dots) and simulated (green lines) non-dimensional streamwise velocity component profiles in cross-sections Nos. 1, 2 and 3 along four longitudinal profiles; (b) comparison of measured and simulated streamwise velocity components.
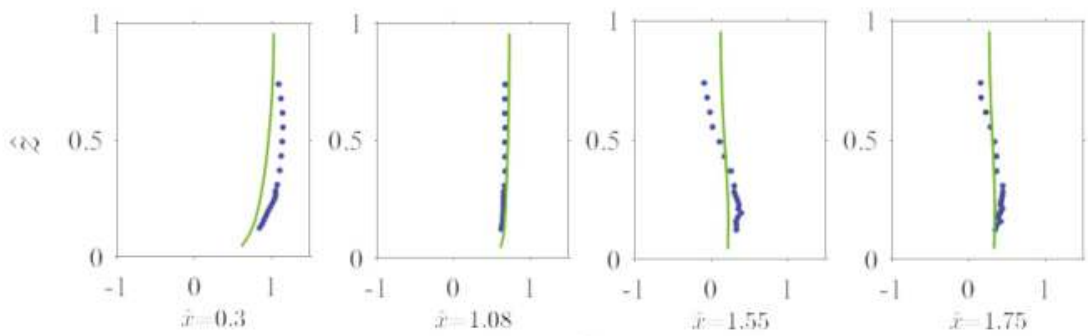

$\hat{u}$

Figure 10. Comparison of measured (blue dots) and simulated (green lines) non-dimensional streamwise velocity component profiles in cross-sections Nos. 1, 3, 7 and 8 along the longitudinal profile $\hat{y}=0.95$.

Specifically, the separation zone was delimited by the streamlines shown in Figure 11, where the time-averaged velocity $\bar{v}_{x y}=\sqrt{\bar{u}^{2}+\bar{v}^{2}}$ in the horizontal plane $\hat{z} \sim 0.5$ was plotted. $L_{s}$ was defined as 
the distance from the separation point to the reattachment point, whereas $B_{S}$ was the distance from the wall to the point belonging to the streamline passing through the reattachment point, in which $\bar{v}$ is null.

For $\alpha=70^{\circ}, L_{S}$ and $B_{S}$ were $48.6 \mathrm{~cm}$ and $6.4 \mathrm{~cm}$, respectively. These two main features were also obtained from the experimental data and were approximately $46 \mathrm{~cm}$ and $6 \mathrm{~cm}$, respectively, showing that the numerical model slightly overestimates $L_{S}$ with a relative error equal to $5.6 \%$. The error is greater for $B_{S}$ and is equal to $-8 \%$, since the predicted width is narrower than the measured one. This discrepancy can be attributed to the effect of the secondary currents, discussed in Section 2.2. Such results were compared with those obtained by Brito et al. [32], who modelled the free surface with the VoF method. In particular, they obtained the following separation zone characteristic sizes: $L_{S}=54 \mathrm{~cm}$ and $B_{S}=8.7 \mathrm{~cm}$. Therefore, the estimations of both $L_{S}$ and $B_{S}$ are more precise in the present study, with respect to that of Brito et al. [32].

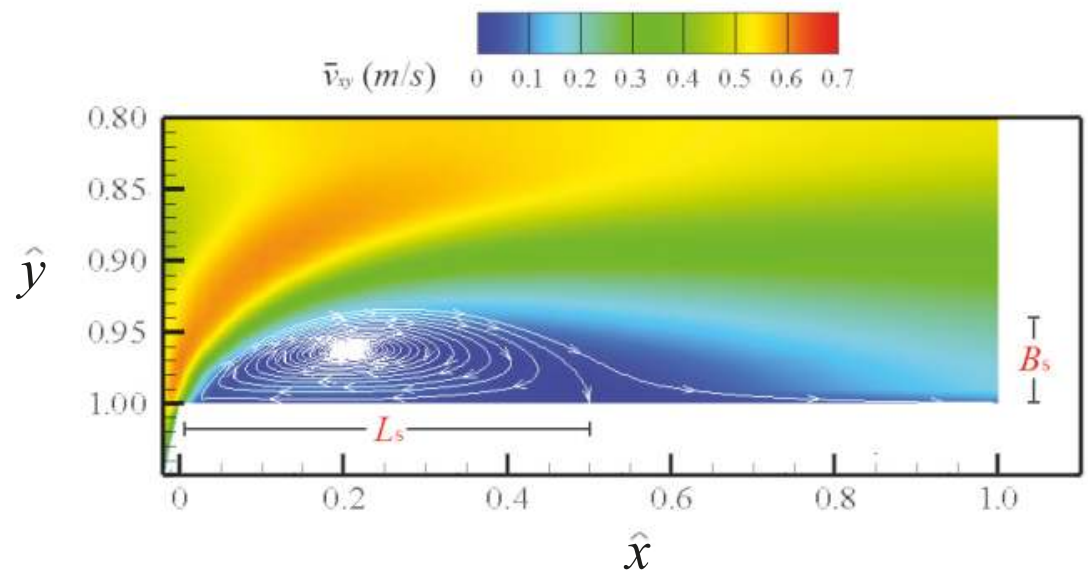

Figure 11. Time-averaged velocity $\bar{v}_{x y}$ in the horizontal plane $\hat{z} \sim 0.5$ for $\alpha$ equal to $70^{\circ}$. Here, $\hat{x}=x / B_{m}$ is the non-dimensional abscissa directed in the streamwise direction centred in the downstream corner of the confluence.

\section{Results and Discussion}

To study the effects of the junction angle on the flow structure of a confluence with fixed concordant beds and low width and discharge ratios, the computational domain created on the basis of the facility used by Birjukova et al. [23] was modified according to nine other different junction angles, ranging from $45^{\circ}$ to $90^{\circ}$ with a step of $5^{\circ}$.

The simulations were carried out using the same $Q_{r}$ of the experimental test in order to compare the obtained results as a function of solely $\alpha$, maintaining constant hydraulic conditions for both channels. The modification of the junction angle implies the change of the constant mass force (per unit mass) that drives the flow into the tributary. Such value is just the slope of the tributary towards the main channel. Therefore, to always guarantee the same value of $Q_{t}$, preliminary and separate simulations of fully developed turbulent flows were performed adapting the constant mass force to each simulated geometry. The main channel flow, upstream to the confluence, was not affected by the changes of the junction angle.

The results are presented by comparing the effects of the junction angle on the retardation zone, the flow deflection zone, the flow separation zone and the maximum streamwise velocity zone. Although these regions are strongly related, hereinafter they are analyzed independently for a better understanding of each involved hydrodynamic process. 


\subsection{Retardation Zone}

The retardation zone within each channel originates from the deflection of flows away from the upstream junction corner. It is a region of reduced velocities, whose size depends on the junction angle. Two particular points, for each of the two incoming channels, characterize this zone and were identified in this work by the respective distances from the upstream junction corner to the points in which the velocity vector deflects from the wall with an angle greater than $1^{\circ}$ (points $P_{m}$ and $P_{t}$, respectively, in the main and tributary channels) (Figure 12a,b). In particular, $x_{s m}$ is the streamwise distance between the upstream junction corner and the point $P_{m}$, while $y_{s t}$ is the lateral distance between the upstream junction corner and the point $P_{t}$.

Figure 12a,b shows such values made non-dimensional with $B_{m}$ for different $\alpha$ angles at elevation $\hat{z} \sim 0.5$. It is possible to note that, as $\alpha$ increases, $\hat{x}_{s m}$ and $\hat{y}_{s t}$ increase quasi-linearly, causing a more extended retardation zone. In addition, for $\alpha$ equal to $45^{\circ}$ and $50^{\circ}$, the retardation zone is confined only within the tributary channel, since $\hat{x}_{s m}$ is equal to 0 . The maximum extension of such region occurs at $90^{\circ}$, where $x_{s m}$ and $y_{s t}$ are about $0.1 \mathrm{~m}$ and $0.09 \mathrm{~m}$, respectively.
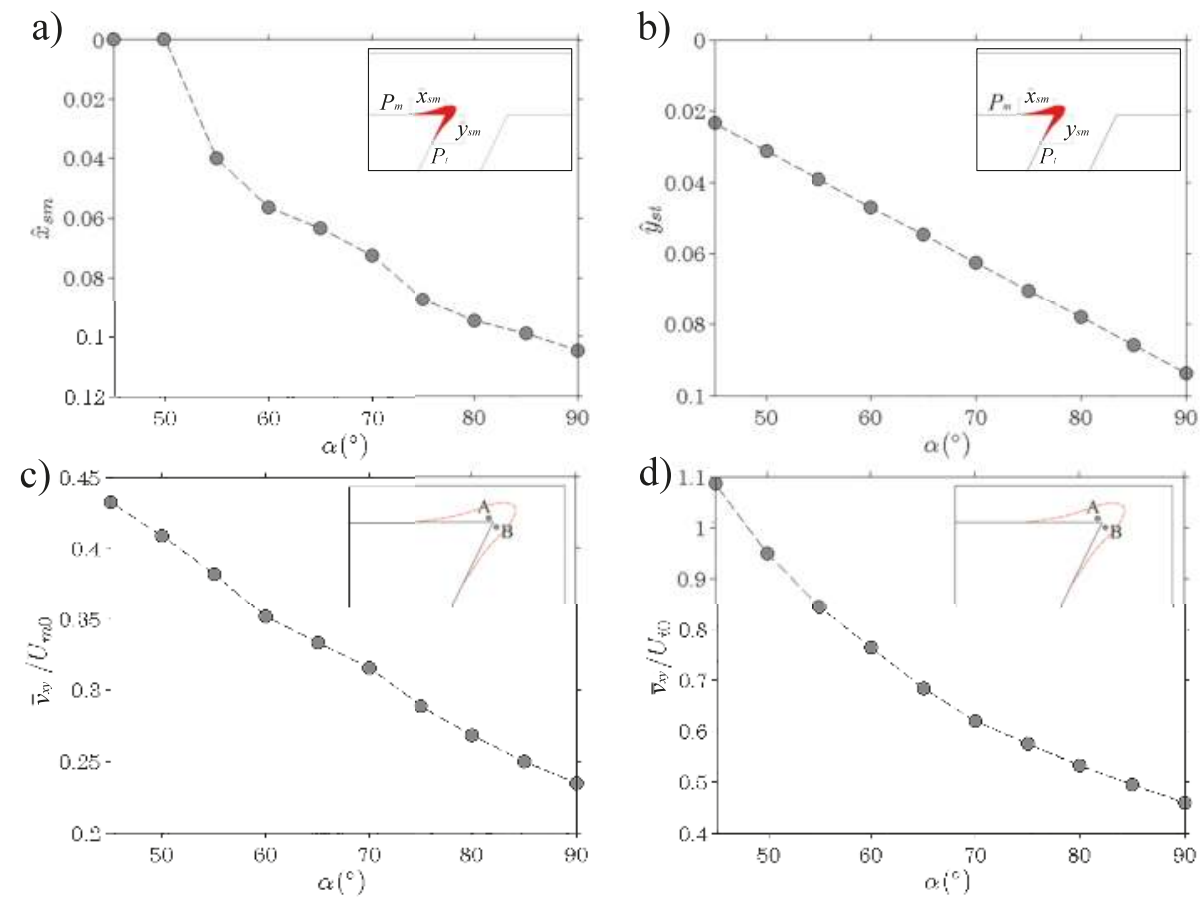

Figure 12. Distributions of (a) $\hat{x}_{s m}$ and (b) $\hat{y}_{s t}$ with respect to $\alpha$ at $\hat{z} \sim 0.5$ and of the relative flow velocity at points (c) A and (d) B within the retardation zone with respect to $\alpha$ at $\hat{z} \sim 0.5$.

Therefore, the higher the junction angle, the more extended the retardation zone and the lower the velocities in this region. In fact, as an example, Figure $12 \mathrm{c}, \mathrm{d}$ shows the relative flow velocity at points A and B located immediately upstream to the junction corner within the main and tributary channels, respectively, and their variation with the confluence angle. The relative flow velocity at point A is expressed as $\bar{v}_{x y} / U_{m 0}$, where $\bar{v}_{x y}=\sqrt{\bar{u}^{2}+\bar{v}^{2}}$ at point $\mathrm{A}$. The relative flow velocity at point $\mathrm{B}$ is expressed as $\bar{v}_{x y} / U_{t 0}$, where $\bar{v}_{x y}=\sqrt{\bar{u}^{2}+\bar{v}^{2}}$ at point $\mathrm{B}$ and $U_{t 0}$ is the mean velocity of the tributary channel (equal to $0.29 \mathrm{~ms}^{-1}$ ). It is evident that the flow retardation rate increases at a higher junction 
angle, since, increasing $\alpha$, a progressively strong retardation of the flow velocity is observed in both the channels.

The results are consistent with the findings of several researchers (e.g., $[1,13,18])$, who, however, focused on the position of a stagnation point only within the tributary channel. Actually, as it was observed in this work, two particular points in the two incoming channels can be taken into account to delimit the extension of the retardation zone. However, they can not be defined as stagnation points since the velocity in that points is not equal to zero.

\subsection{Flow Deflection Zone}

As Gurram et al. [17] observed, assuming that the inflow angle along the junction cross-section is equal to the junction angle is a wrong hypothesis. In fact, the lateral inflow velocity varies from the upstream to the downstream junction corners, increasing towards this latter, as it occurs in curve flow, but always with a smaller angle with respect to $\alpha$. To measure the deflection of the flow on the horizontal plane at the tributary entrance into the confluence, the flow angle $\delta$ was calculated, along the entire cross-section connecting the upstream and downstream junction corners, on the basis of the streamwise and spanwise time-averaged components of the velocity vector: $\delta=\arctan (\bar{v} / \bar{u})$. Then, the angle of deflection was made non-dimensional by dividing by $\alpha$, giving the new variable $\hat{\delta}$.

Figure 13 shows, for each layout, the distribution of the $\hat{\delta}$-angle at the tributary entrance. It is readily noticeable that, close to the bottom, the flow deflection angles assume lower values than those at the surface (especially near the upstream junction corner), where the resulting vector $\left(\bar{v}_{x y}\right)$ tends to maintain the same direction as that of the tributary, but always with $\delta<\alpha$. This trend is more visible at high $\alpha$-angles (from Figure $13 \mathrm{f}-\mathrm{j}$ ), where $\delta$ is about $0.5 \alpha$ at the bottom and near the upstream lateral wall, while it is about $0.9 \alpha$ at the surface near the downstream junction corner.

This means that, as $\alpha$ increases, the confluent channels undergo a progressively greater deflection with respect to the flow direction in the post-confluence channel, as it was observed also by Best [1]. Furthermore, it confirms that, near the left bank of the tributary channel, the degree of flow retardation increases.

It is also evident that at low $\alpha$-values the penetration of the main channel flow into the tributary channel flow never occurs.

Extrapolating the data at elevation $\hat{z} \sim 0.5$, a direct comparison among the different distributions of the deflection angle along the $\hat{x}_{t}$-axis is shown in Figure 14. It is possible to note that all the data collapse within a band, with a slight deviation for the case of $\alpha=45^{\circ}$. In particular, $\hat{\delta}$ increases along the interfacial plane between the tributary and the main channels, assuming the maximum values near the downstream junction corner (as it was observed by Djordjevic [12]), whereas the variability of $\hat{\delta}$ for $\alpha=45^{\circ}$ is limited in the range $0.76 \div 0.83$, owing to the combination of low $\alpha, B_{r}$ and $Q_{r}$, which leads to a more uniform distribution of $\hat{\delta}$ across the interfacial layer between the tributary and the main channel flows.

\subsection{Flow Separation Zone}

The analysis on the tributary flow deflection demonstrates that, at the downstream junction corner, $\delta$ is always lower than $\alpha$, which means that, in correspondence of this point, the velocity vectors have a streamwise component that is neither zero nor negative. Such a behaviour supported the procedure proposed in this study for the definition of the separation zone, which is based on the assumption that the separation point is not coincident with the junction point, but it is located downstream of it. 

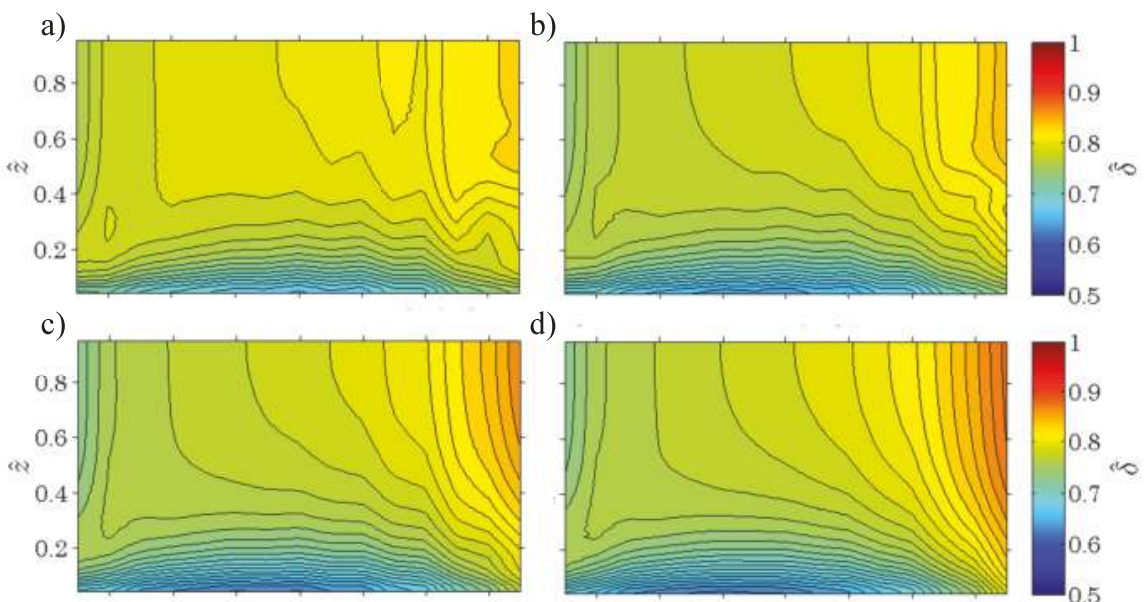

d)

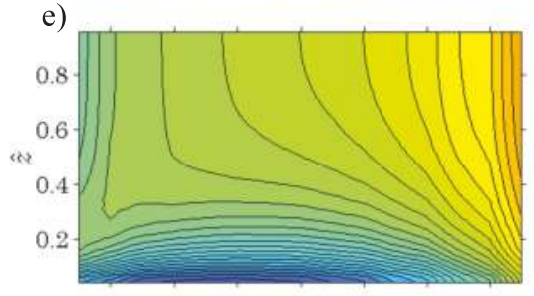

f)
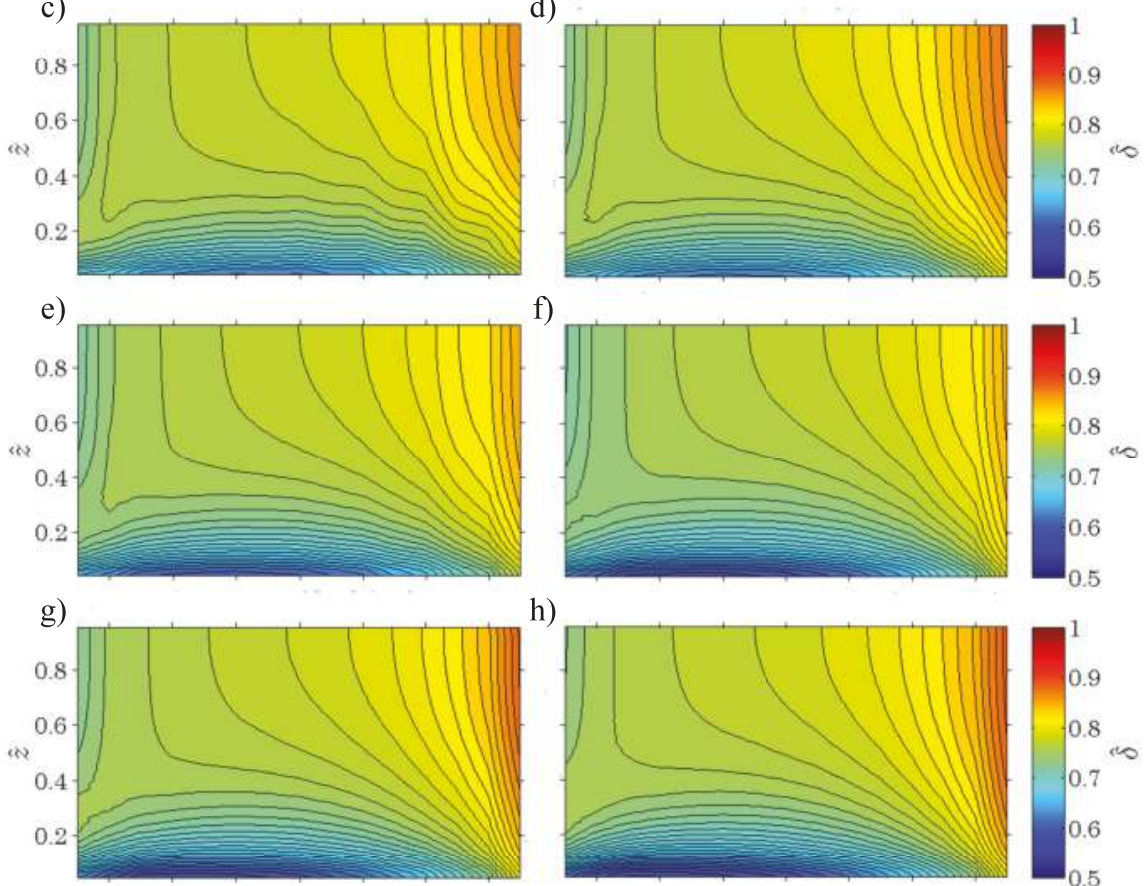

h)
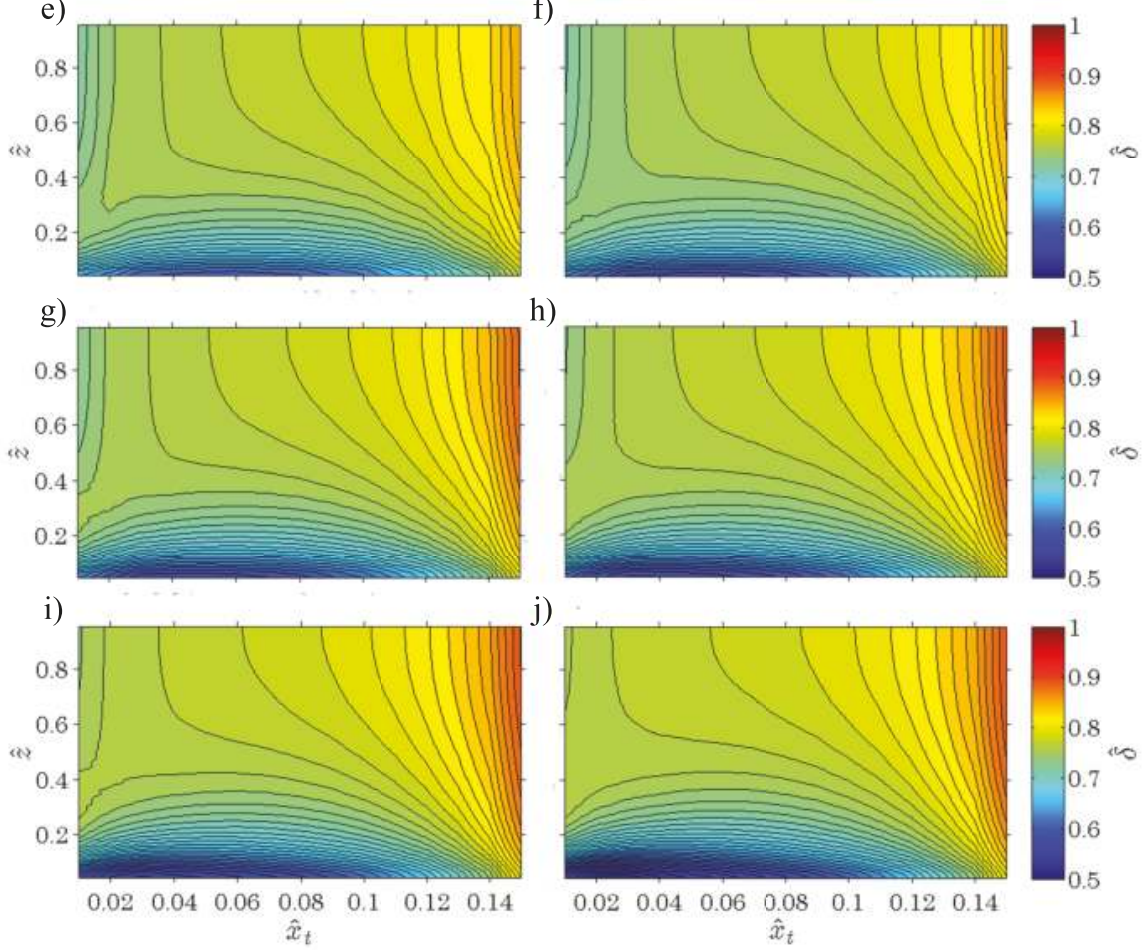

Figure 13. Distribution of the $\hat{\delta}$-angle at the tributary entrance for $\alpha$ equal to (a) $45^{\circ}$, (b) $50^{\circ}$, (c) $55^{\circ}$, (d) $60^{\circ}$, (e) $65^{\circ}$, (f) $70^{\circ}$, (g) $75^{\circ}$, (h) $80^{\circ}$, (i) $85^{\circ}$, (j) $90^{\circ}$. Here, $\hat{x}_{t}=x_{t} / B_{m}$ is the non-dimensional abscissa directed in the streamwise direction centred in the upstream corner of the confluence.

Figure 15 shows the time-averaged velocity $\bar{v}_{x y}$ in the horizontal plane $\hat{z} \sim 0.5$, obtained by the numerical simulations, and the shape of the flow separation zone for the analysed layouts. It is evident that, as $\alpha$ increases, the separation zone becomes longer and wider. This trend is the same of that reported in other studies (see Introduction). For example, Best and Reid [16] found that increasing the 
junction angle and, also, the contribution of the tributary to total discharge results in an increase of the width and length of the flow-separation zone.

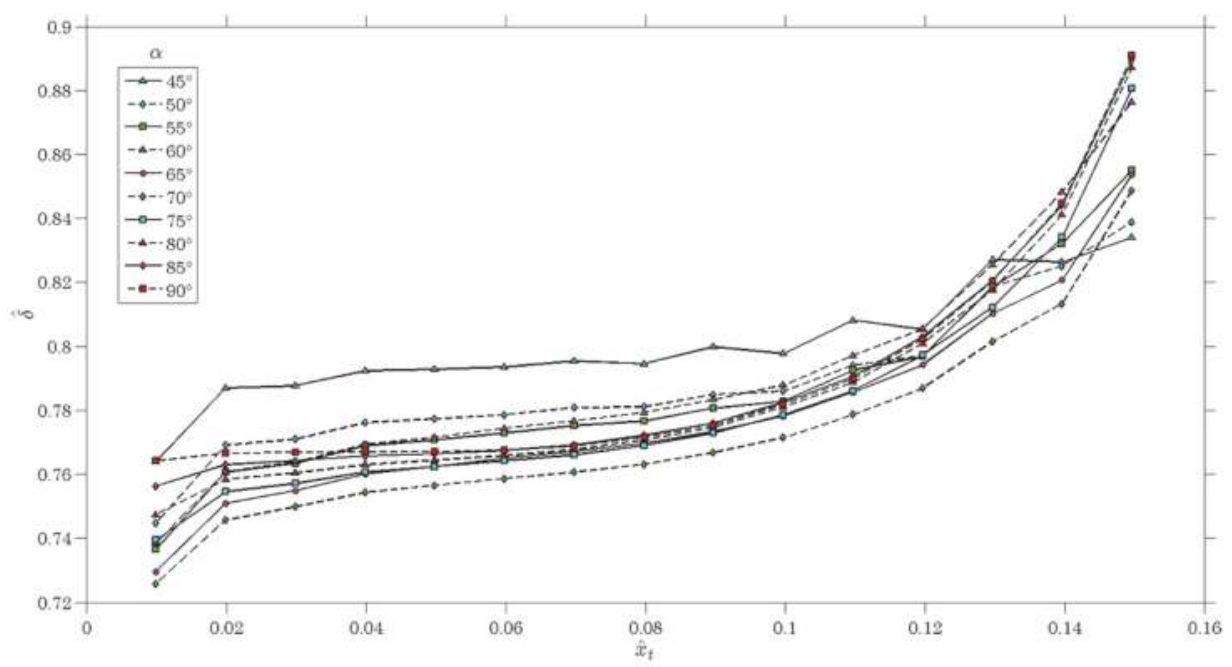

Figure 14. Effects of $\alpha$ on the $\hat{\delta}$-angle distributions at the tributary entrance into the confluence at $\hat{z} \sim 0.5$.

Anyway, this behaviour confirms the findings regarding the flow deflection zone: at the downstream bank of the tributary, the higher the deflection angles (occurring in the case of high junction angles), the stronger the separation downstream of the confluence (as observed by Djordjevic [12]). Furthermore, it is possible to note, especially for low $\alpha$ values, that the separation point is always located beyond the origin of the downstream junction angle, as it was specified previously.

The dimensions $L_{S}$ and $B_{S}$ were calculated for each confluence angle and were plotted in Figure 16a after normalisation with $B_{m}\left(\hat{L}_{s}, \hat{B}_{s}\right)$. Specifically, they are linearly correlated with $\alpha$ according to the equations shown in Figure 16a, with a coefficient of determination $\left(R^{2}\right)$ equal to 0.97 for $\hat{L}_{s}$ and 0.99 for $\hat{B}_{s}$. It is important to highlight that, in contrast with the literature findings, the separation zone does not occupy the half of the width of the post-confluence channel, as it was observed in other studies with high $Q_{r}$. In fact, it is confined within about $13 \mathrm{~cm}$ from the wall (inner bank of the flume), considering that its maximum width occurs at $\alpha=90^{\circ}$. Only Hsu et al. [27], for $B_{r}=1$, obtained a value of $B_{S}$ comparable with that reported here.

For junction angles less than $55^{\circ}$, the separation zone is no longer observed, owing to the low deflection angles with respect to $\alpha$ at the tributary entrance into the confluence. Therefore, it is possible to state that, in the geometrical and hydraulic conditions of the present study, the separation zone starts appearing for $\alpha$ between $50^{\circ}$ and $55^{\circ}$. However, it is important to point out that this result is valid for the particular case of highly channelized confluences, since it was demonstrated by several authors that the flow separation might be suppressed by the gentle curvature of natural confluence corners (e.g., [5]). 

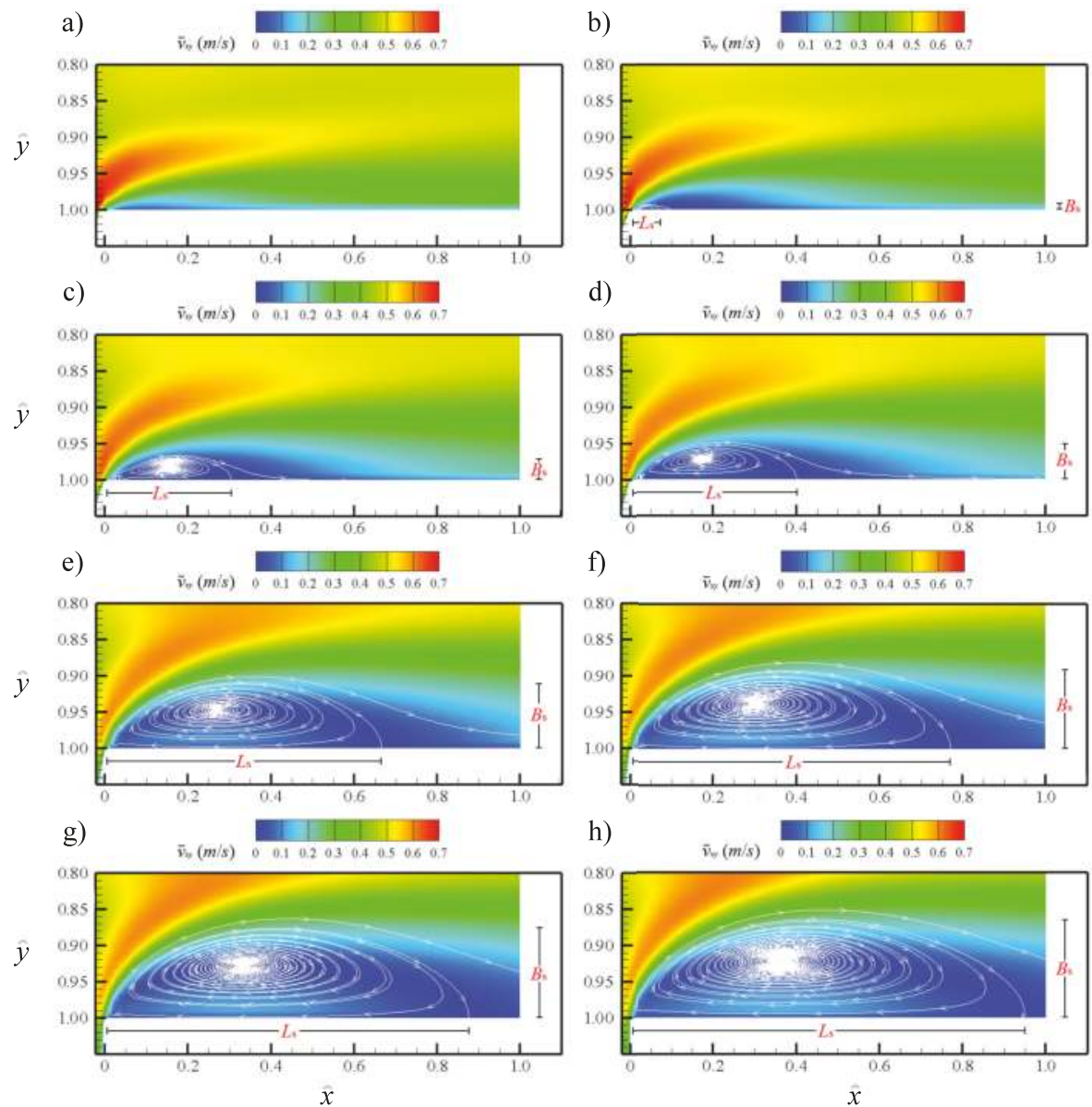

Figure 15. Time-averaged velocity $\bar{v}_{x y}$ in the horizontal plane $\hat{z} \sim 0.5$ for $\alpha$ equal to: (a) $50^{\circ}$; (b) $55^{\circ}$; (c) $60^{\circ}$; (d) $65^{\circ}$; (e) $75^{\circ}$; (f) $80^{\circ}$; (g) $85^{\circ}$; (h) $90^{\circ}$. Here, $\hat{x}=x / B_{m}$ is the non-dimensional abscissa directed in the streamwise direction centred in the downstream corner of the confluence.

\subsection{Contraction Zone and Maximum Streamwise Velocity}

The flow separation causes the establishment of a contraction zone beside it, in which the flow is forced to increase its velocity. The reason behind this fact is the reduction of the effective width of the post-confluence channel. To quantify such reduction, a contraction coefficient was calculated as $C_{c}=\left(B_{m}-B_{s}\right) / B_{m}$ for all the analysed layouts, as shown in Figure 16b. It is illustrated how $C_{c}$ decreases linearly as $\alpha$ increases, since the width of the separation zone increases. The result is coherent with the findings observed in Figure 16a, for the recirculation width $B_{s}$. Aiming at giving a relationship that would be useful in 1D modelling to determine the effective mean velocity in the post-confluence channel, an equation is proposed in Figure 16b, valid for low values of $B_{r}$ and $Q_{r}$. Obviously, $C_{c}$ is equal to 1 for $\alpha$ less than $50^{\circ}$, since no separation zone occurs $\left(B_{S}=0\right)$.

The contraction coefficient can be used for the determination of the head losses $\left(\Delta h_{L}\right)$ at junctions with the Borda-Carnot formula for a sudden flow expansion (e.g., [15]) as $\left(1-C_{c}\right)^{2} \cdot \frac{U_{p c}^{2}}{2 g}$ (where $U_{p c}$ is the mean velocity in the post-confluence channel). In turn, $\Delta h_{L}$ can be used to estimate the 
water level elevations in the separation and contraction zones. Specifically, since $C_{c}$ decreases as $\alpha$ increases, it is expected that the head losses increase as well. This means that a reduced extent of the separation zone will lead to lesser water level differences. Note that the local decrease in water level in correspondence with the separation zone had been noted in many laboratory experiments, including that of Birjukova et al. [23].

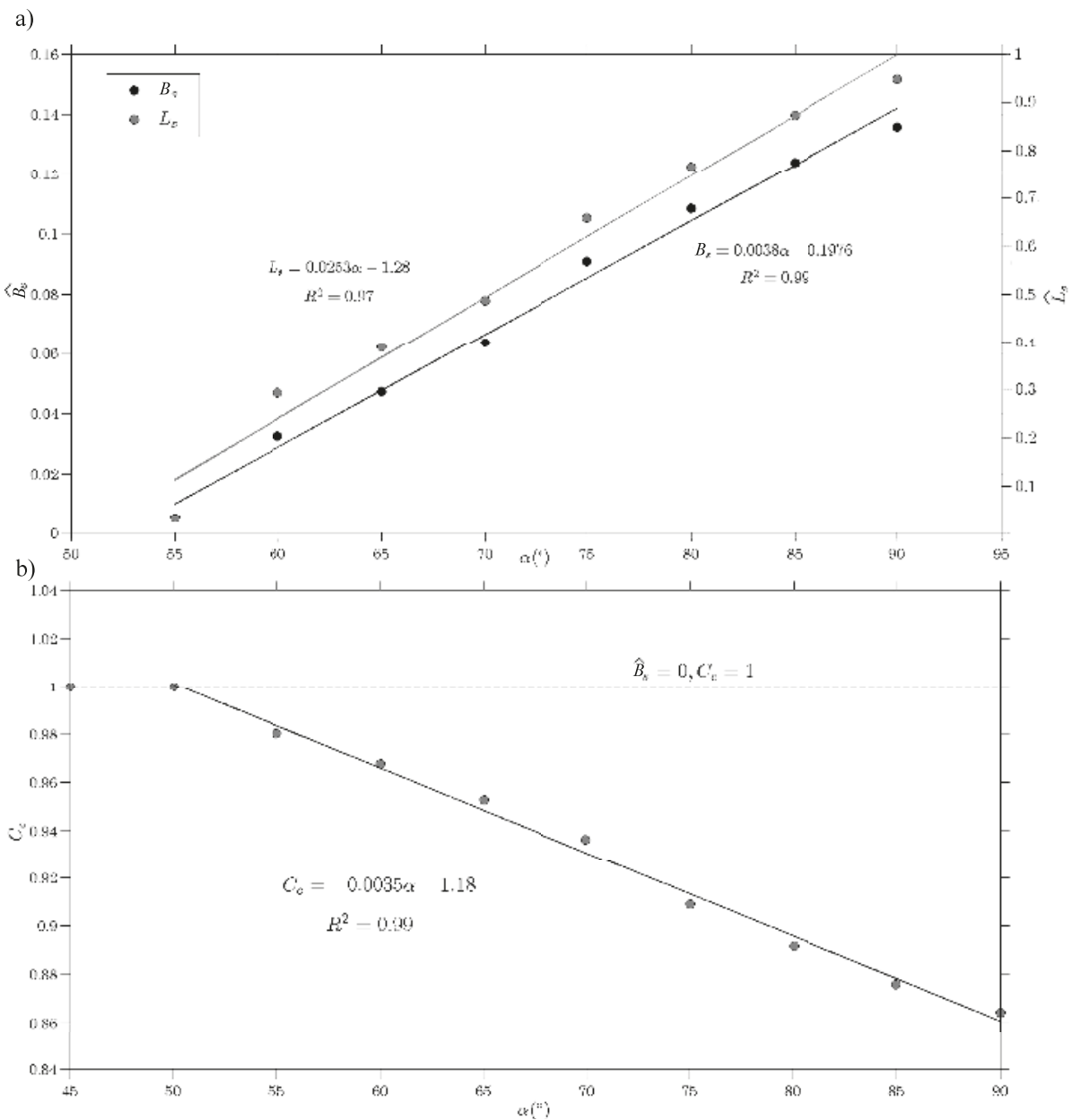

Figure 16. (a) non-dimensional width and length of the separation zone as a function of $\alpha$; (b) distribution of the contraction coefficient as a function of $\alpha$ at $\hat{z} \sim 0.5$.

Table 2 shows the position in the computational domain and the value of the non-dimensional maximum streamwise velocity (normalised with $U_{p c}=0.049 \mathrm{~ms}^{-1}$ ) in the flow field at each analysed layout, $\Delta x$ being the distance from the downstream junction corner and $\Delta y$ the distance from the inner bank of the post-confluence channel to the point in which the streamwise velocity achieves its maximum value.

It is readily noticeable that the maximum velocity occurs at $\alpha=45^{\circ}$ and, then, increasing the junction angle, it starts to decrease up to $\alpha=70^{\circ}$, where a reverse trend leads to an increasing of $\bar{u}$. This tendency could be justified considering the interference induced by the separation zone into 
the main channel flow and also by the increased discharge due to the added tributary flow. In fact, for $\alpha=45^{\circ}$, owing to the low entrance angle of the lateral flow, the tributary flow almost does not interfere with the main channel flow. The separation zone does not exist and, therefore, the zone of maximum flow velocity is confined near the wall $(\Delta y=1 \mathrm{~cm})$ at the downstream junction corner $(\Delta x=0 \mathrm{~cm})$, occupying a very small area. For $\alpha=50^{\circ}$, this area becomes wider and, since the discharge of the tributary flow is not changed, the maximum flow velocity in the post-confluence channel is smaller than for $\alpha=45^{\circ}$. Note that, also in this case, since no separation occurs, the maximum flow velocity zone is confined near the wall $(\Delta y=2 \mathrm{~cm})$ at the downstream junction corner $(\Delta x=0 \mathrm{~cm})$. Increasing $\alpha$, the streamwise velocity component reduces its magnitude because the flow acceleration is limited, owing to the presence of the separation zone that always gets wider. The maximum velocity zone increases its width too. This trend is visible up to $\alpha=70^{\circ}$. It should be highlighted that the numerical simulations are in accordance with the experiment of Birjukova et al. [23], since it was demonstrated that the zone of generally high streamwise velocities is located from $4 \mathrm{~cm}$ to $40 \mathrm{~cm}$ beyond the downstream junction corner for $\alpha=70^{\circ}$. Starting from $\alpha=75^{\circ}$, the streamwise maximum flow velocity increases and it is found at $35 \div 38 \mathrm{~cm}$ from the wall and at $43 \div 50 \mathrm{~cm}$ from the downstream junction corner. This means that such velocity values are not related to the acceleration induced by the lateral flow that approaches the post-confluence channel, which continues to decrease increasing $\alpha$. They are, instead, due to the increased discharge, owing to the tributary flow that should pass through the contraction section.

Table 2. Non-dimensional maximum streamwise velocity component at different junction angles.

\begin{tabular}{cccc}
\hline$\alpha\left(^{\circ}\right)$ & $\Delta x(\mathrm{~m})$ & $\Delta y(\mathrm{~m})$ & $\bar{u} / U_{p c}$ \\
\hline 45 & 0.00 & 0.010 & 1.475 \\
50 & 0.00 & 0.020 & 1.336 \\
55 & 0.10 & 0.070 & 1.238 \\
60 & 0.15 & 0.100 & 1.231 \\
65 & 0.16 & 0.110 & 1.211 \\
70 & 0.20 & 0.130 & 1.205 \\
75 & 0.43 & 0.355 & 1.224 \\
80 & 0.45 & 0.355 & 1.249 \\
85 & 0.49 & 0.380 & 1.272 \\
90 & 0.50 & 0.380 & 1.290 \\
\hline
\end{tabular}

Therefore, as it was discussed and unlike the common opinion or other literature findings (e.g., [1,29]), in the case of a channel confluence with low $B_{r}$ and $Q_{r}$, the maximum velocity does not necessarily occur in the contraction zone, but rather along the inner wall. This clarification is important with respect to possible scouring phenomena that can occur in correspondence of the maximum velocity zone and, thus, of the maximum bed shear stresses, in the case of mobile beds.

\section{Conclusions}

This paper constitutes a novelty in the study of the dynamics of open-channel confluences, since it focuses on a particular type of confluence characterized by fixed concordant beds and low width and discharge ratios. Specifically, it illustrates the development of the flow structure increasing the junction angle from $45^{\circ}$ to $90^{\circ}$. The paper is based on numerical simulations performed with the use of the PANORMUS code adapted ad hoc, which were compared and calibrated with the experiment performed by Birjukova et al. [23] for $\alpha$ equal to $70^{\circ}$. Ten different junction angles were analysed, focusing on the retardation zone in both the main and tributary channels, the deflection of the tributary flow from the junction angle, the extension of the separation zone downstream the channel confluence and the maximum streamwise velocity. The simulation results and analysis suggested that, as $\alpha$ increases: 
1. near the upstream lateral bank of the tributary channel, the degree of flow retardation increases, which implies wider and longer retardation zone and lower velocity in this region. In fact, the streamwise distance between the upstream junction corner and the point $P_{m}$ decreases with increasing $\alpha$, as well as the lateral distance between the upstream junction corner and the point $P_{t}$;

2. the confluent channels undergo a progressively greater deflection with respect to the flow direction in the post-confluence channel. Specifically, for all the junction angles, the deflection of the flow in the horizontal plane at the tributary entrance to the confluence increases along the interfacial plane between the tributary and the main channels, except for $\alpha=45^{\circ}$, where its distribution was more uniform than in the other cases, owing to the combination of low $\alpha, B_{r}$ and $Q_{r}$;

3. the separation zone becomes longer and wider, following a linear relationship, since, at the downstream lateral bank of the tributary, the higher the deflection angles (occurring in the case of high junction angles), the higher the separation downstream of the confluence. Moreover, for $\alpha$ less than $55^{\circ}$, the separation zone is no longer observed, owing to the low discharge ratio and deflection angles with respect to $\alpha$ at the tributary entrance into the confluence;

4. the contraction coefficient decreases linearly, since the width of the separation zone increases. The determination of this coefficient would be useful in 1D modelling to determine the effective mean velocity in the post-confluence channel;

5. the maximum streamwise flow velocity in the whole confluence region does not necessarily increase because it does not always occur in the contraction zone. This fact could be caused by the acceleration induced by the lateral flow that approaches the post-confluence channel, especially in the case of low $\alpha, B_{r}$ and $Q_{r}$.

Acknowledgments: This research was supported by the European Commission, the European Social Fund and the Regione Calabria. The authors are the only ones responsible for this research. The European Commission and the Regione Calabria are not responsible for any use that may be made of the information contained therein.

Author Contributions: Olga B. Canelas and António H. Cardoso designed and performed the laboratory test; Nadia Penna and Mauro De Marchis designed the numerical simulations; Mauro De Marchis and Enrico Napoli carried out the numerical simulations; Nadia Penna, Mauro De Marchis and Roberto Gaudio analysed experimental and numerical results. The finalization of the paper draft was made by Nadia Penna.

Conflicts of Interest: The authors declare no conflict of interest.

\section{References}

1. Best, J.L. Flow dynamics at river channel confluences: Implications for sediment transport and bed morphology. In Recent Developments in Fluvial Sedimentology; Ethridge, F.G., Flores, R.M., Harvey, M.D., Eds.; SEPM Society for Sedimentary Geology: Tulsa, OK, USA, 1987; pp. 27-35.

2. Taylor, E.H. Flow characteristics at rectangular open-channel junctions. J. Trans. 1944, 109, 893-902.

3. Biron, P.; Best, J.L.; Roy, A.G. Effects of bed discordance on flow dynamics at open channel confluences. J. Hydraul. Eng. 1996, 122, 676-682.

4. Leite Ribeiro, M.; Blanckaert, K.; Roy, A.; Schleiss, A.J. Flow and sediment dynamics in channel confluences. J. Geophys. Res. Earth Surf. 2012, 117, doi:10.1029/2011JF002171.

5. Roy, A.G.; Roy, R.; Bergeron, N. Hydraulic geometry and changes in flow velocity at a river confluence with coarse bed material. Earth Surf. Process. Landf. 1988, 13, 583-598.

6. Rhoads, B.L.; Sukhodolov, A.N. Field investigation of three-dimensional flow structure at stream confluences: 1. Thermal mixing and time-averaged velocities. Water Resour. Res. 2001, 37, 2393-2410.

7. Riley, J.D.; Rhoads, B.L.; Parsons, D.R.; Johnson, K.K. Influence of junction angle on three-dimensional flow structure and bed morphology at confluent meander bends during different hydrological conditions. Earth Surf. Process. Landf. 2015, 40, 252-271.

8. Gualtieri, C.; Filizola, N.; de Oliveira, M.; Santos, A.M.; Ianniruberto, M. A field study of the confluence between Negro and Solimões Rivers. Part 1: Hydrodynamics and sediment transport. C. R. Geosci. 2018, $350,31-42$. 
9. Ianniruberto, M.; Trevethan, M.; Pinheiro, A.; Andrade, J.F.; Dantas, E.; Filizola, N.; Santos, A.; Gualtieri, C. A field study of the confluence between Negro and Solimões Rivers. Part 2: Bed morphology and stratigraphy. C. R. Geosci. 2018, 350, 43-54.

10. Biron, P.M.; Ramamurthy, A.S.; Han, S. Three-dimensional numerical modeling of mixing at river confluences. J. Hydraul. Eng. 2004, 130, 243-253.

11. Constantinescu, G.; Miyawaki, S.; Rhoads, B.; Sukhodolov, A.; Kirkil, G. Structure of turbulent flow at a river confluence with momentum and velocity ratios close to 1: Insight provided by an eddy-resolving numerical simulation. Water Resour. Res. 2011, 47, doi:10.1029/2010WR010018.

12. Djordjevic, D. Numerical study of 3D flow at right-angled confluences with and without upstream planform curvature. J. Hydroinform. 2013, 15, 1073-1088.

13. Webber, N.B.; Greated, C. An investigation of flow behaviour at the junction of rectangular channels. Proc. Inst. Civ. Eng. 1966, 34, 321-334.

14. Ramamurthy, A.S.; Carballada, L.B.; Tran, D.M. Combining open channel flow at right angled junctions. J. Hydraul. Eng. 1988, 114, 1449-1460.

15. Schindfessel, L.; Creëlle, S.; De Mulder, T. How Different Cross-Sectional Shapes Influence the Separation Zone of an Open-Channel Confluence. J. Hydraul. Eng. 2017, 143, 04017036.

16. Best, J.L.; Reid, I. Separation zone at open-channel junctions. J. Hydraul. Eng. 1984, 110, 1588-1594.

17. Gurram, S.K.; Karki, K.S.; Hager, W.H. Subcritical junction flow. J. Hydraul. Eng. 1997, 123, 447-455.

18. Shumate, E.D. Experimental Description of Flow at an Open-Channel Junction. Ph.D. Thesis, University of Iowa, Iowa City, IA, USA, 1998.

19. Wang, X.; Wang, X.; Lu, W.; Liu, T. Experimental study on flow behavior at open channel confluences. Front. Archit. Civ. Eng. China 2007, 1, 211-216.

20. Qing-Yuan, Y.; Xian-Ye, W.; Wei-Zhen, L.; Xie-Kang, W. Experimental study on characteristics of separation zone in confluence zones in rivers. J. Hydrol. Eng. 2009, 14, 166-171.

21. Biswal, S.; Mohapatra, P.; Muralidhar, K. Flow separation at an open channel confluence. J. Hydraul. Eng. 2010, 16, 89-98.

22. Schindfessel, L.; Creëlle, S.; Boelens, T.; De Mulder, T. Flow patterns in an open channel confluence with a small ratio of main channel to tributary discharge. In Proceedings of the 7th International Conference on Fluvial Hydraulics (River Flow), Lausanne, Switzerland, 3-5 September 2014; Taylor \& Francis Group: London, UK, 2014; pp. 989-996.

23. Birjukova, O.; Guillen, S.; Alegria, F.; Cardoso, A.H. Three dimensional flow field at confluent fixed-bed open channels. In Proceedings of the 7th International Conference on Fluvial Hydraulics (River Flow), Lausanne, Switzerland, 3-5 September 2014; Crc Press-Taylor \& Francis Group: London, UK, 2014; pp. 1007-1014.

24. Yuan, S.; Tang, H.; Xiao, Y.; Qiu, X.; Zhang, H.; Yu, D. Turbulent flow structure at a 90-degree open channel confluence: Accounting for the distortion of the shear layer. J. Hydro-Environ. Res. 2016, 12, 130-147.

25. Lin, J.; Soong, H. Junction losses in open channel flows. Water Resour. Res. 1979, 15, 414-418.

26. Hsu, C.C.; Lee, W.J.; Chang, C.H. Subcritical open-channel junction flow. J. Hydraul. Eng. 1998, 124, 847-855.

27. Hsu, C.C.; Wu, F.S.; Lee, W.J. Flow at 90 equal-width open-channel junction. J. Hydraul. Eng. 1998, 124, 186-191.

28. Bradbrook, K.; Lane, S.; Richards, K.; Biron, P.; Roy, A. Role of bed discordance at asymmetrical river confluences. J. Hydraul. Eng. 2001, 127, 351-368.

29. Shakibainia, A.; Tabatabai, M.R.M.; Zarrati, A.R. Three-dimensional numerical study of flow structure in channel confluences. Can. J. Civ. Eng. 2010, 37, 772-781.

30. Bonakdari, H.; Lipeme-Kouyi, G.; Wang, X. Experimental validation of CFD modeling of multiphase flow through open channel confluence. In Proceedings of the World Environmental and Water Resources Congress 2011: Bearing Knowledge for Sustainability, Palm Springs, CA, USA, 22-26 May 2011; pp. 2176-2183.

31. Yang, Q.; Liu, T.; Lu, W.; Wang, X. Numerical simulation of confluence flow in open channel with dynamic meshes techniques. Adv. Mech. Eng. 2013, 5, 860431.

32. Brito, M.; Canelas, O.; Leal, J.; Cardoso, A. 3D numerical simulation of flow at a $70^{\circ}$ open-channel confluence. In Proceedings of the V Conferencia Nacional de Mecanica dos Fluidos, Termodinamica e Energia, Porto, Portugal, 11-12 September 2014.

33. Schindfessel, L.; Creëlle, S.; De Mulder, T. Flow patterns in an open channel confluence with increasingly dominant tributary inflow. Water 2015, 7, 4724-4751. 
34. Guillén-Ludeña, S.; Franca, M.; Cardoso, A.; Schleiss, A. Evolution of the hydromorphodynamics of mountain river confluences for varying discharge ratios and junction angles. Geomorphology 2016, 255, 1-15.

35. Napoli, E. PANORMUS Users Manual; University di Palermo: Palermo, Italy, 2011.

36. Rodríguez, J.F.; García, M.H. Laboratory measurements of 3-D flow patterns and turbulence in straight open channel with rough bed. J. Hydraul. Res. 2008, 46, 454-465.

37. Blanckaert, K.; Duarte, A.; Schleiss, A.J. Influence of shallowness, bank inclination and bank roughness on the variability of flow patterns and boundary shear stress due to secondary currents in straight open-channels. Adv. Water Resour. 2010, 33, 1062-1074.

38. Defina, A. Transverse spacing of low-speed streaks in a channel flow over a rough bed. Coherent Flow Struct. Open Channels 1996, 4, 87-99.

39. Barros, J.M.; Christensen, K.T. Observations of turbulent secondary flows in a rough-wall boundary layer. J. Fluid Mech. 2014, 748, doi:10.1017/jfm.2014.218.

40. Willingham, D.; Anderson, W.; Christensen, K.T.; Barros, J.M. Turbulent boundary layer flow over transverse aerodynamic roughness transitions: Induced mixing and flow characterization. Phys. Fluids 2014, 26, 025111.

41. De Marchis, M.; Napoli, E. The effect of geometrical parameters on the discharge capacity of meandering compound channels. Adv. Water Resour. 2008, 31, 1662-1673.

42. Chen, X.; Zhu, D.Z.; Steffler, P.M. Secondary currents induced mixing at channel confluences. Can. J. Civ. Eng. 2017, 44, 1071-1083.

(C) 2018 by the authors. Licensee MDPI, Basel, Switzerland. This article is an open access article distributed under the terms and conditions of the Creative Commons Attribution (CC BY) license (http:/ / creativecommons.org/licenses/by/4.0/). 
Article

\title{
Flow Characteristics in the Wake Region of a Finite-Length Vegetation Patch in a Partly Vegetated Channel
}

\author{
Didem Yılmazer ${ }^{1}$, Ayşe Yüksel Ozan ${ }^{2, *}$ and Kubilay Cihan ${ }^{3}$ \\ 1 Civil Engineering Department, Namık Kemal University, Çorlu 59860, Tekirdag, Turkey; \\ didem_yilmazer@yahoo.com \\ 2 Civil Engineering Department, Adnan Menderes University, Aydın 09010, Turkey \\ 3 Civil Engineering Department, Kırıkkale University, Kırıkkale 71450, Turkey; kubilaycihan@gmail.com \\ * Correspondence: ayse.yuksel@adu.edu.tr; Tel.: +90-256-213-7503 (ext. 3550)
}

Received: 26 February 2018; Accepted: 8 April 2018; Published: 11 April 2018

\begin{abstract}
Aquatic vegetation in rivers and coastal regions controls the flow structure in terms of mean velocity and turbulence. The vegetation in the flow affects the transportation of nutrients, microbes, dissolved oxygen, sediment, and contaminants; therefore, the flow characteristics of different types of vegetation layers should be examined in order to understand the effects of vegetation on the flow structure. In this paper, the effect of the submergence ratio and SVF (Solid Volume of Fraction) of a vegetation patch, which was present across half of the channel in a spanwise direction, on the flow structure at the wake region was examined. For this purpose, different submergence ratios with different SVFs were considered in the experiments, and velocity measurements were performed in the wake region of the vegetation layer with an Acoustic Doppler Velocimeter (ADV). According to the results, the effect of different vegetation heights and SVFs on the velocity distribution was obtained. Moreover, inflectional velocity distribution over the cross-section in the wake region of the vegetation layer was obtained, and it was concluded that jet flow occurred in the non-vegetated half of the channel due to the vegetation layer.
\end{abstract}

Keywords: vegetation patch; wake region; submerged ratio; SVF

\section{Introduction}

Vegetation has an important role in the sustainable development of the aquatic environment since it significantly affects the hydrodynamic characteristics of the flow. By altering the hydrodynamic conditions, submerged aquatic vegetation can dramatically affect the fate and transport of sediment, nutrients, contaminants, dissolved oxygen, and fauna in aquatic systems [1,2]. Vegetation in the aquatic environment is also very important for river management and the river environment. Thus, the investigation of the velocity distribution, turbulence structure as well as mass and momentum exchange between the vegetated and non-vegetated zones is a crucial issue for the river habitat.

In flows through submerged vegetation, the vertical discontinuity of the drag results in strong velocity shear at the top of the canopy and greatly increases the turbulence intensities in this region, relative to unobstructed flow [3-5]. Furthermore, coherent canopy-scale eddies have been observed to dominate vertical momentum fluxes into terrestrial [6-8], and aquatic canopies [5,9].

Vegetation reduces the suspended sediment transport as a result of the local reduction in bed shear stress [10]. Submerged vegetation generates coherent motions at the vegetation edge and such large-scale eddies control the vertical exchange of mass and momentum both within and above the vegetation layer [10]. 
Okamoto and Nezu (2013) [10] emphasized that a key feature of vegetation flow is the generation of a shear layer at the vegetation edge which is similar to the free shear layer. For aquatic vegetation flow, the flow structure has a range of behaviors depending on the submergence depth ratio [10].

Ghisalberti and Nepf (2004) [11] studied the dynamic equilibrium that occurred in a submerged vegetated shear layer and demonstrated that the turbulence kinetic energy in the shear layer was balanced by drag dissipation. They also presented a one-dimensional approximation of a three-dimensional flow for shear layer hydrodynamics.

Ghisalberti and Nepf (2006) [12] performed an experimental study on the structure of coherent vortices and vertical transport in shallow vegetated shear flows using rigid and flexible vegetation models. They implied that environmental flows over porous media, boulder beds, urban landscapes, wind farms, agricultural, and forest canopies were examples of an obstructed shear flow like flow over submerged vegetation. They also figured out that the vortex street in a vegetated shear layer created a pronounced oscillation in the velocity profiles. Moreover, the oscillation in vegetation had the effect of decreasing the amount of the turbulent vertical momentum transportation in the shear layer when compared to the flow over rigid vegetation [12].

Nepf (1999) [2] developed a model that predicted the turbulence intensity and diffusion within emergent vegetation. This model showed that turbulence intensity was dependent on the vegetative drag and for lower densities, which were smaller than 0.01 , the bed-drag and bed-shear production were negligible when compared to their vegetation correspondents. They also found that the turbulence scales were controlled by vegetation geometry, especially for vegetation densities higher than 0.01 [2].

Ben Meftah et al. (2014) [13] presented a new theoretical approach to model the flow pattern within the shear layer in the unobstructed area close to the vegetated area. They validated their model with experiments using rigid emergent vegetative elements for different Reynolds numbers. They showed that the peak of the turbulence intensity and that of the spanwise Reynolds Stress were shifted toward the center of the shear layer.

Ben Meftah and Mossa (2016) [14] focused on the effect of the contraction ratio, which is the ratio between the width of the obstructed and unobstructed area in the flow hydrodynamic structure. They found that the contraction ratio affected the flow hydrodynamic, and also presented an improved modified log-law that predicted the transversal profile of the mean flow velocity at the interface of obstructed and unobstructed areas.

Nepf and Vivoni (2000) [15] performed a study on the transition between submerged and emergent canopy flows. They suggested that the flow within an aquatic canopy could be divided into two regions: upper and lower. In the upper canopy, which is the "vertical exchange zone", vertical turbulence exchange with the overlying water is dynamically significant to the momentum balance and turbulence; and turbulence produced by mean shear at the top of the canopy is important [15]. The lower canopy, the "longitudinal exchange zone", communicates with surrounding water predominantly through longitudinal advection [15]. Nepf and Vivoni (2000) [15] indicated that in the lower layer, turbulence was locally generated by the canopy elements, and the momentum budget was a simple balance of vegetative drag and pressure gradient. They also emphasized that a vertical exchange zone appeared at the top of the canopy and deepened into the canopy as the depth of submergence increased in the submerged canopy.

Poggi et al. (2004) [16] developed a phenomenological model that described the turbulence structure within the canopy shear layer by using Laser Doppler Anemometry (LDA) measurements. They divided the canopy sublayer into three zones: the deep zone, where the Karman vortices drive the flow; the second zone, which is the mixing layer; and the uppermost zone where the classical surface-layer similarity theory was present (Figure 1).

Aquatic vegetation occurs in patches of finite length and different submergence ratios in natural rivers. In such vegetated flows, flow is diverted away from the vegetation patch at the leading edge of the patch [10]. In these areas, a shear layer does not form, and longitudinal advection contributes significantly to mass and momentum transport [10]. 


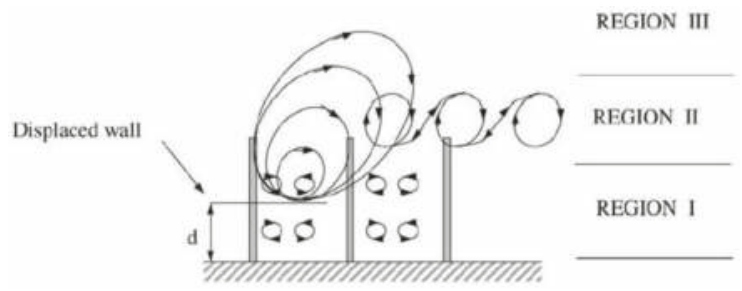

Figure 1. Regions of the canopy sub-layer [16].

Aside from experimental and analytical studies, there have also been numerical studies about vegetation in open channel flows. Brito et al. (2016) [17] studied open channel flows with submerged vegetation floodplains numerically. They modeled the vegetation layer as a porous media and demonstrated that the anisotropic turbulence EARSM (Explicit Algebraic Reynolds Stress Model) model was able to reproduce the secondary currents.

Kang and Choi (2006) [18] described a Reynolds stress model for the numerical simulation of compound open-channel flows with submerged vegetation on the floodplain. They considered various sub-models in the numerical model. Moreover, they also investigated lateral momentum transfer in the compound-open channel and demonstrated that the rate of change of the apparent shear stress increased with vegetation density. Additionally, with the increasing vegetation density on the floodplain, the drag force term was pronounced when compared with the lateral gradient of the apparent shear stress and to reduce the bottom shear stress of its 2D value in the floodplain [18].

The motivation behind this study was to examine the effect of the submergence ratio of the vegetation patch with different vegetation SVFs on the flow structure at the wake region of the patch. For this purpose, different submergence ratios with different SVFs were considered. This study especially focused on the wake region of the vegetation layer at the partly vegetated channel. The velocity measurements in the flow area were conducted with Acoustic Doppler Velocimeter (ADV).

\section{Experimental Method}

Experiments were conducted in an $11 \mathrm{~m}$ long, $1.2 \mathrm{~m}$ wide, and $0.75 \mathrm{~cm}$ deep flume with glass walls in the Civil Engineering Laboratory at Adnan Menderes University (Figures 2 and 3). The flume had an interior re-circulating system providing a steady flow. Figure 2a shows the schematic of the experimental set-up. Flow depths were measured by means of a pointer gauge and the velocity measurements were performed by SONTEK Acoustic Doppler Velocimeter (ADV). Bricks were installed along the cross section of the flume at the entrance and outlet of the flume to provide smooth inlet and outlet conditions.

The entire base of the flume was covered with a plywood sheet and a finite-length submerged vegetation patch was composed with $d=0.01 \mathrm{~m}$ diameter rigid circular plastic rods in a plywood sheet. The rods had two different heights: $h_{v 1}=0.05 \mathrm{~m}$, and $h_{v 2}=0.10 \mathrm{~m}$. The cylinders in the vegetation patch were placed in a staggered, equilateral array. For this geometry, the solid volume fraction was related to the spacing $(s)$ and diameter $(d)$ by White and Nepf, 2007 [19]:

$$
\varnothing=(\sqrt{3} \pi / 6)\left(d^{2} / s^{2}\right)
$$

In these experiments, the vegetation layer was characterized by the solid volume fraction-SVF $\left(\phi_{1}\right)$ and the height of the vegetation layer $\left(h_{v}\right)$. The variable $\phi$ was defined as the ratio of volume occupied by the cylinders to the total volume of vegetation layer. Experiments were performed for two different SVFs, $\phi_{1}=0.01$ and $\phi_{2}=0.08$, and two different vegetation heights, $h_{v 1}=0.05 \mathrm{~m}$ and $h_{v 2}=0.10 \mathrm{~m}$. Here, $\phi_{1}=0.01$ and $\phi_{1}=0.08$ corresponded to $\mathrm{N}_{1}=172 \mathrm{IP} / \mathrm{m}^{2}$, and $\mathrm{N}_{2}=1142 \mathrm{IP} / \mathrm{m}^{2}$ respectively, where IP $/ \mathrm{m}^{2}$ means the individual plant number per square meter. Moreover, the blockage caused by 
the vegetation layer was characterized by its frontal area per volume $\left(V_{0}\right)$, called the vegetation layer density, a $\left(\mathrm{m}^{-1}\right)[20]$. Vegetation density was calculated with the given equation;

$$
a=A / V_{0}=n h_{v} d / h_{v} S
$$

where $A$ is the frontal area of a vegetation element; $h_{v}$ is the vegetation height; $n$ is the number of the vegetation elements occupied in the referred bed area $S$; and $H$ is water depth.
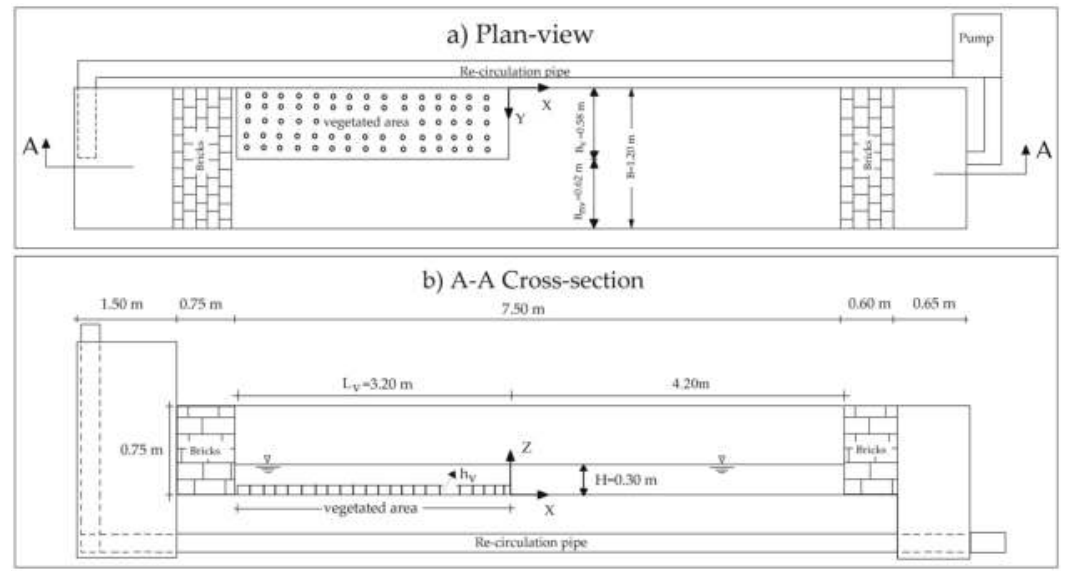

Figure 2. Schematic views of the channel: (a) Plan view of the flume; (b) A-A cross-section of the flume.

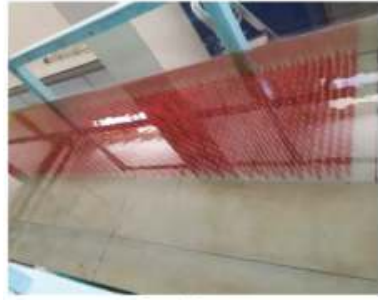

(a)

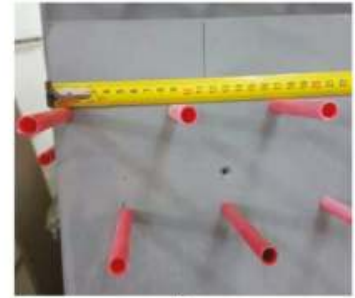

(b)

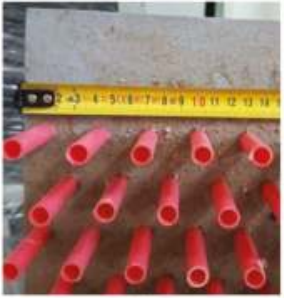

(c)

Figure 3. Photos from experimental set-up: (a) Vegetation layer in the flume; (b) vegetation layer with $\phi_{1}=0.01 ;(\mathbf{c})$ vegetation layer with $\phi_{2}=0.08$.

For all cases, the water depth, $H=0.30 \mathrm{~m}$, and bulk mean velocity were kept constant. The Reynolds Number $\left(\operatorname{Re}=\mathrm{u}_{\mathrm{dm}} H /=41,000\right)$ and Froude Number $\left(\mathrm{Fr}=\mathrm{u}_{\mathrm{dm}} / \sqrt{g H}=0.08\right)$ were calculated using the mean depth averaged velocity $\left(\mathrm{u}_{\mathrm{dm}}\right)$ for the non-vegetated case. Here, $H$ is flow depth; $v$ is the kinematic viscosity; and $g$ is the gravitational acceleration. Mean depth averaged velocity $\left(\mathrm{u}_{\mathrm{dm}}\right)$ for the non-vegetated case was used for the non-dimensionalization of the figures in Section 3. Table 1 shows the hydraulic conditions.

Table 1. Hydraulic conditions.

\begin{tabular}{ccccccc}
\hline Cases & $\mathbf{N}\left(\mathbf{I P} / \mathbf{m}^{2}\right)$ & $\phi$ & $\lambda(\mathbf{a h})$ & $h_{v}(\mathbf{c m})$ & $H / h_{v}$ & $\mathbf{u}_{\mathrm{dm}}(\mathrm{cm} / \mathbf{s})$ \\
\hline h5f1 & 172 & 0.01 & 0.08 & 5 & 6 & 13.6 \\
h5f8 & 1142 & 0.08 & 0.57 & 5 & 6 & 13.6 \\
h10f1 & 172 & 0.01 & 0.17 & 10 & 3 & 13.6 \\
h10f8 & 1142 & 0.08 & 1.14 & 10 & 3 & 13.6 \\
\hline
\end{tabular}


The three instantaneous components of velocity $(\mathrm{u}, \mathrm{v}, \mathrm{w})$, corresponding to the streamwise, spanwise and vertical velocity components in $\mathrm{X}, \mathrm{Y}$ and $\mathrm{Z}$ directions, respectively, were measured with ADV during $120 \mathrm{~s}$ with 3000 sampling number at $25 \mathrm{~Hz}$. Each velocity record was decomposed into time averages $(\bar{u}, \bar{v}, \bar{w})$ and fluctuating components $\left(\mathrm{u}^{\prime}, \mathrm{v}^{\prime}, \mathrm{w}^{\prime}\right)$. The ADV probe was established over the vegetation layer during the experiments. The velocity measurements were performed at $117(=9 \times 13)$ points in the $X Y$ plane (Figure $4 a)$ and six different levels in the $Z$ direction (Figure $4 b$ ). Measurement points along the $X$ and $Y$ directions were placed at $10 \mathrm{~cm}$ intervals and $2.5 \mathrm{~cm}$ in the $\mathrm{Z}$ direction. Only, point Z1 $(=0.05 \mathrm{~cm})$ was placed at the closest point to the wall where the velocity measurement could be performed (Figure $4 b$ ).

ADV measurements sometimes may include scattering data because of using clean water without suspended material and correlation scores. If the correlation factor of measured velocities are very low then velocity measurements can not be reliable. The manufacturer recommends that a $15 \mathrm{db}$ signal-to-noise ratio (SNR) and a correlation coefficients larger than $70 \%$ for high-resolution measurements. Therefore, the poor quality samples in the measured data were removed using $\mathrm{SNR}<15 \mathrm{db}$ and correlation coefficient $<70 \%$ conditions.
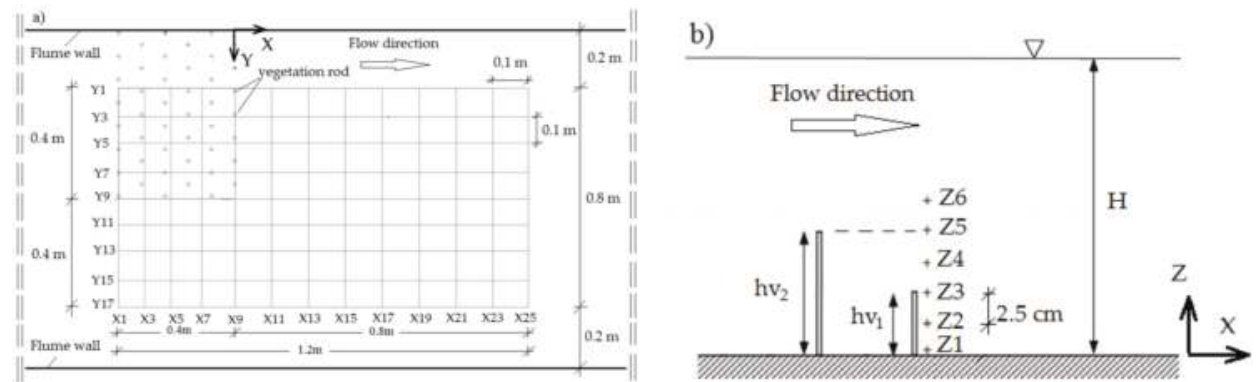

Figure 4. Velocity measurements grid: (a) Plan-view; (b) Side-view.

\section{Results and Discussion}

Figure 5 presents the non-dimensional velocity distributions for the non-vegetated case along the vertical direction at different sections of the channel and logarithmic velocity distribution. It could be seen that the streamwise velocity distribution represented the best fitted logarithmic regression line for the non-vegetated case. The logarithmic line was obtained by using the law [21] for rough boundaries, which is

$$
\frac{u}{u_{*}}=\frac{1}{\kappa} \ln \left(\frac{30 z}{k_{s}}\right)
$$

where $u$ is streamwise point velocity; $u_{*}$ is local shear velocity; $\mathrm{k}$ is von Karman's constant; $z$ is distance from the measurement point to the bed; and $k_{s}$ is the Nikuradse equivalent bed roughness. Velocity profiles, which were plotted on semi-logarithmic paper, fitted the logarithmic law display as a constant gradient [22,23].

Figure 6 presents an example of a measured velocity for non-vegetated case (Figure 6a) and spectral distribution (Figure $6 \mathrm{~b}$ ) for $h_{v 2}=0.10 \mathrm{~m}$ at X13Y3Z2. This point corresponds to the wake region of the vegetated layer. Velocity range was chosen as $\pm 0.3 \mathrm{~m} / \mathrm{s}$ with a velocity accuracy of \pm 1 in the measurements. A signal-to-noise ratio (SNR) higher than $15 \mathrm{~dB}$ is recommended for $25 \mathrm{~Hz}$ and higher sample rates, and the typical threshold for data acceptance is a correlation coefficient higher than $70 \%$ for high-resolution measurements [24]. Therefore, the measured data were filtered with Phase-space threshold despiking $[25,26]$ which is the default filter in winADV and poor quality samples were removed using SNR $<15 \mathrm{~dB}$ and correlation coefficient $<70 \%$. 


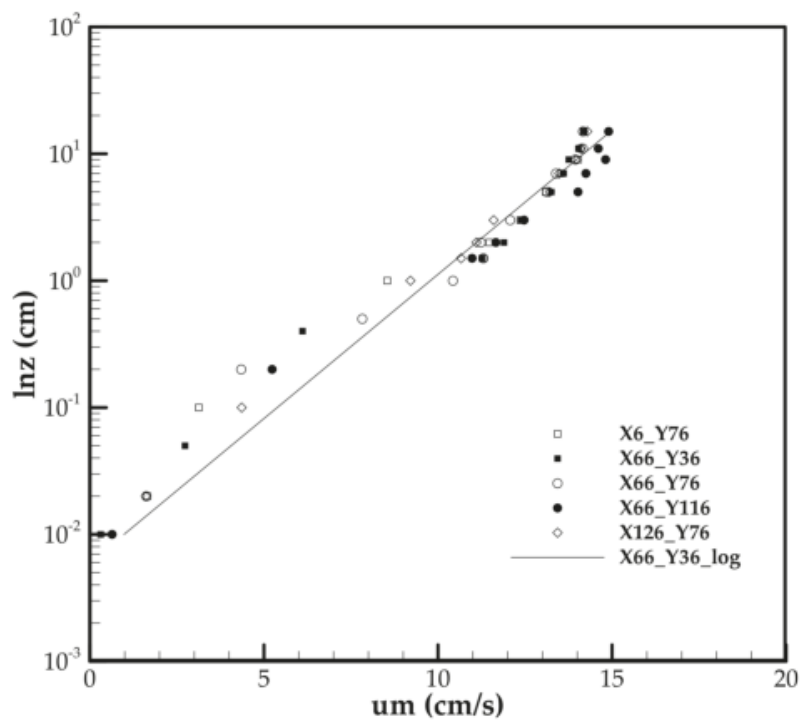

Figure 5. Velocity profiles in semi-logarithmic form for non-vegetated case at different sections of the channel.

In order to explore the distribution of energy in the signal across frequencies, examples of the power spectral densities (PSD) of velocity fluctuations in three directions were plotted in Figure 6b. The spectrum frequency between 0.2 and $0.6 \mathrm{~Hz}$ which indicates the scaling subrange is referred to as the production subrange. This range is characterized by a $-3 / 2$ spectral slope [27]. An inertial subrange with $\mathrm{a}-5 / 3$ power law between 0.9 and $5 \mathrm{~Hz}$ which is in a narrow range was observed. There is transitional subrange between 0.6 and $0.9 \mathrm{~Hz}$ which corresponds to transition between the production subrange and the inertial subrange. In the transition subrange, a transformation of the turbulent energy of large scale motion to energy of small scale motion takes place [27]. The PSD has small values at a frequency highger than $5 \mathrm{~Hz}$, which corresponds to the universal equilibrium range. These results are in agreement with results obtained by Meftah and Mossa [27] and Nezu and Onitsuka [28].

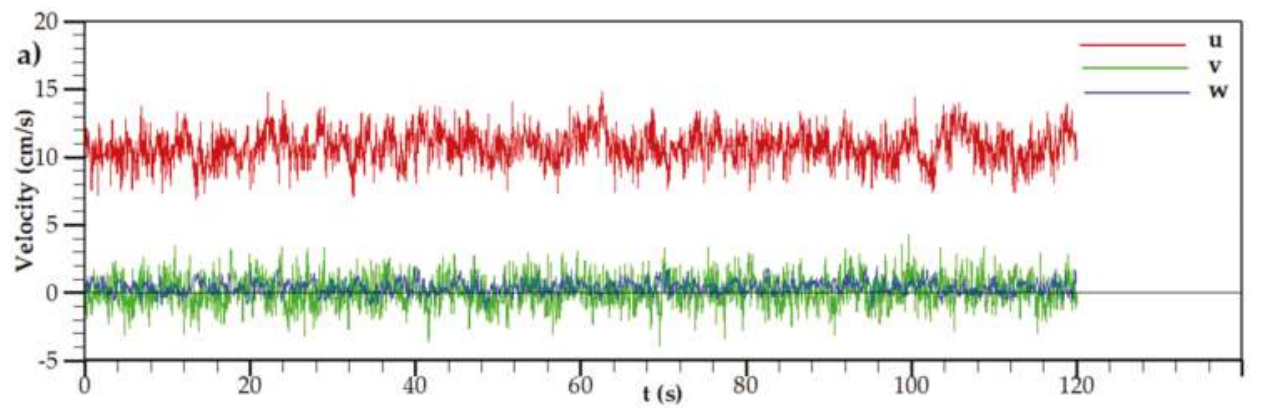

Figure 6. Cont. 


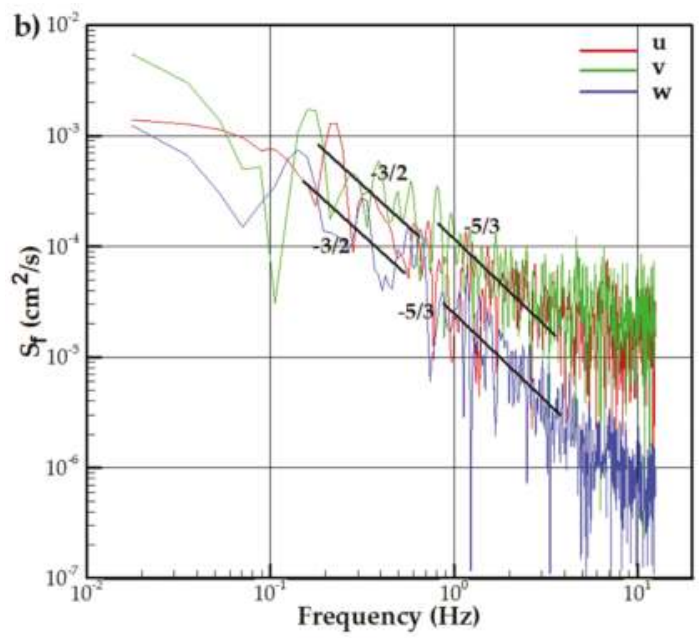

Figure 6. (a) Measured velocity signal at X13Y3Z2 for non-vegetated case; (b) Spectral distribution at $\mathrm{X} 13 \mathrm{Y} 3 \mathrm{Z} 2$ for $h_{v 2}=0.10 \mathrm{~m}$.

Figure 7 shows the non-dimensional streamwise velocity distributions along the vertical direction at different spanwise (Y3, Y9, and $\mathrm{Y} 15)$ and streamwise (X1, X5, X9, X13, X17, and X25) sections for $h_{v 1}=0.05 \mathrm{~m}$ and $h_{v 2}=0.10 \mathrm{~m}$ with $\phi=0.08$, respectively. Here, $\mathrm{Y} 3, \mathrm{X} 1$, and X5 corresponded to the vegetated area whereas $Y 9$ and $X 9$ corresponded to the edge of the vegetation layer (Figure 4a). Additionally, Y15 was placed in the non-vegetated half of the channel whereas X13, X17, and X25 were placed at the wake region of the vegetated layer (Figure 4a). It was obvious that the vegetation layer decreased velocity both inside the vegetation layer and also in the wake region (Figure 7a). Furthermore, the velocity decreased inside and in the wake region of the vegetation layer while it had the same value as the non-vegetated case downstream of the vegetated area (Figure 7b). Moreover, the velocity increased to remain off the vegetation layer (Figure 7c). This increase occurred at about $20 \%$ for $h_{v 2}$ and $10 \%$ for $h_{v 1}$. Furthermore, the velocity difference increased between the vegetated and non-vegetated cases, which was higher for higher vegetation height even when both cases had the same SVF. The main conclusion from these figures was that the velocity decrease occurred in the wake of the vegetation patch whereas velocity increase, which refers to jet flow, occurred along the unobstructed part of the channel.

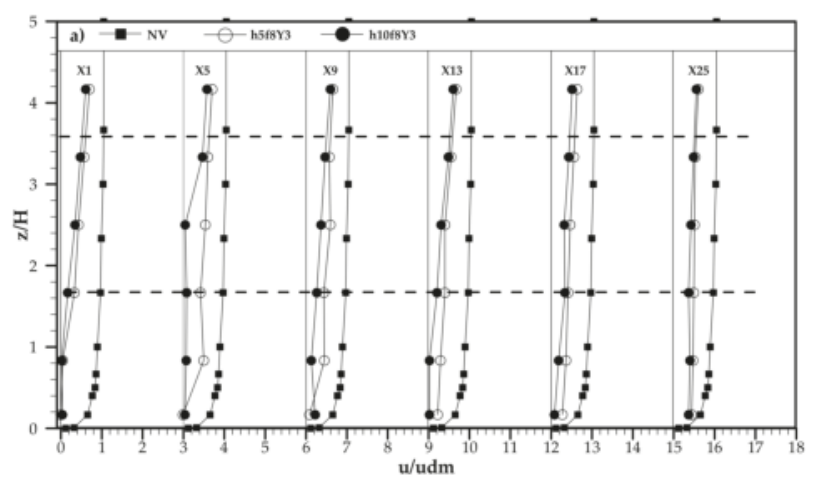

Figure 7. Cont. 

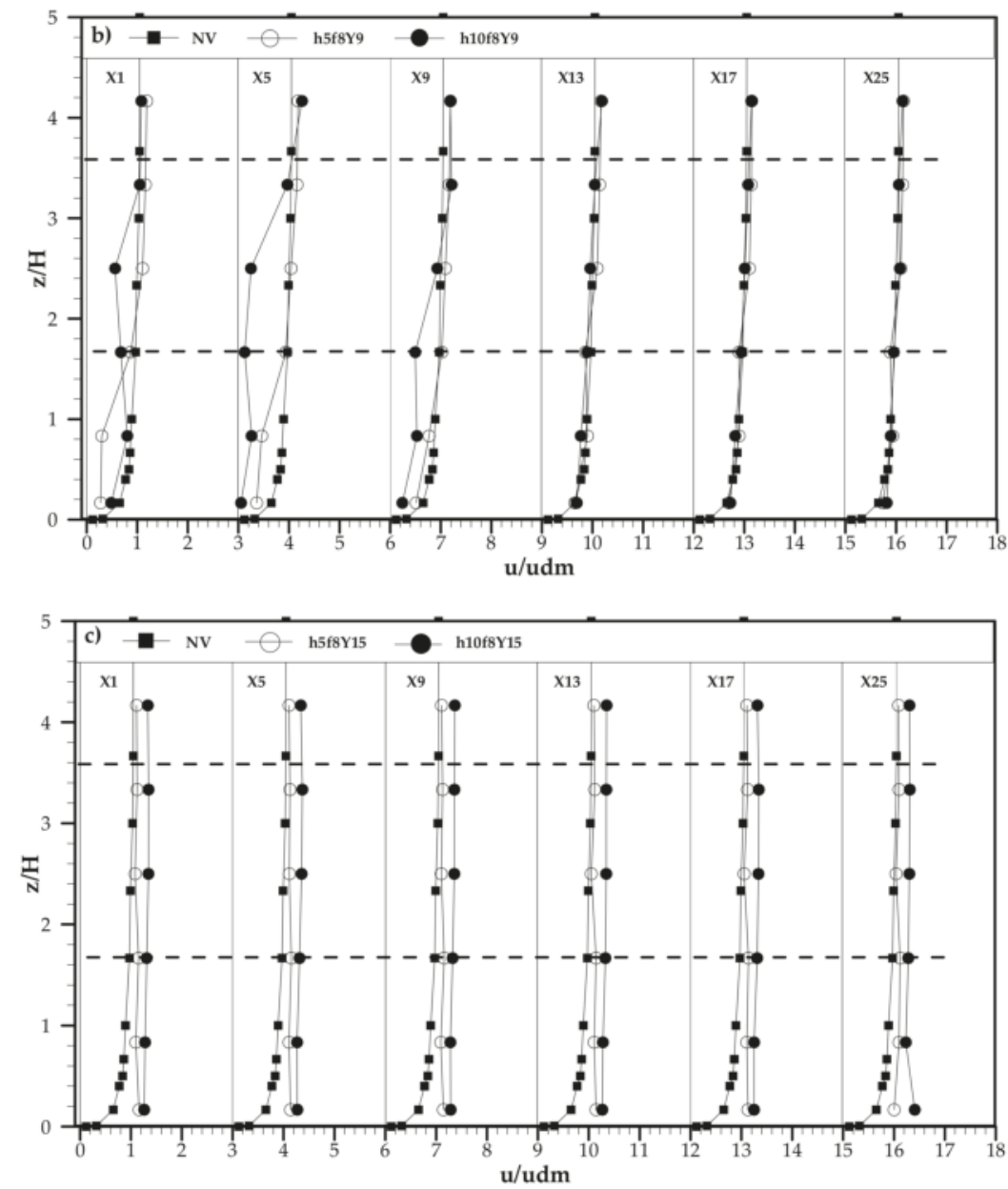

Figure 7. Non-dimensional streamwise velocity distributions along vertical direction at different streamwise sections for both vegetation heights and $\phi=0.08$ : (a) Y3; (b) Y9; (c) Y15.

Yang et al. (2007) [29] studied the flow patterns in a compound channel with vegetated floodplains and used different types of artificial submerged vegetative elements in the experiments distribution on the floodplain. They also found similar velocity distributions for the vegetated flood plain where velocity decreased, and the non-vegetated main channel where velocity increased for compound open-channels.

Figure 8 presents the non-dimensional streamwise velocity distributions at the $\mathrm{Y} 3$ line along the streamwise direction for $h_{v 1}=0.05 \mathrm{~m}$ and $h_{v 2}=0.10 \mathrm{~m}$ with different SVFs. Here, Z2 and Z6 corresponded to the point at the half of the vegetative height, and Z4 and Z6 corresponded to the point over the vegetation layer. It could be seen that low SVF had little effect on the velocity distribution over the vegetation while higher SVF had a large effect, which was an important decrease in velocity distribution inside the vegetation layer for $h_{v 1}=0.05 \mathrm{~m}$. However, with the higher vegetation height $\left(h_{v 2}=0.10 \mathrm{~m}\right)$, the vegetation layer affected the velocity distribution both within and over the vegetation layer. Within the vegetation layer, both SVF values caused a velocity decrease whereas the effect was larger for higher SVF. However, velocity values overlapped to remain off the vegetation layer, especially 
in the wake region for $h_{v}=0.05 \mathrm{~m}$ vegetation height. However, this was not observed for higher vegetation height $\left(h_{v 2}=0.10 \mathrm{~m}\right)$. Velocity decrease occurred in about $50 \%$ of the wake region for higher SVF for both vegetation height cases.
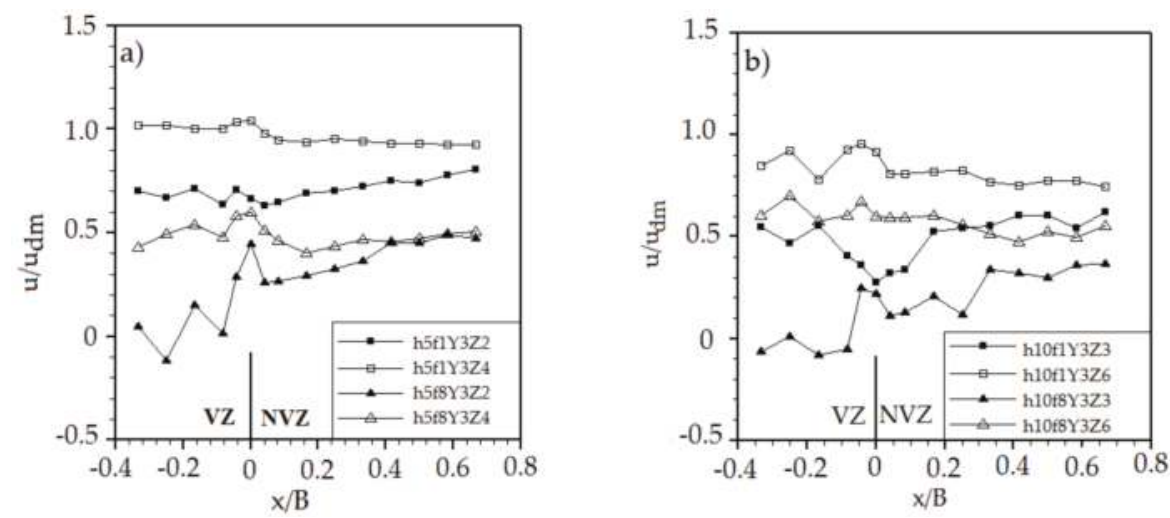

Figure 8. Non-dimensional streamwise velocity distributions at $\mathrm{Y} 3$ line along the streamwise direction for different SVFs, (a) $h_{v 1}=0.05 \mathrm{~m}$; (b) $h_{v 2}=0.10 \mathrm{~m}$.

Figures 9 and 10 show the non-dimensional streamwise velocity distributions along the spanwise direction at different sections of the wake region for $z / h_{v}=0.5$ and $z / h_{v}=1.5$, respectively. The figures are for lower vegetation height $\left(h_{v 1}=0.05 \mathrm{~m}\right)$ and different SVFs. It was found that higher SVF decreased the flow velocity more than the lower SVF for outer layer of the vegetation zone (Figure 10). Apart from this, velocity decrease was similar for both SVFs close to the bed (Figure 9). It was also observed that close to the bed $\left(z / h_{v}=0.5\right)$, the velocity decrease continued further out of the vegetated area $(\mathrm{y} / \mathrm{B} \cong 0.7)$. However, the velocity decrease disappeared at the vegetation edge and velocity started to increase over the vegetation layer along the spanwise direction. The effect of SVF over the vegetation layer can be seen remarkably in Figures 9 and 10. The velocity decrease was still present over the vegetation layer, and the effect of vegetation was still present in the other half of the channel. Moreover, the velocity difference was about $0.5-0.6 \mathrm{u}_{\mathrm{m}}$ for both $\mathrm{Z} 2$ and $\mathrm{Z} 4$ between the vegetated and non-vegetated zones of the flow over the cross-section. However, the main difference was that the velocity was higher than the non-vegetated case at about $1.2 \mathrm{u}_{\mathrm{m}}$ for $\mathrm{Z} 2$ where it was about $1.1 \mathrm{u}_{\mathrm{m}}$ for $\mathrm{Z} 4$ at the non-vegetated zone. This gives an idea about the occurrence of jet flow at the non-vegetated zone of the flow. Additionally, a higher SVF caused a higher drag force, which can be clearly seen in Figure 10 where the higher SVF had smaller velocity values at both zones of the flow. Another important feature obtained from Figure 10 was the inflectional velocity profile over the cross-sections, and the velocity profile had a steep shape for higher SVF over the vegetation layer. This type of velocity distribution also implied that jet flow occurred in the non-vegetated half of the channel. This jet flow was present in all parts of the non-vegetated zone, even starting inside the vegetation layer, which was close to the vegetation edge at $z / h_{v}=1.5$ where it started at $(\mathrm{y} / \mathrm{B} \cong 0.7)$ close to the bed $\left(z / h_{v}=0.5\right)$. Nezu and Onitsuka (2001) [28] performed measurements on partly vegetated open-channel flows and also found similar results over the cross-section. The difference of this study is that the results were given for the wake region. Ikeda et al. [30,31] demonstrated that this type velocity profile showed a generation of coherent horizontal vortex due to an inflectional instability. 

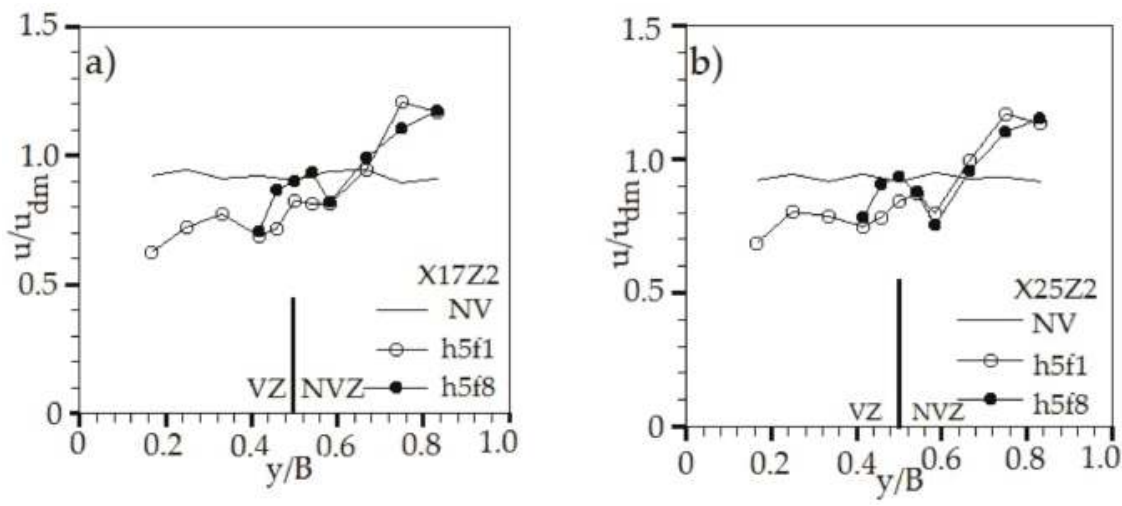

Figure 9. Non-dimensional streamwise velocity distributions along the spanwise direction at $z / h_{v}=0.5$ for $h_{v}=0.05 \mathrm{~m}$ and different SVFs; (a) X17; (b) X25.
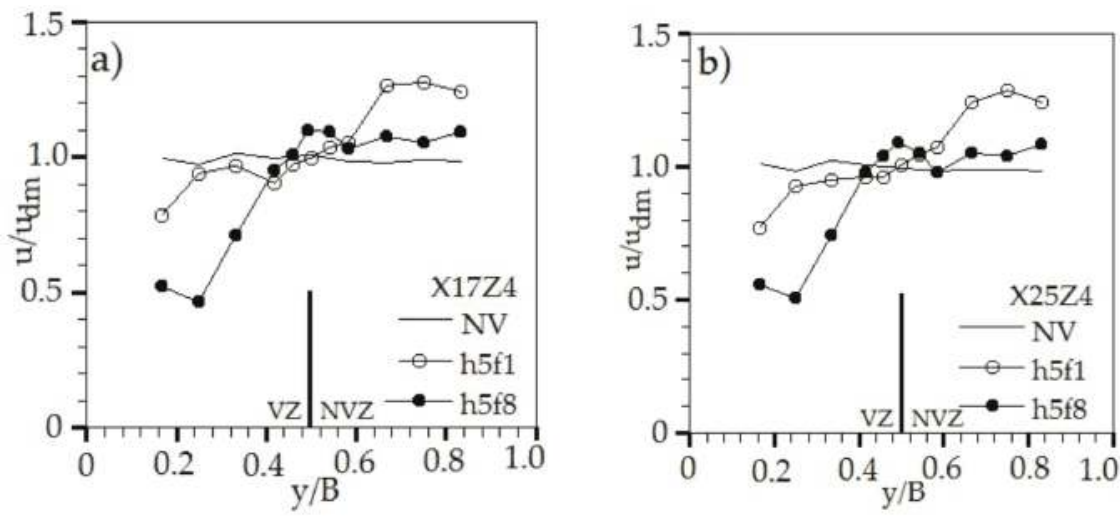

Figure 10. Non-dimensional streamwise velocity distributions along the spanwise direction at $z / h_{v}=1.5$ for $h_{v}=0.05 \mathrm{~m}$ and different SVFs; (a) X17; (b) X25.

Figures 11 and 12 show the non-dimensional streamwise velocity distributions along the spanwise direction at different streamwise sections for $z / h_{v}=0.5$ and $z / h_{v}=1.25$, respectively. The figures are for higher vegetation height $\left(h_{v}=0.10 \mathrm{~m}\right)$ and different SVFs. Figure 11 presents the velocity distribution close to the bed, which was within the vegetation layer, and the velocity distribution here showed similar behavior to the velocity distribution over the vegetation layer for lower vegetation height. Still, there was a steeper inflectional velocity distribution for higher SVF. However, here there was an important difference from lower vegetation height. The difference was that in the non-vegetated zone of the channel, the velocity values were higher for higher SVF than the lower SVF. Moreover, the velocity difference was about $0.7-0.8 \mathrm{u}_{\mathrm{m}}$ for lower SVF where it was 1.0-1.1 $\mathrm{u}_{\mathrm{m}}$ for higher SVF at both Z3 and a Z6 between the vegetated and non-vegetated zones of the flow over the cross-section. Another important result was that the velocity increase ratio was almost the same for both SVF at the non-vegetated zone where the velocity decrease ratio was higher for higher SVF in the vegetated zone, which was about $0.2 \mathrm{u}_{\mathrm{m}}$. The other difference was that the velocity increase started at the junction between the vegetated and non-vegetated zones for higher SVF and at the non-vegetated zone, which was about y/B 0.7 for lower SVF. 

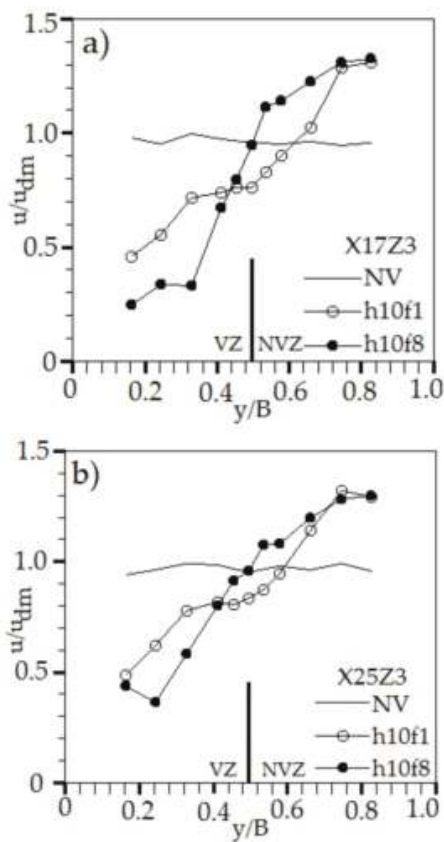

Figure 11. Non-dimensional streamwise velocity distributions along the spanwise direction at $z / h_{v}=0.5$ for $h_{v}=0.1 \mathrm{~m}$ and different SVFs; (a) X17; (b) X25.
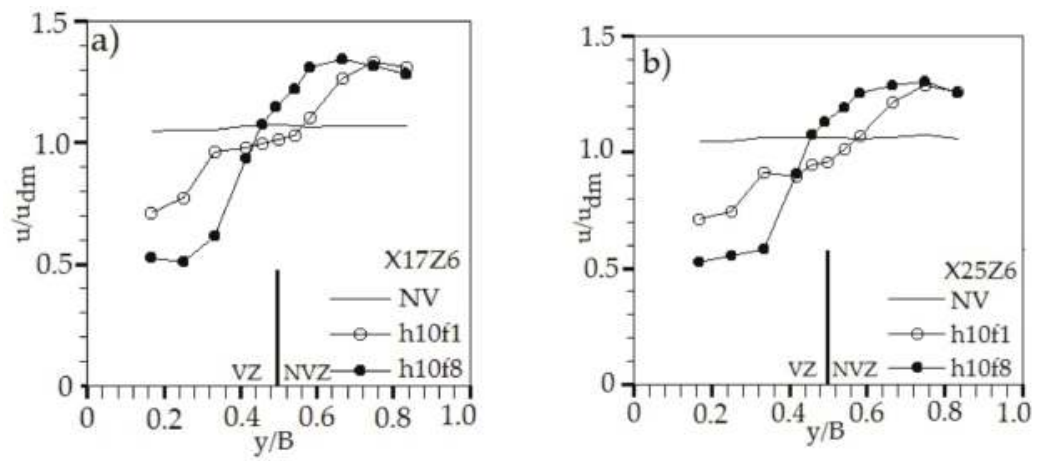

Figure 12. Non-dimensional streamwise velocity distributions along the spanwise direction at $z / h_{v}=1.25$ for $h_{v}=0.1 \mathrm{~m}$ and different SVFs; (a) X17; (b) X25.

Meftah et al. [32] studied on flow patterns in partly obstructed channel. They used a rigid emergent vegetation layer to illustrate the obstruction in the channel. Density of the vegetated area was given as $n=400$ cylinders $/ \mathrm{m}^{2}$ in their studies. They performed mid-depth velocity measurements using ADV in the experiments. They stated that the flow properties depend on contraction ratio $\left(C_{r}\right)$ which is the ratio of the width of the obstructed area to the width of the unobstructed area. The results they obtained in the unobstructed area near the obstructed area and our results in the wake region of vegetation layer (data in Figure 11 where data correspond to the mid-depth of the vegetation layer) were plotted in Figure 13. Here, R1, R2, R3 and R4 correspond to $C_{r}=3.21, C_{r}=1.05, C_{r}=0.36$ and $C_{r}=0.16$, respectively. The contraction ratio was $C_{r}=0.94$ in our study. In Figure 13, $y^{\prime}$ starts at the 
edge of the vegetation layer and $B_{n v}$ is the width of the non-vegetated part. Velocity values near the edge are higher than Meftah et al. [32]'s velocity values. This is because our data were obtained in the wake region of the vegetation layer. Another important point was that an increasing velocity trendline occurred between $\mathrm{y}^{\prime} / \mathrm{B}_{\mathrm{nv}}=0$ and $\mathrm{y}^{\prime} / \mathrm{B}_{\mathrm{nv}}=0.5$ and the horizontal velocity trendline started at $\mathrm{y}^{\prime} / \mathrm{B}_{\mathrm{nv}}=0.5$ for both of the studies. The horizontal velocity trendline of this study corresponds to R3 which has $\mathrm{C}_{\mathrm{r}}=0.36$ contraction ratio.

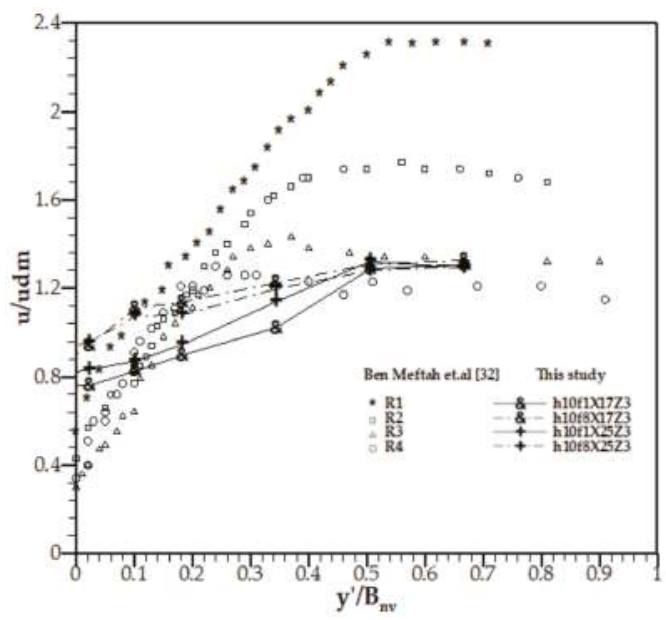

Figure 13. Transversal profiles of non-dimensional velocity distributions versus $\mathrm{y} / \mathrm{B}_{\mathrm{nv}}$.

Figures 14 and 15 show the non-dimensional vertical velocity distributions at the top edge of the vegetation layer along the streamwise direction for $h_{v 1}=0.05 \mathrm{~m}$ and $h_{v 2}=0.1 \mathrm{~m}$, respectively. Here, Y5, Y9, and Y13 corresponded to inside the vegetation layer, edge of the vegetation layer, and non-vegetated half part of the channel in spanwise direction, respectively. Vertical velocity had positive values in the non-vegetated half of the channel for all cases and had higher values for higher SVF $(\phi=0.08)$ than lower SVF $(\phi=0.01)$. Apart from this, the vertical velocity distribution showed different behavior for different SVFs at the vegetation layer. It had negative values along the streamwise direction for lower SVF ( $\phi=0.01$ ). Additionally, vertical velocity was positive in the vegetated layer and negative in the wake region of the vegetated layer and different from zero for higher SVFs in the vegetation layer.
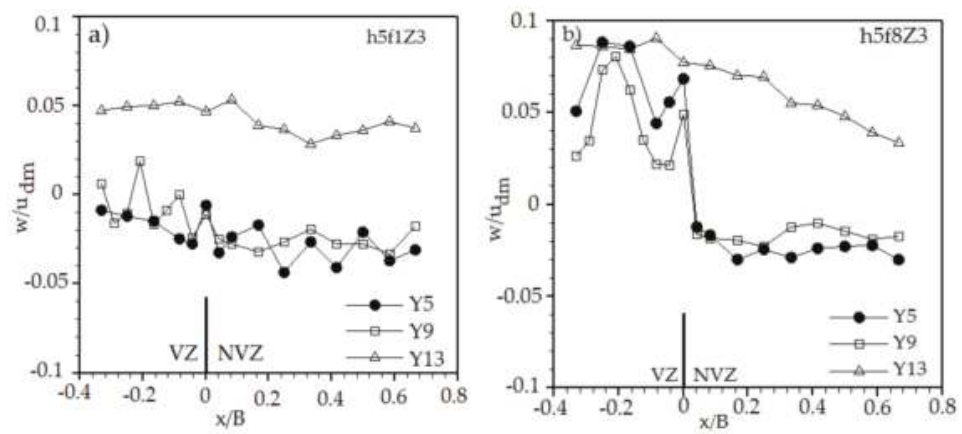

Figure 14. Non-dimensional vertical velocity distributions along the streamwise direction at $z / h_{v}=1$ for $h_{v}=0.05 \mathrm{~m} ;(\mathbf{a}) \phi=0.01 ;(\mathbf{b}) \phi=0.08$. 

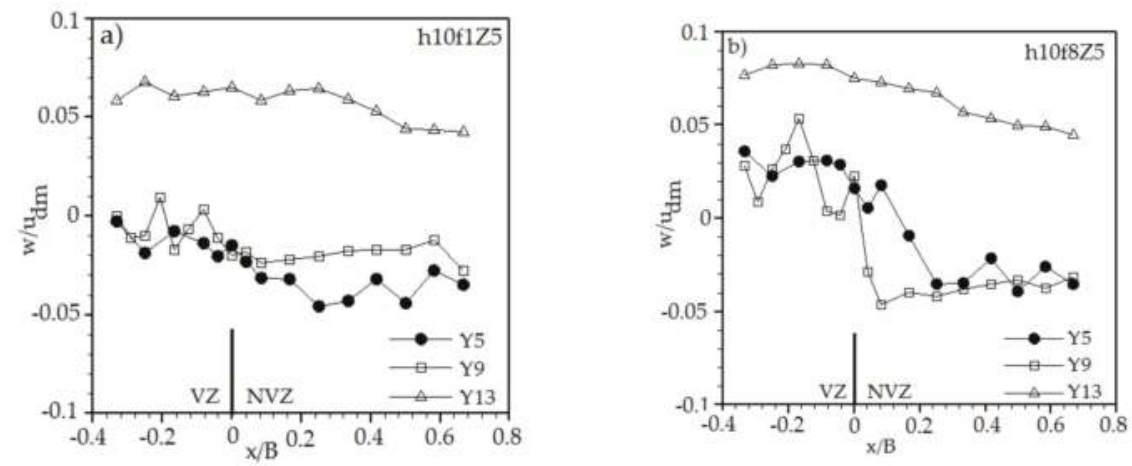

Figure 15. Non-dimensional vertical velocity distributions along the streamwise direction at $z / h_{v}=1$ for $h_{v}=0.1 \mathrm{~m} ;(\mathbf{a}) \phi=0.01 ;(\mathbf{b}) \phi=0.08$.

In Figure 16, the non-dimensional Reynolds Stresses $\left(u^{\prime} w^{\prime}\right)$ at the top edge of the vegetation layer along the $Y 5$ section are shown for all cases. It was evident that higher vegetation SVF caused higher Reynolds Stress, which was significant for higher vegetation height. The Reynolds Stress $\left(u^{\prime} w^{\prime}\right)$ showed a decreasing trend along the wake region for all cases. This result was consistent with the fact that canopies with $\lambda=a h \geq 0.1$ provided sufficient resistance to generate the vegetation shear layer downstream of the patch (see [33]).
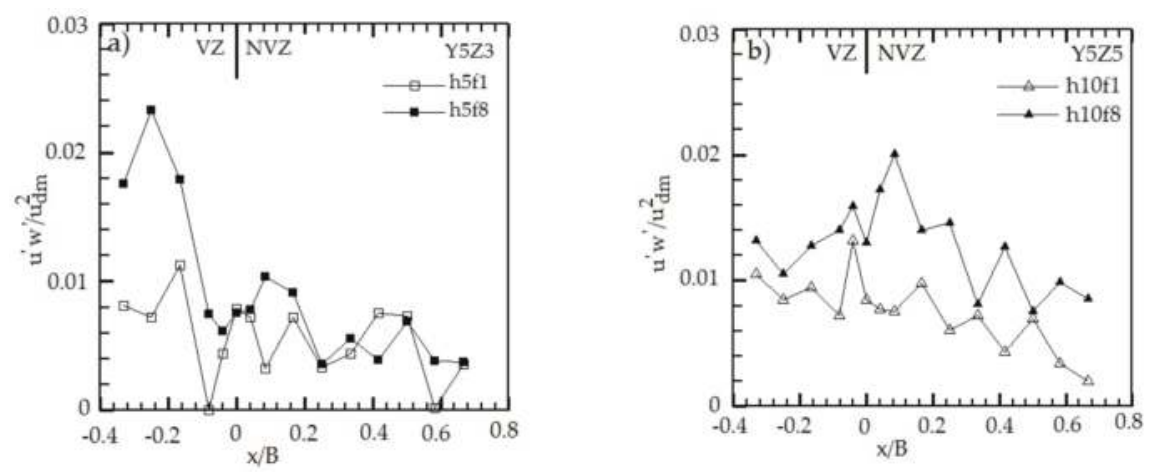

Figure 16. Non-dimensional Reynolds stresses along Y5 section for all cases; (a) $h_{v}=0.05 \mathrm{~m}$; (b) $h_{v}=0.1 \mathrm{~m}$.

White and Nepf (2007) [19] found that the peak of the turbulence intensity in the vegetation layer occurred in the vicinity of the junction between the vegetated and unvegetated parts of the channel at the partially obstructed channels. Here, the effects of vegetation height on the Reynolds stresses $\left(u^{\prime} w^{\prime}\right)$ inside the vegetation and at the wake region were also investigated. For this purpose, the Reynolds stresses at the top edge of the vegetation at two different spanwise directions for denser cases were plotted (Figure 17). In Figure 17, X5 and X19 correspond to inside of the vegetation layer and the wake region of it, respectively. It was observed that in both sections, a higher vegetation layer caused higher shear stress at the top of the vegetation layer. The other important result was that vegetation affected the non-vegetated half of the channel until some point at X5 (Figure 17a). However, the vegetation did not affect the non-vegetated half of the channel in the wake region (Figure 17b). 

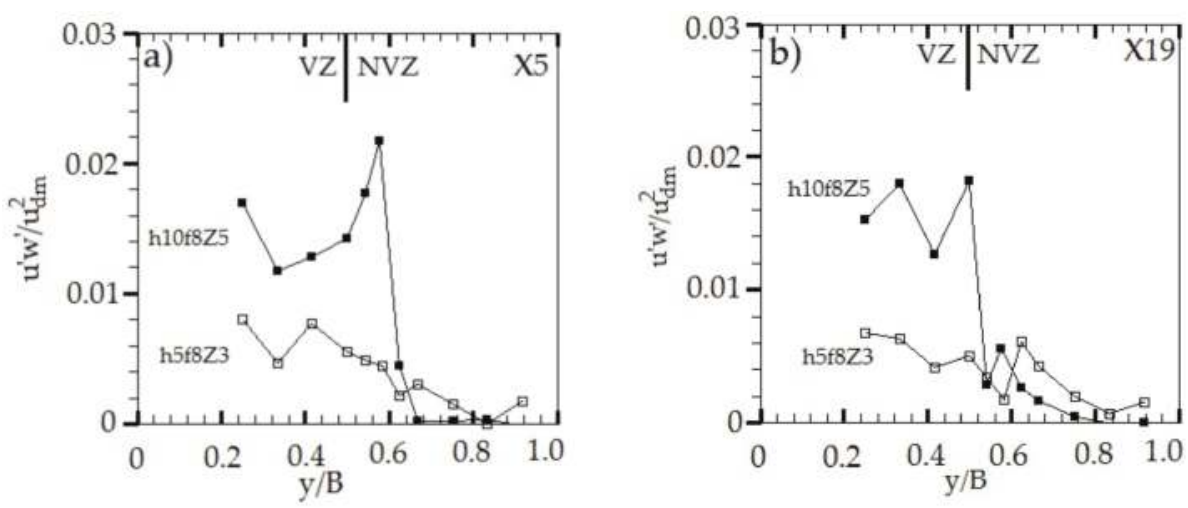

Figure 17. Non-dimensional Reynolds stresses distribution at the top edge of the vegetation layer along the spanwise direction; (a) X5; (b) X19.

\section{Conclusions}

In this study, the effect of a finite-length vegetation patch placed across half of the channel on the flow structure was examined. The study mainly focused on the wake region of the vegetative patch. For this purpose, an experimental study with different submergence ratios and SVFs was carried out.

It was observed that the vegetation layer decreased velocity both inside the vegetation layer and also in the wake region. Additionally, the velocity difference increased between the vegetated and non-vegetated cases, which was higher for higher vegetation height even when both cases had the same SVF. Different vegetation heights affected the velocity distribution in a different way, for example, low SVF had little effect on the velocity distribution over the vegetation layer while higher SVF had a large effect, which was an important decrease in the velocity distribution along the cross-section of the channel for $h_{v 1}=0.05 \mathrm{~m}$ vegetation height. However, with the higher vegetation height $\left(h_{v 2}=0.10 \mathrm{~m}\right)$, the vegetation layer affected the velocity distribution both within and over the vegetation layer. The velocity distribution over the cross-sections had inflectional velocity distribution for both vegetation heights. This type of velocity distribution implied that jet flow occurred in the non-vegetated half of the channel.

In addition, the vertical velocity distribution was also examined, and it was found that different behaviors for different SVFs were present at the vegetation layer. Moreover, vertical velocity was positive in the vegetated layer and negative in the wake region of vegetated layer.

Furthermore, the Reynolds Stress $\left(u^{\prime} w^{\prime}\right)$ distribution was also investigated. It was demonstrated that higher SVF caused higher Reynolds stresses, which was significant for higher vegetation height at the edge of the vegetation layer. The Reynolds Stresses $\left(u^{\prime} w^{\prime}\right)$ also displayed a decreasing trend along the wake region for all cases.

Acknowledgments: The authors would like to acknowledge the funding of the research project (MF-17008) from Adnan Menderes University (ADU). The authors would also like to thank the ADU Civil Engineering Department students Canberk Karacasu and Rıdvan Sarıül who helped during the experiments.

Author Contributions: Didem Yılmazer, Ayşe Yüksel Ozan and Kubilay Cihan conceived and designed the experiments; Didem Yılmazer, Ayşe Yüksel Ozan and Kubilay Cihan performed the experiments; Didem Yılmazer, Ayşe Yüksel Ozan and Kubilay Cihan analyzed the data; Ayşe Yüksel Ozan wrote the paper. All authors read and approved the final manuscript.

Conflicts of Interest: The authors declare no conflict of interest. 


\section{References}

1. Fonseca, M.S.; Kenworthy, W.J. Effects of current on photosynthesis and distribution of seagrasses. Aquat. Bot. 1987, 27, 59-78. [CrossRef]

2. Nepf, H.M. Drag, turbulence, and diffusion in flow through emergent vegetation. Water Resour. 1999, 35, 479-489. [CrossRef]

3. Gambi, M.C.; Nowell, A.R.M.; Jumars, P.A. Flume observations on flow dynamics in Zostera marina (eelgrass) beds. Mar. Ecol. Prog. Ser. 1990, 61, 159-169. [CrossRef]

4. Vivoni, E.R. Turbulence Structure of a Model Seagrass Meadow. Ph.D. Thesis, Massachusetts Institute of Technology, Cambridge, UK, 1998.

5. Wallace, S.; Luketina, D.; Cox, R. Large scale turbulence in seagrass canopies. In Proceedings of the 13th Australasian Fluid Mechanics Conference, Melbourne, Australia, 13-18 December 1998.

6. Raupach, M.R.; Shaw, R.H. Averaging procedures for flow within vegetation canopies. Bound. Layer Meteorol. 1982, 22, 79-90. [CrossRef]

7. Gao, W.; Shaw, R.H.; Paw, U.K.T. Observation of organized structure in turbulent flow within and above a forest canopy. Bound. Layer Meteorol. 1989, 47, 349-377. [CrossRef]

8. Raupach, M.R.; Finnigan, J.J.; Brunet, Y. Coherent eddies and turbulence in vegetation canopies: The mixing layer analogy. Bound. Layer Meteorol. 1996, 78, 351-382. [CrossRef]

9. Ikeda, S.; Kanazawa, M. Three-dimensional organized vortices above flexible water plants. J. Hydraul. Eng. 1996, 122, 634-640. [CrossRef]

10. Okamoto, T.; Nezu, I. Spatial evolution of coherent motions in finite-length vegetation patch flow. Environ. Fluid Mech. 2013, 13, 417-434. [CrossRef]

11. Ghisalberti, M.; Nepf, H.M. The structure of the shear layer in flows over rigid and flexible canopies. Environ. Fluid Mech. 2006, 6, 277-301. [CrossRef]

12. Ghisalberti, M.; Nepf, H.M. The limited growth of vegetated shear layers. Water Resour. Res. 2004, 40, W07502. [CrossRef]

13. Ben Meftah, M.; De Serio, F.; Mossa, M. Hydrodynamic behaviour in the outer shear layer of partly obstructed open channels. Phys. Fluids 2014, 26, 065102. [CrossRef]

14. Ben Meftah, M.; Mossa, M. Partially obstructed channel: Contraction effect on the flow hydrodynamic structure and prediction of the transversal mean velocity profile. J. Hydrol. 2016, 542, 87-100. [CrossRef]

15. Nepf, H.M.; Vivoni, E.R. Flow structure in depth-limited, vegetated flow. J. Geophys. Res. 2000, 105, 28547-28557. [CrossRef]

16. Poggi, D.; Porporato, A.; Ridolfi, L.; Albertson, J.D.; Katul, G.G. The Effect of Vegetation Density on Canopy Sun-Layer Turbulence. Bound. Layer Meteorol. 2004, 111, 565-587. [CrossRef]

17. Brito, M.; Fernandes, J.; Leal, J.B. Porous media approach for RANS simulation of compound open-channel flows with submerged vegetated floodplains. Environ. Fluid Mech. 2016, 16, 1247-1266. [CrossRef]

18. Kang, H.; Choi, S.U. Turbulence modeling of compound open-channel flows with and without vegetation on the floodplain using the Reynolds stress model. Adv. Water Resour. 2006, 29, 1650-1664. [CrossRef]

19. White, B.L.; Nepf, H.M. Shear instability and coherent structures in shallow flow adjacent to a porous layer. J. Fluid Mech. 2007, 593, 1-32. [CrossRef]

20. Kaimal, J.; Finnigan, J. Atmospheric Boundary Layer Flows; Oxford University Press: New York, NY, USA, 1994.

21. Schlichting, H. Boundary Layer Theory, 7th ed.; McGraw-Hill: New York, NY, USA, 1979.

22. Kamphuis, J.W. Determination of sand roughness for fixed beds. J. Hydraul. Res. 1974, 12, 193-203. [CrossRef]

23. Fredsoe, J.; Andersen, K.H.; Sumer, B.M. Wave plus current over a ripple-covered bed. Coast. Eng. 1999, 38, 177-221. [CrossRef]

24. SonTek. ADV Field Technical Manual. In SonTek Technical Documentation; SonTek/YSI Inc.: San Diego, CA, USA, 2001.

25. Goring, D.G.; Nikora, V.I. Despiking Acoustic Doppler Velocimeter Data. J. Hydraul. Eng. 2002, 128, 117-126. [CrossRef]

26. Wahl, T.L. Analyzing ADV data using WinADV. In Proceedings of the Joint Conference on Water Resource Engineering and Water Resources Planning and Management, Minneapolis, MN, USA, 30 July2 August 2000. 
27. Ben Meftah, M.; Mossa, M.A. A modified log-law of flow velocity distribution in partly obstructed open channels. Environ. Fluid Mech. 2016, 16, 453-479. [CrossRef]

28. Nezu, I.; Onitsuka, K. Turbulent structures in partly vegetated open-channel flows with LDA and PIV measurements. J. Hydraul. Res. 2001, 39, 629-642. [CrossRef]

29. Yang, K.; Cao, S.; Knight, D.; Flow, P. Flow patterns in compound channels with vegetated floodplains. J. Hydraul. Eng. 2007, 133, 148-159. [CrossRef]

30. Ikeda, S.; Ohta, K.; Hasegawa, H. Periodic vortices at the boundary of vegetated area along river bank. J. Hydraul. Coast. Environ. Eng. JSCE 1992, 443, 47-54. (In Japanese) [CrossRef]

31. Ikeda, S.; Kanazawa, N.; Ohta, K. Flow over flexible vegetation and 3-D structure of organized vortex associated with honami. J. Hydraul. Coast. Environ. Eng. JSCE 1995, 515, 33-43. (In Japanese) [CrossRef]

32. Ben Meftah, M.; De Serio, F.; Malcangio, D.; Mossa, M. Resistance and boundary shear in a partly obstructed channel flow. In River Flow; Taylor \& Francis Group: London, UK, 2016; pp. 795-801.

33. Nepf, H. Hydrodynamics of vegetated channels. J. Hydraul. Res. 2012, 50, 262-279. [CrossRef]

(C) 2018 by the authors. Licensee MDPI, Basel, Switzerland. This article is an open access article distributed under the terms and conditions of the Creative Commons Attribution (CC BY) license (http:/ / creativecommons.org/licenses/by/4.0/). 
Article

\title{
Wave Height Attenuation and Flow Resistance Due to Emergent or Near-Emergent Vegetation
}

\author{
Paolo Peruzzo ${ }^{1, *}$, Francesca De Serio ${ }^{2}$, Andrea Defina ${ }^{1}$ and Michele Mossa ${ }^{2}$ \\ 1 Department of Civil, Architectural and Environmental Engineering, University of Padua, \\ Via Loredan, 20, 35131 Padova PD, Italy; andrea.defina@dicea.unipd.it \\ 2 Department of Civil, Environmental, Land, Building Engineering and Chemistry, \\ Polytechnic University of Bari, Via Edoardo Orabona, 4, 70126 Bari BA, Italy; \\ francesca.deserio@poliba.it (F.D.S.); michele.mossa@poliba.it (M.M.) \\ * Correspondence: paolo.peruzzo@dicea.unipd.it; Tel.: +39-049-8275659
}

Received: 12 February 2018; Accepted: 27 March 2018; Published: 29 March 2018

\begin{abstract}
Vegetation plays a pivotal role in fluvial and coastal flows, affecting their structure and turbulence, thus having a strong impact on the processes of transport and diffusion of nutrients and sediments, as well as on ecosystems and habitats. In the present experimental study, the attenuation of regular waves propagating in a channel through flexible vegetation is investigated. Specifically, artificial plants mimicking Spartina maritima are considered. Different plant densities and arrangements are tested, as well as different submergence ratios. Measurements of wave characteristics by six wave gauges, distributed all along the vegetated stretch, allow us to estimate the wave energy dissipation. The flow resistance opposed by vegetation is inferred by considering that drag and dissipation coefficients are strictly related. The submergence ratio and the stem density, rather than the wave characteristics, affect the drag coefficient the most. A comparison with the results obtained in the case when the same vegetation is placed in a uniform flow is also shown. It confirms that the drag coefficient for the canopy is lower than for an isolated cylinder, even if the reduction is not affected by the stem density, underlining that flow unsteadiness might be crucial in the process of dissipation.
\end{abstract}

Keywords: wave attenuation; energy dissipation; drag coefficient; flexible vegetation; Spartina maritima

\section{Introduction}

Coastal wetlands and saltmarshes are fundamental for sustaining ecosystems and preserving coasts from erosion and flooding due to storm surge and waves [1-3]. In the last century a widespread reduction in these areas has been observed in countries where short-sighted human activities and an increase of the sea level have strongly affected the morphodynamic equilibrium of intertidal environments $[4,5]$. Since that vegetation plays a fundamental role in the preservation and restoration of this habitat by controlling the coastal hydrodynamics, an effective strategy to counteract this trend is to promote the development of alophyte plants in wetlands. In particular, vegetation attenuates the action of waves, slows down the flow, and promotes diffusion and deposition processes [6-10]. Accordingly, it (i) enhances particle removal and water quality [11-13]; (ii) positively affects the hydrochory and nautochory of seeds and organic matter [14-17]; (iii) reduces sediment erosion and resuspension, while enhancing sediment deposition [18-20]; and (iv) creates preferred paths for tracers' dissipation processes [21-23].

This is one of the main reasons for investigations of the interaction between vegetation and flow. Wave attenuation has been widely investigated in controlled laboratory experiments, where vegetation has often been mimicked by rigid dowels or artificial plants [24-29], as well as in field observations, where it has been limited to cross-shore transects. [30-33]. Despite the large amounts of theoretical and experimental results available in the literature, the interaction of waves with a vegetated canopy 
still deserves further study. In particular, the drag coefficient, which represents/includes the impact of several phenomena such as sheltering, bed interaction, and non-uniform velocity distribution [34], needs to be assessed.

Wave attenuation resulting from wave-vegetation interaction depends on hydrodynamic conditions such as the incident wave height and period, and water depth, as well as on plant properties such as impact area, density, and flexibility. Many studies focused on different species of plants commonly diffused on saltmarshes and along coastal environments, in order to quantify the plant-specific attenuation of the waves, e.g., Laminaria hyberborea, Spartina alternifora, and Macrocystis pyrifera [35-37]. Although Spartina maritima is a common grass in wetlands and saltmarshes [38,39], only a few experimental studies have considered this type of vegetation [40].

The present work describes and discusses the results of an experimental investigation aimed at studying the flow resistance and wave attenuation capability of Spartina maritima. Experiments are carried out in the laboratory under controlled wave conditions; plastic plants mimicking Spartina maritima are arranged on the bottom of the wave-flume and experiments consider either emergent or submerged canopy, as well as different plant density. In order to compare and relate resistance characteristics induced by vegetation (e.g., the drag coefficient) under wave and steady flow conditions, additional experiments are performed in steady flow conditions using the same plastic plants, with varying density.

\section{Materials and Methods}

\subsection{Experimental Setup}

To investigate the wave attenuation induced by Spartina maritima, experiments are carried out in a wave flume $35.0 \mathrm{~m}$ long, $1.0 \mathrm{~m}$ wide, and $1.3 \mathrm{~m}$ deep; an HR Wallingford paddle generates monochromatic waves. At the opposite end of the flume, a wave absorber damps incident waves (see Figure 1). The wave absorber is made of a sloping (1:3) porous metal plate, covered with a needle-punched geogrid, which ensures, in the present experiments, a reflection coefficient smaller than $10 \%$.

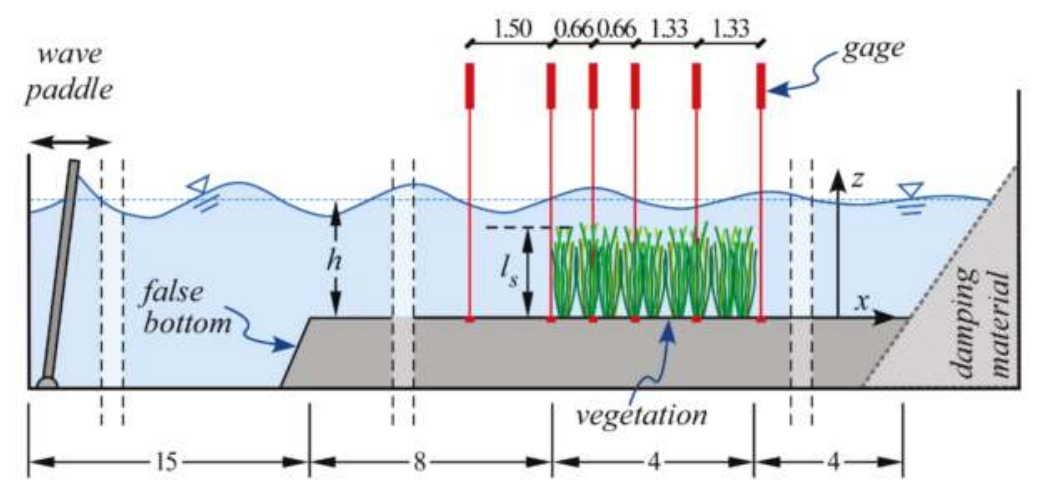

Figure 1. Scheme of the wave apparatus setup. Distances are in meters.

Fifteen meters downstream of the wave generator, a $16 \mathrm{~m}$ long false plane bottom houses the vegetation canopy; the $4 \mathrm{~m}$ long reach occupied by vegetation starts $8 \mathrm{~m}$ downstream from the upstream edge of the false plane. The artificial plants are inserted in adjacent PVC panels, which are drilled in order to fix the main stem of the plant into holes that are equally spaced $4 \mathrm{~cm}$ apart both longitudinally and transversally (see Figure 2a). 


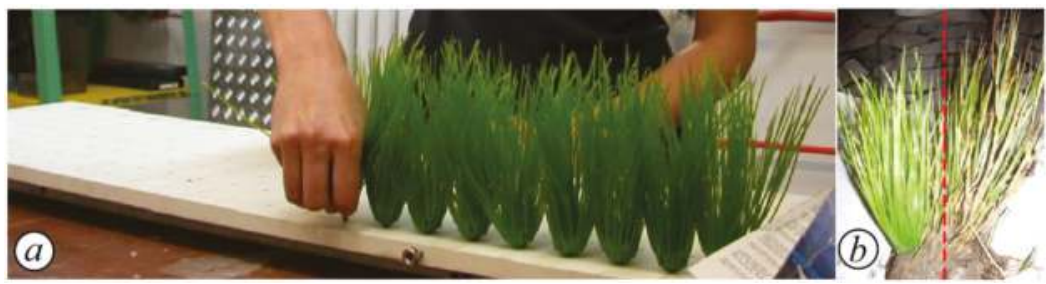

Figure 2. Vegetation used in the experiments. (a) Plant housing into a drilled PVC panel; (b) comparison between silicone plant (left) and Spartina maritima harvested in the Venice Lagoon (right).

The artificial plants chosen for mimicking the real Spartina maritima are the same used in other studies aimed at investigating the transport and diffusion of floating seeds in partially emerged saltmarsh vegetation [41,42]. They are plastic plants $15 \mathrm{~cm}$ high, with $n_{s}=120$ stems having a diameter, $d$, of approximately $2.0 \mathrm{~mm}$. Real Spartina is compared to the present artificial plants in Figure $2 \mathrm{~b}$.

Six wave gauges measure the free surface oscillation in the flume at $20 \mathrm{~Hz}$ sampling rate. Five probes are placed at $0.00 \mathrm{~m}, 0.66 \mathrm{~m}, 1.32 \mathrm{~m}, 2.66 \mathrm{~m}$ and $4.00 \mathrm{~m}$ from the beginning of the vegetated area, and they recorded the wave attenuation along the canopy, whilst the sixth probe, located $1.5 \mathrm{~m}$ upstream of the vegetation field, recorded the approaching wave height, $H_{0}$ (see Figure 1).

Two series of tests are performed. In the first series, waves attenuation is recorded considering four scenarios characterized by different plant configurations with densities $n_{p}=43.75,84.5,156.25$ and 312.5 plant $/ \mathrm{m}^{2}$, arranged as in Figure 3. For each plant configuration, experiments are performed at five water depths, namely $h=10,15,20,25$, and $30 \mathrm{~cm}$. The wave period, $T$, ranges between 0.7 and $1.4 \mathrm{~s}$ and the wave height, $H_{0}$, is selected so that the slope $H_{0} / L$ (with $L$ the wave length) ranges between 0.01 and 0.09 . The hydrodynamic conditions are summarized in Table 1.
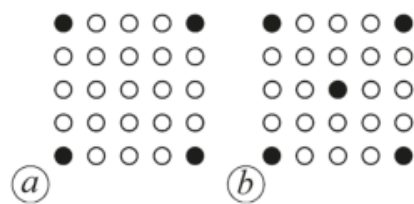

(b)

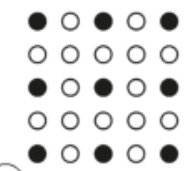

(c)

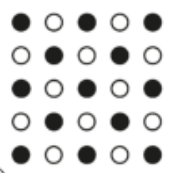

(d)

Figure 3. Plants distribution for the four tested configurations: (a) plants density $n_{p}=43.75$ plant $/ \mathrm{m}^{2}$; (b) plants density $n_{p}=84.5$ plant $/ \mathrm{m}^{2}$; (c) plants density $n_{p}=156.25$ plant $/ \mathrm{m}^{2}$; and (d) plants density $n_{p}=312.5$ plant $/ \mathrm{m}^{2}$.

In the second series of experiments, the configuration having the greatest vegetation density, i.e., $n_{p}=312.5 \mathrm{plant} / \mathrm{m}^{2}$ is investigated in depth. For each of the water depths listed above, nine wave conditions, resulting from the combination of 3 wave periods, $T=0.8,1.0$ and $1.2 \mathrm{~s}$, and three wave slopes, $H_{0} / L=0.03,0.05$ and 0.08 , are investigated (for details see Table 1 ).

Table 1. Summary of the tests in wave condition.

\begin{tabular}{ccccc}
\hline \multicolumn{5}{c}{ Series 1 } \\
\hline $\begin{array}{c}n \\
n_{p}\end{array}$ plant $\left./ \mathrm{m}^{2}\right)$ & $\begin{array}{c}n \\
\left(\mathrm{stem} / \mathrm{m}^{2}\right)\end{array}$ & $\begin{array}{c}h \\
(\mathrm{~cm})\end{array}$ & $\begin{array}{c}T \\
(\mathrm{~s})\end{array}$ & $H_{0} / \mathrm{L}$ \\
\hline 43.75 & 5250 & & & \\
84.50 & 10,140 & $10 \div 30$ & $0.7 \div 1.4$ & $0.01 \div 0.09$ \\
156.25 & 18,750 & & & \\
312.50 & 37,500 & Series 2 & & \\
\hline \multicolumn{5}{c}{$10 \div 30$} \\
\hline 12.50 & 37,500 & $0.8 ; 1.0 ; 1.2$ & $0.03 ; 0.05 ; 0.08$ \\
\hline
\end{tabular}


In order to compare and relate resistance characteristics induced by vegetation under wave and in steady flow conditions, additional experiments are performed in uniform flow condition (UF experiment). These tests are carried out in a $6.0 \mathrm{~m}$ long, $0.3 \mathrm{~m}$ wide, and $0.5 \mathrm{~m}$ high tilting flume, in which the same plastic plants are arranged over a $3 \mathrm{~m}$ long reach. To ensure uniform flow conditions, a magnetic flowmeter accurately measures the bulk flow rate, and constant water depth is achieved by adjusting the bottom slope and a downstream weir.

Four different plant arrangements are investigated, with $n_{p}$ equal to $78.125,156.25,312.5$, and 625 plant $/ \mathrm{m}^{2}$, respectively (see Figure 4). The bulk flow velocity is approximately $6.7 \mathrm{~cm} / \mathrm{s}$ in all experiments, whereas, for each plant density, three water depths are considered, i.e., $h=5 \mathrm{~cm}$, $10 \mathrm{~cm}$, and $15 \mathrm{~cm}$ (Table 2). This means that the UF experiment investigate the behavior of flexible emergent vegetation $\left(l_{s} \geq h\right)$.

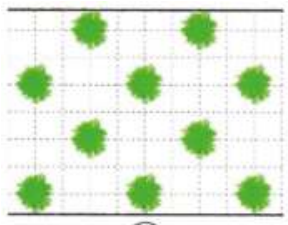

(a)

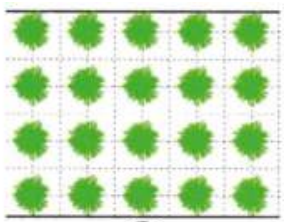

(b)

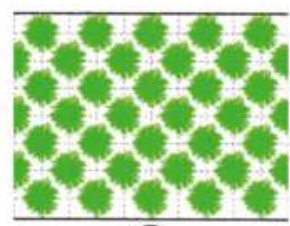

(c)

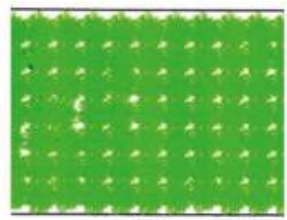

(d)

Figure 4. Plants distribution for the uniform flow tests: (a) plants density $n_{p}=78.125$ plant $/ \mathrm{m}^{2}$; (b) plants density $n_{p}=156.25$ plant $/ \mathrm{m}^{2}$; (c) plants density $n_{p}=312.5$ plant $/ \mathrm{m}^{2}$; and (d) plants density $n_{p}=625$ plant $/ \mathrm{m}^{2}$.

Table 2. Summary of the tests in uniform flow condition.

\begin{tabular}{cccc}
\hline \multicolumn{4}{c}{ Uniform Flow Test } \\
\hline$n_{p}$ & $n$ & $h$ & $T$ \\
$\left(\right.$ plant $\left./ \mathrm{m}^{2}\right)$ & $\left(\mathrm{stem} / \mathrm{m}^{2}\right)$ & $(\mathrm{cm})$ & $(\mathrm{s})$ \\
78.125 & 9375 & & \\
156.25 & 18,750 & $5 ; 10: 15$ & $\sim 6.7$ \\
312.50 & 37,500 & & \\
625.00 & 75,000 & & \\
\hline
\end{tabular}

\subsection{The Theoretical Model}

The energy of waves propagating through and over vegetation is dissipated due to the action of waves on vegetation $[24,26,27]$. The general form of the energy conservation equation is

$$
\frac{\partial E c_{g}}{\partial x}=-\varepsilon_{v}
$$

where $E$ is the wave energy, $c_{g}$ is the wave group velocity and $\varepsilon_{v}$ is the time-averaged rate of energy dissipation per unit length induced by vegetation.

On assuming $c_{g}$ to be constant and recalling that $E=\rho g H^{2} / 8$, with $\rho$ the water density, $g$ the gravity, and $H$ the wave height, the above equation can be rewritten as

$$
\frac{\partial H^{2}}{\partial x}=-\frac{8 \varepsilon_{v}}{\rho g c_{g}}
$$


The dissipation rate $\varepsilon_{v}$ is commonly estimated assuming the linear wave theory $[6,26,29]$. Mendez and Losada [26] obtained the following expression for $\varepsilon_{v}$ :

$$
\varepsilon_{v}=\frac{2}{3 \pi} \rho C_{D} d n\left(\frac{k g}{2 \sigma}\right)^{3} \frac{\sinh ^{3}\left(k l_{s}^{*}\right)+3 \sinh \left(k l_{s}^{*}\right)}{3 k \cosh ^{3}(k h)} H^{3}
$$

where $C_{D}$ is the drag coefficient of the single stem, $l_{s}^{*}$ is the submerged stem height that is equal to $l_{S}$ in submerged condition (i.e., $l_{s} / h \leq 1$ ), and to $h$ in emergent conditions (i.e., $l_{s} / h>1$ ), and $n=n_{p} n_{S}$ is the average stems density, i.e., number of stems per unit area, $k=2 \pi / L$ is the wave number, and $\sigma=2 \pi / T$ is the angular frequency.

In the present experiments all the parameters of Equation (3) are assumed to be constant, hence Equation (2) has the following analytical solution:

$$
H=\frac{H_{0}}{1+\frac{K_{v}}{2} x}
$$

where $H_{0}$ is the incident wave height at $x=0$, corresponding to the upstream cross section of the vegetated reach, and $K_{v}$ is the dissipation coefficient due to the vegetation

$$
K_{v}=\frac{8}{9 \pi} C_{D} d n k \frac{\sinh ^{3}\left(k l_{s}^{*}\right)+3 \sinh \left(k l_{s}^{*}\right)}{\sinh (k h)[\sinh (2 k h)+2 k h]} H_{0}
$$

Alternatively, Kobayashi et al. [6] proposed to linearize the energy equation by approximating $H^{3} \cong H^{2} H_{0}$ in Equation (3). With this assumption, the wave height decreases exponentially, as follows:

$$
H=H_{0} e^{-\frac{K_{v}}{2} x}
$$

Both hyperbolic and exponential models, expressed by Equations (4) and (6) respectively, are commonly adopted in the literature to describe the wave attenuation in the presence of vegetation. Nevertheless, the results of the present investigations clearly show that the linearized solution fits the experimental results better.

The drag coefficient, $C_{D}$, is the only unknown of the problem, hence it can be inferred from experimental data. Specifically, in the present experiments, $K_{v}$ is estimated by minimizing the difference between observed and expected wave attenuation according to Equation (6), Then, $C_{D}$ is estimated from Equation (5).

In the literature, the drag coefficient $C_{D}$ is expressed as a function of either the Reynolds number, $R e$, or the Keulegan-Carpenter number, $K C$. The stem Reynolds number is

$$
R e=\frac{u_{c} d}{v}
$$

where $v$ is the water kinematic viscosity and $u_{c}$ is the horizontal velocity amplitude averaged over the stem height, which can be approximated by the velocity at the top of the canopy, when $k l_{s}^{*}$ is relatively small, i.e.,

$$
u_{c}=\frac{k g H_{0}}{2 \sigma} \frac{\cosh \left(k l_{s}^{*}\right)}{\cosh (k h)}
$$

$u_{c}$ is representative of the velocity magnitude acting on the stem, notwithstanding it is approximately the actual velocity at the top of the canopy, since the vertical velocity profile with submerged vegetation can differ quite a bit from the profile predicted by the linear wave theory [43].

The Keulegan-Carpenter number, also called the period number, is:

$$
K C=\frac{u_{c} T}{d}
$$


Most experimental studies express the drag coefficient as a power law of either $\mathrm{Re}$ or $\mathrm{KC}$ :

$$
C_{D}=\alpha_{R e} \cdot R e^{-\beta_{R e}} \quad, \quad C_{D}=\alpha_{K C} \cdot K C^{-\beta_{K C}}
$$

where $\alpha_{i}$ and $\beta_{i}$ are calibration coefficients.

In the case of vegetated uniform flow, the literature provides many bulk expressions to estimate the drag coefficient, which are based on experimental, theoretical, and computational investigations [25,44-46].

Among the many available models, the simple model created by Nepf [25] for the case of a dense array of emergent rigid cylinders provides reasonable and effective results [46,47]. Therefore, this approach is used in the present study for the estimation of $C_{D}$ in the uniform flow experiments. The force balance in the flow direction is

$$
\frac{\tau_{b}}{\rho}+\frac{1}{2} C_{D} n d^{2} \frac{l_{s}^{*}}{d} U^{2}=g h \frac{\partial h}{\partial x}
$$

where $\tau_{b}$ is the bed shear stress and $U$ is the flow velocity averaged over the stem height. In the present case, the stem density is very high and drag resistance turns out to be much greater than bed shear stress; accordingly, $\tau_{b}$ is neglected in Equation (11). In addition, only the emergent vegetation condition is investigated, so that $U$ is the bulk flow velocity. The choice to perform the UF experiment with only emergent vegetation is essentially due to the large variability of flow velocity in the vertical direction when the vegetation is submerged $[48,49]$. This aspect is not taken into due account by the $C_{D}$ formulation in Equation (11).

The calibration approach used by Mendez and Losada [26] has been chosen for processing the data to obtain $C_{D}$. Based on the calibration approach, the experimental value of $C_{D}$ is estimated from the reduced wave height, which reflects the dissipation of wave energy, using Equations (5) and (6).

\section{Results and Discussion}

Figure 5 shows an example of wave attenuation when the vegetation density is $n_{p}=312.5$ plant $/ \mathrm{m}^{2}$, and the wave period and slope are $T=1 \mathrm{~s}$ and $H_{0} / L=0.08$. The attenuation depends on the ratio between vegetation height and water depth, $l_{s} / h$. Since most energy dissipation occurs in the lower part of the water column obstructed by the stems, the capability of damping turns out to be inversely related to the degree of plant submersion. The overall reduction of $H$ over the $4 \mathrm{~m}$ of Spartina for $l_{s} / h=1.5$, i.e., for partially emergent condition, is nearly $90 \%$ whereas, for $l_{s} / h=0.5$, i.e., for submerged conditions, the reduction is smaller than $50 \%$.
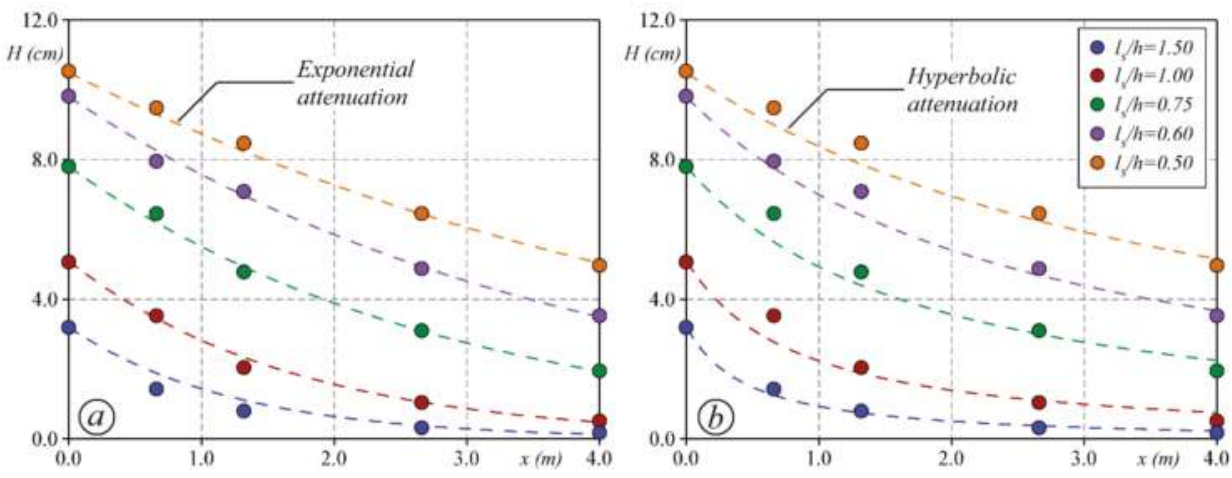

Figure 5. Wave height $H$ along the vegetated reach when density is $n_{p}=312.5 \mathrm{plant} / \mathrm{m}^{2}$ and the relative vegetation height is in the range $0.5 \leq l_{s} / h \leq 1.5$. Wave period and slope are $T=1 \mathrm{~s}$ and $H_{0} / L$ $=0.08$. Circles denote the experimental data, dashed line are the modeled wave attenuation either with (a) the exponential model given by Equation (6); or (b) the hyperbolic model given by Equation (4). 
Figure 5a,b show the experimental data fitted by the exponential law (6) and by the hyperbolic law (4), respectively. The root mean square error due to the hyperbolic law, $R M S E$, is $3.2 \mathrm{~mm}$, while a smaller $R M S E=1.7 \mathrm{~mm}$ is estimated for the exponential law. The comparison between predicted and measured wave height clearly shows that the exponential law better fits the data. For this reason, the exponential model of wave height attenuation is used in the following discussion.

Also, the stem density, $n$, controls wave attenuation, as indicated by Equation (5). Figure 6 shows the estimated coefficient $K_{v}$ as a function of $n$. Here, $K_{v}$ values are the average of all the runs having the same stem density and relative vegetation height, $l_{s} / h$. Interestingly, Figure 6 shows that $K_{v}$ increases less than linearly with $n$, in spite of what was indicated by Equation (5); this important point is discussed later in the text.

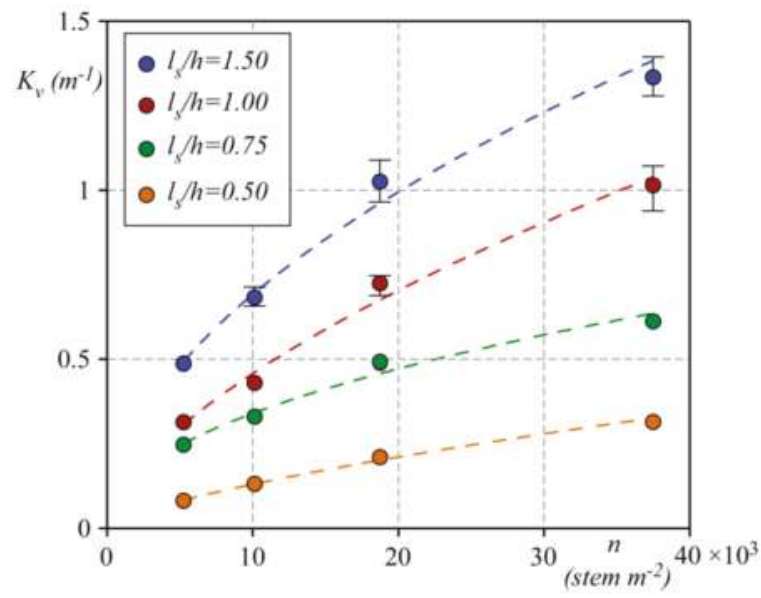

Figure 6. Coefficient of dissipation, $K_{v}$, as a function of stem density, $n$. Average $K_{v}$ are computed for $l_{s} / h=1.5$ (blue circles), $l_{s} / h=1.0$ (red circles), $l_{s} / h=0.75$ (green circles), and $l_{s} / h=0.5$ (orange circles). Bars show the range of variability of $K_{v}$ in the present experiments.

The estimated values for $K_{v}$ are used, with Equation (5), to estimate the stem drag coefficient, $C_{D}$. The latter is shown in Figure 7 as a function of the Reynolds number, $R e$, and the Keulegan-Carpenter number, $K C$. The stem drag coefficient reduces with $R e$ as well as with $K C$ increasing, and can be fitted to the following empirical formulas:

$$
\begin{aligned}
& C_{D}=35.6 \cdot R e^{-0.78} \\
& C_{D}=6.5 \cdot K C^{-0.64}
\end{aligned}
$$

A sensitivity analysis is carried out considering the error due to the measure of the water depth, $h$. Such variation has been estimated at $\pm 0.1 \mathrm{~cm}$ and slightly affects the $C_{D}$ s, whose maximum variation is about $\pm 2 \%$ in the tests performed with the minimum water depth (i.e., $h=10 \mathrm{~cm}$ ).

The coefficients of determination for Equations (12) and (13) are $R^{2}=0.68$ and 0.59 , respectively; hence the regression of computed $C_{D}$ provides a better correlation with $R e$ compared to $K C$. However, given the observed scatter of data, the above relationships cannot be used to safely estimate the drag coefficient.

There are two main reasons for this low accuracy. Firstly, the exact definition of the plant impact area per unit of height. This length is the cylinder diameter when the vegetation is simulated by rigid dowels. In the case of Spartina maritima, which is a porous barrier to the flow where stems can overlap each other, forming a net-like structure [41,42], it is only approximatively equal to the stem diameter. Secondly, the parameter $C_{D}$ considers all the effects that contribute to the overall dissipation 
process, so a single contribution cannot be distinguished, e.g., the extended wake zone within the stems' array [50-52] and stem flexibility [53].

In order to improve the correlation between the measured and predicted $C_{D}$ values, Mendez and Losada [26] suggest replacing $K C$ in Equation (13) with a modified Keulegan-Carpenter number, i.e., $Q_{K C}=K C /\left(l_{s} / h\right)^{0.76}$, which accounts for the impact of submergence. Anderson and Smith [29] extended the same correction to Equation (12) by replacing Re with $Q_{R e}=R e /\left(l_{s} / h\right)^{1.5}$. Close inspection of Figure 7 shows that both these corrections, applied to the present results, are ineffective.

In the present case, some improvement in the estimation of the drag coefficient is achieved by considering the impact of stem density, $n$. In Figure 8 , the estimated drag coefficient, $C_{D}$, is distinguished for each class of relative stem density, $n d^{2}$, and, for each density, the experimental data are fitted by Equation (10). The relatively high values of the coefficients of determination $\left(R^{2}>0.90\right)$ confirm the overall significant improvement. This result proves that the drag experienced by each single stem reduces with increasing dimensionless density $n d^{2}$, i.e., with increasing $n$ in the present experiments. This causes the coefficient $K_{v}$ to increase less than linearly, as noted above (see Figure 6).
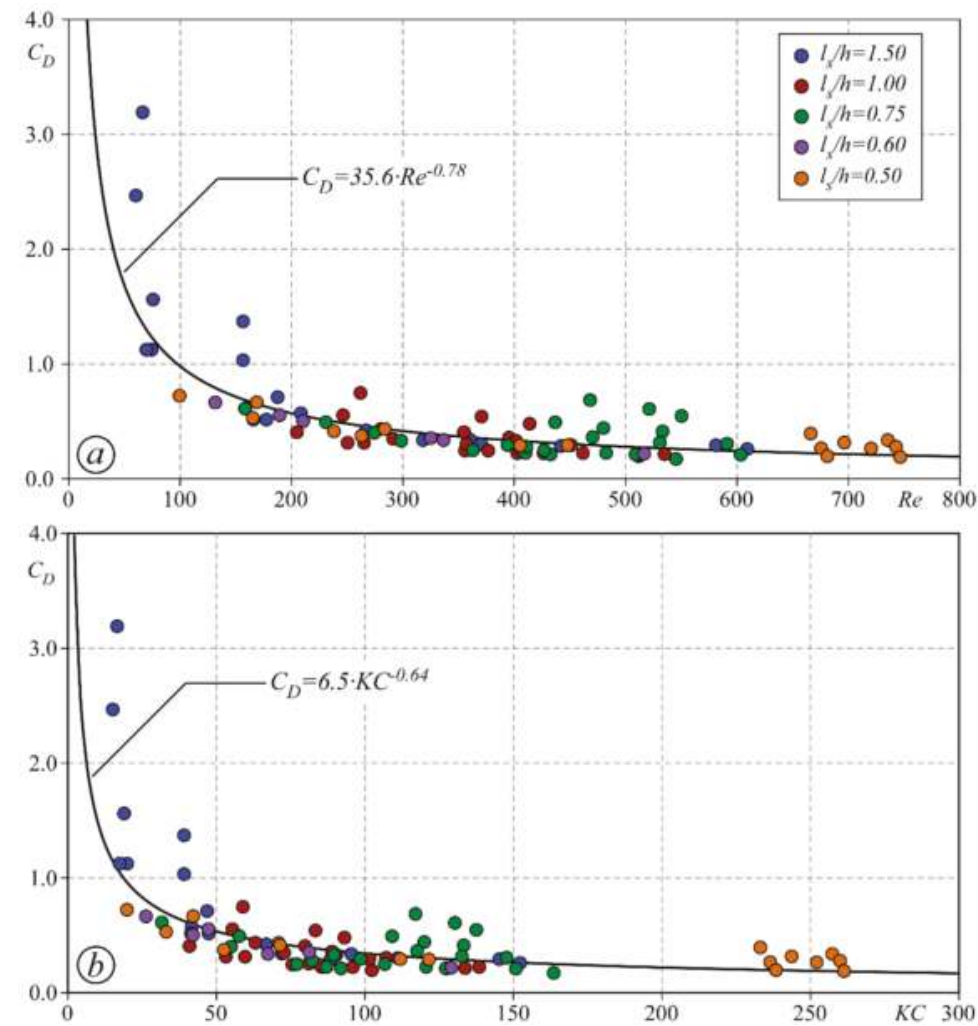

Figure 7. Drag coefficient, $C_{D}$, versus Reynolds number, $R e$, (panel (a)) and Keulegan-Carpenter number, $K C$, (panel (b)). The solid black line represents the regression of experimental data (circles) according to Equation (10). 

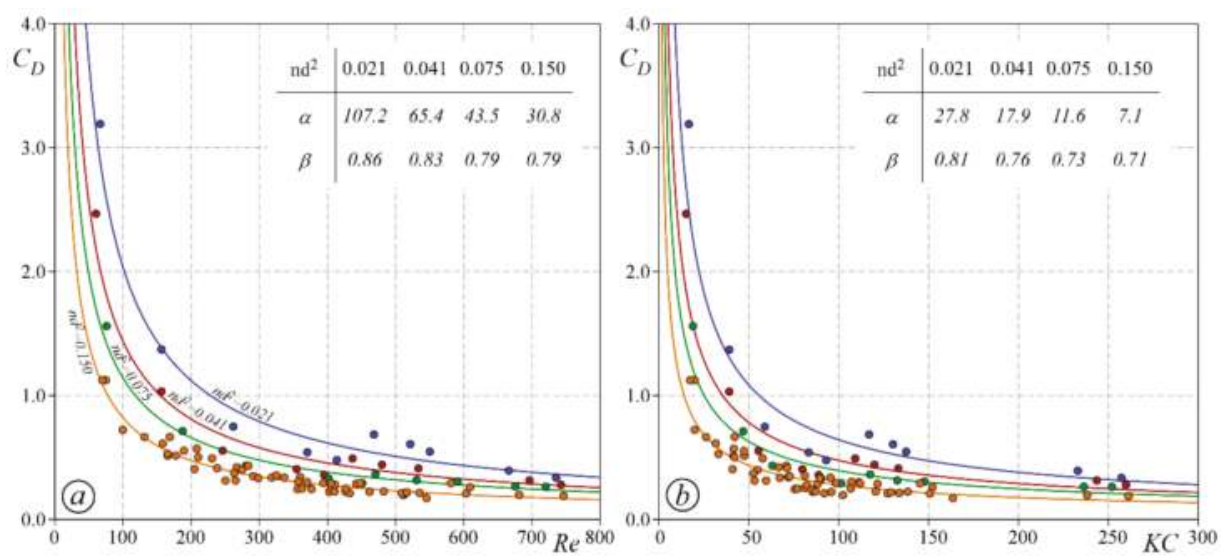

Figure 8. Predicted drag coefficient, $C_{D}$, versus Reynolds number, $R e$, (panel (a)) and Keulegan-Carpenter number, $K C$, (panel (b)) for the four stem densities. The table in the insets gives the coefficients $\alpha$ and $\beta$ to be used in Equation (10).

It is worth noting that the exponent $\beta$ slightly varies with $n d^{2}$, since it ranges between 0.79 and 0.86 for $C_{D}=C_{D}(R e)$ and between 0.71 and 0.81 for $C_{D}=C_{D}(K C)$. On the contrary, the coefficient $\alpha$ significantly reduces when $n d^{2}$ increases (see the coefficient values reported in Figure 8). By forcing $\beta_{i}$ to be constant, the following empirical formulas are found:

$$
\begin{aligned}
& C_{D}=\frac{12.0}{\sqrt{n d^{2}}} \cdot R e^{-0.80} \\
& C_{D}=\frac{4.0}{\sqrt{n d^{2}}} \cdot K C^{-0.80}
\end{aligned}
$$

Figure 9 shows the comparison between the measured drag coefficient and that computed by Equation (14) (panel a) and Equation (15) (panel b). The data are well arranged along the line of perfect agreement, demonstrating that the proposed formulas can explain the measured values well.
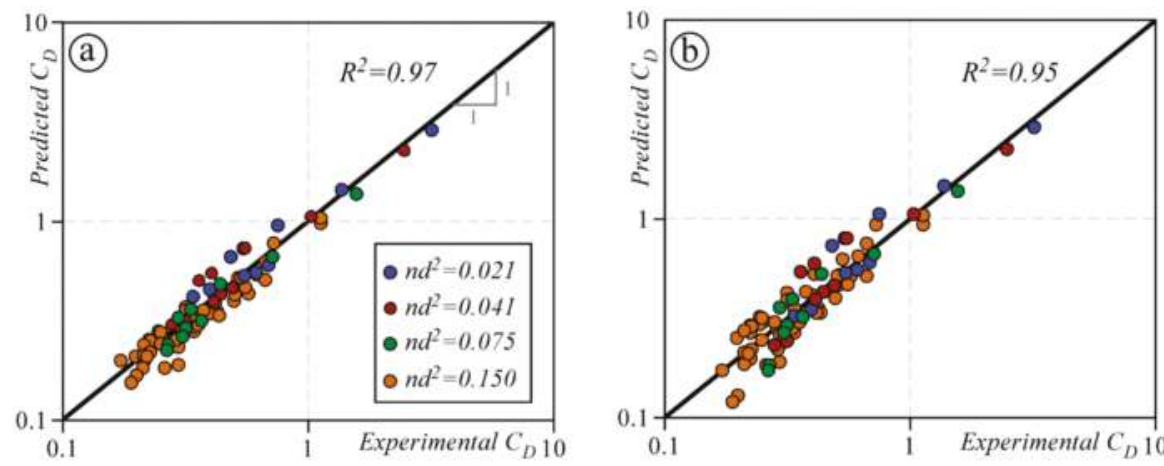

Figure 9. Predicted versus experimental drag coefficient, $C_{D}$; given by Equation (14) (panel (a)) and by Equation (15) (panel (b)). Solid black line indicates perfect agreement between the data and the empirical formulas. 
A relevant observation is that the stem drag coefficient in the present experiments is much smaller than the drag coefficient for an isolated cylinder under uniform flow conditions (by approximately one order of magnitude). This is not a new result (see, e.g., [54]), but the reasons for these small $C_{D} \mathrm{~s}$ are not yet well understood. The observed large drag reduction can be ascribed to either wave flow unsteadiness and/or the relatively high stem density, as observed above, so that wakes interaction dramatically affects $C_{D}$ values [54].

To investigate this circumstance in more depth, we use the results of the experiments performed with uniform flow conditions.

Figure 10 shows the drag coefficient estimated with Equation (11), as it varies with the dimensionless density $n d^{2}$. The drag coefficient $C_{D}$ of a smooth isolated cylinder is affected by the wake structure, and depends only on $R e$, according to many empirical expressions [55]. For the case of a cylinder array, the downstream elements experience lower impact velocity due to the velocity reduction in the wake of the upstream cylinders; hence, the drag coefficient also depends on cylinder spacing and arrangement. Consequently, the downstream cylinders experience reduced drag. This sheltering effect occurs when the average spacing of the cylinders in the array is relatively small [44]. Consistently, Nepf [25] observed that the bulk drag coefficient remains approximately constant up to values of dimensionless density $n d^{2} \cong 0.01$ and then decreases with increasing array density for both random and staggered arrangements; for $n d^{2}=0.1-0.5$, the measured drag coefficient scatters in the range $C_{D}=0.06-0.6$. Consequently the present values of $C_{D}$ are consistent with Nepf's findings. In our UF experiment, values of $C_{D}$ are quite invariant with $n d^{2}$. However, independently of the stem submergence, $C_{D}$ is at a minimum for $n d^{2}=0.075$; this may be due to a slight canalization of the flow promoted by the in-line arrangement of the plants (see Figure $4 \mathrm{~b}$ ). Also, the submergence ratio seems to slightly affect the $C_{D}$ values, perhaps because of the shape of the plants adopted in the tests: their stems gather together in a short basal tuft, thus showing a non-constant impact area along the vertical.

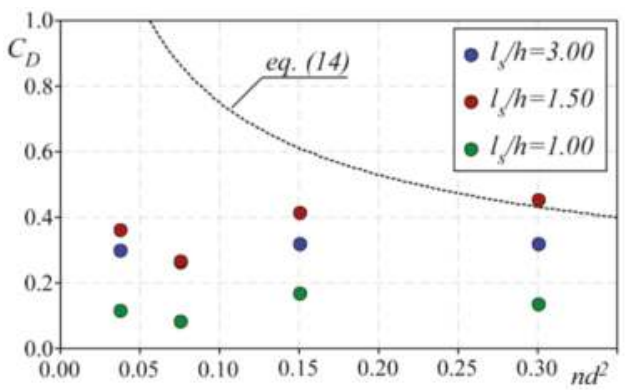

Figure 10. Experimental drag coefficient $C_{D}$ versus dimensionless density $n d^{2}$. Dotted black line shows the drag coefficient predicted by Equation (14) for $R e=135$ in oscillatory flow.

Based on the same artificial vegetation, the comparison between the experimental drag coefficients, one estimated in the presence of waves and the other in uniform flow conditions, could be interesting at this stage. In fact, information derived from both cases could be shared.

The inferred drag coefficients in uniform flow are 4-5 times smaller than the corresponding $C_{D} S$ estimated in wave conditions at the same Reynolds number $\left(C_{D} \cong 0.4-1.2\right.$ by Equation (14) for $n d^{2}=0.04-0.3$ and $\left.R e=135\right)$. The smaller $C_{D}$ values in uniform flow, as well as the dependency of $C_{D}$ values on stem density observed in wave conditions, can be ascribed to the hydrodynamic conditions, and, in particular, to the unsteadiness that prevents the wake zone from fully developing in the canopy.

How to relate the drag coefficients to each other in uniform and wave conditions is still an open question that demands specific investigations, the results of which would be useful for building an in-depth understanding of wave attenuation in wetlands, and hence in the controlling of coastal hydrodynamics. 


\section{Conclusions}

The present work investigated the resistance to flow of flexible vegetation in waves and in uniform flow conditions. The experiments highlighted the role of stem density in the wave attenuation process; the drag experienced by each single stem is reduced as the density increases, while the overall resistance, and hence wave attenuation, increases when stem density increases. The stem sheltering effect is possibly why the measured drag coefficient, both under wave and uniform flow conditions, is smaller than the $C_{D}$ of an isolated cylinder. Interestingly, the drag coefficient measured under wave conditions has the same order of magnitude as that measured in uniform flow, for a comparable Reynolds number and stem density, although the latter turns out to be a little smaller. This circumstance suggests that it could be possible to share knowledge on the drag coefficient from one field to the other; however, this issue needs to be further investigated, possibly by mimicking the vegetation with rigid dowels, in order to reduce the number of degrees of freedom, such as the vegetation flexibility and the non-constant impact area along the vertical.

Both Reynolds $(R e)$ and Keulegan-Carpenter $(K C)$ numbers are found to suitably collapse the measured drag coefficient; the present experimental conditions were such that $R e$ and $K C$ were substantially related to each other, thus preventing us from understanding which of the two non-dimensional numbers is best suited to describe the variability of the drag coefficient. This point also deserves further, specific investigation.

Acknowledgments: We wish to acknowledge Ilaria Bernardi and Silvia Capuzzo for their contribution to the experimental investigation.

Author Contributions: P.P. dealt with the theoretical model and wrote the paper; F.D.S. dealt with the model and reviewed the manuscript; A.D. dealt with the model and conceived the experiments; M.M. reviewed and edited the manuscript.

Conflicts of Interest: The authors declare no conflict of interest.

\section{References}

1. Costanza, R.; d'Arge, R.; De Groot, R.; Farber, S.; Grasso, M.; Hannon, B.; Limburg, K.; Naeem, S.; O’Neill, R.V.; Paruelo, J.; et al. The value of the world's ecosystem services and natural capital. Nature 1997, 387, 253-260. [CrossRef]

2. Fonseca, M.S.; Fisher, J.S.; Zieman, J.C.; Thayer, G.W. Influence of the seagrass, Zostera marina L., on current flow. Estuar. Coast. Shelf Sci. 1982, 15, 351-364. [CrossRef]

3. Fonseca, M.S.; Cahalan, J.A. A preliminary evaluation of wave attenuation by four species of seagrass. Estuar. Coast. Shelf Sci. 1992, 35, 565-576. [CrossRef]

4. Titus, J.G. Rising seas, coastal erosion, and the takings clause: How to save wetlands and beaches without hurting property owners. MD Law Rev. 1998, 57, 1279-1399.

5. Carniello, L.; Defina, A.; D'Alpaos, L. Morphological evolution of the Venice lagoon: Evidence from the past and trend for the future. J. Geophys. Res. Earth Surf. 2009, 114. [CrossRef]

6. Kobayashi, N.; Raichle, A.W.; Asano, T. Wave attenuation by vegetation. J. Waterw. Port Coast. Ocean Eng. 1993, 119, 30-48. [CrossRef]

7. Mork, M. Wave attenuation due to bottom vegetation. Waves Nonlinear Process. Hydrodyn. 1996, 371-382.

8. Möller, I.; Spencer, T. Wave dissipation over macro-tidal saltmarshes: Effects of marsh edge typology and vegetation change. J. Coast. Res. 2002, 36, 506-521.

9. Ghisalberti, M.; Nepf, H.M. Mixing layers and coherent structures in vegetated aquatic flows. J. Geophys. Res. Ocean. 2002, 107. [CrossRef]

10. Ghisalberti, M.; Nepf, H. The structure of the shear layer in flows over rigid and flexible canopies. Environ. Fluid Mech. 2006, 6, 277-301. [CrossRef]

11. Palmer, M.R.; Nepf, H.M.; Pettersson, T.J.R. Accumulation and Removal in Aquatic Systems. Limnol. Oceanogr. 2004, 49, 76-85. [CrossRef] 
12. Hendriks, I.E.; Sintes, T.; Bouma, T.J.; Duarte, C.M. Experimental assessment and modeling evaluation of the effects of the seagrass Posidonia oceanica on flow and particle trapping. Mar. Ecol. Prog. Ser. 2008, 356, 163-173. [CrossRef]

13. Stancanelli, L.M.; Musumeci, R.E.; Cavallaro, L.; Foti, E. A small scale Pressure Retarded Osmosis power plant: Dynamics of the brackish effluent discharge along the coast. Ocean Eng. 2017, 130, 417-428. [CrossRef]

14. Peruzzo, P.; Defina, A.; Nepf, H. Capillary trapping of buoyant particles within regions of emergent vegetation. Water Resour. Res. 2012, 48. [CrossRef]

15. Chang, E.R.; Veeneklaas, R.M.; Buitenwerf, R.; Bakker, J.P.; Bouma, T.J. To move or not to move: Determinants of seed retention in a tidal marsh. Funct. Ecol. 2008, 22, 720-727. [CrossRef]

16. Peruzzo, P.; Defina, A.; Nepf, H.M.; Stocker, R. Capillary interception of floating particles by surface-piercing vegetation. Phys. Rev. Lett. 2013, 111, 164501. [CrossRef] [PubMed]

17. Peruzzo, P.; Pietro Viero, D.; Defina, A. A semi-empirical model to predict the probability of capture of buoyant particles by a cylindrical collector through capillarity. Adv. Water Resour. 2016, 97, 168-174. [CrossRef]

18. Gacia, E.; Granata, T.; Duarte, C. An approach to measurement of particle flux and sediment retention within seagrass (Posidonia oceanica) meadows. Aquat. Bot. 1999, 65, 255-268. [CrossRef]

19. Gacia, E.; Duarte, C.M. Sediment retention by a Mediterranean Posidonia oceanica meadow: The balance between deposition and resuspension. Estuar. Coast. Shelf Sci. 2001, 52, 505-514. [CrossRef]

20. Fauria, K.E.; Kerwin, R.E.; Nover, D.; Schladow, S.G. Suspended particle capture by synthetic vegetation in a laboratory flume. Water Resour. Res. 2015, 51, 9112-9126. [CrossRef]

21. De Serio, F.; Meftah, M.B.; Mossa, M.; Termini, D. Experimental investigation on dispersion mechanisms in rigid and flexible vegetated beds. Adv. Water Resour. 2017. [CrossRef]

22. Mossa, M.; De Serio, F. Rethinking the process of detrainment: Jets in obstructed natural flows. Sci. Rep. 2016, 6, 39103. [CrossRef] [PubMed]

23. Mossa, M.; Meftah, M.B.; De Serio, F.; Nepf, H.M. How vegetation in flows modifies the turbulent mixing and spreading of jets. Sci. Rep. 2017, 7, 6587. [CrossRef] [PubMed]

24. Dalrymple, R.A.; Kirby, J.T.; Hwang, P.A. Wave diffraction due to areas of energy dissipation. J. Waterw. Port Coast. Ocean Eng. 1984, 110, 67-79. [CrossRef]

25. Nepf, H.M. Drag, turbulence, and diffusion in flow through emergent vegetation. Water Resour. Res. 1999, 35, 479-489. [CrossRef]

26. Mendez, F.J.; Losada, I.J. An empirical model to estimate the propagation of random breaking and nonbreaking waves over vegetation fields. Coast. Eng. 2004, 51, 103-118. [CrossRef]

27. Augustin, L.N.; Irish, J.L.; Lynett, P. Laboratory and numerical studies of wave damping by emergent and near-emergent wetland vegetation. Coast. Eng. 2009, 56, 332-340. [CrossRef]

28. Manca, E.; Cáceres, I.; Alsina, J.M.; Stratigaki, V.; Townend, I.; Amos, C.L. Wave energy and wave-induced flow reduction by full-scale model Posidonia oceanica seagrass. Cont. Shelf Res. 2012, 50, 100-116. [CrossRef]

29. Anderson, M.E.; Smith, J.M. Wave attenuation by flexible, idealized salt marsh vegetation. Coast. Eng. 2014, 83, 82-92. [CrossRef]

30. Ifuku, M.; Hayashi, H. Development of eelgrass (Zostera marina) bed utilizing sand drift control mats. Coast. Eng. J. 1998, 40, 223-239. [CrossRef]

31. Moeller, I.; Spencert, T.; French, J.R. Wind wave attenuation over saltmarsh surfaces: Preliminary results from Norfolk, England. J. Coast. Res. 1996, 12, 1009-1016.

32. Moller, I.; Spencer, T.; French, J.R.; Leggett, D.J.; Dixon, M. Wave transformation over salt marshes: A field and numerical modelling study from north Norfolk, England. Estuar. Coast. Shelf Sci. 1999, 49, 411-426. [CrossRef]

33. Mazda, Y.; Kobashi, D.; Okada, S. Tidal-scale hydrodynamics within mangrove swamps. Wetl. Ecol. Manag. 2005, 13, 647-655. [CrossRef]

34. Cassan, L.; Roux, H.; Garambois, P.-A. A Semi-Analytical Model for the Hydraulic Resistance Due to Macro-Roughnesses of Varying Shapes and Densities. Water 2017, 9, 637. [CrossRef]

35. Dubi, A.; Tørum, A. Wave damping by kelp vegetation. Coast. Eng. 1994, 142-156. [CrossRef]

36. Knutson, P.L.; Brochu, R.A.; Seelig, W.N.; Inskeep, M. Wave damping in Spartina alterniflora marshes. Wetlands 1982, 2, 87-104. [CrossRef]

37. Elwany, M.H.S.; O’Reilly, W.C.; Guza, R.T.; Flick, R.E. Effects of Southern California kelp beds on waves. J. Waterw. Port Coast. Ocean Eng. 1995, 121, 143-150. [CrossRef] 
38. Möller, I. Quantifying saltmarsh vegetation and its effect on wave height dissipation: Results from a UK East coast saltmarsh. Estuar. Coast. Shelf Sci. 2006, 69, 337-351. [CrossRef]

39. Silvestri, S.; Defina, A.; Marani, M. Tidal regime, salinity and salt marsh plant zonation. Estuar. Coast. Shelf Sci. 2005, 62, 119-130. [CrossRef]

40. Beach, W.P.; Neumeier, U.; Ciavola, P. Flow Resistance and Associated Sedimentary Processes in a Spartina maritima Salt-Marsh. J. Coast. Res. 2004, 20, 435-447.

41. Defina, A.; Peruzzo, P. Floating particle trapping and diffusion in vegetated open channel flow. Water Resour. Res. 2010, 46. [CrossRef]

42. Defina, A.; Peruzzo, P. Diffusion of floating particles in flow through emergent vegetation: Further experimental investigation. Water Resour. Res. 2012, 48. [CrossRef]

43. Yan, N.I. Drag Forces on Vegetation Due to Waves and Currents. Master's Thesis, Delft University of Technology, Delft, The Netherlands, 2014.

44. Koch, D.L.; Ladd, A.J.C. Moderate Reynolds number flows through periodic and random arrays of aligned cylinders. J. Fluid Mech. 1997, 349, 31-66. [CrossRef]

45. Poggi, D.; Katul, G.G.; Albertson, J.D. A note on the contribution of dispersive fluxes to momentum transfer within canopies. Boundary-Layer Meteorol. 2004, 111, 615-621. [CrossRef]

46. Meftah, M.B.; Mossa, M. Prediction of channel flow characteristics through square arrays of emergent cylinders. Phys. Fluids 2013, 25, 45102. [CrossRef]

47. Meftah, M.B.; De Serio, F.; Mossa, M. Hydrodynamic behavior in the outer shear layer of partly obstructed open channels. Phys. Fluids 2014, 26, 65102. [CrossRef]

48. Defina, A.; Bixio, A.C. Mean flow and turbulence in vegetated open channel flow. Water Resour. Res. 2005, 41. [CrossRef]

49. Poggi, D.; Krug, C.; Katul, G.G. Hydraulic resistance of submerged rigid vegetation derived from first-order closure models. Water Resour. Res. 2009, 45. [CrossRef]

50. Blevins, R.D. Forces on and Stability of a Cylinder in a Wake. ASME J. Offshore Mech. Arct. Eng. 2005, 127, 39-45. [CrossRef]

51. Zdravkovich, M.M.; Pridden, D.L. Interference between two circular cylinders; series of unexpected discontinuities. J. Wind Eng. Ind. Aerodyn. 1977, 2, 255-270. [CrossRef]

52. Tanino, Y.; Nepf, H.M. Laboratory investigation of mean drag in a random array of rigid, emergent cylinders. J. Hydraul. Eng. 2008, 134, 34-41. [CrossRef]

53. Luhar, M.; Nepf, H.M. Flow-induced reconfiguration of buoyant and flexible aquatic vegetation. Limnol. Oceanogr. 2011, 56, 2003-2017. [CrossRef]

54. Trimble, S.; Houser, C.; Trimble, S.; Morales, B. Influence of Blade Flexibility on the Drag Coefficient of Aquatic Vegetation. Estuar. Coast. 2015, 38, 569-577.

55. White, F.M. Viscous Fluid Flow; McGraw-Hill Education: New York, NY, USA, 1991; pp. 335-393.

(c) 2018 by the authors. Licensee MDPI, Basel, Switzerland. This article is an open access article distributed under the terms and conditions of the Creative Commons Attribution (CC BY) license (http:/ / creativecommons.org/licenses/by/4.0/). 
Article

\title{
Wave-Current Interaction: A 2DH Model for Turbulent Jet and Bottom-Friction Dissipation
}

\author{
Sara Pascolo *, Marco Petti and Silvia Bosa \\ Dipartimento Politecnico di Ingegneria e Architettura, University of Udine, 33100 Udine, Italy; \\ marco.petti@uniud.it (M.P.); silvia.bosa@uniud.it (S.B.) \\ * Correspondence: sara.pascolo@uniud.it; Tel.: +39-0432-558-713
}

Received: 28 February 2018; Accepted: 25 March 2018; Published: 27 March 2018

\begin{abstract}
A correct representation of the non-linear interactions between waves and currents is one of the key points when studying the morphological evolution of nearshore environments, in particular close to river mouths or tidal inlets. Undoubtedly, the numerical modelling of similar phenomena can be very complex and computationally demanding, given the size of the domains. In the present paper, a two-dimensional horizontal (2DH) numerical model is applied to investigate the hydrodynamics of a turbulent jet current interacting with frontal waves, preparatory to the study of morphodynamical processes. The purpose is to reproduce accurately the turbulence of the current flow, which develops in both vertical and horizontal planes, even with the simplifications of depth-averaged velocities. Moreover, the bottom shear stress induces a mechanism of dissipation, which acts both on the jet hydrodynamics and on the wave field. Significant attention is given to this process, which turns out to be crucial in shallow waters. The present model, based on classic shallow-water equations and wave action balance, is applied to a literature test. Comparisons with theoretical and numerical outcomes are shown, the latter obtained with a quasi-3D model.
\end{abstract}

Keywords: turbulent jet; wave-current interaction; spectral dissipation; bottom friction; numerical model; hydrodynamic model; spectral model

\section{Introduction}

The morphological evolution of a river delta or a tidal inlet is the result of the interaction between jet-like current dynamics, tides and wind wave-induced processes. Sediment transport mechanisms, resulting from this interaction, have important effects on the equilibrium configurations of the bottom of channels and of the near inlet region. These processes are governed by the non-linear interaction between currents and gravity waves, one of the main focuses of maritime and coastal hydraulic research.

Theoretical and experimental studies on waves propagating with and against a current have been widely conducted [1-4], highlighting the effects on wave kinematics (Doppler effect and changes in the wave number and frequency due to shoaling and refraction) and dynamics (changes in wave steepness and wave-action conservation). In turn, the current field is affected by the mutual interaction, through forces generated by the radiation stress tensor of wave fluctuations and increased bed shear stress. In this regard, the bottom friction coefficient of currents can be enhanced significantly by waves [5-8].

In the nearshore region, characterized by shallower water depth, the interaction between currents and waves with the bottom is one of the main processes that affects the hydrodynamic field.

A river- or tidal-induced current, debouching into the sea, can be regarded as a plane turbulent jet characterized by longitudinal velocity decay and an expansion of its cross section [9]. Several authors have investigated the velocity distribution of a turbulent jet. One of the main contributions is by Abramovich [10], who described the basic principles of a free flow, referring to Prandtl's hypothesis of 
free turbulence on the horizontal plane. This theory establishes the form of the similarity function and thus the velocity profile in the jet cross section [11]. Moreover, Öszoy and Ünlüata [12] examined with an analytical approach a turbulent jet, which exits from tidal inlets. They considered several factors: the lateral entrainment, the bathymetric changes and, in particular, the role played by bottom friction. The authors started from widely used depth-averaged equations, thus becoming a reference also for comparison with experimental or numerical results.

Giger et al. [13] and Adami and Milan [14] discussed, from both theoretical and experimental points of view, the effects of bottom friction on the reduction of the longitudinal velocity. They found that this phenomenon had no influence on the crosswise distribution. This outcome is in contrast with the analytical results of Öszoy and Ünlüata [12]. Ismail and Wiegel [15] are the first authors who investigated the behavior of a shallow plane turbulent jet in the presence of opposing surface gravity waves, pointing out the role of the radiation stress components on the flow field.

In nearshore environments, waves have a dominant role that considerably affects the morphodynamics [16,17]. It follows that the correct prediction of the wave field is fundamental to a proper estimate of the morphodynamic changes near the coast.

A key aspect in the spectral transformation of waves, as they propagate from deep to shallow water, is the dissipation of wave energy caused by interaction with the bottom. In particular, the friction coefficient has been observed to vary significantly as a function of both the wave Reynolds number and the relative roughness; the expressions proposed for the calculation of the friction coefficient are numerous and different [18], but precise indications on how to apply them are not always provided. These different formulations can be used in similar contexts, but they can give diverse results, both in the wave field and in the bottom shear-stress values, depending on the wave period. In fact, the wave period may not remain constant in the nearshore propagation, in particular if waves interact with currents. The known Doppler effect can be more or less pronounced according to the angle of incidence between waves and current, for instance in the two cases of an ebbing flow from a river mouth or a tidal inlet, and a longshore current. With the period, the relative roughness also changes and, consequently, the amount of wave-energy dissipation in the presence of shallow depths. The particular aspect of the variability of the wave period and the consequent effects on the bottom friction dissipation in the wave-current interaction, has not been previously dealt with.

It is clear at this point that the bottom strongly conditions the non-linear mechanisms of the wave-current interaction, considerably affecting the current and the wave field, and the bottom shear stress, with important effects on the morphological evolution of littorals or river and tidal inlets. The complexity of the phenomenon necessarily requires dedicated numerical modelling able to investigate hydrodynamic and morphodynamic processes involving estuarine and tidal environments.

A numerical approach needs the development of an appropriate model that should be computationally efficient and able to solve complex hydrodynamic interactions with relative simplicity. In tidal and coastal domains, a widely used methodology consists of coupling different modules [19-24]: a hydrodynamic model based on classical shallow water equations with 1D, 2D or a quasi-3D approach and a spectral wave model that reproduces nearshore wave fields, taking into account all the input and dissipation-energy terms.

It has been largely established that two-dimensional horizontal (2DH) models are useful to study the morphological evolution of littorals or nearshore bottoms requiring large domains of application. Moreover, 2DH models can reproduce the observed coastal flow field satisfactorily [22,25].

A quasi-3D model is undoubtedly more complete, but at the same time the improvement in the estimation of vertical eddy viscosity involves a greater computational effort mainly due to the following aspects: the vertical discretization of the domain, which can become demanding in very extensive domains such as coastal or tidal environments, and the calibration process of related parameters. In particular, the last condition is more pronounced when considering the interaction with the vertical profile of wave orbital velocity and radiation stresses, the distribution of which is an on-going debate $[26,27]$. Furthermore, the logarithmic description of the mean current velocity profile was 
experimentally found to be valid also in its interaction with waves [28], confirming the validity of a 2DH approach.

Following these considerations and due to the very good hydrodynamic results obtained in literature, Petti et al. [29] presented and applied an efficient 2DH model to a jet current expanding into a shallow basin in the presence of opposing frontal waves. The authors obtained good agreement with both theoretical and numerical results computed by means of a quasi-3D model [30]. The role played by radiation stress forces and the increasing bottom shear stresses in the wave-current interaction was examined [31]. In particular, near the jet inlet, these two mechanisms seem to have the same effect on the flow field, while at greater distances, the contribution to the decay of the longitudinal current velocity due to bottom shear stress is dominant.

In the present paper and starting from these results, the authors investigate the effects induced by bottom shear stresses on the spectral propagation of the waves against the jet current. As indicated above, the bottom dissipation is crucial to the correct prediction of both the wave kinematics and the flow field. In several applications the choice of spectral wave dissipation is not adequately motivated, and sometimes even omitted. For these reasons, the authors intend to verify how the relative roughness affects the wave characteristics, the current velocities, and the bottom shear stress due to the spatial variability of the relative wave period so as to improve the comprehension of the interaction phenomenon, also in the perspective of a morphodynamic application.

Therefore, different approaches used in the computation of the spectral wave dissipation by bottom friction are examined and compared to each other.

In Section 2 the numerical scheme is briefly presented, and in Section 3 the benchmark test is described. Section 4 reports a discussion of the numerical results obtained.

\section{Numerical Scheme}

In this work, a bidimensional hydrodynamic model has been coupled with the spectral model SWAN (Simulating WAves Nearshore) [32].

The hydrodynamic model solves the classical shallow-water equations:

$$
\frac{\partial \mathbf{U}}{\partial t}+\nabla \cdot[\mathbf{F}, \mathbf{G}]-\nabla \cdot\left[\mathbf{F}_{t}, \mathbf{G}_{t}\right]=\mathbf{S}
$$

with:

$$
\begin{gathered}
\mathbf{U}=[h, U h, V h]^{T} ; \mathbf{S}=\left[0,-g h \frac{\partial z_{b}}{\partial x}-\frac{\tau_{m x}}{\rho}+\frac{F_{x}}{\rho},-g h \frac{\partial z_{b}}{\partial y}-\frac{\tau_{m y}}{\rho}+\frac{F_{y}}{\rho}\right]^{T} ; \\
\mathbf{F}=\left[U h, U^{2} h+\frac{g h^{2}}{2}, U V h\right]^{T} ; \mathbf{F}_{t}=\left[0,2 v_{t} h \frac{\partial U}{\partial x}, v_{t} h\left(\frac{\partial U}{\partial y}+\frac{\partial V}{\partial x}\right)\right]^{T} ; \\
\mathbf{G}=\left[V h, U V h, V^{2} h+\frac{g h^{2}}{2}\right]^{T} ; \mathbf{G}_{t}=\left[0, v_{t} h\left(\frac{\partial U}{\partial y}+\frac{\partial V}{\partial x}\right), 2 v_{t} h \frac{\partial V}{\partial y}\right]^{T}
\end{gathered}
$$

$(t, x, y)$ being temporal and horizontal spatial coordinates; $\mathbf{U}$ the variable vector; $(\mathbf{F}, \mathbf{G})$ and $\left(\mathbf{F}_{t}, \mathbf{G}_{t}\right)$ the vectors of advective and turbulent fluxes; and $\mathbf{S}$ the source term vector. Moreover, $h$ is the water depth; $(U, V)$ the mean velocities over the depth in $x$ - and $y$ - directions; $g$ the gravity acceleration; $\rho$ the water density; $z_{b}$ the bottom height; and $v_{t}$ the horizontal eddy viscosity coefficient. $\left(F_{x}, F_{y}\right)$ are the forces due to wave radiation stresses, which depend on the radiation stress tensor $S_{i j}$ as:

$$
\left[F_{x}, F_{y}\right]^{T}=-\left[\frac{\partial S_{x x}}{\partial x}+\frac{\partial S_{y x}}{\partial y}, \frac{\partial S_{x y}}{\partial x}+\frac{\partial S_{y y}}{\partial y}\right]^{T} .
$$


$\left(\tau_{m x}, \tau_{m y}\right)$ are the components along $x$ and $y$, respectively, of the mean bottom shear stress $\tau_{m}$, due to the interaction of current and wave motion. Among all the theories available in literature which combine their effect, in the present paper the formula proposed by Soulsby [31] has been preferred [33]:

$$
\tau_{m}=\tau_{c}\left[1+1.2\left(\frac{\tau_{w}}{\tau_{c}+\tau_{w}}\right)^{3.2}\right]
$$

Here, the stresses due to the current $\tau_{c}$ and to the waves $\tau_{w}$ are evaluated as:

$$
\tau_{c}=\rho \frac{g n^{2}\left[(U h)^{2}+(V h)^{2}\right]}{h^{7 / 3}} ; \tau_{w}=\frac{\rho f_{w} U_{w}^{2}}{2} ; f_{w}=1.39\left(\frac{A}{k_{n} / 30}\right)^{-0.52} ; A=\frac{U_{w} T}{2 \pi}
$$

with $n$ the Manning coefficient; $U_{w}$ the maximal bottom orbital velocity; $T$ the relative wave peak period; $A$ the horizontal semi-orbital excursion at the bottom; $k_{n}$ the equivalent roughness length; and $f_{w}$ the wave friction factor [31].

The horizontal eddy viscosity coefficient $v_{t}$ is evaluated by means of a Smagorinsky approach, as:

$$
v_{t}=c_{s}{ }^{2} l_{c}^{2} \sqrt{2\left(\frac{\partial U}{\partial x}\right)^{2}+2\left(\frac{\partial V}{\partial y}\right)^{2}+\left(\frac{\partial U}{\partial y}+\frac{\partial V}{\partial x}\right)^{2}}
$$

with $c_{S}$ a numerical coefficient and $l_{c}$ a characteristic length.

The structure of (8) is similar to $v_{t}$ as evaluated following Prandtl's theory, assuming the product $c_{S} \cdot l_{c}$ as the mixing length. In this context, Abramovich [10] found that the mixing length in a free turbulent jet is proportional to the half width of the jet itself $b$, which varies linearly along the longitudinal axis. In this way, $c_{S}$ can be considered as the proportional constant and $l_{c}$ as the half width of the jet. Aiming to adopt a magnitude of $b$, representative of the whole jet field, its value measured at the inlet $b_{0}$ has been chosen. The coefficient $c_{s}$ has been calibrated in previous tests, based on the experiments carried out by Ismail and Wiegel [15], starting from some values proposed by Abramovich. Finally, $c_{S}$ has been assumed equal to 0.2 and $l_{c}$ equal to $b_{0}$.

The numerical integration of Equation (1) is carried out by means of a well-balanced second order accurate finite volume method, based on the Harten-Lax- van Leer-Contact (HLLC) Riemann solver associated with a hydrostatic variable reconstruction that assures the scheme is well balanced also in wet and dry conditions [34-36].

The wave parameters are evaluated through SWAN [32], an open source finite difference model that solves the wave action density balance equation:

$$
\frac{\partial N}{\partial t}+\frac{\partial c_{x} N}{\partial x}+\frac{\partial c_{y} N}{\partial y}+\frac{\partial c_{\vartheta} N}{\partial \vartheta}+\frac{\partial c_{\sigma} N}{\partial \sigma}=\frac{S_{t o t}}{\sigma}
$$

where $N$ is the wave action density, defined as $E / \sigma$, and it is preferred over wave energy $E$ since it is conserved in the presence of a current field [3]. The propagation velocities in Cartesian and spectral spaces $\left(c_{x}, c_{y}, c_{\sigma}, c_{\vartheta}\right)$, can be written as:

$$
\begin{gathered}
\left(c_{x}, c_{y}\right)=\frac{d \mathbf{x}}{d t}=\mathbf{c}_{\mathbf{g}}+\mathbf{U}_{\mathbf{c}}=\frac{1}{2}\left[\left(1+\frac{2 k h}{\sinh 2 k h}\right)\right] \frac{\sigma \mathbf{k}}{k^{2}}+\mathbf{U}_{\mathbf{c}} \\
c_{\sigma}=\frac{\partial \sigma}{\partial t}=\frac{\partial \sigma}{\partial h}\left(\frac{\partial h}{\partial t}+\mathbf{U}_{\mathbf{c}} \cdot \nabla h\right)-c_{g} \mathbf{k} \cdot \frac{\partial \mathbf{U}_{\mathbf{c}}}{s} \\
c_{\vartheta}=\frac{d \vartheta}{d t}=-\frac{1}{k}\left(\frac{\partial \sigma}{\partial h} \frac{\partial h}{\partial m}+\mathbf{k} \cdot \frac{\partial \mathbf{U}_{\mathbf{c}}}{\partial m}\right)
\end{gathered}
$$


$\sigma$ being the relative radiant frequency; $\vartheta$ the spectral wave direction; $\mathbf{k}$ the wave number vector; $c_{g}$ the wave group velocity vector; $\mathbf{U}_{\mathbf{c}}$ the current velocity vector $(U, V)$; and $(s, m)$ a local system of coordinates, respectively $s$ in the direction $\vartheta$ and $m$ perpendicular to $s$. $S_{t o t}$, the term on the right side of (9), is the source term that takes into account all physical processes including energy generation by wind, dissipation through whitecapping, bottom friction and depth-induced wave breaking, and wave-wave non-linear transfer.

Equation (9) can also represent phenomena like shoaling or refraction, which are related to non-linear interaction and dissipation during wave propagation in shallow water and in the presence of changing water levels and current fields. Among the theories implemented in SWAN to evaluate the dissipation due to bottom friction, in the present paper the approaches by Collins [37] and Madsen et al. [38] are considered, which express the source term due to bottom friction with the same structure and a linear dependence by a bottom-friction coefficient $C_{f}$. Collins [37] assumes it as constant and equal to 0.015 , while in Madsen et al. [38] $C_{f}$ is a function of a wave friction factor $f_{w M}$ related to the equivalent bottom roughness height:

$$
C_{f}=\frac{f_{w M}}{\sqrt{2}} ;\left\{\begin{array}{cc}
\frac{1}{4 \sqrt{f_{w M}}}+\log _{10}\left(\frac{1}{4 \sqrt{f_{w M}}}\right)=-0.08+\log _{10}\left(\frac{A}{k_{n}}\right) & \text { if } A / k_{n} \geq 1.57 \\
f_{w M}=0.3 & \text { if } A / k_{n}<1.57
\end{array}\right.
$$

SWAN and the hydrodynamic module interact through currents, radiation stress and bottom shear stress. In the present work they have been coupled at discrete time steps $\left(\Delta t_{\text {int }}\right)$. This means that they run separately one after the other, so that wave-radiation stress, wave parameters $\left(U_{w}\right.$ and $\left.T\right)$, and current fields are updated and interchanged with each other.

For each run, the water level and current velocities resulting from the hydrodynamic model are set as input data in SWAN and, on the other hand, the forces due to wave-radiation stresses, the maxima of the orbital motion near the bottom, and the wave relative peak period resulting from SWAN are set as input data in the hydrodynamic model.

Each module works on its own computational grid and the results, to be transferred from one module to the other, are interpolated on an exchange grid.

The whole process is managed by a main program that runs hydrodynamic and SWAN modules alternatively, as suggested by the flow chart depicted in Figure 1 , until the simulation ends.

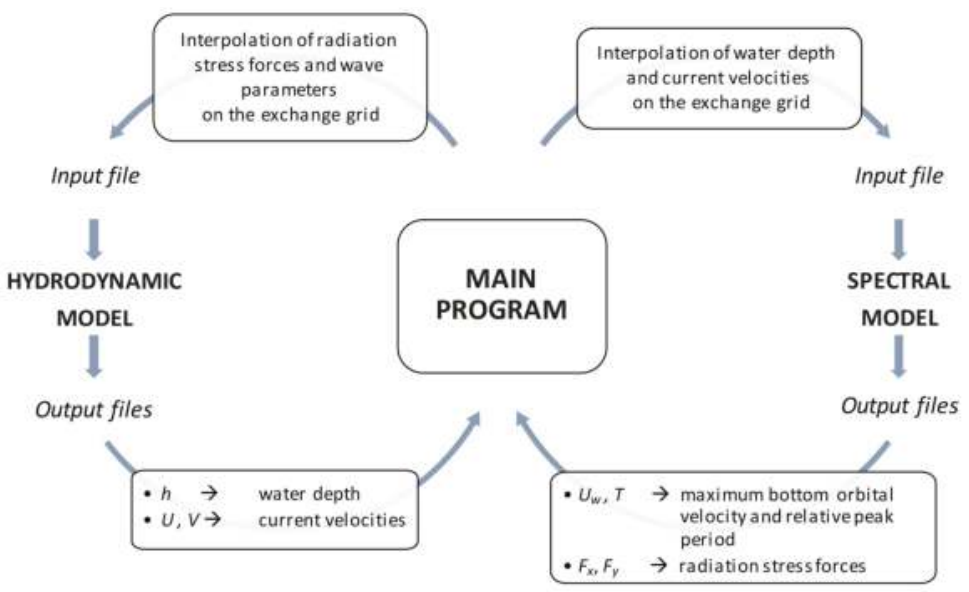

Figure 1. Flow chart of the coupling process between the hydrodynamic model and the spectral model managed by the main program. 


\section{Simulation Setup}

The test case is that proposed by Petti et al. [29] in comparison with Nardin et al. [30]: a jet issuing in a rectangular basin and interacting with an opposing wave field. As depicted in Figure 2, three different computational grids are built: one mesh for the hydrodynamic model, one for SWAN, and one for the data exchange.

The hydrodynamic mesh is slightly different from that of Petti et al. [29]: it consists of a rectangular channel river inlet entering into a $3000 \mathrm{~m}$ large and $2000 \mathrm{~m}$ long basin (Figure 2). The elements are rectangular, with a size of $5 \mathrm{~m}$ in the x-direction (along the longitudinal axis of the jet) while in the $\mathrm{y}$-direction (along the cross section of the jet) the cell dimension linearly increases from $5 \mathrm{~m}$ in a band close to the river centerline up to $50 \mathrm{~m}$ in the farthest part of the domain. This guarantees a higher density of grid cells in the central region, where the main characteristics of the flow field develop. Different grid resolutions have been tested, finding differences in the hydrodynamic numerical results in the order of a few percentage points. For this reason, the numerical results can be considered independent of grid size.

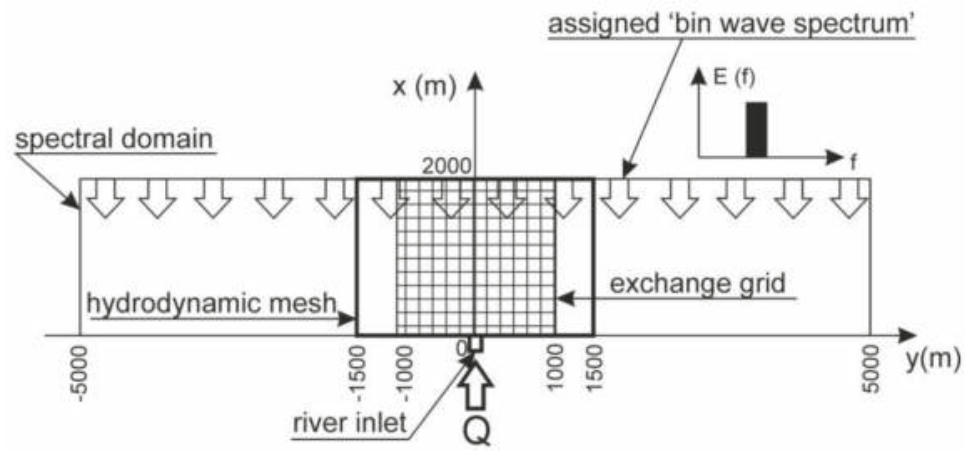

Figure 2. Computational grids. A "bin wave spectrum" is specified at the open sea boundary of the spectral domain, locating energy $\mathrm{E}(\mathrm{f})$ in one frequency bin closest to a corresponding assigned peak period. $Q$ is the constant discharge set at $x=-200 \mathrm{~m}$.

The basin has a constant depth of $3 \mathrm{~m}$. A constant discharge of $3.8 \mathrm{~m}^{3} / \mathrm{sm}$ is assigned to the boundary located at $x=-200 \mathrm{~m}$, along a width of $100 \mathrm{~m}$, which coincides with the initial width of the jet. Along the boundary near the jet inlet, a wall boundary condition is assigned, while a mean sea water level coinciding with the still water level is imposed on the remaining sides of the basin.

The resulting velocity downstream of the inlet is $1.4 \mathrm{~m} / \mathrm{s}$, corresponding to the velocity indicated in Nardin et al. [30]. A constant Manning coefficient of $0.018 \mathrm{~s} / \mathrm{m}^{1 / 3}$ is adopted for the hydrodynamic computational mesh, coherent with the value of Chezy coefficient declared by Nardin et al. [30].

The SWAN cartesian computational grid is regular with squared elements of $10 \mathrm{~m}$ sides. It has the same water depth but it is larger than the hydrodynamic domain to keep the lateral boundaries sufficiently away from the central area where the wave-current interaction is investigated. Three configurations of waves are imposed at the open sea boundary of the domain, located at $x=2000 \mathrm{~m}$ : a "bin wave spectrum", one for each simulation, is specified, locating energy in one frequency bin closest to a corresponding peak period of respectively $3 \mathrm{~s}, 5 \mathrm{~s}$ and $8 \mathrm{~s}$ (Figure 2). The chosen values of the peak period are typical of wind waves generated in fetch-limited and shallow-depth basins, such as the Northern Adriatic Sea and its lagoons. The same significant wave height, equal to $0.5 \mathrm{~m}$, is assigned in all the performed simulations of wave-current interaction. In the remaining sides of the SWAN mesh, outflow boundary conditions were imposed (i.e., waves could leave the area freely). 
SWAN is set in non-stationary mode to follow the temporal evolution of the interaction between the waves against the jet current. The physics commands are set up in order to reproduce the steepening induced by currents [1-4], that can lead to white-capping or, in the limited condition given by the shallow depth, to depth-induced breaking; this last term is modelled in the form proposed by Battjes and Janssen with a constant breaking parameter equal to 0.78 [39].

The wave decay by bottom friction is taken according to the formulations of Collins [37] and Madsen et al. [38] as described in Section 2. Using the Madsen approach, an equivalent roughness-length scale of the bottom of $0.025 \mathrm{~m}$ is taken. Petti et al. [29] set the dissipation term for bottom friction conforming to the empirical JONSWAP model for wind-sea conditions [40].

The lowest and the highest discrete frequencies used in the calculation are respectively set equal to $0.05 \mathrm{~Hz}$ and $0.595 \mathrm{~Hz}$. The spectral directions cover the full circle with a resolution of $6^{\circ}$.

For better comprehension, the list of the performed simulations with corresponding acronym and characteristics is reported in Table 1.

Table 1. List of the simulations performed.

\begin{tabular}{cccccc}
\hline Test & Model & Hs $(\mathbf{m})$ & Tp $(\mathbf{s})$ & Spectral Bottom Friction & Wave-Current Interaction \\
\hline jet alone & hydrodynamic & - & - & - & - \\
T3C & coupled & 0.5 & 3 & Collins & complete ${ }^{1}$ \\
T3M & coupled & 0.5 & 3 & Madsen & complete \\
T5 & coupled & 0.5 & 5 & No friction & complete \\
T5C_rds & coupled & 0.5 & 5 & Collins & Collins \\
T5C & coupled & 0.5 & 5 & Madsen & complete \\
T5M & coupled & 0.5 & 5 & JONSWAP & complete \\
T5J & coupled & 0.5 & 5 & Collins & complete \\
T8C & coupled & 0.5 & 8 & Madsen & complete \\
T8M & coupled & 0.5 & 8 & complete \\
\hline
\end{tabular}

The last computational grid, named the "exchange grid", is the smallest and represents the part of the domain in which the hydrodynamic and the spectral model interact. It is a regular grid with squared elements of $10 \mathrm{~m}$ sides for a total length of $2000 \mathrm{~m}$ in both plane directions. The domain, inside which the jet develops, coincides with that of Petti et al. [29] and Nardin et al. [30].

The interaction between waves and current is performed alternating hydrodynamic and spectral modules every $\Delta t_{\text {int }}=300 \mathrm{~s}$ until reaching a steady-state condition.

\section{Results and Discussion}

The hydrodynamics of the jet current alone, without the interaction with a superimposed wave field, is well interpreted by the numerical model [29]. The assumption of mean velocity and, thus, of a logarithmic profile in the vertical section, subtended by the hypothesis of fully developed turbulent flow, is suitable to interpret the behavior of a river or a tidal flow debouching into a large shallow basin.

Following Abramovich [10], two main properties characterize a free turbulent jet current in the main region or otherwise called ZOEF (zone of established flow): the exponential decay of longitudinal velocity and the self-similarity profile of the velocity in cross sections. In shallow depth, bottom friction enhances the velocity decrease, whereas the self-similar profile is maintained. In this regard, Öszoy and Ünlüata [12] proposed the analytical expression for the longitudinal velocity for different values of bottom friction and suggested faster spreading rates of the jet.

In the present test, the hydrodynamic results of the jet alone are compared with the results obtained in the analogous test carried out by Petti et al. [29] and Nardin et al. [30] by means of a quasi-3D numerical model that computes the vertical eddy viscosity with a standard $\mathrm{k}-\varepsilon$ closure scheme and the horizontal eddy viscosity with a large eddy simulation technique. In Figure 3 the normalized longitudinal velocity along the jet centerline is reported. 


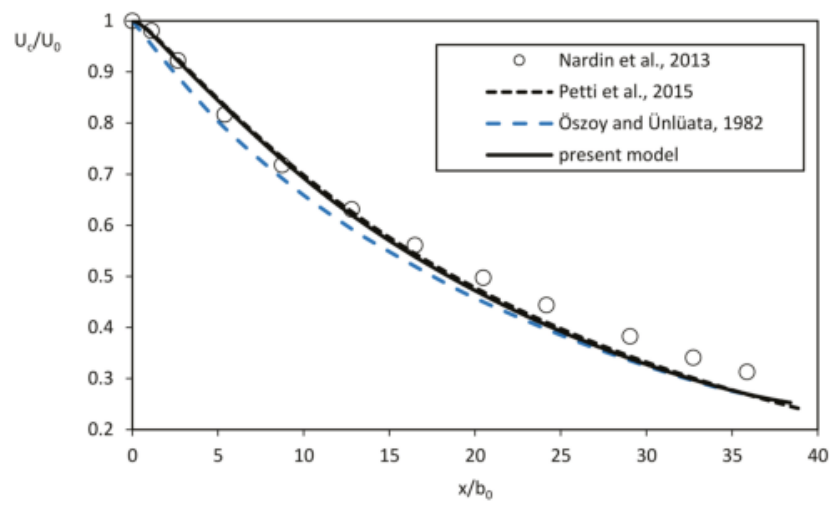

Figure 3. Longitudinal velocity $U_{c}$, taken along the jet centerline, normalized with the velocity at the jet inlet, $\mathrm{U}_{0}$. In the abscissa, $\mathrm{x}$ is the distance from the start of the zone of established flow (ZOEF) normalized with the half-width of the inlet, $b_{0}$.

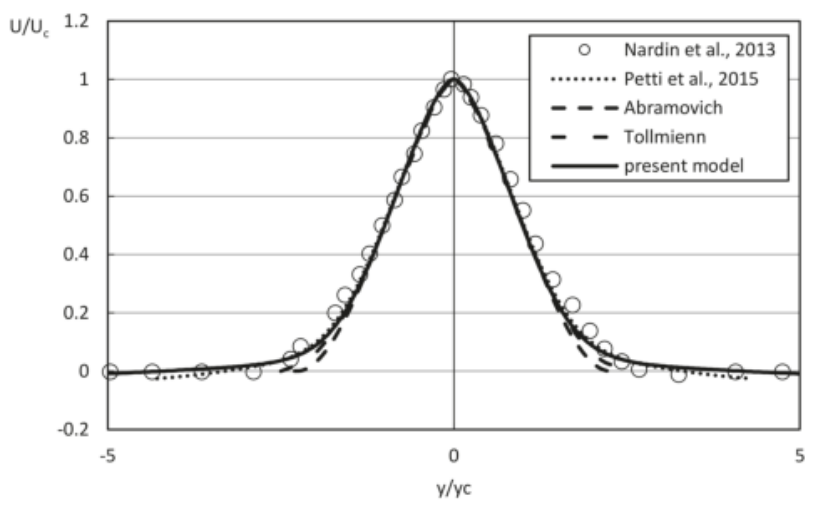

Figure 4. Longitudinal velocity $\mathrm{U}$, taken along a transverse transect $780 \mathrm{~m}$ from the jet inlet, normalized with the centerline velocity, $U_{c}$. In the abscissa, $y$ is the distance from the jet axis and $y_{c}$ is the local half-width of the jet, corresponding to the location where the mean velocity is equal to half the centerline velocity $\mathrm{U}_{\mathrm{c}}$.

The very small differences compared to the previous test [29] could be ascribed to the different boundary conditions used in the present paper. In order to maintain a constant level inside the domain, Petti et al. [29] inserted a crosswise weir on the opposite side of the hydrodynamic mesh compared to the jet inlet, while in the present simulation a fixed water level has been imposed. This last solution is an improvement because it requires a less extensive domain and its applicability is supported by the results obtained. The comparison is also made with the theoretical profile of Öszoy and Ünlüata [12] defined by means of an analytical approach based on depth-averaged shallow water equations, confirming the $2 \mathrm{DH}$ model herein used.

Similar considerations can be made about the normalized velocity profile, taken along a cross section and depicted in Figure 4; the two theoretical self-similar profiles proposed by Schlichting and Tollmienn for a free turbulent jet in the main region [10], are shown for comparison with numerical results.

The graph shows that the self-similar profile is maintained along transverse transects but with a greater spread due to bottom friction, according to Öszoy and Ünlüata [12]. This statement justifies 
the increased width at the base of the section of the numerical curves instead of theoretical ones without friction.

Figures 3 and 4 show good agreement of the present model with numerical quasi-3D and analytical results [29], with the great advantage that it is computationally less expensive than a quasi-3D model.

Petti et al. [29] analyzed the effects of an opposing wave field imposed on the previously described hydrodynamic steady-state condition. The same test is herein repeated, assigning a wave bin spectrum with a peak period of $5 \mathrm{~s}$ at the open sea boundary of the domain (Figure 2).

In order to verify the effects of the bottom friction in the wave propagation and in the subsequent interaction with the jet current, diverse tests are performed using different formulations of spectral energy dissipation, as summarized in Table 1.

The most important hydrodynamic evidence compared to the previous simulation is a more pronounced decay of the longitudinal velocity, as depicted in Figure 5, where velocity linear contours are compared in the two cases of the jet flow alone and the jet interacting with waves. For both conditions, the present current field is completely analogous to that reported by Nardin et al. [30].

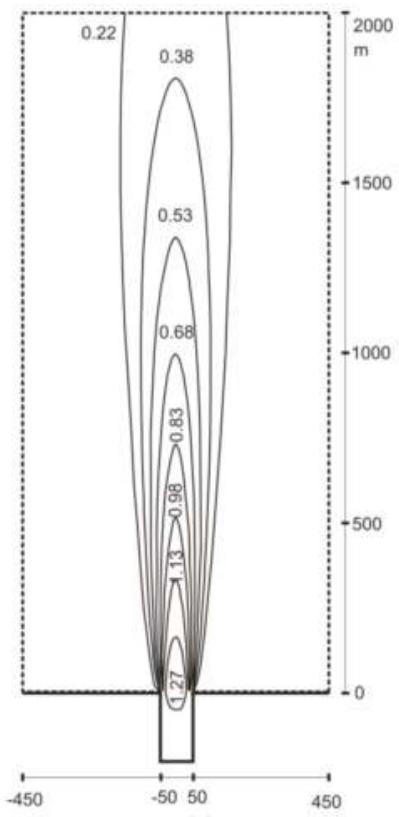

(a)

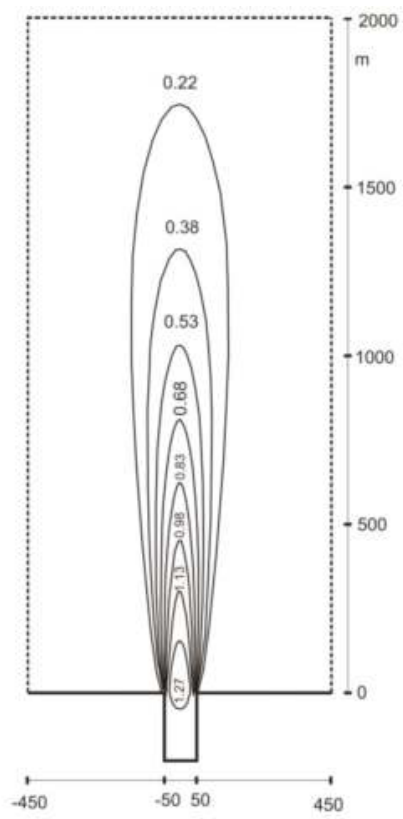

(b)

Figure 5. Linear contours of longitudinal velocity (m/s): (a) jet alone; (b) jet interacting with waves according to T5M simulation.

The flow field develops on the horizontal plane with the prevalent component of the current velocity aligned with the jet axis and a lateral spreading due to the transverse transfer of momentum, which involves formation of eddies on the jet sides. A characteristic feature of a turbulent jet, coherent with both theory and experimental results [10], is the smallness of the transverse velocity components in any section of the jet, compared to the longitudinal ones. In the cases analyzed, the maximum value reached by the $y$-directed transverse velocity is about $7 \mathrm{~cm} / \mathrm{s}$, corresponding to $5 \%$ of the velocity at the inlet. For this reason, the waves propagating against the jet current are weakly refracted and remain substantially collinear, at least in the domain portion where the interaction with the current velocities is more important, as pointed out in Figure 6a. 
With respect to this, the major changes in the wave field are observed in a narrow region around the jet centerline, where the current vorticity is greater. As can be seen in Figure $6 \mathrm{~b}, \mathrm{c}$, the main evidence is the steepening of the waves as they approach the jet inlet, where current velocities are higher, due to the increase of wave height and a simultaneous progressive reduction of the relative period as predicted by the Doppler effect. The obtained results are completely analogous to those found by Nardin et al. [30].

Given the underlined peculiarities of both the wave and the flow field, the analysis focuses on the main variables extracted along the jet centerline, starting from the normalized longitudinal velocity of the jet, depicted in Figures 7 and 8. In Figure 9 the normalized longitudinal velocity is taken along a cross section.

As can be appreciated from the graphs, the spectral friction scheme adopted is important and is fundamental for the prediction of the bottom shear stresses, which are also responsible for sediment transport mechanisms. This aspect, in several numerical applications of nearshore wave-current interaction [20-22], is not specified.

The major evidence compared to the simulation without wave-current interaction, is the more pronounced decay of longitudinal velocity of the current caused by the increase of the global bottom shear stress due to the wave motion, which leads to the definition of $\tau_{\mathrm{m}}$ [31].

In particular, two aspects emerge from the relative comparison of the results: the first is a larger velocity reduction obtained by the present model compared to Petti et al. [29] and Nardin et al. [30] numerical results. Petti et al. [29] have already registered a higher steepening of waves to that calculated by Nardin et al. [30], who do not specify the frictional scheme setup.

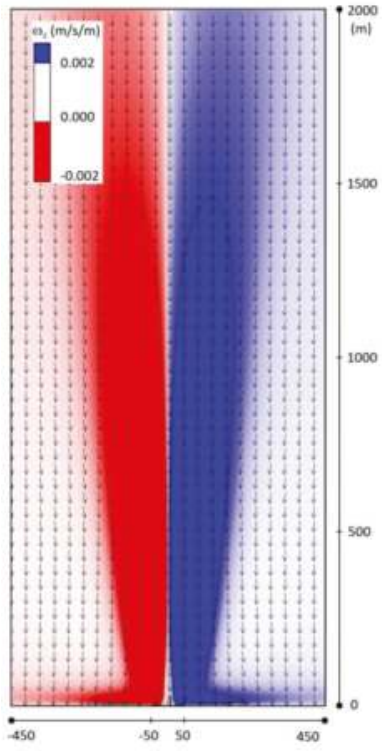

(a)

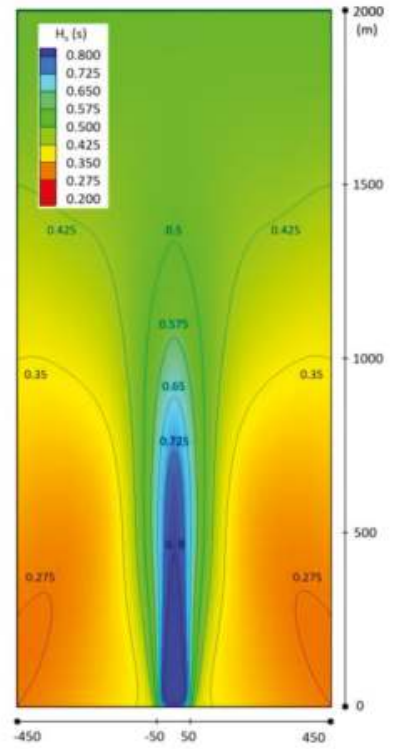

(b)

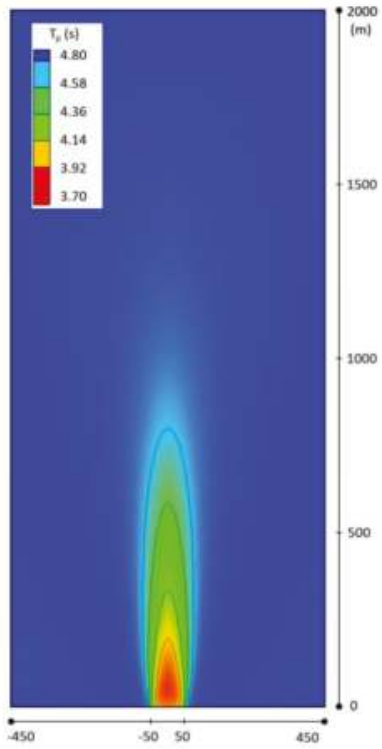

(c)

Figure 6. Variations obtained in the simulation T5M of the following parameters: (a) the z-directed component of the current vorticity, defined as the curl of the flow velocity vector, with superimposed arrows representing the mean direction of the waves; (b) the significant wave height $\mathrm{H}_{\mathrm{s}}$; (c) the relative peak period $T_{p}$. 


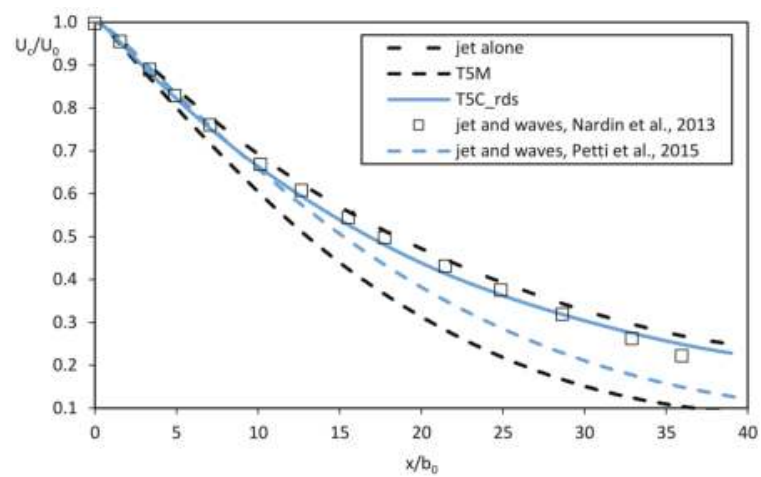

Figure 7. Normalized longitudinal velocity, along the jet centerline, in the case of jet alone and with the superimposed wave propagation with a period of $5 \mathrm{~s}$.

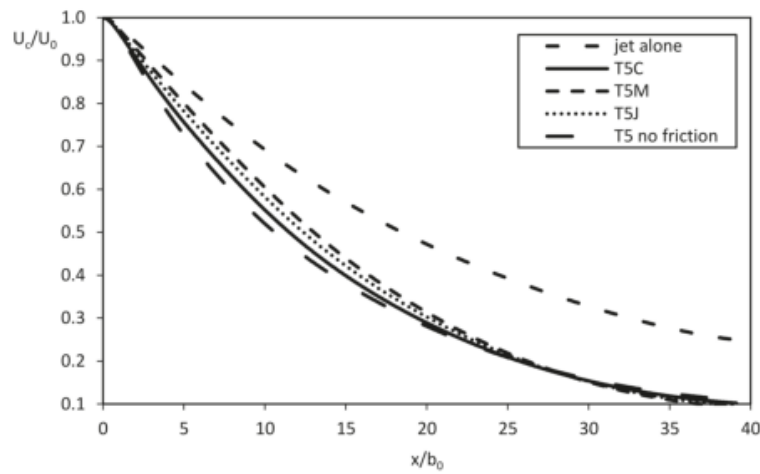

Figure 8. Normalized longitudinal velocity, along the jet centerline, in the case of jet alone and with the superimposed wave propagation with a period of $5 \mathrm{~s}$, applying different schemes of the spectral bottom friction dissipation.

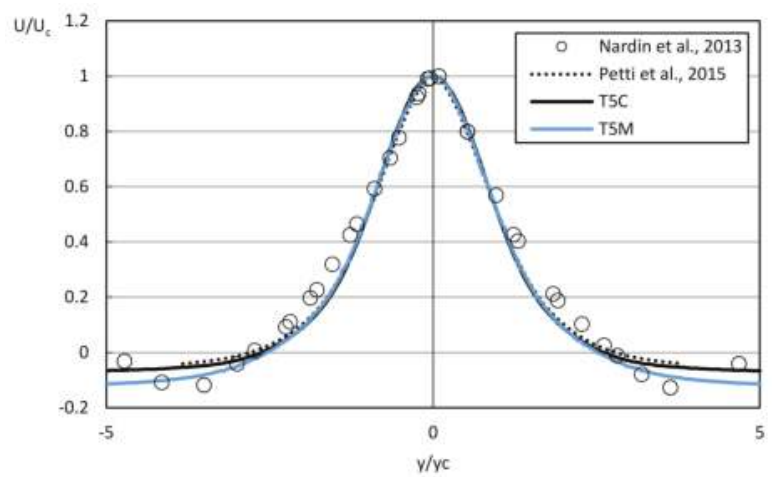

Figure 9. Normalized longitudinal velocity, in a cross section taken at $780 \mathrm{~m}$ from the jet inlet in the case of jet with the superimposed wave propagation with a period of $5 \mathrm{~s}$. 
Following Kamphuis [41], the bottom-friction coefficient $C_{f}$ is a function of both the wave Reynolds number, $\operatorname{Re}=A U_{w} / v$, where $v$ is the kinematic viscosity coefficient, and the relative roughness $A / k_{n}$. A wave rough turbulent motion near the bed, in which $C_{f}$ depends only on the relative roughness, can be distinguished from a laminar or transitional one. Hasselmann and Collins [42], using wave spectra measured offshore of Panama City, have indicated a constant value of $C_{f}$ equal to 0.015 . This value has been widely used, also in recent modelling, even though it has a great variability, experimentally verified [18], as found by Madsen et al. [38], who obtained the expression (13) as a function of the relative roughness. Figure 10a,b show that, as the relative roughness decreases, the bottom friction coefficient considerably increases.

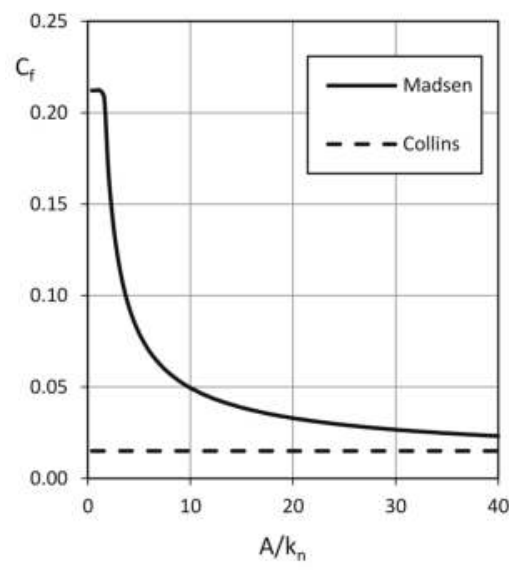

(a)

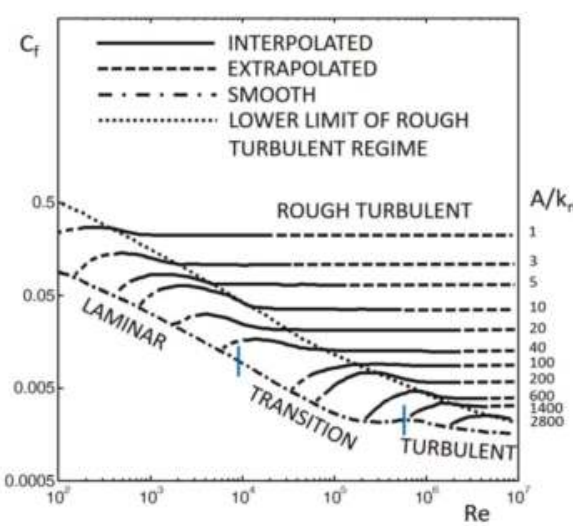

(b)

Figure 10. (a) Comparison between the constant value of the bottom friction coefficient $C_{f}$ assumed by Collins [37] and that computed by Madsen et al. [38] as a function of the relative roughness $A / k_{n}$; (b) friction coefficient diagrams proposed after Kamphuis [18,41].

Figure 11 shows values of the Reynolds number and the relative roughness obtained in the present simulations performed. These values are compatible with a wave rough turbulent flow at the bottom, confirming the validity of the bottom-friction approaches applied.

However, in Madsen formulation, the spectral energy dissipation is increased by the progressive steepening of the waves against the jet current and consequently the enhancement of the bottom-friction coefficient. This explains the higher longitudinal velocity in the T5M simulation compared to T5C (Figure 8).

In the mechanisms of interaction between the waves against current, a gradient of radiation stress tensor also arises from the steepening of the waves and, thus, additional hydrodynamic forces enter into the motion equations.

Petti et al. [29] have already highlighted that in the first region of the spreading jet, up to $x / b_{0}=10$, the contribution to the decay of the velocity due to radiation stress and bottom shear stress is similar, due to the presence of major gradients of radiation stress toward the inlet. For this purpose, the simulation T5C has also been performed taking into account only the radiation stress contribution and neglecting the wave-bottom shear stress in the computation of $\tau_{m}$. The results are very close to that of Nardin et al. [30], as can be seen in Figure 7. 


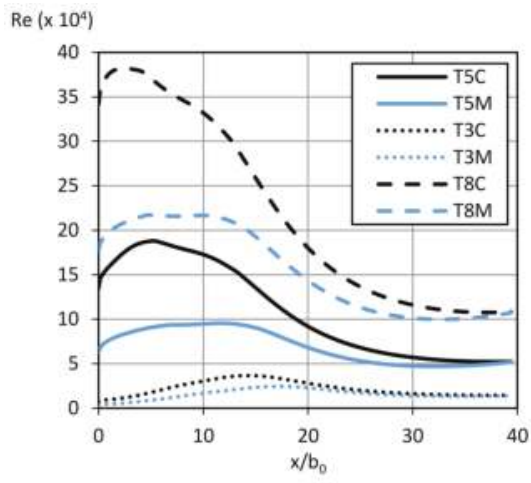

(a)

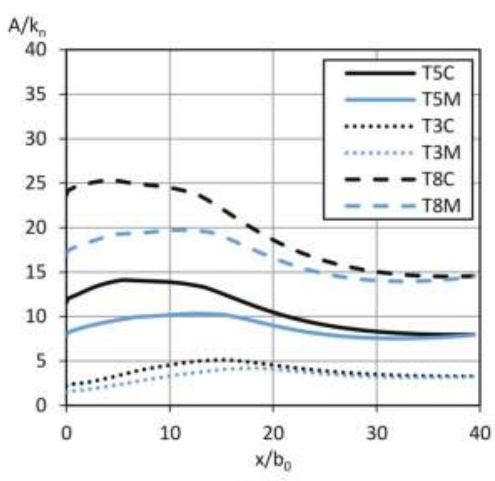

(b)

Figure 11. Simulations of wave-current interaction for different peak periods ( $3 \mathrm{~s}, 5 \mathrm{~s}$ and $8 \mathrm{~s}$ ) and assuming Collins and Madsen approaches: (a) Reynolds numbers and (b) relative roughness $A / \mathrm{k}_{n}$, along the centerline of the jet current.

To check if a different wave period can further influence the jet hydrodynamics, simulations with, respectively, a peak period of $3 \mathrm{~s}$ and $8 \mathrm{~s}$ have also been carried out. In analogy with the above, the results in term of normalized longitudinal current velocity are reported in Figures 12 and 13.

The comparison between the simulations with the different assigned periods does not highlight significant differences in the jet flow field; the curves relating to the same bottom friction model are practically overlapping. However, the same cannot be said if the wave bed shear stress (Figure 14a) and the maximum derived from the wave-current interaction (Figure 14b) are investigated.

According to Soulsby [31], the maximum bed shear stress is expressed as follows:

$$
\tau_{\max }=\left[\left(\tau_{m}+\tau_{w} \cos \phi\right)^{2}+\left(\tau_{w} \sin \phi\right)^{2}\right]^{1 / 2}
$$

$\phi$ being the angle between the current velocity and the direction of wave travel. This quantity is fundamental in morphodynamics because it is responsible for the lifting of sediments from the bottom when it exceeds the threshold bed shear stress for the onset of motion.

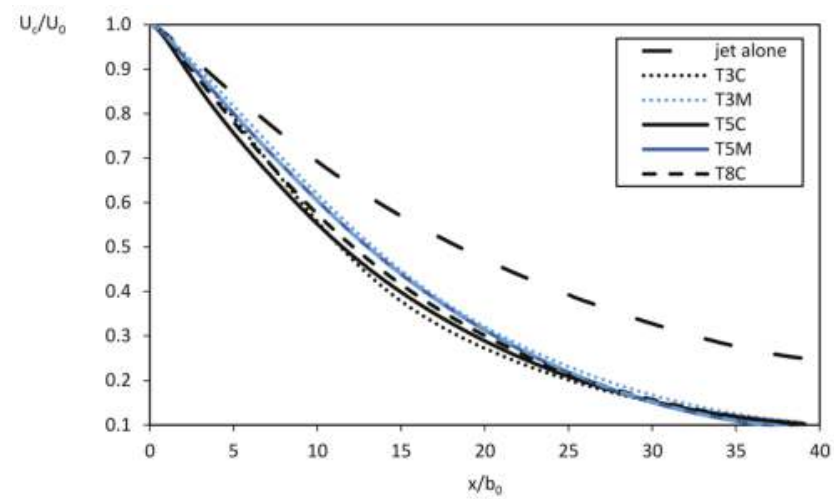

Figure 12. Normalized longitudinal velocity, along the jet centerline, in the case of jet alone and with the superimposed wave propagation with peak periods of $3 \mathrm{~s}, 5 \mathrm{~s}$ and $8 \mathrm{~s}$. 


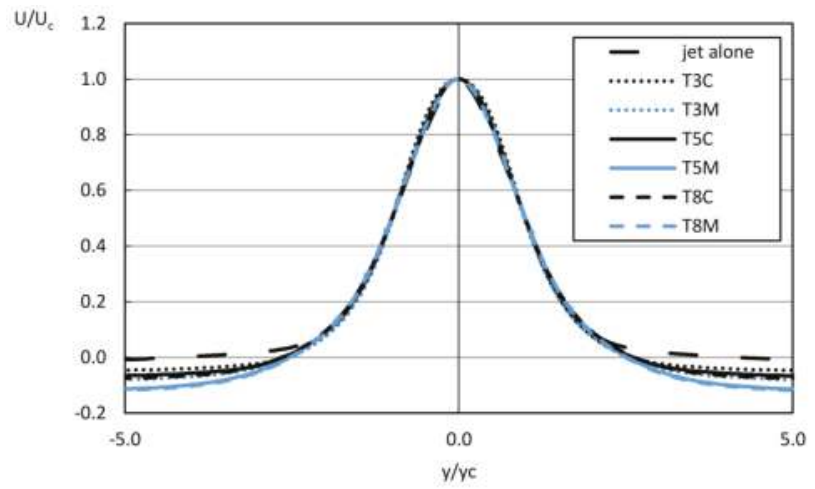

Figure 13. Normalized longitudinal velocity, in a cross section taken at $780 \mathrm{~m}$ from the jet inlet in the case of jet alone and with the superimposed wave propagation with peak periods of $3 \mathrm{~s}, 5 \mathrm{~s}$ and $8 \mathrm{~s}$.

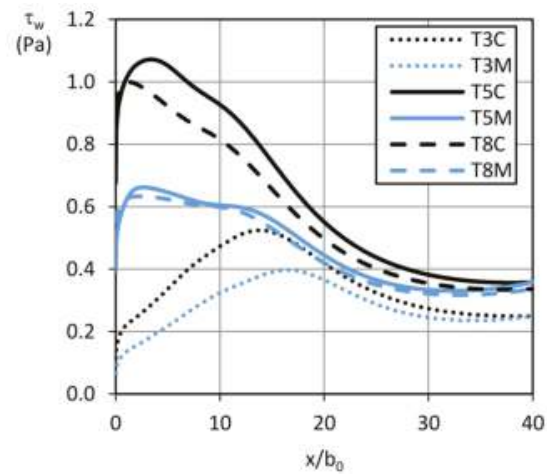

(a)

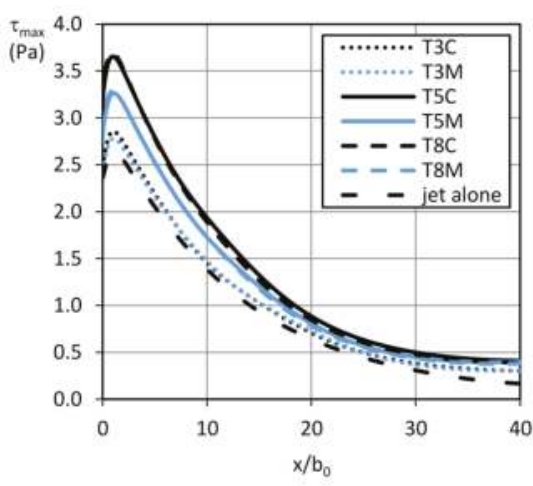

(b)

Figure 14. Bottom shear stresses along the jet centerline: (a) $\tau_{w}$ in the performed simulations; (b) comparison between the maximum bottom shear stress of the jet alone with those computed in the interactions with waves.

If the wave bed shear stresses are considered, a slight dependence on the period is noticed. Increasing the periods, the wave bed shear stresses are higher and reach the maximum value closer to the jet inlet. Moreover, comparing the results with the same period, great differences are found related to the bottom friction scheme, consistent with the previous results.

The maximum bottom shear stress, deriving from the wave-current interaction, is significantly affected by greater values of the wave period. The configurations performed with a peak period of $3 \mathrm{~s}$ do not show particular differences either among themselves or with the bottom shear stress of the jet alone. The contribution of wave shear stress to the maximum bottom shear stress is small for low periods at the assigned depth. Instead, $\tau_{w}$ related to $5 \mathrm{~s}$ and $8 \mathrm{~s}$ peak period, increase $\tau_{\max }$ considerably and differently if the bottom friction coefficient $C_{f}$ is left to vary with the relative bottom roughness [38].

This outcome can be very important in modelling hydrodynamic nearshore processes, especially in shallow water depth environments, where the interaction with bottom plays a crucial role, both on currents and waves, as can be seen from the numerical results described above. 
For this purpose, in a context, for instance, close to a tidal inlet or an estuarine environment, spectral energy dissipation presents great variability related to the peak period, which can change significantly in the presence of strong gradients of the velocities due to the Doppler effect. This mechanism depends on the angle between current and waves: the Doppler effect is not as evident in the case of quasi-orthogonal incidence, as occurs for the longshore currents and the waves propagating nearshore, but it becomes important if current and waves are quite collinear, as for river or tidal jets. For these reasons, it is difficult to consider that the frictional coefficient can be regarded as constant over the whole domain.

The effects of this dissipation are particularly expressed on the bottom shear stresses, responsible for sediment resuspension and motion and the morphological evolution of the bottom itself.

\section{Conclusions}

In the present work, a simple 2DH numerical model has been applied to a literature test $[29,30]$ reproducing a river jet current issuing into a large shallow basin and interacting with frontal waves. The model couples a hydrodynamic numerical model with the spectral model SWAN and it is suitable for reproducing the interaction between waves and currents typical of estuarine and coastal areas. Moreover, the known computational advantages of 2D modelling allow it to be applied to large domains.

The outcomes have been compared to both analytical and numerical results, the latter being obtained with a model that adopts a more complex representation of the vertical and horizontal eddy viscosity. The comparison shows that the present model provided a very adequate representation of the hydrodynamics of a turbulent jet confined to the bottom.

In detail, the role played by bottom friction has been investigated and it has been recognized as the dominant process in the decay of the jet longitudinal velocity and its greater spreading. Bottom friction dissipation becomes even more important in the correct reconstruction of the nearshore wave field, above all in shallow-depth contexts.

The simulations of the jet current interaction with opposing travel waves carried out have shown that, in the spectral mechanism of dissipation energy, the proven dependence of the bottom friction coefficient with the relative roughness at the bed can change the wave field significantly. Consequently, the interaction with current can also affect hydrodynamics.

To this end, two different theories implemented in SWAN to evaluate the dissipation due to bottom friction have been considered: the Collins approach [37], which assumes the bottom friction coefficient as constant, and the Madsen scheme [38], which takes into account the variability of the same coefficient in contexts of wave rough turbulent motion at the bed.

The effects of these two approaches are quite sensitive to the period of waves, which can change in the domain due to shoaling and Doppler mechanisms generated by currents. In particular, bottom friction has a dominant role in the calculation of the maximum shear stress, which is responsible for bed-erosion processes. The stress is significantly increased by greater values of the wave period and of the current velocity. Above all, it is conditioned by the assumed spectral dissipation scheme.

From a general point of view, both of the theories analyzed are suitable for calculating energy dissipations that wind waves, propagating on shallow domains, undergo due to interaction with the bed. However, near a river delta or a tidal inlet, where currents can be strong and quite collinear compared to the mean wave direction, relative roughness can vary, consequently affecting the friction coefficient and the related bottom shear stress. The Madsen formulation [38] takes this variability into account, as opposed to the Collins scheme [37], and for this reason it results as preferable for interpreting the interaction phenomenon.

Author Contributions: All the authors contributed equally to this work.

Conflicts of Interest: The authors declare no conflict of interest. 


\section{References}

1. Longuet-Higgins, M.; Stewart, R. The changes in amplitude of short gravity waves on steady non-uniform currents. J. Fluid Mech. 1961, 10, 529-549. [CrossRef]

2. Peregrine, D.H. Interaction of Water Waves and Currents. Adv. Appl. Mech. 1976, 16, 9-117. [CrossRef]

3. Jonsson, I.G.; Skougaard, C.; Wang, J.D. Interaction between Waves and Currents. Coast. Eng. 1970, 1, 489-507. [CrossRef]

4. Wolf, J.; Prandle, D. Some observations of wave-current interaction. Coast. Eng. 1999, 37, 471-485. [CrossRef]

5. Grant, W.D.; Madsen, O.S. Combined wave and current interaction with a rough bottom. J. Geophys. Res. 1979, 84, 1797-1808. [CrossRef]

6. Fredsøe, J. Turbulent Boundary Layer in Wave-current Motion. J. Hydraul. Eng. 1984, 110, 1103-1120. [CrossRef]

7. Christoffersen, J.B.; Jonsson, I.G. Bed friction and dissipation in a combined current and wave motion. Ocean Eng. 1985, 12, 387-423. [CrossRef]

8. Soulsby, R.L.; Hamm, L.; Klopman, G.; Myrhaug, D.; Simons, R.R.; Thomas, G.P. Wave-current interaction within and outside the bottom boundary layer. Coast. Eng. 1993, 21, 41-69. [CrossRef]

9. Wang, F.C. The dynamics of a river-bay-delta system. J. Geophys. Res. 1984, 89, 8054-8060. [CrossRef]

10. Abramovich, G.N. The Theory of Turbulent Jets; MIT Press: Cambridge, MA, USA, 1963.

11. Schlichting, H. Boundary Layer Theory; McGraw-Hill: New York, NY, USA, 1960.

12. Özsoy, E.; Ünlüata, Ü. Ebb-tidal flow characteristics near inlets. Estuar. Coast. Shelf Sci. 1982, 14, $251-263$. [CrossRef]

13. Giger, M.; Dracos, T.; Jirka, G.H. Entrainment and mixing in plane turbulent jets in shallow water. J. Hydraul. Res. 1991, 29, 615-641. [CrossRef]

14. Adami, A.; Milan, G. Effetto della scabrezza delle pareti di contenimento sulla diffusione turbolenta di un getto piano. In Proceedings of the XVI Convegno di Idraulica e Costruzioni Idrauliche, Torino, Italy, 25-27 September 1978.

15. Ismail, N.M.; Wiegel, R.L. Opposing wave effect on momentum jets spreading rate. J. Waterw. Port. Coast. Ocean Eng. 1983, 109, 465-483. [CrossRef]

16. Bhattacharya, J.P.; Giosan, L. Wave-influenced deltas: Geomorphological implications for facies reconstruction. Sedimentology 2003, 50, 187-210. [CrossRef]

17. Ashton, A.D.; Giosan, L. Wave angle control of delta evolution. Geophys. Res. Lett. 2011, 38. [CrossRef]

18. Hsiao, S.V.; Shemdin, O.H. Bottom Dissipation in Finite-Depth Water Waves. Coast. Eng. 1978, 24, 434-448. [CrossRef]

19. Bolaños-Sanchez, R.; Riethmüller, R.; Gayer, G.; Amos, C.L. Sediment Transport in a Tidal Lagoon Subject to Varying Winds Evaluated with a Coupled Current-Wave Model. J. Coast. Res. 2005, 21, e11-e26. [CrossRef]

20. Warner, J.C.; Sherwood, C.R.; Signell, R.P.; Harris, C.K.; Arango, H.G. Development of a three-dimensional, regional, coupled wave, current, and sediment-transport model. Comput. Geosci. 2008, 34, 1284-1306. [CrossRef]

21. Rusu, L.; Bernardino, M.; Guedes Soares, C. Modelling the influence of currents on wave propagation at the entrance of the Tagus estuary. Ocean Eng. 2011, 38, 1174-1183. [CrossRef]

22. Nahon, A.; Bertin, X.; Fortunato, A.B.; Oliveira, A. Process-based 2DH morphodynamic modeling of tidal inlets: A comparison with empirical classifications and theories. Mar. Geol. 2012, 291, 1-11. [CrossRef]

23. Dodet, G.; Bertin, X.; Bruneau, N.; Fortunato, A.B.; Nahon, A.; Roland, A. Wave-current interactions in a wave-dominated tidal inlet. J. Geophys. Res. Oceans 2013, 118, 1587-1605. [CrossRef]

24. Sutherland, J.; Walstra, D.J.R.; Chesher, T.J.; van Rijn, L.C.; Southgate, H.N. Evaluation of coastal area modelling systems at an estuary mouth. Coast. Eng. 2004, 51, 119-142. [CrossRef]

25. Tambroni, N.; Ferrarin, C.; Canestrelli, A. Benchmark on the numerical simulations of the hydrodynamic and morphodynamic evolution of tidal channels and tidal inlets. Cont. Shelf Res. 2010, 30, 963-983. [CrossRef]

26. Mellor, G.L. The Depth-Dependent Current and Wave Interaction Equations: A Revision. J. Phys. Oceanogr. 2008, 38, 2587-2596. [CrossRef]

27. Bennis, A.; Ardhuin, F. Comments on "The Depth-Dependent Current and Wave Interaction Equations: A Revision". J. Phys. Oceanogr. 2011, 41, 2008-2012. [CrossRef] 
28. Kemp, P.; Simons, R. The interaction of waves and a turbulent current: Waves propagating against the current. J. Fluid Mech. 1983, 130, 73-89. [CrossRef]

29. Petti, M.; Bosa, S.; Pascolo, S.; Lubrano, F. Hydrodynamic Wave Effects on the River Delta: An Efficient 2HD Numerical Model. In Proceedings of the Fifteenth International Conference on Civil, Structural and Environmental Engineering Computing, Prague, Czech Republic, 1-4 September 2015; CIVIL-COMP Press: Prague, Czech Republic, 2015.

30. Nardin, W.; Mariotti, G.; Edmonds, D.A.; Guercio, R.; Fagherazzi, S. Growth of river mouth bars in sheltered bays in the presence of frontal waves. J. Geophys. Res. Earth Surf. 2013, 118, 872-886. [CrossRef]

31. Soulsby, R.L. Dynamics of Marine Sands: A Manual for Practical Applications; Thomas Telford Publications: London, UK, 1997; p. 249.

32. Booij, N.; Ris, R.C.; Holthuijsen, L.H. A third-generation wave model for coastal regions, Part I, Model description and validation. J. Geophys. Res. 1999, 104, 7649-7666. [CrossRef]

33. Bosa, S.; Petti, M.; Lubrano, F.; Pascolo, S. Morphodynamic Model Suitable for River Flow and Wave-Current Interaction. IOP Conf. Ser. Mater. Sci. Eng. 2017, 245. [CrossRef]

34. Audusse, E.; Bouchut, F.; Bristeau, M.-O.; Klein, R.; Perthame, B. A fast and stable well-balanced scheme with hydrostatic reconstruction for shallow water flows. SIAM J. Sci. Comput. 2004, 25, 2050-2065. [CrossRef]

35. Liang, Q.; Marche, F. Numerical resolution of well-balanced shallow water equations with complex source terms. Adv. Water Resour. 2009, 32, 873-884. [CrossRef]

36. Toro, E.F. Shock-Capturing Methods for Free-Surface Shallow Flows; John Wiley \& Sons: New York, NY, USA, 2001.

37. Collins, J.I. Prediction of shallow water spectra. J. Geophys. Res. 1972, 77, 2693-2707. [CrossRef]

38. Madsen, O.S.; Poon, Y.-K.; Graber, H.C. Spectral wave attenuation by bottom friction: Theory. In Proceedings of the 21th International Conference on Coastal Engineering, Costa del Sol-Malaga, Spain, 20-25 June 1988; pp. 492-504.

39. Holthuijsen, L.H. Waves in Oceanic and Coastal Waters; Cambridge University Press: Cambridge, UK, 2007.

40. Hasselmann, K.; Barnett, T.P.; Bouws, E.; Carlson, H.; Cartwright, D.E.; Enke, K.; Ewing, J.; Gienapp, H.; Hasselmann, D.E.; Kruseman, P.; et al. Measurements of Wind Wave Growth and Swell Decay during the Joint North Sea Wave Project (JONSWAP); Deutches Hydrographisches Institut: Hamburg, Germany, 1973; pp. 1-95.

41. Kamphuis, W. Friction Factor under Oscillatory Waves. J. Waterw. Harbors Coast. Eng. Div. 1975, 101, 135-144.

42. Hasselmann, K.; Collins, J.I. Spectral dissipation of finite-depth gravity waves due to turbulent bottom friction. J. Mar. Res. 1968, 26, 1-12.

(C) 2018 by the authors. Licensee MDPI, Basel, Switzerland. This article is an open access article distributed under the terms and conditions of the Creative Commons Attribution (CC BY) license (http:/ / creativecommons.org/licenses/by/4.0/). 
Article

\title{
Experimental and Numerical Investigation of Pre-Breaking and Breaking Vorticity within a Plunging Breaker
}

\author{
Diana De Padova ${ }^{1, *}$, Maurizio Brocchini ${ }^{2}$, Federica Buriani ${ }^{3}$, Sara Corvaro ${ }^{2}$, \\ Francesca De Serio ${ }^{1}$, Michele Mossa ${ }^{1}$ and Stefano Sibilla ${ }^{4}$ \\ 1 Department of Civil, Environmental, Land, Building Engineering and Chemistry (DICATECh), \\ Polytechnic University of Bari, Via E. Orabona 4, 70125 Bari, Italy; francesca.deserio@poliba.it (F.D.S.); \\ michele.mossa@poliba.it (M.M.) \\ 2 Dipartimento di Ingegneria Civile, Edile e dell'Architettura, Università Politecnica delle Marche, \\ Via Brecce Bianche 12, 60131 Ancona, Italy; m.brocchini@univpm.it (M.B.); s.corvaro@univpm.it (S.C.) \\ 3 Department of Mathematical Sciences, Loughborough University, Leicestershire LE11 3TU, UK; \\ f.buriani@lboro.ac.uk \\ 4 Department of Civil Engineering and Architecture (DICAr), University of Pavia, Via Ferrata 3, \\ 27100 Pavia, Italy; stefano.sibilla@unipv.it \\ * Correspondence: diana.depadova@poliba.it
}

Received: 18 February 2018; Accepted: 23 March 2018; Published: 26 March 2018

\begin{abstract}
The characteristics of vorticity induced just prior and at the splash-down of a plunging breaker on a 1:10 planar slope have been studied using wave flume experiments and numerical simulations. Laboratory experiments involved detailed measurements in the outer surf zone of both fluid velocities below trough level, achieved by a fibre-optic laser-Doppler anemometer, and water surface elevations, obtained by an ultrasound probe. A Weakly-Compressible Smoothed Particle (WCSPH) model, coupled with a two-equation model for turbulent stresses, has been employed for the numerical simulations. A thorough calibration of the SPH's numerical parameters has been first performed, through comparison between numerical and experimental wave elevation and velocity data. Then, considering that time-averaged laboratory data shows a significant vorticity beneath the free surface in the wave pre-breaking region, the vorticity generation mechanism has been thoroughly analyzed by means of the numerical model. In the attempt of explaining the generation of vorticity as induced by flow separation, we also inspected the role of the streamwise flow deceleration and surface-parallel vorticity flux. In analogy with the case of spilling breakers a cause-effect relation has been observed between streamwise flow deceleration and vorticity generation. Numerical findings are positively confirmed by the experimental results.
\end{abstract}

Keywords: smoothed particle hydrodynamics models; physical modelling; plunging breaking waves; vorticity

\section{Introduction}

Turbulence and undertow currents play an important role in surf-zone mixing and transport processes; therefore, their study is fundamental for the understanding of nearshore dynamics and the related planning and management of coastal engineering activities.

Pioneering studies were carried out by [1-3], who qualitatively described the features of plunging breakers in the outer region of the surf zone. More detailed information on the velocity field under plunging breakers can be found in the experimental works by, among others, refs. [4-7] and more recently by [8-10]. In these works, single-point measurement techniques, such as hot-wire anemometry 
and Laser Doppler Anemometry, were used to provide maps of the flow field in a time-averaged or ensemble-averaged sense.

The advent of non-intrusive measuring techniques, such as Particle Image Velocimetry (PIV) provided accurate and detailed instantaneous spatial maps of the flow field. Moreover, by correlating spatial gradients of the measured velocity components, the instantaneous vorticity maps could be deduced.

Measurements of vorticity using planar PIV were obtained, among others, by [11], who carried out experiments on waves breaking on a submerged breakwater on a 1:100 sloped beach, and by [12], who gained information on the overall characteristics of the $2 \mathrm{D}$ flow field of breaking waves in shallow water and proved the existence of oblique descending vortices, previously observed by [13]. An extensive investigation on the surf-zone of breaking waves over a sloping beach, also performed with a PIV, was carried out by [14], who estimated the longshore vorticity. A volumetric particle-tracking system was used by [15] to study the vorticity dynamics during wave breaking on a fixed bar on a rigid plane slope.

Many attempts have been made to identify and examine the source of vorticity in breaking waves [16,17], especially for the case of the spilling waves [18], which most commonly occur, but this topic is still debated and deserves further study. In the breaking wave, the main region of vorticity generation is at the free surface, while seabed vorticity is much smaller. Longuet-Higgins [19] explained this vorticity generation in terms of the combined effect of surface curvature and zero-shear stress boundary conditions at the interface, with a Stokes layer initially confining the generated vorticity, which in a subsequent stage escapes and fills the domain. Also, Lin and Rockwell [20] attributed the vorticity generation to the sharp curvature of the interface, where a flow separation occurs. Complementary to these findings, Dabiri and Gharib [21] experimentally deduced that the major source of vorticity in spilling breakers was attributable to the free surface fluid deceleration before breaking, together with an increase of fluid pressure on the back of the wave, pushing the crest.

What is clear is the fundamental link between near-surface vorticity generation and the subsequent degeneration into the near-surface turbulence of a breaking wave. The mechanism governing this connection still needs a thorough analysis, especially for the lesser investigated plunging breakers. In particular, the vorticity field by a plunging breaker has received less attention than that due to a spiller because spilling waves are representative of storm waves and of bore jumps, thus they frequently occur. However, the same spilling breaker may be regarded as a locally (at wave crest) weak plunging breaker, with the rotational flow generated by the plunger spreading down along the front of the wave and forming a roller [16]. The added value of our study with respect to previous investigations is our focusing on the plunging breaking wave, where the high curvature due to the steepening of the wave and the related flow shearing naturally induce a strong vorticity and related turbulence.

In the perspective to investigate these aspects, numerical models represent a useful tool: the dynamics of breaking-induced mean vorticity was numerically analysed by numerous authors, including [22,23]. If grid-based numerical methods can suffer from certain limitations when used to study such violent free surface flows, particle meshless methods have instead the potential to provide a comprehensive description of the full processes associated with wave breaking, whilst they can accurately capture the water surface profile during such processes.

Among the meshless methods, Smoothed Particle Hydrodynamics (SPH) is at present effective in solving several fluid-dynamic problems with highly nonlinear deformation, such as wave breaking and impact [24,25]; hydraulic jumps [26-29]; long waves, e.g., floods, tsunamis and landslide submersions [30]; oscillating jets inducing breaking waves [31].

In view of the above, the main aim of our paper is to bring to the attention the cause-effect relation between surface convective deceleration and vorticity generation in plunging breakers. Such a goal is pursed by using both experimental and numerical means. First, Laser Doppler Anemometer (LDA) velocity measurements have been used to detect the time-averaged vorticity field in the pre-breaking region of a plunging breaker. Then, a calibration of the SPH model has been performed, by comparing 
experimental and numerical velocity and elevation time series obtained at different locations along the channel. Finally, SPH simulations have been used in the surf zone (i) to inspect and detail the vorticity field, evaluating, in particular, its temporal evolution; (ii) to relate the vorticity field and the free surface dynamics, in particular with the flow surface-parallel deceleration.

\section{Experimental Set $U p$}

Experimental investigations were carried out in a two-dimensional wave channel in the Coastal Engineering Laboratory (LIC) of the DICATECh of the Polytechnic University of Bari. The wave channel was $2.42 \mathrm{~m}$ wide and $45.20 \mathrm{~m}$ long. A uniform and fixed slope 1:10 started at $32.58 \mathrm{~m}$ from the paddles. The wave generating system is a piston-type one, with paddles producing the desired wave by providing a translation of the water mass, according to the proper input signal. Due to its dimension the channel can be considered a large-scale facility, with minimized scale effects. A picture and a sketch of the wave channel are shown in Figures 1 and 2.

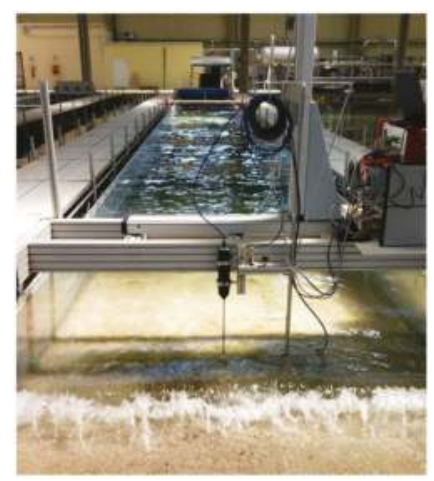

Figure 1. Frontal view of the wave channel in the Coastal Engineering Laboratory (LIC) of the Polytechnic University of Bari. The array with the measurement instrumentations is in the foreground.

Table 1 shows the main characteristics of the tested regular wave near the paddles, i.e., wave height $H_{s}$, wave period $T_{p}$ and wavelength $\mathrm{L}$ according to the Airy theory. The mean water depth was $d$. Considering the Irribarren breaking number $\xi_{b}$, the wave field is characterized by a plunging breaker $\left(\xi_{b}=0.71\right)$. The breaking section was located at a distance of $39.48 \mathrm{~m}$ from the wave paddle and the depth at breaking was around $11 \mathrm{~cm}$.

Table 1. Experimental parameters of the analysed regular wave.

\begin{tabular}{cccc}
\hline$H_{s}(\mathbf{c m})$ & $T_{P}(\mathrm{~s})$ & $L(\mathrm{~m})$ & $d(\mathrm{~m})$ \\
\hline 10 & 2 & 5.10 & 0.80 \\
\hline
\end{tabular}

Water surface elevations and velocities were measured at fourteen different locations along the centreline axis of the wave channel. Along each vertical profile, velocity measurements were acquired starting from the still water level. The measured points were $1 \mathrm{~cm}$ vertically spaced in the most superficial layer (for a thickness of $4 \mathrm{~cm}$ ) and $0.5 \mathrm{~cm}$ vertically spaced in the remaining water column. The sketch of Figure 2 shows the four sections named 0, 1, 2, and 3, used in the present work for the SPH model calibration, and the pre-breaking region where further ten shoreward sections were investigated, being $1 \mathrm{~cm}$ longitudinally spaced (see Table 2). The cross-shore direction is given by the $x$ axis and the vertical by the $\mathrm{z}$ axis. 
Velocity measurements were performed at each vertical profile along almost the entire water column by means of a 2D LDA (by Dantec). Only for the bottom layer, where the LDA could not be used for technical limitations, an Acoustic Doppler Velocimetry system (ADV vertical looking Vectrino Velocimeter by Nortek) was adopted (Figure 3), characterized by an accuracy of $1 \mathrm{~mm} / \mathrm{s}$ for the examined velocity range and a sampling frequency of $25 \mathrm{~Hz}$. At the same time and location, water surface elevation was measured by an ultrasonic system UltraLab (by General Acoustics), with an accuracy of $2 \mathrm{~mm}$. Data processing has included numerical filtering of wave elevation, despiking of ADV signal and filtering of the LDA acquisition based on a high signal to noise ratio.

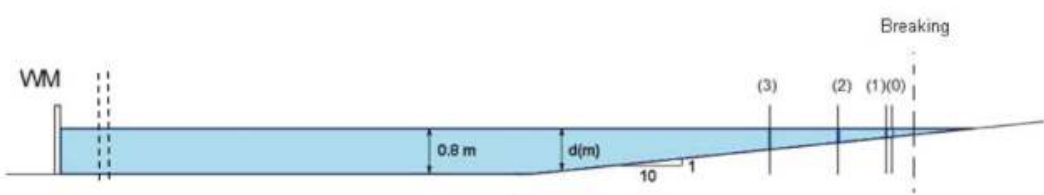

(a)

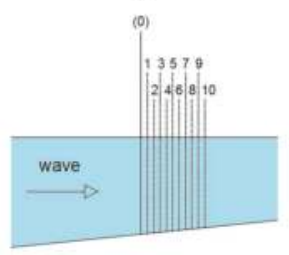

(b)

Figure 2. (a) Sketch of the wave channel with location of (a) the four investigated sections, used to calibrate the numerical model; and (b) zoomed view of the region where further ten measurement sections, used for the vorticity analysis, were located.

Table 2. Location of the measurements sections used for the code calibration and water depths.

\begin{tabular}{ccc}
\hline Investigated Section & Distance from Paddles $(\mathbf{m})$ & $\boldsymbol{d}(\mathbf{m})$ \\
\hline Section 0 & 39.18 & 0.14 \\
Section 1 & 39.08 & 0.15 \\
Section 2 & 38.24 & 0.23 \\
Section 3 & 37.04 & 0.35 \\
\hline
\end{tabular}

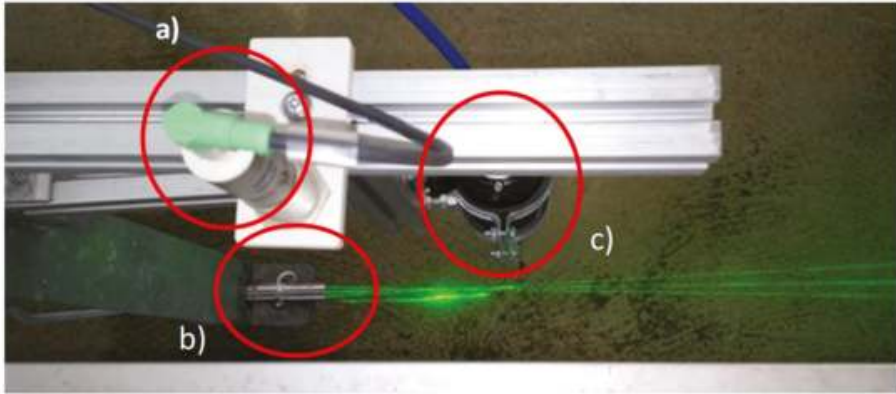

Figure 3. Top view of the measurement instruments: (a) ultrasound probe; (b) LDA and (c) ADV. The ADV and the LDA measurement volume are vertically aligned. 


\section{SPH Numerical Method}

$\mathrm{SPH}$ is a meshless, Lagrangian method where the fluid domain is represented by nodal points that are scattered in space with no grid structure and move with the fluid. A general description of the SPH can be found in [32-34]. The specific features of the SPH method here used are detailed in $[26,35]$.

Vertically-2D simulations have been performed through a Weakly Compressible SPH (WCSPH) approach, in which an artificial compressibility is introduced to solve explicitly in time the equations of motion of an incompressible fluid. Monaghan [33] demonstrated that the error associated with the use of a compressible formulation for the incompressible free-surface water flow is bounded to $1 \%$, provided the local numerical Mach number be everywhere lower than 0.1 : here, this condition is ensured by means of an artificial speed of sound in water of $30 \mathrm{~ms}^{-1}$. Such artificial speed of sound is not related to the physical speed of sound, which would be two orders of magnitude higher. It is introduced to avoid the extremely small time steps that would arise from the Courant stability condition, owing to the explicit scheme used for time integration. Monaghan [33] demonstrated that no unphysical compressibility effects arise if the local (numerical) Mach number, i.e., the ratio of the local velocity to the artificial speed of sound, is everywhere lower than 0.1 . Numerical tests on flapping jets [31] have shown that this limit may be even stricter (0.05). Here, given that the highest velocities in the computational domain are lower than $1 \mathrm{~m} / \mathrm{s}$, a uniform value of $30 \mathrm{~m} / \mathrm{s}$ was used for the artificial speed of sound, in order to guarantee a local Mach number smaller than 0.035 .

The Reynolds-averaged Navier-Stokes (RANS) equations are implemented by an SPH semi-discrete form as:

$$
\left\{\begin{array}{c}
\left\langle\frac{\mathrm{D} \rho_{i}}{\mathrm{D} t}\right\rangle=\sum_{j} m_{j}\left(\boldsymbol{v}_{i}-\boldsymbol{v}_{j}\right) \cdot \widehat{\nabla} W_{i j} \\
\left\langle\frac{\mathrm{D} v_{i}}{\mathrm{D} t}\right\rangle=-\sum_{j} m_{j}\left(\frac{p_{i}}{\rho_{i}^{2}}+\frac{p_{j}}{\rho_{j}^{2}}\right) \nabla W_{i j}+\sum_{j} m_{j}\left(\mathcal{T}_{i}-\mathcal{T}_{j}\right) \cdot \widehat{\nabla} W_{i j}+\mathbf{g} \\
p_{i}-p_{0}=c_{i}^{2}\left(\varrho_{i}-\rho_{0}\right) \\
\mathcal{T}_{i}=\mu_{T_{i}} \mathcal{S}_{i}
\end{array}\right.
$$

where the angled brackets indicate the SPH particle approximation, written for each particle $i$ having mass mi. Summation is extended to all particles $j$ at a distance from $i$ smaller than $2 \mathrm{~h}$, i.e., lying within the circle where the $\mathrm{C} 2$ Wendland kernel function $W_{i j}$ is defined [36].

In Equation (1), $v=(u, v)$ is the velocity vector, $p$ is pressure, $\rho$ is density, $g$ is the gravity acceleration vector, $\mathcal{T}$ is the turbulent shear stress tensor, $c$ is the speed of sound in the weakly compressible fluid, $\mu_{T}$ is the dynamic eddy viscosity, $\mathcal{S}$ is the rate-of-strain tensor and the subscript 0 denotes a reference state for pressure computation. All variables are assumed to be Reynolds averaged.

A standard $k-\varepsilon$ turbulence model [37] has been used to obtain the eddy viscosity as $\mu_{T}=c_{\mu} \frac{k^{2}}{\varepsilon}$ : the equations for the turbulent kinetic energy $k$ and for the turbulent dissipation rate $\varepsilon$ in the SPH semi-discrete form [26,35] are:

$$
\begin{gathered}
\frac{D k_{i}}{D t}=P_{k_{i}}+\frac{1}{\sigma_{k}} \sum_{j} m_{j} \frac{v_{T_{i}}+v_{T_{j}}}{\rho_{i}+\rho_{j}} \frac{k_{i}+k_{j}}{r_{i j}^{2}+0.01 h^{2}} \boldsymbol{r}_{i j} \cdot \nabla \widehat{W}_{i j}-\varepsilon_{i} \\
\frac{D \varepsilon_{i}}{D t}=C_{\varepsilon_{1}} \frac{\varepsilon_{i}}{k_{i}} P_{k_{i}}+\frac{1}{\sigma_{\varepsilon}} \sum_{j} m_{j} \frac{v_{T_{i}}+v_{T_{j}}}{\rho_{i}+\rho_{j}} \frac{\varepsilon_{i}+\varepsilon_{j}}{r_{i j}^{2}+0.01 h^{2}} \boldsymbol{r}_{i j} \cdot \nabla \widehat{W}_{i j}+C_{\varepsilon_{2}} \frac{\varepsilon_{i}}{k_{i}} \sum_{j} \frac{m_{j}}{\rho_{j}} \varepsilon_{j} \widehat{W}_{i j}
\end{gathered}
$$

where $P_{k}$ is the production of turbulent kinetic energy depending on the local rate of deformation and $v_{T}$ is the kinematic eddy viscosity. Like in [38], the values originally proposed by [37] for the model constants $\left(\sigma_{k}=1, \sigma_{\varepsilon}=1.3, C_{\varepsilon 1}=1.44, C_{e 2}=1.92\right)$ have been maintained.

Notation $\widehat{\nabla} W_{i j}$ in Equations (1) and (2) indicates the gradient of $W_{i j}$, renormalized through a procedure which enforces consistency on the first derivatives to the 1st order [39], leading to a 2nd-order accurate discretization scheme in space. The kernel renormalization is applied everywhere, 
apart from the pressure gradient term, where the form originally proposed by [33] is retained to guarantee momentum conservation.

The semi-discrete system (Equations (1) and (2)) is integrated in time by a 2nd-order two-stage $\mathrm{XSPH}$ explicit algorithm [38], where each particle is moved according to a velocity

$$
\boldsymbol{v}_{i}^{X}=\varphi \sum_{j} \frac{m_{j}}{\rho_{j}} \boldsymbol{v}_{j} \widehat{W}_{i j}+(1-\varphi) \boldsymbol{v}_{i}^{n+1}
$$

where $\varphi$ is a velocity smoothing coefficient and $v_{i}{ }^{n+1}$ is the value obtained by solution of the second equation in (1).

A pressure smoothing procedure is also applied to the difference between the local and the hydrostatic pressure values [26] and contributes to reduce the numerical noise that affects the WCSPH owing to high frequency acoustic waves [40]. This approach proved to be effective in SPH analyses of different free-surface flows [35] and constitutes a valid alternative to other methods, such as $\delta$-SPH [40], where a numerical diffusive term for density is added to the continuity equation, or filtering of the high-frequency pressure oscillations is used [41,42].

Finally, wall boundary conditions are imposed through the ghost particle method [43], i.e., by mirroring the positions of the inner SPH particles beyond the wall and by assigning to the latter the proper velocities and pressure conditions to obtain a Dirichlet condition for the velocity and a Neumann condition for the pressure.

\section{Numerical Tests and Calibration of SPH Parameters}

In this section the SPH model is employed to simulate the breaking wave on a sloping plane. See Table 1 for the experimental data.

The numerical domain was $22.5 \mathrm{~m}$ long and $1 \mathrm{~m}$ high, shorter than the laboratory channel. Such shorter domain was chosen to reduce the computational cost without influencing the quality of the numerical solution, as shown by $[25,26]$.

The offshore boundary condition has been modelled by a numerical wave paddle. i.e., by a moving-wall boundary condition imposed by ghost particles, whose motion has been forced to obtain the frequency and amplitude of the wave paddle needed to generate the desired sinusoidal wave. Section 0 is located at $x=14.6 \mathrm{~m}$ from the numerical wave paddle (thus the paddle is the origin of the $x$ reference system, $x=0$ ). The initial water depth is set equal to $0.80 \mathrm{~m}$.

The choice of the initial particle spacing $\Sigma$ depends on the physical process of the problem and on the desired computational accuracy and efficiency. The accuracy of particle methods is influenced by the initial particle spacing $\Sigma$, as well as by the smoothing length $\eta$. Both parameters must be taken into account when attempting to improve the resolution of the numerical simulation. It has been shown that the efficiency of the SPH kernel function depends also on the choice of the $\eta / \Sigma$ ratio [44] and that a value $\eta / \Sigma \geq 1.2$ should be preferred [45]. Maintaining the value of $\eta / \Sigma=1.5$ constant, the effect of particle resolution on the quality of the numerical results has been investigated and a convergence analysis carried out (Figure 4). Simulations have been performed by choosing a coarse and a fine initial particle spacing $(\Sigma)$; in particular, the 2D flow has been simulated by discretizing the computational domain through a value of particle spacing $\Sigma$ varying from to 0.08 to $0.02 \mathrm{~m}$.

The related number of SPH particles in the computational domain ranges from about 1500 to 24,000 , respectively. The simulations have been performed with a velocity smoothing coefficient in the XSPH scheme $\varphi$ equal to 0.02 (Equation (3)). Figure 4 shows the comparison between the numerical crest heights and the laboratory measurements at the chosen locations (Figure 2), by using the three different spatial resolutions reported above. The large spatial dimension of the SPH particles can also help explain the differences between the experimental data and the SPH results. In particular, Figure 4 shows the smoothing effect of a too low particle resolution $(\Sigma=0.08 \mathrm{~m}, \mathrm{~N}=1500)$ on the description of the breaking wave. The simulation with the lowest resolution cannot adequately predict 
the experimental results. Figure 4 also shows that a value of particle spacing $\Sigma$ equal to $0.02 \mathrm{~m}(\mathrm{~N}=$ $24,000)$ leads to a significant improvement of the simulated results in term of wave heights. Therefore, the reference SPH simulation was performed with particle spacing $\Sigma$ equal to $0.02 \mathrm{~m}$.

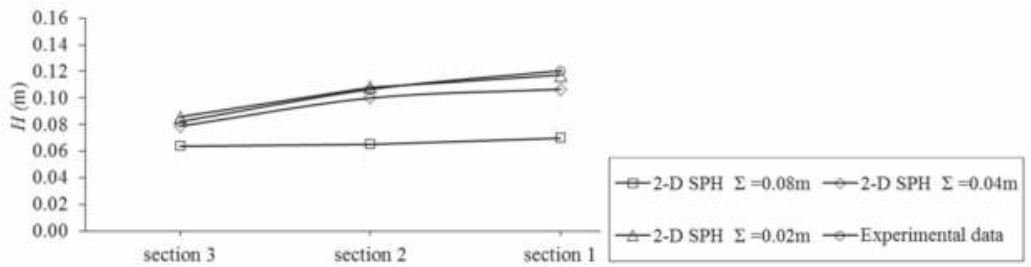

Figure 4. Effect of the initial particle resolution $\Sigma$ on the numerical simulation.

The effect of the velocity smoothing parameter has also been investigated. Simulations with an initial particle spacing $\Sigma=0.02 \mathrm{~m}$ have been run with two different $\varphi$ values, equal to 0.01 and 0.02 , respectively. While the computed values of crest height appear to be almost independent from $\varphi$, the computed horizontal $(u)$ and vertical $(v)$ velocity components at the investigated sections (Figure 2) are underestimated when increasing the smoothing coefficient. With a very low value of the velocity smoothing parameter $(\phi<0.01)$, the SPH method is unstable and one manifestation is that each of the particles begins to move chaotically for an excessive velocity. As an example, in Figure 5 both laboratory and numerical velocities at vertical Sections 2 and 3 are plotted, referring to the point located at $1 \mathrm{~cm}$ from the free surface. Therefore, the value $\varphi=0.01$, which guarantees the stability of the SPH solutions without affecting the quality of the numerical results, has been chosen for the following runs.
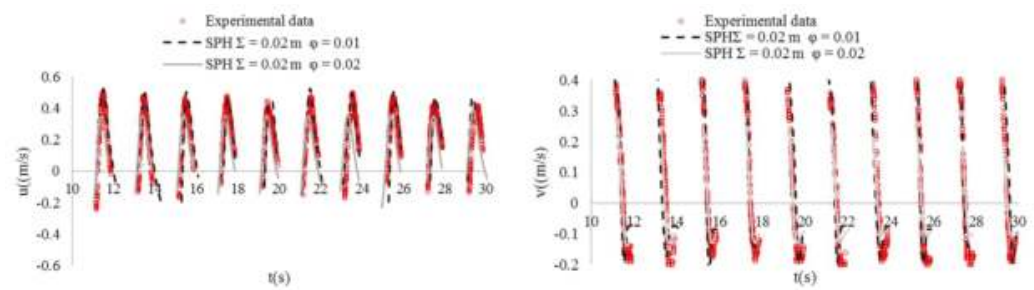

(a)
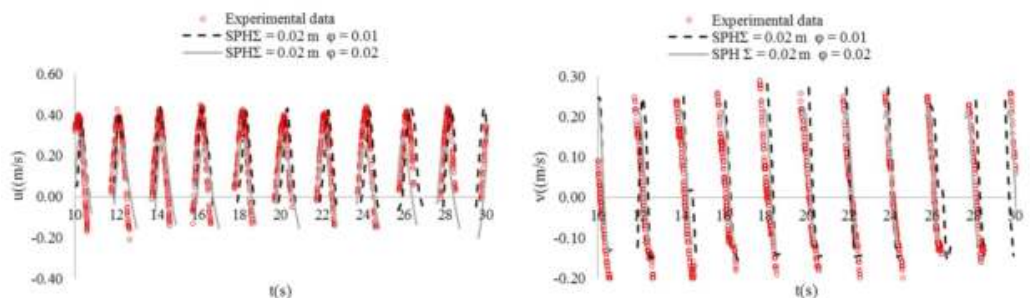

(b)

Figure 5. Effect of the velocity smoothing parameter $\phi_{\mathrm{V}}$ on the numerical simulations: computed and measured horizontal and vertical velocities in (a) Section 2 and (b) Section 3.

After this sensitivity analysis, the time series of the horizontal and vertical velocity components have been compared at Section 0 for all the investigated vertical locations. The agreement between 
the calibrated numerical results and the laboratory measurements is fairly good: Figure 6 illustrates a representative comparison, referring to both a superficial (Figure 6a) and a near-bed point (Figure 6b). Near the channel bed local effects (i.e., bed not perfectly smooth) could affect the vertical velocity component, this leading to a relatively poorer agreement (Figure 6b).
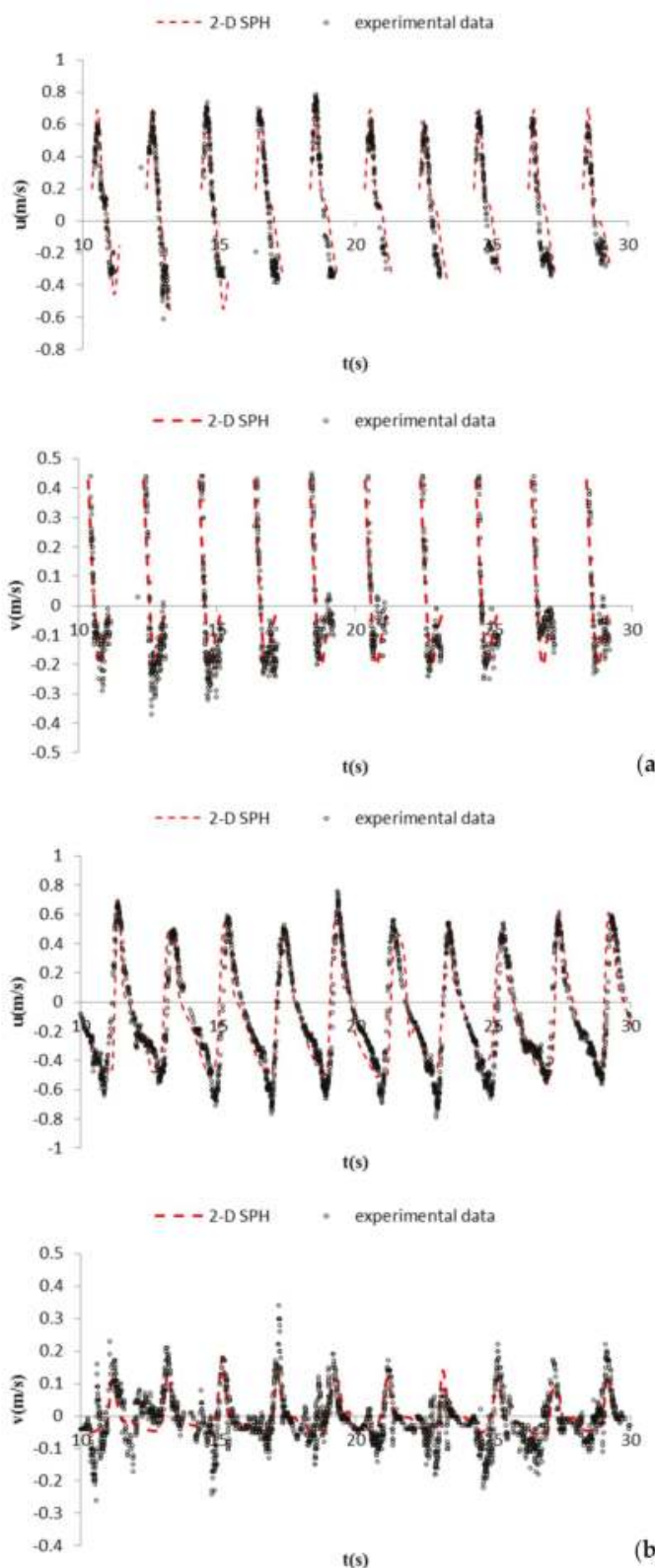

Figure 6. Time series of the horizontal and vertical velocity components at Section 0 , (a) near the surface, at $1 \mathrm{~cm}$ from the SWL and (b) near the seabed, at $10 \mathrm{c} \mathrm{m}$ from the SWL. 
A global assessment of the model performances has been made by using the Wilmott index [46]:

$$
I_{w}=1-\frac{\sum_{k=1}^{N}\left(X_{C_{k}}-X_{m_{k}}\right)^{2}}{\sum_{k=1}^{N}\left[\left|X_{C_{k}}-\overline{X_{m}}\right|+\left|X_{m_{k}}-\overline{X_{m}}\right|\right]^{2}}
$$

a statistical parameter in which $X c$ and $X m$ are the modelled and measured values, respectively, while the bar denotes the average of the modelled and measured values. $I_{W}$ takes a value of 1 , when a perfect agreement exists between the measured and modelled values, while a value of $I_{W}$ close to 0 denotes a complete discrepancy between numerical and experimental results. For the present run, all points placed along the vertical of Section 0 have been considered (sum for $k$ between 1 and $N$ ), providing values of $I_{W}$ equal to 0.95 and 0.90 for the horizontal and vertical velocity components, respectively.

\section{Investigation on the Vorticity Generation}

The characteristics of the vorticity characterizing the pre-breaking and breaking stages of the plunger have been studied both experimentally and numerically by means of the calibrated SPH numerical model. The vorticity is defined as

$$
\omega=\left(\frac{\partial U}{\partial z}\right)-\left(\frac{\partial V}{\partial x}\right)
$$

This has been computed using both the experimental data and the numerical output in the pre-breaking region of the plunging breaker, i.e., onshore Section 0 (Figure 2). $U$ and $V$ in Equation (5) are the time-averaged horizontal and vertical velocity components. Figure $7 \mathrm{a}, \mathrm{b}$ displays the time-averaged velocity vectors superposed to the map of mean vorticity, respectively for the experimental data and the numerical solution.

Experimental values of vorticity have been evaluated for all the measured points (about 220 points). The averaging time for the experimental data is the whole acquisition period, i.e., about $80 \mathrm{~s}$, and for the numerical data is $60 \mathrm{~s}$. For a comparison in terms of time-averaging, this number of waves (i.e., $\sim 40$ and 30 for the experimental and numerical case, respectively) has been proved sufficient, a steady condition being reached. A very good match is observed between the experimental data and the numerical solution, both for the velocity profiles and for the vorticity field. The time-averaged velocities highlight the undertow structure (middle and lower part of the water column), while in the region above the wave through the flow displays a significant seaward drift, associated to the wave crest that is evolving to give a plunging jet.

Both experimental and numerical data show that most of the vorticity is due to the pre-breaking, free surface dynamics, with a much weaker contribution from the seabed shear. Figure 7a,b displays large positive (clockwise) vorticity over most of the upper water column, while, some smaller, negative (counterclockwise) vorticity characterizes the lower part of the water column, seaward of the breaking point, which approximately occurs at about $30 \mathrm{~cm}$ shoreward of Section 0 (Figure 2).

The positive vorticity of the upper water column has a background value in the range [0-3 Hz], while local maxima reach even $7 \mathrm{~Hz}$. This is about the same order of magnitude of the wave phase speed divided by the local water depth, also found by [12] outside the aerated region. Negative background vorticity in the lower part of the water column is in the range [0-2 Hz] while local maxima are observed around $-4 \mathrm{~Hz}$. The cyclic pattern illustrated in Figure 7a,b (both upper and lower) is only due to the interpolation spacing used for plotting (equal to the spacing used in the computation of $\omega$ ).

In order to better characterize the vorticity field, the numerical study has been focused to the time evolution of the vorticity field. Three reaches have been examined along the channel, as shown in Figure 8, and the numerical results are illustrated for three different times, i.e., before, during and after the breaking event. Specifically, we identify the breaking event as the impact of the plunging jet with the free surface. This also means that the conditions referenced as "before breaking" and "after breaking" occur $T / 4$ before and $T / 10$ after splash-down, respectively, as identified by inspecting the 
instants preceding and following the impact of the jet on the surface. Therefore, the "after breaking" condition displays features due to the jet impact. The three analysed reaches partly overlap and move following the wave. In this numerical case, the vorticity is computed using instant values of the horizontal and vertical velocity in Equation (5). Vorticity contour lines-black giving positive and red giving negative vorticity, respectively-are superposed to the velocity vectors, giving size and direction of the velocity field (Figure $8 \mathrm{a}-\mathrm{c}$ ).
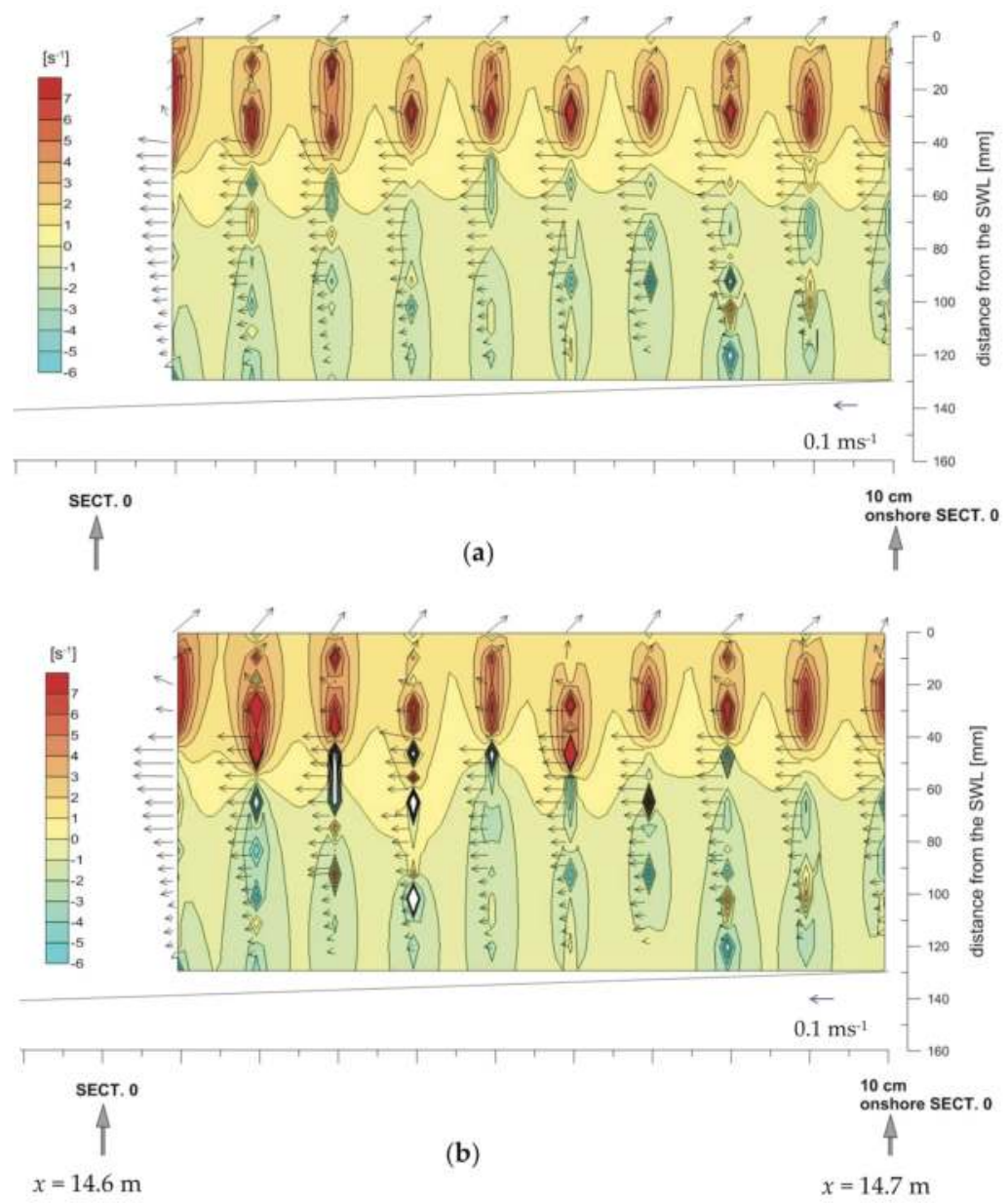

Figure 7. (a) Experimental and (b) numerical mean velocity vertical profiles superposed to the map of the mean vorticity. Values of the $x$ coordinates are given with reference to the numerical wave paddle (see Section 4).

Figure 8 a highlights that before breaking very low negative vorticity is locally present in all the three reaches, especially near the bottom in reach 3, and spreads towards the surface in reach 2 and 1. Since the previous wave has just moved along the same reaches, with a fast-moving crest and the opposing slower-moving water below the trough, these negative values still present in the channel should be regarded as the residual effect of the previous wave. During breaking (Figure 8b), due to the impact of the jet on the surface, flow separation occurs and positive vorticity is generated and 
propagates with the uprush flow, while negative vorticity forms in the downwash flow and slowly spreads in the return flow along the channel. After breaking (Figure 8c), positive vorticity persists near the surface in reaches 2 and 3, while negative vorticity rapidly decreases.
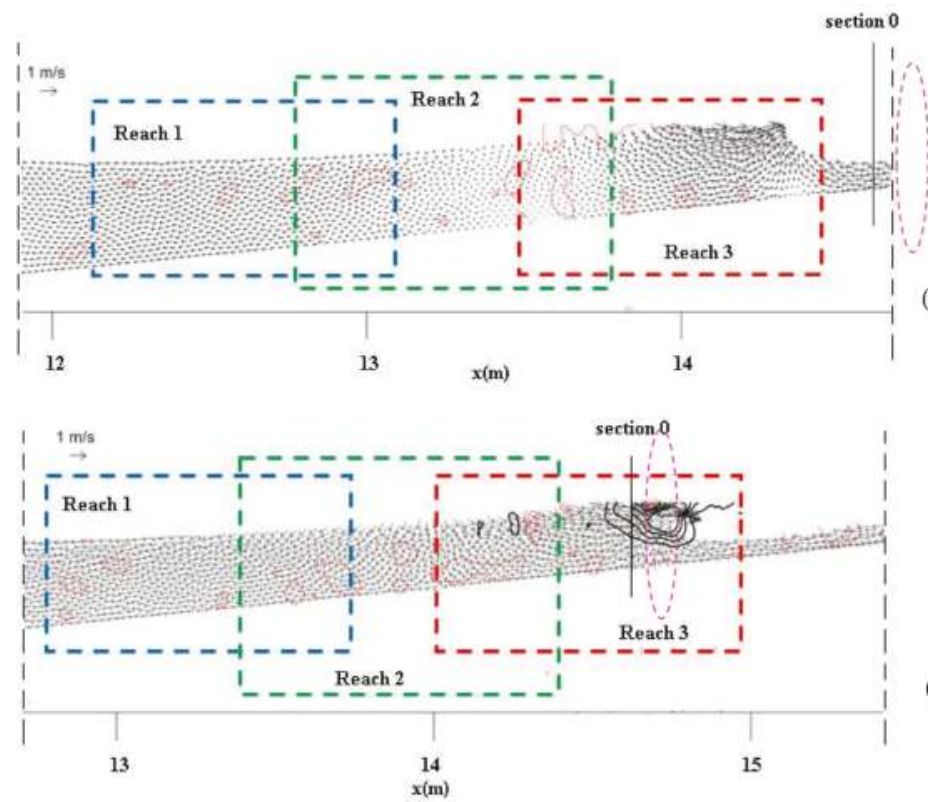

(b)

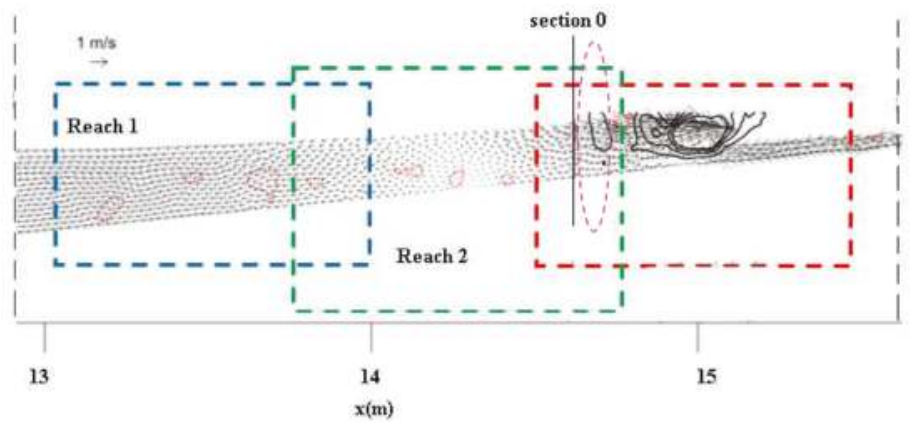

(c)

Figure 8. Sketch of the flow region with indication of the flow reaches where the vorticity field has been investigated in detail: (a) before; (b) during and (c) after breaking. The increment for each negative (red dashed lines) and positive (black solid lines) contour line is $1 \mathrm{~s}^{-1}$. The dashed oval shows the region where the sections for the experimental analysis of vorticity were located.

We analysed this vorticity generation in the light of flow separation at the free surface (see, as reference, the case of flow separation at a hydraulic jump illustrated by [47], i.e., in the light of fluid deceleration). Hence, we regard the analysis of fluid deceleration at a spilling breaker by [21] as a useful reference for our analysis. Dabiri and Gharib [21], verified that the vorticity was also convected due to the sharp velocity gradient of the fluid near the free surface with respect to the fluid below. To better explore this aspect, we also computed the surface parallel convective acceleration, $U_{s} \partial U_{s} / \partial s$ which also represents a fundamental contribution to the flux of near-surface vorticity as shown by Equation (8) of [21]. 
In fact, Dabiri and Gharib [21] demonstrate that the dominant terms in the vorticity flux of surface-parallel vorticity through the surface are the gravity and the deceleration terms.

Assuming that the gravity term remains constant, Dabiri and Gharib [21] theoretically derived that near-surface vorticity increases with increasing deceleration term.

Figure 9 show the comparison between the instantaneous map of vorticity and of the surface parallel convective acceleration, along reach 3 and for the upper half of the channel. The flow acceleration due to the convective term $U_{s} \partial U_{s} / \partial s$ has been computed taking as $\mathrm{s}$ a curvilinear coordinate running to the free-surface, so that it is accurate near the mean surface where the streamlines closely follow the surface. As shown by [47], the mean surface-parallel velocities were calculated as:

$$
U_{s}=U \cos \theta+V \sin \theta
$$

where $\theta$ is the angle that the tangent to the mean surface makes with the horizontal axis.

A flow deceleration occurs in the same location where peaks of positive vorticity appear. Referring to reach 3 during the breaking event, the vorticity reaches its maximum near the surface where the wave plunges (Figure 9a) and where also the surface-parallel convective deceleration, or vorticity flux (see [47]) strongly increases (Figure 9b). This vorticity does not decay rapidly away from the mean surface. Rather, the flow remains rotational along the whole depth.

For a direct, though qualitative, comparison with Figure 7, the marked box in Figure 9a delimits the corresponding experimental and numerical areas.

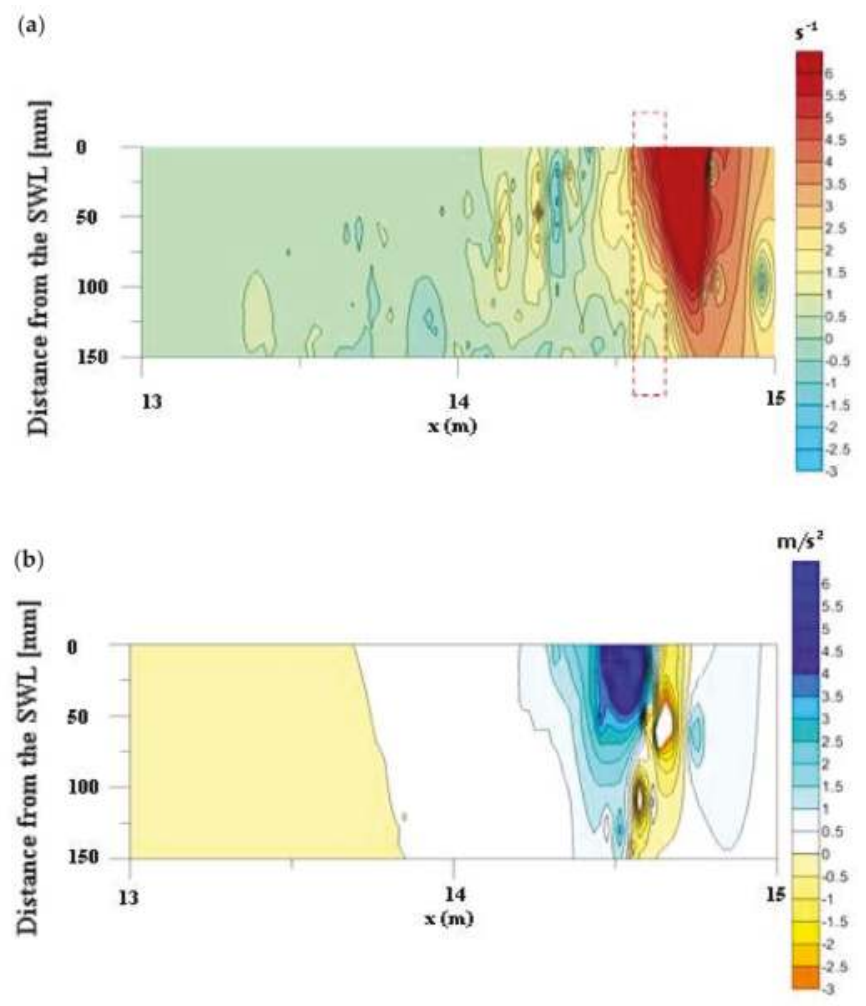

Figure 9. Reach 3, at breaking: (a) vorticity map, with the red box area to be compared with Figure 7a,b, (b) surface-parallel convective acceleration map. 
In more detail, Figure 10 shows both the numerically-computed, surface-parallel deceleration term and the surface velocity $U_{s}$ along reach 3 , at the points nearest to the free surface, respectively before, during and after breaking. Trends observed in the spatial maps of Figure 9 are confirmed.
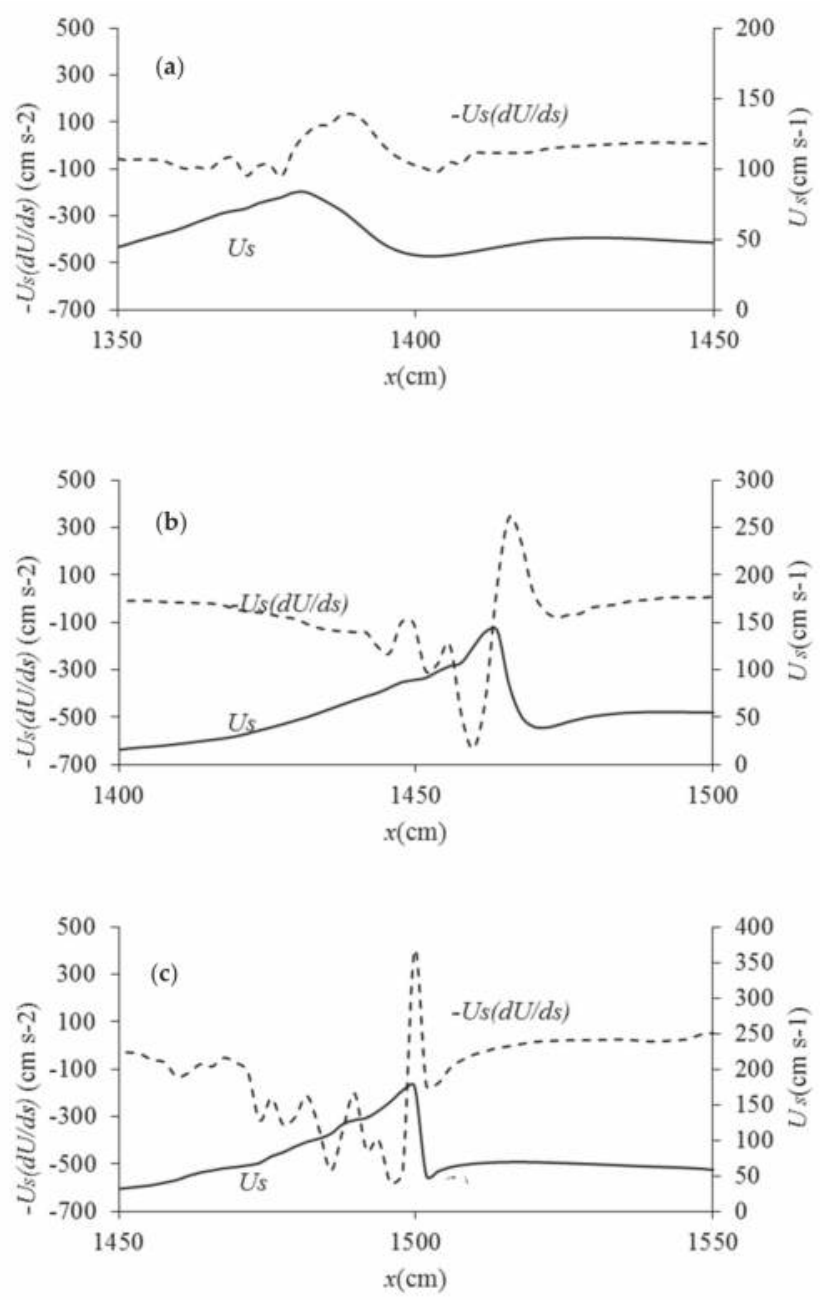

Figure 10. Free surface velocity and deceleration term (a) before, (b) during and (c) after breaking for reach 3 .

The deceleration term and the surface velocity along reach 3 before breaking (Figure 10a) do not display any evident peak, meaning that at this stage and location there is no significant flux of vorticity. This is consistent with the map of Figure 8a, where in reach 3 no new positive vorticity is generated. During breaking (Figure 10b), the deceleration term is characterized by a peak at $x=14.6 \mathrm{~m}$. It is preceded by a peak in the streamwise velocity $(x=14.57 \mathrm{~m})$, while the minimum streamwise velocity is downstream, at $x=14.7 \mathrm{~m}$. In other words, at the location of the maximum flow deceleration a large amount of vorticity is injected. Breaking is detected around $x=14.9 \mathrm{~m}$. In Figure 10c, the peaks of the 
streamwise velocity and of the deceleration term are very close, being the first at $x=14.98 \mathrm{~m}$ and the second at $x=15 \mathrm{~m}$. The minimum value of $U_{s}$ is at $x=15.07 \mathrm{~m}$.

The jet has moved onshore reaching its extreme evolution, with maximum velocity and vorticity, then rapidly impacts the free surface, inducing a flow reversal in the underlying water column. Again, the largest deceleration coincides with the peak of vorticity flux, suggesting that this behaviour is robust.

Both during and after breaking the streamwise velocity sharply decreases downstream the location where the deceleration peaks are observed. Nevertheless, contrarily to what observed by [21] for a spilling breaker, our findings do not show stagnation points for the streamwise velocity. The positive vorticity already observed in Figure $8 b, c$ is located beneath the surface and concentrated at the same coordinates of maximum deceleration.

Similarly, to the case of a spilling breaker [21] and hydraulic jump [47], a cause-effect relation between near-surface flow deceleration and vorticity flux is thus experimentally proved also for the plunging case, this being a novel result with respect to previous studies. Once clarified this analogy with the spilling case, we also note some differences.

In particular, Dabiri and Gharib [21] observed an important surface-parallel flow deceleration at the toe of the spiller but excluded that the sharp curvature of the free surface could be the main cause of wave breaking, while Misra et al. [47] correlated vorticity generation with sharp free surface curvature changes and surface-parallel adverse pressure gradients. For the present plunging breaker, surface-parallel convective deceleration is observed at the wave crest bulge, with maximum immediately downstream of the sharp crest velocity reduction. Therefore, our findings, with some similarity to [47], lead us to think that a strong free surface deformation associated with near-surface flow deceleration induces an important generation of vorticity in the near surface flow, and leads to the plunger splash-down.

The deceleration term and the surface velocity were examined also for the experimental and numerical data, referring to time-averaged quantities (Figure 11) acquired onshore of Section 0, to confirm the interpretation of the mean flow vortical dynamics shown above. In a time-averaged sense, Figure 11a,b shows a peak of the deceleration term located $3 \mathrm{~cm}$ onshore of Section 0 . Even this observation is consistent with the higher positive near-surface vorticity assessed and plotted in Figure $7 \mathrm{a}, \mathrm{b}$, thus confirming what numerically obtained.

These results can be of interest for different stakeholders because vorticity generation and spreading produce effects on the: (i) evolution of bed topography, as a result of a complex interaction between flow and sediment particles along the bed, being wave-breaking turbulence responsible for most of the sediment suspension through breaking injected eddies; (ii) air-water mixing, this being fundamental for ocean-atmosphere exchanges; (iii) mixing and transport of solutes, these being fundamental for water quality management purposes. Finally, information on the link between vorticity generations and turbulence spreading is also useful for numerical modeling, enabling improved calculations of water mixing over the entire water column and possibly limiting the computational efforts.

\section{Conclusions}

Vorticity generation in the pre-breaking and breaking zone of a plunger was examined in this study. Experimental measurements of fluid velocities, carried out in the wave channel of the LIC laboratory of the Polytechnic University of Bari, highlighted the presence of positive vorticity beneath the surface of the breaking wave. On the contrary negative vorticity was localized near the flume bed. This observation was the result of a time-average operation on velocity data assessed by single point measurement techniques, i.e., LDA and ADV.

To thoroughly investigate the vorticity field under the breaker and its temporal evolution, a numerical model was used. Specifically, a Weakly-Compressible SPH (WCSPH) scheme, which includes a two-equation $k-\varepsilon$ turbulence model, was applied. Preliminarily, the SPH model was validated 
by means of both elevation and velocity laboratory data, providing a successful agreement between numerical results and measurements.
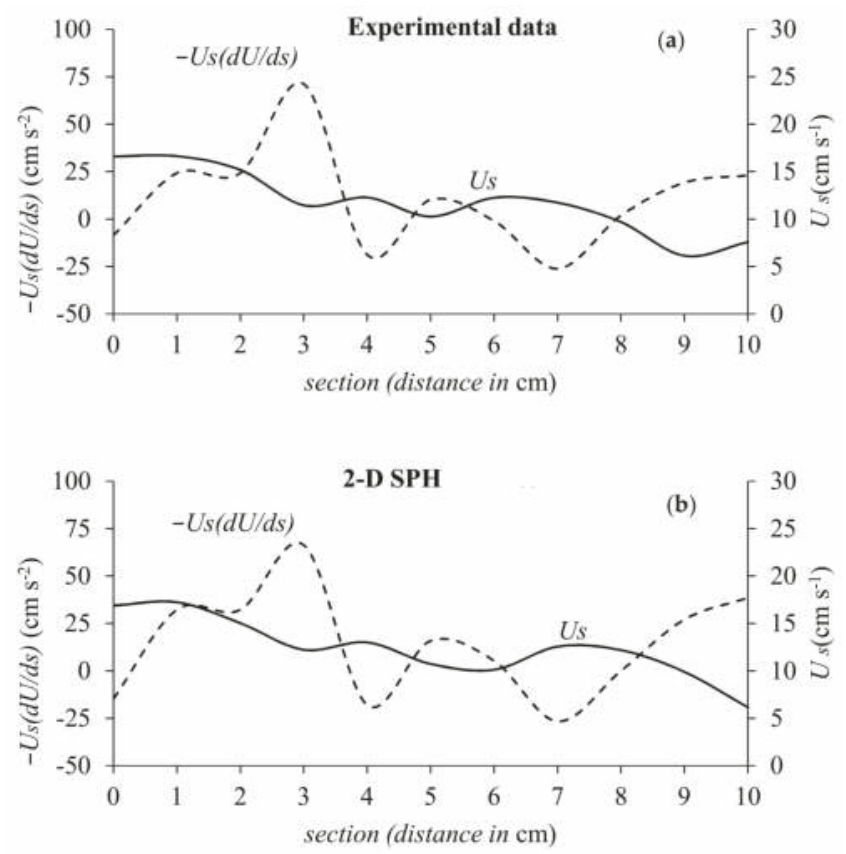

Figure 11. Free-surface velocity and deceleration term for (a) experimental and (b) numerical data.

Then, the validated SPH model run to detail the vorticity field under the plunging breaker and to shed some light on the vorticity source. Three frames along the channel were examined, during three different instant times, i.e., before, during and after breaking. Numerical vorticity maps clearly show that new positive vorticity is generated beneath the free surface during and after breaking. The comparison between time-averaged experimental and numerical vorticity provided an excellent overall similarity.

Successively, starting from the instantaneous numerical streamwise velocity, the surface-parallel convective acceleration was computed, considering that it is a fundamental contribution to the flux of near-surface vorticity [21,47]. Its analysis shows that a flow deceleration occurs in the same locations where peaks of positive vorticity appear. This result is furtherly confirmed by the investigation of the surface-parallel deceleration in the points nearest to the free surface, respectively before, during and after breaking. Also, in this case, both during and after breaking, the largest deceleration coincides with the peak of vorticity flux, suggesting that this behaviour is robust. The novelty of our study is the detection for the plunging breaker of a cause-effect relation between near-surface flow deceleration and vorticity flux, similarly to the case of a spilling breaker [21] and a hydraulic jump [47].

However, differently from [21], our findings do not show stagnation points for the streamwise velocity. Moreover, in the present plunging breaker, the surface-parallel convective deceleration is observed at the wave crest bulge. With some similarity with the deductions by [47], this specific result lead us to think that both strong free surface deformation and near-surface flow deceleration induce an important generation of vorticity in the near surface flow, and lead to the plunger splash-down.

Analysis is underway to quantitative detail the relation between near-surface flow deceleration and vorticity injection into a plunger, with reference to what typically observed in spillers. 
Acknowledgments: The experiments described in the present study have been carried out in the frame of the GII (Hydraulic Italian Group) Placement in Water Engineering of the year 2015. The authors acknowledge the LIC technicians' contribution.

Author Contributions: S.S. developed the SPH numerical code; D.D.P. and S.S. used the numerical code and analyzed the numerical results; F.D.S. and M.M. conceived the experimental set up; F.B. and F.D.S. carried out the experiments; all the authors contributed analysis tool, wrote, discussed and reviewed the manuscript.

Conflicts of Interest: The authors declare no conflict of interest.

\section{References}

1. Peregrine, D.H. Breaking waves on beaches. Annu. Rev. Fluid Mech. 1983, 15, 149-178. [CrossRef]

2. Basco, R.D. A qualitative description of wave breaking. J. Waterw. Port Coast. Ocean Eng. ASCE 1985, 111, 171-188. [CrossRef]

3. Battjes, J.A. Surf-zone dynamics. Annu. Rev. Fluid Mech. 1988, 20, 257-293. [CrossRef]

4. Stive, M.J.F. Velocity and pressure field of spilling breakers. In Proceedings of the 17th International Coastal Engineering Conference, Sydney, Australia, 23-28 March 1980; pp. 547-566.

5. Hattori, M.; Aono, T. Experimental study on turbulence structures under breaking waves. Coast. Eng. Jpn. 1985, 28, 97-116.

6. Ting, F.C.K.; King, J.T. Dynamics of surf-zone turbulence in a strong plunging breaker. Coast. Eng. 1995, 24, 177-204. [CrossRef]

7. Longo, S. Turbulence under spilling breakers using discrete wavelets. Exp. Fluids 2003, 43, 181-191. [CrossRef]

8. Stansby, P.k.; Feng, T. Kinematics and depth-integrated terms in surf zone waves from laboratory measurement. J. Fluid Mech. 2005, 529, 279-310. [CrossRef]

9. De Serio, F.; Mossa, M. Experimental study on the hydrodynamics of regular breaking waves. Coast. Eng. 2006, 53, 99-113. [CrossRef]

10. De Serio, F.; Mossa, M. A laboratory study of irregular shoaling waves. Exp. Fluids. 2013, 54, 15-36. [CrossRef]

11. Petti, M.; Quinn, P.A.; Liberatore, G.; Easson, W.J. Wave velocity field measurements over a submerged breakwater. Coast. Eng. 1995, 1, 525-539.

12. Chang, K.A.; Liu, P.L.F. Velocity, acceleration and vorticity under a breaking wave. Phys. Fluids 1998, 10, 327-329. [CrossRef]

13. Nadaoka, K.; Hino, M.; Koyano, Y. Structure of the turbulent flow field under breaking waves in the surf zone. J. Fluid Mech. 1989, 204, 359-387. [CrossRef]

14. Kimmoun, O.; Branger, H. A particle image velocimetry investigation on laboratory surf-zone breaking waves over a sloping beach. J. Fluid Mech. 2007, 588, 353-397. [CrossRef]

15. Chiapponi, M.; Cobos, M.A.; Losada, M.A.; Longo, S. Cross-shore variability and vorticity dynamics during wave breaking on a fixed bar. Coast. Eng. 2017, 127, 119-133. [CrossRef]

16. Duncan, J.H. Spilling breakers. Annu. Rev. Fluid Mech. 2001, 33, 540-550. [CrossRef]

17. Qiao, H.; Duncan, J.H. Gentle spilling breakers: Crest flow-field evolution. J. Fluid Mech. 2001, 439, 57-85. [CrossRef]

18. Longo, S. Vorticity and intermittency within the pre-breaking region of spilling breakers. Coast. Eng. 2009, 56, 285-296. [CrossRef]

19. Longuet-Higgins, M.S. Capillary rollers and bores. J. Fluid Mech. 1992, 240, 659-679. [CrossRef]

20. Lin, J.C.; Rockwell, D. Instantaneous structure of a breaking wave. Phys. Fluids. 1994, 6, 2877-2879. [CrossRef]

21. Dabiri, D.; Gharib, M. Experimental investigation of the vorticity generation within a spilling water wave. J. Fluid Mech. 1997, 330, 113-139. [CrossRef]

22. Lin, P.; Liu, P.L.F. Turbulence transport, vorticity dynamics, and solute mixing under plunging breaking waves in surf zone. J. Geophys. Res. Oceans 1998, 103, 15677-15694. [CrossRef]

23. Christensen, E.D.; Deigaard, R. Large eddy simulation of breaking waves. Coast. Eng. 2001, 42, 53-86. [CrossRef]

24. Dalrymple, R.A.; Rogers, B.D. Numerical modelling of waves with the SPH method. Coast. Eng. 2006, 53, 141-147. [CrossRef]

25. De Padova, D.; Dalrymple, R.A.; Mossa, M. Analysis of the artificial viscosity in the smoothed particle hydrodynamics modelling of regular waves. J. Hydraul. Res. 2014, 52, 836-848. [CrossRef] 
26. De Padova, D.; Mossa, M.; Sibilla, S.; Torti, E. 3D SPH modelling of hydraulic jump in a very large channel. J. Hydraul. Res. 2013, 51, 158-173. [CrossRef]

27. De Padova, D.; Mossa, M.; Sibilla, S. SPH modelling of hydraulic jump oscillations at an abrupt drop. Water 2017, 9, 790. [CrossRef]

28. De Padova, D.; Mossa, M.; Sibilla, S. SPH numerical investigation of characteristics of hydraulic jumps. Environ. Fluid Mech. 2017. [CrossRef]

29. De Padova, D.; Mossa, M.; Sibilla, S. SPH numerical investigation of the characteristics of an oscillating hydraulic jump at an abrupt drop. J. Hydrodyn. 2018, 30, 106-113. [CrossRef]

30. Capone, T.; Panizzo, A.; Monaghan, J.J. SPH modelling of water waves generated by submarine landslides. J. Hydraul. Res. 2010, 48, 80-84. [CrossRef]

31. Espa, P.; Sibilla, S.; Gallati, M. SPH simulations of a vertical 2-D liquid jet introduced from the bottom of a free-surface rectangular tank. Adv. Appl. Fluid Mech. 2008, 3, 105-140.

32. Gomez-Gesteira, M.; Rogers, B.D.; Darlymple, R.A.; Crespo, A.J.C. State-of-the-art of classical SPH for free-surface flows. J. Hydraul. Res. 2010, 48, 6-27. [CrossRef]

33. Monaghan, J.J. Smoothed particle hydrodynamics. Annu. Rev. Astron. Astrophys. 1992, 30, 543-574. [CrossRef]

34. Violeau, D. Fluid Mechanics and the SPH Method: Theory and Applications; Oxford University Press: Oxford, UK, 2012.

35. De Padova, D.; Mossa, M.; Sibilla, S. SPH numerical investigation of the velocity field and vorticity generation within a hydrofoil-induced spilling breaker. Environ. Fluid Mech. 2016, 16, 267-287. [CrossRef]

36. Wendland, H. Piecewise polynomial, positive definite and compactly supported radial functions of minimal degree. Adv. Comput. Math. 1995, 4, 389-396. [CrossRef]

37. Launder, B.E.; Spalding, D.B. The numerical computation of turbulent flows. Comput. Methods Appl. Mech. Eng. 1974, 3, 269-289. [CrossRef]

38. Violeau, D.; Issa, R. Numerical modelling of complex turbulent free-surface flows with the SPH method: An overview. Int. J. Numer. Methods Fluids 2007, 53, 277-304. [CrossRef]

39. Sibilla, S. An algorithm to improve consistency in smoothed particle hydrodynamics. Comput. Fluids. 2015, 118, 148-158. [CrossRef]

40. Antuono, M.; Colagrossi, A.; Marrone, S. Numerical diffusive terms in weakly-compressible SPH schemes. Comput. Phys. Commun. 2012, 183, 2570-2580. [CrossRef]

41. Meringolo, D.D.; Colagrossi, A.; Marrone, S.; Aristodemo, F. On the filtering of acoustic components in weakly-compressible SPH simulations. J Fluids Struct. 2017, 70, 1-23. [CrossRef]

42. Aristodemo, F.; Tripepi, G.; Meringolo, D.D.; Veltri, P. Solitary wave-induced forces on horizontal circular cylinders: Laboratory experiments and SPH simulations. Coast. Eng. 2017, 129, 17-35. [CrossRef]

43. Randles, P.W.; Libersky, L.D. Smoothed particle hydrodynamics: some recent improvements and applications. Comput. Methods Appl. Mech. Eng. 1996, 139, 375-408. [CrossRef]

44. Dehnen, W.; Aly, H. Improving convergence in smoothed particle hydrodynamics simulations without pairing instability. Mon. Not. R. Astron. Soc. 2012, 425, 1068-1082. [CrossRef]

45. De Padova, D.; Dalrymple, R.A.; Mossa, M.; Petrillo, A.F. An analysis of SPH smoothing function modelling a regular breaking wave. In Proceedings of the International Conference XXXI Convegno Nazionale di Idraulica e Costruzioni Idrauliche, Perugia, Italy, 9-12 September 2008; p. 182.

46. Willmott, C.J. On the validation of models. Phys. Geogr. 1981, 2, 184-194.

47. Misra, S.K.; Kirby, J.T.; Brocchini, M.; Veron, F.; Thomas, M.; Kambhamettu, C. The mean and turbulent flow structure of a weak hydraulic jump. Phys. Fluids 2008, 20, 035106. [CrossRef]

(C) 2018 by the authors. Licensee MDPI, Basel, Switzerland. This article is an open access article distributed under the terms and conditions of the Creative Commons Attribution (CC BY) license (http:/ / creativecommons.org/licenses/by/4.0/). 


\title{
Advanced 3D Mapping of Hydrodynamic Parameters for the Analysis of Complex Flow Motions in a Submerged Bedrock Canyon of the Tocantins River, Brazil
}

\author{
Gustavo Tomas ${ }^{1, *}$, Tobias Bleninger ${ }^{1}$, Colin D. Rennie ${ }^{2}$ and Henrique Guarneri ${ }^{1}$ \\ 1 Federal University of Paraná (UFPR), Post-Graduate Program on Water Resources and Environmental \\ Engineering (PPGERHA), Caixa Postal 19011, Curitiba 81531-990, Brazil; tobias.bleninger@gmail.com (T.B.); \\ henriqueguarneri@gmail.com (H.G.) \\ 2 Department of Civil Engineering, University of Ottawa, 161 Louis Pasteur St., Ottawa, ON K1N 6N5, \\ Canada; Colin.Rennie@uottawa.ca \\ * Correspondence: gustavopachecotomas@gmail.com
}

Received: 1 February 2018; Accepted: 20 March 2018; Published: 23 March 2018

\begin{abstract}
Validation and scaling of sophisticated physical and numerical fluvial hydraulic models to real field conditions are limited by temporal and spatial constraints of field measurement technologies. These limitations increase when analyzing hydraulic properties of complex river forms such as submerged bedrock canyons. The analysis of flow under these conditions has demonstrated non-linear behavior, strong secondary circulation and a high level of turbulence. The objective of this paper is to obtain and analyze turbulence and secondary current information for flow analysis, by evaluating the spatial distribution of bed shear stress and eddy viscosity with acoustic Doppler current profiler (ADCP) measurements. The real field-scale case study was the Lourenço Rock Canyon in Tocantins River, Brazil. A total of 10,531 velocity profiles were measured with an ADCP adapted to a moving boat. The data were post-processed by decomposing the velocity data measurements and calculating the standard deviations. Three different methods are compared for bed shear stress: total kinetic energy (TKE), law of the wall and the depth-slope product. The eddy viscosity was estimated with the Boussinesq approach. The initial data processing confirmed turbulence and flow velocity characteristics described in similar literature findings. The results for the bed shear stress for the canyon area were 8 times greater than the outside and for eddy viscosity, 10 times higher. For the surroundings area, the eddy viscosity showed reasonable results with values around the regular $1.0 \mathrm{~m}^{2} / \mathrm{s}$ used for rivers. It is concluded that turbulent values can be calculated to better represent physical processes with the intention to improve hydrodynamic numerical models calibrations.
\end{abstract}

Keywords: bedrock canyon; ADCP; eddy viscosity; bed shear stress; spatial analysis

\section{Introduction}

The understanding of complex flow features, as a consequence of complex geometries or hydrodynamic boundary conditions, is very important for the analysis of transport of substances or sediment in rivers and coastal waters, as well as for navigability analysis in waterway projects. The study of those features generally involves sophisticated physical and numerical modeling approaches [1]. Unfortunately, validation with and scaling to real field conditions are still challenging, due to temporal or spatial constraints of field measurement technologies. Whereas lab or numerical data are available in high frequency and high spatial resolution (e.g., particle image velocimetry (PIV) and laser Doppler anemometry (LDA) [2], field data are usually measured at fixed points or along transects, and in the past were limited to mean velocity measurements (no turbulence quantities). 
Also, regarding extraordinary geometries, such as deep and narrow bedrock canyons, the conventional modeling laws are untested and are most probably not applicable.

Nowadays, acoustic technologies are more accessible and improved, presenting considerable advances in the quality of the field measurements. Compared to intrusive and punctual devices (current meter), acoustic instruments, such as the acoustic Doppler current profiler (ADCP), have been used in conventional, hydrological river measurements (discharge, depth, velocity), yielding increased accuracy and greater spatial and temporal resolution due to hardware improvements providing better accuracy for positioning, but also having several frequencies combined in the same devices, as well as being robust equipment for field deployment, especially compared to optical (e.g., LSPIV) techniques [3]. Recently, higher spatial resolutions were obtained by mapping individual ADCP velocity profiles over large flow areas, as presented by Flener et al. [4] or Jamieson et al. [5], where the latter included measured sections with 5 and $20 \mathrm{~m}$ spacing upstream and downstream of two different wing dikes for three different discharges.

Using the Doppler effect of acoustic waves scattered back from particles within the water column, $\mathrm{ADCP}$ is proven to measure velocity, depth, and water discharge accurately with high resolution $(\mathrm{cm})[6]$. Besides the depth and the mean velocity components, additional data inherent to the acoustic measuring method can be analyzed, such as the backscatter intensity and velocity standard deviation. For example, ADCP data have been used to derive other flow characteristics, such as mixing coefficients [7-9], sediment transport rates [5], and moving bed velocity [10]. The flow velocity information has also been used for profile analysis and mapping of integrated or characteristic quantities, such as bed shear stress and depth-averaged velocity [6,11]. Moreover, acoustic backscatter signal and its statistics can be used for a suspended sediment transport analysis [12]. The velocity standard deviation information (velocity's second-moment statistics), can be explored to obtain turbulence quantities [13,14], which will be the focus of this article. This parameter is especially of interest when analyzing and modeling complex flow situations, where strong and large secondary currents exist (3D effects), creating non-conventional turbulence distributions (non-logarithm velocity profiles), thus limiting resistance law-based approaches or hydrostatic modeling concepts.

Bedrock Rivers can present morphology variations of width and depth over small distances caused by climatic variations, abrasion, cavitation or tectonic movements. Relatively narrow and deep canyons emerge in locations of active bedrock incision, sometimes in association with tectonic fault lines [15]. Narrow canyons have flow characteristics (hydraulic radius, velocities, and turbulent effects) vastly different from less confined reaches, with the presence of vertical accelerations and secondary currents that influence the shape of the velocity profiles. Analyzing the flow in canyons of the Fraser River (Canada), Venditti et al. [16] described the flow as nonlinear and with strong secondary circulation and high levels of turbulence. At the entrance to canyon pools, they observed a plunging core of high velocity flow, which suppressed the maximum velocity below the free surface.

The objective of this paper is to obtain turbulence and secondary current information for flow analysis and model calibration in complex flow environments in a peculiar and morphologically unique submerged bedrock canyon of the Tocantins River in Brazil, a tributary of the Amazon estuary. As the canyon reach is considered an obstruction point for navigation within the important Tocantins waterway, recent studies were undertaken to evaluate and plan river engineering projects for Brazil's National Transport Infrastructure Department (DNIT).

The spatial distributions of bed shear stress $\left(\tau_{0}\right)$ and eddy viscosity $\left(v_{t}\right)$ were assessed in this river reach, based on extensive ADCP measurements collected from a moving boat to better understand the river dynamics in this submerged bedrock canyon. Using this real field-scale case study, three different approaches were used to calculate bed shear stress: the total kinetic energy (TKE) method, law of the wall method and the depth-slope product method. With the results of the TKE method, the eddy viscosity was estimated with the Boussinesq approach. 


\section{Materials and Methods}

\subsection{Bedrock Canyon of Tocantins River}

The study area is named Lourenço's Rock Canyon (Figure 1), a reach of Tocantins River, one of the largest and most important rivers in Brazil. Located in northern Brazil, the Tocantins River is the main river of the Tocantins-Araguaia Basin, the second largest hydrographic basin in the country with $967,059 \mathrm{~km}^{2}$, smaller only than the Amazon River Basin.

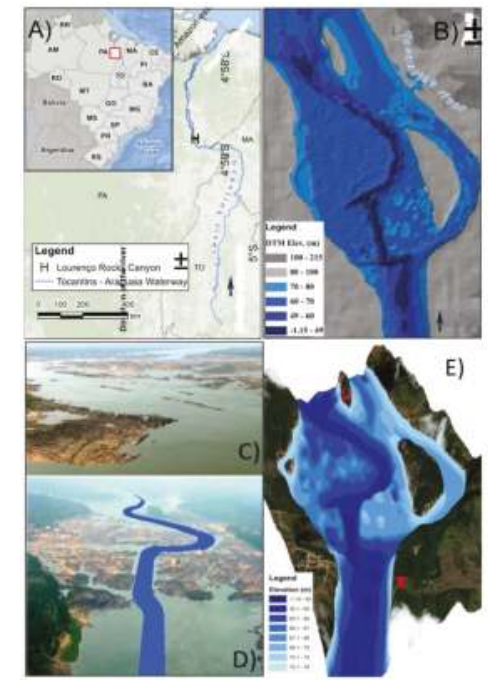

Figure 1. (A) Geographic location of the Tocantins River and the canyon location at Lourenço's Rock; (B) Bathymetrical map (above sea level); (C) Image looking downstream during low flow condition; (D) Image looking upstream during low flow conditions and indicating the navigation channel; (E) 3D bathymetrical visualization (vertical exaggeration $=15$ ) overlaid on a satellite image and $(\mathbf{C}, \mathbf{D})$ viewing directions.

The Tocantins-Araguaia waterway is one of the main transportation routes of Brazilian products for export, such as minerals and agricultural commodities. Nowadays, the Lourenço Rock Canyon is considered one of the most significant obstructions to navigation in the Tocantins River. Despite the high depth, the canyon path presents a sharp curve and the available width is not adequate for safe navigation during the drought season and for the commonly used vessels. Moreover, through the flood season, the canyon induces strong shear flows, making navigation more difficult and less safe. According to DNIT [17,18], the Brazilian Government is investing more than U\$150 million in engineering projects to improve the navigation channel in the study area.

The submerged bedrock canyon is $6 \mathrm{~km}$ long, and its depth can reach $60 \mathrm{~m}$ (upstream and downstream of the canyon reach the river has a depth of $7 \mathrm{~m}$ ). The annual mean flow in the reach is $11,800 \mathrm{~m}^{3} / \mathrm{s}$, with high seasonal variations ranging from 1898 to $45,717 \mathrm{~m}^{3} / \mathrm{s}$. During the flood season, the river width is around $1.5 \mathrm{~km}$, with the presence of a few rocky outcrops, high velocities and strong turbulent flow (large shear layers and longitudinal vortices). During the drought season, the flow is confined along the canyon area (150 $\mathrm{m}$ width), exposing the river bedrock at the reach. Although having smaller diameters, the presence of vortices is constant, even under low flow conditions.

According to the Brazilian Mineral Resources Research Company [19], the river reach is located between two tectonic domains, the Couto Magalhães Formation (part of the Tucuruí Group) and the Bacajá Domain (part of the Transamazonas Province). The river bed is composed of sandstone Rock 
from the Itapecuru (K12it), Pastos Bons (J2pb) and Grájau (K1g) formations on the undifferentiated fractured basement $(\mathrm{Fr})$. The canyon's origins can be associated with the Tucuruí Fault, caused by tectonic movements that generated a series of structural features in the lithologies and later migmatites, which also induced a dynamic metamorphic event in the rocks under green-schist facies conditions.

\subsection{Equipment and Field Data}

In April (flood season) and October (drought season) 2015, several flows and velocity measurements were performed in Lourenço's Rock Canyon using an acoustic Doppler current profiler (ADCP), model M9, series SN-3754 of Sontek Inc. (San Diego, CA, USA) [20]. The equipment was installed with fixed support on a moving boat, and the installation depth of the transducer was $0.47 \mathrm{~m}$ below the water surface. The equipment was coupled to a real-time kinematic (RTK) global positioning system providing sub-decimeter accuracy for the measurement coordinates.

As the boat traverses the sampling area, each vertical depth profile (ensemble) is subdivided into several cells (bins), and the velocity profile for each compass axis (easting, northing, and vertical) is calculated for each of the four inclined beams. An internal compass and tilt sensor provide compensation for boat movements. As only three beams are necessary to compute the three velocity components, additional information can be obtained when using ADCPs with four transducers (similar to the one used here), where the fourth beam provides a redundant measurement of vertical velocity, from which velocity heterogeneities and turbulence variations may be quantified. The ADCP can be operated in 3 different modes: NarrowBand, Pulse to Pulse and Broadband [21]. In NarrowBand mode, the transducers emit, in a short period (e.g., 1 s), several pulses (pings) and the water velocity is calculated based on statistical analysis of the received signals. As a result, for every ensemble profile measurement, a short time series is produced along each axis in each depth cell, with a mean velocity $(\langle\mathrm{u}\rangle,\langle\mathrm{v}\rangle,\langle\mathrm{w}\rangle)$ and a standard deviation $\left(\sigma_{\mathrm{x}}, \sigma_{\mathrm{y}}, \sigma_{\mathrm{z}}\right)$. Some ADCP brands supply all the velocity samples from the time series, allowing the user to calculate the statistics; however, the M9 only provides the results of mean velocity and standard deviation, which will be used here.

The ADCP M9 is a double frequency instrument, with four transducers ( $25^{\circ}$ from vertical) at both $1 \mathrm{MHz}$ and $3 \mathrm{MHz}$. The frequency and thus cell sizes are changed automatically on-the-fly depending on the local water depth, if not configured otherwise. Along the sections in the Lourenço's Rock Canyon, the shallow margins were usually measured with a frequency of $3 \mathrm{MHz}$, and the deeper canyon with $1 \mathrm{MHz}$, both in Narrowband mode. The vertical dimension of the measurement cells $\left(\Delta \mathrm{Z}_{\text {cel }}\right)$ varied between $0.1-0.5 \mathrm{~m}$ for the $3 \mathrm{MHz}$ and $0.5-2.0 \mathrm{~m}$ for the $1 \mathrm{MHz}$. The horizontal sampling dimension is determined as a function of the boat's movement in time, and the data acquisition frequency $(\mathrm{f}=1 \mathrm{~s})$. Also, there is a vertical beam of $0.5 \mathrm{MHz}$ in the middle of the instrument head to improve bed level measurements. The Sontek M9 maximum depth for velocity measurements is $40 \mathrm{~m}$, and some parts of the canyon were more than $50 \mathrm{~m}$ deep, thus resulting in larger blanking areas near the bottom. Nonetheless, the bed level was measured throughout the canyon using the vertical beam.

Although measurements are available for both flood and drought season, only the data of the flood situation have been processed so far regarding the methods presented in this manuscript. Thus, all following results are based on the flood situation only, being the more interesting one in terms of the observable features and processes.

The mean discharge during the April 2015 flood flow situation was $18,275 \mathrm{~m}^{3} / \mathrm{s}$. Several transversal sections and a longitudinal zig-zag type of measurement were undertaken along Lourenço's Rock Canyon during a short period (short enough to have discharge changes of less than $3 \%$ ). The measurement resulted in 10,531 velocity profiles (ADCP ensembles) at different points of the canyon, each having on average about 14 cells, thus resulting in approximately 140,000 3D velocity vectors. Figure 2 shows the location of the spatial measurements. Three Elevation Reference (ER) stations were installed and geo-referenced along the study area using the Global Navigation Satellite System (GNSS). The ADCP's compass was calibrated three times during the measurement. The water surface elevation measured at each point is presented in Figure 2, and the resulting mean water surface 
slope $\left(\mathrm{S}_{\mathrm{WS}}\right)$ was $2.36 \times 10^{-4} \mathrm{~m} / \mathrm{km}$. A detailed bathymetry measurement of the area was available from previous measurements in 2012, with both single and multi-beam echosounder equipment. A DTM (Digital Terrain Model) was created (Figure 1), combining the bathymetry data with SRTM (Shuttle Radar Topographic Mission) data to complete the margin information.

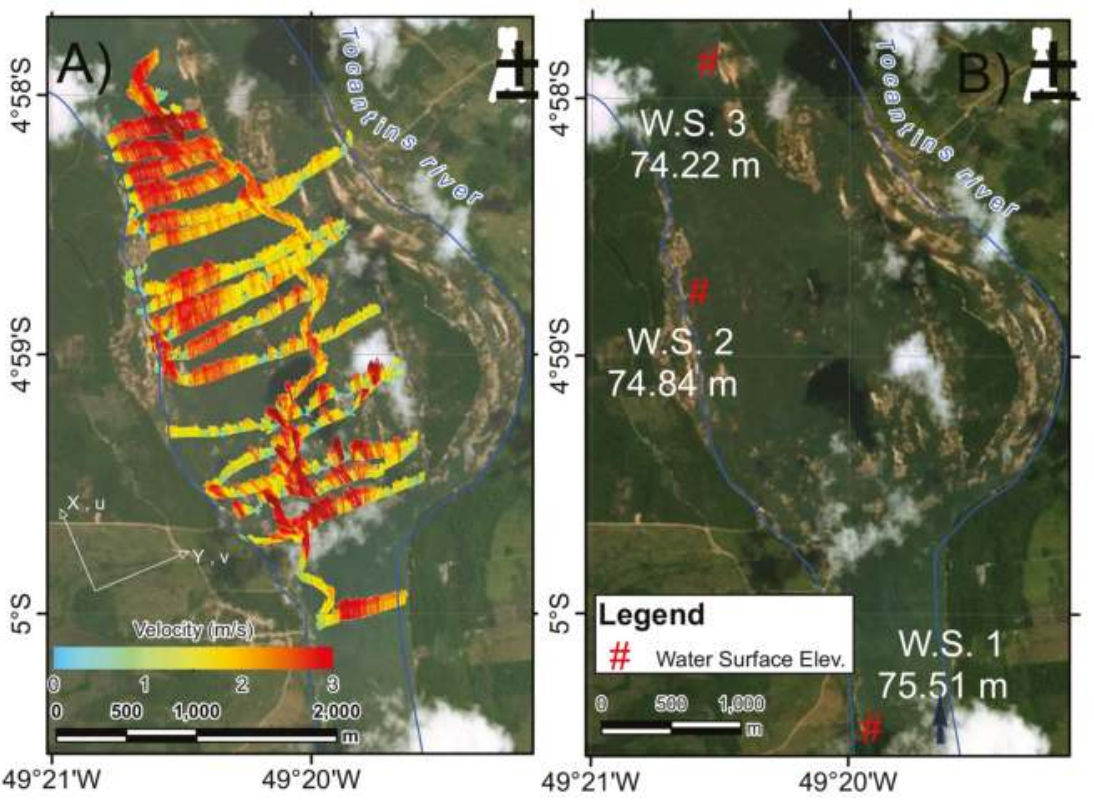

Figure 2. Measurements in Lourenço's Rock Canyon; (A) ADCP trajectories with colored depth-averaged velocity vectors (April 2015); (B) River banks during high flow are shown in blue, and the location of the three gaging stations with measured values of the water surface elevation (April 2015) is presented.

\subsection{Initial ADCP Data Processing}

The equipment's software Riversurveyor [20] was used to export all the velocity profiles into Matlab [22] file formats. The data coordinate system was ENU (East-North-Up), the depth reference used was the vertical beam (VB) and the boat velocity used as reference the bottom track (BT) feature. Subsequent post-processing was done using a recently programmed toolbox, VelMap [23]. The toolbox can decompose the velocity data measurements by rotation as a function of an angle chosen by the user. For the study area, an angle equal to $337^{\circ}$ clockwise from North (angle $=0^{\circ}$ ), was considered the longitudinal flow direction (x-axis), and $247^{\circ}$ as the transversal (y-axis. This fixed coordinate system has been chosen here due to the large heterogeneities in the flow and thus difficulties in defining a consistent main flow direction. The chosen angle, however, corresponds to the overall mean velocity direction. Moreover, the toolbox uses additional parameters (SNR - signal to noise ratio, STD—standard deviation) for further processing, following instrument specifications.

Many factors affect the uncertainty of ADCP measurements, such as instrument noise and configuration, velocity fluctuations due to flow turbulence, bottom track errors, compass errors and GPS errors [6]. According to Sontek [24], the standard deviation of the velocity data can be attributed to installation and calibration problems, acoustic noise from the equipment, and velocity fluctuations from the local turbulence. Following $[25,26]$, installation and calibration errors were considered negligible, and the standard deviation for each velocity component decomposed as:

$$
\sigma^{2}=\sigma_{t}^{2}+\sigma_{n}^{2}
$$


where $\sigma$ is the standard deviation from the velocity data $(\mathrm{m} / \mathrm{s}) ; \sigma_{t}$ is the velocity fluctuation from the local turbulence $(\mathrm{m} / \mathrm{s}) ; \sigma_{\mathrm{n}}$ is the acoustic noise effect from the equipment on the standard deviation $(\mathrm{m} / \mathrm{s})$. The acoustic noise is a result of the physical process by which sound waves are dispersed by the solid particles in the water. To estimate the turbulent part of the standard deviation, previous approaches calculated the acoustic noise using different assumptions. Vachtman and Laronne [27] considered the acoustic noise equal to the minimum variance of calculated water velocities for all depth cells $\left(\sigma_{\mathrm{n}}{ }^{2}=\sigma^{2} \mathrm{~min}\right)$. Another approach was presented by Rennie and Church [6], who applied Equation (1), but used the average ADCP error velocity $\left(\sigma_{\text {error }}\right)$, instead of the acoustic noise. The average ADCP error velocity considered other uncertainties and can be calculated by the velocity difference $\left(V_{d}\right)$ from 2 vertical velocity measurements and the horizontal velocity error $\left(V_{e}\right)$. According to Sontek [24], although being a random effect, it can be assumed that acoustic noise follows a Gaussian distribution. The magnitude of the noise can vary according to the equipment frequency $(F)$, the vertical dimension of the cell $\left(\Delta \mathrm{Z}_{\mathrm{cel}}\right)$ and the number of pings $(\mathrm{N})$ emitted in the period measured per cell. For a standard 3D ADCP measurement, the acoustic noise can be estimated with Equation (2).

$$
\sigma_{\mathrm{n}}=235\left(\mathrm{~F} \Delta \mathrm{Z}_{\mathrm{cel}} \mathrm{N}^{0.5}\right)^{-1}
$$

Developed by the same manufacturer, Equation (2) was defined for older ADCP models than the M9. Conferring with Sontek [28], there is not yet a specific noise equation for the M9. It is herein assumed that the results from Equation (2) can be used as the best approximation of the noise from the ADCP data.

All results can be visualized as velocity magnitudes along a cross-section for each mean velocity component $(\langle\mathrm{u}\rangle,\langle\mathrm{v}\rangle,\langle\mathrm{w}\rangle)$, related velocity fluctuations from the local turbulence $\left(\sigma_{\mathrm{tx}}, \sigma_{\mathrm{ty}}, \sigma_{\mathrm{tz}}\right)$ and computed flow parameters (turbulent kinetic energy $k$ and shear stress $\tau$ ), as shown in Figures 5-7, and explained in the following section. The velocity distributions show the part of the canyon with highest velocities and highest longitudinal velocities occurring in the deeper part of the channel. These effects follow the observations by Venditti et al. [16], where similar flow features were observed for a bedrock canyon of the Fraser River.

The strongest transversal velocity fluctuations occur in the canyon section. The variations of transversal and vertical velocity components indicate large and strong secondary currents induced by the canyon walls. The secondary currents in the horizontal plane were even visible as strong (boat was shaking), and large (several meters) surface vortices (Figure 3) also manifested as strong transversal velocity fluctuations. Normally, these large vortices are difficult to observe in natural rivers. However, these vortices can frequently be viewed along the canyon section during the flood season, reinforcing the river reach peculiarity.

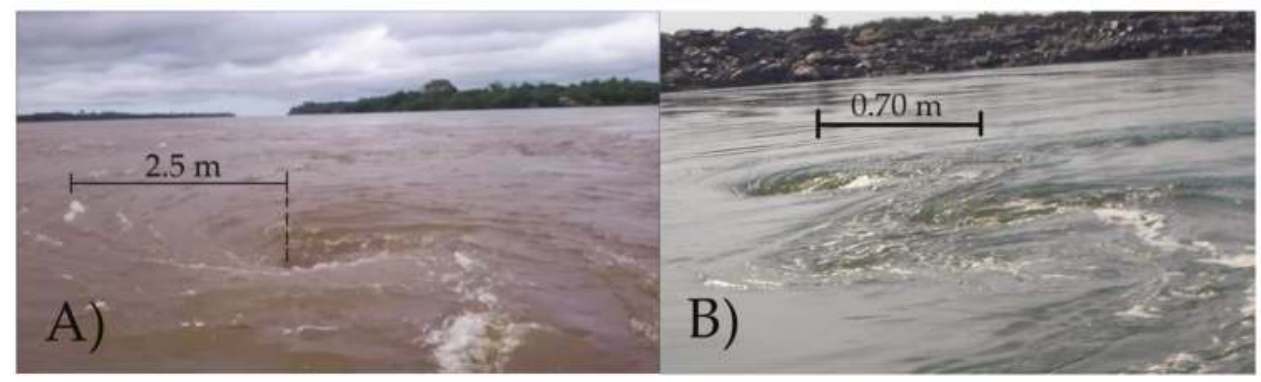

Figure 3. Large vortices in the canyon shear-layer where steep bathymetrical gradients exist; (A) Flood event-April 2015; (B) Drought event-October 2015. 


\section{Theory and Analytical Methods}

This section briefly reviews the different methods applied to calculate the local bed shear stress and eddy viscosity distributions, including discussion of details and assumptions.

ADCPs operated from moving vessels have been used in previous studies to estimate spatial flow characteristics. Flener et al. [4], Rennie and Church [6], Rennie and Millar [10], Sime et al. [11], Szupianyet et al. [29], Guerrero and Lamberti [30], are reference studies that mapped some flow characteristics, such as shear velocity, shear stress, suspended and bedload sediment transport. However, in some river structures, such as meanders, bifurcations and submerged canyons, these characteristics are more difficult to estimate because of the high turbulence levels and the nonlinear velocity profiles along the river area [31]. Three different methods to calculate the bed shear stress were compared here to evaluate the flow characteristic of the measured reach.

\subsection{TKE Method}

All the velocities can be split into mean components $(\langle\mathrm{u}\rangle,\langle\mathrm{v}\rangle,\langle\mathrm{w}\rangle)$ and fluctuating components $\left(\mathrm{u}^{\prime}, \mathrm{v}^{\prime}, \mathrm{w}^{\prime}\right)$ using Reynolds decomposition, where the parentheses denote the time-averaged value, and the prime symbol the turbulent fluctuations. Results of the TKE method relate the intensity of velocity fluctuations $\left(\mathrm{u}^{\prime}, \mathrm{v}^{\prime}, \mathrm{w}^{\prime}\right)$ to turbulent kinetic energy $(\mathrm{k})$, as described in Equation (4). Based on the hypothesis that the Reynolds stress and the variances in the region near the bottom are practically constant and proportional to the shear velocity $\left(\mathrm{u}^{*}\right)$, this method has been applied to ADCP data in studies by Lu and Lueck [21], Nystrom et al. [32], Hurther and Lemmin [33], Vachtman and Laronne [27], Liu and $\mathrm{Wu}$ [34]. Therefore, the shear stress was calculated as:

$$
\begin{gathered}
\tau_{0}=\mathrm{C}_{0} \mathrm{TKE}=\mathrm{C}_{0} \varrho \mathrm{k} \\
\mathrm{k}=\left(<\mathrm{u}^{\prime 2}>+<\mathrm{v}^{\prime 2}>+<\mathrm{w}^{\prime 2}>\right) / 2
\end{gathered}
$$

where $\mathrm{C}_{0}$ is a dimensionless constant (i.e., $\mathrm{C}_{0}=0.18-0.21$ ) [13]; $\varrho$ is the water density (i.e., $\varrho=1 \mathrm{~g} / \mathrm{cm}^{3}$ at $15^{\circ} \mathrm{C}$ ). Using the ADCP in Narrowband mode, it is possible to combine the Standard Deviation (STD, Equation (1)) from the velocity data (for each component), and the Reynolds Decomposition to determine the temporal root mean square of the velocity fluctuations $\left(<\mathrm{u}^{\prime 2}>\right)$.

$$
\sigma_{\mathrm{tx}}=\left(\mathrm{N}^{-1} \sum(\mathrm{u}-<\mathrm{u}>)^{2}\right)^{0.5}=\left(<(\mathrm{u}-<\mathrm{u}>)^{2}>\right)^{0.5}=\left(<\mathrm{u}^{\prime 2}>\right)^{0.5}
$$

By rewriting Equation (4) with the other equations and directions, the shear stress for each cell $\left(\tau_{0 \text {-TKEcel }}\right)$ can be calculated using the velocity STD components of the ADCP data.

$$
\tau_{0-\mathrm{TKE} e \mathrm{l}}=\mathrm{C}_{0} \varrho\left(\sigma_{\mathrm{tx}}^{2}+\sigma_{\mathrm{ty}}^{2}+\sigma_{\mathrm{tz}}^{2}\right) / 2
$$

The bed shear stress $\left(\tau_{0-\mathrm{TKE}}\right)$, calculated by the TKE method, can be determined by the integration of the shear stress calculated in each cell ( $\left.\tau_{0 \text {-TKEcel }}\right)$ of the vertical measurement (ensemble). For the vertical average the variance sum law was applied, where the covariance of the velocity time series between each cell should be calculated; however without the samples, this study considered that the sum of the covariance among the ensemble is equal to zero. Togneri et al. [35] used the same consideration to compare the ADCP observations with a 3D model simulation. Besides, Lu and Lueck [21] calculated the distribution (histogram) of the covariance in velocity profiles from an ADCP fixed to the bottom of a tidal channel at a depth of $30 \mathrm{~m}$, during 20-min intervals in 2 different flow events. The results showed a balance between positive and negative covariance, with a mean near to zero.

The numbers of samples in each cell measurement is another relevant point. Depending on the size and duration of the turbulent features to be measured, longer averaging times are required, requiring 
either anchored boat measurements [11] or slow boat movements. In the present study, the slow boat moving method with large spatial distribution was chosen to capture large-scale turbulent variations.

\subsection{Law of the Wall Method}

The log law is often used to estimate shear stress in open channels and rivers, as presented in Keulegan [36]. Based on the assumption that the velocity profile in open channel flows has a logarithmic shape, the "Law of the Wall" can be described by the von Karman-Prandtl Equation.

$$
\mathrm{U} / \mathrm{u}^{*}=1 / \mathrm{k} \ln \left(\mathrm{z} / \mathrm{z}_{0}\right)
$$

where $\mathrm{k}$ is the von Karman constant (i.e., $\mathrm{K}=0.40$ ); $\mathrm{U}$ is the velocity at height $\mathrm{z}$ above the bed; $\mathrm{u}^{*}$ is the shear velocity, and $\mathrm{z}_{0}$ is the roughness length. For natural rivers, considered as a hydraulically rough flow, Equation (7) can be described using the characteristic roughness length-scale $\left(\mathrm{k}_{\mathrm{s}}\right)$.

$$
\mathrm{U}=\left(\mathrm{u}^{*} / \mathrm{k}\right) \ln (\mathrm{z})+\left(\mathrm{u}^{*} / \mathrm{k}\right) \ln \left(30 / \mathrm{k}_{\mathrm{s}}\right)
$$

The shear velocity and the roughness length-scale can be estimated using the velocity profile measurement by the ADCP and fitting the data to the following equation.

$$
\mathrm{U}=\mathrm{m} \ln (\mathrm{z})+\mathrm{b}
$$

where $\mathrm{m}=\left(\mathrm{u}^{*} / \mathrm{k}\right) ; \mathrm{b}=\left(\mathrm{u}^{*} / \mathrm{k}\right) \ln \left(30 / \mathrm{k}_{\mathrm{s}}\right)=\mathrm{m} \ln \left(30 / \mathrm{k}_{\mathrm{s}}\right)$.

The relationship between shear velocity and bed shear stress $\left(\tau_{0-\log }\right)$, by the log-law, is given by:

$$
\tau_{0-\log }=\varrho\left(\mathrm{u}^{*}\right)^{2}
$$

According to Pope et al. [37], this method is only valid for steady, uniform, one-dimensional flow and its application to natural streams does have some limitations. Afzalimehr and Rennie [38] comment that the method does not hold in general and can only be considered an approximate representation of experimental data. For measurements at a single point, long time-series data are required for proper averaging. In the present study, the velocity distributions do not seem to follow a logarithmic profile, thus it was expected that this method is not applicable in detail.

Figure 4 shows a typical vertical profile of longitudinal velocities as measured in the canyon. The profile shows the features observed in the cross-section visualizations, with high velocities in deep channel regions. Unfortunately, the depth limitations of the equipment resulted in a large blanking region close to the bed. Regardless, the profile does not visually follow a log law. To minimize the velocity non-linearity problem, each velocity profile was separated in 2 parts: a non-uniform (near the water surface) and a log part (starting at the maximum velocity point in the profile). Using this assumption, only the data below the maximum velocity point were considered for the fitting processes. Several fits also resulted in negative velocities close to the bed, limiting this method further, thus not following it for the subsequent calculations. 


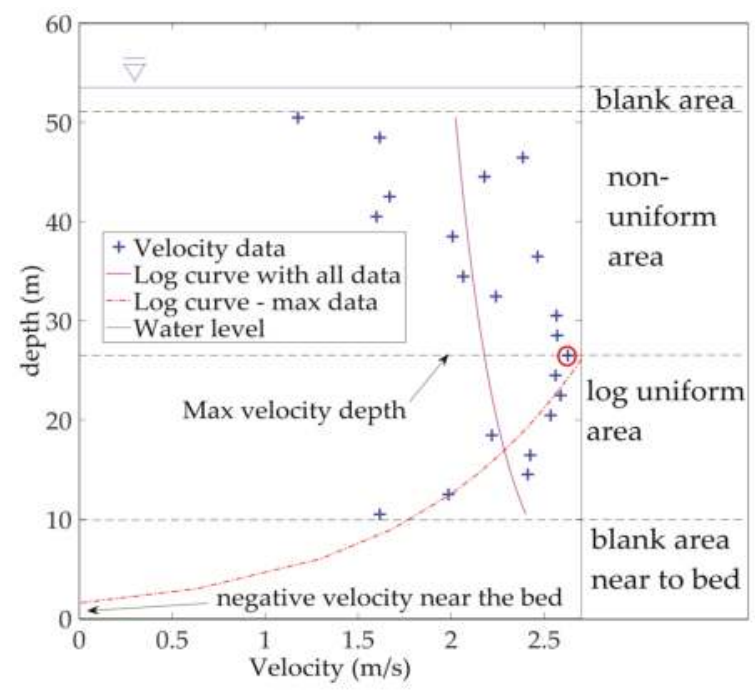

Figure 4. Measured vertical velocity profile of longitudinal velocity $(+)$, and fitted logarithmic curves using a different number of measured points.

\subsection{Depth-Slope Product Method}

The mean boundary shear stress equation considers a steady and uniform flow and is generally used in sediment transport studies to estimate the channel stability. In large rivers, with small slopes, it is possible to assume that the bed slope $\left(\mathrm{S}_{0}\right)$ is parallel to the energy slope $\left(\mathrm{S}_{\mathrm{e}}\right)$ and the water level slope $\left(\mathrm{S}_{\mathrm{ws}}\right)$, and the hydraulic radius $\left(R_{h}\right)$ nearly equals the transect-averaged water depth $(\langle h\rangle)$. The application of these assumptions within the mean boundary shear stress equation is often referred to as the "depth-slope product", as presented in:

$$
\tau_{0-\mathrm{ds}}=\varrho \mathrm{g} R_{h} \mathrm{~S}_{\mathrm{e}}=\varrho g<h>\mathrm{S}_{0}
$$

where $\tau_{0 \text {-ds }}$ is bed shear stress, by the depth-slope product method; $g$ is the gravitational acceleration (i.e., $g=9.81 \mathrm{~m}^{2} / \mathrm{s}$ ); $\langle h\rangle$ is the transect-averaged water depth $(\mathrm{m}) ; \mathrm{S}_{\mathrm{e}}$ is the local energy slope. The transect-averaged water depth is a standard result of the ADCP measurement, and the bed slope $\left(\mathrm{S}_{0}\right)$ was considered equal to the water surface slope $\left(\mathrm{S}_{\mathrm{ws}}\right)$ measured in the field. Because of the depth difference, the ADPC cross-section data were subdivided, selecting a mean hydraulic radius of the canyon area and another value for the rest of the cross-section. Unfortunately, the simplifications presented in the depth-slope product method (steady and uniform flow) are an unrealistic representation of the spatial data condition of the measurements in Lourenço's Rock Canyon. Nonetheless, Sime et al. [11] considered it worthwhile to compare the method with other results because of its high usability. However, it has not been followed further in this manuscript.

\subsection{Boussinesq's Hypothesis}

The concept adopted in Reynolds averaging of the Navier-Stokes (RANS) created a non-linear term of the Navier-Stokes equation, called the "Reynolds stress". The term represents the influence of the turbulent fluctuations in the momentum distribution in the mean flow [39]. The coordinate system of the Reynolds stress is presented in Table 1 [40]. 
Table 1. Reynold Stress notation.

\begin{tabular}{ccccc}
\hline Measurement & Stress Component & Plane & Direction & Velocity Gradient \\
\hline$-\varrho<\mathrm{u}^{\prime} \mathrm{w}^{\prime}>$ & $\tau_{\mathrm{xz}}^{\prime}$ & $\mathrm{yz}$ & $\mathrm{z}$ & $\partial<\mathrm{w}>/ \partial \mathrm{x}$ \\
& $\tau_{\mathrm{zx}}^{\prime}$ & $\mathrm{yx}$ & $\mathrm{x}$ & $\partial<\mathrm{u}>/ \partial \mathrm{z}$ \\
$\left.-\varrho<\mathrm{u}^{\prime} \mathrm{v}^{\prime}\right\rangle$ & $\tau_{\mathrm{xy}}^{\prime}$ & $\mathrm{yz}$ & $\mathrm{y}$ & $\partial<\mathrm{v}>/ \partial \mathrm{x}$ \\
& $\tau_{\mathrm{yx}}^{\prime}$ & $\mathrm{zx}$ & $\mathrm{x}$ & $\partial<\mathrm{u}>/ \partial \mathrm{y}$ \\
$-\varrho<\mathrm{v}^{\prime} \mathrm{w}^{\prime}>$ & $\tau_{\mathrm{yz}}^{\prime}$ & $\mathrm{zx}$ & $\mathrm{z}$ & $\partial<\mathrm{w}>/ \partial \mathrm{y}$ \\
& $\tau_{\mathrm{zy}}^{\prime}$ & $\mathrm{yx}$ & $\mathrm{y}$ & $\partial<\mathrm{v}>/ \partial \mathrm{z}$ \\
\hline
\end{tabular}

With the extra term, the equations of motion for turbulent flow can never form a closed system in which the number of equations is equal to the number of unknowns. This is called the turbulence closure problem and can be solved by eddy-viscosity models (EVM), which use Boussinesq's hypothesis.

Boussinesq's hypothesis was formulated in 1877 and is based on the analogous relationship between the viscous stresses of the laminar regime and the turbulent one, and it is assumed that the turbulent stresses are proportional to the mean velocity gradient of the flow in a similar manner as the viscous stresses (Newton's Viscosity Law) [41]. This proportionality factor was named the eddy viscosity $\left(v_{t}\right)$ and is not a physical property but is dependent on the current flow condition and turbulence levels. The correlation between the turbulent shear stress of the flow and the eddy viscosity (for the $\mathrm{x}$ and $\mathrm{z}$ coordinate axes) is described by:

$$
\tau^{\prime}{ }_{x z} / \varrho=-<u^{\prime} w^{\prime}>=v_{t}(\partial<u>/ \partial z+\partial<w>/ \partial x)-2 / 3 \delta_{x z} k
$$

where $\tau^{\prime}{ }_{x z}$ is the turbulent shear stress ( $x$ and $z$-axes), in $\mathrm{kg} / \mathrm{ms}^{2} ;\left\langle\mathrm{u}^{\prime} \mathrm{w}^{\prime}\right\rangle$ is the Reynolds Stress component ( $\mathrm{x}$ and $\mathrm{z}$-axes); $v_{\mathrm{t}}$ is the eddy viscosity, in $\mathrm{m}^{2} / \mathrm{s} ; \mathrm{k}$ is the turbulent kinetic energy, in $\mathrm{m}^{2} / \mathrm{s}^{2}$; $\delta$ is the dimensionless Kronecker delta.

In natural rivers, the shear stress is the force of moving water against the bed, and the turbulent effects are the principal component $\left(\tau \approx \tau^{\prime}\right)$. According to Vachtman and Laronne [32], from the computed turbulent kinetic energy $(\mathrm{k})$, based on the average of the velocity variances for a given depth above the bed, the tridimensional turbulent shear stress $\left(\tau^{\prime}\right)$ can be estimated using [13,42]:

$$
\tau^{\prime}=\mathrm{C}_{1} \mathrm{TKE}
$$

where $C_{1}$ is a dimensionless constant (i.e., $C_{1} \approx 0.19-0.21$ ). The specific value of $C_{1}$ may not apply at all levels within the water column because it requires confirmation before using the method universally [13]. Nonetheless, this paper considered the turbulent shear stress for the water column $\left(\tau^{\prime}\right.$ ensemble $)$ equal to bed shear stress, by the TKE method $\left(C_{1}=C_{0}=0.20\right)$.

The velocity gradients and a $3 \mathrm{D}$ vorticity field were calculated using the ADCP velocity data. However, the transversal distance between the points was on average $1.5 \mathrm{~m}$, and the longitudinal reached $200 \mathrm{~m}$ (distance between transects). Because of the longitudinal distance between the ADCP data, it is reasonable to set the terms with longitudinal distance to zero. Considering the vertical average and applying Boussinesq's hypothesis, the turbulence eddy viscosity (for each ensemble) can be calculated as:

$$
v_{\text {t ensemble }}=\tau_{\text {ensemble }}^{\prime} /\left(\varrho \omega_{\text {ensemble }}\right)
$$

where $v_{\text {t ensemble }}$ is the turbulence eddy viscosity, in $\mathrm{m}^{2} \mathrm{~s}^{-1}, \tau^{\prime}$ ensemble is shear stress, by the TKE method $\left(\mathrm{Nm}^{-2}\right)$; $\omega$ ensemble is the vertical average of the $3 \mathrm{D}$ vorticity along the cross-section $\left(\mathrm{s}^{-1}\right)$.

Numerical models are generally used to complement field data for further analysis. The most accessible and common models are Reynolds averaged Navier-Stokes (RANS) models, which require either the modeling or the definition of values for eddy viscosity. Usually, constant values are used, and simulations are calibrated using varying roughness values. However, under complex flow 
situations, the calibration process can result in non-realistic roughness coefficients, thus non-realistic results for simulations beyond the calibrated regime. Under such conditions, varying eddy viscosity values may provide better flow representations, as adopted in Cheng et al. [39].

\section{Results}

The previously described methods have been applied to all measured velocity profiles to obtain shear stress and eddy viscosity distributions for the whole river section. First, a cross-sectional result will be shown, to illustrate the differences between shallow, regular river sections, compared to the deep canyon section. Those are followed by mapped results for the whole region.

\subsection{Single Cross-Section}

The section shown in Figure 5 is approximately $1400 \mathrm{~m}$ wide and has depths varying between $5 \mathrm{~m}$ in the shallow part down to $55 \mathrm{~m}$ in the canyon. The canyon itself is only $150 \mathrm{~m}$ wide in most of the sections. Figure 6A-F show only a part of the cross-section, between of the distances 700 to $1100 \mathrm{~m}$, focusing on the canyon area.

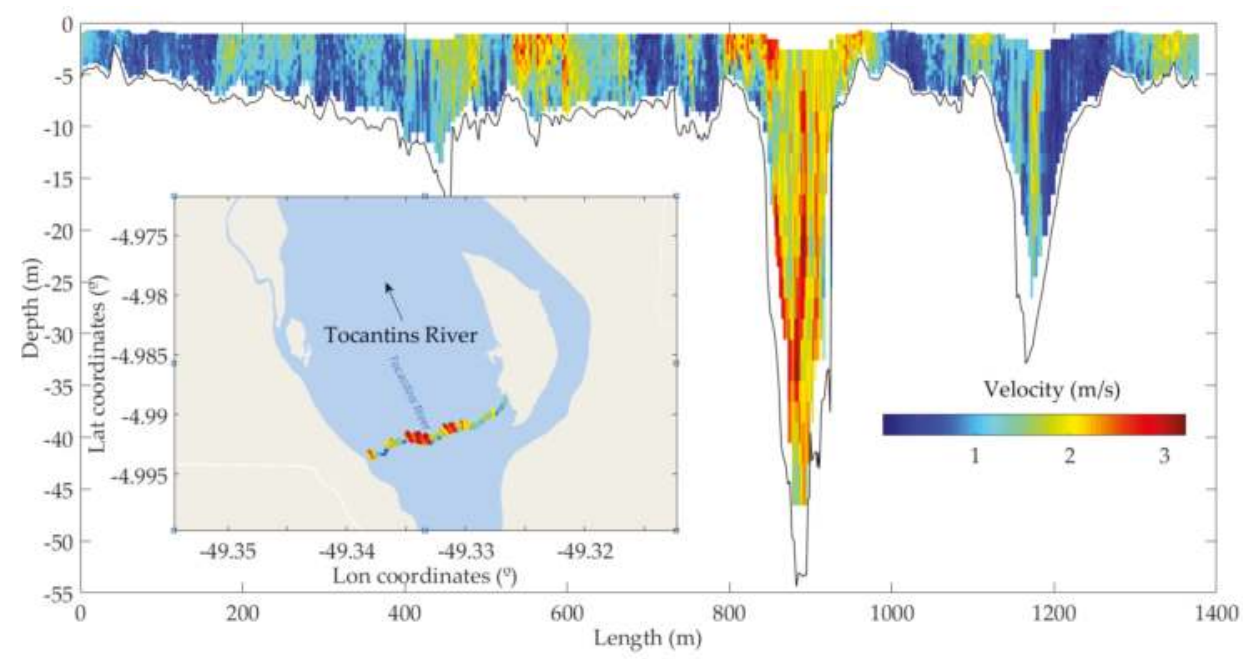

Figure 5. Flow velocity profiles over a full cross-section (3D Velocity magnitude) (April 2015).

Applying the TKE method, the velocity data and standard deviations were used to compute the turbulent kinetic energy $(\mathrm{k})$ in all measured cells. For situations where the instrument noise was higher than the turbulence fluctuations, the $\mathrm{k}$ value was considered equal to zero. Afterward, all values were interpolated (natural neighbor) on an equidistant vertical grid, to eliminate changing vertical cell sizes due to frequency shifts and to extrapolate the blanking area. The value used was the ADCP maximum vertical cell size in the canyon $(2 \mathrm{~m})$. The horizontal grid sizes were maintained (i.e., varying according to boat speed). Afterward, shear stress and eddy viscosity were computed for each cell using Equations (5) and (13). The shear stress results for the TKE method in the canyon area are shown in Figure 7.

For the horizontal analysis, a comparison between the bed shear stress of the three methods is presented in Figure 8. For the TKE method, the vertical-average was used to represent each ensemble. 

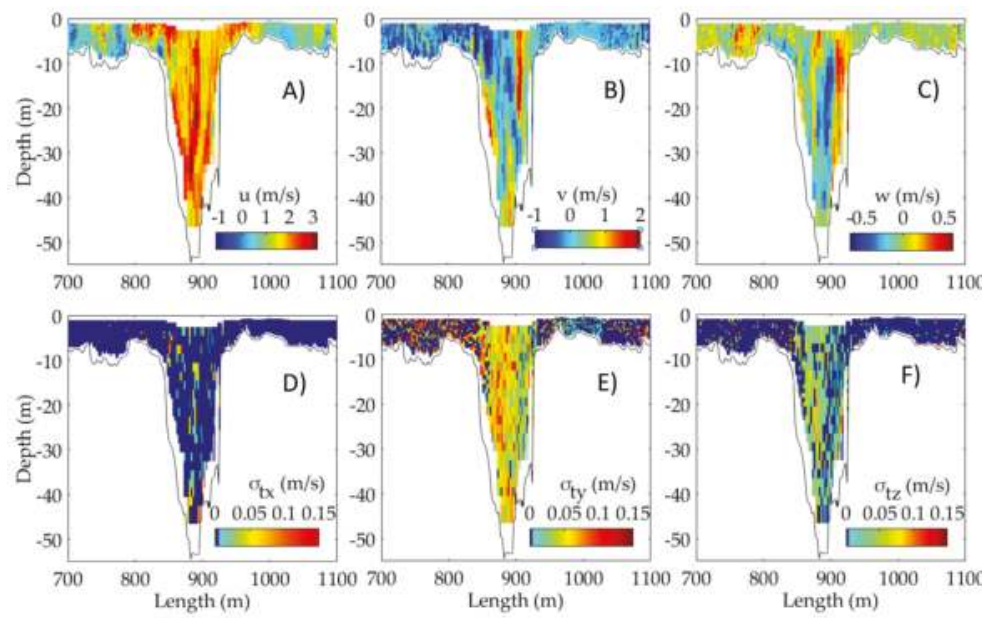

Figure 6. Flow velocity profiles over the canyon part (April 2015); (A-C) Magnitude of mean velocity components $<\mathrm{u}>$ (longitudinal), $<\mathrm{v}>$ (transversal), $<\mathrm{w}>$ (vertical) over cross-section; (D-F) Magnitude of turbulent velocity fluctuations.
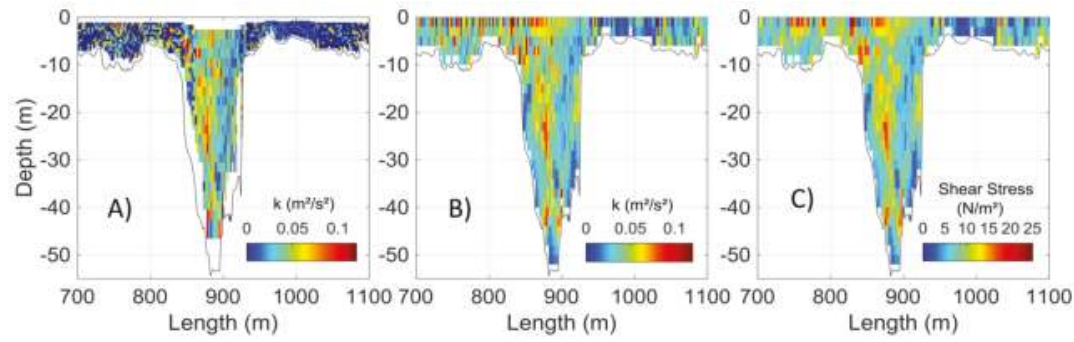

Figure 7. Turbulent kinetic energy (k) before (A) and after (B) vertical interpolation; (C) Shear-stress distribution using the TKE method.

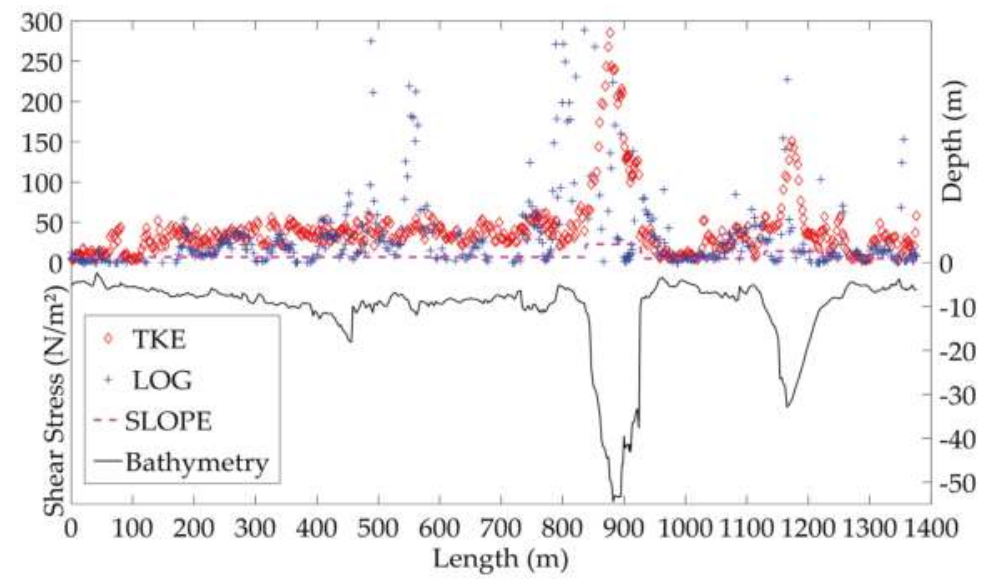

Figure 8. Bed shear stress distribution along the cross-section, using the TKE method, the log-law method, and the depth-slope product method. 
The velocity information was processed further to identify secondary currents by computing vorticity maps over the interpolated cross-sectional velocity distributions (Figure 9). Those showed large-scale structures at the canyon walls where surface vortices were also mostly present. The large vortices most probably are induced by the shear effects of the strongly inclined canyon walls. Those secondary currents seem to be responsible for pushing the water at high velocities from the surface down into deeper regions of the canyon, creating non-logarithm velocity profiles.

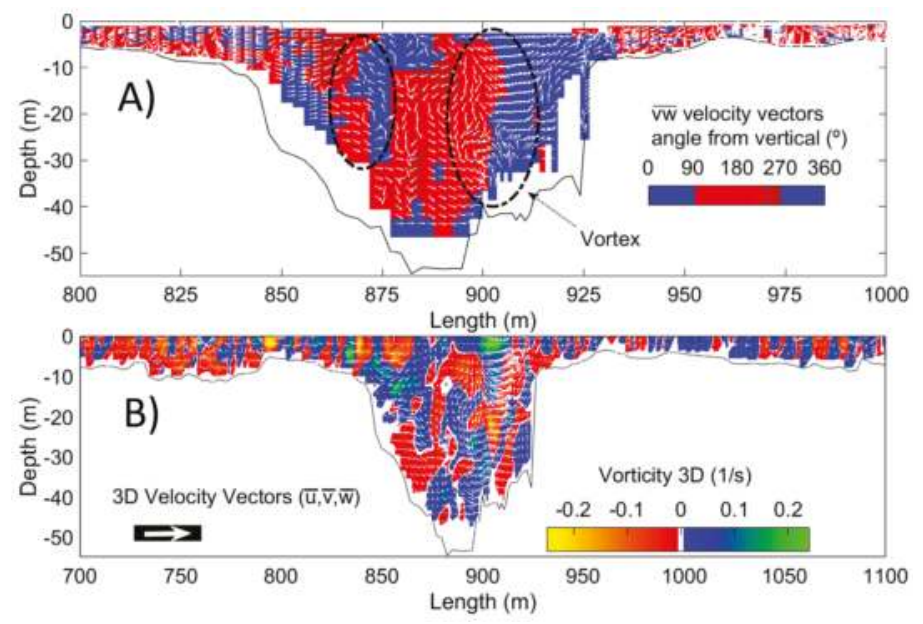

Figure 9. (A) Distribution plot of the $\langle\mathrm{vw}>$ direction (angle from up) resulting velocity in the vertical plane $\left(\langle\mathrm{vw}\rangle=\left(\langle\mathrm{V}\rangle^{2}+\langle\mathrm{W}\rangle^{2}\right)^{0.5}\right)$ and schematized identification of vortex structure; (B) Vorticity $(\omega)$ of the canyon section part.

Applying Boussinesq's hypothesis, the spatial eddy viscosity distribution indicates a strong turbulent effect within the canyon (Figure 10). These high values are different from the homogeneous values obtained for the rest of the cross-section.

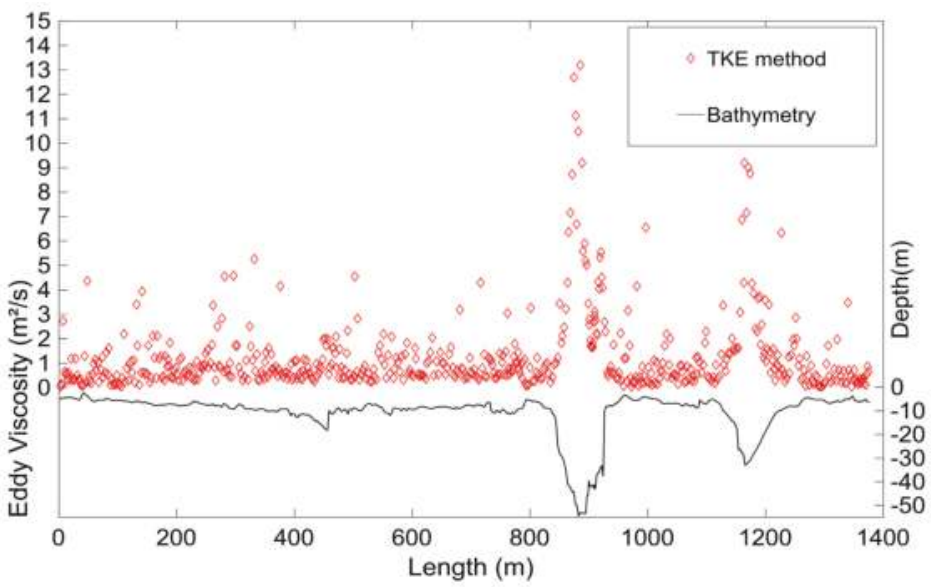

Figure 10. Eddy viscosity distribution over the cross-section, using the TKE method. 


\subsection{Spatial Distribution}

The previously described processing was done for all ADCP profiles over the whole river section. The mapping was done on a regular rectangular grid with cells of $10 \mathrm{~m} \times 30 \mathrm{~m}$ (transversal $\times$ longitudinal), oriented in the mean flow direction ( $337^{\circ}$ from North). Combining the water level measurements with the bathymetry, the whole wetted region was determined by cutting a water level surface with the digital terrain model of the bathymetry. The data were then extrapolated to regions not measured. The details of the spatial interpolation are presented in Appendix A. The principal results are shown in Figure 11.

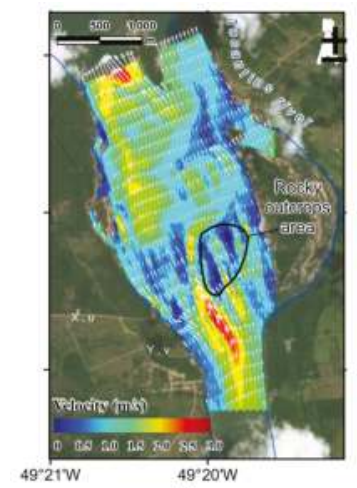

(A)

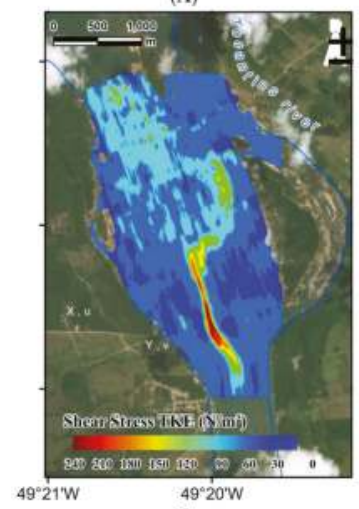

(C)

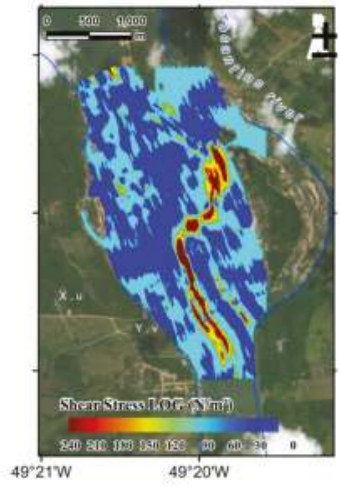

(B)

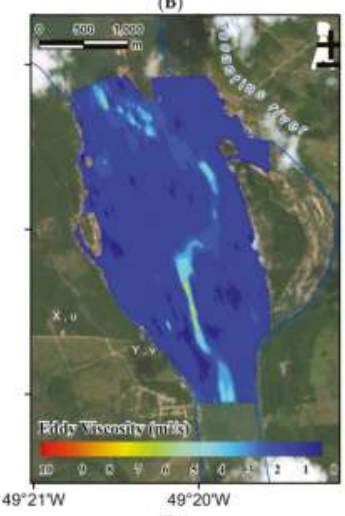

(D)

Figure 11. (A) Velocity magnitude and vector direction within the river section; (B) Shear stress distribution using the log method; (C) Shear stress distribution using the TKE method; (D) Eddy viscosity distribution based on the shear stress computed by TKE method.

\section{Discussion and Conclusions}

The measured velocity profiles in the submerged canyon showed irregular flow features, characterized by high flow velocities in the deeper part of the canyon, and large secondary currents developing at the canyon side walls. Analyzing the sections velocity field, the higher vorticity values happened around the longitudinal axis. The initial processing of the data showed high velocity fluctuations in the vertical and transversal axis, representing the strong presence of turbulence along the canyon.

The turbulent quantities associated with the ADCP data indicated a large internal shear occurring within the canyon. Those effects have been summarized by computing the bed shear stress and eddy viscosity distributions and showing distinct regions with significant differences. The shear stress 
values from the depth-slope product method were considered lower than the real flow conditions, reaffirming the method limitations for high turbulence flow. The assumption used in the law of wall method assisted the curve-fitting process for non-logarithm velocity profiles. However, because of the blank area near the bottom, some curves were fitted considering negative velocities near the bottom, resulting in higher values for the bed shear stress along the canyon and some outside parts. The results from the TKE method were considered most realistic for the canyon area, with shear stress values similar to those presented in other studies with similar conditions [13]. The most significant shear stress difference happened in the canyon upstream, with values 8 times greater than outside.

The eddy viscosity distributions show reasonable results with values around the regular $1.0 \mathrm{~m}^{2} / \mathrm{s}$ used for rivers. In most of the areas of the stretch, the values were between 0.8 and $1.8 \mathrm{~m}^{2} / \mathrm{s}$. In the canyon, analyzing each section, some points reached values higher than $20 \mathrm{~m}^{2} / \mathrm{s}$. For spatial analysis, the higher value considered was 10 times higher than the other parts of the stretch.

The spatial interpolation was able to show the horizontal variation of shear-stress and eddy viscosity distributions. However, due to the longitudinal distance between the cross-sections, it was not possible to represent the velocity gradients at the $x$-direction $(\partial<v>/ \partial x$ and $\partial<w>/ \partial x)$.

The turbulent quantified values can be used with the intention to improve hydrodynamic numerical model calibrations (turbulence models) and better representation of physical processes. However, it is important to highlight the assumptions and the methodology limitations described in this paper, such as the short velocity time series and the interpolation.

To improve future field measurements, we recommend adding extra points with fixed ADCP measurements along each section to evaluate the shear stress results with an extensive time series. To improve the vertical analysis, equipment with a different frequency to check the velocity near the bottom should be used. For longitudinal analysis, the maximum distance should be only 2 times the transversal distance data.

For future analysis, a sensitivity grid test is recommended, comparing different cell sizes or applying a non-regular grid. In addition, different interpolation methods can be tested. The next steps will be to compare the results of the flood and drought season (already measured, but not processed) and to evaluate their applicability to hydrodynamic models.

Acknowledgments: The authors acknowledge the financial support for the field studies from Brazil's National Transport Infrastructure Department (DNIT), and the continuous support from the Transport Infrastructure Technical Institute (ITTI) of the Federal University of Paraná (UFPR), and Coordination for the Improvement of Higher Education Personnel (CAPES) regarding scholarships. We also acknowledge the participating professor provided by Brazil's National Council for Scientific and Technological Development (CNPq), as well as additional funding from the Araucaria Foundation for the travel expenses.

Author Contributions: G.T. and T.B. proposed and elaborated the presented idea and performed the field measurements. C.D.R. encouraged G.T. to investigate the law of the wall method and supervised the findings of this work, and provided scientific background information; G.T. performed the data post-processing and the methods; H.G. performed the spatial interpolation. G.T. wrote the manuscript with support from T.B., C.D.R. and H.G.

Conflicts of Interest: The authors declare no conflict of interest.

\section{Appendix A}

For the spatial interpolation of the flow's parameters, a regular rectangular grid was developed with cells of $10 \mathrm{~m} \times 30 \mathrm{~m}$ (transversal $\times$ longitudinal). Considering the intrinsic anisotropic patterns of river systems, the grid follows the general longitudinal flow direction of the stretch ( $337^{\circ}$ from North). However, the canyon's curvature varies along the stretch, resulting in different longitudinal angles of the main flow compared to the canyon's thalweg. This characteristic results in difficult interpolation inside the canyon with coarse point density.

A similar situation happens with spatial analysis in meandering rivers, where the spatial grid does not follow the longitudinal flow direction. For a proper interpolation of a river's main channel longitudinal characteristics, Merwade et al. [43] suggested a coordinate transformation from the typical 
(E, N) coordinate system to one that follows the river's main channel. The transformation consists of attributing to every point a $(\mathrm{s}, \mathrm{d})$ coordinate, where $\mathrm{s}$ is the distance of a cross-section to an arbitrary point that starts at the river's center path or thalweg, and d the distance from a point to the intersection of the central path with the cross-section to which it belongs. The coordinate transformation can be applied to ADCP sample points using the AnyForm QGIS plugin [44]. The algorithm uses the basic concept of the method's implementation described by Guarneri [45] and described by the Equation (A1).

$$
[\mathrm{s}, \mathrm{d}]=\left[\mathrm{S}_{\mathrm{b}}+|\mathrm{PB}| \cos (\alpha), \psi(|\mathrm{PB}| \sin (\alpha)]\right.
$$

where [] represents a matrix and bold font the vector system; $S_{b}$ is the longitudinal distance of $B$ from the arbitrary beginning; $B$ is the planimetric coordinate $\left[E_{B}, N_{B}\right]$ of the upstream point; $P$ is the planimetric coordinate $\left[\mathrm{E}_{\mathrm{P}}, \mathrm{N}_{\mathrm{P}}\right]$ of the surveyed point; $\alpha$ is the angle between the vectors $|\mathrm{CP}|$ and $|\mathrm{CB}|$; $\mathrm{C}$ is the planimetric coordinate $\left[\mathrm{E}_{\mathrm{C}}, \mathrm{N}_{\mathrm{C}}\right]$ of the downstream point; $\psi$ is a parameter that equals -1 for points on the right side and 1 for points to the left.

For this paper, the spatial interpolation was divided into 2 steps. First, the interpolation occurs over the deepest parts of the canyon, using the samples with bathymetry ADCP data less than $63 \mathrm{~m}$ above sea level. The grid was linearized by the canyon's thalweg (s-line), and the samples were interpolated with kriging interpolation. The results were re-transformed to a regular grid and combined with the other ADCP points as an input for the second step. Figure A1 presents the activity order from the first step. The second step is a simple kriging interpolation with an angle anisotropy of $337^{\circ}$ from North and an aperture angle tolerance of $20^{\circ}$. Figure A2 shows the input and output from the second step for the ADCP bathymetry data. The same interpolation procedure was applied to all variables presented in the paper.

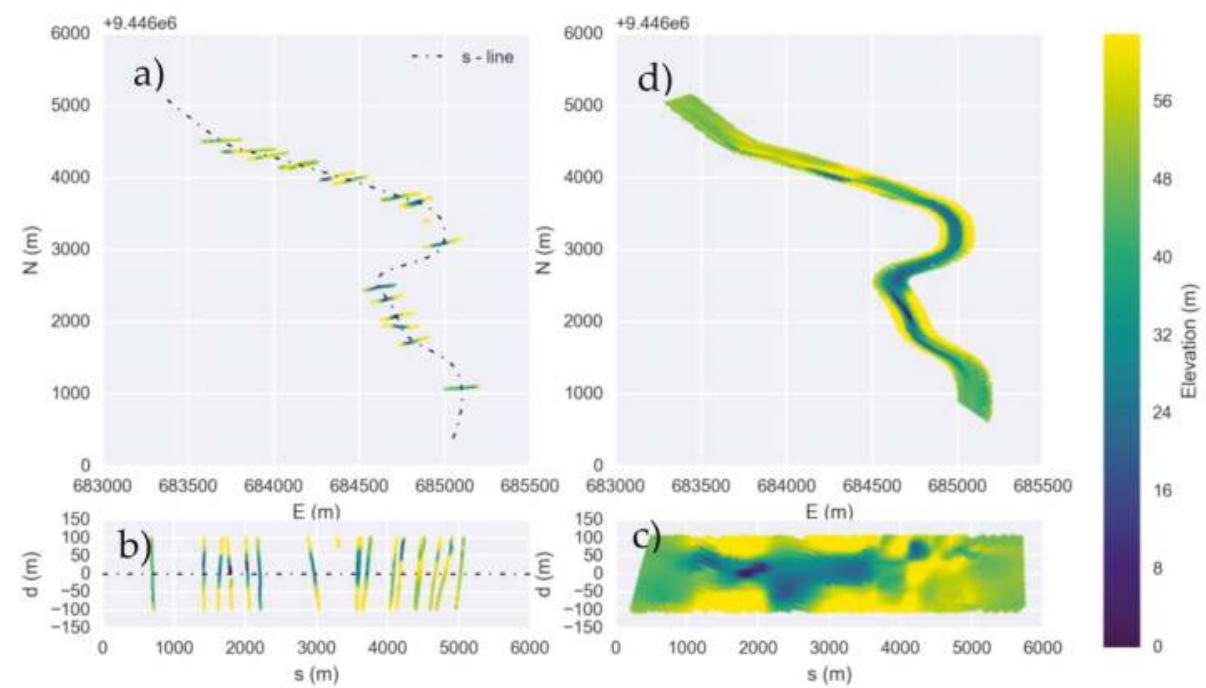

Figure A1. (a) Bathymetry data (below $63 \mathrm{~m}$ ) in a regular coordinate system grid (E-N); (b) Bathymetry data transformed in the s-d grid (by s-line direction); (c) Kriging interpolation using the s-d grid; (d) Interpolated bathymetry grid along the canyon central path in a regular grid. 


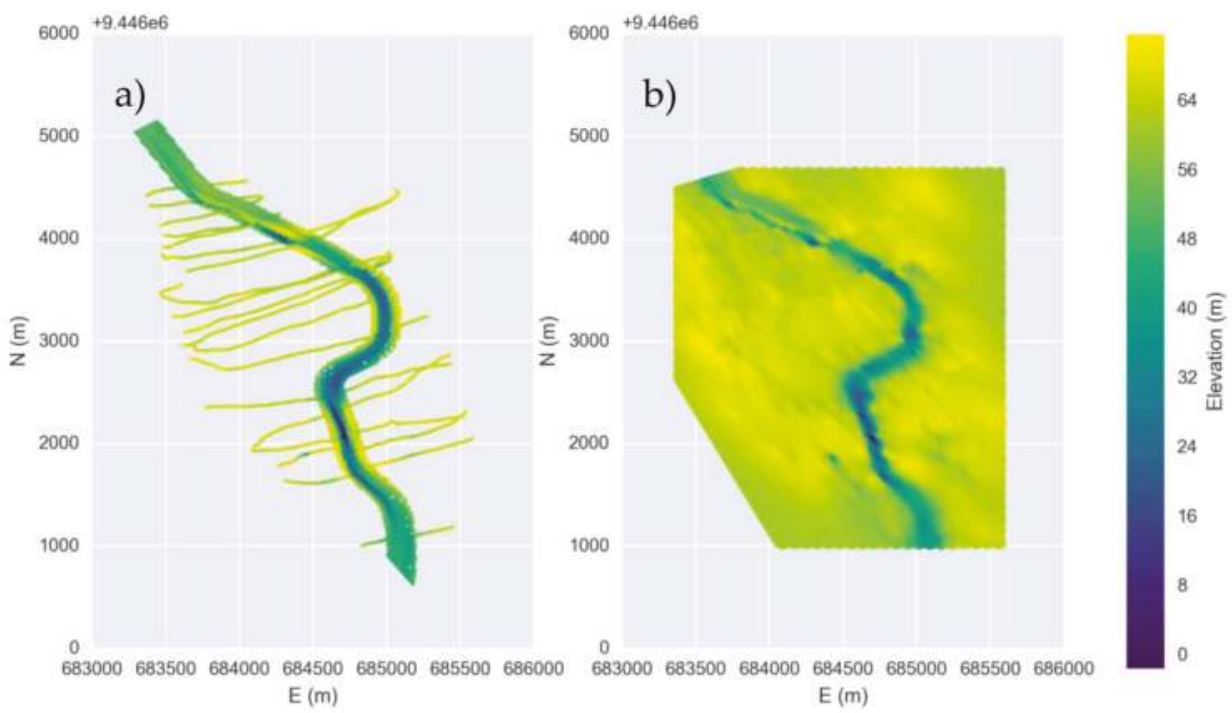

Figure A2. (a) All the Bathymetry data and the results from the first step at the coordinate system grid $(\mathrm{E}-\mathrm{N})$ were used as an input for the second step; (b) Bathymetry results from kriging interpolation after the second step.

\section{References}

1. Constantinescu, G.; Kashyap, S.; Tokyay, T.; Rennie, C.D.; Townsend, R.D. Hydrodynamic processes and sediment erosion mechanisms in an open channel bend of strong curvature with deformed bathymetry. J. Geophys. Res. Earth Surf. 2013, 118, 480-496. [CrossRef]

2. Aberle, J.; Rennie, C.D.; Admiraal, D.; Muste, M. Experimental Hydraulics: Methods, Instrumentation, Data Processing and Management: Volume II: Instrumentation and Measurement Techniques; CRC Press: Boca Raton, FL, USA, 2017; ISBN 9781351658034.

3. Pilechi, A.; Mohammadian, A.; Rennie, C.D.; Zhu, D.Z. Efficient method for coupling field data and numerical modeling for the estimation of transverse mixing coefficients in meandering rivers. J. Hydraul. Eng. 2016, 142, 0001116. [CrossRef]

4. Flener, C.; Wang, Y.; Laamanen, L.; Kasvi, E.; Vesakoski, J.M.; Alho, P. Empirical modeling of spatial 3D flow characteristics using a remote-controlled ADCP system: Monitoring a spring flood. Water 2015, 7, 217-247. [CrossRef]

5. Jamieson, E.C.; Rennie, C.D.; Jacobson, R.B.; Townsend, R.D. 3-D flow and scour near a submerged wing dike: ADCP measurements on the Missouri River. Water Resour. Res. 2011, 47, W07544. [CrossRef]

6. Rennie, C.D.; Church, M. Mapping spatial distributions and uncertainty of water and sediment flux in a large gravel bed river reach using an acoustic Doppler current profiler. J. Geophys. Res. 2010, 115, F03035. [CrossRef]

7. Carr, M.L.; Rehmann, C.R. Measuring the dispersion coefficient with acoustic doppler current profilers. J. Hydraul. Eng. 2007, 133. [CrossRef]

8. Shen, C.; Niu, J.; Anderson, E.J.; Phanikumar, M.S. Estimating longitudinal dispersion in rivers using acoustic Doppler current profilers. Adv. Water Resour. 2010, 33, 615-623. [CrossRef]

9. Kim, D. Assessment of longitudinal dispersion coefficients using acoustic Doppler current profilers in large river. J. Hydro-Environ. Res. 2012, 6, 29-39. [CrossRef]

10. Rennie, C.D.; Millar, R.G. Measurement of the spatial distribution of fluvial bedload transport velocity in both sand and gravel. Earth Surf. Process. Landf. 2004, 29, 1173-1193. [CrossRef] 
11. Sime, L.C.; Ferguson, R.I.; Church, M. Estimating shear stress from moving boat acoustic Doppler velocity measurements in a large gravel bed river. Water Resour. Res. 2007, 43, W03418. [CrossRef]

12. Wall, G.R.; Nystrom, E.A.; Litten, S. Use of an ADCP to compute suspended-sediment discharge in the tidal Hudson River, New York. U.S. Geological Survey Scientific Investigations Report. 2006. Available online: https://pubs.usgs.gov/sir/2006/5055/ (accessed on 8th July 2017).

13. Kim, S.C.; Friedrichs, C.T.; Maa, J.P.; Wright, L.D. Estimating bottom stress in tidal boundary layer from acoustic Doppler velocimeter data. J. Hydraul. Eng. 2000, 126, 399-406. [CrossRef]

14. De Serio, F.; Mossa, M. Analysis of mean velocity and turbulence measurements with ADCPs. Adv. Water Resour. 2015, 81, 172-185. [CrossRef]

15. Hancok, G.S.; Anderson, R.S.; Whipple, K.X. Beyond power: Bedrock river incision process and form. In Rivers Over Rock: Fluvial Processes in Bedrock Channels; Tinkler, K., Wohl, E.E., Eds.; American Geophysical Union: Washington, DC, USA, 1998; Volume 3, pp. 35-60.

16. Venditti, J.G.; Rennie, C.D.; Bomhof, J.; Bradley, R.W.; Little, M.; Church, M. Flow in bedrock canyons. Nature 2014, 513, 534-537. [CrossRef] [PubMed]

17. DNIT. Licitação Para Derrocamento do Pedral do Lourenço, na Hidrovia do Tocantins. 2016. Available online: http:/ / www1.dnit.gov.br/editais/consulta/resumo.asp?NUMIDEdital=5682 (accessed on 16th October 2017). (In Portuguese)

18. Tomas, G.P.; Werner, J.; Ratton, P.; Bleninger, T.; Godoy, P. Modelagem Hidrodinâmica Para o Derrocamento do Rio Tocantins_-Pedral do Lourenço; XXI Simpósio Brasileiro de Recursos Hídricos; Associação Brasileira de Recursos Hídricos: Brasília, Brazil, 2015. (In Portuguese)

19. Content Protection for Recordable Media (CPRM). Mapas Geológicos Estaduais-Pará. Companhia de Pesquisa de Recursos Minerais; GEOBANK, 2012. Available online: http://geobank.cprm.gov.br (accessed on 25th January 2016). (In Portuguese)

20. SonTek. RiverSurveyor S5/M9 System Manual: Firmware Version 1.0; SonTek Inc.: San Diego, CA, USA, 2010.

21. Lu, Y.; Lueck, R.G. Using a broadband ADCP in a tidal channel. Part II: Turbulence. J. Atmos. Ocean. Technol. 1999, 16, 1568-1579. [CrossRef]

22. MATLAB. Matlab and Statistics Toolbox Release 2016a; The MathWorks, Inc.: Natick, MA, USA, 2016.

23. Tomas, G.P.; Hopker, R.; Frigo, A.L.; Bleninger, T. Velocity Mapping Toolbox for Sontek M9 ADCP Data. River Flow; Taylor \& Francis Group: Iowa City, IA, USA, 2016; pp. 1571-1576.

24. SonTek. ADP Introductory Documentation. Acoustic Doppler Profiler Technical Documentation; SonTek Inc.: San Diego, CA, USA, 2000.

25. Rennie, C.D. Uncertainty of ADCP spatial velocity distributions. In Proceedings of the 6th International Symposium on Ultrasonic Doppler Methods for Fluid Mechanics and Fluid Engineering, Prague, Czech Republic, 9-11 September; 2008.

26. Greene, A.D.; Hendricks, P.J.; Gregg, M.C. Using an ADCP to Estimate Turbulent Kinetic Energy Dissipation Rate in Sheltered Coastal Waters. J. Atmos. Oceanic Technol. 2015, 32, 318-333. [CrossRef]

27. Vachtman, D.; Laronne, J.B. Flow structure over bed irregularities in a straight cohesive open channel. J. Hydraul. Eng. 2011, 137, 1335-1346. [CrossRef]

28. SonTek; (San Diego, CA, USA). Personal communication, 2017.

29. Szupiany, R.N.; Amsler, N.L.; Best, J.L.; Parsons, D.R. Comparison of fixed- and moving -vessel flow measurements with an aDp in a large river. J. Hydraul. Eng. 2007, 133, 1299-1309. [CrossRef]

30. Guerrero, M.; Lamberti, A. Flow field and morphology mapping using ADCP and multibeam techniques: Survey in the Po River. J. Hydraul. Eng. 2011, 137. [CrossRef]

31. Muste, M.; Yu, K.; Spasojevic, M. Practical aspects of ADCP data use for quantification of mean river flow characteristics: Part 1. Moving-vessel measurements. Flow Meas. Instrum. 2004, 15, 1-16. [CrossRef]

32. Nystrom, E.A.; Oberg, K.A.; Rehmann, C.R. Measurement of turbulence with acoustic Doppler current profilers-Sources of error and laboratory results. In Hydraulic Measurements and Experimental Methods, Proceedings of the Hydraulic Measurements and Experimental Methods 2002, Estes Park, CO, USA, 28 July-1 August 2002; American Society of Civil Engineers (ASCE): Reston, VA, USA; pp. 346-355.

33. Hurther, D.; Lemmin, U. A correction method for turbulence measurements with a 3D acoustic Doppler velocity profiler. J. Atmos. Ocean. Technol. 2001, 18, 446-458. [CrossRef]

34. Liu, H.; Wu, J. Estimation of bed shear stresses in the pearl river estuary. China Ocean Eng. 2015, 29, 133-142. [CrossRef] 
35. Togneri, M.; Lewis, M.; Neill, S.; Masters, I. Comparison of ADCP observations and 3D model simulations of turbulence at a tidal energy site. Renew. Energy. 2017, 114, 273-282. [CrossRef]

36. Keulegan, G.H. Laws of turbulent flow in open channels. J. Res. Natl. Bur. Stand. 1938, 21, 707-741. [CrossRef]

37. Pope, N.D.; Widdows, J.; Brinsley, M.D. Estimation of bed shear stress using the turbulent kinetic energy approach-A comparison of annular flume and field data. Cont. Shelf Res. 2006, 26, 959-970. [CrossRef]

38. Afzalimehr, H.; Rennie, C.D. Determination of bed shear stress in gravel-bed rivers using boundary-layer parameters. Hydrol. Sci. J. 2009, 54, 147-159. [CrossRef]

39. Cheng, R.T.; Ling, C.H.; Gartner, J.W. Direct Measurements of Turbulence Properties by a BB-ADCP in Bottom Boundary Layer. In Interactions between Estuaries, Coastal Seas and Shelfs Seas; TERRAPUB: Tokyo, Japan, 2000; pp. 37-55.

40. Jamieson, E.C.; Rennie, C.D.; Townsend, R.D. Turbulence and vorticity in a laboratory channel bend at equilibrium clear-water scour with and without stream barbs. J. Hydrol. Eng. 2013, 139, 259-268. [CrossRef]

41. Schmitt, F.G. About Boussinesq's turbulent viscosity hypothesis: Historical remarks and a direct evaluation of its validity. Comptes Rendus Mec. 2007, 335, 617-627. [CrossRef]

42. Stapleton, K.R.; Huntley, D.A. Seabed stress determination using the inertial dissipation method and the turbulent kinetic energy method. Earth Surf. Process. Landf. 1995, 20, 807-815. [CrossRef]

43. Merwade, V.; Maidment, D.; Hodges, B. Geospatial representation of river channels. J. Hydrol. Eng. 2005, 10, 243-251. [CrossRef]

44. Guarneri, H. Methods for the Analysis and Design of Inland Waterways and Application for the Paraguay River Waterway. Master's Thesis, Universidade Federal do Paraná, Curitiba, Brazil, March 2017.

45. Guarneri, H. AnyForm-Anisotropic Transformation-QGIS Plugin. GitHub Repos. 2017. Available online: https://github.com/henriqueguarneri/qgis-anyform-plugin (accessed on 21 November 2017).

(C) 2018 by the authors. Licensee MDPI, Basel, Switzerland. This article is an open access article distributed under the terms and conditions of the Creative Commons Attribution (CC BY) license (http:/ / creativecommons.org/licenses/by/4.0/). 


\title{
Dip Phenomenon in High-Curved Turbulent Flows and Application of Entropy Theory
}

\author{
Donatella Termini $^{1, *}$ and Tommaso Moramarco ${ }^{2}$ \\ 1 Dipartimento di Ingegneria Civile, Ambientale e Aerospaziale, University of Palermo, Viale delle Scienze, \\ 90128 Palermo, Italy \\ 2 Research Institute for Geo-Hydrological Protection, CNR, Via Madonna Alta, 126, 06128 Perugia, Italy; \\ Tommaso.Moramarco@irpi.cnr.it \\ * Correspondence: donatella.termini@unipa.it; Tel.: +39-328-727-4471
}

Received: 7 February 2018; Accepted: 9 March 2018; Published: 12 March 2018

\begin{abstract}
The estimation of velocity profile in turbulent open channels is a difficult task due to the significant effects of the secondary flow. The present paper investigates the mechanism of the velocity-dip phenomenon, whereby the location of the maximum velocity appears to be below the free surface. Previous studies conducted in straight channels relate the mechanism of the velocity-dip phenomenon to secondary flow induced by anisotropy of turbulence. This work focuses on high-curved channels where the secondary motion, which is also induced by the channel's curvature, evolves along the bend. The width-to-depth ratio, $B / h$, is one of the most important parameters that are affecting the secondary motion entity. In particular, the present study aims to investigate the evolution of the velocity-dip along the bend for two values of the width-to-depth ratio and the applicability of the entropic model for the dip-phenomenon estimation. The results show that the velocity-dip is more accentuated for low values of the width-to-depth ratio, where the secondary motion plays a fundamental role in the distribution of the downstream flow velocity, although the velocity-dip is also present when the aspect ratio is higher than 10 . Furthermore, the velocity profiles that were estimated by applying the entropic model are in good agreement with the measured ones, especially for $B / h<10$.
\end{abstract}

Keywords: rivers; meanders; turbulence; secondary motion; prediction

\section{Introduction}

The understanding of flow characteristics in different bed and plane shape conditions is very important in hydraulic engineering practice. In fact, the identification of such characteristics is useful in understanding the nature of turbulence and consequent transport processes.

Literature shows (among others [1,2]) that the turbulent structure in open-channel flows can be divided into three sub-regions: a wall region, which is controlled by the inner variables (flow viscosity, local shear velocity, distance normal to the boundary); an intermediate region, which is controlled both by wall and free surface proprieties; a free surface region, which is controlled by outer variables (flow depth, maximum or mean velocity).

The log law is universally accepted for the velocity distribution in the inner region; experiments (see in [2]) show that in the outer region the velocity data deviate from the log law. To express the velocity distribution in both the inner and outer regions, some authors ([3-7]) suggested including an additional term, called the wake term.

Some researchers $([8,9])$, analyzing the velocity field and the importance of secondary currents over three-dimensional bedforms, also highlighted a deviation of flow from the logarithm behavior.

The effect of the free surface on turbulence is particularly important $([10,11])$. Literature widely reports on the velocity-dip phenomenon due to the position of the maximum velocity below the 
water surface (among others [12,13]). Although experiments (among others [13]) demonstrate that the velocity-dip phenomenon occurs if the aspect ratio of the channel width to the water depth, $B / h$, is less than 5 (see as an example in [14]), some authors (among others $[15,16]$ ) have highlighted that it occurs near the side-walls, even if $B / h$ is large. Generally, the velocity-dip increases passing from the channel axis to the side-walls. Yan et al. [17] experimentally investigated the influence of the distance from the sidewalls on the dip-position, for different values of the aspect ratios. Their results substantially validated the classification of wide and narrow open-channel flows [13], identifying two different regions in the flow field: the strong sidewall region, where the dip-position is affected only by the distance from the sidewalls; the weak sidewall region, where the dip-position is affected both by the distance from the sidewalls and by the aspect ratio.

Thus, in summary, different factors (such as the walls roughness, the distance from the sidewalls, the aspect ratio, the free-surface effect) could affect the formation and position of the velocity-dip.

The log law cannot account for the velocity-dip phenomenon and different formulations to describe the vertical distribution of longitudinal velocities in the narrow channels (i.e., for low width-to-depth ratios) can be found in literature. As an example, Yang et al. [16] proposed a dip-modified log law depending on a dip correction factor; Bonakdari et al. [18] and Absi [19] presented new formulations for the velocity distribution and dip phenomenon based on the Navier-Stokes equations. Kundu [20] presented an analytical model to predict the dip-position through the maximization of the entropy function and assuming the dip as a random variable. As discussed in [20], Kundu's model allows for the estimation of the dip-position with an accuracy that is similar to that obtained by using Yang et al.' [16] formulation.

However, the suggested models require different parameters that limit their application. Furthermore, the major part of studies that were conducted to investigate the dip phenomenon considered uniform flows in straight channels; the dip phenomenon was often related to the secondary currents, which transport low momentum fluids from the near bank region to the center and high momentum fluids from the free surface to the bed $([2,21,22])$. Recently, Yang and Lee [23], analyzing the kinematic behavior of non-uniform (accelerating/decelerating) straight flows, suggested to describe the velocity-dip phenomenon through the Reynolds shear stress distribution. More recently, Moramarco et al. [24] presented a new methodology to estimate the velocity profile and the velocity-dip starting from the surface velocity evaluation. Such a methodology was based on the entropic theory, and thus, on the linear relationship between the maximum velocity, $u_{\max }$, and the mean flow velocity, $u_{m}$, through a dimensionless function $\Phi(M)$, depending on the entropic parameter, $\mathrm{M}$ ([25-29]).

The point is that the mechanism of dip phenomenon is not well known still today. More research on the velocity-dip mechanism and its relation with secondary motion is needed.

In such a context, the objective of the present work is to gain some insight into the relation between the velocity-dip and secondary motion in accelerating/decelerating flows. In particular, this paper focuses on high-curvature channels, where the variation of the turbulent activity of flow is strongly correlated with the evolution of the secondary motion, which also plays a fundamental role in the distribution of the downstream flow velocity ([30-32]). The study is motivated by the fact that meandering bends have radii of curvature continuously variable in stream-wise direction so that convective flow accelerations occur and the entity of secondary motion changes from cross-section to cross-section (see as an example in [32]). As literature shows ([33,34]), the width-to-depth ratio is one of the most important parameters affecting the entity of the secondary motion in a meandering stream. As an example, the experiments that were conducted by Hooke ([35]) highlighted that the "helix strength" increases as the ratio $B / h$ decreases; in a large amplitude sine-generated channel, Whiting and Dietrich ([36]) found that the effect of flow convective accelerations is more important than that of secondary circulation in "wide" meandering streams, which are characterized by $B / h>10([13,34,37])$. Termini ([33]), analysing the flow pattern for two values of the width-to-depth ratio in a large amplitude sine-generated laboratory flume, confirmed the aforementioned behavior. 
This behavior may influence the shape of the velocity profiles, and thus the entity of the velocity-dip phenomenon, along the bend.

Thus, the specific purpose of this paper is twofold: (1) to analyze the velocity-dip phenomenon along the bend for two values of the width-to-depth ratio; and, (2) to explore the applicability of the entropic model ([24]) to estimate the velocity profile and the velocity-dip phenomenon, starting from the acknowledgment of the surface velocity.

\section{Materials and Methods}

\subsection{Experimental Dataset}

The data used in the present work were collected in the ambit of previous studies that aimed to analyze the kinematic characteristics of flow along a high-amplitude meandering channel. Details of the experimental setup and measurements conditions can be found in previous works (see as an example in [33]) and only features of particular interest for the following analyses are briefly presented here.

The channel follows the sine-generated curve with a deflection angle at the inflection section $\theta_{0}=110^{\circ}$ (see Figure 1).

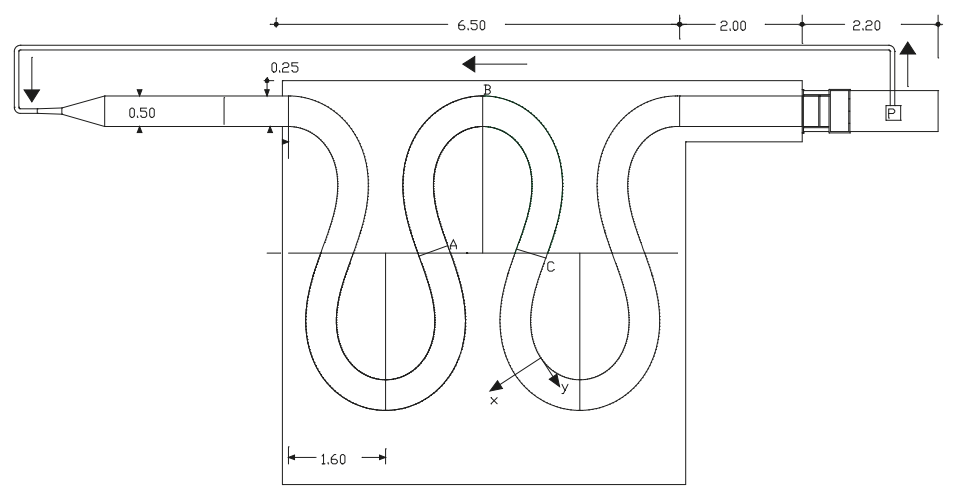

Figure 1. Experimental apparatus.

The channel cross-section is rectangular with width $B=0.50 \mathrm{~m}$; the fixed vertical sidewalls are of plexiglass and the bed, with longitudinal centerline slope $S=0.371 \%$, is flat and made of quartz sand $\left(d_{50}=\right.$ medium sediment diameter $=0.65 \mathrm{~mm}, \sigma_{g}=$ geometric standard deviation $\left.=1.34\right)$. The meandering channel is around $25 \mathrm{~m}$ long in order to accommodate two meander wavelengths; the upstream and downstream ends of the meandering channel are connected to two straight channels $3 \mathrm{~m}$ and $2 \mathrm{~m}$ long, respectively.

In the present paper, the data collected during two runs conducted with two different values of flow discharge have been used: Run 1 was conducted with a flow discharge of $Q=0.019 \mathrm{~m}^{3} / \mathrm{s}$ (channel-averaged flow depth $h=5.5 \mathrm{~cm} ; B / h=9.09<10$ ); Run 2 was conducted with a flow discharge $Q=0.007 \mathrm{~m}^{3} / \mathrm{s}$ (channel-averaged flow depth $h=3.0 \mathrm{~cm}, B / h=16.67>10$ ). Herein, in accordance with other authors ([13,34]), $B / h<10$ indicates "small" width-to-depth ratio and $B / h>10$ indicates "large" width-to-depth ratio.

During each run, the water surface was measured using a Profiler Indicator PV09 (Delft Hydraulics) with precision of $0.1 \mathrm{~mm}$. The instantaneous local longitudinal and transverse velocity components were measured in cross-sections spaced about $50 \mathrm{~cm}$ or so apart, starting from the first inflection section of the meandering channel (see Figure 1), by using a two-dimensional (2D) side-looking Acoustic Doppler Velocimeter (ADV) manufactured by SonTek Inc. (San Diego, CA, USA), in points spaced of $1-1.5 \mathrm{~mm}$ along the verticals of nine transverse abscissas symmetrically to the 
channel axis (see details in [33]). Bearing in mind the specific aim of the present work, our attention is focused only on the longitudinal velocity component. Furthermore, in the present work attention concerns only two consecutive inflection sections (sections A and C of Figure 1) and the apex section B of Figure 1.

\subsection{Pertinent Aspects and Summary of Previous Results}

Before proceeding further, it seems important to briefly summarize here some peculiar results of investigations performed in the same meandering flume of that considered in this work:

- $\quad$ Termini ([33]) conducted experiments under the hydraulic conditions that are considered in the present work in order to examine the effect of the continuously changing channel's curvature on flow pattern. She verified that, because of the changing channel curvature, the flow accelerates near the outer bank from the beginning of the bend to the inflection section downstream and decelerates near the inner bank. The flow accelerated zone is more evident for $B / h>10$ than for $B / h<10$. Such a different behaviour is caused by the secondary circulation that develops more significantly in the case of $B / h<10$ than in the case of $B / h>10$, and that attenuates the effect of the convective acceleration close to the outer bank (see also in [31]).

- Termini and Moramarco ([29]) investigated the effect of the downstream variation of the channel's curvature on the applicability of the linear entropic relationship between the maximum velocity, $u_{\max }$, and the mean flow velocity, $u_{m}$, through a dimensionless parameter $\Phi(M)$. As result, they observed that the ratio $u_{m} u_{\max }$, and thus the value of the parameter $\Phi(M)$, varies along the bend. In particular, Termini and Moramarco ([29]) demonstrated that, in contrast to what observed in straight channels ([28]) where $\Phi(M)$ assumed a value that was almost constant and on average equal to 0.65 , along the meandering bend the parameter $\Phi(M)$ increases passing from the inflection section to the apex section and decreases from the apex section to the inflection section downstream, assuming a value of around 0.8 close to the apex section and of around 0.6 at the inflection section. This behavior can be observed from Figure 2, where the values of the parameter $\Phi(M)$, as obtained by Termini and Moramarco ([29]), along the bend are reported.

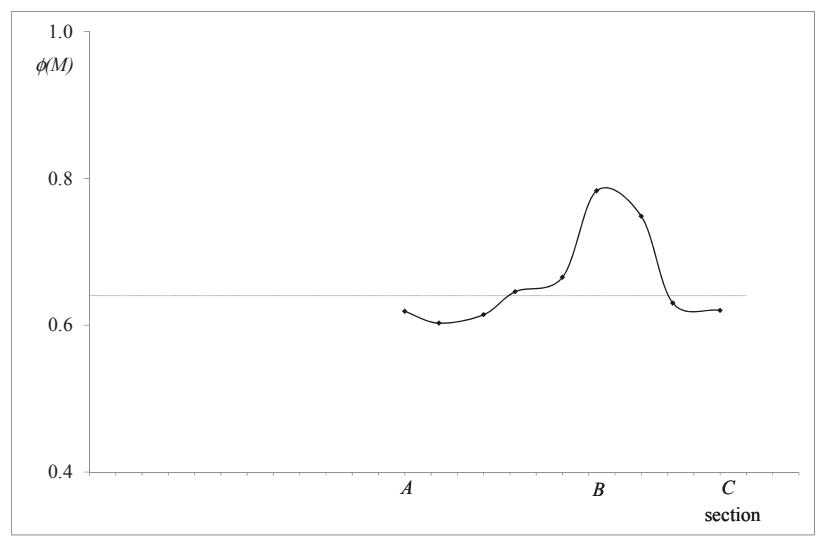

Figure 2. Values of $\Phi(M)$ obtained by Termini and Moramarco ([20]) along the meandering bend.

\section{Results}

\subsection{Measured Velocity Profiles and Effect of the Aspect Ratio on the Velocity-Dip}

The time-averaged longitudinal velocity, $u$, was estimated for each measurement point by using the series of the measured instantaneous longitudinal velocity component. Thus, the 
$u$-profiles (hereafter denoted as "measured profiles") were estimated for each measurement vertical. Then, the distance $\delta$ (dip) between the maximum of flow velocity below the water surface was evaluated for each vertical measured profile.

From the analysis of all the measured profiles appeared clear that the dip-phenomenon occurs for both the width-to-depth ratios. But, the value of the dip decreases as the width-to-depth ratio increases. As an example, Figure 3 shows the velocity profiles that were measured in peculiar verticals (i.e., where the dip was evident) of the examined sections for both the aspect ratios. Furthermore, it was observed that, while in Run 1 (i.e., for $B / h<10$ ) the velocity-dip is evident in all the measured profiles, in Run 2 (i.e., for $B / h>10$ ), the velocity-dip is especially evident in the regions that are close to the banks. This behavior is consistent with previous literature findings obtained in straight channels (among others $[2,13]$ ).

The entity of the distance $\delta$ (dip) varies along the bend. This behavior can be observed from Table 1 , where the values of the distance $\delta$ determined for each measured profile as percentage of the local flow depth are reported for both runs, and from Figure 4, which compares the $\delta$-values that were estimated in the apex section and in the inflection sections for both the width-to-depth ratios. From Table 1 and Figure 4, it can be noted that the velocity-dip is more evident when the aspect ratio $B / h$ is less than 10 , but it is also present when the aspect ratio $B / h$ is higher than 10 . In particular, as Table 1 shows, in average, the dip assumes a value of around $53 \%$ of the flow depth in Run 1 (i.e., for $B / h<10$ ) and of around $34 \%$ of the flow depth in Run 2 (i.e., for $B / h>10$ ).
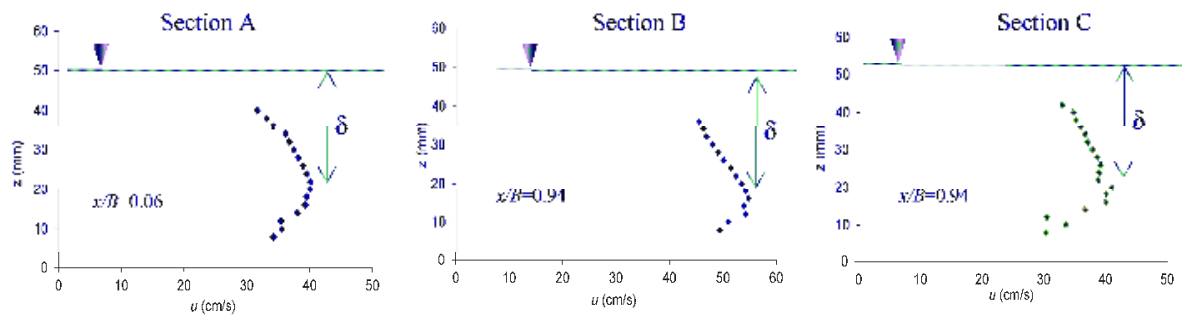

(a)
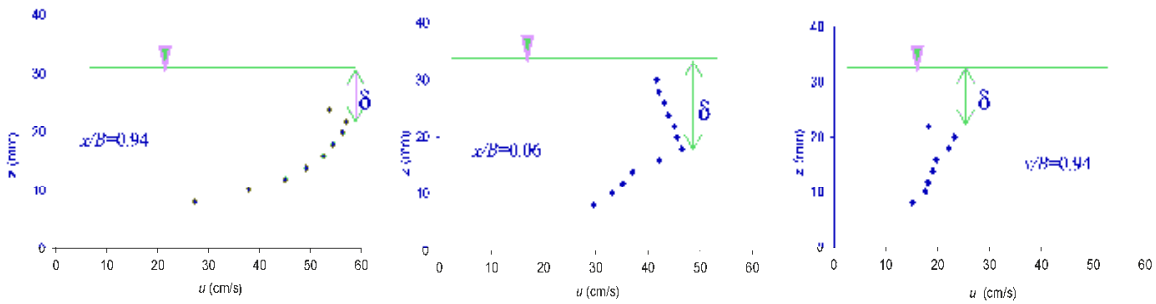

(b)

Figure 3. Measured profiles at peculiar verticals of the examined sections: (a) Run $1(B / h<10)$; (b) Run $2(B / h>10) ; x / B$ indicates the relative distances from the left (outer) bank.

This is probably due to the fact that, as literature demonstrates ([32,35]), the entity of the secondary circulation increases as the ratio $B / h$ decreases. Thus, the observed behavior also indicates that the evolution of the velocity-dip phenomenon along the bend is strongly related to the evolution of the cross-sectional secondary motion along the bend itself.

Figure 4 also indicates that, for $B / h<10$, the velocity-dip always assumes significant values: at the inflection section upstream, the velocity-dip has the higher values that are close to the left (outer) bank; at the apex section high values of the velocity-dip occur almost in the whole cross section until that at the inflection section downstream $\delta$ assumes the higher values close to the right (inner) 
bank. For $B / h>10$, the velocity-dip assumes always very small values and the higher values of the velocity-dip can be found close to the left (outer) bank at the apex section.

Table 1. $\delta$-values estimated for each measured profile as percentage of the local flow depth.

\begin{tabular}{|c|c|c|c|c|c|c|c|c|c|}
\hline \multicolumn{10}{|c|}{$\delta$ av $(\%)=53$} \\
\hline$B / h<10$ & & & & $x / B$ & & & & & \\
\hline Section & 0.06 & 0.17 & 0.28 & 0.39 & 0.5 & 0.61 & 0.72 & 0.83 & 0.94 \\
\hline A & 57.36 & 61.24 & 52.94 & 52.85 & 52.94 & 53.40 & 53.85 & 47.47 & 56.52 \\
\hline B & 53.33 & 43.56 & 35.94 & 43.40 & 44.55 & 58.19 & 52.48 & 60.35 & 64.99 \\
\hline C & 39.62 & 47.26 & 51.81 & 52.94 & 57.20 & 57.69 & 61.83 & 61.17 & 60.78 \\
\hline \multicolumn{10}{|c|}{$\delta$ av $(\%)=34$} \\
\hline$B / h>10$ & & & & $x / B$ & & & & & \\
\hline Section & 0.06 & 0.17 & 0.28 & 0.39 & 0.5 & 0.61 & 0.72 & 0.83 & 0.94 \\
\hline A & 43.22 & 48.39 & 0.00 & 47.37 & 35.90 & 35.90 & 0.00 & 38.27 & 36.60 \\
\hline B & 50.00 & 35.86 & 20.97 & 37.69 & 35.48 & 26.42 & 37.50 & 36.62 & 44.44 \\
\hline C & 32.89 & 32.20 & 24.91 & 40.48 & 33.55 & 34.21 & 34.64 & 33.77 & 32.89 \\
\hline
\end{tabular}
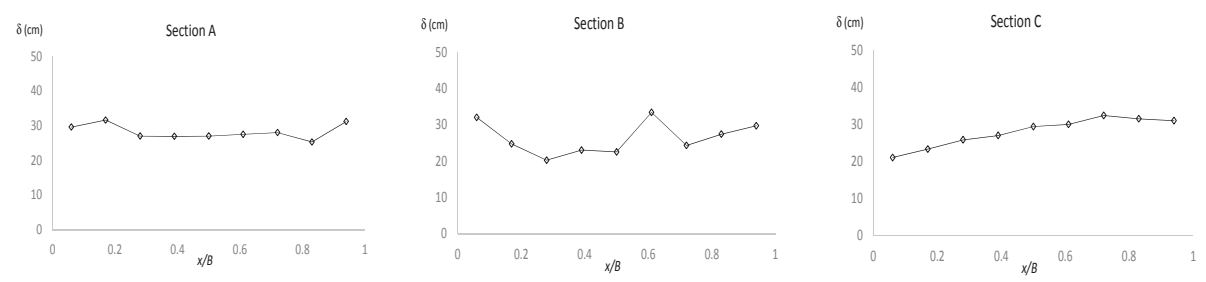

(a)
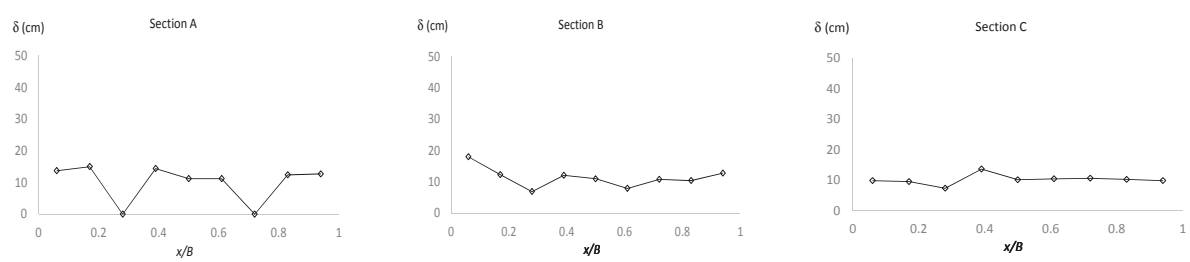

(b)

Figure 4. Velocity-dip phenomenon in each examined section: (a) $B / h<10$; (b) $B / h>10$.

\subsection{Application of the Entropic Model to Estimate the Velocity-Dip Phenomenon}

Recently, Moramarco et al. ([24]) developed an iterative procedure to estimate the velocity profile and the velocity-dip based on the entropy profile, which can be written as:

$$
u(x, z)=\frac{u_{\max , v}}{M} \ln \left[1+\left(e^{M}-1\right) \frac{z}{D-\delta} \exp \left(1-\frac{z}{D-\delta}\right)\right]
$$

with

$$
u_{\max , v}=\frac{u_{\text {surf }}}{\frac{1}{M} \ln \left[1+\left(e^{M}-1\right) \delta e^{1-\delta}\right]}
$$


where $u_{\max , v}$ is the maximum velocity along the each measurement vertical, $D$ is the water depth, $z$ is the distance of the velocity point from the bed, $x$ is the distance of the considered vertical from the left bank, and $M$ is the entropic parameter, which can be estimated by using the entropic relation ([25]):

$$
u_{m}=\left(\frac{e^{M}}{e^{M}-1}-\frac{1}{M}\right) u_{\max }=\phi(M) u_{\max }
$$

where $u_{\max }=\max \left[u_{\max , v}\right]$ which is located at $z$-axis. The velocity-dip is estimated by using the modified expression proposed by Yang et al. ([16]), written as ([24]):

$$
\delta^{\prime}(x)=a_{p}+\delta(x) \text { with } \delta=1+1.3 e^{-x / D}
$$

where $a_{p}$ is an iterative parameter representing the dip at $z$-axis, and which is updated at each iteration, $p$, by adding a constant value $(0.001 \mathrm{~m})$, and starting from the initial value $a_{1}=0.0001$.

By inspecting Equations (1)-(4), it is clear that, in contrast to other formulations that are proposed in literature to estimate the velocity profile and the velocity-dip (among others [4,12,13]), the aforementioned procedure only depends on parameters that have a clear physical meaning and are easily measurable.

Starting from the observed surface velocity, $u_{\text {surf }}$, and the knowledge of $\Phi(M)$, the maximum velocity $u_{\max , v}$ and the velocity-dip are computed, respectively, through Equations (2) and (4) by initializing $a_{p}=a_{1}$. Then, the velocity profiles are computed through Equation (1) and the maximum velocity $u_{\max }=\max \left[u_{\max , v}\right]$ is assessed. Once the velocity profiles are identified, the mean flow velocity, $u_{m}$, can be assessed by applying the velocity area method ([38]) at the estimated velocity profiles; therefore, the ratio $\Phi_{\text {com }}\left(M_{1}\right)=\frac{u_{m}\left(a_{1}\right)}{u_{\max }\left(a_{1}\right)}$ can be computed by Equation (3). The iterative procedure ends when the optimal value of $\Phi_{c o m}\left(M_{p}\right)$ is achieved in accordance with:

$$
\Phi\left(M_{o b s}\right)-\Phi\left(M_{p}\right)=\text { minimum }
$$

Thus, for each measurement vertical and for each run, the velocity profiles (hereon called as "estimated velocity profiles") have been determined by applying the aforementioned iteration procedure and starting from the acknowledgment of the measured surface velocity. Figure 5 shows, as an example, the comparison between the measured and the estimated velocity profiles in the selected measurement verticals. From this figure, it can be observed that the estimated velocity profiles are in good agreement with the measured ones. This is especially evident in the case of $B / h<10$.
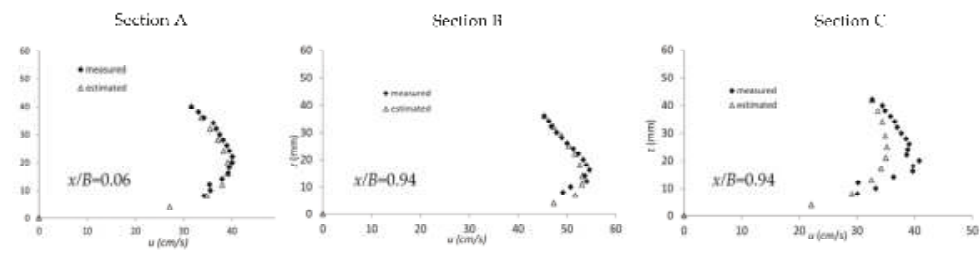

(a)
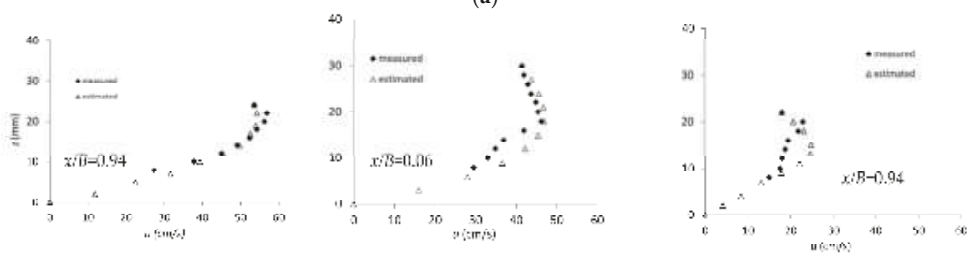

(b)

Figure 5. Comparison between measured and estimated profiles: (a) $B / h<10$; (b) $B / h>10$. 
The good fit between all of the $\delta$-values determined from the estimated velocity profiles and those that were obtained from the measured velocity profiles has been verified by using the root mean squared error $(\sigma)$ as indicator. It is:

$$
\sigma=\sqrt{\frac{\sum_{i=1, N}\left(\delta_{i, m}-\delta_{i, e}\right)^{2}}{N}}
$$

where $\delta_{i, m}$ and $\delta_{i, e}$ are the $\delta$-values determined, respectively, from the measured and from the estimated profiles, $N$ is the total number of measurements that are considered for each profile. Figure 6 reports the pair of values $\left(\delta_{i, m}, \delta_{i, e}\right)$ and the line of perfect agreement (bisector line). The error bar is defined by the value of $\sigma$. As shown in Figure 6, with a few exceptions, the points arrange around the bisector line and the $\sigma$ is quite low in comparison to the magnitude of the measured values.

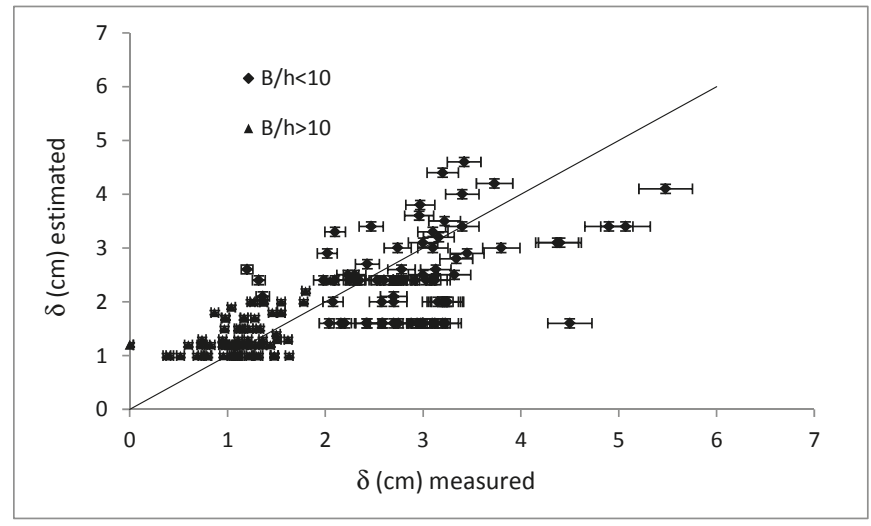

Figure 6. Comparison between estimated and measured $\delta$-values.

\section{Discussion and Concluding Remarks}

The mechanism of the dip phenomenon is investigated in a high-amplitude meandering channel, which is characterized by radii of curvature continuously variable in stream-wise direction, and, as previous findings indicate (see as an example [31,32]), convective flow accelerations occur and the entity of secondary motion changes along the bend modifying the turbulent activity at the channel's boundaries.

As it should be clear from the "Introduction", previous researches that were conducted in straight channels relate the velocity-dip to the secondary currents occurring especially in narrow channels where the side-walls effect is strong. Experiments (see among others in $[2,13]$ ) show that in narrow channels the side-walls produce anisotropy of turbulence but the velocity-dip is also determined by the free-surface effect. In wide channels, there is a central area where the side-wall effect disappears and only the free-surface effect occurs.

The results obtained in this work have shown that the velocity-dip phenomenon is more accentuated for low aspect ratio $(B / h<10)$, but it is also present for large aspect ratio $(B / h>10)$. In particular, in the first case, the velocity-dip, $\delta$, assumes always significant values, but it varies along the bend. In particular, at the inflection section upstream it is more accentuated close to the left (outer) bank; then at the apex section high values of the velocity-dip occur almost in the whole cross section until that at the inflection section downstream $\delta$ assumes the higher values close to the right (inner) bank. For high aspect ratio, the velocity-dip assumes always very small values and, in accordance with results obtained in straight channels, the dip-phenomenon assumes the lower values especially in the 
central region of the cross section. Furthermore, the variation of the $\delta$-values along the bend that were observed for high aspect ratio is less evident than that observed for low aspect ratio.

Termini ([32]), by using data collected in the same channel as that considering in the present work for $B / h<10$, verified that, as the curvature increases along the bend, the advective momentum transport by the cross-circulation increases determining the deformation of the vertical distribution of the downstream flow velocity (see also in [30]). This behavior suggests that the evolution of the velocity-dip phenomenon along the bend is strongly related to the evolution of the cross-sectional secondary motion.

The results have also shown that the entropic model allows for adequately estimating the velocity-dip, especially in the case of $B / h<10$. This allows for us to evaluate the velocity profile and the dip phenomenon, starting from the acknowledgment of the measured surface velocity and by considering the easily measurable parameters.

In conclusion, the reported results confirm that the velocity-dip varies along the channel depending on the variation of the secondary motion; the velocity-dip could be easily estimated through the application of the entropy model.

Based on the obtained results, future research will be oriented towards estimating the relation between the secondary motion and the entropy-evaluated velocity-dip.

Author Contributions: For research articles with several authors, a short paragraph specifying their individual contributions must be provided. The following statements should be used "D.T. conceived and designed the experiments; D.T. performed the experiments; T.M. contributed providing numerical tool; D.T. and T.M. analyzed the data; D.T. and T.M. wrote the paper." Authorship must be limited to those who have contributed substantially to the work reported.

Conflicts of Interest: The authors declare no conflict of interest.

\section{References}

1. Song, T.; Graf, W.H. Velocity and turbulence distribution in unsteady open-channel flows. J. Hydraul. Eng. 1996, 122, 141-154. [CrossRef]

2. Nezu, I.; Nakagawa, H. Turbulence in Open-Channel Flows; Balkema, A.A., Ed.; CRC Press: Rotterdam, The Netherlands, 1993.

3. Coles, D. The low of the wake in the turbulent boundary layer. J. Fluid Mech. 1956, 1, 191-226. [CrossRef]

4. Finley, P.J.; Phoe, K.C.; Poh, C.J. Velocity measurements in a thin turbulent water layer. La Houille Blanche 1966, 6, 713-721. [CrossRef]

5. Kironoto, B.A.; Graf, W.H. Turbulence Characteristic in Rough Uniform Open-Channel Flow; Laboratoire de Researches Hydrauliques, Ecole Polytechnique Federale: Lausanne, Switzerland, 1994.

6. Guo, J.; Julien, P.Y. Application of the modified log-wake law in open-channels. J. Appl. Fluid Mech. 2008, 1, 17-23.

7. Cioffi, F.; Gallerano, F. Velocity and concentration profiles in a channel with movable end erodible bed. J. Hydraul. Res. 1991, 29, 387-401. [CrossRef]

8. Da Silva, M.F.; Kanani, A. A study of large-scale horizontal; in alluvial streams with a view towards its morphological consequences. In Free Surface Flows and Transport Processes, GeoPlanet: Earth and Planetary Sciences; Springer: Berlin, Germany, 2018; pp. 1-25.

9. Najafabadi, E.F.; Afzalimehr, H.; Rowinski, P.M. Flow structure through a fluvial pool-riffle sequence-Case study. J. Hydro-Environ. Res. 2018, 19, 1-15. [CrossRef]

10. Flores, O.; Riley, J.J.; Horner-Devine, A.R. On the dynamics of turbulence near a free surface. J. Fluid Mech. 2017, 821, 248-265. [CrossRef]

11. Troiani, G.; Cioffi, F.; Casciola, C. Free surface-vorticity interactions in an open channel flow. J. Hydraul. Eng. 2004, 130, 313-323. [CrossRef]

12. Steffler, P.M.; Rajaratnam, N.; Peterson, A.W. LDA measurements in open channel flow. J. Hydraul. Eng. ASCE 1985, 111, 119-130. [CrossRef]

13. Nezu, I.; Rodi, W. Open channel flow measurements with a laser Doppler anemometer. J. Hydraul. Eng. ASCE 1986, 112, 335-355. [CrossRef] 
14. Greco, M.; Moramarco, T. Influence of bed roughness and cross section geometry on medium and maximum velocity ratio in open-channel flow. J. Hydraul. Eng. ASCE 2016, 142, 06015015. [CrossRef]

15. Sarma, K.V.N.; Lakshminarayana, P.; Lakshmana Rao, N.S. Velocity distribution in smooth rectangular open channel. J. Hydraul. Eng. ASCE 1983, 109, 270-289. [CrossRef]

16. Yang, S.Q.; Tan, S.K.; Lim, S.Y. Velocity distribution and dip phenomenon in smooth uniform open channel flow. J. Hydraul. Eng. ASCE 2004, 130, 1179-1186. [CrossRef]

17. Yan, J.; Tang, H.; Xiao, Y.; Li, K.; Tian, Z. Experimental study on influence of boundary on location of maximum velocity in open channel flows. Water Sci. Eng. 2011, 4, 185-191.

18. Bonakdari, H.; Larrarte, F.; Lassabatere, L.; Joannis, C. Turbulent velocity profile in fully-developed open channel flows. Environ. Fluid Mech. 2008, 8, 1-17. [CrossRef]

19. Absi, R. An ordinary differential equation for velocity distribution and dip-phenomenon in open channel flows. J. Hydraul. Res. 2011, 49, 82-89. [CrossRef]

20. Kundu, S. Prediction of velocity-dip-position over the entire cross section of open channel flows using entropy theory. Environ. Earth 2017, 76, 363. [CrossRef]

21. Stearns, E.P. A reason why the maximum velocity of water flowing in open channels is below the surface. Trans. ASCE 1983, 7, 331-338.

22. Cardoso, A.H.; Graf, W.H.; Gust, G. Uniform flow in a smooth open channel. J. Hydraul. Res. 1989, 27, 603-616. [CrossRef]

23. Yang, S.Q.; Lee, J.W. Reynolds shear stress distributions in a gradually varied flow. J. Hydraul. Res. 2007, 45, 462-471. [CrossRef]

24. Moramarco, T.; Barbetta, S.; Tarpanelli, A. From Surface Flow Velocity Measurements to Discharge Assessment by the Entropy Theory. Water 2017, 9, 120. [CrossRef]

25. Chiu, C.L. Application of Entropy Concept in open-channel flow study. J. Hydraul. Eng. ASCE 1991, 117, 615-628. [CrossRef]

26. Xia, R. Relation between mean and maximum velocities in a natural river. J. Hydraul. Eng. ASCE 1997, 123, 720-723. [CrossRef]

27. Moramarco, T.; Saltalippi, C.; Singh, V.P. Estimation of mean velocity in natural channel based on Chiu's velocity distribution equation. J. Hydraul. Eng. ASCE 2004, 9, 42-50. [CrossRef]

28. Moramarco, T.; Termini, D. Entropic approach to estimate the mean flow velocity:experimental investigation in laboratory flumes. Environ. Fluid Mech. 2015, 15, 1163-1179. [CrossRef]

29. Termini, D.; Moramarco, T. Application of entropic approach to estimate the mean flow velocity and Manning roughness coefficient in a high-curvature flume. Hydrol. Res. Dec. 2016, 2016. [CrossRef]

30. Blanckaert, K.; Graf, W.H. Momentum transport in sharp open-channel bends. J. Hydraul. Eng. ASCE 2004, 130, 186-198. [CrossRef]

31. Termini, D.; Piraino, M. Experimental analysis of cross-sectional flow motion in a large amplitude meandering bend. Earth Surf. Process. Landf. 2011, 36, 244-256. [CrossRef]

32. Termini, D. Momentum transport and bed shear stress distribution in a meandering bend: Experimental analysis in a laboratory flume. Adv. Water Resour. 2015. [CrossRef]

33. Termini, D. Experimental observations of Flow and Bed processes in a Large-amplitude Meandering Flume. J. Hydraul. Eng. ASCE 2009, 135, 575-587. [CrossRef]

34. Yalin, M.S. River Mechanics; Pregamon Press: London, UK, 1992.

35. Hooke, R.L. Distribution of sediment transport and shear stress in a meander bend. J. Geol. 1975, 83, 543-565. [CrossRef]

36. Whiting, P.J.; Dietrich, W.E. Experimental Studies of Bed Topography and Flow Patterns in Large-Amplitude Meanders, 1. Observations. Water Resour. Res. 1993, 29, 3605-3614. [CrossRef]

37. Da Silva, A.M.F.; El-Tahawy, T.; Tape, W.D. Variations of flow pattern with sinuosity in sine-generated meandering streams. J. Hydraul. Eng. ASCE 2006, 132, 1003-1014. [CrossRef]

38. Herschy, R.W. Streamflow Measurement; Elsevier: London, UK, 1985.

(C) 2018 by the authors. Licensee MDPI, Basel, Switzerland. This article is an open access article distributed under the terms and conditions of the Creative Commons Attribution (CC BY) license (http:/ / creativecommons.org/licenses/by/4.0/). 
Article

\title{
Turbulence Measurement of Vertical Dense Jets in Crossflow
}

\author{
Mouldi Ben Meftah * and Michele Mossa \\ Department of Civil, Environmental, Land, Building Engineering and Chemistry, Polytechnic University of Bari, \\ Via E. Orabona 4, 70125 Bari, Italy; michele.mossa@poliba.it \\ * Correspondence: mouldi.benmeftah@poliba.it; Tel.: +39-080-596-3508
}

Received: 29 January 2018; Accepted: 6 March 2018; Published: 8 March 2018

\begin{abstract}
Turbulence measurement of a dense jet perpendicularly issued into a crossflow is investigated experimentally. The flow-velocity components were extensively measured with a high frequency Acoustic Doppler Velocimeter (ADV) system, whereas, a Micro Scale Conductivity Temperature instrument was used to measure the jet salinity. Special attention is given to understand the jet flow-structures in the flow symmetry plane. The flow velocity-fields, the jet trajectory, the turbulence intensities, the turbulent kinetic energy, the turbulent length scales, and the dispersion coefficients have been analyzed. The flow velocity-fields show that the dense jet is characterized by two distinct regions: an ascending region, of jet-like mixing, and a descending region of plume-like mixing. In this study, a new scaling approach of the jet trajectories, based on the jet characteristic length scales, is proposed, leading to an empirical closed-form expression to predict the jet trajectory. The turbulence analysis shows that the jet is accompanied by high levels of flow-turbulence intensities and large kinetic energy production. The results of the turbulent length scales indicate that the ambient flow-field, without jet effect, is an isotropic process. However, in the jet flow-field, a significant spatial-variation of the turbulent length scales was observed, indicating an anisotropic process. The trends of the dispersion coefficients follow those of the turbulent length scales. In comparison with the ambient flow, the jet flow-field shows a decrease of the longitudinal dispersion coefficient and an increase of the vertical one, leading to the increase of the jet width.
\end{abstract}

Keywords: dense jet; current flow; velocity; trajectory; turbulence; dissipation

\section{Introduction}

The mixing of discharged fluids with a surrounding natural fluid is of primary concern for a wide range of engineering and environmental applications, i.e., the discharge of wastewater in receiving natural water bodies, the ascending motions of thermals in the atmosphere, the dynamic of volcanic eruption columns, the fuel injection in combustion chambers, the engineering propulsion systems, the propagation of smoke in free or enclosed spaces [1]. Brine discharge from desalination plants, as an example, is the concern of many countries around the world. Many nations, having very scarce water resources or suffer water shortages, are being forced to develop alternative sources derived from seawater desalination to meet the demand for freshwater. The brine is typically discharged into a receiving water body (coastal water, ocean, river, lake) as a turbulent jet (Figure 1) [2], reflecting a number of complex hydrodynamic phenomena and affecting the ecosystem services. Discharge systems need to be well designed to achieve a rapid mixing of the discharged brine, reducing environmental impacts. Therefore, a good knowledge of the interaction between a discharge system (e.g., dense jet) and a receiving water environment is required to promote best environmental management practices. 


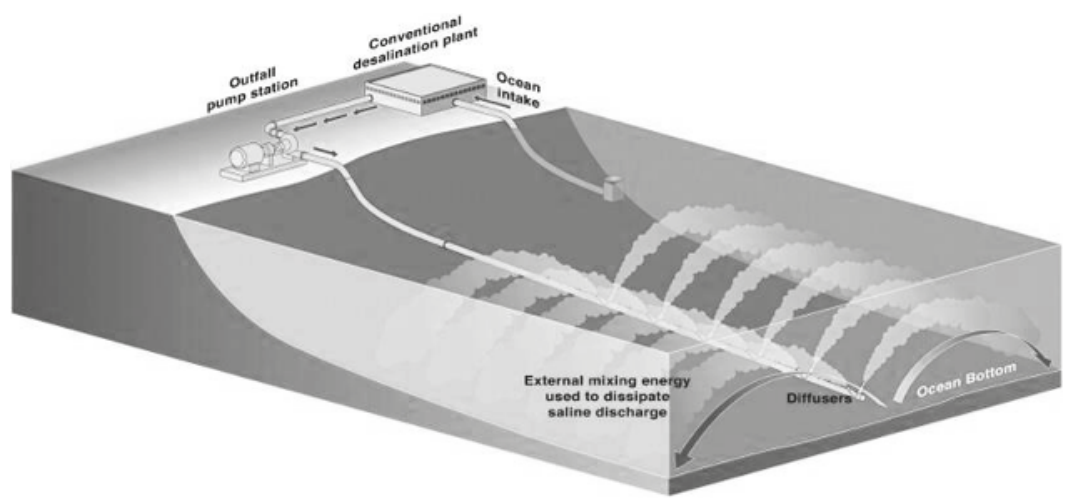

Figure 1. Schema of a brine discharge system at a desalination plant [2].

The effluent jet properties are usually different than the environmental water properties, i.e., the temperature, the density, the salinity, the chemical composition, the buoyancy-induced rise or fall of the discharged flow. Since all components of aquatic ecosystems are very sensitive to any water quality changes, a rapid and maximum dilution of the jet discharge within the ambient flow is a key solution to keep the ecosystem healthy and sustainable. Mixing of turbulent jets within surrounding environment was the topic of numerous experimental, analytical and numerical studies [3-18].

Using a three-dimensional laser-induced fluorescence (3DLIF), Gungor and Roberts [19] carried out a series of laboratory experiments on the dilution of vertical dense jets in a flowing current. The investigators observed that the jet reveals very complex flows, spatially varying and depend on the current speeds $u_{r} F$, where $u_{r}$ is the ratio of ambient to jet velocity and $F$ is the jet densimetric Froude number. These complex phenomena make the jet difficult to numerically manage. Thanks to the extensive jet-concentration measurements, the authors obtained a detailed three-dimensional picture of the jet structure within the surrounding environmental flow. They indicated that, at low speeds $\left(u_{r} F<0.5\right)$, the jet descending flow is strongly asymmetric and of complex hydrodynamic structure, experiencing a sharp curvature at its terminal rise height. At $u_{r} F=0.9$, a slight asymmetry occurs in the ascending region, whereas, an almost vertical symmetry of the jet was observed in the descending region. Recently, Ben Meftah et al. [18], analyzing the same data of the present study but focusing on the vortical structure of the jet, definitely confirmed the formation of counter-rotating vortex pair (CRVP) through both the ascending and descending jet regions, a topic of conjecture in many previous studies without any experimental demonstration.

Both studies of Gungor and Roberts [19] and Ben Meftah et al. [18] indicated that, at high speeds $\left(u_{r} F>2\right)$, the jet undergoes rapid dispersion in the receiving water body by the effect of the ambient flow-turbulence. This significantly reduces the negative-buoyancy effect of the jet, leading to the disappearance of the descending region. At $u_{r} F>2$, the dense jet almost behaves as a momentum jet discharged into a crossflow $[5,14,20,21]$.

Analysis of vortex system of turbulent jets, issuing into a crossflow [5,6,22-25], suggested that the CRVP increase entrainment of the crossflow into the jet, leading to better jet mixing. Kaminski et al. [26] depicted that, at small scale, the agents of entrainment are turbulent eddies, which form a mixing layer between the jet and its surrounding flow. The diffusion/mixing processes strongly depend on the strain rates and/or scalar dissipation rates. Danckwerts [27] indicated that the mixing process is a breaking-up of a clump of fluid from large scales to smaller scales, leading to differences in scalar concentration values. The author pointed out that the mixing degree in flow fields can be mainly expressed by two statistically-defined quantities: a scale and an intensity of segregation. In the case of turbulent flows, the scale and intensity of segregation are analogous to the turbulent 
length-/time-scale and the turbulence intensity, respectively. The determination of such scales requires adequate measurements/simulations of the flow turbulence. Recently, Galeazzo et al. [28] have focused on the study of turbulent mixing within a jet in crossflow, comparing experimental results with those of numerical simulations. The authors used a combination between planar particle image velocimetry and laser induced fluorescence to measure the turbulent velocity and concentration flow fields. The comparison of their experimental results with simulated results, using RANS and LES, showed that the mean jet velocity field was well described, however, the turbulent quantities, such as the Reynolds stresses and the predicted turbulent mixing, were not in good agreement with the experiments.

Due to the complexity of turbulent jet flow structure and its challenging numerical simulation, in the present study, we will experimentally try to examine some turbulence characteristic parameters of a dense round jet vertically discharged into a flowing current. The flow velocity-fields, the jet trajectory, the turbulence intensity, the turbulent kinetic energy, the turbulent length-scales, and the dispersion coefficients will be specifically analyzed. These parameters are very helpful for validation and calibration of numerical models of dense jets.

\section{Theoretical Analysis}

An inclined dense jet discharged into a flowing current $[19,29,30]$ can almost be characterized by two distinct phases: (i) a rapid ascent phase; and (ii) a gradually descent phase [18]. During the ascent phase, the jet behaves as a momentum pure jet discharged into a crossflow. With a small value of $u_{r}$, the jet is only weakly affected near the exit, penetrating uprightly into the crossflow before bending over. During the descent phase, the jet changes to a kind of negatively buoyant jet/plume; its velocity significantly reduces, the downward buoyant forces cause the discharge to gradually fall back and its trajectory impacts the bottom at a downstream distance $x_{i}$. Near the bottom, the discharge laterally spreads in all directions, forming a bottom layer of spreading density current of thickness $z_{L}$.

Figure 2 shows a definition sketch of a typical dense jet discharged normally to a main shallow flow of depth $H$. The jet consists of a vertical (of an angle relative to the horizontal $\theta=90^{\circ}$ ) round nozzle, of a diameter $D$ and a port height $z_{0}$, which releases effluent of an initial density $\rho_{0}$ into a channel crossflow of fluid density $\rho_{a}$, with $\rho_{a}<\rho_{0}$. The jet discharges at an initial velocity $U_{0}$ in a uniform channel/ambient flow of mean velocity $U_{a}$. The effluent consists of saltwater solution of initial conductivity $c_{0}$ and that of the ambient flow is $c_{a}, c_{a}<c_{0}$. Figure 2 also shows the jet trajectory. In Figure $2, z_{t}$ indicates the maximum rise height of the jet trajectory occurred at the downstream distance $x_{t}$. All the basic symbols and the system of coordinates are clearly indicated in Figure 2 .

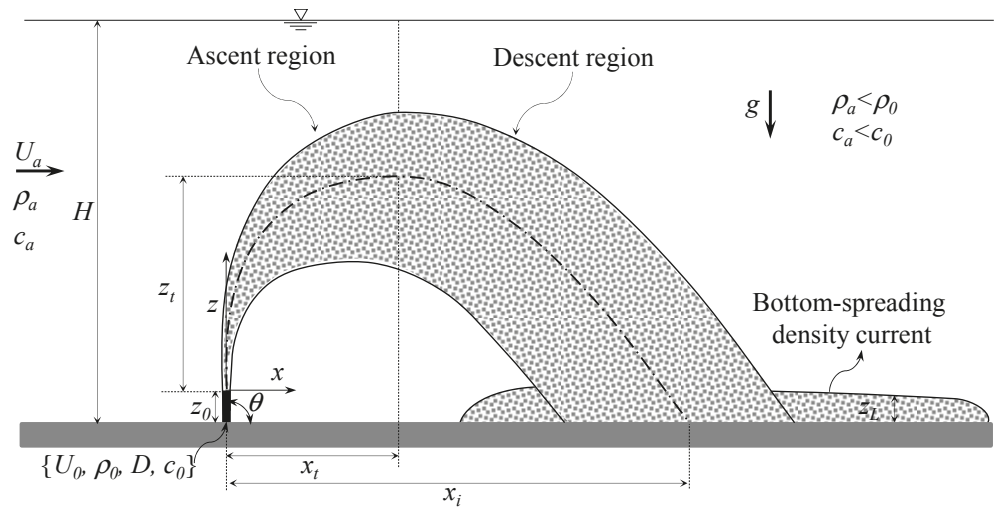

Figure 2. Definition sketch of the vertical dense jet in shallow water. The jet trajectory (jet axis or centerline) is indicated by the dashed-dot curve. 
The exact equations describing a negative buoyant jet flow are generally the unsteady Navier-Stokes and the concentration equations. Direct numerical resolution of turbulent jet flow, at the small-scale motions, remains a challenge for engineers and scientists. The statistical approach, however, is more feasible, averaging the motion equations over time. The continuity equation is:

$$
\frac{\partial\left(\rho U_{i}\right)}{\partial x_{i}}=0
$$

The momentum equations are:

$$
\rho\left(\frac{\partial U_{i}}{\partial t}+U_{j} \frac{\partial U_{i}}{\partial x_{j}}\right)=\frac{\partial}{\partial x_{j}}\left(\mu \frac{\partial U_{i}}{\partial x_{j}}+\tau_{i j}\right)-\frac{\partial p}{\partial x_{i}}+\rho g_{i}
$$

The time-averaged concentration equation, neglecting the molecular diffusion against the turbulent diffusion, is:

$$
\frac{\partial C}{\partial t}+\frac{\partial U_{i} C}{\partial x_{i}}=-\frac{\partial}{\partial x_{i}}\left(U_{i}^{\prime} C^{\prime}\right)=\frac{\partial}{\partial x_{i}}\left(K_{i} \frac{\partial C}{\partial x_{i}}\right)
$$

where $\rho$ is the fluid density, $U_{i}(=U, V, W)$ are the mean velocity components in the $x_{i}(=x, y, z)$ directions, in which $x, y$ and $z$ are the streamwise, the spanwise and the vertical directions, respectively, $t$ is the time, $\mu$ is the dynamic viscosity, $\tau_{i j}=-\rho U_{i}{ }^{\prime} U_{j}^{\prime}$ is the time-averaged stress of $u_{i}^{\prime} u_{j}^{\prime}(t)$ over the length of the time series, $p$ is the fluid pressure, $g^{\prime}=\left[\left(\rho_{0}-\rho\right) / \rho\right] g$ is the reduced gravity, $g$ is the gravity acceleration, $C$ is the mean fluid concentration, $U^{\prime} C^{\prime}$ is the time-averaged concentration transport by turbulent velocity fluctuations $u_{i}^{\prime} c(t)$ over the length of the time series and $K_{i}\left(=K_{x}, K_{y}, K_{z}\right)$ is the dispersion coefficient in the $x_{i}(=x, y, z)$ directions, respectively. The instantaneous velocity and concentration are defined as $u_{i}(t)=U_{i}+u_{i}{ }^{\prime}(t)$ and $c(t)=C+c^{\prime}(t)$, where $u_{i}=(u, v, w)$ in the $x, y$ and $z$ directions, respectively, and $u_{i}{ }^{\prime}=\left(u^{\prime}, v^{\prime}, w^{\prime}\right)$ is its fluctuation, while $c^{\prime}$ is the concentration fluctuation. The second member of the left-hand side of Equation (3) describe the advection of the solute in the three directions. The dispersion coefficient in the right-hand side of Equation (3) aggregate all the turbulent diffusion in the three directions.

At high Reynolds numbers, the turbulence is assumed to be at equilibrium, i.e., the production term is of order of the dissipation term. The turbulent kinetic energy of velocity fluctuations cascades from large to small scales of motion. In the analysis of the flow-dispersion interaction, the turbulent kinetic energy (TKE) is important in determining the turbulent dispersion coefficient and thus the mass transport [31]. At equilibrium, the transport equation of the turbulent kinetic energy is expressed as:

$$
\frac{\partial k}{\partial t}+\frac{\partial U_{i} k}{\partial x_{i}}=-\frac{\partial}{\partial x_{i}}\left(\frac{1}{\rho} U_{i}^{\prime} P^{\prime}+U_{i}^{\prime} k^{\prime}\right)=\frac{\partial}{\partial x_{i}}\left(D_{i} \frac{\partial k}{\partial x_{i}}\right)
$$

where $k=1 / 2 U_{i}{ }^{\prime} U_{i}{ }^{\prime}$ is the time-averaged turbulent kinetic energy of $1 / 2 u_{i}{ }^{\prime} u_{i}{ }^{\prime}(t)$ over the length of the time series, $U_{i}{ }^{\prime} P^{\prime}$ is the time-averaged pressure diffusion of $u_{i}{ }^{\prime} p^{\prime}(t), U_{i}{ }^{\prime} k^{\prime}$ is the time-averaged turbulent transport of $1 / 2 u_{i}^{\prime} u_{i}^{\prime} u_{i}^{\prime}(t)$ and $D_{i}$ is the turbulent diffusion coefficient.

The dispersion coefficients, $K_{i}$, and the turbulent diffusion coefficients, $D_{i}$, are strongly flow dependent, varying with the flow field, especially in the case of a dense jet discharged into a flowing current. These coefficients are properties of the flow. Therefore, the obvious question that arises for any numerical simulation of the dense jet is: what are the values of these coefficients in any particular situation and positions within the jet/ambient flows and how do they depend on the measured mean properties of the flow.

Since the dimensions of $D_{i}$ must be length squared per time, lending to a product of a velocity scale and a length scale, a physical meaningful expression of $D_{i}$ can be obtained as: 


$$
D_{i} \sim(k)^{\frac{1}{2}} L_{i}
$$

where $L_{i}$ is a characteristic eddy length scale and $(k)^{1 / 2}$ is a turbulent velocity scale.

Analogous to the expression of $D$ in Equation (5), it is also possible to assume that the net dispersion coefficients of Equation (3) could be proportional to:

$$
K_{i} \sim(k)^{\frac{1}{2}} L_{i}
$$

On another hand, by assuming that the Boussinesq approximation is valid and the flow is fully turbulent, the initial properties of an inclined dense jet discharged into a cross-flow is characterized by its discharge volume flux $Q_{0}$, its momentum flux $M_{0}$ and its buoyancy flux $B_{0}$, defined as:

$$
Q_{0}=U_{0} A_{0} ; M_{0}=Q_{0} U_{0} ; B_{0}=g^{\prime} Q_{0}
$$

where $A_{0}=\pi D^{2} / 4$ is the jet source area. These fluxes can be combined with the ambient velocity, $U_{a}$, to provide some relevant length scales, such as:

$$
l_{M}=\frac{M_{0}^{3 / 4}}{B_{0}^{1 / 2}} ; \quad l_{m}=\frac{M_{0}^{1 / 2}}{U_{a}} ; \quad l_{Q}=\frac{Q_{0}}{M_{0}^{1 / 2}}
$$

Briefly, $l_{M}$ is the jet-to-plume length and measures the relative importance of the initial momentum flux against the buoyancy flux, differentiating the region of jet-like mixing dominance from the region of buoyancy dominance (for $z / L_{m}<<1$, as an example, the initial momentum effect will be dominant over the bouncy effec), $l_{m}$ is the jet-to-crossflow length scale and measures the relative importance of the initial excess momentum flux to the ambient flow-velocity (for $z / l_{m}<<1$, the jet velocity is so much higher than the ambient velocity and then a momentum jet will be vertically grown within the ambient flow), and $l_{Q}$ is the discharge length scale and indicates the distance over which the volume flux of the entrained ambient fluid becomes approximately equal to the initial volume flux (for $z / l_{Q}<10$, the jet diameter will have a direct effect on the flow characteristics) [19,32].

Defining a velocity scale [19] as:

$$
U_{c}=\frac{B_{0}^{1 / 2}}{M_{0}^{1 / 4}}
$$

For vertical jets ( $\theta$ is invariant) discharged in large channels (with no effect of side walls on the jet), any dimensionless jet property $\Phi$ can be expressed as:

$$
\Phi=f\left(\frac{l_{M}}{l_{Q}}, \frac{U_{a}}{U_{c}}\right)
$$

The ratio $l_{M} / l_{Q}$ in Equation (10) is proportional to the jet densimetric Froude Number $F$ as:

$$
\frac{l_{M}}{l_{Q}}=\left(\frac{\pi}{4}\right)^{-1 / 4}\left(\frac{U_{0}}{\left(D g^{\prime}\right)^{1 / 2}}\right)^{1}=\left(\frac{\pi}{4}\right)^{-1 / 4} F
$$

According to Gungor and Roberts [19], the dynamical effect of the ambient current is mainly determined by the ratio of the ambient velocity to the characteristic velocity $U_{a} / U_{c}$, which is also equal to $l_{M} / l_{m}$. For round jet nozzle this ratio can be expressed as:

$$
\frac{U_{a}}{U_{c}}=\left(\frac{\pi}{4}\right)^{-1 / 4} u_{r} F
$$


Equations (10)-(12) clearly indicate that all the dependent geometric scales of the flow, such as $x_{t}$, $x_{i}, z_{t}$ can therefore be written as:

$$
\frac{x_{t}}{D F}, \frac{x_{i}}{D F}, \frac{z_{t}}{D F}=f\left(F, u_{r} F\right)
$$

taking into account that $l_{M}$ is proportional to $D F$.

Gungor and Roberts [19] and Roberts and Toms [33] observed that, for $l_{Q}<<l_{M}(F>>1)$, the dynamic effect of the source volume flux becomes negligible, and then $F$ does not appear as an individual variable. After these assumptions Equation (13) becomes:

$$
\frac{x_{t}}{D F}, \quad \frac{x_{i}}{D F}, \quad \frac{z_{t}}{D F}=f\left(u_{r} F\right)
$$

\section{Experimental Method}

Experiments were carried out in a closed circuit flume, specifically designed for the study of buoyant jets, at the Coastal Engineering Laboratory (LIC) of the Department of Civil, Environmental, Land, Building Engineering, and Chemistry of the Polytechnic University of Bari (Italy). The system consists of a rectangular channel made of glass, $15 \mathrm{~m} \mathrm{long}, 4 \mathrm{~m}$ wide and $0.4 \mathrm{~m}$ deep (Figure 3). The fresh water at ambient temperature is supplied from a downstream big metallic tank, by means a centrifugal electro-pump to an upstream steel tank. The upstream tank is equipped with a side-channel spillway with adjustable height in order to maintain constant flow-head condition. The overflowing water reaches again the downstream tank. The pumped and overflowed flow rates are measured by means two electromagnetic flow meters, mounted on the channel system. To create a smooth flow transition, damping inlet turbulence, from the upstream tank to the flume, a set of stilling grids were installed in the outlet of the upstream tank.

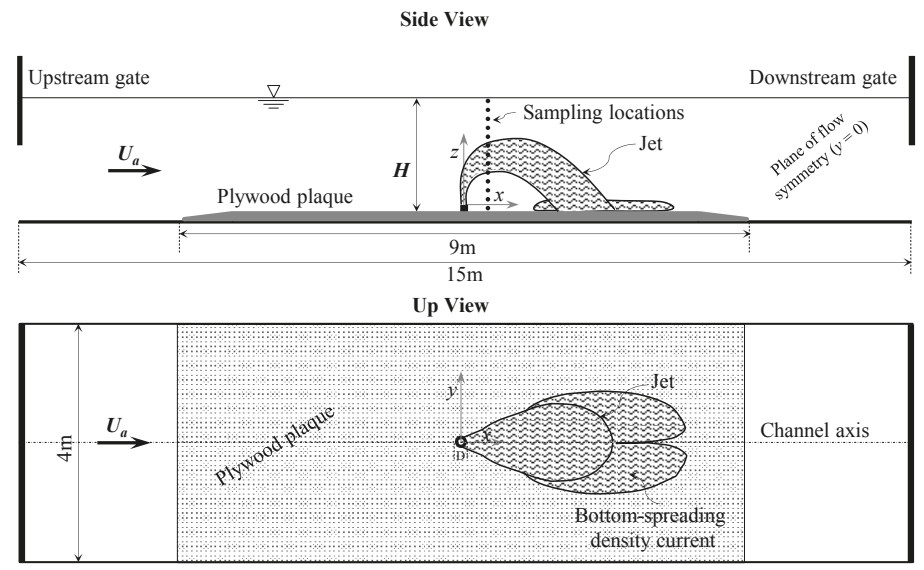

Figure 3. General sketch of the laboratory flume with the jet flow. For the sake of simplicity, only one vertical profile is qualitatively presented to show the sampling locations, the same sampling locations are repeated at different downstream locations $x / D$ along the plane of flow symmetry.

The second part of the laboratory model consists of a dense jet hydraulic system. To mix tap/fresh water with salt $(\mathrm{NaCl})$, a large cylindrical storage tank made by fiberglass, of maximum volume $6 \mathrm{~m}^{3}$, is used. The tank is equipped with four compressed air jets, installed on two levels at opposite positions, to mix the salt with the fresh water. The saltwater is pumped through a pipeline to the jet nozzle. This pipeline is equipped with a regulating valve and a magnetic flow meter to provide a well-defined flow discharge. The jet nozzle is of diameter $D=10 \mathrm{~mm}$, vertically mounted in the flume center at a port height $z_{0}=10 \mathrm{~mm}$ above the flume bottom. 
In order to elucidate the three-dimensional characteristics of the vertical dense jet, both the velocity and the salinity flow fields were measured at several sampling locations along the plane of flow symmetry $(y=0)$ and at different cross-sections downstream the jet source $(x=0)$. Since the grid spacing of samples is $\mathrm{O}(1 \mathrm{~cm})$, the measuring instruments are attached to a semi-automatic cross-beam to accurately move and align the different probes at each sampling location. The salinity field was measured by means of a Micro Scale Conductivity Temperature Instrument (MSCTI) by Precision Measurement Engineering (PME), designed to measure the temperature and electrical conductivity of water solutions and moving fluids, containing conductive ions. Before starting acquiring data, the probe could be calibrated using a salt solution, of known salinity, prepared in laboratory. At each measurement location, 1000 acquisitions of the water conductivity were carried out at $20 \mathrm{~Hz}$. The velocity data were collected using a 3-D Acoustic Doppler Velocimeter (ADV)-Vectrino system, developed by Nortek, for $60 \mathrm{~s}$ at a sampling rate of $150 \mathrm{~Hz}$. The sampling volume of the ADV was located $5 \mathrm{~cm}$ below the transmitter probe. The Vectrino was used with a velocity range equal to $\pm 0.30 \mathrm{~m} / \mathrm{s}$, a measured velocity accuracy of $\pm 0.5 \%$, a sampling volume of vertical extent of $7 \mathrm{~mm}$. For high-resolution measurements, the manufacturer recommends a $15 \mathrm{db}$ signal-to-noise ratio (SNR) and a correlation coefficient larger than $70 \%$. The acquired data were filtered based on the Tukey's method and the bad samples (SNR $<15 \mathrm{db}$ and correlation coefficient $<70 \%$ ) were also removed. Additional details concerning the channel setup and the ADV operations can be found elsewhere in [34-38].

The initial experimental conditions and some parameters of the performed experimental runs are reported in Table 1, where $T$ is the water temperature. In addition to the experimental data of the present study, in Table 1, the data obtained by Gungor and Roberts [19] for ten other different configurations, of various values of $u_{r} F$, are also illustrated.

Table 1. Initial experimental conditions and parameters of the investigated runs.

\begin{tabular}{|c|c|c|c|c|c|c|c|c|c|c|c|c|c|c|}
\hline & \multirow[t]{2}{*}{ Runs } & \multirow[t]{2}{*}{$\begin{array}{c}H \\
(\mathrm{~cm})\end{array}$} & \multirow[t]{2}{*}{$\begin{array}{c}T \\
\left({ }^{\circ} \mathrm{C}\right)\end{array}$} & \multirow[t]{2}{*}{$\begin{array}{c}U_{a} \\
(\mathrm{~cm} / \mathrm{s})\end{array}$} & \multirow[t]{2}{*}{$\begin{array}{c}U_{0} \\
(\mathrm{~cm} / \mathrm{s})\end{array}$} & \multirow[t]{2}{*}{$u_{r}(-)$} & \multirow[t]{2}{*}{$F(-)$} & \multirow[t]{2}{*}{$u_{r} F(-)$} & \multicolumn{2}{|c|}{$x_{t} /(D F)(-)$} & \multicolumn{2}{|c|}{$x_{i} /(D F)(-)$} & \multicolumn{2}{|c|}{$z_{t} /(D F)(-)$} \\
\hline & & & & & & & & & ${ }^{1}$ Sal. & ${ }^{2}$ Vel. & ${ }^{1}$ Sal. & ${ }^{2}$ Vel. & ${ }^{1}$ Sal. & ${ }^{2}$ Vel. \\
\hline \multirow{5}{*}{ 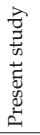 } & R1 & 36 & 16 & 4.17 & 31.83 & 0.131 & 7.7 & 1.006 & 1.28 & 1.52 & 4.16 & 4.29 & 1.51 & 1.57 \\
\hline & $\mathrm{R} 2$ & 36 & 16 & 4.17 & 38.20 & 0.109 & 9.8 & 1.071 & 1.48 & 1.41 & 4.07 & 4.34 & 1.46 & 1.62 \\
\hline & $\mathrm{R} 3$ & 36 & 14 & 4.17 & 44.56 & 0.093 & 11.4 & 1.069 & NI & 1.68 & NI & 4.25 & NI & 1.59 \\
\hline & $\mathrm{R} 4$ & 36 & 14 & 4.17 & 50.93 & 0.082 & 13.1 & 1.069 & NI & 1.48 & NI & 4.27 & NI & 1.60 \\
\hline & R5 & 28 & 13 & 8.93 & 31.83 & 0.280 & 8.2 & 2.287 & NI & NI & NI & $\mathrm{NI}$ & NI & $\mathrm{NI}$ \\
\hline \multirow{10}{*}{ 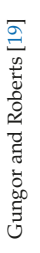 } & DJV01 & NI & NI & NI & $\mathrm{NI}$ & 0.025 & 20.9 & 0.522 & NI & NI & 2.87 & NI & NI & NI \\
\hline & DJV02 & NI & NI & NI & NI & 0.025 & 20.1 & 0.511 & NI & NI & 3.01 & NI & NI & NI \\
\hline & DJV03 & NI & NI & NI & NI & 0.044 & 20.7 & 0.915 & NI & NI & 6.12 & NI & NI & NI \\
\hline & DJV04 & NI & NI & NI & NI & 0.010 & 23.0 & 0.233 & NI & NI & 1.16 & NI & NI & NI \\
\hline & DJV05 & NI & NI & NI & NI & 0.010 & 22.5 & 0.232 & NI & NI & 1.49 & NI & NI & NI \\
\hline & DJV06 & NI & NI & NI & NI & 0.036 & 19.0 & 0.692 & NI & NI & 5.24 & NI & NI & NI \\
\hline & DJV07 & NI & NI & NI & NI & 0.010 & 23.7 & 0.243 & NI & NI & 1.36 & NI & NI & NI \\
\hline & DJV08 & NI & NI & NI & NI & 0.017 & 21.6 & 0.373 & NI & NI & NI & NI & NI & NI \\
\hline & DJV09 & NI & NI & NI & NI & 0.010 & 21.5 & 0.220 & NI & NI & 1.32 & NI & NI & NI \\
\hline & DJV10 & NI & NI & NI & NI & 0.010 & 20.9 & 0.213 & NI & NI & 0.75 & $\mathrm{NI}$ & NI & NI \\
\hline
\end{tabular}

Notes: ${ }^{1}$ Salinity; ${ }^{2}$ Velocity. NI stands for not identified.

\section{Results and Discussion}

\subsection{Jet Velocity Fields}

Since turbulence is the most important mechanism that leads to substantial mixing of the jet within the surrounding environment, dense measurements, in high frequency, of the ambient/jet flow velocity-field were carried out. Figures 4 and 5 , as an example, show the vertical profiles of the flow velocity at different downstream locations, $x / D$, away from the jet source. The profiles refer to runs $R 1$ and $R 2$ and represent the streamwise time-averaged velocity component, $U$, and the vertical time-averaged velocity component, $W$, both normalized by $U_{a}$. All of them were obtained in the plane of flow symmetry $(y=0)$. On Figures 4 and 5 , the jet axis positions, location of maximum jet 
velocity (velocity resultant of $U$ and $W$ ), are indicated by horizontal arrows. Note that, due to the flow symmetry, the spainwise velocity, $V$, is theoretically expected to be null and therefore it has not any physical significance in this plane.

The trend of the jet axis, shown by the arrows on Figures 4 and 5 , clearly indicates that the jet initially penetrates upward within the ambient current, attains a terminal rise height at the downstream distance $x_{t}$ (Figure 2) and then it inclines downward, showing two distinct characteristic regions: an ascending region followed by a descending one [18]. Figures $4 \mathrm{a}$ and $5 \mathrm{a}$ show that the trend of the vertical profile of $U / U_{a}$ gradually changes with the increase of $x / D$. In the ascending region $(x / D<12$ and 15 , respectively for $\mathrm{R} 1$ and $\mathrm{R} 2$ ), the $U / U_{a}$-profiles show two characteristic maxima: an absolute maximum, located within the jet flow-field slightly above the jet axis, and a local maximum, appeared in the jet wake-like region.

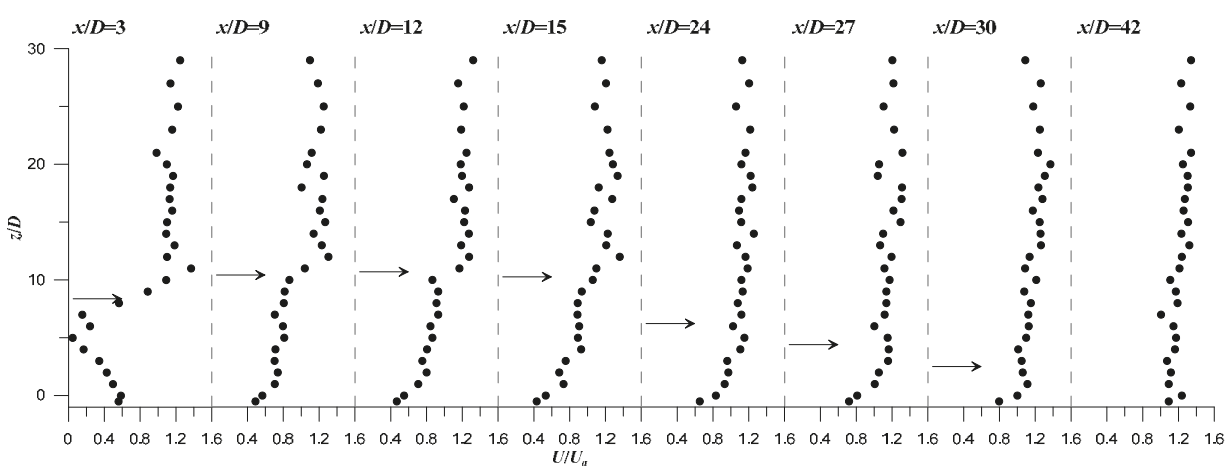

(a)

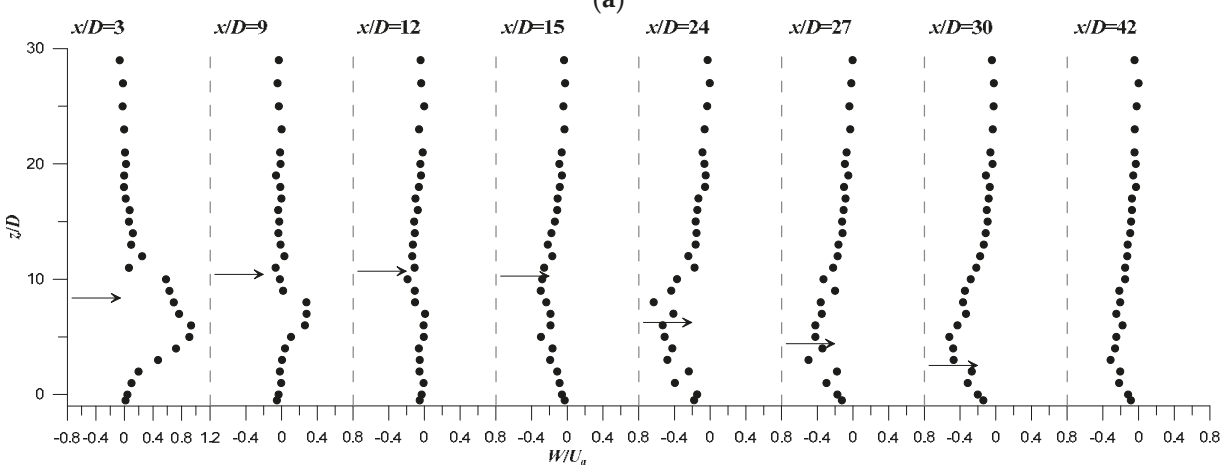

(b)

Figure 4. Normalized velocity profiles in the plane of flow symmetry $(y=0)$ of run R1 at $u_{r}=0.131$ and $u_{r} F=1.006$ : (a) $U / U_{a} ;$ (b) $W / U_{a}$. The horizontal arrow on the profiles indicate the position of the jet axis.

This finding is in good agreement with results obtained by Ben Meftah et al. [14] and Sherif and Pletcher [39] for momentum jets in crossflow. This suggests that, in the ascending region, the jet is primarily driven by momentum flux, behaving like a vertical non-buoyant jet in crossflow $[5,14,39]$. The velocity profiles in Figures $4 \mathrm{a}$ and 5 a show that the absolute maximum of $U / U_{a}$, in the jet flow-field, always appears slightly above the jet axis. The magnitudes of both maxima gradually decrease as $x / D$ increases, as clearly shown by the profiles of run R2. In the wake region, close to the jet source, the jet velocity $U / U_{a}$ experiences very small and negative values. At $x / D=3$, as an example, $U / U_{a} \approx 0.05$ and -0.1 with $\mathrm{R} 1$ and $\mathrm{R} 2$, respectively. This confirms the wake vortices formation at the inner part 
of the jet, as also mentioned in the previous study by Fric and Roshko [40]. For all the profiles at any position $x / D, U / U_{a}$ shows an almost constant value of order $1.2 U_{a}$, over the flow depth starting from the jet outer-boundary up to the free-surface flow. These results are in complete agreement with those obtained by Ben Meftah et al. [14] and Sherif and Pletcher [39], reflecting simply the vertical distribution of $U / U_{a}$ in the ambient current without the jet effects. In the descending region, the two velocity-maxima are less pronounced, and the $U / U_{a}$-profiles, at $x / D>30$, resemble a classical vertical profile of mean velocity in an open channel flow.

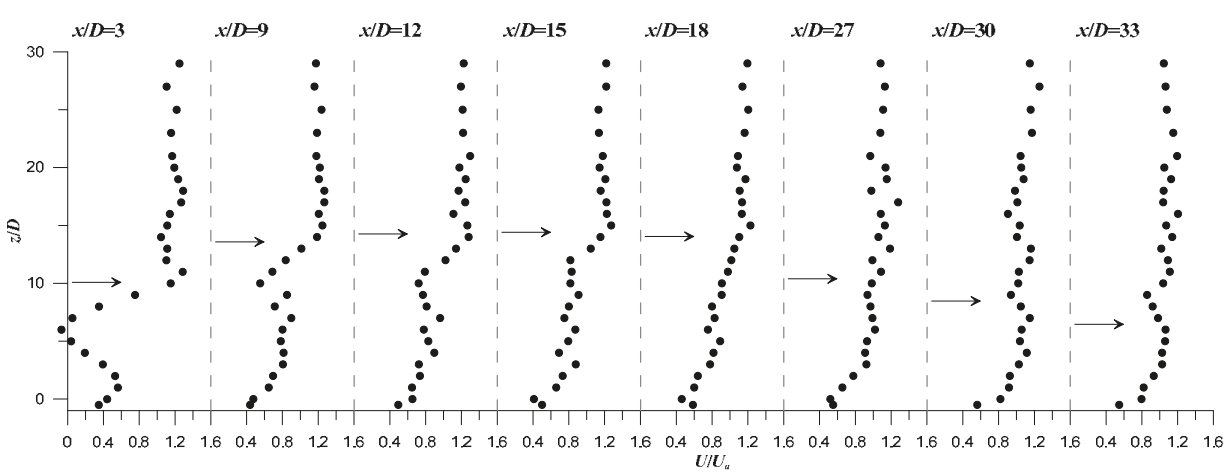

(a)

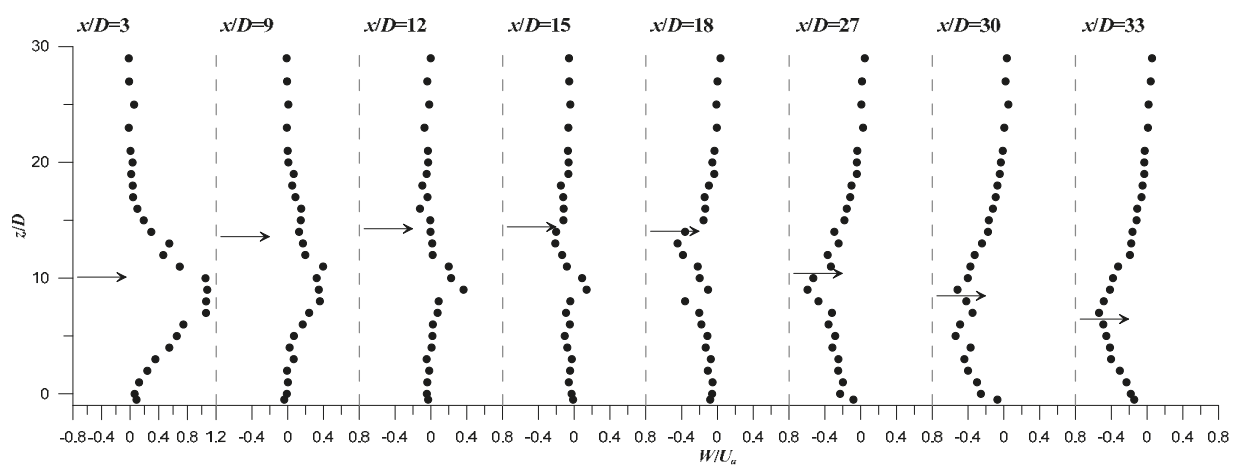

(b)

Figure 5. Normalized velocity profiles in the plane of flow symmetry $(y=0)$ of run R2 at $u_{r}=0.109$ and $u_{r} F=1.071:(\mathbf{a}) U / U_{a} ;(\mathbf{b}) W / U_{a}$.

Unlike the streamwise velocity component, the $W / U_{a}$-profiles are differently developed over the flow depth. They always experience a single peak below the jet axis. In the ascending region, $W / U_{a}$ shows positive values which gradually decrease as going further away from the jet source, similar to what happens with momentum jets in crossflows [14,39]. At the downstream distance $x_{t}$ (Figure 2), where the jet flow practically becomes horizontal, $W / U_{a}$ significantly reduces. In the descending region, $W / U_{a}$ shows negative values, indicating that the jet bends downward.

The jet trajectory is one of the most important parameter characterizing a jet discharged into a crossflow. It principally reflects the ability of the jet to penetrate within the ambient flow, revealing its spreading/mixing process. Despite the numerous previous studies conducted on turbulent jets, there is no recognized closed-form expression of the jet trajectory. Indeed, it was always observed some scatters between the different proposed scaled jet trajectories. According to Hasselbrink and Mungal [20], this scattering is partly due to the definitions of the jet trajectory and partly from opinions 
on the best form of the correlating equation. In the present study, the jet trajectory is defined as the curve formed by the locus of maximum velocity at the different downstream positions $x / D$, along the plane of flow symmetry, which is usually known as the jet axis, or the locus of the maximum concentration along the same plane, usually known as the jet centerline.

For momentum jets, Margason [41], as an example, reviewing several correlations of jet-trajectory coordinates, concluded that much of the revised data could be collapsed well by normalizing the jet-trajectory coordinates $x$ and $z$ with the ratio $D / u_{r}$, leading to a simple power-law in the form of $u_{r} z / D=\alpha\left(u_{r} x / D\right)^{\beta}$, where $\alpha$ and $\beta$ are coefficient determined experimentally.

Figure 6 shows the jet trajectories of runs R1 to R5 scaled with $D / u_{r}$ and plotted in $\log -\log$ scales. It is important to note here that these trajectories refer to the jet axis, as above defined. In addition to the data of the present study, the jet centerlines (as above defined) obtained by Gungor and Roberts [19] for a dense jet of five different values of $u_{r} F$ are also plotted. On Figure 6 we also plot some predicted and experimental trajectories obtained in previous studies [41-43] for momentum jets.

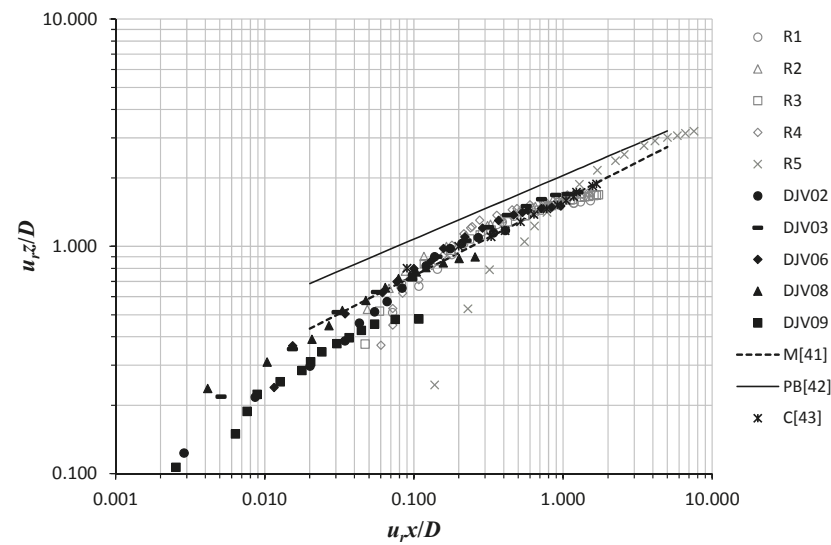

(a)

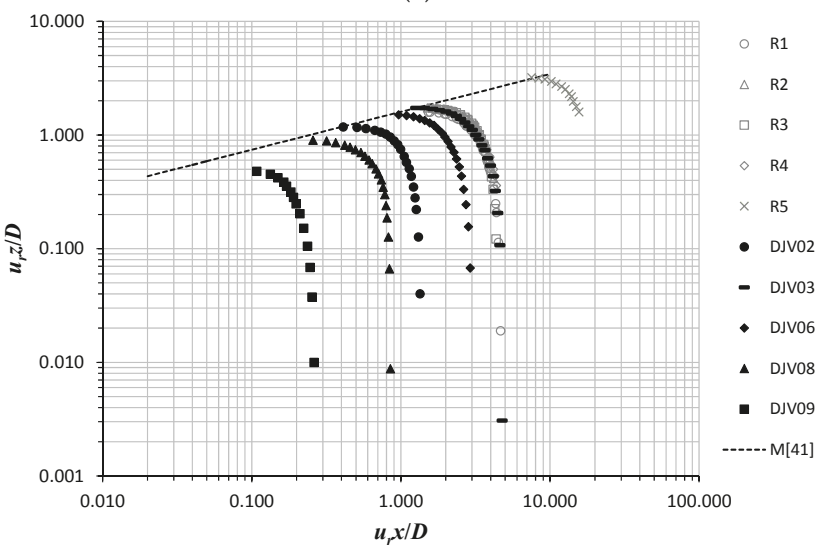

(b)

Figure 6. Normalized jet trajectory: (a) ascending region; (b) descending region. R1 to R5 represent the jet axis of the present study, DJV02 to DJV09 are the jet centerlines of Gungor and Roberts [19], M [41] is a similar power-law of momentum jet trajectory proposed by Margason [41], PB [42] is a similar power-law of momentum jet trajectory proposed by Pratte and Baines [42], and C [43] are experimental data of momentum jet trajectory obtained by Chochua et al. [43]. 
In the ascending region, as shown in Figure 6a, the dense jet trajectories of the present study and those of Gungor and Roberts [19] almost exhibit the same power-law exponent. The data of the different trajectories tend to collapse onto a single curve, following the data of Margason [41], Pratte and Baines [42], and Chochua et al. [43]. The almost similarity between the different jet trajectories in Figure 6a clearly indicates that the dense jet in the ascending region behaves as a momentum jet, and therefore its trajectory seems to be predictable by a simple power-law as proposed by Margason [41].

Figure $6 \mathrm{~b}$ shows the normalized jet trajectories in the descending region. It can be clearly seen the significant gap between the different trajectories. In the descending region, the scaling of the jet trajectory by $D / u_{r}$ does not show similarity between the different runs. On the other hand, the examination of Figure $6 b$, indicates that the trajectories are not randomly scattered between them, but they show a kind of systematic gaps. The jet trajectories show a "manure fork-shape" of inclined "root", following the power-law (dashed line on the figure) proposed by Margason [41], and parallel "tines", as shown by the different trajectories. The distance between the "tines" seems proportional to $u_{r} F$. When $u_{r} F$ increases, the jet trajectory linearly translates (along almost the dashed line on the figure) to the right. For comparable values of $u_{r} F$, such as the case in runs R1 to R4 of the present study and in run DJV03 of Gungor and Roberts [19] (Table 1), the jet trajectories collapse onto an almost single curve. The absence of similarity between the jet trajectories at different values of $u_{r} F$ is related to the choice of the scaling mode, where the scaling with $D / u_{r}$ is not appropriate to represent all the trajectories by a typical profile.

Figure $6 \mathrm{~b}$ also indicates that the maximum rise height (ending and beginning of the ascending and descending regions, respectively) of the jet trajectories appeared at different longitudinal and vertical coordinates. This points out that, despite the collapse of the jet trajectories in Figure 6a onto an almost single curve, the scaled profiles in the ascending region, really, suffer an incomplete similarity. Therefore, the application of a power-law as proposed by Margason [41] can simply predict the tendency of the jet trajectory in the ascending region, but not the exact profile. The incomplete similarity between the jet trajectories, following the scaling mode proposed for momentum jets, makes us think of another compatible scaling rule. In a recent study, Gungor and Roberts [19] suggested that, based on dimensional analysis of the initial characteristic physical properties of a dense jet, the jet trajectory coordinates could be scaled by $D F$. Nevertheless, the authors observed that the scaling of the jet trajectories by $D F$ leads to one "universal" trajectory for only experiments that have the same values of $u_{r} F$, as also observed in Figure $6 \mathrm{~b}$ scaling the jet trajectory by $D / u_{r}$. Once again, it can be noted that the scaling of the jet trajectories by $D F$ is also not appropriate to represent the trajectories of different $u_{r} F$-values by a typical profile.

At $u_{r} F<2$, it was observed that the dense jet is characterized by an ascending and a descending regions $[14,19]$ and its trajectory is fundamentally described by three characteristic length scales (Figure 2) $x_{i}, x_{t}$ and $z_{t}$ (Table 1). In the present study, we will try to rescale the jet trajectory as $z / z_{t}$ vs. $x / x_{t}$, in the ascending region, and $z / z_{t}$ vs. $\left[1+\left(x-x_{t}\right) /\left(x_{i}-x_{t}\right)\right]$, in the descending region. Figure $7 \mathrm{a}$ illustrates both the rescaled jet axis, for all the experimental runs illustrated in Table 1 of the present study (except that of run R 5 at $u_{r} F>2$, where the jet undergoes rapid dispersion by the effect of the ambient flow-turbulence), and the jet centerline for runs R1 and R2. The results of the present study are also compared to the centerline trajectories obtained by Gungor and Roberts [19]. Figure 7a clearly shows that all the jet trajectories (axis and centerline), of the present study and those of Gungor and Roberts [19], perfectly collapse onto a single profile. In the ascending region, at $x / x_{t}<0.03$, it can be noted a slight data scattering, due to the effect of the potential core region of the jet. The rescaling of the jet trajectories by $x_{t}, x_{i}$ and $z_{t}$ leads to a typical trajectory for all experiments with complete independence of $u_{r} F$ (ranging almost between 0.2 and 1.1 in the present study). This typical trajectory is predictable by the following proposed closed-form expression: 


$$
\begin{cases}\frac{z}{z_{t}}=-0.95\left(\frac{x}{x_{t}}\right)^{2}+1.60\left(\frac{x}{x_{t}}\right)+0.35 & \text { for } \frac{x}{x_{t}} \leq 1 \\ \frac{z}{z_{t}}=-0.95\left(1+\frac{x-x_{t}}{x_{i}-x_{t}}\right)^{2}+1.60\left(1+\frac{x-x_{t}}{x_{i}-x_{t}}\right)+0.35 & \text { for } \frac{x}{x_{t}} \geq 1\end{cases}
$$

In order to make Equation (15) easily practicable, expressions of $x_{i}, x_{t}$ and $z_{t}$ can be found, based on the dimensional analysis as depicted in Equation (14). Figure $7 \mathrm{~b}-\mathrm{d}$ show, respectively, the normalized jet characteristic lengths $x_{i} / D F, x_{t} / D F$ and $z_{t} / D F$ plotted versus $u_{r} F$. Based on the experimental data of the present study and that of Gungor and Roberts [19], $x_{i}, x_{t}$ and $z_{t}$, for the jet centerline, can be approximately predicted by the following semi-empirical equations:

$$
\begin{gathered}
\frac{x_{i}}{D F}=5.20\left(u_{r} F\right) \\
\frac{x_{t}}{D F}=1.50\left(u_{r} F\right) \\
\frac{z_{t}}{D F}=-0.84\left(u_{r} F\right)+2.60
\end{gathered}
$$

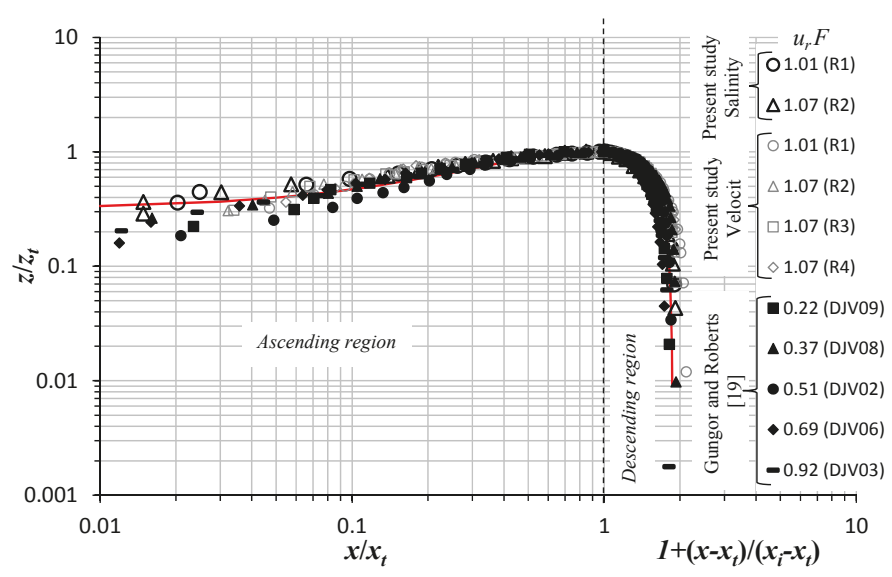

(a)

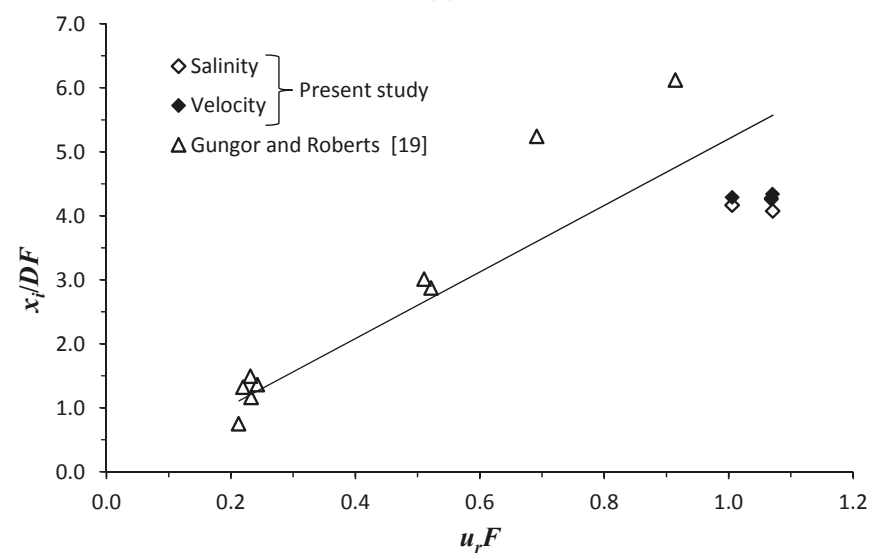

(b)

Figure 7. Cont. 


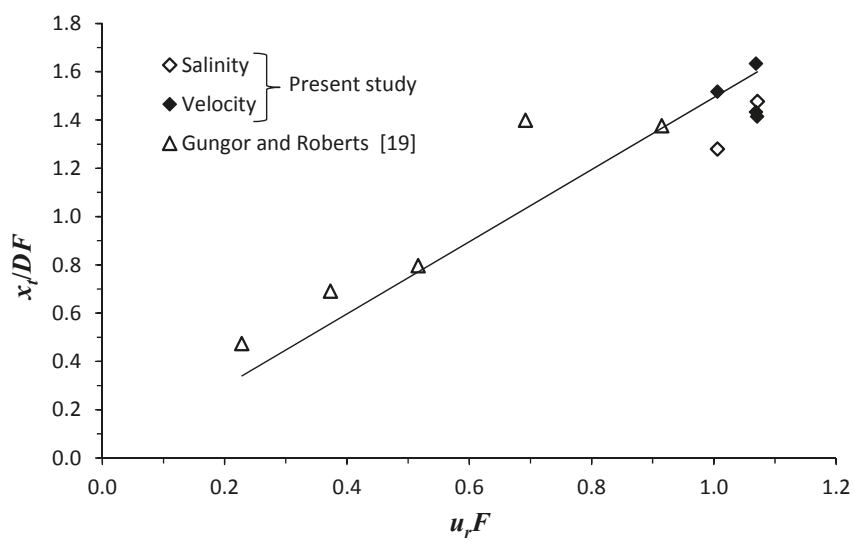

(c)

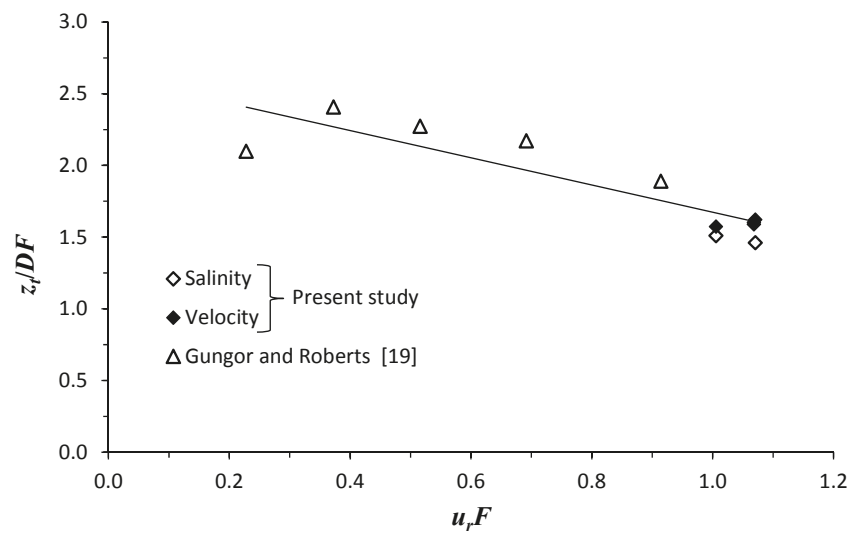

(d)

Figure 7. Jet trajectory analysis: (a) scaling of jet trajectories based on the characteristic length scales $x_{i}$, $x_{t}$ and $z_{t}$. The solid red curve represents the solution of Equation (15); (b) $x_{i} / D F$ versus $u_{r} F$; (c) $x_{t} / D F$ versus $u_{r} F ;(\mathbf{d}) z_{t} / D F$ versus $u_{r} F$. The solid regression lines on (b-d) refer to the data of jet concentration of the present study and that of Gungor and Roberts [19].

For the jet axis, it is suggested the use of Equations (16)-(18), keeping almost the same value of $x_{t}$ and increasing that of $x_{i}$ and of $z_{t}$ by almost $8 \%$, since the jet axis appears slightly above the jet centerline.

The advantage of the proposed scaling approach, represented by Equations (15)-(18), is its simplicity. It is relatively easy to predict the trajectory of any dense jet vertically discharged into a flowing current, of $u_{r} F$ ranging almost between 0.2 and 1.1. This approach can be extended with vertical dense jets of $u_{r} F<2$ and for inclined dense jets, which is the subject of a future study. Indeed, such approach is very useful to many engineering and environmental applications such as the brine discharge from desalination plants or dense wastewater discharge.

\subsection{Turbulence Intensity Associated with the Jet Flow-Field}

This section focuses on the analysis of the jet flow-field-turbulence intensities. The turbulence intensity is defined as the ratio of the standard deviation of the flow velocity fluctuation to the ambient mean velocity $U_{a}$. Hereafter, we indicate by $U^{\prime} / U_{a}, V^{\prime} / U_{a}$ and $W^{\prime} / U_{a}$, the streamwise, the spanwise 
and the vertical flow turbulence intensities, where $U^{\prime}, V^{\prime}$ and $W^{\prime}$ are the standard deviation of the streamwise, the spanwise and the vertical flow velocity component fluctuations, respectively.

Figures 8 and 9 , as an example, illustrate the vertical profiles of $U^{\prime} / U_{a}$ and $W^{\prime} / U_{a}$, at different downstream positions $x / D$ of runs R1 and R2. The data refer to the plane of flow symmetry $(y=0)$. Due to the flow symmetry, the spanwise velocity is theoretically expected to be null and therefore $V^{\prime}$ has not any physical significance in this plane. $U^{\prime} / U_{a}$ shows the largest values in the ascending region. In this region, $U^{\prime} / U_{a}$ almost shows an absolute peak near the jet axis. In general, according to previous studies, this peak is a distinct off-axis peak, where the turbulence undergoes a slight decrease at the jet axis. This behavior is less pronounced with the current profiles because the vertical profiles are not perfectly orthogonal to the jet axis. Figures 8 a and 9a show that $U^{\prime} / U_{a}$ significantly increases in the jet flow-field as compared to that in the ambient flow-field (upper part of profiles). Moreover, it can be noted that $U^{\prime} / U_{a}$ gradually decays as $x / D$ increases. It shows a maximum value equal to $0.83,0.93$, 1.05 and 1.17 at $x / D=3$, and $0.45,0.47,0.49$ and 0.61 at $x / D=12$, for runs $\mathrm{R} 1$ to $\mathrm{R} 4$, respectively. These values clearly indicate that $U^{\prime} / U_{a}$ increases as $u_{r}$ decreases. In the descending region, $U^{\prime} / U_{a}$ continues to decrease monotonically. Far from jet source, at $x / D>27, U^{\prime} / U_{a}$ shows values comparable to those of the ambient flow-field.
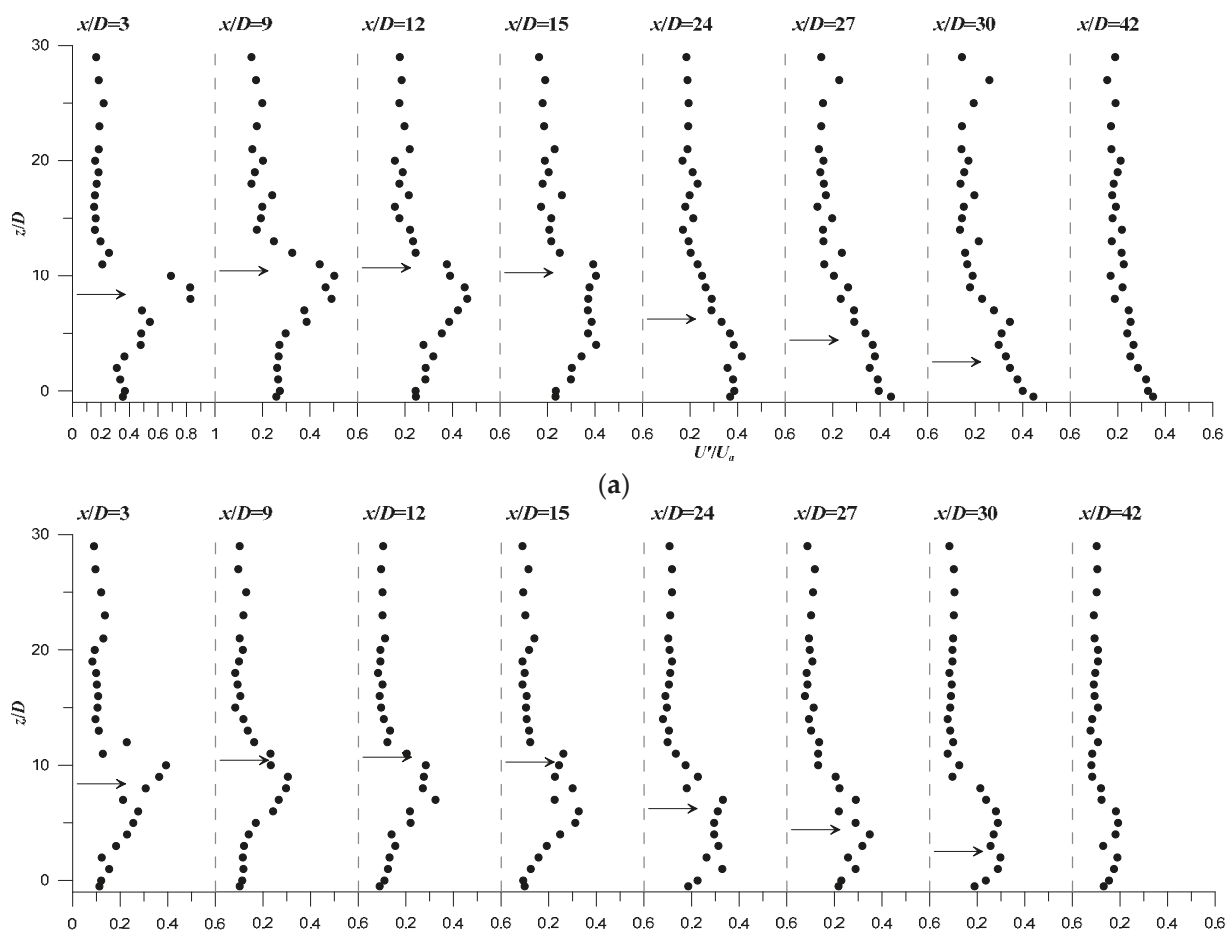

(a)

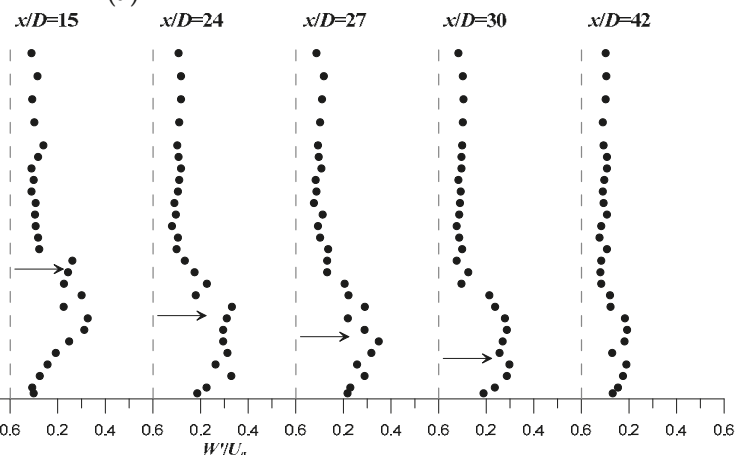

(b)

Figure 8. Vertical profiles of the flow turbulence intensity at different downstream positions $x / D$ along the plane of flow symmetry $(y=0)$ of run R1: (a) $U^{\prime} / U_{a}$; (b) $W^{\prime} / U_{a}$. The horizontal arrow on the profiles indicate the position of the jet axis. 


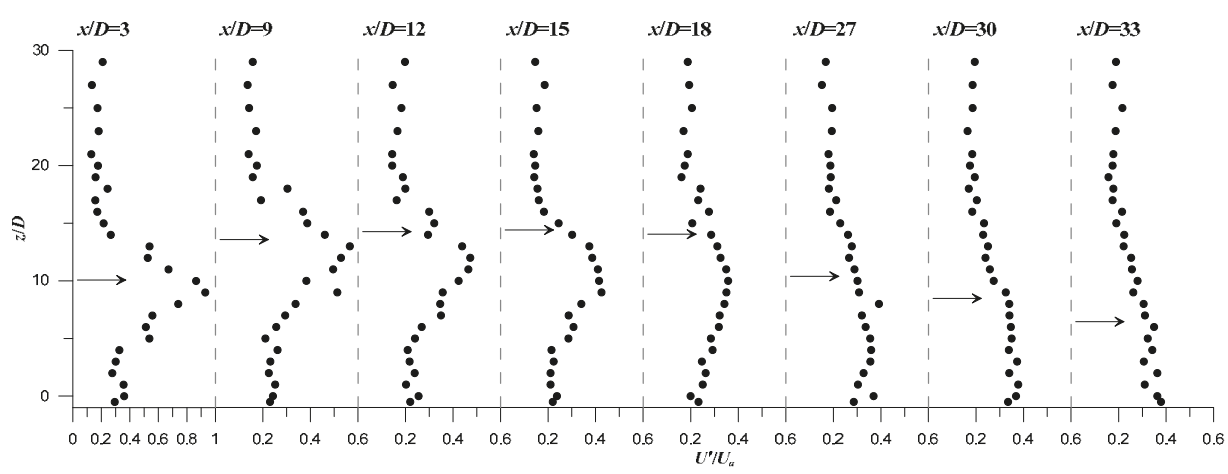

(a)

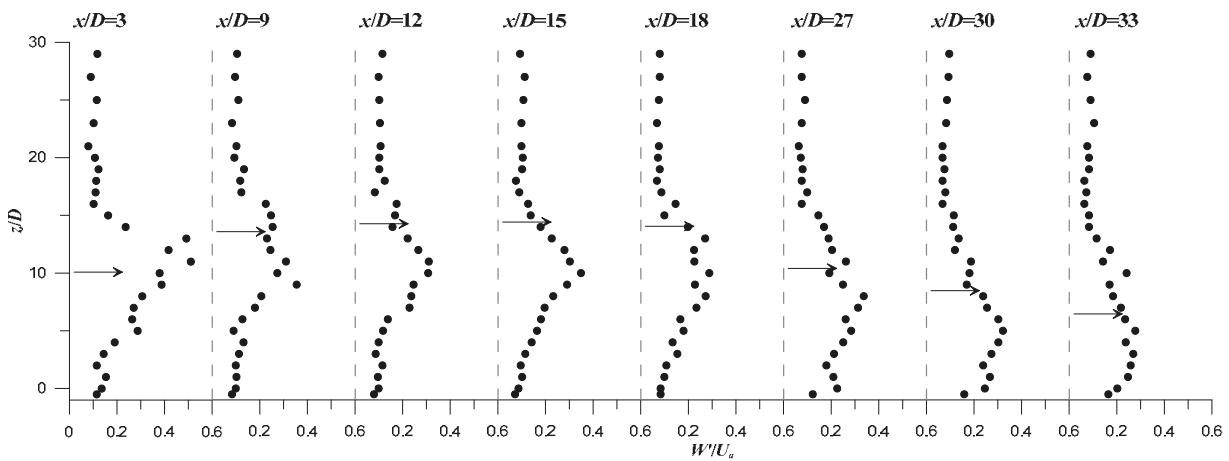

(b)

Figure 9. Vertical profiles of the flow turbulence intensity at different downstream positions $x / D$ along the plane of flow symmetry $(y=0)$ of run R2: (a) $U^{\prime} / U_{a} ;(\mathbf{b}) W^{\prime} / U_{a}$.

Figures $8 \mathrm{~b}$ and $9 \mathrm{~b}$ show that the highest values of $W^{\prime} / U_{a}$ take place in the jet flow-field. $W^{\prime} / U_{a}$ also shows an absolute peak below the jet axis. In the ascending region, $W^{\prime} / U_{a}$ decreases as $x / D$ increases, attains minimum values at the maximum rise height position, as shown at $x / D=9$ and 12 , and then starts to slightly increase in the descending region with the increase of $x / D$. Outside the jet flow-field, independently of $x / D, W^{\prime} / U_{a}$ for both runs R1 and R2 shows almost constant values of $\mathrm{O}(0.10)$ over the flow depth.

In order to get more information on the jet-turbulence-intensity behavior, an analysis of the maximum-turbulence-intensity decay/rise as a function of the downstream distance from the jet exit is examined for runs R1 to R4. Figure 10a,b show, respectively, a plot of $U_{m}^{\prime} / U_{a}$ and $W_{m}^{\prime} / U_{a}$ against $x / x_{t}$, where the subscribed $m$ indicates the maximum value of $U^{\prime} / U_{a}$ and $W^{\prime} / U_{a}$ on the vertical profiles of Figures 8 and 9. Figure 10a shows that, for all runs, $U_{m}^{\prime} / U_{a}$ continuously decays as a function of $x / x_{t}$, regardless its appearance in the ascending $\left(x \leq x_{t}\right)$ or in the descending regions $\left(x \geq x_{t}\right)$. Figure 10a indicates that $U_{m}^{\prime} / U_{a}$ decays more rapidly in the ascending region than in the descending region. As going further downstream, the decrease-rate of $U_{m}^{\prime} / U_{a}$ as a function of $x / x_{t}$ significantly reduces.

In the ascending region, $W_{m}^{\prime} / U_{a}$ behaves in a similar way as $U_{m}^{\prime} / U_{a}$. It strongly decays in the ascending region, attaining a minim value at $x=x_{t}$ (Figure 2). In the descending region, however, $W_{m}^{\prime} / U_{a}$ shows a gradual increase as $x / x_{t}$ increases and then starts to decrease again, as clearly shown with R1 for $x / x_{t}>2.5$. The decay and rise of $W_{m}^{\prime} / U_{a}$ are related to the vertical-velocity behavior, which shows a decrease of its magnitude along the ascending region as $x / D$ increases, attains almost null values at $x_{t}$ and then starts to increase along the descending region, as above discussed (Figures 4 and 5). 


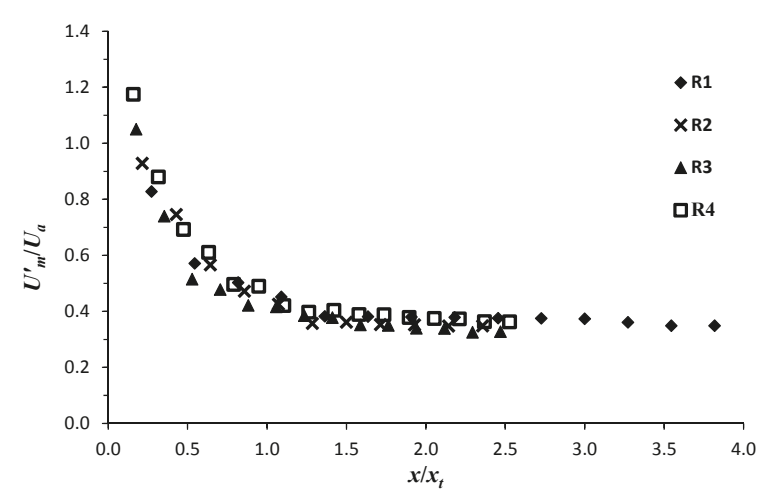

(a)

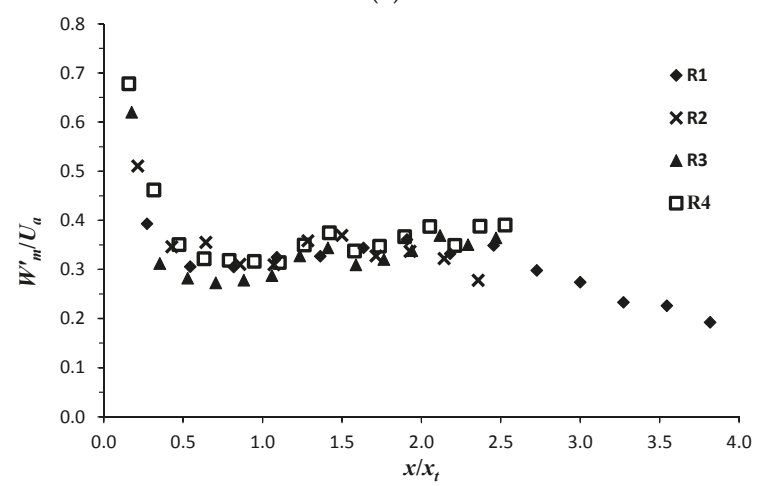

(b)

Figure 10. Decay/rise of the maximum turbulence intensity as a function of the downstream position $x / x_{t}:(\mathbf{a}) U_{m}^{\prime} / U_{a} ;(\mathbf{b}) W_{m}^{\prime} / U_{a}$.

Figure 11 displays, as an example, the vertical profiles of the time-averaged turbulent kinetic energy, $k$, normalized by $U_{a}{ }^{2}$, at different downstream positions $x / D$ from the jet exit. The data refer to runs R1 and R2. Figure 11 clearly highlights a significant increase of $k / U_{a}{ }^{2}$ within the jet flow-field. Outside the jet flow-field, $k / U_{a}{ }^{2}$ experiences almost constant values over the ambient flow-depth. It is of $\mathrm{O}(0.02)$ above the jet outer-boundary, and $\mathrm{O}(0.04)$ below the jet inner-boundary. Figure 11 indicates that the largest values of $k / U_{a}{ }^{2}$ take place below the jet axis, as indicated by the horizontal arrows on the figure. This indicates that the jet wake-region is a location of maximum turbulent energy production.

The examination of Figure 11 also reveals that the production of turbulent kinetic energy is locally variable with jet flow-field. $k / U_{a}{ }^{2}$ monotonically decreases as $x / D$ increases, reaching values comparable to those obtained in the ambient flow at considerable downstream positions $x / D$, as shown on Figure $11 \mathrm{a}$ at $x / D=42$. In Figure 12 we plot the maximum values of $k / U_{a}{ }^{2}$ versus $x / x_{t}$ for runs R1 to R4. Figure 12 shows that $k_{m} / U_{a}^{2}$ strongly reduces along the ascending region as $x / x_{t}$ increases. It is reduced by up to $65 \%$ at $x=x_{t}$ as compared to its value at $x / x_{t}=0.16$. In the descending region, $k_{m} / U_{a}^{2}$ shows a very slight increase until $x / x_{t}=2.5$, and then returns to decrease again, but very gradually. 


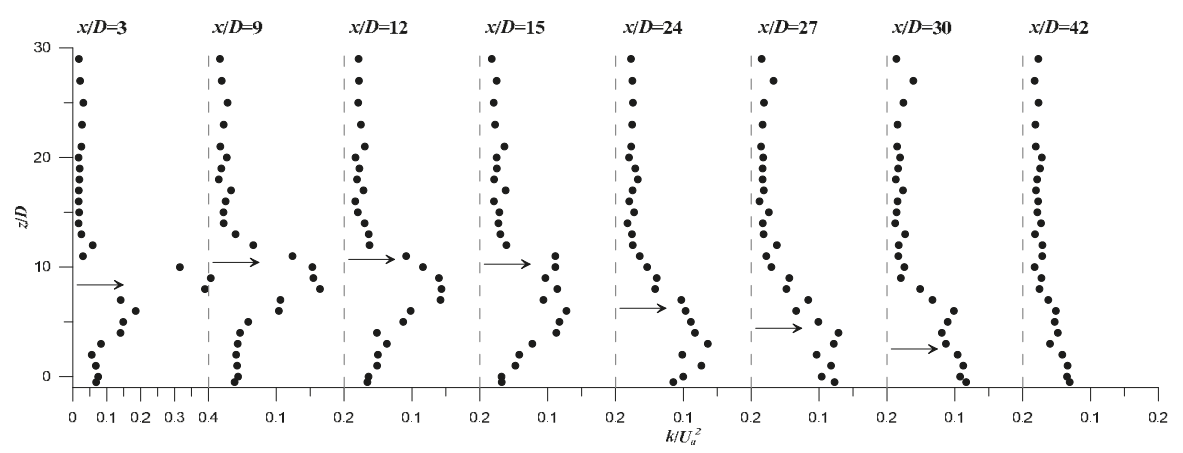

(a)

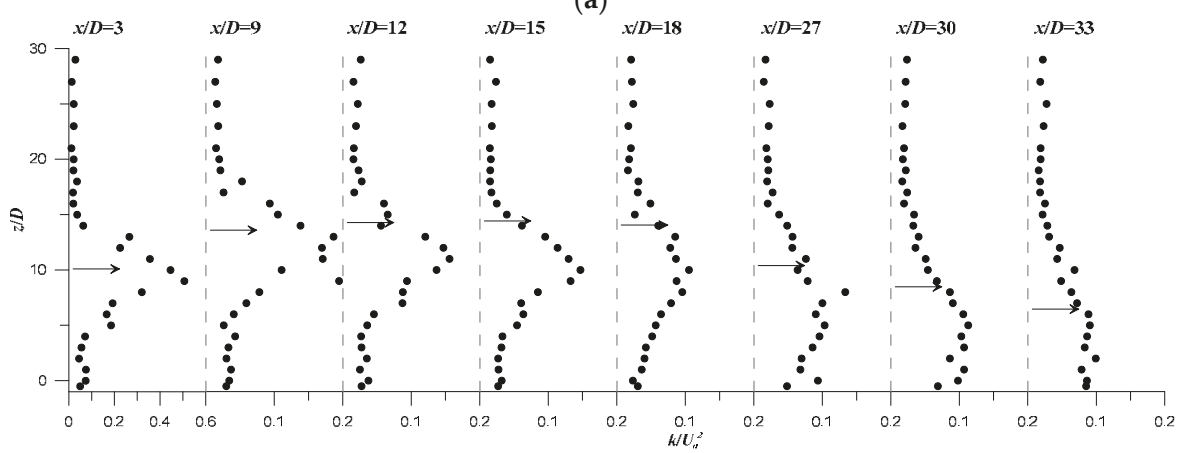

(b)

Figure 11. Vertical profiles of the normalized time-averaged turbulent kinetic energy at different downstream positions $x / D$ : (a) run R1; (b) run R2. The horizontal arrows on the profiles indicate the position of the jet axis.

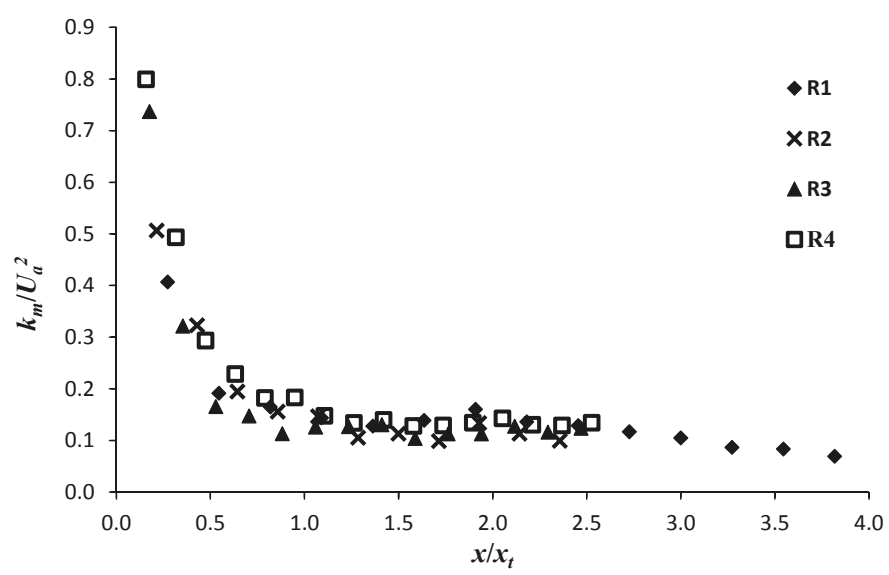

Figure 12. Decay of the maximum turbulent kinetic energy $k / U_{a}^{2}$ as a function of $x / x_{t}$.

\subsection{Turbulent Length Scales and Dispersion Coefficients}

Determination of the largest scales in a turbulent flow is of crucial importance for experimental and numerical investigations, defining suitable domain-dimensions (area or volume) for computation. 
As above shown in Equations (5) and (6), both the turbulent diffusion and net dispersion coefficients, are proportional to a characteristic eddy length-scale of the turbulent flow. In the present study, the characteristic eddy length-scale $L_{i}$ is simply calculated as the integral time scale $T_{i}$ times the local time-averaged velocity $U_{i}$, where $T_{i}$ is computed integrating the autocorrelation of the measured instantaneous flow velocities.

Figures 13 and 14 illustrate the vertical profiles of the integral length-scales $L_{x}$ and $L_{z}$, normalized by the jet diameter $D$, at different downstream position $x / D$. The data always refer to run R1 and R2, respectively. The profiles are presented in semi-logarithmic plot for a better visualization of the data. The most important observation from Figures 13 and 14 is the significant spatial-variation of $L_{x}$ and $L_{z}$ in the jet flow-field, which is more pronounced in the ascending region. In the ambient flow-region, above the jet outer-boundary or at $x / D=42$ (with small jet effect) in R1, $L_{x}$ and $L_{z}$ are nearly constant over the flow depth. They are, respectively, equal to almost $10 D$, a value of order the channel flow depth $H$, and $0.5 D$. This seems reasonable for the ambient flow where the vertical-velocity component is very small as compared with the streamwise component.
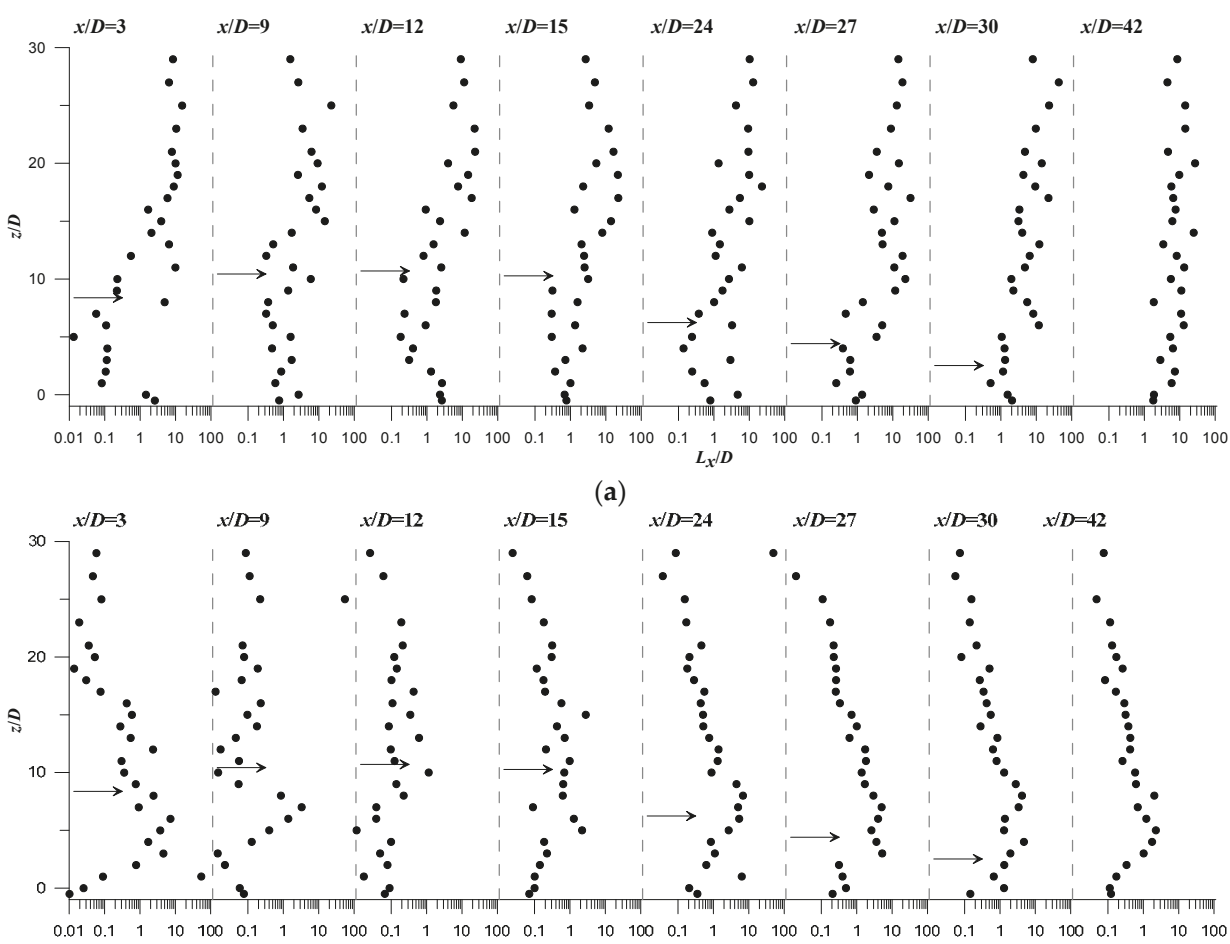

(a)

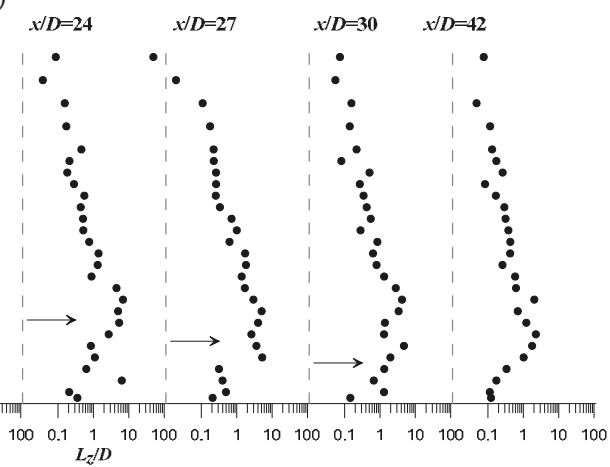

(b)

Figure 13. Vertical profiles of the normalized turbulent length scales at different downstream positions $x / D$ at the plane of flow symmetry $(y=0)$ of run R1: (a) $L_{x} / D ;(\mathbf{b}) L_{z} / D$. The horizontal arrow on the profiles indicate the position of the jet axis. 

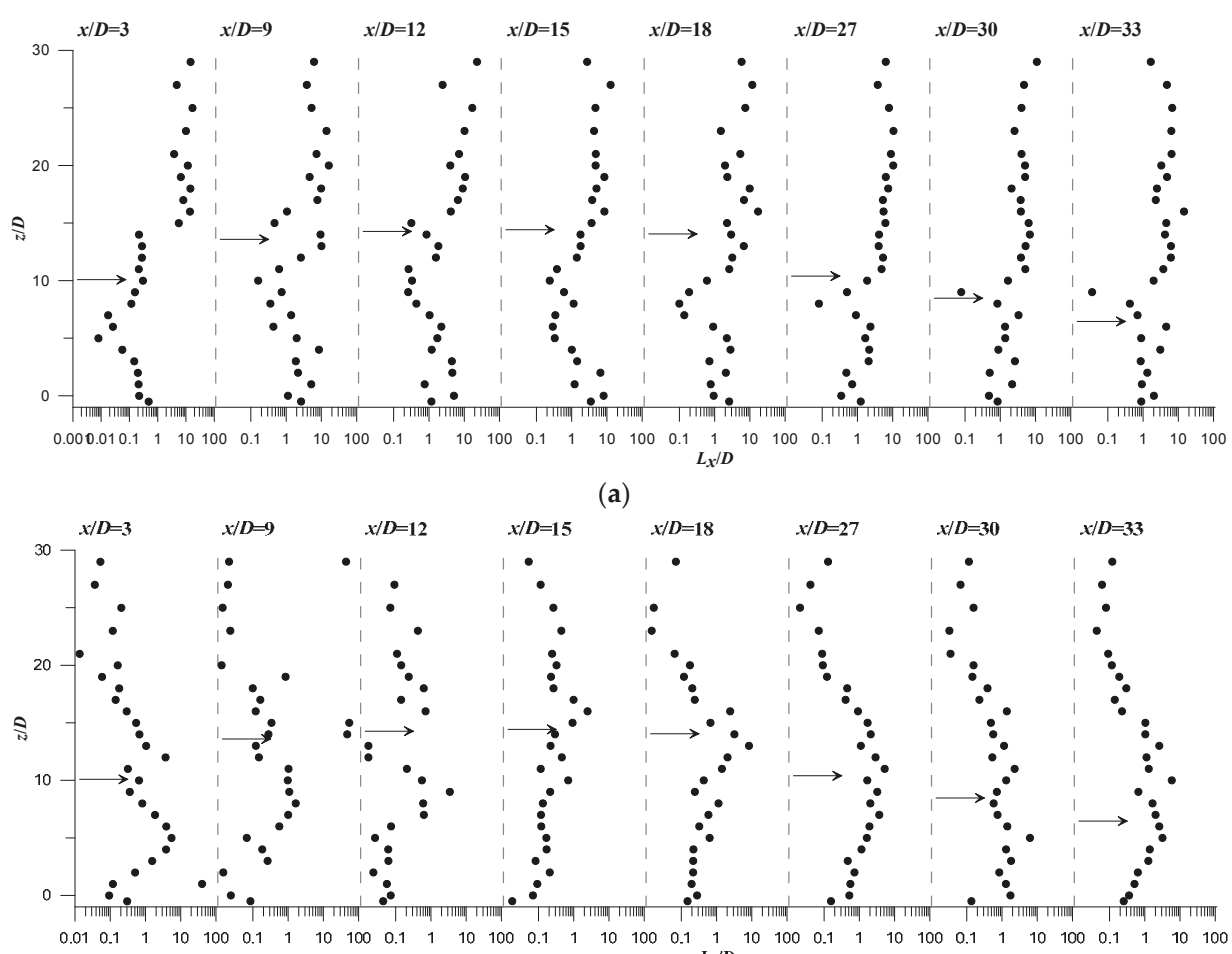

(a)

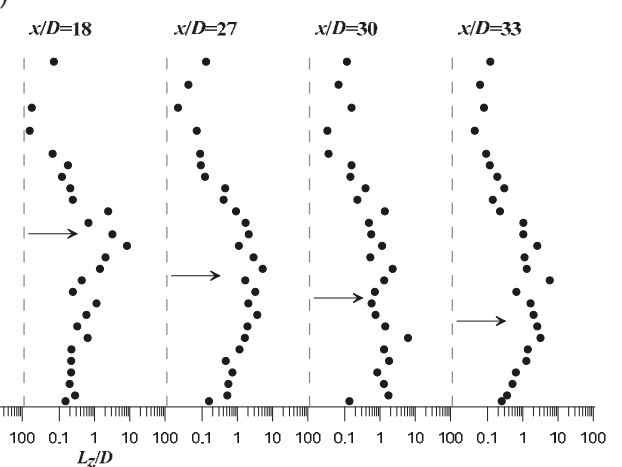

(b)

Figure 14. Vertical profiles of the normalized turbulent length scales at different downstream positions $x / D$ at the plane of flow symmetry $(y=0)$ of run R2: (a) $L_{x} / D ;(\mathbf{b}) L_{z} / D$.

In the jet flow-field, $L_{x}$ decrease as compared to that obtained in the ambient flow-field. $L_{x}$ shows a typical trend over the flow depth, as represented by the profile at $x / D=9$ for both runs R1 and R2. This profile shows that $L_{x}$ reduces from a values of $\mathrm{O}(10 D)$, above the jet outer-boundary, to a value of $\mathrm{O}(D)$ in the jet upper-region, increases at the jet axis and then again decreases to a scale of $O(D)$ in the wake-like region. Figures 13 and 14 indicate that the jet wake region is the location of minimum $L_{x}$-scales. As $x / D$ increases, the typical trend of $L_{x}$ gradually changes and disappears at large values of $x / D$, resembling a profile in the ambient flow-field. Figures 13 and 14 indicate that $L_{z}$ behaves contrary to $L_{x}$. It increases in the jet upper-region, attaining values of order 3 to $5 D$, decreases at the jet axis and then significantly increases in the wake-like region, as clearly shown in Figure $14 \mathrm{~b}$ at $x / D=3$ to 12 .

Figures 15 and 16 depict the vertical profiles of $k^{\frac{1}{2}} L_{x} / U_{a} D$ and $k^{\frac{1}{2}} L_{x} / U_{a} D$, order of magnitude of the normalized dispersion coefficients $K_{x}$ and $K_{z}$, at different downstream position $x / D$, for runs R1 and R2. According to previous studies [31,44], the net dispersion coefficient can be generally determined as $K_{i}=\alpha k^{\frac{1}{2}} L_{i}$, where the scale factor $\alpha$ will differ between the vertical and horizontal diffusion and differ from a location to another within the jet flow-field. Following Nepf [44], the scale factor is of O(1). Figures 15 and 16 show that $K_{x}$ and $K_{z}$ roughly behave like $L_{x}$ and $L_{z}$, respectively. These observation support the assumption that turbulent diffusion is proportional to the turbulent eddies length-scales. The change in eddy scales controls the turbulent diffusion, such that the longitudinal dispersion $K_{x}$ is reduced in the jet flow-field, despite the fact that the turbulence intensity is increased, as shown in Figures 8, 9 and 11a. This finding is in good agreement with that observed by Nepf [44] for turbulent flow dispersion in vegetated channel. The decrease of the longitudinal dispersion $K_{x}$, as compared 
to that of the ambient flow-field, is somewhat recovered by an increase of the vertical dispersion $K_{z}$, increasing the jet width, and consequently the transversal dispersion $K_{y}$ could be also increased. As a conclusion, the results of the present study demonstrate that the turbulent dispersion of the jet flow is an anisotropic process. It is strongly influenced by the jet hydrodynamic structure itself. In the jet flow-field, the longitudinal dispersion $K_{x}$ decreases enough as compared to that in the ambient flow-field, promoting an increase of the vertical dispersion $K_{z}$, and consequently an increase of the jet width occurs.
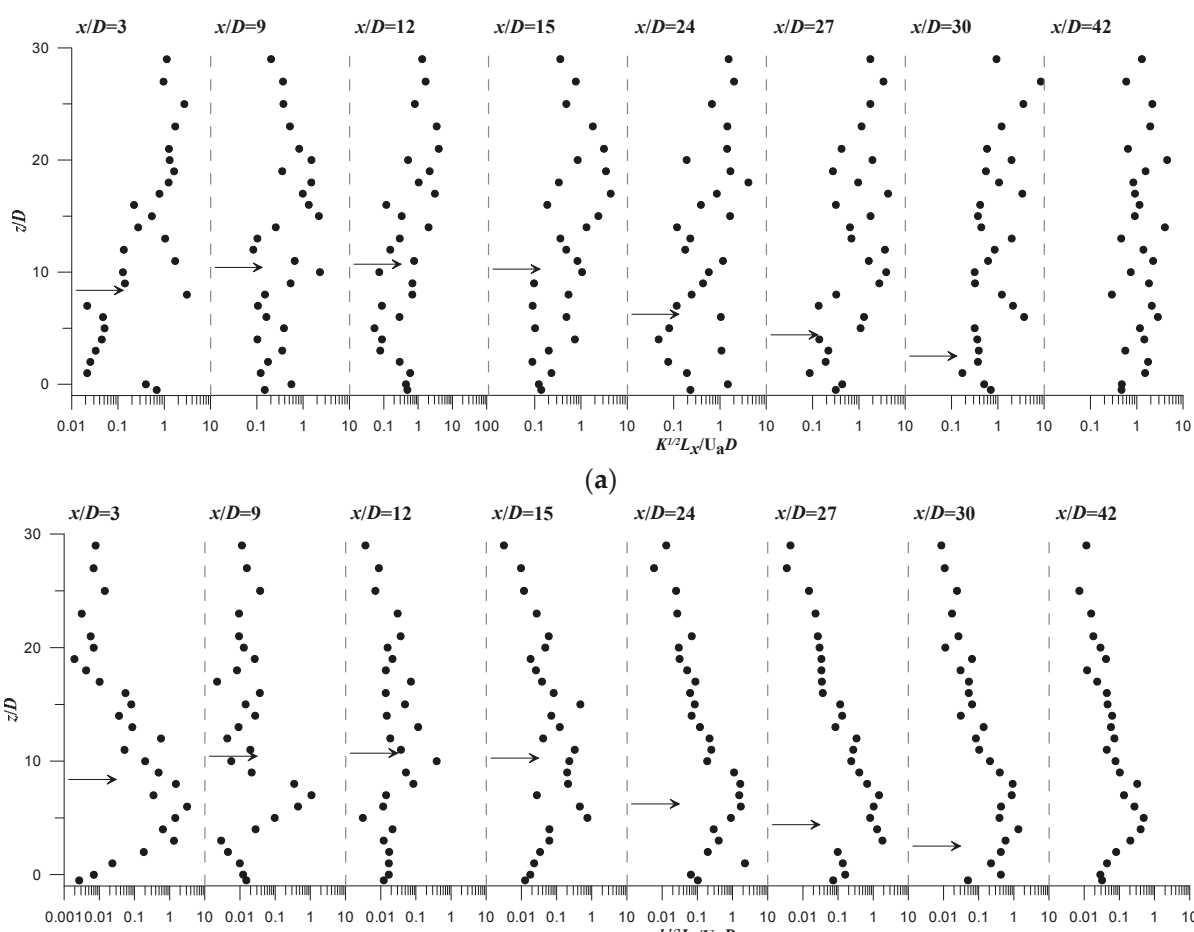

(a)

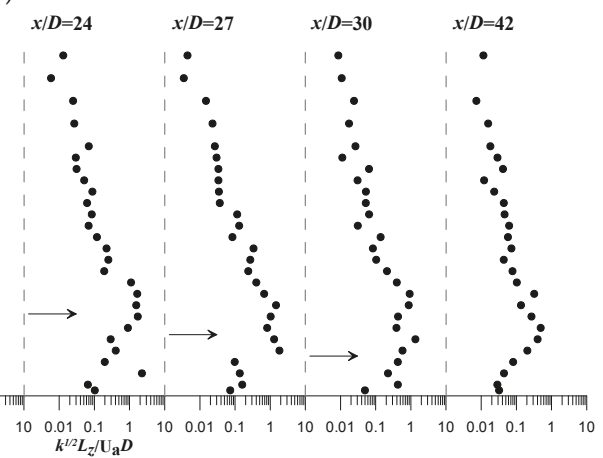

(b)

Figure 15. Trend of the turbulent dispersion coefficient $K_{x}$ and $K_{z}$ at different downstream positions $x / D$ at the plane of flow symmetry $(y=0)$ of run R1: (a) $k_{x} \sim k^{\frac{1}{2}} L_{x} / U_{a} D ;(\mathbf{b}) k_{z} \sim k^{\frac{1}{2}} L_{z} / U_{a} D$. The horizontal arrow on the profiles indicate the position of the jet axis. 

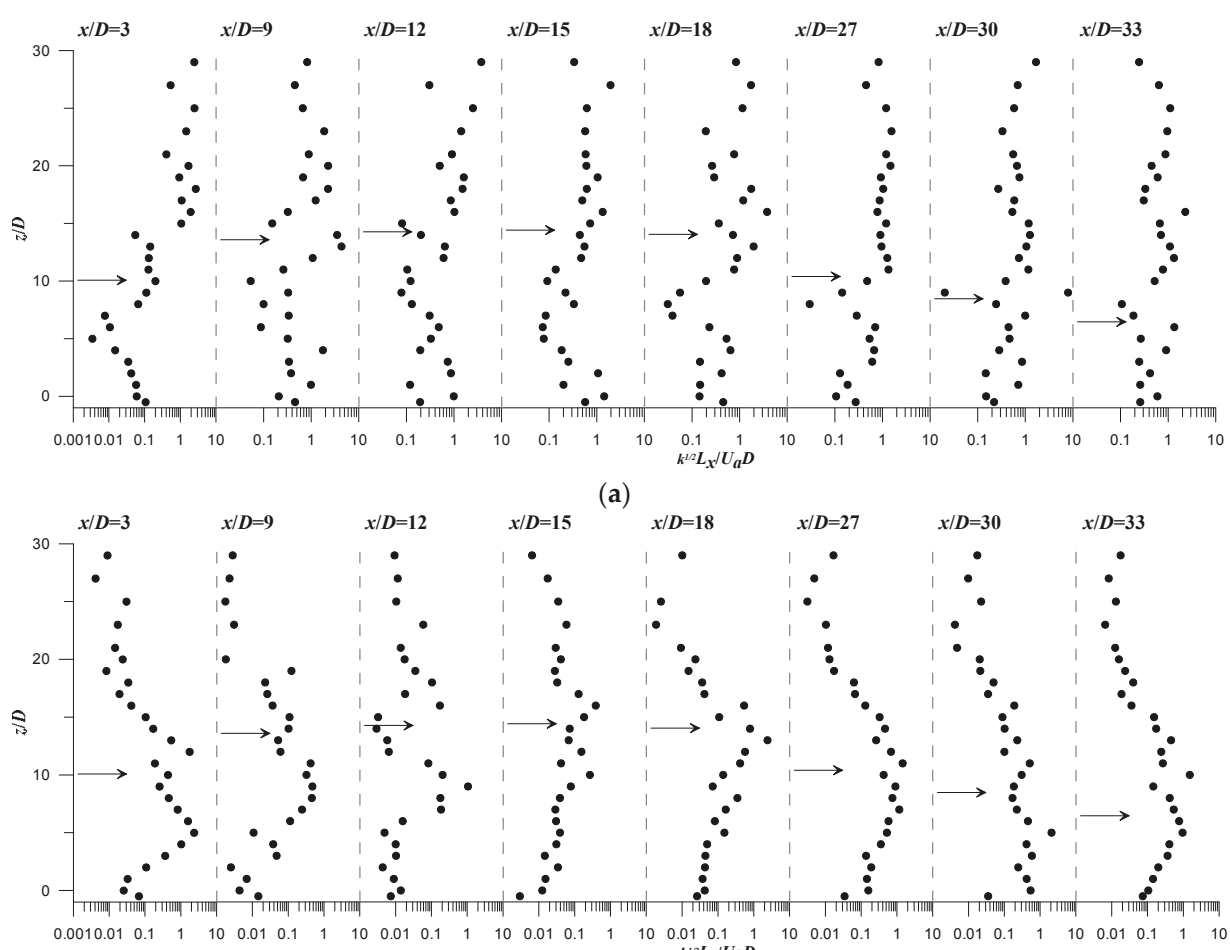

(a)
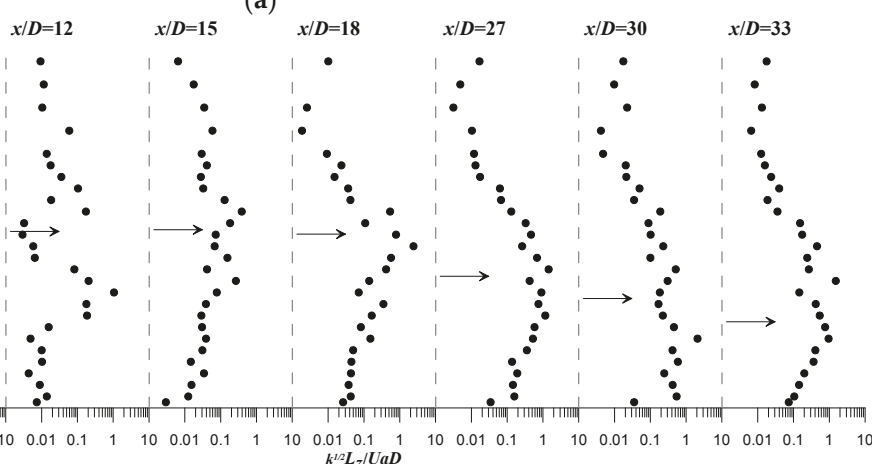

(b)

Figure 16. Trend of the turbulent dispersion coefficient $K_{x}$ and $K_{z}$ at different downstream positions $x / D$ at the plane of flow symmetry $(y=0)$ of run R2: (a) $k_{x} \sim k^{\frac{1}{2}} L_{x} / U_{a} D ;(\mathbf{b}) k_{z} \sim k^{\frac{1}{2}} L_{z} / U_{a} D$.

\section{Conclusions}

With population growth, urbanization, and industrial expansion the quantity of waste/brackish water discharge into natural water bodies in continuously increasing. In coastal environments, as an example, the brine of desalination plants is usually discharged as a turbulent jet flow, producing complex hydrodynamic phenomena within the surrounding ambient and affecting the ecosystem. Discharge systems need to be well designed to reduce their environmental impact. Therefore, a good knowledge of the interaction between effluent discharge and surrounding flow-field is required to promote best environmental management practices. This manuscript focuses on the analysis of flow turbulence structures that develop when a dense round jet, consisting of a saltwater solution, is injected perpendicularly into a flowing current of fresh water.

At $u_{r} F<2$, the measured flow velocity fields show that the dense jet is characterized by two distinct regions: a rapidly ascending region and a gradually descending region. In the ascending region, the buoyancy effect is dominated by the jet momentum flux and the mixing is jet-like, analogously to momentum jets. In the descending region, the jet flow is buoyancy-driven and the mixing is plume-like.

In this study, a new scaling approach of the jet trajectories, based on the jet characteristic length scales $x_{i}, x_{t}$ and $z_{t}$ (Figure 2), is proposed. This new rescaling rule enables all the trajectories to collapse onto a typical profile, independent of the current speed parameter $u_{r} F$, leading to an empirical closed-form expression as depicted in Equation (15). By proposing empirical expressions of $x_{i}, x_{t}$ and $z_{t}$, the new scaling approach become very practical and easily applicable to predict the trajectory of any dense jet, of $u_{r} F$ ranging between 0.2 and 1.1, vertically discharged into a flowing current. 
The detailed turbulence data reported in this study indicate that the strength of both the streamwise and the vertical turbulence intensities significantly increases in the jet flow-field as compared to the ambient flow-field. The largest values of the turbulence intensities consistently occur in the ascending region. The experimental results also reveal that the turbulence intensities increase with the decrease of $u_{r}$-ratio. The streamwise turbulence intensity shows a rapid decay along the ascending region and very gradually decay in the descending region. The vertical turbulence intensity, however, rapidly decays in the ascending region, attains a minimum value at $x=x_{t}$, gradually rises in the beginning of the descending region and then undergoes again a decay as going further downstream. The time-averaged turbulent kinetic energy also shows a significant increase in the jet flow-field. The maximum production of turbulent kinetic energy takes place in the jet wake-region.

The distribution, in the plane of flow symmetry, of the longitudinal and vertical integral length scales, respectively, indicates that the ambient flow-field is isotropic process. In the jet flow-field, the turbulent lengths scales show a significant spatial-variation, indicating an anisotropic process. The trends of the turbulent dispersion coefficients $K_{x}$ and $K_{z}$ follow those of the turbulent length scales $L_{x}$ and $L_{z}$, respectively. The change in eddy scales controls the turbulent diffusion of the jet flow. In comparison with the ambient flow-field, $K_{x}$ shows an enough reduction in the jet flow-field, however, an increase of the vertical dispersion $K_{z}$ occurs, leading to the increase of the jet width.

Acknowledgments: The experiments were carried out at the Coastal Engineering Laboratory of the Department of Civil, Environmental, Land, Building Engineering, and Chemistry of the Polytechnic University of Bari, Italy.

Author Contributions: Mouldi Ben Meftah performed the experiments, analyzed the data and wrote the paper; Michele Mossa contributed suggestions and reviewed the manuscript.

Conflicts of Interest: The authors declare that they have no conflicts of interest.

\section{References}

1. Van Reeuwijk, M.; Salizzoni, P.; Hunt, G.R.; Craske, J. Turbulent transport and entrainment in jets and plumes: A DNS study. Phys. Rev. Fluids 2016, 1, 074301. [CrossRef]

2. Voutchkov, N. Overview of seawater concentrate disposal alternatives. Desalination 2011, 273, $205-219$. [CrossRef]

3. McDougall, T.J. Negatively buoyant vertical jets. Tellus 1981, 33, 313-320. [CrossRef]

4. Rathgeber, D.E.; Becker, H.A. Mixing between a round jet and a transverse turbulent pipe flow. Can. J. Chem. Eng. 1983, 61, 148-157. [CrossRef]

5. Andreopoulos, J.; Rodi, W. Experimental investigation of jets in a crossflow. J. Fluid Mech. 1984, 138, $93-127$. [CrossRef]

6. Broadwell, J.E.; Breidenthal, R.E. Structure and mixing of a transverse jet in incompressible flow. J. Fluid Mech. 1984, 148, 405-412. [CrossRef]

7. Gu, R.; Stefan, H.G. Stratification dynamics in wastewater stabilization ponds. Water Res. 1995, 29, $1909-1923$. [CrossRef]

8. Huq, P.; Dhanak, M.R. The bifurcation of circular jets in crossflow. Phys. Fluids 1996, 8, 754. [CrossRef]

9. Cotel, A.J.; Breidenthal, R.E. Jet detrainment at a stratified interface. J. Geophys. Res. Atmos. 1997, 102, 23813-23818. [CrossRef]

10. Jirka, G.H. Improved Discharge Configurations for brine effluents from desalination plants. J. Hydraul. Eng. 2008, 134, 116-120. [CrossRef]

11. Marugán-Cruz, C.; Rodríguez-Rodríguez, J.; Martínez-Bazán, C. Negatively buoyant starting jets. Phys. Fluids 2009, 21, 117101. [CrossRef]

12. Roberts, D.A.; Johnston, E.L.; Knott, N.A. Impacts of desalination plant discharges on the marine environment: A critical review of published studies. Water Res. 2010, 44, 5117-5128. [CrossRef] [PubMed]

13. Bashitialshaaer, R. An experimental study to improve the design of brine discharge from desalination plants. Am. J. Environ. Prot. 2013, 2, 176-182. [CrossRef]

14. Ben Meftah, M.; De Serio, F.; Malcangio, D.; Mossa, M.; Petrillo, A.F. Experimental study of a vertical jet in a vegetated crossflow. J. Environ. Manag. 2015, 164, 19-31. [CrossRef] [PubMed] 
15. Dai, C.; Jia, L.; Zhang, J.; Shu, Z.; Mi, J. On the flow structure of an inclined jet in crossflow at low velocity ratios. Int. J. Heat Fluid Flow 2016, 58, 11-18. [CrossRef]

16. Nada, S.A.; Fouda, A.; Elattar, H.F. Parametric study of flow field and mixing characteristics of outwardly injected jets into a crossflow in a cylindrical chamber. Int. J. Therm. Sci. 2016, 102, 185-201. [CrossRef]

17. Mossa, M.; Ben Meftah, M.; De Serio, F.; Nepf, H.M. How vegetation in flows modifies the turbulent mixing and spreading of jets. Sci. Rep. 2017, 7, 6587. [CrossRef] [PubMed]

18. Ben Meftah, M.; Malcangio, D.; De Serio, F.; Mossa, M. Vertical dense jet in flowing current. Environ. Fluid Mech. 2018, 18, 75-96. [CrossRef]

19. Gungor, E.; Roberts, P.J.W. Experimental studies on vertical dense jets in a flowing current. J. Hydraul. Eng. 2009, 135, 935-948. [CrossRef]

20. Hasselbrink, E.F.; Mungal, M.G. Transverse jets and jet flames. Part 1. Scaling laws for strong transverse jets. J. Fluid Mech. 2001, 443, 1-25. [CrossRef]

21. Hasselbrink, E.F.; Mungal, M.G. Transverse jets and jet flames. Part 2. Velocity and OH field imaging. J. Fluid Mech. 2001, 443, 27-68. [CrossRef]

22. Fearn, R.; Weston, R.P. Vorticity associated with a jet in a cross flow. AIAA J. 1974, 12, 1666-1671. [CrossRef]

23. Cortelezzi, L.; Karagozian, A.R. On the formation of the counter-rotating vortex pair in transverse jets. J. Fluid Mech. 2001, 446, 347-373. [CrossRef]

24. Marzouk, Y.M.; Ghoniem, A.F. Vorticity structure and evolution in a transverse jet. J. Fluid Mech. 2007, 575, 267-305. [CrossRef]

25. Karagozian, A.R. Transverse jets and their control. Prog. Energy Combust. Sci. 2010, 36, 531-553. [CrossRef]

26. Kaminski, E.; Tait, S.; Carazzo, G. Turbulent entrainment in jets with arbitrary buoyancy. J. Fluid Mech. 2005, 526, 361-376. [CrossRef]

27. Danckwerts, P.V. The definition and measurement of some characteristics of mixtures. Appl. Sci. Res. 1952, A3, 279-296. [CrossRef]

28. Galeazzo, F.C.C.; Donnert, G.; Habisreuther, P.; Zarzalis, N.; Valdes, R.J.; Krebs, W. Measurement and Simulation of Turbulent Mixing in a Jet in Crossflow. J. Eng. Gas Turbines Power 2011, 133, 061504. [CrossRef]

29. Cipollina, A.; BonFigurelio, A.; Micale, G.; Brucato, A. Dense jet modelling applied to the design of dense effluent diffusers. Desalination 2004, 167, 459-468. [CrossRef]

30. Abessi, O.; Roberts, P.J.W. Dense Jet Discharges in shallow water. J. Hydraul. Eng. 2016, 142. [CrossRef]

31. De Serio, F.; Ben Meftah, M.; Mossa, M.; Termini, D. Experimental investigation on dispersion mechanisms in rigid and flexible vegetated beds. Adv. Water Resour. 2017. [CrossRef]

32. Abessi, O.; Saeedi, M.; Davidson, M.; Zaker, N.H. Flow Classification of Negatively Buoyant Surface Discharge in an Ambient Current. J. Coast. Res. 2012, 278, 148-155. [CrossRef]

33. Roberts, P.J.W.; Toms, G. Inclined Dense Jets in Flowing Current. J. Hydraul. Eng. 1987, 113, 323-340. [CrossRef]

34. Ben Meftah, M.; De Serio, F.; Mossa, M.; Pollio, A. Experimental study of recirculating flows generated by lateral shock waves in very large channels. Environ. Fluid Mech. 2008, 8, 215-238. [CrossRef]

35. Ben Meftah, M.; Mossa, M.; Pollio, A. Considerations on shock wave/boundary layer interaction in undular hydraulic jumps in horizontal channels with a very high aspect ratio. Eur. J. Mech. B Fluids 2010, 29, 415-429. [CrossRef]

36. Ben Meftah, M.; Mossa, M. A modified log-law of flow velocity distribution in partly obstructed open channels. Environ. Fluid Mech. 2016, 16, 453-479. [CrossRef]

37. Ben Meftah, M.; Mossa, M. Prediction of channel flow characteristics through square arrays of emergent cylinders. Phys. Fluids 2013, 25, 045102. [CrossRef]

38. Ben Meftah, M.; De Serio, F.; Mossa, M. Hydrodynamic behavior in the outer shear layer of partly obstructed open channels. Phys. Fluids 2014, 26, 065102. [CrossRef]

39. Sherif, S.A.; Pletcher, R.H. Measurements of the flow and turbulence characteristics of round jets in crossflow. J. Fluids Eng. 1989, 111, 165-171. [CrossRef]

40. Fric, T.F.; Roshko, A. Vortical structure in the wake of a transverse jet. J. Fluid Mech. 1994, 279, 1-47. [CrossRef]

41. Margason, R.J. The Path of a Jet Directed at Large Angles to a Subsonic Stream; N.A.S.A., TN.D. 4919; Langley Research Center: Hampton, VA, USA, 1968.

42. Pratte, B.D.; Baines, W.D. Profiles of the round turbulent jet in a crossflow. J. Hydraul. Eng. Div. ASCE 1967, 92, 53-64. 
43. Chochua, G.; Shyy, W.; Thakur, S. A computational and experimental investigation of turbulent jet and crossflow interaction. Numer. Heat Transf. Part A Appl. 2000, 38, 557-572. [CrossRef]

44. Nepf, H.M. Drag, turbulence, and diffusion in flow through emergent vegetation. Water Resour. Res. 1999, 35, 479-489. [CrossRef]

(C) 2018 by the authors. Licensee MDPI, Basel, Switzerland. This article is an open access article distributed under the terms and conditions of the Creative Commons Attribution (CC BY) license (http://creativecommons.org/licenses/by/4.0/). 
Article

\title{
On the Hydrodynamic Geometry of Flow-Through versus Restricted Lagoons
}

\author{
Nikolaos Th. Fourniotis ${ }^{1,2, *}$, Georgios M. Horsch ${ }^{1}$ and Georgios A. Leftheriotis ${ }^{1}$ \\ 1 Department of Civil Engineering, University of Patras, University Campus, 26500 Patras, Greece; \\ ghorsch@upatras.gr (G.M.H.); gleytheriot@upatras.gr (G.L.A.) \\ 2 Department of Civil Engineering, Technological Educational Institute of Western Greece, \\ Megalou Alexandrou 1, 26334 Patras, Greece \\ * Correspondence: nfou@upatras.gr; Tel.: +30-2610-996519
}

Received: 19 January 2018; Accepted: 17 February 2018; Published: 25 February 2018

\begin{abstract}
The classification of a lagoon as a restricted lagoon is shown to depend not solely on its geometry but also on the tidal hydraulics. By numerically simulating the tidal exchange of two lagoons of similar geometrical dimensions, the Nidova lagoon and the Papas lagoon, in Western Greece, subject to very similar tidal forcing, applied to the two tidal inlets in the first case and three in the second, very different residence times are found, namely 2.5 days for the Nidova and 25-30 days for the Papas lagoon. This large difference is attributed to the fact that whereas the Papas lagoon functions as a typical restricted lagoon, in which the water renewal is achieved by mixing in the lagoon of the tidal prism water exchanged within a tidal cycle, the Nidova lagoon functions as a flow-through system because of the differential arrival of the tide at its two tidal inlets. It is suggested that this way of enhancing the flushing rate of a lagoon be considered, whenever possible, when creating a new tidal inlet to the lagoon.
\end{abstract}

Keywords: flow-through system; tidal inlets; residence time; coastal lagoon; MIKE 3 FM (HD \& TR); MIKE 21 FM (HD)

\section{Introduction}

Coastal lagoons are particularly important for fisheries and extensive or intensive aquaculture in many areas of the world, since marine fish species migrate towards lagoons, which provide favorable conditions for feeding and shelter [1]. Mediterranean lagoons, specifically, are important for aquaculture activities, and make a crucial contribution to the fishery economies in many countries. In Greek waters, important fishing and extensive aquaculture activities have been developed in the majority of lagoons (30 in the Aegean and 46 on the Ionian coast), occupying a total area of about 34,500 ha [2]. The fishery exploitation is based on traditional barrier fish traps. These are permanent entrapment devices and the catches are based on the species-specific inshore-offshore seasonal or ontogenic fish migration [3]. The majority of these extensive lagoon systems are located in western and northern Greece. The largest is the Messolonghi lagoonal complex located in western Greece, comprising six lagoons. The Messolonghi lagoon system covers about 15,000 ha [4] (p. 97), while the Papas lagoon (Araxos Cape, Achaia, Greece) has a mean size of 4500 ha [5].

In recent years many lagoon ecosystems have been affected by natural and mostly anthropogenic influences, e.g., hydrologic and hydrodynamic alterations, sources of pollution, sedimentation of tidal inlets/mouths, and regional infrastructure work. These changes gradually lead to alterations in the physical, chemical, and biochemical parameters of these complex ecosystems, which in turn may directly affect fish production and ecosystem dynamics [6,7]. The concentration of the corresponding substances in the lagoon ecosystems is generally dependent on hydrodynamic circulation and water renewal time, which are the crucial factors for decisions with regard to lagoon restoration design and 
management actions, aiming at the improvement of environmental conditions and fishery exploitation. An innovative method of data analysis has been presented in recent studies, focusing on similar coastal basins in Southern Italy $[8,9]$. The aim of these studies was to interpret hydrodynamic processes associated with these water bodies by identification and evaluation of hydrodynamic and meteorological data.

Among the physical parameters that affect the water quality of coastal lagoons, renewal of water has long been identified as a key parameter and has been made the basis of the early classification by Kjerfve [10] in choked, restricted, and leaky systems according to the degree of water exchanged with the adjacent coastal ocean. More recently, the water renewal time has been proposed as the basis for the classification of lagoons [11]. As a result of the importance of water renewal in lagoons, the creation of new artificial tidal inlets has been proposed and applied as a solution in several instances (see e.g., [12]).

The question then arises: is the amount of water exchanged in each tidal cycle the basic parameter-with all other parameters kept constant-determining the flushing time? Or, to rephrase the question, are two lagoons of similar dimensions and under the same tidal forcing expected to have similar flushing rates, or is it possible to have widely varying differences in flushing rates? The answer is provided by a real-life example of two lagoons situated in Western Greece, of similar dimensions and under very similar tidal forcing, namely the Nidova lagoon, part of the complex of the wider Messolonghi lagoon, and the Papas lagoon, both communicating with the Gulf of Patras. The former has a flushing rate on the order of a few days and the latter of over a month. In what follows, we analyze why the former, having only two tidal inlets, does not behave as a typical, restricted lagoon, as one might expect from mere inspection of its geometry, because the hydrodynamics circumvent, so to speak, the topography and render the lagoon a flow-through basin, whereas the latter, having three tidal inlets, behaves like a typical restricted lagoon and, possibly as a result, suffers recurring dystrophic crises.

\section{Materials and Methods}

\subsection{Description of the Study Areas}

The Nidova lagoon is embedded between the Messolonghi (south) and the Aetoliko (north) greater lagoons (Figure 1a). The lagoon is approximately $1.5 \mathrm{~km}^{2}$ in surface area, with a mean depth of $1.20 \mathrm{~m}$ and a maximum depth of $3 \mathrm{~m}$ at the western part of the basin. It is elongated in plan view, $2000 \mathrm{~m}$ long, approximately, with a perimeter of $5000 \mathrm{~m}$. The lagoon communicates to the north with the deeper Aetoliko lagoon via arc stone bridges, while to the south it is connected to the Messolonghi lagoon by a dredged trench (i.e., an underwater channel that is deeper than its surrounding waters) approximately $50 \mathrm{~m}$ wide, $1000 \mathrm{~m}$ long, and 1-3 m deep at mean lagoon level. At the western part of the lagoon an embankment with a road on it exists, linking the city of Aetoliko with the Dolmas islet in the south, where the Waste Water Treatment Plant of Aetoliko City operates. This embankment precludes exchange of Nidova waters with the Messolonghi lagoon waters from the west, so that the exchange between Nidova and Messolonghi takes place exclusively south of Nidova.

Tidal water enters from the Gulf of Patras to the Messolonghi lagoon from the south, and proceeds to Nidova through the dredged trench, while continuing to move into the Messolonghi lagoon, in parallel to the embankment up to the Aetoliko lagoon, and enters the Aetoliko lagoon through the arc stone bridges. The Nidova lagoon communicates with the Aetoliko lagoon (in the north) though the continuation of the arc stone bridges. During the last few years phytoplankton biomass blooms have formed in the restricted Nidova basin and cover almost the entire surface of the basin during the spring and early summer.

The Papas Lagoon is embedded within the northwestern coast of the Peloponnese, adjacent to the Gulf of Patras (Figure 1b). The lagoon is of elongated shape, with its main axis along the NW-SE direction. It is $5 \mathrm{~km}$ long, $1 \mathrm{~km}$ wide on average, and covers an area of about $6.2 \mathrm{~km}^{2}$ [13]. It is a deep water body, having a mean depth of $1.8 \mathrm{~m}$ and a maximum depth of $5 \mathrm{~m}$. It is connected to the Gulf of Patras with three stable tidal inlets, the length and width of which lie within 160-260 m and 25-50 m, 
respectively. During the winter, it is supplied with fresh water by a small draining stream discharging into the lagoon's southeastern part, which borders cultivated land [14].

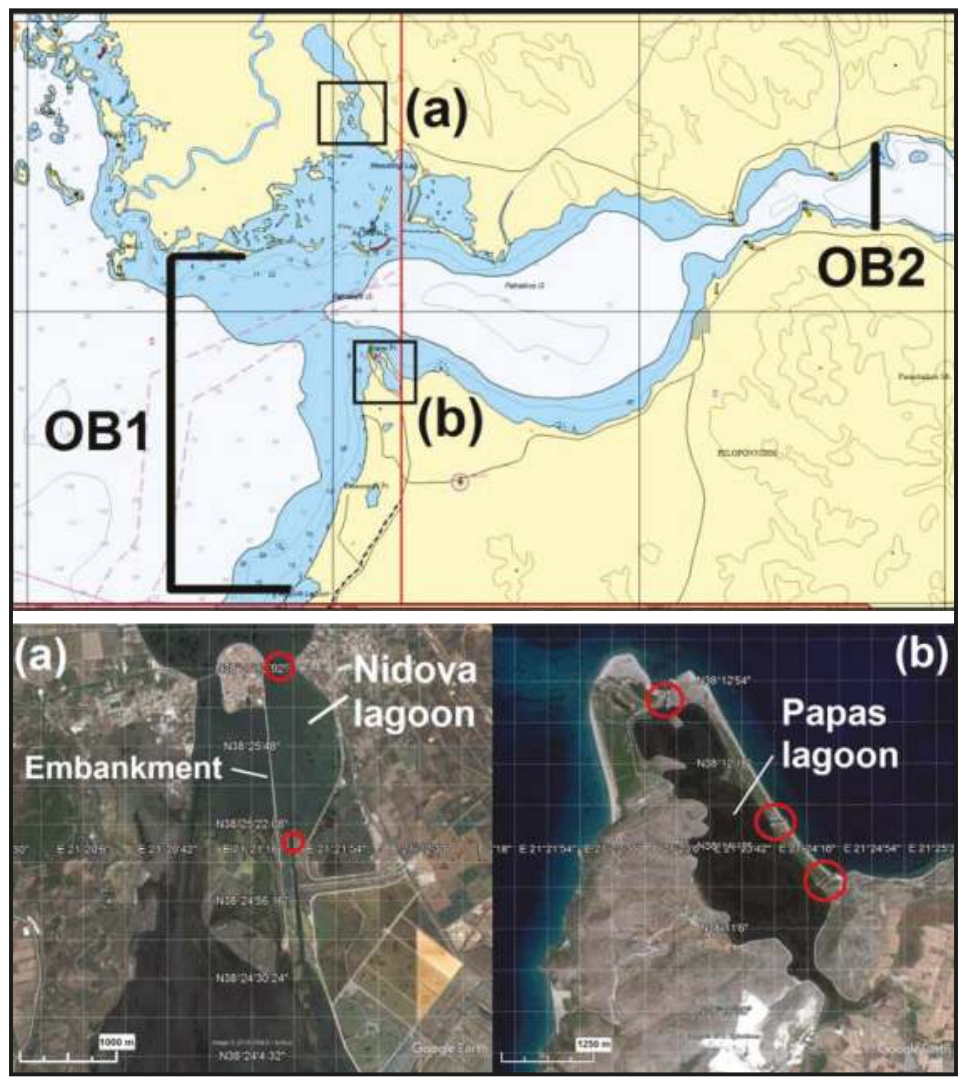

Figure 1. Upper panel: General map of the wider area of the Gulf of Patras in western Greece (adapted from MIKE C-MAP, 2018, [15]). The open boundaries have been marked with black lines. Land area is given in yellow; sea area is given in blue and white (blue for depths $<50 \mathrm{~m}$ and white for depths $>50 \mathrm{~m}$ ). Detail of the areas of interest is given in the lower panel for (a) the Nidova lagoon (Google Earth, 12-08-2017) and (b) the Papas lagoon (Google Earth, 15-07-2016) [16]. The locations of tidal inlets are denoted with red circles.

The lagoon is subject to extensive fish exploitation and aquaculture. It is naturally eutrophic, with no anthropogenic influences on the waterfront. Within the last 35 years, nine dystrophic crises have been reported, followed by mass fish mortality and benthic fauna; these occurred during the summer months of the years 1979, 1984, 1987, 1996, 1997 [17], 2004, 2010, and 2012 [18]. The dystrophic crises have been related to the decomposition of large beds of macro algae [14].

Both lagoons are protected by the Ramsar Convention, and the Nidova lagoon is also included in the Natura 2000 network. The proper management of the lagoons, which is of primary importance to local fishermen, includes plans for dredging operations, creation of inlets, or other infrastructure works in the area, with the aim of improving the hydrodynamic circulation and water renewal of these ecosystems. 


\subsection{The Hydrodynamic Code and the Advection-Diffusion Code}

The simulations presented herein have been performed using the commercially available CFD (Computational Fluid Dynamics) code MIKE21 and MIKE3 Flow Model FM (where FM stands for flexible mesh). The MIKE3 Flow Model FM has been used for three-dimensional flow simulation in the Messolonghi-Aetoliko lagoon. It is a modeling system developed by the Danish Hydraulic Institute (DHI), based on a finite volume and an unstructured mesh approach. The hydrodynamic module (HD) extracts numerical solutions from the three-dimensional continuity, momentum, temperature, salinity, and density equations. The momentum equations are used in the incompressible, Reynolds-averaged form of the Navier-Stokes equation (RANS), invoking the Boussinesq assumption and the hypothesis of hydrostatic pressure in the vertical. The turbulence closure is achieved using the Smagorinsky formulation for diffusion in the horizontal and the standard $\mathrm{k}-\varepsilon$ model in the vertical direction. To account for the Coriolis force, an f-plane has been used. The free surface is taken into account using a sigma-coordinate transformation. The spatial discretization of the primitive equations is performed using a cell-centered finite volume method. In the horizontal plane an unstructured grid is used, while in the vertical direction the discretization is structured. The elements are prisms or bricks whose horizontal faces are triangles or quadrilateral elements, respectively. A Riemann solver is used for computation of the convective fluxes in vertical interfaces, which makes it possible to handle discontinuous solutions. For convective fluxes in horizontal interfaces, two schemes are available: a low order (based on upwinding) and a high order. The low-order scheme requires considerable less time than the high-order scheme. Use is made of the high-order scheme for the accurate simulation of the advection-diffusion simulations (see Section 2). For the time integration a semi-implicit approach is used, where the horizontal terms are treated explicitly and the vertical terms implicitly [19].

The MIKE 21 FM (HD) model has been used for simulations of the tidal propagation in the Gulf of Patras, thus providing the boundary conditions both at the entrance of the Messolonghi-Aetoliko lagoon (in the NW part of the Gulf) and at the tidal inlets of the Papas lagoon (in the SW part of the Gulf). The model simulates unsteady two-dimensional flow in one layer of vertically homogeneous fluid, by having the equations for the conservation of mass and momentum integrated over the vertical [20]. We note that the entrances of both the Papas lagoon and the Messolonghi lagoon are at a safe distance from the Rio-Antirio straits, where the presence of stratification influences local hydrodynamics [21]. Thus, the depth-averaged simulations produce adequate boundary conditions for the three-dimensional simulations of flow in both lagoons.

Finally, the advection and diffusion of the conservative tracer, applied in the Nidova and Papas lagoons, is simulated utilizing the CFD code MIKE 3 FM Transport Module (TR). This modeling system simulates the spreading and fate of dissolved or suspended substances in an aquatic environment under the influence of the fluid transport and the associated diffusion processes. The hydrodynamic basis for the Transport Module (TR) is calculated with the Hydrodynamic Module (HD) [22].

\subsection{Computational Domain and Grid}

Due to a lack of free surface time series measurements at the entrance of the tidal inlets of the Nidova and the Papas lagoons, which would have been adequate data as boundary conditions for flow simulations, the only alternative is the computation of such data. The only locations that are suitable for boundaries of numerical simulations and where, at the same time, the harmonic constituents of the tide are available, are both ends of the Gulf of Patras, one of which is close to the southern opening of the Messolonghi lagoon and the tidal inlets of the Papas lagoon [23]. It would have been numerically inefficient to perform fully three-dimensional simulations in a domain comprising of an area as large and deep as the Gulf of Patras (135 m maximum depth) together with the lagoonal systems, which includes extensive areas with depth less than $0.5 \mathrm{~m}$, to focus on a small section of the entire Messolonghi-Aetoliko lagoon, namely the Nidova lagoon. Thus, it was decided to resort to two-dimensional, depth-averaged simulations in a domain similar to the one used by [24] covering the entire area of the Gulf of Patras to extract boundary conditions for the three-dimensional simulations 
in the Messolonghi-Aetoliko lagoon. Such two-dimensional simulations are adequate, in terms of tidal propagation, even in summer conditions when thermal stratification is set in the lagoon ecosystems.

\subsubsection{The Nidova Lagoon}

The numerical domain for the three-dimensional simulations covers the entire Messolonghi-Aetoliko lagoonal system and a small part of the adjacent open waters of the northwestern part of the Gulf of Patras (i.e., the coastal waters in front of Messolonghi lagoon). This domain was discretized using a numerical grid consisting of a three-zone triangular mesh in the horizontal (Figure 2). In the first zone, covering the northwestern part of the gulf of Patras, the characteristic length is up to $400 \mathrm{~m}$ offshore. In the second zone, the characteristic length is $200 \mathrm{~m}$ and covers the greater area of the Messolonghi-Aetoliko lagoon complex, upstream and downstream of the area of interest, and in the third zone the length dimension varies from 20 to $50 \mathrm{~m}$ and covers the transitional part between the Messolonghi and Aetoliko lagoons, where the area of the Nidova lagoon is embedded.

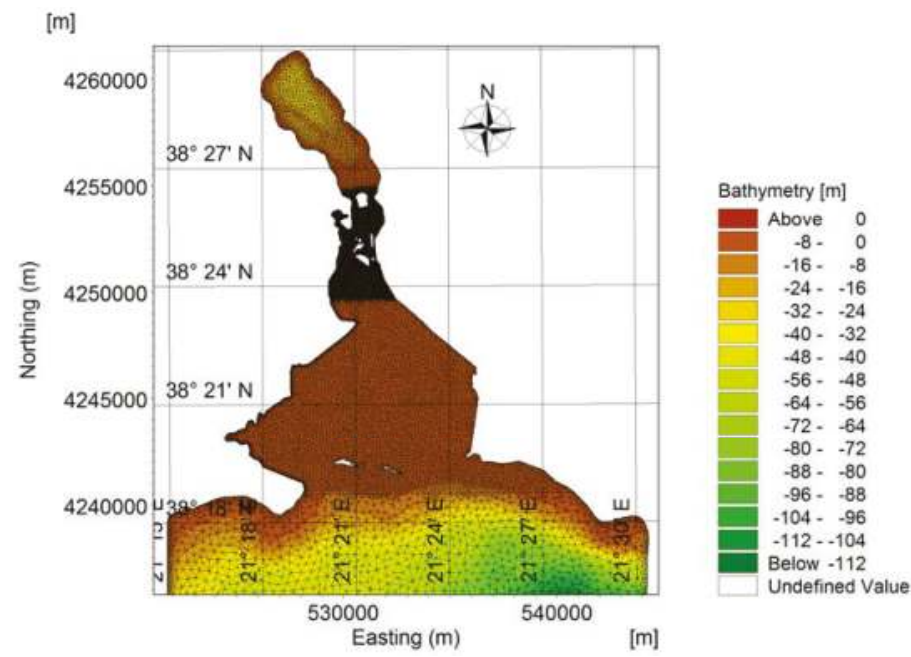

Figure 2. Bathymetric map of the Messolonghi, Aetoliko, and Nidova lagoons, with isobaths shown every $8 \mathrm{~m}$. Superimposed is the unstructured triangular mesh. The coordinate system refers to UTM-34. In the middle of the domain a finer mesh covers the wider area of the Nidova lagoon.

The goal of the present study is to compare how the residence time of semi-enclosed water bodies (i.e., lagoons) depends upon the details of their geometry. Since the evaluation of residence times is based on simulations of numerical tracers' transport, it was found necessary, in order to increase the accuracy, to create a third (even smaller) domain (Figure 3), consisting of the entire Nidova lagoon, the Aetoliko lagoon, and part of the surrounding Messolonghi lagoon area. It is within this truncated domain that the Nidova waters are estimated to remain when exchanged with adjacent waters of the Messolonghi and Aetoliko lagoons under tidal forcing, at least for the time span of interest. Again, the free-surface-time-series boundary conditions for this truncated domain are to be obtained from flow simulations in the immediately larger domain (Figure 2). In the truncated domain, the numerical grid consists of 10 equidistant layers in the vertical, and a horizontally varied mesh lying between 10 and $20 \mathrm{~m}$. This domain proved adequate for simulations covering at least 40 days, which, as verified a posteriori, greatly exceeds the water residence time for the Nidova lagoon.

Finally, to produce a grid on which the numerical simulations would require a reasonable amount of computer time, it was found necessary to merge in one opening all the openings of the series 
of arches supporting the stone bridge located at the junction of the Nidova and Aetoliko lagoons. The reason for this approximation is that the span of the arches is barely a few meters, so discretization of all the arches would require a mesh of characteristic length on the order of $30 \mathrm{~cm}$ or so, which is not practical. This practice gave rise to the discretization depicted in Figures 2 and 3.

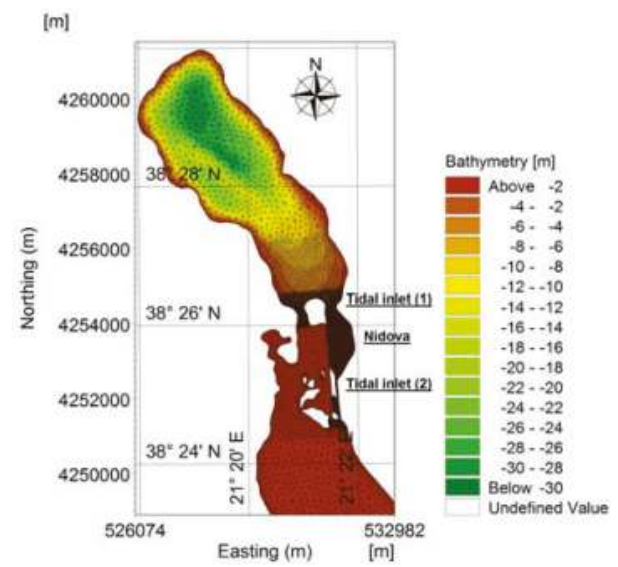

Figure 3. Truncated domain for the wider area of the Nidova lagoon, covering the Aetoliko lagoon and part of the Messolonghi lagoon.

\subsubsection{The Papas Lagoon}

Concerning the Papas lagoon, a truncated computational domain was formed covering the entire lagoon and the adjacent open waters of the southwestern part of Gulf of Patras, consisting of three zones in the horizontal (Figure 4).

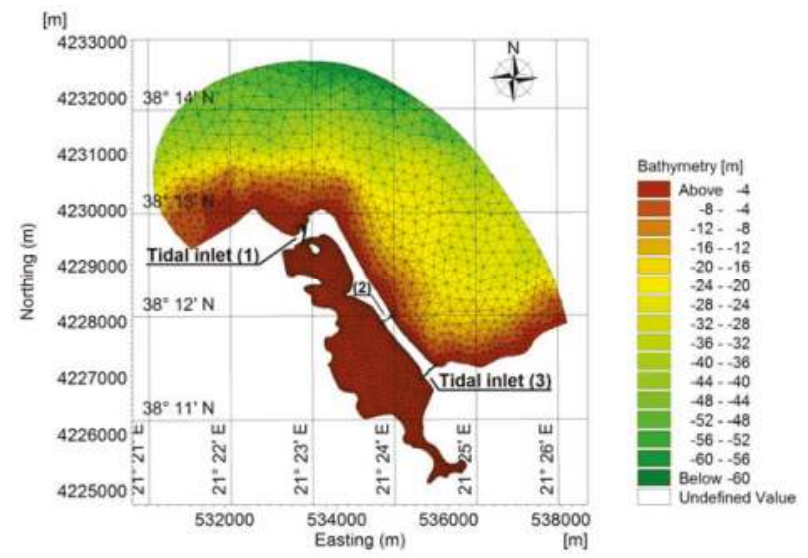

Figure 4. Truncated domain for the wider area of the Papas lagoon, covering part of the Gulf of Patras.

In the first zone, grid triangles have a characteristic length of up to $300 \mathrm{~m}$ offshore, and cover the southwestern part of the gulf of Patras, which is included in the simulation domain. In the second zone, the characteristic length varies from 20 to $100 \mathrm{~m}$ and covers the greater area of the Papas lagoon, which is the area of main interest and, finally, in the third zone, the cells' dimension varies from 5 to $20 \mathrm{~m}$ and covers the tidal inlets of the lagoon as well as the transitional part between the 
open gulf's waters and the lagoon. This truncated domain is designed so that its periphery is very nearly an equitidal line, and, as in the case of Nidova, was made large enough so that waters initially inside the Papas lagoon and later exchanged with Gulf of Patras waters will remain inside this domain during the whole length of the simulations, as verified by the numerical tracer experiments. The grid consists of 10 equidistant layers in the vertical.

\subsection{Boundary and Initial Conditions}

In all cases considered herein, the coastline has been defined as an impermeable, zero normal velocity boundary, while the bottom is a no-slip (via wall functions), impermeable boundary. For the first domain where the Nidova lagoon is located, the bottom roughness was set equal to $0.01 \mathrm{~m}$ and care was taken to ensure that the geometry of the bottom cell was compatible with the requirement of fully rough flow, assuming the minimum flow depth to be $0.4 \mathrm{~m}$. In the transitional part, i.e., in the interior of Messolonghi lagoon, a higher bottom roughness was considered in order to represent the vegetated bottom of the shallow lagoon. At the southern end of the computational domain lies the Gulf of Patras: it is from this boundary that the tide enters the lagoons, namely OB1 (Figure 1). To formulate an elevation time series for the location of this open boundary, results from the two-dimensional simulations of the Gulf of Patras were used in the simulations that follow. For the secondary truncated domain (Figure 3), used for the experiments with the tracer, tidal boundary conditions were extracted from the previously conducted simulations of the intermediate domain (Figure 2).

Concerning the Papas lagoon, tidal elevation time series that resulted from the two-dimensional simulations of the Gulf of Patras were used as open boundary conditions in the simulations that follow (Figure 4).

Initial conditions were needed in all four regions: (a) the Aetoliko lagoon at the northern of the domain, (b) the Messolonghi lagoon in the central part, (c) the opening (OB1) at the northeastern part of the Gulf, and (d) the Papas lagoon. Mean temperature and salinity profiles were imposed as initial conditions throughout the Aetoliko basin, based on the field measurements at four stations covering the longitudinal axis of the lagoon in a NW-SE direction, measured in July 2010 [25], since these measurements showed little horizontal variation. Since this dataset included no measurements in the Messolonghi lagoon, field measurements performed by [26] in the same summer period, i.e., July 2006, were used. Finally, the required profiles for the Gulf of Patras were taken as in [21].

Concerning the Papas lagoon, the simulations were performed considering barotropic flow in the basin even for the summer months, when the Gulf's waters (outside the Papas lagoon) were found to be stratified [27]. This seems justified because in the shallow waters of the Gulf, within the domain chosen, previous simulations show that the stratification is destroyed and the waters become nearly isothermal and these waters are of constant salinity. Further, according to measurements of [28], stratification does not seem to be important in the lagoon during the summer months. Therefore, the flow, both into the lagoon and in the near vicinity outside it, can be taken as homogeneous.

\subsection{The Tracer}

In this section we present the method we applied to estimate the residence time of the Nidova and Papas lagoonal ecosystems. Various transport time scales have been used in the literature to quantify the renewal of waters in natural systems (e.g., [29]). The present work has been stimulated by research in coastal lagoons that suffer from recurring dystrophic crises, the inception of which has been observed to occur locally before spreading to the entire lagoon [18]. Therefore, it is desirable to aim at calculating a time scale characterizing the local transport rate rather than an integral time scale characterizing the overall renewal of the entire lagoon. For the Papas lagoon, the latter has been carefully calculated in the form of flushing time by [14], who based their calculations on water quality measurements in the lagoon. We note that even though they call their time scale a residence time, we have referred to it as a flushing time since herein we use the definition of [29] for flushing time as an integral scale, and retain the local character they give to the term residence time. 
More precisely, for the purposes of the calculation, we define as residence time the time needed for the concentration of a conservative, passive tracer to fall to $1 / \mathrm{e}(\sim 37 \%)$ of its initial value (see for example [30,31]). By defining the initial concentration of the conservative numerical tracer to be equal to 1 inside and 0 outside the lagoon, we are thus able to calculate the residence time at each point inside the lagoon by following the evolution of the concentration of the tracer as determined by the advection-diffusion equation. This information should be useful in the analysis of the very complex problem of the inception of the recurring dystrophic crises that plague especially the Papas lagoon. We note, however, that the e-folding value is a mere convention and that in different applications the use of different cutoffs may be required. This is especially true for research concerning the causes of the dystrophic crises, a topic not well understood at this time.

\section{Results and Discussion}

\subsection{Hydrodynamic Characteristics of the Lagoon Ecosystems}

Both the Nidova and the Papas lagoon communicate with the Gulf of Patras: the former is situated in the northwest, whereas the latter is in the southwest of the Gulf (Figure 1), and are therefore subject to similar tidal forcing, in terms of amplitude, because the distance of both locations from the Rio-Antirio Straits, where the semidiurnal tide entering the Gulf of Patras from the Ionian Sea undergoes the reflection [24], is nearly the same. In the following summary of the salient features of the hydrodynamic forcing of the lagoons, we confine our attention to the purely tidal flow, ignoring wind-induced effects, since this is the most severe situation that arises, in terms of water renewal, in both lagoons.

An instance of the velocity field induced by flood tide in the Messolonghi-Aetoliko lagoonal system is depicted in Figure 5, where it can be observed that surface velocities range between 5 and $12 \mathrm{~cm} / \mathrm{s}$, except in locations where the depth is very shallow. The high velocities are especially pronounced at the sand bars, i.e., in sites where tidal inlets are formed, at the entrance of the Messolonghi lagoon (from the Gulf of Patras), and are crucial for fish migration into the lagoon ecosystem.

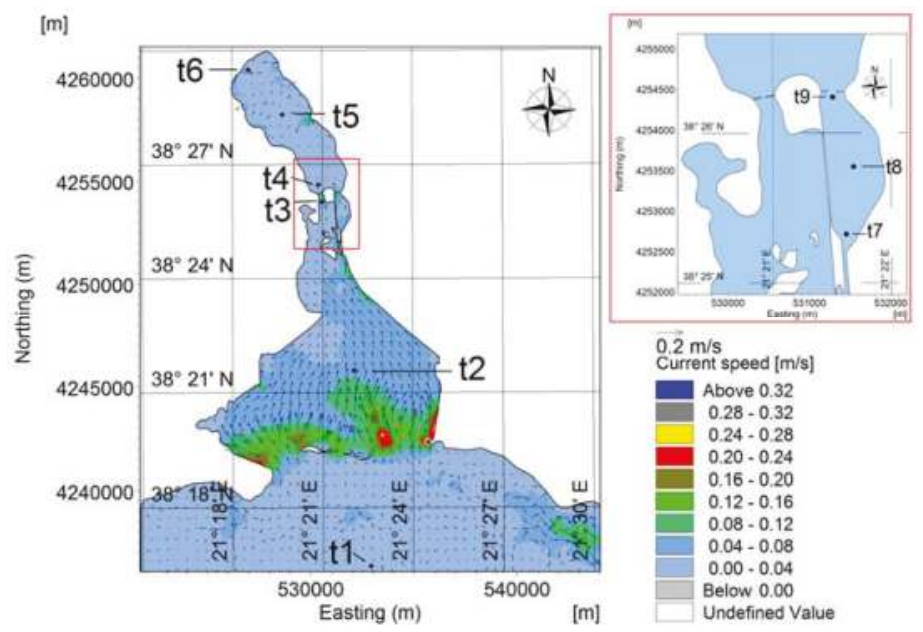

Figure 5. The surface flow field during flood tide into the Messolonghi-Aetoliko lagoonal complex. At the right upper corner, detail of the wider area of Nidova lagoon is shown, and numerical stations t7, $\mathrm{t} 8$, and $\mathrm{t} 9$ are marked.

The very shallow tidal flow in the Messolonghi and Nidova lagoons is controlled by high bottom friction. This is documented in Figure 6, where it is shown that, as we proceed from the Gulf of Patras towards the Aetoliko lagoon through the Messolonghi lagoon, there is a decrease in the amplitude of 
the tide and a delay. The latter becomes manifest as a phase difference between the time series at the entrance of the Messolonghi lagoon (which is also the entrance of the underwater trench that leads to the Southern entrance of Nidova) and at the stone bridges, which delineate the northern end of the Messolonghi lagoon but also of the Nidova lagoon (with the interpolation of the Aetoliko island). Thus, the Gulf of Patras tide reaches the northern end of the Nidova lagoon via the Aetoliko lagoon, the surface of which undergoes oscillations in unison, because of its relatively large depth (from $12 \mathrm{~m}$ in the southern part up to 30-35 $\mathrm{m}$ in the northern part). This differential path the tide of the Gulf of Patras has to take in order to reach the two ends of the Nidova lagoon results in the development of a differential amplitude and a phase lag and therefore a pressure gradient along the Nidova lagoon (Figure 7). This seems to be the key factor that causes the Nidova lagoon to function as a flow-through lagoon, with a concomitantly short time of water renewal, as we shall see in the following.

This analysis was tested by performing a simulation in which we closed the tidal inlet that connects the Nidova and Aetoliko lagoons, so as to block the flushing phenomenon. The result was, anticipating Section 3.2 below, that the residence time for this blocked Nidova lagoon increased to 20 days, as compared to the less than three days when flushing is allowed to occur.
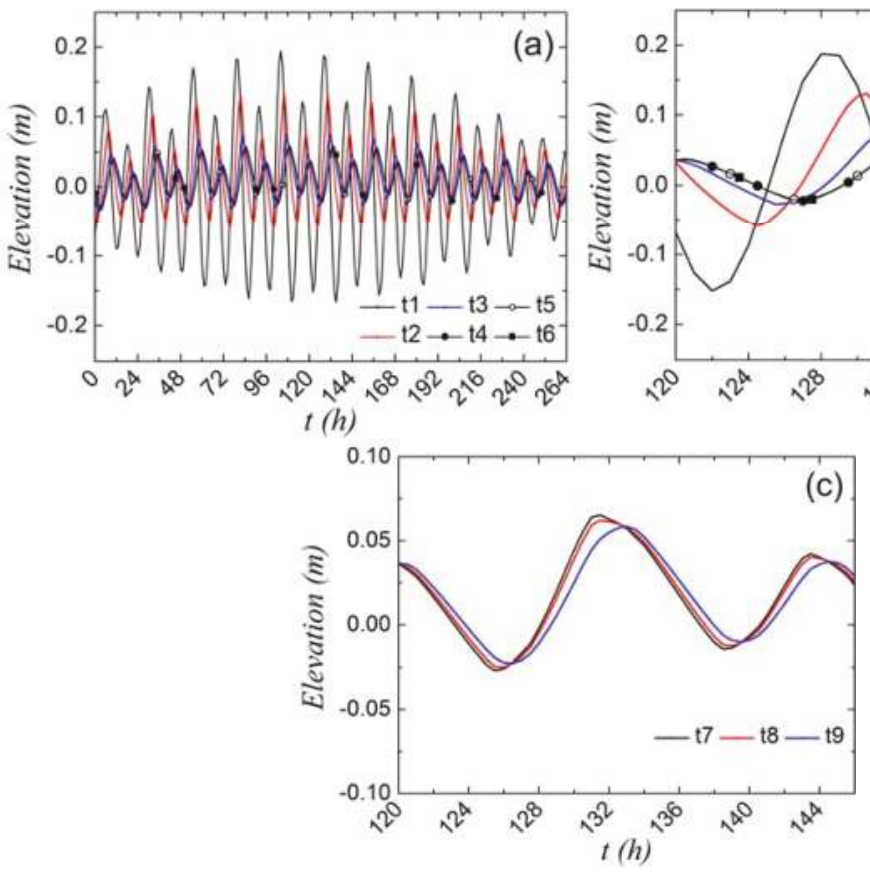

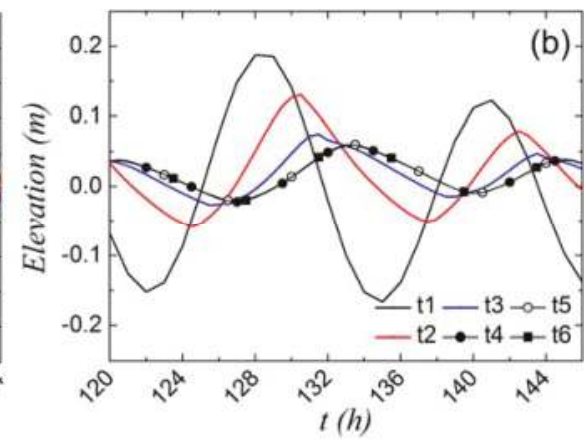

(c) $t(h)$ 


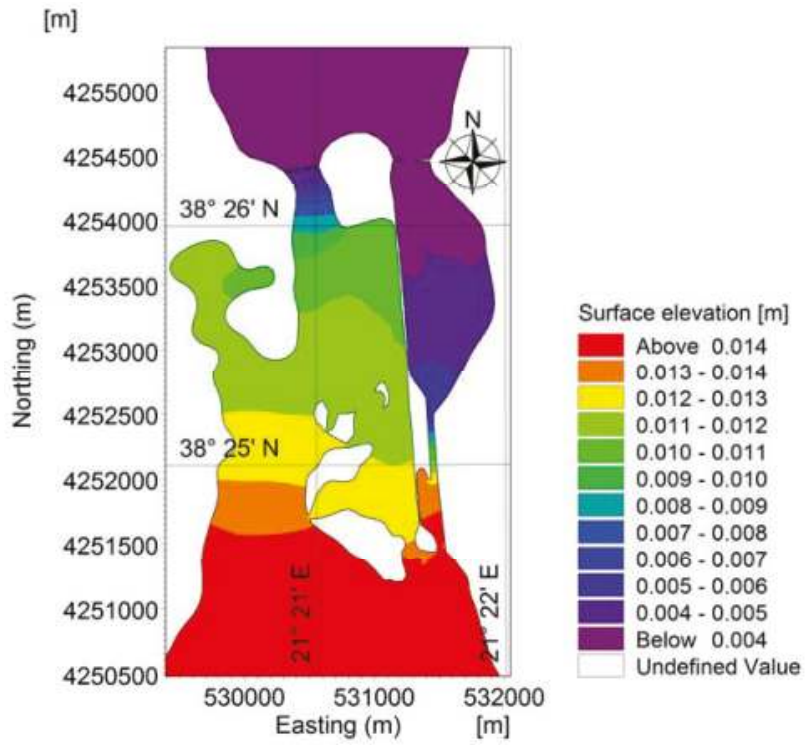

Figure 7. Sea surface formation during the tidal propagation in the wider area of Nidova lagoon.

If we were to confine our attention to its geometry alone, we would have to classify Nidova, with its two tidal inlets as a restricted lagoon and would have expected, accordingly, its water renewal to be rather slow. The fact that the two inlets of the lagoon are situated on opposite sides of its long axis does not by itself dictate the functioning of the lagoon as a flow-through system, because, as we shall see, nearly the same geometrical setting exists for the Papas lagoon, but there the hydrodynamics impose a radically different forcing. This fact has not been reported in the literature before, and therefore needs closer examination as to the exact circumstances in which it occurs. It seems, however, to be a variation of the famous, in the history of science, precedent of the quasi-periodic currents created by the differential arrival at the Strait of Euripus (between mainland Greece and the island of Evvoia (or Euboea) of the tide moving around Evvoia [32,33]. These currents have been famous since ancient times because they were associated with the name of Aristotle, who, living his last years in Evvoia, became fascinated with them, though he only had partial success in explaining the phenomenon [34,35].

The specific values for the tidal circulation in the Nidova lagoon are as follows. The incoming water, i.e., the exchange flowrate (Figure 8), was computed to range between $8 \mathrm{~m}^{3} / \mathrm{s}$ (neap tides) and $24 \mathrm{~m}^{3} / \mathrm{s}$ (spring tide), with an intermediate value of $\sim 11 \mathrm{~m}^{3} / \mathrm{s}$ (e.g., mean tidal range). Maximum flow velocities of tidal currents of $0.5 \mathrm{~m} / \mathrm{s}$ and $0.4 \mathrm{~m} / \mathrm{s}$ occur in the dredged trench during the flood and ebb phases of the tidal action, respectively. Water velocities were found to diminish rapidly in the submerged open channel, with values of $0.20-0.30 \mathrm{~m} / \mathrm{s}$ at the free surface, while near the bottom these values were decreased to $0.05-0.1 \mathrm{~m} / \mathrm{s}$. In contrast, at the opposite side of the lagoon, i.e., in the neighborhood of the arc stone bridges, a water flow velocity of approximately $0.2 \mathrm{~m} / \mathrm{s}$ developed. To summarize, the numerical predictions show that the Nidova lagoon is being flushed through the south dredged trench, transferring tidal waters from the greater Messolonghi lagoon to the deeper Aetoliko lagoon in the north and back to the Gulf of Patras. 


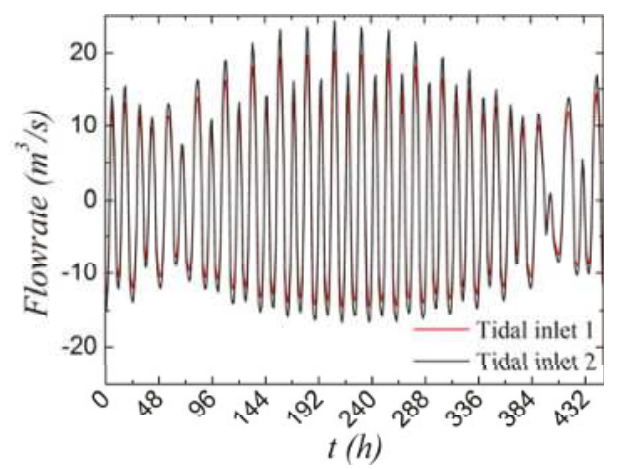

Figure 8. The hydraulic exchange flowrate between the Nidova lagoon and the surrounding waters (i.e., Aetoliko lagoon - tidal inlet 1, Messolonghi lagoon - tidal inlet 2).

In contrast to the Nidova lagoon, the Papas lagoon is subject to a straightforward tidal forcing. It has three tidal inlets: two in the eastern side and one in the northern side of the lagoon, and all of them are exposed immediately to the semidiurnal tide of the Gulf of Patras. The distances between them are small so that both the amplitude and the phase of the tide is practically the same in all three. An instance of the velocity field induced by flood tide in the lagoonal system is depicted in Figure 9, where it can be observed that surface velocities range between 0.5 and $20 \mathrm{~cm} / \mathrm{s}$, except in locations where the flow is strongly affected by the presence of the inlets. The high velocities are especially pronounced at the three inlets and in front of these, since the tidal currents through the narrow inlets flowing into the wide lagoon form a tidal jet, often called a mushroom jet, as it generates a vortex pair. When the tide reverses (ebb flow), the pattern of the flow resembles a radial flow to a sink [36]. The hydraulic exchange flowrate between the lagoon and the Gulf's open waters, via the three tidal inlets, is given in Figure 10. It can be seen that the exchange flowrate for the three inlets ranges between $4-6 \mathrm{~m} 3 / \mathrm{s}$ (neap tides) and $7-15 \mathrm{~m} 3 / \mathrm{s}$ (spring tide), with an intermediate value of 5-10 $\mathrm{m} 3 / \mathrm{s}$ (e.g., mean tidal range).

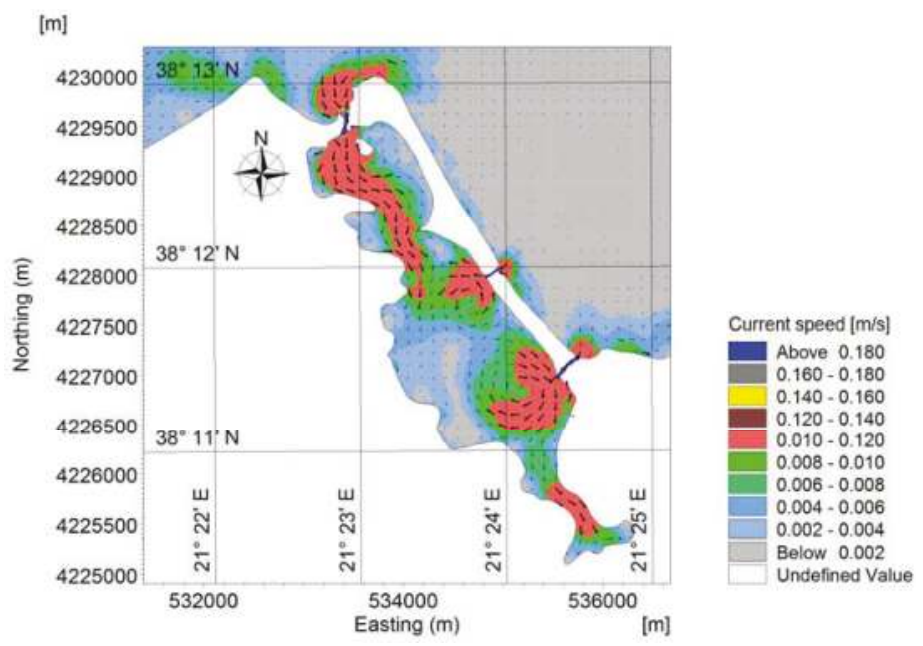

Figure 9. Cyclonic and anticyclonic gyres that form in the vicinity of tidal inlets in Papas lagoon during the flood tide. 


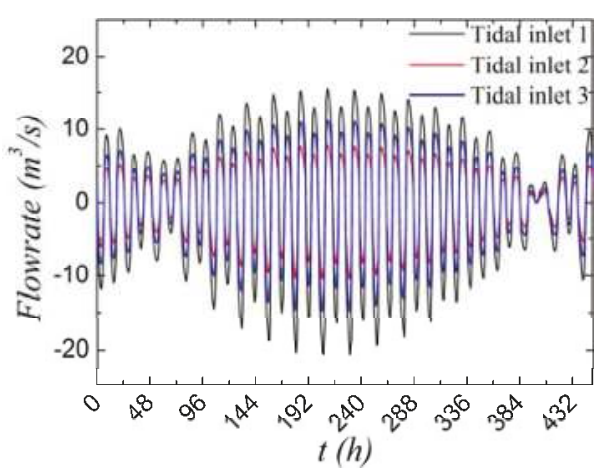

Figure 10. The hydraulic exchange flowrate between the Papas lagoon and the Gulf of Patras.

\subsection{Residence Time of the Lagoons}

\subsubsection{The Nidova Lagoon}

Based on the above description of the tidal hydrodynamics of the Nidova basin, it is clear that the renewal mechanism of its waters differs substantially from the mechanism based on the mere introduction of a volume of water equal to the tidal prism at the first half of the tidal cycle, recirculation and mixing of that volume within the lagoon, and subsequent exit of an almost equal amount in the second half of the tidal cycle. This is because the water introduced from the southern tidal inlet moves northwards and in part exits from the northern inlet into the Aetoliko lagoon, where it mixes with the Aetoliko lagoon waters before proceeding to the second half of the cycle.

To produce a value for the residence time scale, the procedure described in Section 2.5 was followed. The result is summarized in Figure 11, from which it follows that renewal is achieved in less than three days.

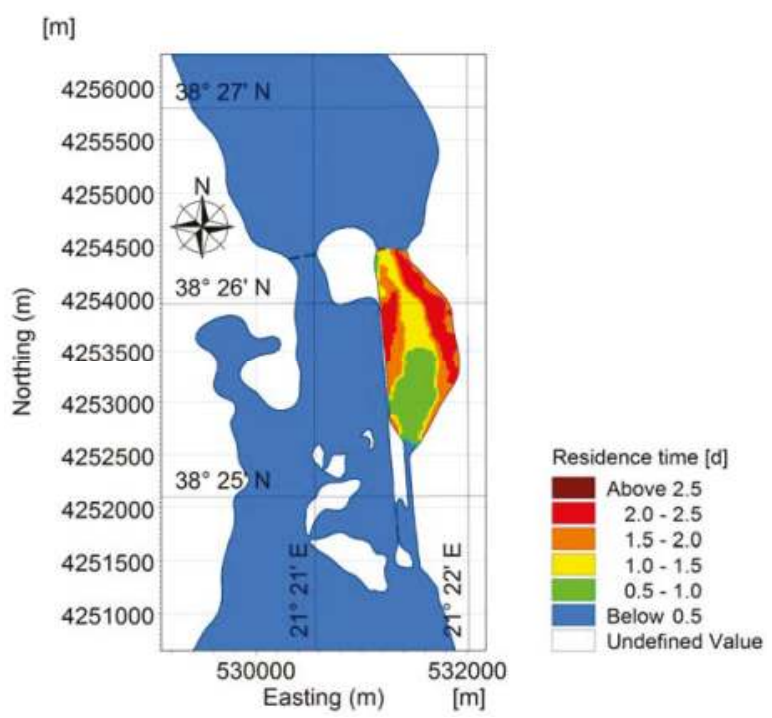

Figure 11. Spatial distribution of the residence time of the conservative tracer for the Nidova lagoon. Values below 0.5 refer to 0 initial concentration. 
It should be stressed again that this specific value depends strongly on the definition of the threshold under which the tracer's value is considered small, so that the timescale value should be used with great care and in conjunction with the desired application. A further note of caution is in order for the specific site we are analyzing. From the above description, it follows that waters that exit the Nidova lagoon mix with the Aetoliko lagoon waters before re-entering in Nidova. Thus, the time scale determined is meaningful as a short-term scale. This is because we base our calculation on the decrease of a substance introduced once into the Nidova lagoon. If, however, substances such as agricultural fertilizers are being introduced intermittently into the Aetoliko lagoon, the result might be a more or less well mixed water volume of a substantial contaminant that would be recirculated within the Aetoliko-Nidova-Messolonghi lagoonal system within each tidal cycle. This might be the reason why, in spite of its impressively low residence time, recurring thick algae blooms have been observed to develop during the last few years in the Nidova lagoon.

The note of caution concerning the definition of the residence time using the standard e-folding value becomes concrete in the following simulated scenario. The effect on residence time of removing the embankment separating the Nidova lagoon from the Messolonghi lagoon was examined numerically. From a purely hydrodynamic point of view, removing the embankment enhanced the circulation, making possible the development of a gyre encompassing both the Messolonghi and the Nidova lagoons (Figure 12). The residence time, however, was not significantly changed, still remaining below three days.

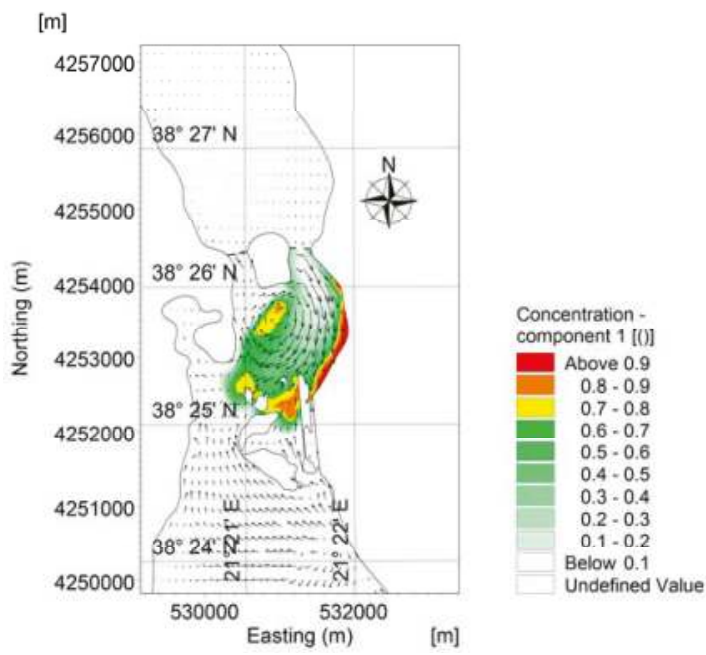

Figure 12. Spatial distribution of the concentration of the conservative tracer for the Nidova lagoon, after the embankment's removal, under the development of an anti-cyclonic gyre.

The above analysis rests on a very important condition that should be underlined. The dredged trench, connecting the southern end of the Nidova lagoon with the Messolonghi lagoon, should remain clear of transported material so as to ensure that the Gulf of Patras-Messolonghi-Nidova-Aetoliko system remains a flow-through system.

\subsubsection{The Papas Lagoon}

The numerical results showed that the residence time in the water body exhibits a strong spatial variation. In the region located in front of the inlets, rapid mixing occurs and these areas have the shortest residence time ( $\sim 5$ days). The southern, somewhat isolated shallow region and also the deeper central-western region, which is relatively far from the inlets, seems to remain at a near 
stagnation state for significant time intervals, having a residence time of more than 40 days (Figure 13). The hydrodynamic gyres due to tidal action cause a conservative tracer to be trapped between the two openings in the eastern side of the lagoon. In the middle area of the eastern openings, a rather unexpectedly high residence time appears. This area seems to be at a near stagnation state, having a residence time of approximately $25-30$ days. It is worth noting that it is in this area, i.e., in the vicinity of tidal inlets, that the dystrophic crisis occurred in June 2012 [18], showing that the high residence time in this area is not incompatible with the local initiation of dystrophic phenomena.

Finally, it is worth noting that the northern tidal inlet and the lower of the two eastern inlets are geometrically in nearly opposite positions. This lagoon, however, does not function as a flow-through lagoon because the tidal hydrodynamics do not favor it. In other words, the tide arrives simultaneously at all the inlets, with no substantial difference in amplitude. This is the reason why the opening of the middle inlet in 1992 [18] made only a limited contribution to the renewal of the waters of the Papas lagoon.

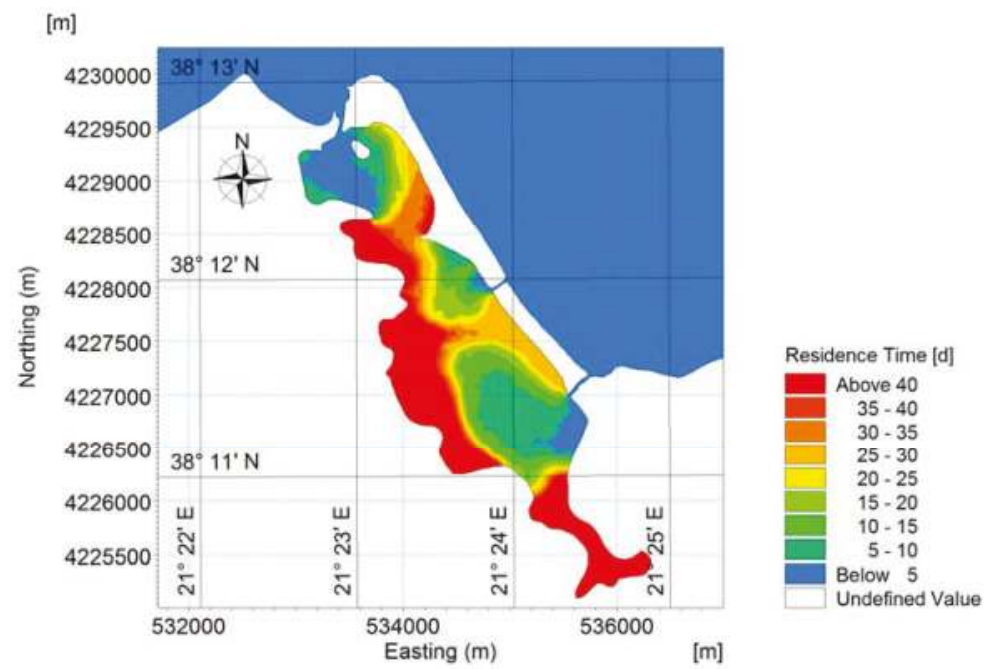

Figure 13. Spatial distribution of the residence time of the passive tracer for the Papas lagoon. Values below 5 outside the lagoon refer to 0 initial concentration.

\section{Conclusions}

Restricted lagoons suffer from long residence times, which result because of the limited tidal hydraulic exchange between the lagoon and the adjacent open water body (the ocean). The present study documented, however, that this state is not solely a result of the geometry of the lagoon, but also of the intricacies of the tidal hydrodynamics, making credible the term "hydrodynamic geometry". This term is meant to signify that if the geometry is in the right relation with the tidal forcing applied at different tidal inlets of the lagoon, the result may be the creation of a flow-through system, which flushes the lagoon waters considerably more efficiently that the slow process of tidal prism exchange and mixing in successive tidal cycles. An example of the former is the Nidova lagoon, and the Papas lagoon of the latter: both are situated in Western Greece and forced by the same tide of the Gulf of Patras. The creation of strong currents because of the differential arrival of the tide at different locations is not as exotic as it might seem since it has also been known to happen in open waters, as in the well-known Evripos straits. This advantage, in terms of flushing rate, should therefore be taken into account whenever possible when deciding the opening of an additional tidal inlet in a lagoon in order to enhance its flushing rate. 
Acknowledgments: Useful discussions with Professors Yannis Cladas, of the Technological Educational Institute of Western Greece, and Constantin Koutsikopoulos, of the University of Patras, are gratefully acknowledged. Publication costs were provided by the Hydraulic Engineering Laboratory, Department of Civil Engineering, University of Patras.

Author Contributions: Nikolaos Th. Fourniotis and Georgios M. Horsch conceived and designed the numerical experiments, carried out the analysis and interpretation of the results, and wrote the paper; Nikolaos Th. Fourniotis and Georgios A. Leftheriotis performed the numerical simulations and analyzed the numerical results.

Conflicts of Interest: The authors declare no conflict of interest.

\section{References}

1. Colombo, G. Lagoons. In The Coastline; Barnes, R.S.K., Ed.; John Wiley and Sons: New York, NY, USA, 1977; pp. 63-81.

2. Tsihrintzis, V.A.; Sylaios, G.K.; Sidiropoulou, M.; Koutrakis, E.T. Hydrodynamic modeling and management alternatives in a Mediterranean, fishery exploited, coastal lagoon. Aquac. Eng. 2007, 36, 310-324. [CrossRef]

3. Koutsikopoulos, C.; Dimitriou, E.; Rogdakis, Y.; Katselis, G.; Pagoni, S.; Kentrou, A.; Koutrakis, E.; Cladas, Y.; Economidis, P.S. A typology of the Hellenic lagoons based on physical and socio-economic aspects on their fisheries characteristics. In Proceedings of the International Conference on Lagoons and Coastal Wetlands in the Global Change Context, Impacts and Management Issues, Venice, Italy, 26-28 April 2004; pp. 26-28.

4. Cataudella, S.; Crosetti, D.; Massa, F. Mediterranean Coastal Lagoons: Sustainable Management and Interactions among Aquaculture, Capture Fisheries and the Environment; Food and Agriculture Organization of the United Nations (FAO) Studies and Reviews No. 95; FAO: Rome, Italy, 2015; p. 278.

5. Dimitriou, E.; Economidis, P.S.; Athanasopoulos, T.; Kapareliotis, A.; Katselis, G.; Koutrakis, E.; Koutsikopoulos, K.; Liourdi, M.; Manios, V.; Roussi, A.; et al. Study of the organization and function of fishery use in lagoons. PESCA, ICHTHIKA S.A., Department of Fish-farming and Inland Waters, Hellenic Ministry of Agriculture, 2001; Unpublished work.

6. Corsi, F.; Ardizzone, G.D. Some environmental conditions affecting yellow eels catchability. Oebalia 1985, 11, 561-571.

7. Chauvet, C. Manuel Sur I Amenagement Des Peches Dans Les Lagunes Cotieres La Bordigue Mediterraneenne Fao Document Technique Sur Les Peches 290; Food and Agriculture Organization of the United Nations (FAO): Rome, Italy, 1988; p. 77.

8. Armenio, E.; De Serio, F.; Mossa, M. Analysis of data characterizing tide and current fluxes in coastal basins. Hydrol. Earth Syst. Sci. 2017, 21, 3441-3454. [CrossRef]

9. De Serio, F.; Mossa, M. Meteo and hydrodynamic measurements to detect physical processes in confined shallow seas. Sensors 2018, 18, 280. [CrossRef] [PubMed]

10. Kjerfve, B. Comparative oceanography of coastal lagoons. In Estuarine Variability; Kennedy, V.S., Ed.; Academic Press: New York, NY, USA, 1986; pp. 63-81.

11. Andréfouët, S.; Pagés, J.; Tartinville, B. Water renewal time for classification of atoll lagoons in the Tuamotu Archipelago (French Polynesia). Coral Reef. 2001, 20, 399-408. [CrossRef]

12. Aubrey, D.G.; Giese, G.S. Formation and Evolution of Multiple Tidal Inlets; American Geophysical Union: Washington, DC, USA, 1993.

13. Papatheodorou, G.; Avramidis, P.; Fakiris, E.; Christodoulou, D.; Kontopoulos, N. Bed diversity in the shallow water environment of Pappas Lagoon in Greece. Int. J. Sediment Res. 2012, 27, 1-17. [CrossRef]

14. Krasakopoulou, E.; Pagou, K. Seasonal steady-state budgets of nutrients and stoichiometric calculations in an Eastern Mediterranean lagoon (Papas Lagoon-Greece). Mediterr. Mar. Sci. 2011, 12, 21-41. [CrossRef]

15. DHI. MIKE C-MAP, Extraction of World Wide Bathymetry Data and Tidal Information. DHI Software, Version 2014. Available online: https://www.mikepoweredbydhi.com/download/mike-by-dhi-2014 (accessed on 15 January 2018).

16. Google Earth Pro, Version 7.3.0.3832. Available online: http://www.google.com/earth/download/ge/ agree.html (accessed on 17 January 2018).

17. National Centre for Marine Research (NCMR). Monitoring of the Papas Lagoon (Cape Araxos/Achaia) Ecosystem-Management and Protection Proposal; Final Technical Report; Pagou, K., Ed.; NCMR: Athens, Greece, 2000; p. 190. 
18. Cladas, Y.; Papantoniou, G.; Bekiari, V.; Fragkopoulu, N. Dystrophic crisis event in Papas Lagoon, Araxos Cape, Western Greece in the summer 2012. Mediterr. Mar. Sci. 2016, 17, 32-38. [CrossRef]

19. DHI. MIKE 3 FLOW MODEL FM. Hydrodynamic Module-User Guide. DHI Software. p. 138. Available online: https://www.mikepoweredbydhi.com/download/mike-by-dhi-2014 (accessed on 15 January 2018).

20. DHI. MIKE 21 FLOW MODEL FM. Hydrodynamic Module-User Guide. DHI Software. p. 132. Available online: https://www.mikepoweredbydhi.com/download/mike-by-dhi-2014 (accessed on 15 January 2018).

21. Fourniotis, N.T.; Horsch, G.M. Baroclinic circulation in the Gulf of Patras (Greece). Ocean Eng. 2015, 104, 238-248. [CrossRef]

22. DHI. MIKE 3 FLOW MODEL FM. Transport Module-User Guide. DHI Software. p. 46. Available online: https://www.mikepoweredbydhi.com/download/mike-by-dhi-2014 (accessed on 15 January 2018).

23. Achilleopoulos, P. Tides in Gulfs and Straits of Western Greece. Ph.D. Thesis, University of Patras, Patras, Greece, 1990.

24. Horsch, G.M.; Fourniotis, N.T. Wintertime Tidal Hydrodynamics in the Gulf of Patras, Greece. J. Coastal Res. 2017, 33, 1305-1314. [CrossRef]

25. Avramidis, P.; Bekiari, V.; Christodoulou, D.; Papatheodorou, G. Sedimentology and water column stratification in a permanent anoxic Mediterranean lagoon environment, Aetoliko lagoon, western Greece. Environ. Earth Sci. 2015, 73, 5687-5701. [CrossRef]

26. Marazioti, C.; Flessia, G.; Vlachos, P.; Koutrouli, E.; Ntaikou, I.; Antonopoulou, G.; Pakou, C.; Kalfas, K.; Kornaros, M.; Lyberatos, G. On the environmental State of the Mesologgi Lagoon in Greece. Fresen. Environ. Bull. 2010, 19, 1151-1164.

27. Papailiou, D.D. Oceanographical Study in Patras Gulf Waters for Pollution Management; University of Patras, Department of Mechanical Engineering: Patras, Greece, 1982; Volume 2, p. 161. (In Greek)

28. Cladas, Y.; Papantoniou, G.; Ketsilis, V.; Fragopoulou, N. Dystrophic crisis event Papas Lagoon (Araxos Achaia). In Proceedings of the 15th Panhellenic Ichthyologists' Conference, Acquatic Ecosystems: Uses, Impact and Management, Thessaloniki, Greece, 2013; pp. 10-13.

29. Monsen, N.; Cloern, J.; Lucas, L.; Monismith, S. A comment on the use of flushing time, residence time and age as transport time scales. Limnol. Oceanogr. 2002, 47, 1545-1553. [CrossRef]

30. Stamou, A.I.; Loverdou, L.; Matsoukis, C.; Albanis, T.; Gkesouli, A. Modeling renewal times in Amvrakikos gulf, Greece. Global NEST J. 2012, 14, 386-392.

31. Ranjbar, M.H.; Zaker, H.N. Numerical modeling of general circulation, thermohaline structure, and residence time in Gorgan Bay, Iran. Ocean Dyn. 2018, 68, 35-46. [CrossRef]

32. Eginitis, D. The problem of the tide of Euripus. Astron. Nachr. 1929, 236, 321-328. [CrossRef]

33. Tsimplis, M.N. Tides and sea-level variability at the Strait of Euripus. Estuar. Coast. Shelf Sci. 1997, 44, 91-101. [CrossRef]

34. Eginitis, D. The problem of the tide of Euripus. Pragmatiai Acad. Athens 1929, A, 127.

35. Gill, A.E. Atmosphere-Ocean Dynamics; Academic Press: San Diego, CA, USA, 1982; p. 662.

36. Li, Y.; Wolanski, E.; Zhang, H. What processes control the net currents through shallow straits? A review with application to the Bohai strait, China. Estuar. Coast. Shelf Sci. 2015, 158, 1-11. [CrossRef]

(C) 2018 by the authors. Licensee MDPI, Basel, Switzerland. This article is an open access article distributed under the terms and conditions of the Creative Commons Attribution (CC BY) license (http:/ / creativecommons.org/licenses/by/4.0/). 
Article

\title{
Invariants of Turbulence Reynolds Stress and of Dissipation Tensors in Regular Breaking Waves
}

\author{
Sandro Longo ${ }^{1, *}$, Maria Clavero ${ }^{2}$, Luca Chiapponi ${ }^{1}$ and Miguel A. Losada ${ }^{2}$ \\ 1 Dipartimento di Ingegneria e Architettura (DIA), Università di Parma, Parco Area delle Scienze, 181/A, \\ 43124 Parma, Italy; luca.chiapponi@unipr.it \\ 2 Instituto Interuniversitario de Investigación del Sistema Tierra (IISTA), Universidad de Granada, \\ Avda. del Mediterráneo s/n, 18006 Granada, Spain; mlosada@ugr.es (M.C.); mlosada@ugr.es (M.A.L.) \\ * Correspondence: sandro.longo@unipr.it; Tel.: +39-0521-905157
}

Received: 30 September 2017; Accepted: 10 November 2017; Published: 16 November 2017

\begin{abstract}
A series of measurements in a flume with a particle-tracking system in three dimensions applied to breaking waves is used to analyse the structure of turbulence with a full set of variables that usually are available only in numerical simulations. After extracting turbulence, in addition to the standard analysis aiming to quantify the fluxes, i.e., the time-average and the phase-average levels of turbulence and vorticity (details are given in two former papers), a more in-depth description of the structure of turbulence Reynolds stress tensor is given, focussing on the invariants evolution in time and in the vertical. A relation between the components of the Reynolds stress tensor and of the dissipation tensor is depicted. This relation is finalised to possible models of turbulence in breaking waves.
\end{abstract}

Keywords: breaking waves; turbulence invariants; laboratory experiments

\section{Introduction}

Breaking waves are rarely adopted as a flow field for modelling turbulence due to the unsteadiness and to the complexity of the phenomena taking place, enhanced by vorticity and turbulence generation and evolution during breaking and near the bottom. While the phenomena at the bottom are relevant in shallow water, the phenomena near the free surface are always relevant, even though with different level of importance depending on the stage of breaking evolution. Vorticity injection is addressed (i) to surface curvature plus the effects of boundary conditions at the interface (Longuet-Higgins, 1992 [1]); (ii) to the sharp curvature of the interface (Lin and Rockwell, 1994 [2]); (iii) to deceleration of the free surface before breaking (Dabiri and Gharib, 1997 [3]). Vorticity is often accompanied by turbulence even in cases where the classical air entrapment of the breaker is not observed. Turbulence is the final stage of energy transfer in sea gravity waves and marks the evolution of a low entropy system (gravity waves in non breaking conditions) to a high entropy system (broken waves). This evolution is accompanied by several phenomena of practical interest, like enhanced diffusion of heat, chemicals, gases, pollutants, currents generation, action on the sand bottom to activate sediment transport, or spray diffusion in the atmosphere. We notice that the energy budget of the incoming waves is generally not dominated by dissipation at viscous level (the final stage of turbulence action at a molecular scale, with the generation of heat), but is also based on transfer toward large scale features as long-shore and cross-shore currents, free (unbounded) long waves escaping offshore, edge waves trapped in the nearshore, and macro eddies at the wave length scale. In this respect, the analysis of turbulence structure in breaking waves helps the comprehension of all these new flow field structures, but at the same time it is more complex with respect to many other turbulence fields. Very recent contributions to the description of the flow field structure in waves and breaking waves are due to Grue and Kolaas, (2017) [4], who applied particle tracking velocimetry (in two-dimensional space, 2D) in non-breaking periodic laboratory 
waves to estimate the Lagrangian drift velocity, and to Smith et al., (2017) [5], who studied plunging breakers on a sloping beach (in 2D) with Particle Image Velocimetry, paying attention to air bubbles geometry and dynamics. The threshold of onset of breaking is still under scrutiny, since there are numerous phenomena affecting the process. In this respect, the analysis by Barthelemy et al., (2015) [6], has been recently extended by Saket et al., (2017) [7] to include deep water wave groups with or without wind action.

The models of turbulence generation and evolution are based on observations, detailed measurements, and direct numerical simulation (DNS) (see also Deike et al., 2016 [8] and the recent paper by Deike et al., 2017 [9]). The dissipation in unsteady breaking waves has been parametrized by Drazen et al., (2008) [10], who found a dissipation per unit length of breaking crest proportional to $\rho_{w} g c^{5}$, being $\rho_{w}$ the mass density of water, $g$ the gravity acceleration, and $c$ the phase speed. A huge effort in measuring the flow field characteristics in breaking surface waves and in analysing the turbulence characteristics (in 2D) is documented in Drazen and Melville, (2009) [11]. In particular, many models for turbulence have been developed by analysing the structure of the Reynolds stress tensor and of the dissipation tensors, which can be obtained only with DNS. These tensors carry information about the "componentality" of turbulence, i.e., the strength of turbulence with direction, and are local estimators. Other tensors are non-local estimators and depict the "dimensionality" of turbulence and account for the space structure of fluctuations (Reynolds, 1989 [12], Stilyanou et al., 2015 [13]). The study of the invariants of these tensors is of interest since their relative strength can be adopted to develop models based on a functional dependence.

The availability of three-dimensional measurements of fluid velocity with particle tracking methodology allows for the first time a direct evaluation of these tensors. The aim of the present work is (i) the identification of the level of isotropy/anisotropy in the turbulence by analysing the Reynolds stress tensor and the dissipation tensor; and (ii) the individuation of the functional dependence between the components of these tensors. A feature of anisotropic Reynolds stress flows is the presence of organized flow structures. It is true in wall boundary layers (Robinson, 1991 [14]) and in free surface boundary layers (Longo, 2010 [15]), and is expected to be true also in the region beneath the free surface in breaking waves.

In the present work we focus on the structure of turbulence. The experimental layout, the experiments, and the data analysis are detailed in Clavero et al., (2016) [16], and are shortly described herein. The vorticity dynamics has been analysed in depth in Chiapponi et al., (2017) [17].

The paper is structured as follows. Section 2 briefly describes the experimental layout and the experiments, Section 3 explains the methodology for data analysis. Section 4 is devoted to the analysis of the Reynolds stress and dissipation tensors. Section 5 gives the Conclusions.

\section{Experimental Set-Up and Experiments}

A series of experiments were planned in the wave flume of the Andalusian Institute for Earth System Research (IISTA), Universities of Granada and Cordoba, in Granada. An artificial slope of 1:10 with a berm of stones and plastic blocks was realized in order to force the breaking section, see Figure 1, with a still-water depth in front of the paddle of $43 \mathrm{~cm}$. Reflection was controlled with an active absorption system (AWACS).

A three-dimensional (3D) Particle Tracking Velocimetry system (V3V by TSI Inc., USA) was used for measuring the velocity in a volume of $\approx 14 \times 14 \times 10 \mathrm{~cm}^{3}$. The volume of measurement was enlightened by a laser, and three cameras ( $2048 \times 2048$ pixels) generated three pairs of 12-bit images, with a time delay between two images of each pair of $100 \mu \mathrm{s}$. The frequency of V3V was $7.25 \mathrm{~Hz}$, with 10 or 13 shots per each period for waves with $T=1.5 \mathrm{~s}$ and $2.0 \mathrm{~s}$, respectively. Each experiment contained 10 cycles, with a total of 100 and 130 shots. The overall accuracy in velocity measurements is $0.1 \mathrm{~cm} / \mathrm{s}$ for the cross-shore and the vertical direction, and $0.4 \mathrm{~cm} / \mathrm{s}$ for the alongshore velocity component. 

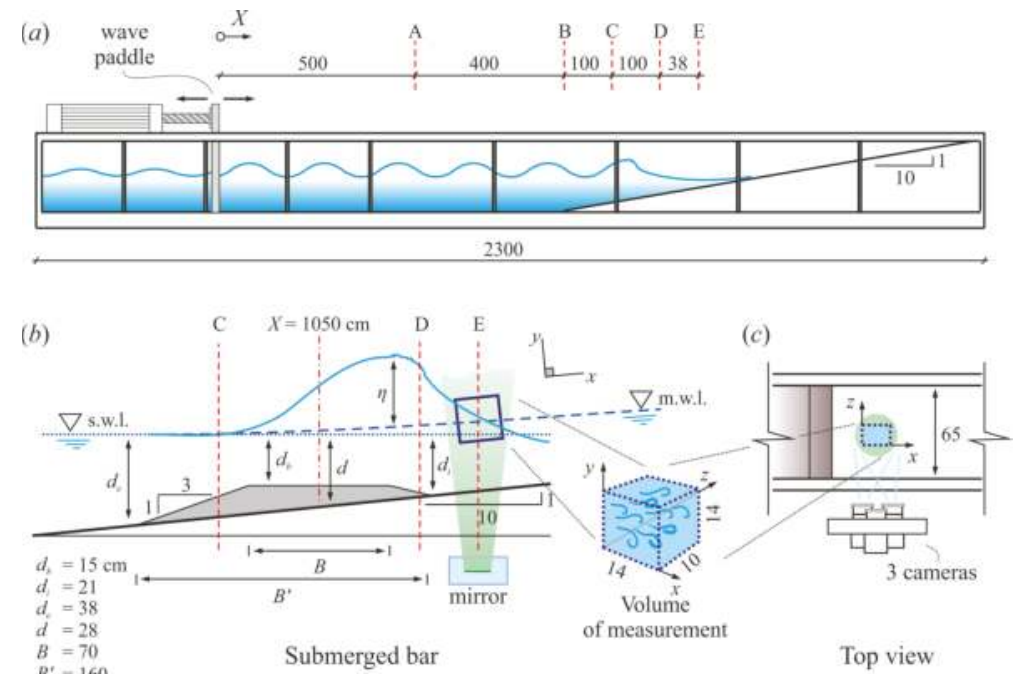

Figure 1. The experimental flume adopted for the tests: (a) side view of the flume; (b) layout of the bar and of the volume of measurement; (c) top view showing the cameras of the V3V system. The still water depth in the mid section of the bar $(X=1050 \mathrm{~cm})$ is $d=28 \mathrm{~cm}$. The dot line indicates the still water level, the dashed line is the mean water level $\bar{\eta}$ (wave set-up or set-down). Dimensions are in centimeters.

The surface elevation during tests was measured in several sections using Ultrasonic probes (UltraLab ${ }^{\circledR}$ USL 80D by General Acoustics, Kiel, Germany, sensor model USS635), with an accuracy on the instantaneous water level measurements equal to $\pm 0.5 \mathrm{~mm}$.

The main parameters of the experiments are listed in Table 1.

Table 1. Parameters of the tests. $H_{0}$ is the target wave height (almost coincident with the generated wave height), $T$ is the wave period and $H_{0} / L_{0}$ is the deep-water wave steepness. $\xi_{0}=\tan \alpha / \sqrt{H_{0} / L_{0}}$ is the Iribarren number ( $\alpha$ is the bed slope), $h=d+\bar{\eta}$ is the mean water depth in the section of measurements, $\bar{\eta}$ is the wave set-up, and $H_{b-r m s}, H_{b-1 / 3}$, and $H_{b-\text { max }}$ are the root-mean-square wave height, the mean of the highest third of the waves, and the maximum wave height, respectively, all referred to as the statistics of the breakers. $d_{i}$ and $d_{e}$ are the still-water depth at the internal and external toe of the bar, respectively, $B$ and $B^{\prime}$ are the width of the crest and the total width of the bar. The still-water depth in front of the paddle is $43 \mathrm{~cm}$ and the breaking section is $\mathrm{D}$ at $X \approx 1138 \mathrm{~cm}$, with a still-water depth $d=19.2 \mathrm{~cm}$.

\begin{tabular}{cccccccccccccc}
\hline Exp. & $\begin{array}{c}H_{0} \\
(\mathbf{c m})\end{array}$ & $\begin{array}{c}T \\
(\mathbf{s})\end{array}$ & $H_{0} / L_{0}$ & $\xi_{0}$ & $\begin{array}{c}h \\
(\mathbf{c m})\end{array}$ & $\begin{array}{c}\bar{\eta} \\
(\mathbf{c m})\end{array}$ & $\begin{array}{c}H_{b-r m s} \\
(\mathbf{c m})\end{array}$ & $\begin{array}{c}H_{b-1 / 3} \\
(\mathbf{c m})\end{array}$ & $\begin{array}{c}H_{b-m a x} \\
(\mathbf{c m})\end{array}$ & $d_{i} / L_{0}$ & $d_{e} / L_{0}$ & $B / L_{0}$ & $B^{\prime} / L_{0}$ \\
\hline 1b & 6 & 1.5 & 0.017 & 0.765 & 19.2 & 0.0 & 5.7 & 6.2 & 6.3 & 0.060 & 0.109 & 0.199 & 0.456 \\
2b & 7 & 1.5 & 0.020 & 0.708 & 19.4 & 0.2 & 7.4 & 8.1 & 8.7 & & & \\
3b & 8 & 1.5 & 0.023 & 0.662 & 19.6 & 0.4 & 8.0 & 8.6 & 8.8 & & \\
4b & 9 & 1.5 & 0.026 & 0.624 & 19.7 & 0.5 & 8.7 & 9.2 & 9.9 & & \\
5b & 10 & 1.5 & 0.028 & 0.592 & 19.8 & 0.6 & 9.2 & 9.9 & 10.4 & & \\
6b & 6 & 2 & 0.010 & 1.020 & 19.4 & 0.2 & 6.1 & 6.3 & 6.5 & 0.034 & 0.061 & 0.112 \\
7b & 7 & 2 & 0.011 & 0.944 & 19.4 & 0.2 & 7.6 & 8.3 & 8.7 & & \\
8b & 8 & 2 & 0.013 & 0.883 & 19.5 & 0.3 & 9.0 & 9.6 & 10.4 & & \\
9b & 9 & 2 & 0.014 & 0.833 & 19.6 & 0.4 & 10.3 & 11.2 & 12.8 & & \\
\hline
\end{tabular}




\section{Data Analysis and Visualization}

The raw signal was elaborated by applying operators expressing different kind of averages. The phase-average for the variable $a$ is

$$
\widetilde{a}=\frac{\sum_{i=1}^{N} a(t+i T)}{N},
$$

where $T$ is the period of the signal and $N$ is the number of cycles. The time-average is defined as

$$
\bar{a}=\frac{\sum^{N} a}{N}
$$

and the phasic-average is defined as

$$
<a>=\frac{\sum^{N} a \phi}{\sum^{N} \phi},
$$

where $\phi=1$ and $\phi=0$ if water is present or absent, respectively. The relative volume of water is $\alpha=\sum^{N} \phi / N$ and $\alpha=1$ below the (minimum) trough level, and $\alpha=0$ above the (maximum) crest level. The phasic-average is always greater than and equal to the time average if $\alpha<1$ and $\alpha=1$, respectively.

The phasic-phase-average is also defined as

$$
<\widetilde{a}>=\frac{\sum_{i=1}^{N} a(t+i T) \phi}{\sum^{N} \phi} .
$$

For simplicity and for more consistency, in the present analysis we refer to "vertically integrated" data, meaning data phasic-averaged in the vertical, and "cycle integrated" equivalent to a time-average or a phasic-average according to the indications. Figure 2 shows the shape of the wave at the breaking section for all the experiments.

(a)

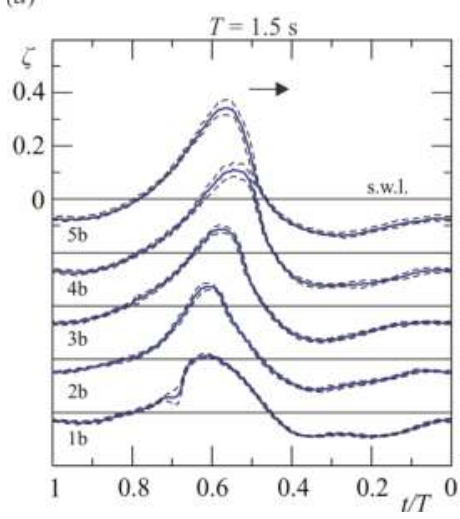

(b)

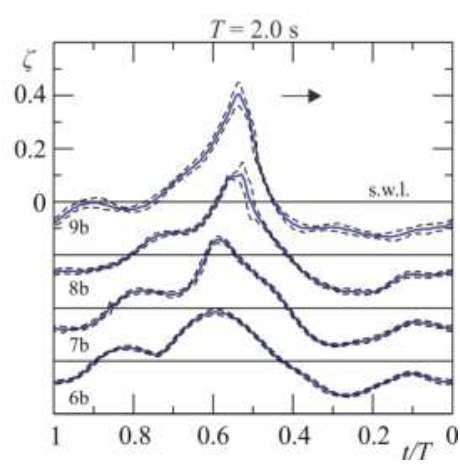

Figure 2. Wave profiles in the section of velocity measurements (Section D) for (a) the tests with $T=1.5 \mathrm{~s}$, and $(\mathbf{b})$ the tests with $T=2.0 \mathrm{~s}$. The dashed lines limit the \pm 1 standard deviation band for the sample of 10 wave cycles during velocity acquisition. 
The first problem is the separation of periodic (organized) components and fluctuating (turbulent) components. First (i) a principal orthogonal decomposition (POD) is applied to the time series of the spatial measured velocity in order to detect the most energetic contributions; (ii) then a cut-off number of components is chosen in order to eliminate disturbances and the reconstructed time series with a limited number of modes is used for the computations. The details are reported in Clavero et al., (2016) [16]. The results are presented as average in the cross-shore and alongshore directions, obtaining a single profile in the vertical per shot as average of $\approx 800$ profiles. If the results are further phase averaged over 10 and 13 cycles, each profile in the vertical is the average of $\approx 8000$ and $\approx 10,500$ profiles, a very large sample which guarantees an adequate accuracy of the statistical estimators.

Figure 3 shows the instantaneous measured velocity, the phase-averaged and the fluctuating velocity for a single snapshot of Experiment $6 \mathrm{~b}$.
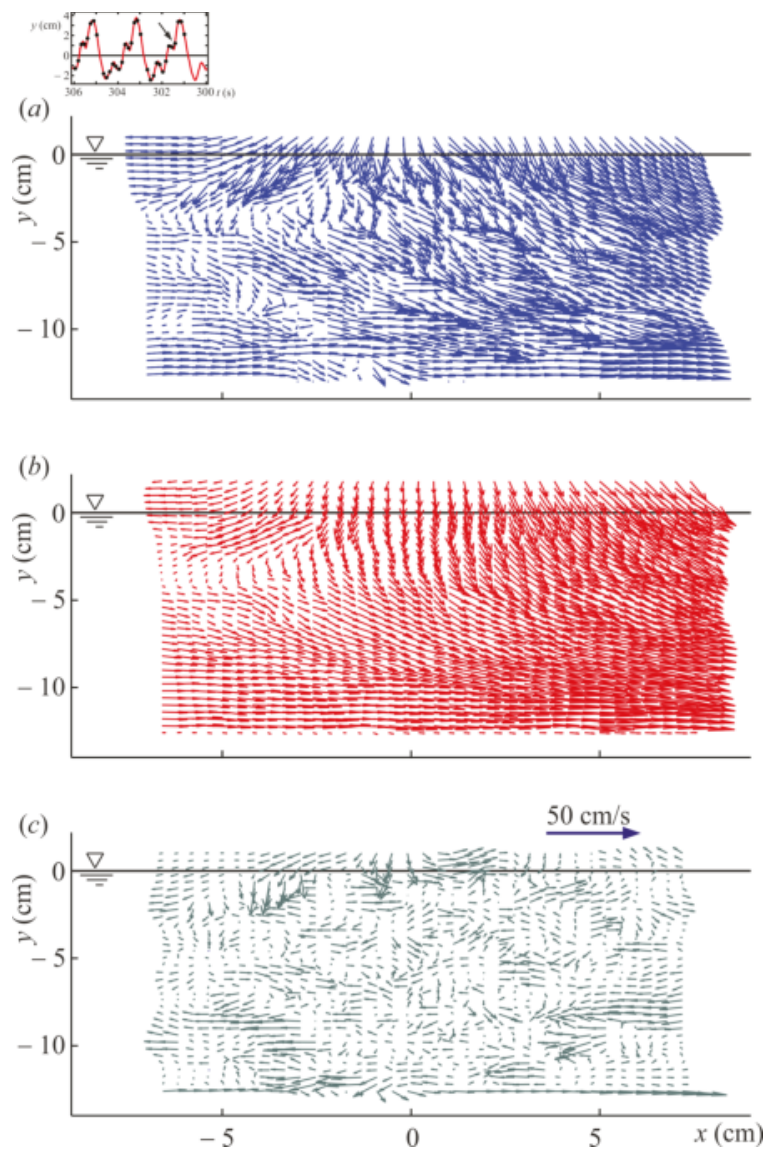

Figure 3. Experiment $6 \mathrm{~b}$, a single snapshot in a sequence of 13 shots of the first measured wave cycle. (a) Instantaneous velocity in the midplane of the flume $(z=0)$; (b) phase-averaged velocity; (c) velocity vectors difference (fluctuating velocity) between the instantaneous velocity and the phase-averaged velocity. Only velocity components in the $x-y$ plane are shown. The inset depicts the surface elevation time series, with the symbols indicating the time of the shot. (modified from Clavero et al., 2016 [16], with permission). 


\section{Turbulent Stress Invariants Analysis and Relation with the Dissipation Tensor}

The anisotropy Reynolds stress tensor is defined as

$$
a_{i j}=\frac{u_{i}^{\prime} u_{j}^{\prime}}{2 k}-\frac{1}{3} \delta_{i j}
$$

where $k=(1 / 2) u_{i}^{\prime}, u_{i}^{\prime}$ is the turbulent kinetic energy, $i, j=1,2,3$ are the spatial components and $\delta_{i j}=0$ if $i \neq j, \delta_{i j}=1$ if $i=j$ is the Kronecker delta. Perfect isotropy gives a tensor with null diagonal elements.

Figure $4 \mathrm{a}, \mathrm{b}$ show the most representative elements $a_{i j}$ for the four phases across breaking, for Experiment $6 \mathrm{~b}$ and Experiment $9 \mathrm{~b}$, respectively (the less and the most energetic breaking conditions).

In Experiment 6b, turbulence is largely oriented in plane 1-2 (cross-shore-vertical), with the 3rd component (longshore) carrying a modest fraction of the total kinetic energy. The changes of the structure due to breaking are modest and mainly limited to an increment of turbulent kinetic energy (TKE) in the 3-direction. In Experiment $9 \mathrm{~b}$ there is a clear evolution of the tensor before and after breaking. In phase 2 a significant axisymmetric structure develop with axis along the 3-direction.
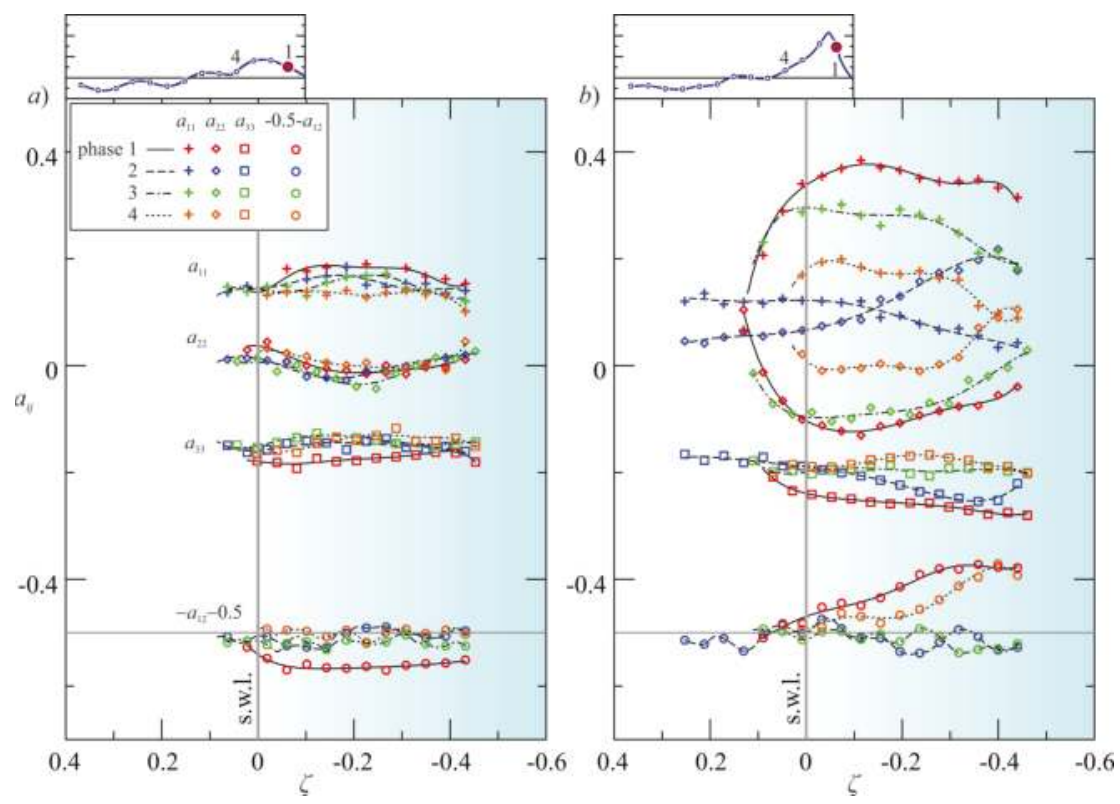

Figure 4. The main elements of the anisotropy tensor $a_{i j}$ for four phases across breaking. (a) Experiment $6 \mathrm{~b}$; (b) Experiment $9 \mathrm{~b}$. The element $-a_{12}$ is shifted downward, and only one experimental data of two is shown for an easy visualization.

A useful tool to analyse the structure of turbulence is given by the Lumley triangle, which contours all the Reynolds anisotropy stress tensor invariants (Lumley, 1978 [18]). For an incompressible flow, the three invariants of the anisotropy tensor are

$$
\begin{aligned}
& I_{1}=u_{i}^{\prime} u_{i}^{\prime}, \\
& I_{2}=\frac{1}{2}\left(u_{i}^{\prime} u_{j}^{\prime}\right)\left(u_{j}^{\prime} u_{i}^{\prime}\right), \\
& I_{3}=\operatorname{det}\left(u_{i}^{\prime} u_{j}^{\prime}\right),
\end{aligned}
$$


and they are bounded by limits related to the state of turbulence. For instance, $I_{2}=I_{3}=0$ is the definition of isotropic turbulence; while $I_{3}=3 / 27, I_{2}=-1 / 3$ represents one-component turbulence, and $I_{2}=-3\left(1 / 27+I_{3}\right)$ represents two-component turbulence. In most cases the modified expressions for the invariants, $I_{2 a}=-2 I_{2}$ and $I_{3 a}=3 I_{3}$, are adopted.

A different synthetic representation of the level of anisotropy of turbulence is obtained through the eigenvalues of the anisotropy Reynolds tensor. Let $\lambda_{1}, \lambda_{2}$, and $\lambda_{3}$ be the three eigenvalues in descending order, the tensor can be expressed as a linear combination of the three tensors of a basis (see Banerjee et al., 2007 [19]) with coefficients given by

$$
\begin{aligned}
& C_{1 c}=\lambda_{1}-\lambda_{2}, \\
& C_{2 c}=2\left(\lambda_{2}-\lambda_{3}\right), \\
& C_{3 c}=3 \lambda_{3}+1,
\end{aligned}
$$

and satisfying the normalizing condition $C_{1 c}+C_{2 c}+C_{3 c}=1$. These coefficients are used to plot a barycentric map according to the following transformation (see Banerjee et al., 2007 [19])

$$
\begin{aligned}
& x=C_{1 c} x_{1 c}+C_{2 c} x_{2 c}+C_{3 c} x_{3 c}, \\
& y=C_{1 c} y_{1 c}+C_{2 c} y_{2 c}+C_{3 c} y_{3 c},
\end{aligned}
$$

where $\left(x_{1 c}, y_{1 c}\right),\left(x_{2 c}, y_{2 c}\right),\left(x_{3 c}, y_{3 c}\right)$ are the coordinates of three arbitrary basis points. Usually the barycentric map is an equilateral triangle.

Figure 5 shows the structure of turbulence in Experiment $6 \mathrm{~b}$ represented in a Lumley map and in a barycentric map, with data averaged in horizontal layers $0.4-\mathrm{cm}$ thick, for the first four phases of the first wave cycle. Breaking appears between phase 2 and phase 3 . In all of the analysed phases, turbulence has three different components and is anisotropic. At the wave crest and near the free surface, the turbulence is almost one-component turbulence, with only the cross-shore component.

Figure 6 shows the structure of turbulence in Experiment 9b, with breaking between phase 1 and phase 2. Again, turbulence is three-component and in general the distance from isotropy is smaller for the deepest levels, toward the bottom. An exception is phase 3, after breaking, in which the levels near the free surface have a degree of isotropy larger than that of the levels toward the bottom. The scenario is coherent with the turbulence in the breaker being mainly one dimensional (in the $x$ direction) and with a fast return to isotropy. This fast return can be attributed partially to the fluctuating pressure acting to redistribute the energy amongst the three components, and partially to the transport toward the bottom of the most energetic components, which in turn are responsible of the increase in anisotropy in the deep layers. This mechanism of exchange of anisotropy between different vertical layers can be observed only for the highest waves of the set of experiments (Experiment $5 b$ -not shown- and Experiment 9b) and is much less evident for the less energetic breakers.

An anisotropy measurement suggested by Lumley and Newman (1977) [20] is $F=1+9 I_{2}+27 I_{3}$, which varies between $F=0$ for a two-component turbulence, and $F=1$ for the three-component isotropic turbulence. Figure 7 shows the invariant function $F$ for two experiments, five phases across breaking, first cycle. 
(a)

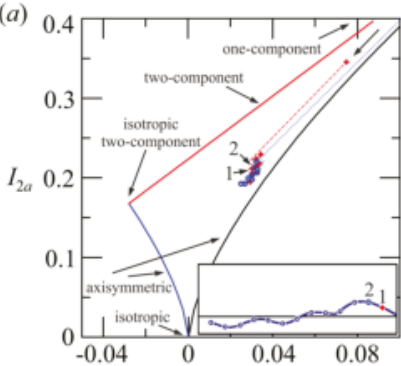

(c)

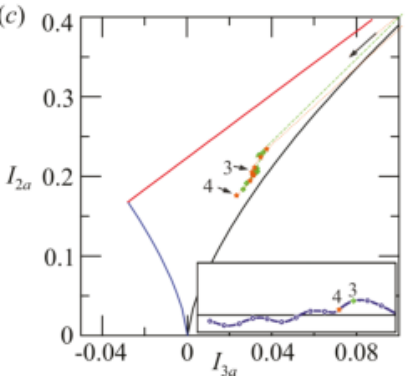

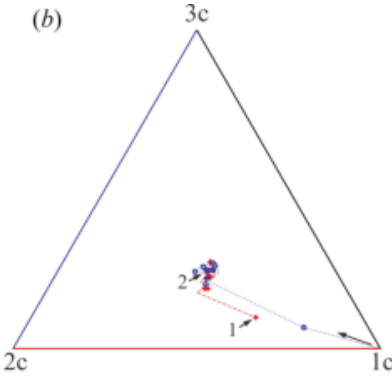

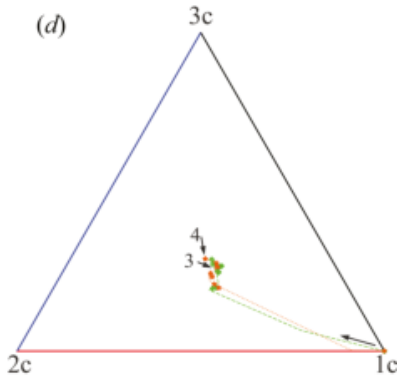

Figure 5. Experiment $6 \mathrm{~b}$, first four phases (crosses, circles, squares and stars), first wave cycle. (a-c) Lumley map; (b-d) barycentric map based on scalar metrics which are functions of eigenvalues of the second order stress tensor describing turbulence. The values are averaged in horizontal layers. The insets show the phase, the arrows indicate the deepest point, with data $0.4 \mathrm{~cm}$ apart. Only one point of three is plotted for a clear visualization.

(a)

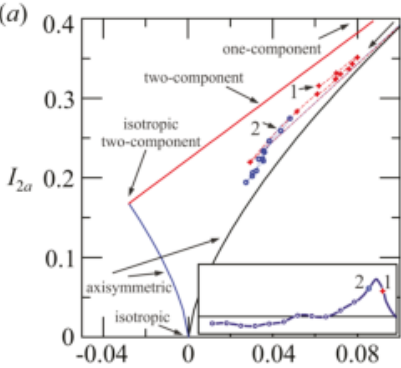

(c)

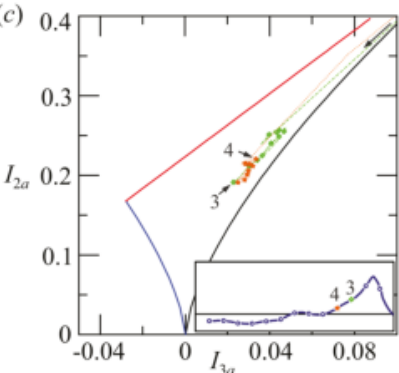

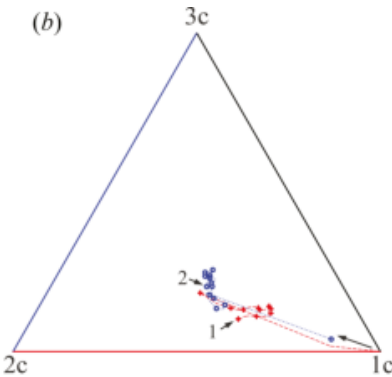

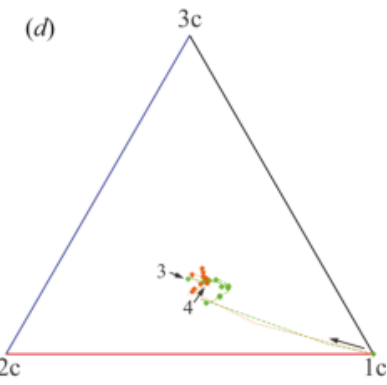

Figure 6. Experiment 9b, see Figure 5 for caption. 

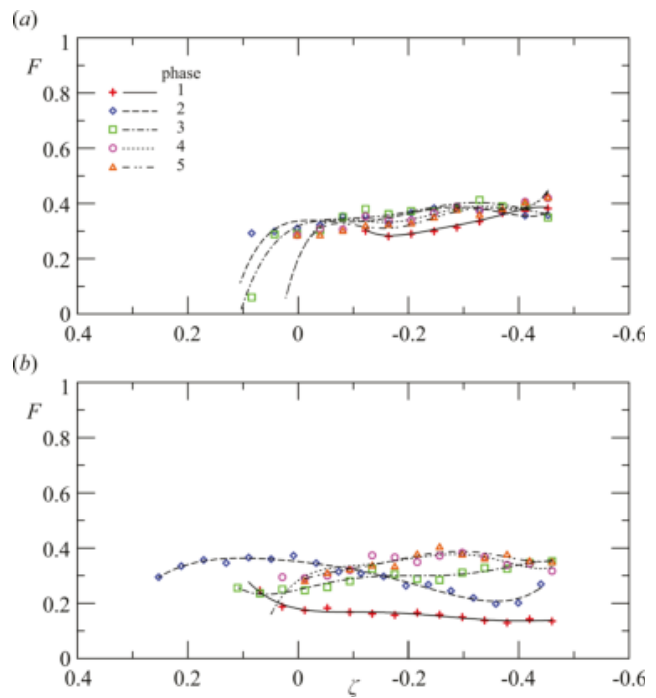

Figure 7. Invariant function $F=1+9 I_{2}+27 I_{3}$ for the first five phases. (a) Experiment $6 \mathrm{~b}$; (b) Experiment 9b.

The flow organization reasonably influences the small scale turbulence structures, inducing isotropy or anisotropy in the dissipation rate (Antonia et al., 1994 [21], Mansour et al., 1988 [22]). The structure of dissipation rate is depicted by the following tensor

$$
\epsilon_{i j}=2 v u_{i l}^{\prime} u_{j l}^{\prime}
$$

with its anisotropic form

$$
e_{i j}=\frac{\epsilon_{i j}}{2 \epsilon}-\frac{1}{3} \delta_{i j}
$$

where $v$ is the kinematic viscosity and $\epsilon=(1 / 2) \epsilon_{i i}$ is the average dissipation rate of TKE.

This tensor has been considered as a significant element in the attempts to model Reynolds stresses, since $a_{i j}$ and $e_{i j}$ are likely to be related at least in some categories of flow. Figure 8 show the main components of the anisotropic dissipation tensor for two experiments. Again for the most intense breaking wave the dissipation tensor is largely anisotropic, with the main term in the cross shore direction immediately after breaking. The overall relation between the two tensors is depicted in Figure 9, where the invariant function $F$ for the anisotropy Reynolds tensor $a_{i j}$ is compared with the corresponding invariant $F_{\text {diss }}=1+9 I_{2}+27 I_{3}$ for the anisotropy dissipation tensor $e_{i j}$. For Experiment $1 \mathrm{~b}-6 \mathrm{~b}$ (low energy breaking) there is a proportionality between $F$ and $F_{\text {diss }}$, with dissipation more isotropic $\left(F_{\text {diss }} \rightarrow 1\right)$ than turbulence, and with $F_{\text {diss }} \approx 1.85 F$. For Experiments $5 \mathrm{~b}-9 \mathrm{~b}$ (high energy breaking) the data are much more dispersed. The dissipation tensor is still more isotropic than turbulence, but there is no evident correlation with the level of isotropy of turbulence tensor. Surprisingly, the coherence is still present only for data measured above the still water, in the breaker crest. All these analyses refer to macroturbulence, since the data resolution in space and time of the measurements does not allow an in-depth analysis of the behaviour at the microscales.

Several data in literature show that a relationship holds between the components of the anisotropy Reynolds tensor and of the anisotropy dissipation tensor. The shape of the relation is strictly dependent on the flow field and has been described as linear in wall boundary layer (see Antonia et al., 1994 [21]). Also in the present experiments a linear function is forecast for the low energy breaker in Figure 10a-c, with a coefficient of proportionality almost equal for the three main components. We expect that the 
coefficient is function of the ratio between the turbulence microscale $\lambda$ and the integral macroscale $\Lambda$ (see, e.g., Tennekes and Lumley, 1972 [23], Longo, 2010 [15]), which in the present experiments is related to the intensity of the breaker. However, very energetic breakers show a poor coherence between the components of the two tensors, see Figure 10d-f.
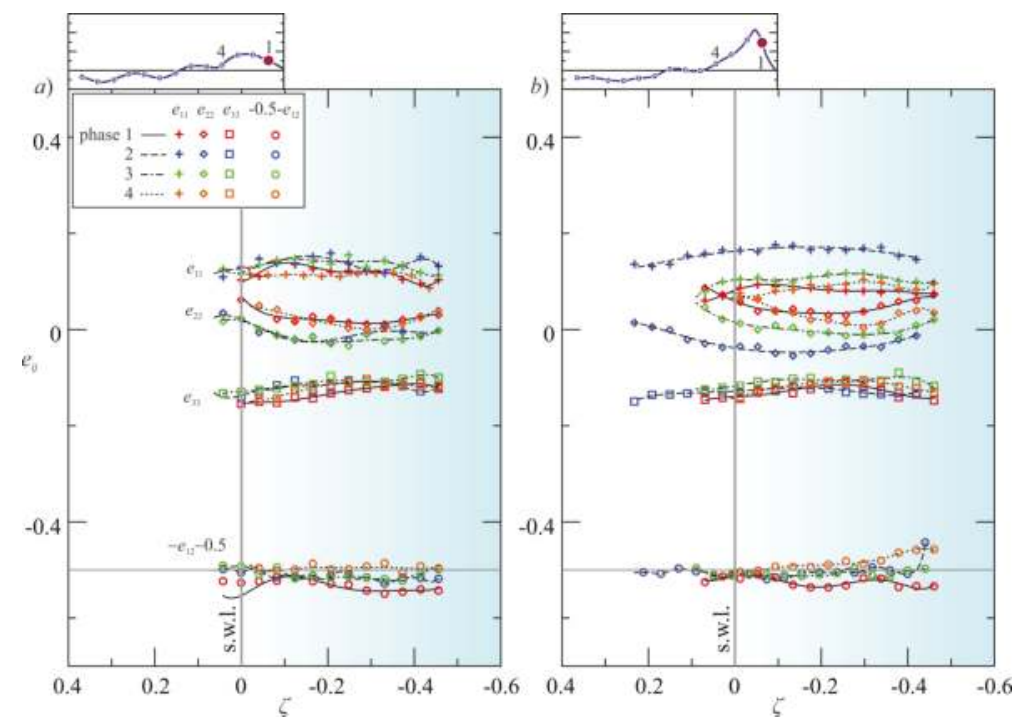

Figure 8. The main elements of the dissipation anisotropy tensor $e_{i j}$ for four phases across breaking. (a) Experiment 6b; (b) Experiment $9 \mathrm{~b}$. The element $-e_{12}$ is shifted downward, and only one experimental data of two is shown for an easy visualization.

(a)

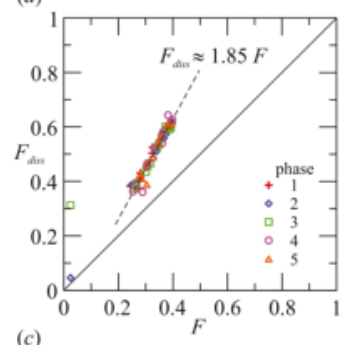

(c)

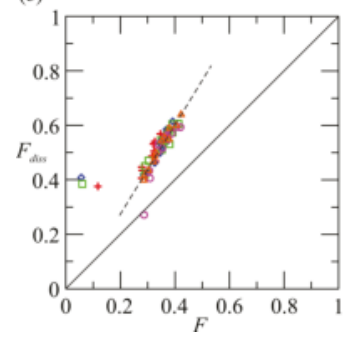

(b)

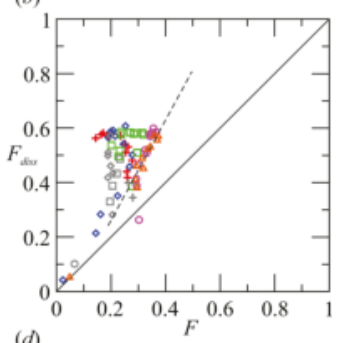

(d)

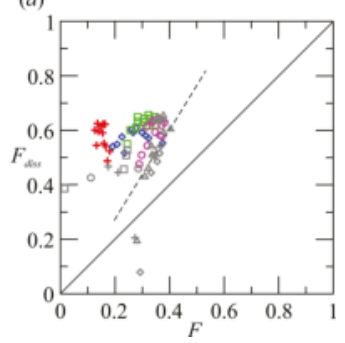

Figure 9. Comparison of the invariant function of the turbulence and of the dissipation tensors for the first five phases. (a) Experiment 1b; (b) Experiment 2b; (c) Experiment 6b; (d) Experiment $9 \mathrm{~b}$. The dashed curve is the interpolating line. Gray symbols refer to measurements above the still water level. 
(a)

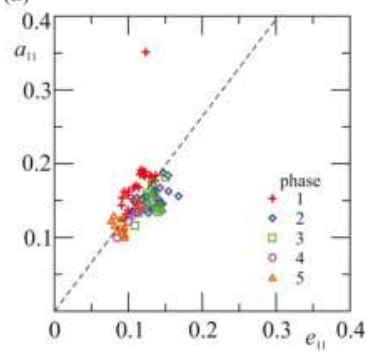

(d)

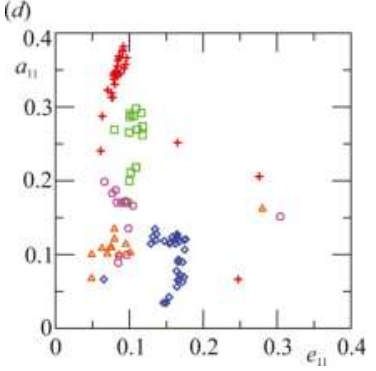

(b)

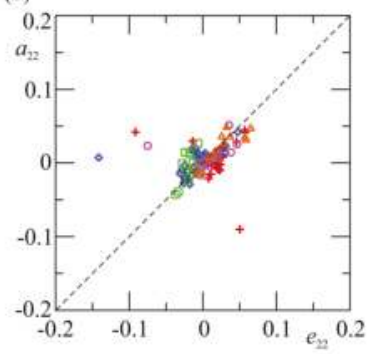

(e)

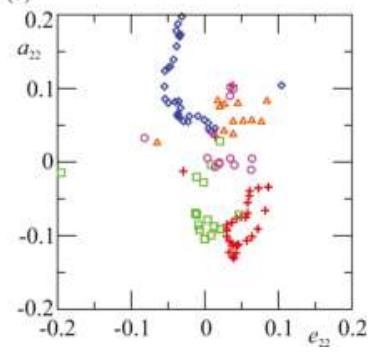

(c)

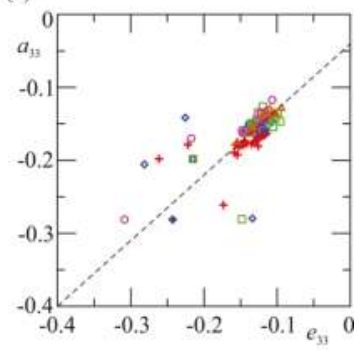

(f)

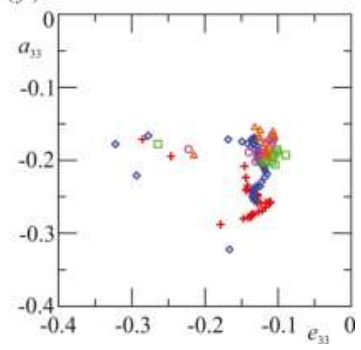

Figure 10. Relation between $a_{i i}$ and $e_{i i}$. (a-c) Experiment 6b; (d-f) Experiment 9b. The dashed curves are the interpolating lines.

\section{Conclusions}

We have analysed the anisotropy of the Reynolds stress and of the dissipation tensors with a unique dataset of measurements in breaking waves. The anisotropy is quantified by the invariants and is relatively high during all phases and more evident for the most energetic breakers. For low energy breakers, anisotropy is minor in the dissipation tensor than in the Reynolds stress tensor, with $F_{\text {diss }} \approx 1.85 F$ ( $F \rightarrow 1$ means perfect isotropy). For high energy breakers, the dissipation tensor still has an adequate level of isotropy, the Reynolds stress tensor is biased toward anisotropy.

The relationship between the main components of the two tensors is a simple proportionality for low energy breaker. That means that some classical models of turbulence are still applicable. For high energy breakers the relationship looks much more complex, and no simple model can be suggested.

The unique availability of these experimental data has allowed an in depth analysis following the same path adopted for DNS data. However, we expect that the dissipation tensor is affected by the limited space resolution of the data, and both tensors are affected by the limited time resolution, hence the presented results should be evaluated in the light of these limitations.

Beyond the application of well known models for turbulence estimation, there is the important novelty of huge experimental data set for calibrating these models. This data set are a step ahead toward the detailed interpretation of the fundamental mechanisms of turbulence generation, evolution and dissipation.

As a development of data analysis there is the evaluation of the "dimensionality" elements of turbulence. This is left to future work.

Author Contributions: S.L. and L.C. conceived and designed the experiments and performed the experiments with M.C.; S.L., L.C., M.C. and M.A.L. analyzed the data and wrote the paper.

Conflicts of Interest: The authors declare no conflict of interest. 


\section{References}

1. Longuet-Higgins, M. Capillary rollers and bores. J. Fluid Mech. 1992, 240, 659-679.

2. Lin, J.; Rockwell, D. Instantaneous structure of a breaking wave. Phys. Fluids 1994, 6, 2877-2879.

3. Dabiri, D.; Gharib, M. Experimental investigation of the vorticity generation within a spilling water wave. J. Fluid Mech. 1997, 330, 113-139.

4. Grue, J.; Kolaas, J. Experimental particle paths and drift velocity in steep waves at finite water depth. J. Fluid Mech. 2017, 810, doi:10.1017/jfm.2016.726.

5. Smith, L.; Jensen, A.; Pedersen, G. Investigation of breaking and non-breaking solitary waves and measurements of swash zone dynamics on a $5^{\circ}$ beach. Coast. Eng. 2017, 120, 38-46.

6. Barthelemy, X.; Banner, M.L.; Peirson, W.L.; Fedele, F.; Allis, M.J.; Dias, F. On the local properties of highly nonlinear unsteady gravity water waves. Part 2. Dynamics and onset of breaking. arXiv 2015, arXiv:1508.06002v1.

7. Saket, A.; Peirson, W.L.; Banner, M.L.; Barthelemy, X.; Allis, M.J. On the threshold for wave breaking of two-dimensional deep water wave groups in the absence and presence of wind. J. Fluid Mech. 2017, 811, 642-658.

8. Deike, L.; Melville, W.K.; Popinet, S. Air entrainment and bubble statistics in breaking waves. J. Fluid Mech. 2016, 801, 91-129.

9. Deike, L.; Pizzo, N.; Melville, W.K. Lagrangian transport by breaking surface waves. J. Fluid Mech. 2017, 829, 364-391.

10. Drazen, D.A.; Melville, W.K.; Lenain, L. Inertial scaling of dissipation in unsteady breaking waves. J. Fluid Mech. 2008, 611, 307-332.

11. Drazen, D.A.; Melville, W.K. Turbulence and mixing in unsteady breaking surface waves. J. Fluid Mech. 2009, 628, 85-119.

12. Reynolds, W.C. Effects of rotation on homogeneous turbulence. In Proceedings of the 10th Australasian Fluid Mechanics Conference, Melbourne, Australia, 11-15 December 1989; Volume 1, pp. KS2.1-KS2.6.

13. Stylianou, F.; Pecnik, R.; Kassinos, S. A general framework for computing the turbulence structure tensors. Comput. Fluids 2015, 106, 54-66.

14. Robinson, S.K. Coherent motions in the turbulent boundary layer. Annu. Rev. Fluid Mech. 1991, 23, 601-639.

15. Longo, S. Experiments on turbulence beneath a free surface in a stationary field generated by a Crump weir: Free surface characteristics and the relevant scales. Exp. Fluids 2010, 49, 1325-1338.

16. Clavero, M.; Longo, S.; Chiapponi, L.; Losada, M. 3D flow measurements in regular breaking waves past a fixed submerged bar on an impermeable plane slope. J. Fluid Mech. 2016, 802, 490-527.

17. Chiapponi, L.; Cobos, M.; Losada, M.A.; Longo, S. Cross-shore variability and vorticity dynamics during wave breaking on a fixed bar. Coast. Eng. 2017, 127, 119-133.

18. Lumley, J. Computational modelling of turbulent flows. Adv. Appl. Mech. 1978, 18, 123-176.

19. Banerjee, S.; Krahl, R.; Durst, F.; Zenger, C. Presentation of anisotropy properties of turbulence, invariants versus eigenvalue approaches. J. Turbul. 2007, 8, N32.

20. Lumley, J.L.; Newman, G.R. The return to isotropy of homogeneous turbulence. J. Fluid Mech. 1977, 82, 161-178.

21. Antonia, R.A.; Djenidi, L.; Spalart, P.R. Anisotropy of the dissipation tensor in a turbulent boundary layer. Phys. Fluids 1994, 6, 2475-2479.

22. Mansour, N.N.; Kim, J.; Moin, P. Reynolds-stress and dissipation-rate budgets in a turbulent channel flow. J. Fluid Mech. 1988, 194, 15-44.

23. Tennekes, H.; Lumley, J. A First Course in Turbulence; Cambridge University Press: Cambridge, UK, 1972. 

MDPI

St. Alban-Anlage 66

4052 Basel

Switzerland

Tel. +41 616837734

Fax +41 613028918

www.mdpi.com

Water Editorial Office

E-mail: water@mdpi.com www.mdpi.com/journal/water

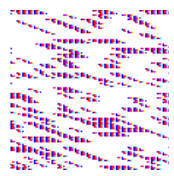



MDPI

St. Alban-Anlage 66

4052 Basel

Switzerland

Tel: +41 616837734

Fax: +41 613028918 